\title{
THE ISOLATION OF \\ BIOLOGICALLY ACTIVE SECONDARY METABOLITES FROM NEW ZEALAND MARINE ORGANISMS.
}

\author{
by \\ Robert Alexander Keyzers
}

VICTORIA UNIVERSITY OF WELLINGTON Te Whare Wānanga o te Ūpoko o te Ika a Māui

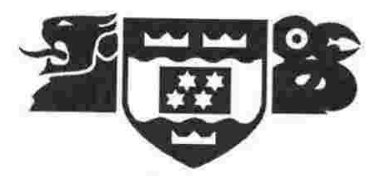

\author{
A thesis \\ submitted to Victoria University of Wellington \\ in fulfilment of the \\ requirements for the degree of \\ Doctor of Philosophy \\ in Chemistry.
}

Victoria University of Wellington 2003 


\section{Abstract}

An improved protocol for the screening of marine sponges using cyclic loading, PSDVB, and both 1D and 2D NMR spectroscopy is described. Using this new methodology, 51 sponges were screened. Further investigations were carried out on seven of the 51 organisms, resulting in the isolation of several known and eight novel compounds. Clathriols A (32) and B (33) are novel sterols isolated from the sponge Clathria lissosclera. Both 32 and 33 possess the rare $14 \beta$ stereochemistry, a feature only naturally occurring in marine sponges. Both are also moderate anti-inflammatory compounds. Ten spongian diterpenes were isolated from the New Zealand sponge Chelonaplysilla violacea, six of which are novel. Cadlinolides C (138) and D (139) are similar to several previously reported compounds while pourewic acid A (140), 15-methoxypourewic acid B (141), methylpourewate B (142) and pourewanone (143) have unique structural features and are of biogenetic significance. Pourewanone (143) is the first example of a formate isolated from the marine environment. Several of the novel diterpenes exhibit moderate anti-inflammatory activity.

A potent dinoflagellate toxin was partially purified from cultures of the producing organism, Karenia brevisulcata. K. brevisulcata is a new dinoflagellate species implicated in a large toxic algal bloom in Wellington Harbour, New Zealand, which formed during the summer of 1997/1998. Although the toxin could not be identified, some of the functionality present, and several possible substructures, is proposed. The biological activity of the toxin is also described. 


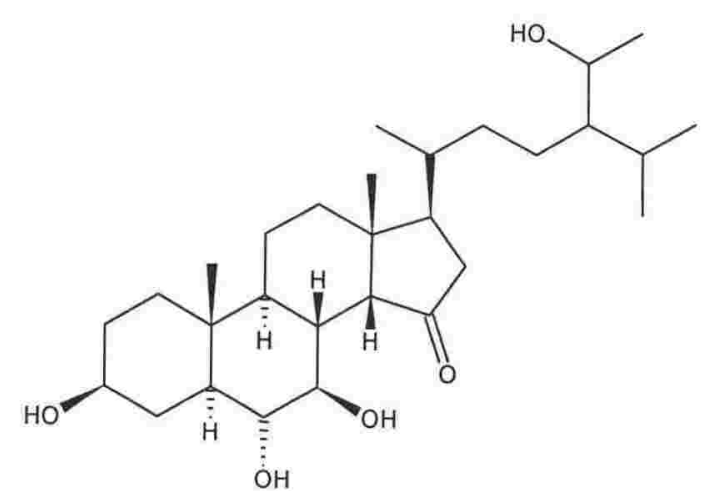

(32)

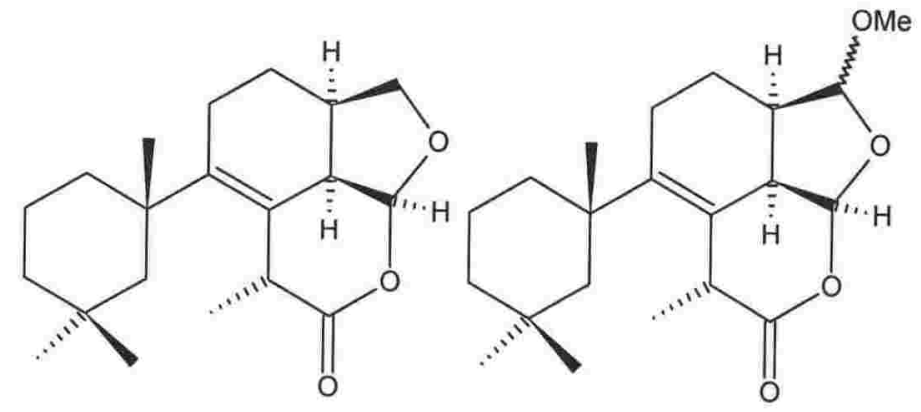

(138)

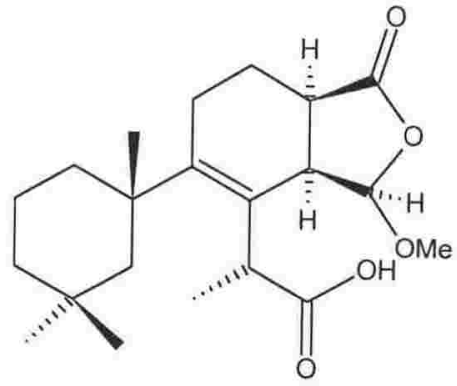

(141)

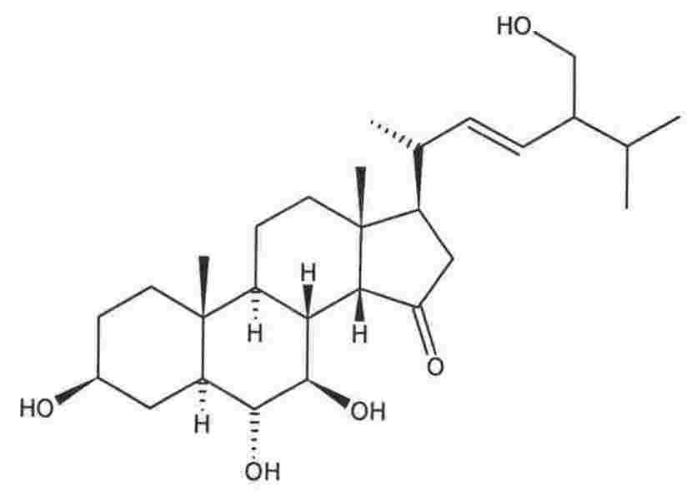

(33)

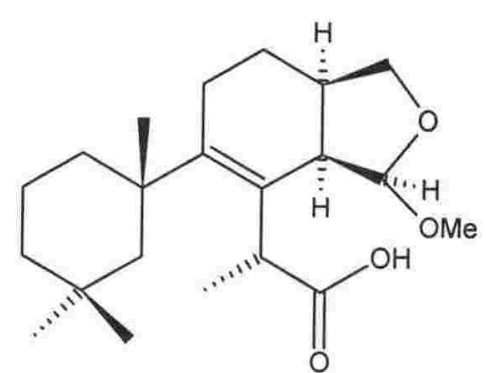

(140)<smiles>C[C@H](C(=O)O)C1=C([C@]2(C)CCCC(C)(C)C2)CC[C@H](COC=O)C1=O</smiles>

(143) 
Dedicated to my Mum,

For keeping me sane throughout my $\mathrm{PhD}$, while you had your own, much more important things to deal with. You are amazing. 


\section{"He who overseas everything also created very many poisonous fish, and in this way he punishes those who seek them."}

A comment about the punishment meted out upon those who seek to find bioactive marine natural products.*

The most important part my studies at Victoria are without a doubt the people that have been a part of my life during my time as a student.

First I would like to thank Dr Peter Northcote for his time and patience at guiding me through my tumultuous time as a post-graduate scientist. Peter, you have been a fantastic supervisor. More importantly, I have no qualms in saying that you are also a great friend. Thank you very much.

Next would have to be Dr Penny Truman (ESR) for all her support, enthusiasm and knowledge, and for performing all the N2A assays for KBT. Thanks also to David Stirling (ESR) for all the help, support, and advice with KBT.

I would also like to thank our major collaborators on several projects. In no particular order, Mr Mike Page (NIWA) for all the diving and sponge work, Drs Vicky Webb and Hoe Chang, and Ms. Sarah Allen, (NIWA), for all the help with KBT. Thanks also to Dr Patrick Holland and Ms. Veronica Beuzenberg (Cawthron Institute) also for help, advice, and materials, for KBT. Thanks to Dr John Miller and his students (School of Biological Sciences) as well as Dr Mike Berridge and An Tan (Malaghan Institute of Medical Research) for bioassays of the various sponge metabolites described. Thank you to both Dr Brent Copp (University of Auckland) and Professor John Blunt (University of Canterbury) for running various NMR spectra for me throughout the course of my PhD studies.

A vote of thanks to Dr. Belinda Glasby for the identification of Clathria lissosclera and to Professor Pat Bergquist for the identification of Chelonaplysilla violacea.

\footnotetext{
'Scheuer, P. J. Accounts of Chemical Research 1977, 10, 33-39

Halstead, B. W. Poisonous and Venomous Marine Animals of the World; United States Government Printing Office: Washington D.C., 1965; Vol. 2, p 162.
} 
Next I have the great pleasure to thank all my fellow post-graduate students for their friendship and help. A very special thanks to my partner-in-crime is so many things, Laine Cousins. Thanks to John Ryan for help with editing, NMR trouble-shooting, and debates about commas. Thanks to all my other colleagues, in no particular order, Drs Antony Fake, Mike Richardson, Thomas Borrmann, and Lyndon West, Peter Bickers, Steve Mackey, Greta Moraes, Ben Clark, Andy McFarlane, Craig Milestone, Kirsten Edgar, and Wendy Popplewell.

Thanks to all the academic and support staff at VUW including Professors Brian Halton, John Spencer, and Jim Johnston, Dr John Hoberg, Rhys Batchelor, Oleg Zubkov, Rhyl Singleton, Jenny Hall, Dave Stead, Dave Gilmour, Alan Rennie, Grant Franklin, Ying Shang, Bill Leck, Sally Wisheart, Teresa Gen, and Jackie King.

I would like to especially thank my family and friends. Dad, for all of the expert editorial skills which he supplied without complaint as well as for all the discussions on a wide range of topics which we have had, especially during my time at home while writing this tome. Mum for just being there and supplying the important emotional (and gastric!) support. Pete Junior and Amanda for their thoughts from the other side of the world. Also, thanks to my two second families, the Freemans and the McCabes. I must also thank those friends that aren't part of SCPS. Thanks to Marc, Dave, Becky, Steve, Sue, Allison, Olivia, Bruce, Duncan, Sarah, and Amanda. All of you have all helped keep me human.

Most important of all, I would like to take this opportunity to thank my partner Lucy for all the love, support, and good times that we've had together throughout the last few years. Thank you for everything, especially when times were hard and you helped me keep in touch with the real world.

Finally, I would like to thank the School of Chemical and Physical Sciences, the Curtis Gordon Research Scholarships Committee, the Victoria University Science Faculty Leave and Grants Committee, ESR, NIWA, and the New Zealand Marine Sciences Society, for financial support, and also the Victoria University Library for help with reference materials.

Thank you one and all. 
Abstract:
Dedication:
Acknowledgments:
Table of Contents:
List of Figures:
List of Schemes:
Gist of Tables:

\section{Chapter 1: Introduction}

1. Natural Products Chemistry and History................................................. 1

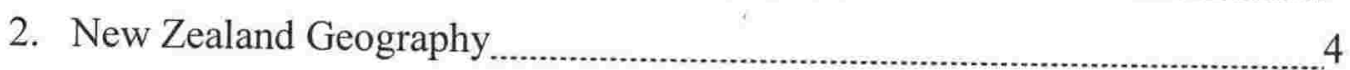

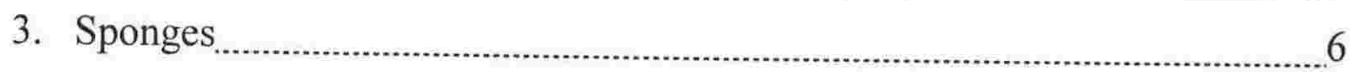

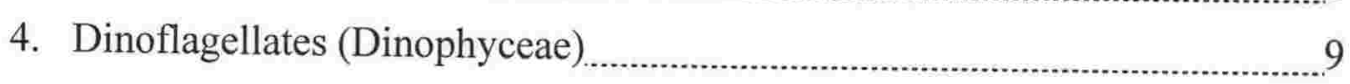

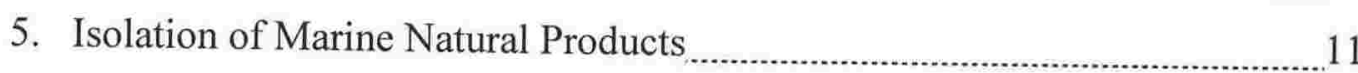

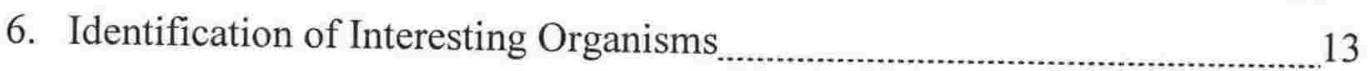

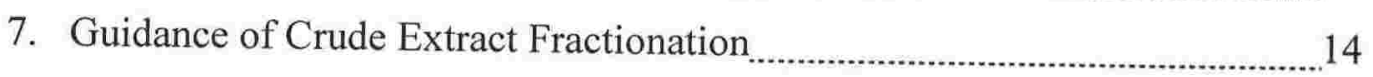

8. Methods Used in the Isolation of Secondary Metabolites _........................... 15

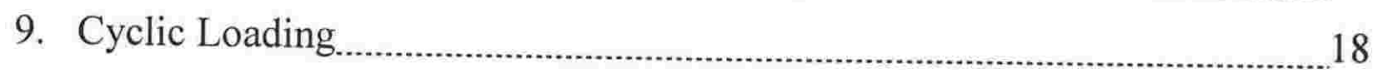

\section{Chapter 2: Sponge Screening and Results}

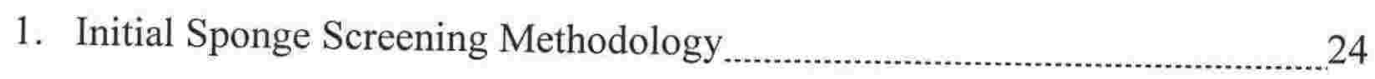

2. Revised Sponge-Screening Protocol

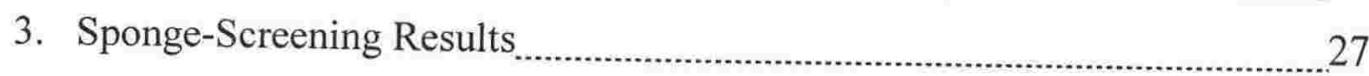

4. Conclusions of the Revised Sponge-Screening Protocol _............................... 42

\section{Chapter 3: Clathriols A and B: Novel polyoxygenated $14 \beta$ sterols isolated} from the sponge Clathria lissosclera

1. Steroid Biosynthesis 44

2. Sponge Sterols.

3. $14 \beta$ Sterols

4. Clathria lissosclera.

5. Clathriol A. 
6. Clathriol B 67

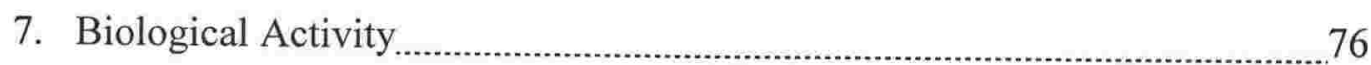

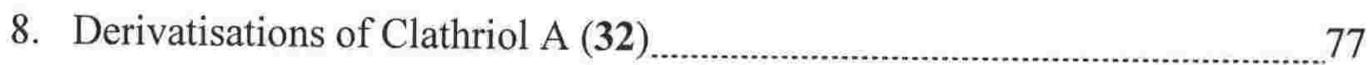

9. Other Secondary Metabolites Isolated from the Genus Clathria …................ 78

\section{Chapter 4: Spongian Diterpene Constituents of the Marine Sponge}

\section{Chelonaplysilla violacea}

1. Dictyoceratid and Dendroceratid Sponges

2. Diterpenes from Dictyoceratid and Dendroceratid Sponges 87

3. Miscellaneous Diterpenes 87

4. Spongian Diterpenes 89

5. Rearranged Spongian Diterpenes. 92

6. Taxonomic Considerations 98

7. Biogenesis of Spongian Diterpenes and Spongian Derived Skeletons 99

8. Novel Spongian Diterpenes from Chelonaplysilla violacea 103

9. Cadlinolide $\mathrm{C}$ 107

10. Cadlinolide D 113

11. Pourewic Acid A 119

12. 15-Methoxypourewic Acid B. 124

13. Methylpourewate B 130

14. Pourewanone 135

15. Biogenesis of the Isolated Spongian Diterpenes 140

16. Biological Activity. 147

17. Other Metabolites from the Genus Chelonaplysilla. 148

\section{Chapter 5: Isolation of a Marine Toxin Produced by the}

\section{Dinoflagellate Karenia brevisulcata}

1. Dinoflagellate Toxin Classes

2. Paralytic Shellfish Poisoning (PSP).

3. Neurotoxic Shellfish Poisoning (NSP)

4. Diarrhetic Shellfish Poisoning (DSP).

5. Amnesic Shellfish Poisoning (ASP)

6. Other Dinoflagellate Toxins

7. Structural Considerations

8. Dinoflagellate Toxins and New Zealand

9. Karenia Brevisulcata 
10. Bioassays Used to Guide Isolation 163

11. Culture Harvesting. 165

12. Stability Tests. 166

13. Isolation 167

14. Spectral Analysis of KBT. 172

15. Biological Activity of KBT 182

16. Further Work on KBT 184

Chapter 6: Concluding Remarks 186

\section{Chapter 7: Experimental}

1. General Methods 189

2. Revised Sponge-Screening Protocol

3. Sponges Selected for Further Investigation

4. Isolation of $5 \alpha, 8 \alpha$-epidioxy-6-ene-24-R-ethylcholesta-3 $\beta$-ol (7) and a Sphingolipid

5. Isolation of a Tribromobisindole Compound

6. Isolation of (10Z)- and (10E)-hymenialdisine (12) and (13) and a Related Compound

7. Isolation of Clathriol A (32) and Clathriol B (33) 196

8. Per-acetylation of Clathriol A (32) 197

9. Base Induced Epimerisation of Clathriol A (32) 198

10. $\mathrm{NaBH}_{4}$ Reduction of Clathriol A (32) 198

11. Isolation of Diterpene Metabolites from Chelonaplysilla violacea 199

12. Methylation of Pourewic Acid A (140). 203

13. Basic Harvesting of Karenia brevisulcata 204

14. Improved Harvesting of Karenia brevisulcata 205

15. Optimised Isolation of KBT 205

16. Stability of KBT to Acids and Bases. 207

17. Effect of Acid or Base on Elution from PSDVB 207

18. Use of the Amino Ion Exchange Column 209

19. Use of the CBA Ion Exchange Column 209

Appendix 1: NMR spectra of clathriol A (32) 210

Appendix 2: NMR spectra of clathriol B (33). 218

Appendix 3: NMR spectra of cadlinolide C (138) 222 
Appendix 4: NMR spectra of cadlinolide D (139) 228

Appendix 5: NMR spectra of pourewic acid A (140) 230

Appendix 6: NMR spectra of 15-methoxy pourewic acid B (141). 236

Appendix 7: NMR spectra of methylpourewate B (142) 238

Appendix 8: NMR spectra of pourewanone (143). 241

Appendix 9: NMR spectra of Wellington Harbour Toxin 248

References: 252 


\section{List of Figures}

\section{Chapter 1}

1. Map of New Zealand 5

2. SEM image of Protoceratium reticulatum showing transverse and posterior groves 10

3. A marine algal bloom in Hawkes Bay, New Zealand. 11

4. Diagrammatic representation of the cyclic loading process 21

\section{Chapter 2}

1. COSY and HSQC NMR screen of MNP0196 30

2. COSY and HSQC NMR screen of MNP0241 31

3. COSY and HSQC NMR screen of MNP0352 33

4. Substructures of a brominated bisindole compound isolated from Zyzzya sp. 34

5. COSY and HSQC NMR screen of MNP0355 35

6. COSY and HSQC NMR screen of MNP0707 37

7. COSY and HSQC NMR screen of MNP0090 40

8. COSY and HSQC NMR screen of MNP0979 41

\section{Chapter 3}

1. Stereochemistry of cholesterol 46

2. Hydrographic chart of Three Kinds Islands 52

3. Physical structure of Clathria lissosclera 54

4. Up-field portion of the ${ }^{1} \mathrm{H}$ NMR spectrum of clathriol A (32) indicating severe spectral overlap at $300 \mathrm{MHz}$

5. Selected COSY and HMBC correlations used to establish substructure I of clathriol A (32)

6. COSY correlations used to establish substructure II of clathriol A (32)

7. Selected correlations establishing the -3-(4-methylpentan-2-ol) side chain (substructure II) of clathriol A (32)

8. Selected $\mathrm{HMBC}$ correlations connecting $\mathrm{CH}_{3}-18$ and $\mathrm{CH}_{3}-19$ to substructure I of clathriol A (32)

9. Selected $\mathrm{HMBC}$ correlations connecting $\mathrm{CH}_{3}-21$ to substructure I of clathriol A (32)

10. Selected correlations establishing the connectivity of rings A, B and C of clathriol A (32) 
11. Selected correlations connecting rings $\mathrm{A}, \mathrm{B}$ and $\mathrm{C}$ with substructure II of clathriol A (32)

12. Establishment of the final connectivity of clathriol A (32)

13. Observed NOEs establishing stereochemistry of ring A of clathriol A (32).63

14. Observed NOEs establishing stereochemistry of ring B of clathriol A (32)..64

15. Observed NOEs establishing stereochemistry of C-14 and rings $\mathrm{C}$ and D of clathriol A (32).

16. W-coupling observed in the COSY spectrum between $\mathrm{H}-14$ and $\mathrm{H}-12 \beta$ of clathriol A (32)

17. Selected COSY and 1D-TOCSY correlations establishing spin system I of clathriol B (33)

18. Selected COSY correlations establishing spin system II of clathriol B (33).69

19. Selected HMBC correlations connecting spin systems I and II of clathriol B (33)

20. Selected COSY and HMBC correlations establishing the final connectivity of clathriol B (33)

21. Selected NOE correlations establishing the relative stereochemistry of the tetracyclic portion of clathriol B (33) 73

22. Selected NOE correlations establishing the relative stereochemistry of C-20 of clathriol B (33)

\section{Chapter 4}

1. Hydrographic chart of Stephens Island 104

2. Skeleton and appearance of Chelonaplysilla violacea 106

3. Establishment of 1,3,3-trimethylcyclohexyl substructure of cadlinolide C (138)

4. Establishment of the tetrahydrofuran substructure of cadlinolide C (138) $\ldots . .108$

5. Establishment of the side chain of cadlinolide C (138).

6. Establishment of final connectivity of cadlinolide C (138)

7. NOE correlations and stereochemistry of tetrahydrofuran ring of cadlinolide C (138)

8. Selected NOE correlations establishing the stereochemistry of the 1,3,3-trimethylcyclohexyl and lactone rings of cadlinolide C (138).

9. Establishment of 1,3,3-trimethylcyclohexyl substructure of cadlinolide D (139).

10. Establishment of the tetrahydrofuran ring of cadlinolide D (139). 
11. Establishment of final connectivity of cadlinolide D (139). 116

12. Selected NOE correlations establishing the stereochemistry of the 1,3,3-trimethylcyclohexyl and lactone rings of cadlinolide D (139)

13. Suggested stereochemistry of tetrahydrofuran ring of the major epimer of cadlinolide D (139).

14. Selected $\mathrm{HMBC}$ correlations establishing the final connectivity of pourewic acid $A(140)$

15. Selected NOE correlations establishing the stereochemistry of the tetrahydrofuran ring of pourewic acid A (140)

16. Selected NOE correlations establishing the stereochemistry of the side chain and the 1,3,3-trimethylcyclohexyl ring of pourewic acid A (140)

17. Negative ion HRESIMS spectrum of 15-methoxypourewic acid B (141).

18. Establishment of $\gamma$-lactone substructure of 15-methoxypourewic acid B (141)

19. Establishment of the final connectivity of 15-methoxypourewic acid B (141)

20. Establishment of the stereochemistry of 15-methoxypourewic acid B (141) 128

21. Establishment of the side chain of methylpourewate B (142).

22. Selected correlations establishing the final connectivity of methylpourewate B (142)

23. NOE correlations establishing the stereochemistry of methylpourewate B (142).

24. Establishment of the methylene chain substructure of pourewanone (143)....136

25. Establishment of the final connectivity of pourewanone (143) 137

\section{Chapter 5}

1. Hydrographic chart of Wellington Harbour and southern Wairarapa Coast 160

2. SEM image of Karenia brevisulcata. 160

3. N2A cells 165

4. Elution profile of $\mathrm{KBT}$ after running the pilot sample on a diol column 169

5. ${ }^{1} \mathrm{H}-{ }^{13} \mathrm{C}$ one-bond correlations of common algal toxins 177

6. Selected NMR chemical shifts of palytoxin (164) 178

7. Proposed NMR chemical shifts of a 1,4-disubstituted $\Delta^{2,3}$-dihydrofuran ring in KBT

8. NMR chemical shifts of a 1,2-dioxene ring 
9. Selected NMR chemical shifts of C-CTX-1 (166) 181

10. Possible substructures and NMR chemical shifts of KBT 182

\section{Chapter 7}

1. Elution profile from the large diol column 206

2. Comparison of toxicity recovered from the diol MPLC separations 207

3. Graph of two repeat injections of KBT under neutral conditions using PSDVB 208

4. Graph of the re-injection of a fraction from each peak of activity under neutral conditions using PSDVB. 208 


\section{List of Schemes}

\section{Chapter 3}

1. Biosynthesis of lanosterol (15)

2. Oxidative demethylation of lanosterol (15) to form cholesterol (16) 45

3. Alkylation of steroid side chains

4. The isolation of clathriol A (32) and clathriol B (33) from Clathria lissosclera

\section{Chapter 4}

1. Phylogenic relationship of Chelonaplysilla violacea $\ldots . \ldots \ldots$

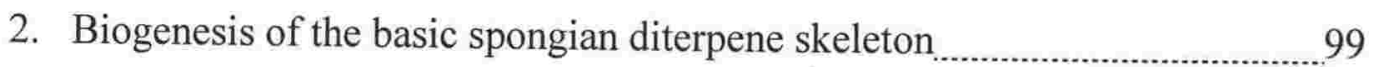

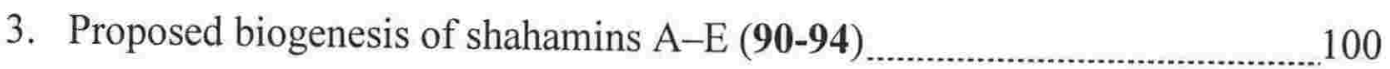

4. Proposed biogenesis of shahamins F-J (97-101)

5. Proposed biogenesis of $(\mathbf{1 0 2}) \ldots$

6. Proposed biogenesis of the norrisolide compounds (103-105) ........................101

7. Proposed biogenesis of gracilin A (118) and related compounds

8. Proposed biogenesis of $(\mathbf{1 2 0})$ and related compounds ..................................... 103

9. Isolation scheme for spongian diterpenes from Chelonaplysilla violacea $\mathrm{sp}$. (part 1)

10. Isolation scheme for spongian diterpenes from Chelonaplysilla violacea $\mathrm{sp}$. (part 2)

11. Proposed biogenesis of cadlinolides A-D (132-133, 138-139).

12. Proposed biogenesis of pourewic acid A (140), 15-methoxypourewic acid B (141), and methylpourewate B (142)

13. Proposed biogenesis of pourewanone (143)

14. Alternative biogenesis of pourewanone (143)

15. Proposed biogenesis of muricenones A and $B(\mathbf{1 4 4}, \mathbf{1 4 5})$

16. Proposed biogenesis of cyclomegistine (146)

\section{Chapter 5}

1. Proposed biosynthesis of brevetoxin B (151)

2. Phylogenic relationship of Karenia brevisulcata

3. Optimised large-scale isolation scheme for KBT 


\section{List of Tables}

\section{Chapter 2}

1. Summary of the sponges screened during this study. 28

\section{Chapter 3}

1. ${ }^{13} \mathrm{C}(75 \mathrm{MHz})$ and ${ }^{1} \mathrm{H}(300 \mathrm{MHz})$ NMR data $\left(\mathrm{CD}_{3} \mathrm{OD}\right)$ of clathriol $\mathrm{A}(32) \ldots 66$

2. ${ }^{13} \mathrm{C}(75 \mathrm{MHz})$ and ${ }^{1} \mathrm{H}(300 \mathrm{MHz})$ NMR data $\left(\mathrm{CDCl}_{3}\right)$ of clathriol B (33)...... 75

\section{Chapter 4}

1. ${ }^{13} \mathrm{C}(75 \mathrm{MHz})$ and ${ }^{1} \mathrm{H}(300 \mathrm{MHz})$ NMR Data $\left(\mathrm{CDCl}_{3}\right)$ of cadlinolide $\mathrm{C}$ (138)

2. ${ }^{13} \mathrm{C}(75 \mathrm{MHz})$ and ${ }^{1} \mathrm{H}(300 \mathrm{MHz})$ NMR Data $\left(\mathrm{CDCl}_{3}\right)$ of cadlinolide D (139)

3. ${ }^{13} \mathrm{C}(75 \mathrm{MHz})$ and ${ }^{1} \mathrm{H}(300 \mathrm{MHz})$ NMR Data $\left(\mathrm{CDCl}_{3}\right)$ of pourewic acid A (140)

4. ${ }^{13} \mathrm{C}(75 \mathrm{MHz})$ and ${ }^{1} \mathrm{H}(300 \mathrm{MHz})$ NMR Data $\left(\mathrm{CDCl}_{3}\right)$ of 15-methoxypourewic acid B (141).

5. ${ }^{13} \mathrm{C}(75 \mathrm{MHz})$ and ${ }^{1} \mathrm{H}(300 \mathrm{MHz})$ NMR Data $\left(\mathrm{CDCl}_{3}\right)$ of methylpourewate B (142)

6. ${ }^{13} \mathrm{C}(100 \mathrm{MHz})$ and ${ }^{1} \mathrm{H}(300 \mathrm{MHz})$ NMR Data $\left(\mathrm{CDCl}_{3}\right)$ of pourewanone (143)

7. Anti-inflammatory activity of several isolated spongian diterpenes 148

\section{Chapter 5}

1. Observed ${ }^{1} \mathrm{H}$ and ${ }^{13} \mathrm{C}$ NMR resonances of $\mathrm{KBT}$ (500 MHz, $\mathrm{D}_{6}$-DMSO) .......175 


\section{Glossary}

Amberchrom

Amino

$\mathrm{C}_{18}$

CBA

$\mathrm{CDCl}_{3}$

$\mathrm{CD}_{3} \mathrm{OD}$

$\mathrm{CH}_{2} \mathrm{Cl}_{2}$

COSY

d

$\mathrm{D}_{2} \mathrm{O}$

$\mathrm{D}_{5}-\mathrm{C}_{5} \mathrm{H}_{5} \mathrm{~N}$

D $_{6}$-DMSO

DEPT

Diol

DMSO

DQF-COSY

ELISA

ESR

$\mathbf{E t}_{2} \mathbf{O}$

EtOAc

fMLP

1D-gNOESY

$\mathrm{H}_{2} \mathrm{O}$

HMBC

HOMO2D $J$

HOHAHA

HP-20

HP-20S

HPLC

HRESIMS

HSQC

IC $_{50}$
PSDVB stationary support (Tosohaas)

Amine derivatised silica gel ( $\left.\mathrm{R}-\mathrm{Si}\left(\mathrm{CH}_{2}\right)_{3} \mathrm{NH}_{2}\right)$

Octadecyl derivatised silica gel (R-Si $\left(\mathrm{CH}_{2}\right)_{17} \mathrm{CH}_{3}$ ) (also ODS)

CarBoxylic $\left.\underline{\text { Acid derivatised silica gel (R-SiCH}}{ }_{2} \mathrm{COOH}\right)$

Deuterated chloroform

Deuterated methanol

Dichloromethane (Methylene chloride)

${ }^{1} \mathrm{H}-{ }^{1} \mathrm{H} \underline{\text { COrrelation }} \underline{\text { Spectroscop}} \underline{Y}$

Doublet

Deuterated $\mathrm{H}_{2} \mathrm{O}$

Deuterated Pyridine

Deuterated dimethyl sulfoxide

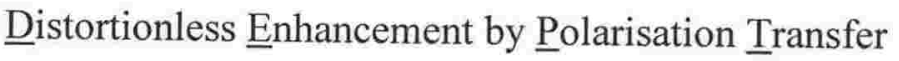

Diol derivatised silica gel

( $\left.\mathrm{R}-\mathrm{Si}\left(\mathrm{CH}_{2}\right)_{2} \mathrm{CH}_{2} \mathrm{OCH}_{2} \mathrm{CH}(\mathrm{OH}) \mathrm{CH}_{2} \mathrm{OH}\right)$

DiMethyl $\underline{\text { Sulfoxxide }}$

Double Quantum $\underline{\text { Filtered }}{ }^{1} \mathrm{H}-{ }^{1} \mathrm{H} \underline{\text { COrrelation }} \underline{\text { Spectroscop}} \underline{Y}$

Enzyme Linked Immuno- $\underline{\text { Sorbent }} \underline{\text { Asssay }}$

Institute of Environmental $\underline{\text { Science and }}$ Research Ltd.

Diethyl ether

Ethyl acetate

N-Formyl-Methionine-Leucine-Phenylalanine

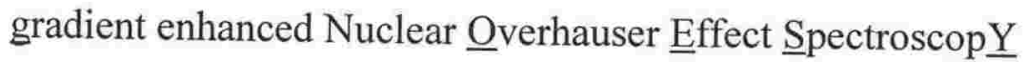

Water

Heteronuclear Multiple Bond $\underline{\text { Correlation }}$

Homonuclear $J$-resolved 2D experiment

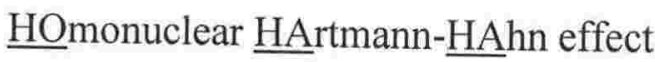

PSDVB stationary support (Mitsubishi)

PSDVB stationary support (Supelco)

$\underline{\text { High }}$ Pressure (Performance) Liquid $\underline{\text { Chromatography }}$

$\underline{H}$ igh $\underline{R}$ esolution Electro- Spray Ionisation $\underline{\text { Mass }} \underline{\text { Spectrometry }}$

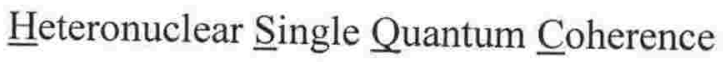

$50 \%$ Inhibitory Concentration 


\begin{tabular}{|c|c|}
\hline IP & IntraPeritoneal \\
\hline IR & InfraRed \\
\hline$J$ & Scalar coupling constant \\
\hline KBT & $\underline{\text { Karenia }}$ Brevisulcata Toxin \\
\hline LC-MS & 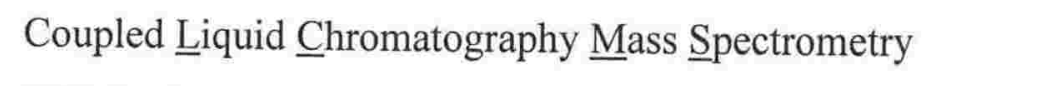 \\
\hline $\mathbf{L D}_{50}$ & $50 \%$ Lethal Dose \\
\hline $\mathrm{Me}_{2} \mathrm{CO}$ & Acetone (Propanone) \\
\hline $\mathrm{MeOH}$ & Methanol \\
\hline MNP number & $\underline{\text { Marine }} \underline{\text { Natural }}$ Products number \\
\hline MPLC & 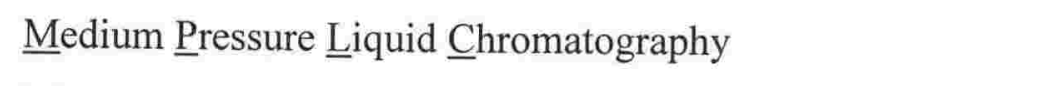 \\
\hline MS & $\underline{\text { Mass }} \underline{\text { Spectrometry }}$ \\
\hline mult. or $\mathbf{m}$. & Multiplet \\
\hline$m / z$ & Mass to charge ratio \\
\hline $\mathbf{N} 2 \mathrm{~A}$ & Murine Neuroblastoma Assay \\
\hline NIWA & $\underline{\text { National Institute for } \underline{W} \text { ater and } \underline{A} \text { tmospheric research }}$ \\
\hline NMR & Nuclear Magnetic Resonance \\
\hline NOE & 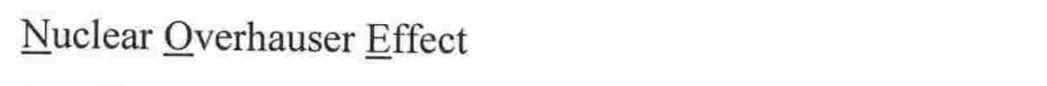 \\
\hline ODS & 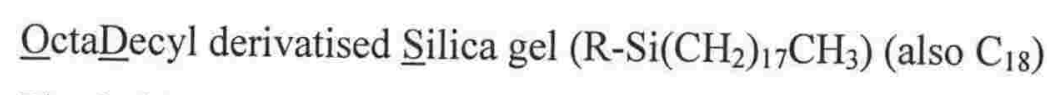 \\
\hline PMA & Phorbol Myristate $\underline{\text { Acetate }}$ \\
\hline ppm & $\underline{\text { Parts }} \underline{\text { Per }} \underline{\text { Million }}$ \\
\hline PSDVB & $\underline{P}$ oly( Styrene-DiVinylBenzene) \\
\hline $\mathbf{q}$ & Quartet \\
\hline ROESY & 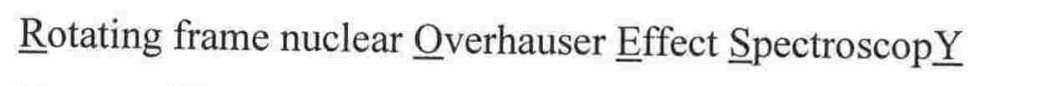 \\
\hline RP & $\underline{\text { Reversed-Phase }}$ \\
\hline $\mathbf{s}$ & Singlet \\
\hline SEM & 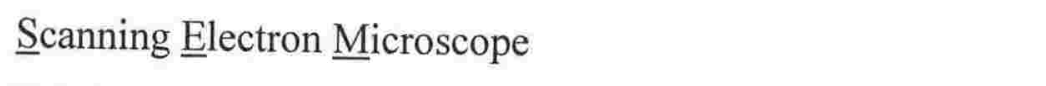 \\
\hline t & Triplet \\
\hline TLC & Thin Layer $\underline{\text { Chromatography }}$ \\
\hline TOCSY & TOtal Correlation $\underline{\text { Spectroscop}} \underline{Y}$ (HOHAHA) \\
\hline TU & Toxicity $\underline{\text { nnit }}$ \\
\hline UV & UltraViolet \\
\hline VLC & Vacuum Liquid Chromatography \\
\hline${ }^{1} \mathrm{H}$ NMR & Proton $\underline{\text { Nuclear }}$ Magnetic $\underline{\text { Resonance }}$ \\
\hline${ }^{13} \mathrm{C}$ NMR & Carbon-13 uulear $\underline{\text { Magnetic }}$ Resonance \\
\hline$\delta$ & Chemical shift (ppm) \\
\hline
\end{tabular}




\section{Chapter One}

\section{Introduction}

\subsection{Natural Products Chemistry and History}

Mankind has a long tradition of utilising natural resources for a variety of applications, including extracting plants for medicines and dyes, using inorganic minerals for building and paints, and smelting of ores to obtain pure metals for tools. ${ }^{1-3}$ The use of plant extracts as medicines, in particular, has fascinated and intrigued all societies. Every ancient civilisation used natural resources to treat illness and disease. ${ }^{1,4}$ As man's understanding of chemistry has progressed throughout the ages, so too has the desire to understand the formulation of the active principles of medicinally beneficial plant extracts. It is the goal of natural products chemists to isolate and elucidate the structures of these active principles.

Use of terrestrial herbs to treat illness has a long-standing history. The marine environment, however, has played very little role in traditional medicines due to the difficulty in collecting flora and fauna in all but the most shallow of waters. There is some evidence, however, that various ethnic groups did have an understanding of the biological activities associated with certain marine organisms, even if these compounds were not used to treat disease [e.g. Hawaii's "Limu make o Hana" ("Deadly seaweed of Hana"), the ancient Egyptians recognition of the poisonous puffer fish Tetradon stellatus during the Fifth dynasty ( 2700 B.C.), or, as most species of toxic fish do not possess true scales, the Biblical decree not to eat fish without scales, (Deuteronomy 14: 9-10, 1451 B.C.)]. ${ }^{4-6}$ Natural products from marine organisms remained largely undiscovered until the early 1940s when Jacques Cousteau designed 
the first inexpensive and reliable SCUBA apparatus. ${ }^{1,7}$ Easier access to shallow subtidal environs allowed the maturing science of terrestrial natural products to diversify into a totally new area of study. The study of marine natural products blossomed in the 1970s and the number of novel metabolites reported each year has been steadily increasing until only very recently. ${ }^{8,9}$

All living organisms produce an enormous variety of organic molecules for a large array of uses. Primary metabolites are those molecules produced by all organisms and include lipids, proteins, amino acids, and carbohydrates. Such metabolites are primarily the subject of study of biochemistry. ${ }^{2,10,11}$ Secondary metabolites are those molecules that are produced by individual species for specific end uses. The biological role of a secondary metabolite can be difficult to identify but often includes defence against predation or encroachment..$^{2,10,12}$ It has been suggested that secondary metabolites may have evolved as a means to consume primary metabolites surplus to the organisms requirements. ${ }^{13}$ Secondary metabolites are often very complex chemical entities and are therefore metabolically expensive to produce, implying that they must provide some kind of ecological advantage to the producing organism. ${ }^{12}$ Biologically active secondary metabolites need to be able to travel throughout a target organism's body in order to reach the intended sites of action. They therefore need to be able to exist in both hydrophilic and hydrophobic environs and so are usually of intermediate polarity, in order to transverse both aqueous and lipid environments. ${ }^{11}$

The biological effects of marine derived secondary metabolites, especially those produced for defensive measures, can be very different in mammals than they are in other marine organisms; some may have therapeutic effects against mammalian diseases or illnesses, which is why marine natural products are currently targeted as potential therapeutic agents. ${ }^{2,10,12,14}$ Although no isolated marine natural product is currently 
available as a pharmaceutical, there is a strong basis for using natural products to treat disease. Between 1983 and 1994, the United States of America Food and Drug Administration Agency approved 522 new pharmaceuticals for human use. Although only $30(5.7 \%)$ of these were true natural products, $127(24.3 \%)$ were semi-synthetic derivatives of natural products whilst $46(8.8 \%)$ were synthetic but based upon natural product templates, all of which implies that $39 \%$ of all new pharmaceuticals introduced between 1983 and 1994 were natural products based. ${ }^{15}$ Of the 92 anti-cancer compounds commercially available up to 1994,57 were derived from natural origins. ${ }^{1,15}$ Moreover, in 1996 eight of the top twenty selling pharmaceuticals were derived from natural sources. ${ }^{16}$

Currently, there are approximately thirteen marine natural product based pharmaceuticals in clinical development. Twelve of these are derived from marine invertebrates. ${ }^{9}$ Several anti-viral and anti-cancer compounds were isolated from the Caribbean sponge Cryptotheca crypta in the 1950s. Synthetic analogues of these were developed leading to the anti-viral agent Ara-A (1) and the clinically used anti-cancer drug Ara-C (2). ${ }^{1,17}$ Bryostatin-1 (3), a macrolide isolated from the bryozoan Bugula neritina, has advanced into phase II clinical trials. ${ }^{1,15,17}$ The first marine natural product to enter clinical testing was didemnin $B$ from the tunicate Trididemnum solidum. Unfortunately, didemnin B was significantly toxic whilst showing irreproducible activity against a variety of tumours in phase II trials. Several other metabolites with microtubule stabilising activity similar to taxol are currently under further investigation. ${ }^{1,17}$ 


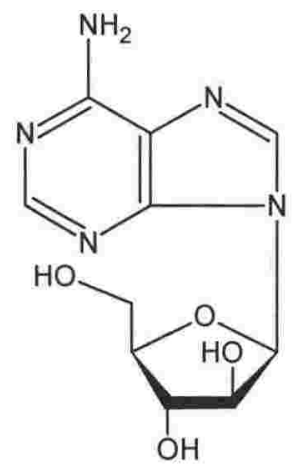

(1)

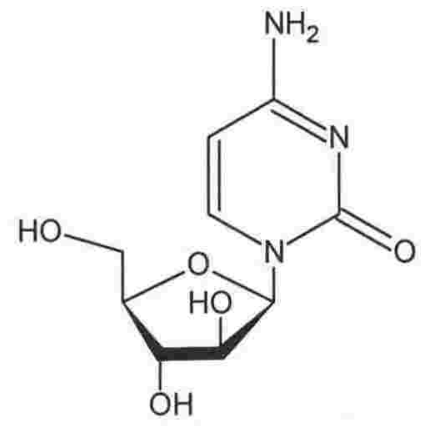

(2)

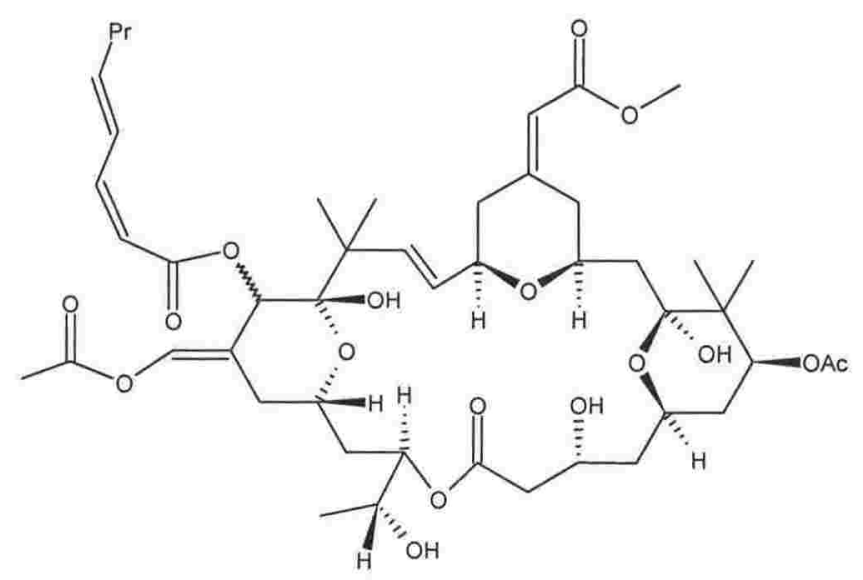

(3)

\subsection{New Zealand Geography}

New Zealand is an archipelago (see figure 1.1), found in the South Pacific Ocean, that has long been isolated from all other landmasses. This isolation has allowed New Zealand to develop a unique flora and fauna that is quite distinct from any other. Prominent New Zealand palaeobiologist Sir Charles Fleming believed that the terrestrial forests of New Zealand bear more resemblance to the Mesozoic forests of Gondwanaland than to other contemporary wooded areas. ${ }^{18-20}$ This unique ecology extends to New Zealand's marine environment. 


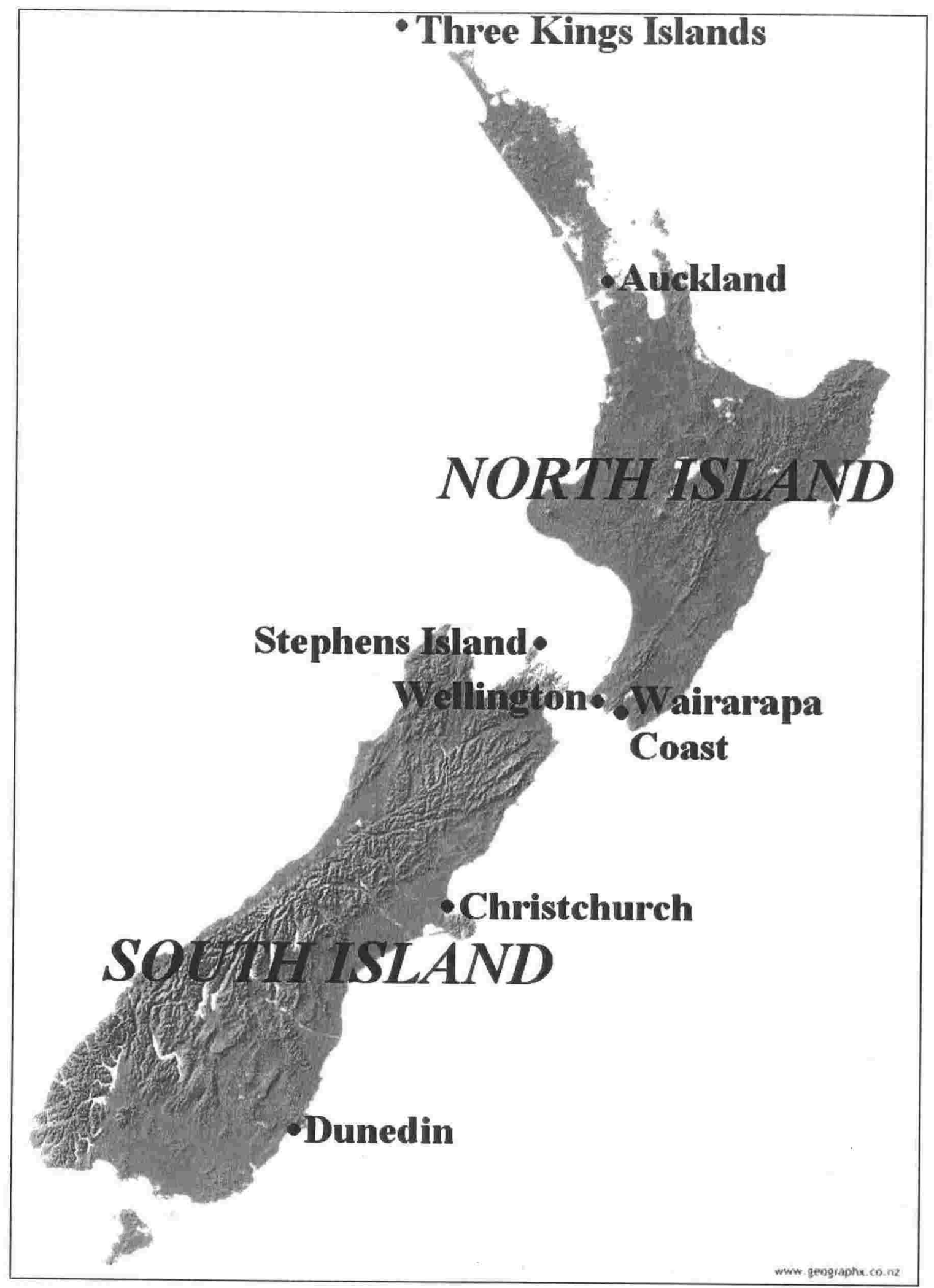

Figure 1.1 Map of New Zealand. (Courtesy of GeoGraphX Mapping, New Zealand) 
During the course of this study, secondary metabolites were isolated from both marine sponges (multicellular aquatic animals) and dinoflagellates (unicellular aquatic plants). Examples of the biology of each are described below.

\subsection{Sponges}

Sponges, which constitute the entire phylum Porifera, are the oldest and simplest multicellular animals (kingdom Metazoa). ${ }^{21-23}$ In fact, sponges are so simplistic that it was not until the late Eighteenth century that they were commonly accepted as animals. $^{21-23}$ The Porifera are considered to belong to an ancient phylum, as representatives of all modern groups of sponges were present and widespread in the early to mid Cambrian era 500 million years ago. ${ }^{21,23}$ Currently the phylum is divided into three classes, 27 sub-classes, 25 orders, 127 families and over 683 genera. $^{23}$ Currently, there are 7000 species of formally described living sponges worldwide, although it is conservatively estimated that there at least 15,000 species extant. ${ }^{22,23}$

Sponges do not possess many of the normal features associated with other Metazoans. For example, sponges do not have any truly individual tissues or organs, nor do they possess circulatory, digestive, or nervous systems. ${ }^{21-24}$ Poriferans are sedentary filter feeders. To feed, sponges use specialised flagellated cells (choanocytes) to pump water in the body via ostia, through a series of channels, and out through circular surface oscules. Within these channels are a series of successively finer filters that catch food particles for the animal to consume. ${ }^{21-23}$ Interspersed between the choanocytes and the outer pinacoderm, which protects the sponge from the outside environment, is the connective tissue known as mesohyl. It is within the mesohyl that the skeletal component of the sponge resides. ${ }^{21-24}$ 
The taxonomy of sponges is a long-standing and difficult problem. Many of the common markers used to differentiate between species in other phyla, such as colour, shape, or size, cannot be applied, as sponges grow in indeterminate patterns similar to many plants. The size, appearance, and morphology of a sponge, is dependent on external factors such as water current, nutrient levels, the presence of symbionts, as well as substrate composition and slope. ${ }^{21-23}$

Other markers for the identification of sponges have taken precedence over those mentioned. In particular, the skeletal content of the mesohyl is predominantly used to identify different sponge specimens. Sponge skeletons are generally comprised of a collagen-type fibre known as spongin, which may or may not be combined with inorganic spines. ${ }^{21-23}$ The inorganic spines, which are made of either calcium carbonate or silica, are known as spicules, the size, shape, and composition of which is often a diagnostic feature of an individual species and is now commonly used to distinguish between different sponge specimens. ${ }^{21-23}$

It was once thought that spicules were used for both protection and structure within the sponge skeleton. ${ }^{21-23}$ There is a recent body of evidence, however, that suggests that sponges rely less on spicules for defence than might first be imagined. There are many examples of physical predation upon sponges by more advanced members of the animal kingdom, including attacks against species possessing the hardest skeletons known. ${ }^{21,25-}$ ${ }^{32}$ It is now thought that sponges rely more heavily upon biochemical means of defence against predation and encroachment. ${ }^{21}$ This is in keeping with the long evolutionary time-scale within which sponges have been able to develop novel secondary metabolic means of defence. ${ }^{21,23}$ 
Other methods currently used to differentiate sponges include identification of individual sponge genomes and also, of more interest to the natural products chemist, chemotaxonomy. Chemotaxonomy is the use of secondary metabolic composition as a taxonomic marker for different organisms. It has been used to differentiate between sponges of different orders, families, and genera, but is too general to discern between species. $^{22,23,33}$

Phylum Porifera is divided into three main classes, the Demospongiae, the Calcarea and the Hexactinellida. The Demospongiae, also known as the siliceous sponges, usually possess spicules made from silica. The Demospongiae represent $85 \%$ of all the described sponges with approximately 6000 species within 15 orders, 88 families and around 500 genera. The class also contains all of the sponge orders devoid of any spicules. $^{21-23,34}$ Demosponges are found in many environs worldwide, including the eight families of sponges that live in freshwater. Most Demosponges, however, are marine based and are found between tidal $(0 \mathrm{~m})$ and hadal $(>6000 \mathrm{~m})$ depths. ${ }^{21-24,34}$

The Calcarea, or calcareous sponges, possess spicules composed exclusively of calcium carbonate. ${ }^{21-23,34}$ The Calcarea represent $7 \%$ of all known sponges with approximately 500 described species in five orders, 22 families and 75 genera. Most are inconspicuous as they are often small and lightly coloured. They are usually found in sheltered and shallow environs $(<1000 \mathrm{~m})$, predominately in tropical waters where they associate with coral reefs. ${ }^{21-23,34}$

The final class, the Hexactinellida or glass sponges, possess six-rayed siliceous spicules (hexacts) that are found individually or fused together. The spicules of Hexactinellid sponges tend to be much longer than those of the other two classes and often make up a larger proportion of the sponge body than the organic component of the animal tissue. ${ }^{21-}$ 
${ }^{23,34}$ The morphology of the Hexactinellid sponges is so different from that of the Demospongiae or the Calcarea that some taxonomists believe that Hexactinellid sponges should form their own sub-phylum distinct from the other two classes, or maybe even form a different phylum altogether. ${ }^{22,23}$ The Hexactinellida are mostly found in deeper water $(>200 \mathrm{~m})$. There are currently about 500 described species distributed in five orders, 17 families and 118 genera. $^{21-23,34}$

It should be noted that the taxonomy of sponges at the genus and species level is very fluid and there is often disagreement between taxonomists as to the phylogenic placement of various individual sponges and even phyletic, class, ordinal, or familial relationships are often disputed. ${ }^{23}$

\subsection{Dinoflagellates (Dinophyceae)}

Dinoflagellates are microscopic, single-celled algae of phylum Chromophycota (kingdom Plantae). Chromophycota is a diverse phylum of photosynthetic plants covering nine separate classes, grouped together only because they all contain chlorophylls $a$ and $c$ but not chlorophyll $b .^{35}$ Dinoflagellates have also been regarded as being part of kingdom Protozoa (phylum Sarcomastigophora, class Dinoflagellata) rather than as an aquatic plant. ${ }^{35-37}$ This debate has led some to believe that dinoflagellates are phylogenically on the border between prokaryotes and eukaryotes; they are therefore at times referred to as "mesokaryotes". 38,39

The dinoflagellates themselves are small (usually $5 \mu \mathrm{m}$ to $2 \mathrm{~mm}$ in size), motile, cellular bodies possessing two whip-like tails or flagella; one is usually found wrapped in a transverse groove around the body while the second is used for movement and travels 
posteriorly behind the cell (see figure 1.2). ${ }^{40}$ Many different forms of dinoflagellate are known; the class comprises fourteen orders and 48 families. ${ }^{35,41}$

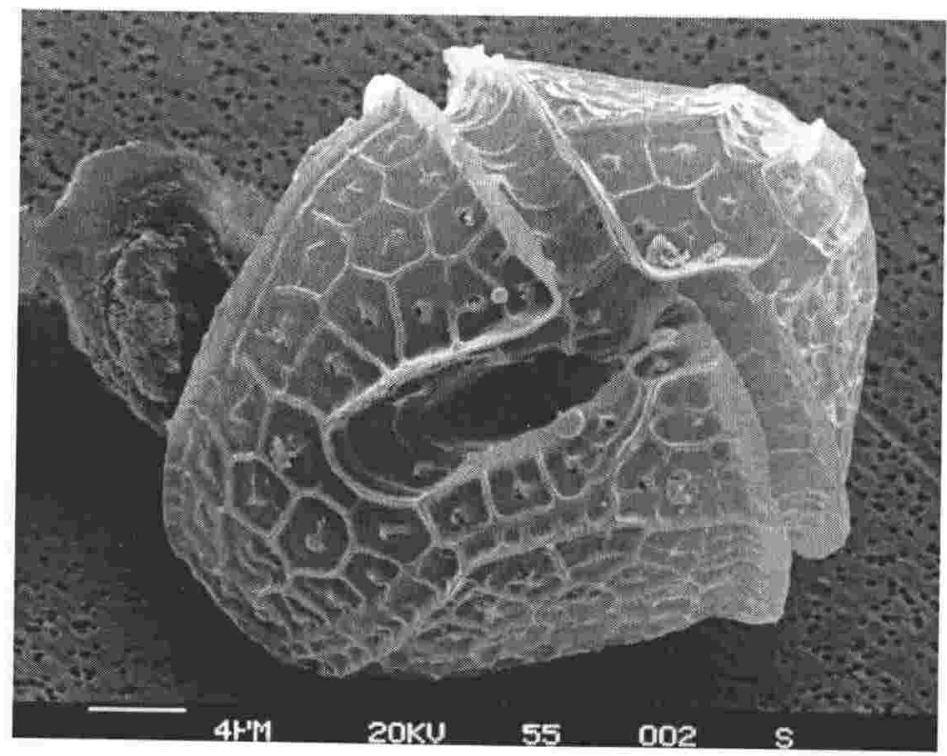

Figure 1.2 SEM image of Protoceratium reticulatum showing transverse and posterior groves. (Courtesy of Dr D. Stirling, ESR, 1998)

Under the right environmental conditions, dinoflagellates can undergo a phase of accelerated reproduction or "bloom" where the concentration of algal cells can rapidly increase from approximately $10-100$ cells/L of seawater to $10^{5}-10^{8}$ cells/L in only a matter of days. ${ }^{37,40}$ The conditions when this happens usually correspond to a change of season from winter to spring, or from spring to summer, when there is a substantial increase in light, water temperature, and nutrient level. Blooms usually require a time of settled weather as strong winds can disperse the cells if they are close to the surface of the sea (see figure 1.3). ${ }^{37,40}$ 


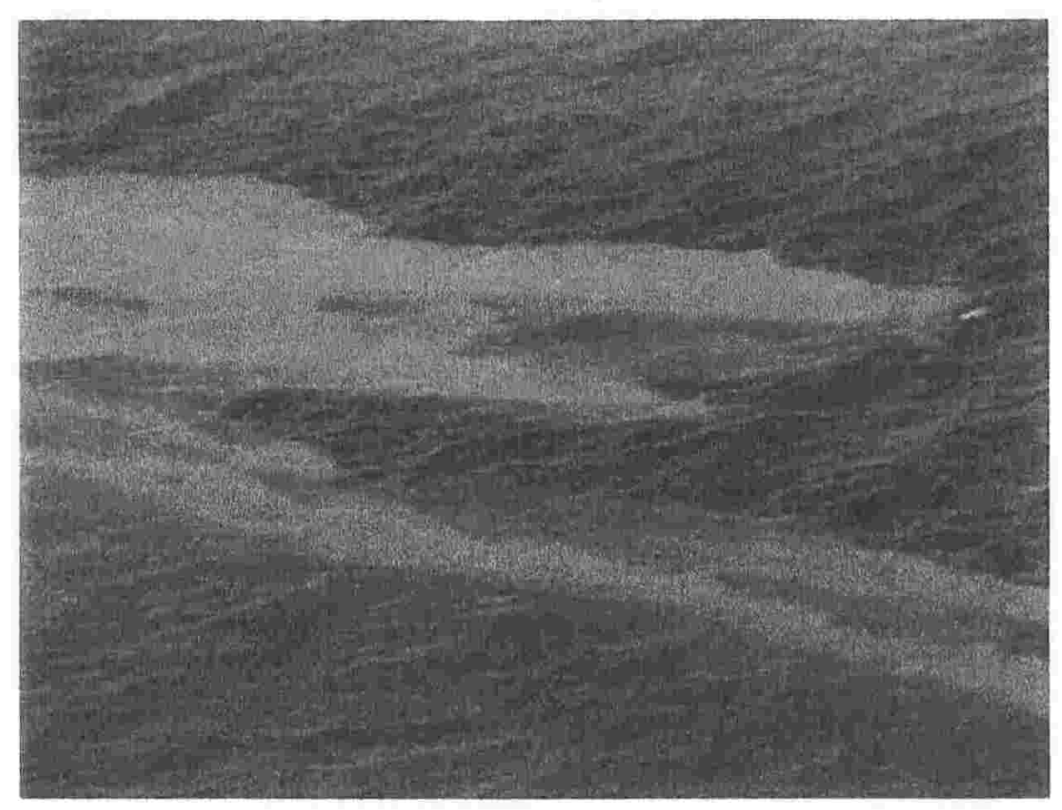

Figure 1.3 A marine algal bloom in Hawkes Bay, New Zealand. (Courtesy of Mr N. Watson, Hawkes Bay District Health Boards, 2003)

Mass mortalities of aquatic life can follow algal blooms for two reasons. First, the large changes in cell number can result in a massive change in the concentration of dissolved $\mathrm{O}_{2}$ in the seawater. Fish may die, often after a bloom has collapsed when a massive depletion of dissolved $\mathrm{O}_{2}$ (anoxia) causes asphyxiation. ${ }^{41}$ Second, although most species of dinoflagellate are harmless, some species produce toxins that when secreted into the surrounding environment, or introduced into the food chain, can cause the death of other organisms. In some cases, the toxins are not dangerous to the consumer, but rather they are accumulated in the tissues of the consuming organism and can intoxicate predators higher in the food chain. ${ }^{37,39,40,42}$ Alexandrium, Dinophysis, Karenia, and Karlodinium, are examples of well known toxin-producing dinoflagellate genera. ${ }^{39,42-45}$

\subsection{Isolation of Marine Natural Products}

The goal of marine natural products chemistry is to isolate novel chemical structures, preferably with interesting and useful biological activities. By their very nature, 
biologically active secondary metabolites are often present in concentrations of less than $10 \mathrm{mg} / \mathrm{kg}$ of the sample organism, therefore isolating these compounds is expensive, difficult, and time consuming. ${ }^{9}$ To maximise the chances of achieving this goal, three questions must be answered.

1. How does one select an organism with which to work?

2. How does one purify (fractionate) the desired secondary metabolite to allow identification?

3. How does one track the isolation of the interesting secondary metabolite from an extract of the selected organism?

Selection is based upon a screening test performed on an extract of the organism, in order to determine if there are any interesting secondary metabolites present. If there are, the extract is subjected to a series of purification steps (chromatography) to separate the compound from all the other chemicals present in the crude extract. Testing is carried out at each stage of the isolation process, in order to confirm the presence of the target metabolite. Once the compound is pure, it is then identified.

These steps are intimately linked. The screening method used to identify which extracts contain novel secondary metabolites is often the method used to test fractions generated throughout an isolation. A screen may require initial fractionation to semi-purify an extract before testing. After each step in an isolation procedure, fractions generated will be tested to check that the compound of interest is still present. Screening, testing of fractions to guide isolations, and methods for fractionation, will each be discussed below. 
Extracts of many marine organisms will contain novel biologically active secondary metabolites, whilst many others will be devoid of any interesting compounds, or they may only contain previously reported metabolites. In order to decide which organisms contain novel secondary metabolites, a preliminary screen needs to be performed on an extract of the organism to assess the presence of any novel metabolites. If possible, it is also desirable to identify if the interesting metabolites present have been previously reported (structure dereplication).

There are several strategies available to maximise the likelihood of selecting an organism that contains novel secondary metabolites. First, a screen targeting a unique biological activity may be used. This may not be advantageous, however, as known compounds can be active in many different bioassay systems. Second, unusual organisms that have not been previously examined could be targeted, as they may possess a unique biochemical makeup. Finally, chemical testing using various chromatographic separations prior to screening, or use of different spectroscopic screens, can select for certain functional groups that often are found in novel structures. This method can also maximise the chance of identifying known compounds at an early stage.

Historically, screening of organisms for interesting metabolites revolved primarily around observed thin layer chromatography (TLC) signatures using a variety of running solvents and visualisation aides ( $R_{f}$ values, UV absorbances and fluorescences, characteristic chars, etc). ${ }^{46}$ More recently, high-throughput screening using a variety of biological assays has become prevalent. Many different in vitro bioassays focussing on different molecular targets, including anti-cancer, anti-fungal, anti-bacterial and anti- 
inflammatory agents, are commonly used to test crude extracts of marine organisms. An organism is generally selected for further study if a crude extract from it has reached or exceeded a certain level of biological activity (usually measured as the concentration required to achieve a certain percentage kill or inhibition of growth e.g. $\mathrm{LD}_{50}$ or $\left.\mathrm{IC}_{50}\right) .1,16,17,47,48$

\subsection{Guidance of Crude Extract Fractionation}

To utilise bioassay-guided fractionation, an extract of the organism selected by the screening process is fractionated using standard separation technologies. After each step in the isolation, all the fractions generated are biologically tested to monitor the activity through the isolation procedure. A compound isolated in this manner will always be biologically active.

Bioassay guided fractionation does suffer from several disadvantages. Bioassay processing time can be significant, meaning that the isolation process is often very slow. Testing at each stage can be very expensive, especially when many fractions requiring testing may be generated at any stage of the isolation procedure. Valuable mass of an active compound is sacrificed at each stage for testing, reducing the amount of material available for the identification of the purified metabolite. ${ }^{6}$ Identification of known compounds is impossible with a purely bioassay guided fractionation. Bioassays themselves are of necessity focussed very narrowly and often go through "boom and bust" cycles of popularity. This means that compounds isolated using a particular assay may be of less therapeutic importance if the assay drops from popularity or usefulness. Whole body in vivo assays are inherently more useful from a therapeutic perspective than in vitro assays, as they test biological activity in a whole organism rather than in one cellular target. This can be misleading as the activity as a whole could be due to a 
mixture of biologically active metabolites affecting multiple linked cellular targets, rather than one single compound. In vivo assays are generally slow, more expensive and difficult to perform, and require more material than in vitro cell-, enzyme-, or receptor-based assays. Often, when an extract triggers an in vivo based screen, a surrogate in vitro assay is used to guide the isolation. This may guide the researcher to a compound that is not active in the original in vivo whole organism assay. The investigator is totally dependant on one assay to monitor and guide them through the isolation procedure. If there are any inconsistencies or problems with the assay, the efficiency of the isolation may be compromised. ${ }^{48,49}$ Finally, most bioassays are qualitative and therefore recovery of the active compounds can be difficult to quantify at each step. Also, those assays that can be quantified often exhibit large levels of uncertainty in the final result (up to $\pm 50 \%$ ), making estimates of recovery difficult to determine.

Two contrasting approaches for the guidance of an isolation were used in this study. The first was an NMR based screen, followed by the NMR guided isolation of metabolites from marine sponge extracts, the results of which are detailed in chapters two, three, and four. The bioassay-guided fractionation of an algal toxin was also performed and is described in chapter five.

\subsection{Methods Used in the Isolation of Secondary Metabolites}

Regardless of the approach taken to guide an isolation procedure, a series of successive separations must be carried out on a crude extract of the organism, in order to purify the interesting secondary metabolites present. There are many methods available for the separation of a target secondary metabolite from the unwanted material in the crude extract of a marine organism. It is estimated that an extract of $1 \mathrm{~kg}$ of sponge (wet 
weight) can contain more than $40 \mathrm{~g}$ of polar salts, proteins and sugars, over $5 \mathrm{~g}$ of non-polar fats and steroids, and often less than $10 \mathrm{mg}$ of the target secondary metabolites. ${ }^{50}$ Any attempt to modify the polarity of a crude extract will result in the precipitation of either the polar or the non-polar components, depending upon how the polarity is modified. ${ }^{50}$

The most challenging part of the fractionation of secondary metabolites will often be at the very start of an isolation procedure, due in large part to the vast array of compounds of differing polarities found in the crude extract. Methods developed to fractionate crude extracts include liquid/liquid partitioning, column chromatography using different stationary phases, and vacuum liquid chromatography (VLC).

Liquid/liquid partitioning is a commonly used procedure where the crude extract is shaken together with an immiscible solvent; ideally the target metabolite concentrates preferentially in one of the two layers dependant upon its solubility in each. The layer containing the interesting metabolite can be partitioned several times using different solvent systems. Many systems of immiscible solvents have been developed to separate a wide range of target molecules. ${ }^{51-53}$ The advantages of liquid/liquid partitioning are that only common glassware is required, the procedure can be carried out on a large laboratory scale (0-2 kg wet weight sponge), and only common solvents are normally needed. Liquid/liquid partitioning is not perfect and there are several problems associated with its use. First, large volumes of potentially environmentally damaging halogenated solvents are often used. Second, stable emulsions of the immiscible solvents are often formed. Third, the target metabolites may be spread over more than one solvent fraction, reducing the resolution of the separation. ${ }^{54}$ 
Flash chromatography (pressure assisted chromatography) using silica gel (hydrated silica, a normal-phase chromatographic stationary phase) is often used as the first step of an isolation procedure. Silica gel is best utilised to separate non-polar compounds and is therefore of limited use when trying to fractionate mid-polarity biologically active metabolites from crude extracts containing compounds of widely varying polarity. ${ }^{55}$ Furthermore, polar molecules can irreversibly bind to the substrate, potentially reducing recovery of materials that may very well be present in only small amounts.

VLC was designed to help fractionate crude extracts and is a variation of flash chromatography using silica gel. The crude extract is loaded onto a small column in a volatile non-polar solvent, which is then removed by an applied vacuum. The column is then sequentially batch-eluted under vacuum with solvents of increasing polarity. This method is designed for normal-phase stationary packing materials only and is therefore only suitable for fractionating non-polar compounds present in small amounts. Chromatographic resolution of the technique is also limited due to the size and shape of the column used. ${ }^{56}$

Blunt and Munro developed the usage of octadecyl derivatised silica gel (ODS or $\mathrm{C}_{18}$ ) for the reversed-phase fractionation of crude extracts. The crude extract is added to a small amount of $\mathrm{C}_{18}$, and the solvent removed under vacuum. This loaded stationary phase is then transferred, either dry or as a slurry, onto a larger column, which is then eluted with solvents of decreasing polarity. ${ }^{54}$ The method is a vast improvement upon traditional techniques as it is faster than multiple liquid/liquid partitioning steps, shows better chromatographic resolution, uses inexpensive glassware and, by using reversedphase substrates, polar molecules will not generally bind to the packing material whilst allowing better separation of biologically active mid-polarity secondary metabolites. ${ }^{54}$ 
The method does suffer, however, from the fact that large amounts of extract can be difficult to concentrate onto the solid support. Also, the process of eluting the column can lead to stripping of the bonded-phase from the stationary support, reducing reusability of the substrate. As well, basic compounds do not separate well on $\mathrm{C}_{18}{ }^{57}$ To prepare the $\mathrm{C}_{18}$ stationary phase, acidic silanol groups on the surface of the silica gel are reacted with $n$-octadecyltrichlorosilane, and then with trimethylchlorosilane. ${ }^{58}$ If this reaction does not go totally to completion, any uncapped sites will retain their acidic character and can therefore ionically associate with organic cations (e.g. protonated amines), leading to an almost irreversible binding of the metabolite to the substrate. Very strong non-polar solvents are needed to elute these compounds from the $\mathrm{C}_{18}$ stationary phase. $^{57}$

\subsection{Cyclic Loading}

West and Northcote developed cyclic loading in 1996 to help in the fractionation of crude extracts. Cyclic loading utilises poly(styrene-divinylbenzene) (PSDVB) resin, a reversed-phased stationary phase that deviates from the use of silica gel, $\mathrm{C}_{18}$, liquid/liquid partitions, etc, in the initial fractionation of crude extracts. ${ }^{50}$ It was originally developed for processing extracts of the sponge Mycale hentscheli in an ongoing screening programme. $M$. hentscheli produces the biologically active compounds mycalamide A (4), pateamine (5) and peloruside (6). ${ }^{59-61}$ All three metabolites vary in both polarity and functionality, and therefore are difficult to concentrate into one sample for analysis. Pateamine (5), in particular, is very sensitive to changes in $p \mathrm{H}^{50,62}$ 


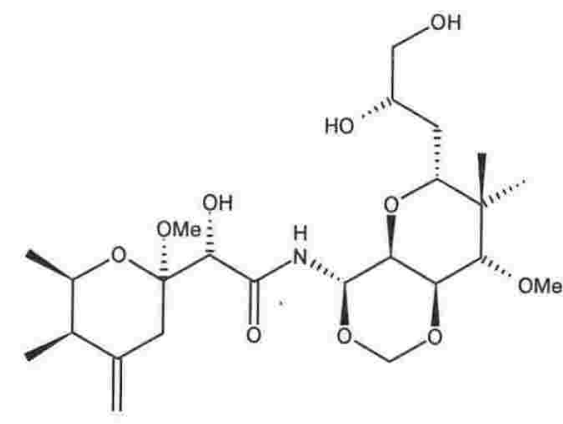

(4)

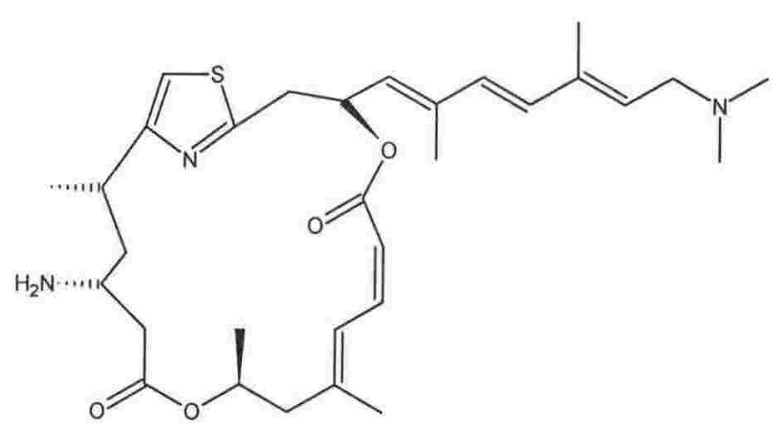

(5)

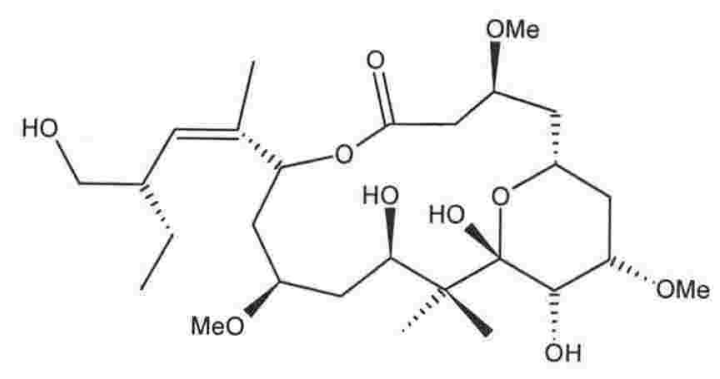

(6)

PSDVB is a rigid, macroporous, cross-linked polymer support that is inexpensive and reusable. It lacks any polar sites and so does not suffer from irreversible binding of polar solutes. It has been found that PSDVB is very useful for the separation of basic compounds and quaternary ammonium salts. ${ }^{63}$ PSDVB is chemically inert in most organic solvents, can be used at a wide range of $p \mathrm{H}$ values ( $p \mathrm{H} 1-13)$, and can withstand solutions of high ionic strength. These characteristics allow for a wide range of potential applications.

Cyclic loading addresses the difficulty of loading crude extracts on reversed-phase supports. The method involves the sequential dilution of a crude extract with $\mathrm{H}_{2} \mathrm{O}$ in order to increase its polarity, thereby forcing less polar molecules to absorb to the stationary phase (although conceptually this can be extended to diluting polar extracts with increasing amounts of non-polar solvents to cyclic load onto a normal-phase substrate). Initially, the crude extract of an organism is passed through a column of PSDVB, after which it is diluted with $\mathrm{H}_{2} \mathrm{O}$. Under normal circumstances, addition of 
$\mathrm{H}_{2} \mathrm{O}$ to a crude extract will result in the precipitation of any non-polar fats, triglycerides, and steroids present. Addition of $\mathrm{H}_{2} \mathrm{O}$ will not cause precipitation in this case, as the non-polar metabolites will have already absorbed to the stationary phase and will no longer be present in the diluted extract. ${ }^{50}$ The diluted extract is then passed through the same column again, with the mid-polarity compounds absorbing to the packing material. The eluent is then be diluted further and is passed back through the same column again. The process of dilution and passing through the column is repeated until all compounds of interest are absorbed onto the column stationary phase. In most cases, the target mid-polarity secondary metabolites will have been absorbed once the extract has been diluted four fold and been passed through the column (see figure 1.4). ${ }^{50}$

The major drawback of cyclic loading is that the volume of solvent passed through the column increases at each step. For example, if a sponge sample is extracted twice with $1 \mathrm{~L} \mathrm{MeOH}$ and is cyclic loaded in two dilution steps to $25 \% \mathrm{MeOH} / \mathrm{H}_{2} \mathrm{O}$, then a total of $14 \mathrm{~L}$ would have been passed through the column $[(2 \times 1 \mathrm{~L})+(1 \times 4 \mathrm{~L})+(1 \times 8 \mathrm{~L})]$. This is time consuming, and often requires the use of large glassware. 


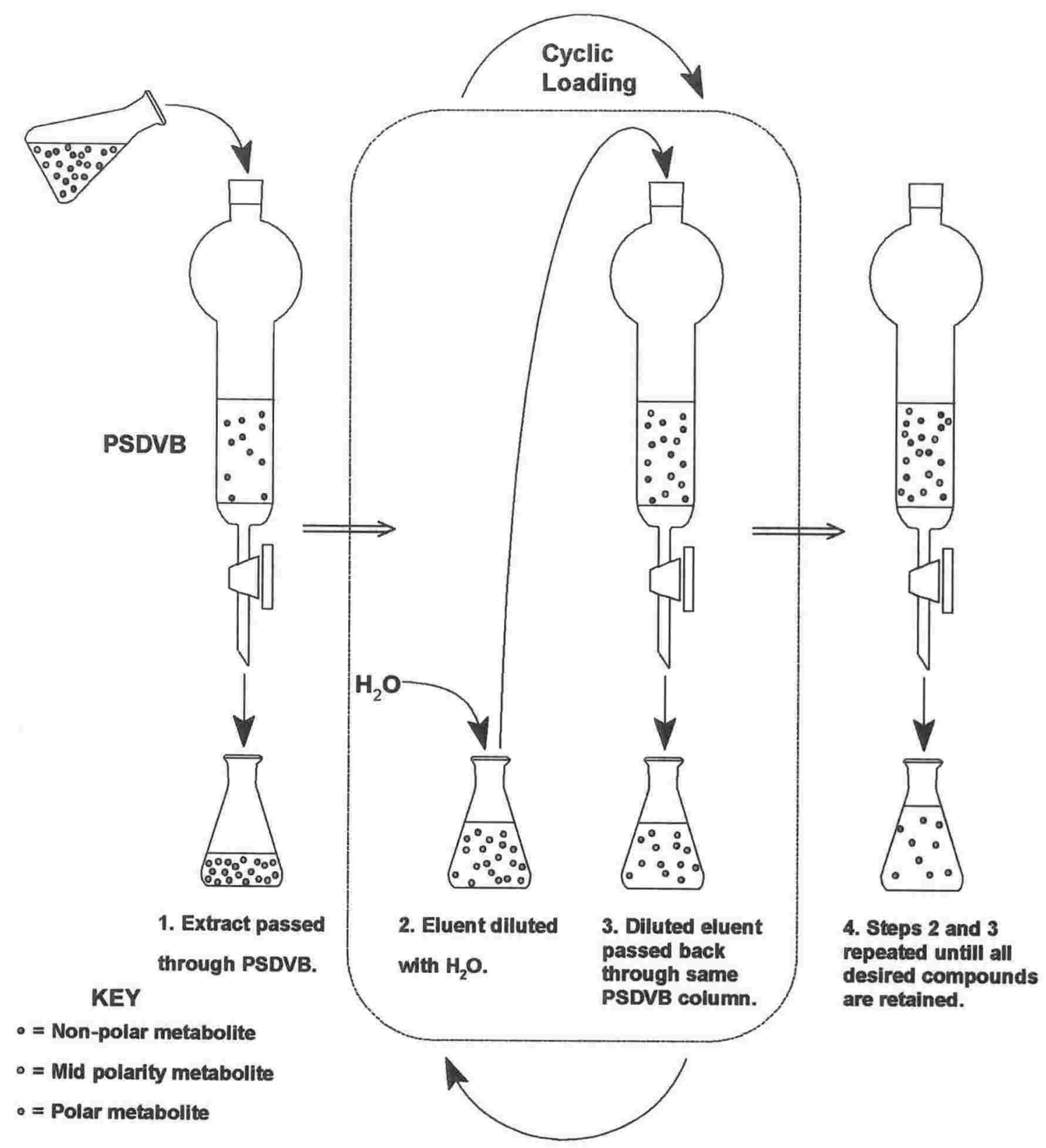

Figure 1.4 Diagrammatic representation of the cyclic loading process.

The disadvantage of increasing volume is far out-weighed by the advantages of the method. No concentration of the crude extract is required prior to loading. No liquid/liquid partition is performed. The solvents used $\left(\mathrm{MeOH}, \mathrm{Me}_{2} \mathrm{CO}, \mathrm{H}_{2} \mathrm{O}\right)$ are environmentally safe and inexpensive to supply in bulk. Finally, the method is easily scalable from analytical through to industrial scale. ${ }^{50,64}$ 
Once the column has been loaded, it is eluted using a stepped or gradient system of increasing organic solvent $\left(\mathrm{MeOH}\right.$ or $\left.\mathrm{Me}_{2} \mathrm{CO}\right)$ in $\mathrm{H}_{2} \mathrm{O} .{ }^{50}$ The majority of the unwanted mass of a crude extract is made up of polar salts, sugars and proteins, or non-polar fats and steroids, while most of the target biologically active secondary metabolites will be of mid-polarity, and so will elute between the polar and non-polar compounds from a reversed-phase support. This exploits the "mass window", the region of lowest eluted mass yet with the highest proportion of biologically active secondary metabolites

Conceptually, cyclic loading is the opposite of chromatography as the technique's aim is to change the polarity of the mobile phase to force target compounds to sequentially absorb to the stationary support. Fine separation is generally not achieved as the crude extract is loaded over the entire length of the column in a manner similar to VLC, reducing chromatographic resolution during elution. The aim of the technique, however, is not to achieve great chromatographic separation, but is to allow easy fractionation of a crude extract of a marine organism into a few discrete samples at the first stage of an isolation scheme, separate from the majority of unwanted compounds and therefore separate from the majority of the mass. The fractions containing the target metabolites can then be further purified using standard separation techniques.

Variations of the cyclic loading method have also been developed. Backloading is often used during an isolation procedure. Fractions eluted from reversed-phase chromatographic supports are usually mixtures of $\mathrm{H}_{2} \mathrm{O}$ and an organic solvent, which are difficult to concentrate as they often "bump" when placed under vacuum. To backload, the eluted fraction is cyclic loaded onto a smaller PSDVB column which can then be eluted with a small volume of pure organic solvent. This has the effect of concentrating the sample into a smaller volume of solvent that is easier to evaporate without bumping than the original $\mathrm{H}_{2} \mathrm{O}$ /organic solvent mix. ${ }^{50}$ Another common 
technique is to cyclic load a fraction onto a small amount of PSDVB which is then be transferred onto a larger column as a slurry. The loaded PSDVB can then be chromatographically separated in a similar way to Blunt and Munro's $\mathrm{C}_{18}$ method. ${ }^{50,54}$ These methods have become standard procedures used in Victoria University's Marine Natural Products Laboratory for the separation of crude extracts in both the screening of organisms, as well as in the fractionation of larger sample extracts. 


\section{Chapter Two}

\section{Sponge Screening and Results}

2.1 Initial Sponge Screening Methodology

Rather than relying upon external bioassays to identify sponges containing interesting secondary metabolites, West and Northcote instigated an in-house ${ }^{1} \mathrm{H}$ NMR protocol for the screening of semi-purified extracts. Of primary importance to the screening protocol was structural novelty, with biological activity a secondary consideration. It was assumed that novel structures often have novel activities that can be determined after identification, rather than using biological activity to track the isolation of a secondary metabolite. The protocol was required to be both cheaper and quicker than what was available through use of out-sourced bioassays. ${ }^{50}$ It has been noted previously that in-house methods are preferable to external assays for speed, economy, and accuracy. ${ }^{48}$

West and Northcote's method used cyclic loading to help semi-purify a crude sponge extract until a sufficient amount of the sample could be dissolved in a single solvent for analysis by ${ }^{1} \mathrm{H}$ NMR. Approximately $100 \mathrm{~g}$ of wet weight sponge was extracted with $\mathrm{MeOH}$. The extract was cyclic loaded onto PSDVB that was washed with $\mathrm{H}_{2} \mathrm{O}$ to remove any polar salts and carbohydrates. The column was then eluted with $30 \%$ $\mathrm{Me}_{2} \mathrm{CO} / \mathrm{H}_{2} \mathrm{O}, 75 \% \mathrm{Me}_{2} \mathrm{CO} / \mathrm{H}_{2} \mathrm{O}$, and finally $\mathrm{Me}_{2} \mathrm{CO}$. The $75 \% \mathrm{Me}_{2} \mathrm{CO}$ fraction was then backloaded onto a smaller PSDVB column and then eluted with $\mathrm{Me}_{2} \mathrm{CO}$. This sample was evaporated to dryness and analysed by ${ }^{1} \mathrm{H}$ NMR in $\mathrm{CDCl}_{3} .{ }^{50}$

It was assumed that the secondary metabolites most likely to be biologically active are of intermediate polarity and would generally elute from the PSDVB column in the $75 \% \mathrm{Me}_{2} \mathrm{CO} / \mathrm{H}_{2} \mathrm{O}$ fraction. If analysis of the ${ }^{1} \mathrm{H}$ NMR screen of a sponge indicated the 
presence of novel secondary metabolites within the extract, fractionation of a bulk sample extract of the sponge was initiated using NMR guided fractionation. Once a metabolite was isolated and its structure elucidated, it was submitted for biological evaluation in any bioassays available. The range of bioassays available to test pure compounds is far greater in number than those suitable for screening crude extracts, and the cost is generally lower due to the smaller number of samples submitted. Using this strategy, West isolated eight novel metabolites, seven clerodane diterpenes, one of which exhibited marked anti-inflammatory activity, and a biologically inactive sulfamic acid indolo $[3,2-a]$ carbazole. $^{50}$

Although West's protocol was successful for the screening and ${ }^{1} \mathrm{H}$ NMR guided fractionation of crude sponge extracts, it suffered from a limited spectral window. Most of the semi-purified extracts screened still contained large amounts of fats, steroids, and other primary metabolites, the signals of which obscured most other resonances from 0.5 to $5.5 \mathrm{ppm}$ in the ${ }^{1} \mathrm{H}$ NMR spectrum. As a consequence, all the metabolites that West detected contained olefins, furans, or aromatic rings, and so were readily observed in the deshielded (> $5.5 \mathrm{ppm})$ portion of the ${ }^{1} \mathrm{H}$ NMR spectrum. ${ }^{50}$ Many biologically active compounds will not possess these structural features, therefore they would not have any significant ${ }^{1} \mathrm{H}$ NMR signature in the spectral window available in West's protocol. The current study expanded upon West's method by utilising 2D NMR experiments (COSY and HSQC) to better identify interesting secondary metabolites in semi-purified sponge extracts.

\subsection{Revised Sponge-Screening Protocol}

A standard protocol was established in order to screen extracts of target sponges. The pre-screen semi-purification remained the same as that used by West. ${ }^{50}$ After 
semi-purification, the screen sample was analysed using three different NMR experiments; ${ }^{1} \mathrm{H}$, COSY and HSQC. Initially, all screens were performed using both $\mathrm{CDCl}_{3}$ and $\mathrm{CD}_{3} \mathrm{OD}$ as NMR solvents. Early results indicated that more resonances attributable to interesting secondary metabolites were observed in samples dissolved in $\mathrm{CD}_{3} \mathrm{OD}$ therefore the use of $\mathrm{CDCl}_{3}$ was abandoned in order to save time.

Once a suitable number of screens had been generated, all the results obtained were collated, and mask sheets showing the positions of correlations from common primary metabolite resonances were generated for both the COSY and HSQC spectra. For analysis, a COSY or HSQC spectrum generated from a sponge screen was overlaid on the relevant mask sheet, and the position of any novel signals identified. The ${ }^{1} \mathrm{H}$ resonances of a secondary metabolite that had the same chemical shift as those of a common primary metabolite would generally show novel correlations in the COSY and/or HSQC spectra. These correlations would allow for a unique spectral signature to be identified for the interesting metabolic product, irrespective of the primary metabolites present. Once all interesting correlations had been identified in the 2D NMR experiments, the results from the ${ }^{1} \mathrm{H}, \mathrm{COSY}$ and HSQC NMR spectra were considered. The screen was then assigned a rating based upon the novelty (position) of any unusual resonances and correlations, the number of novel signals observed, the amount of material present as estimated by the strength of the novel resonances, and also by the availability of the raw sponge material both in terms of frozen sponge available and ease of recollection.

If a sponge extract was deemed worthy of further investigation, the $30 \% \mathrm{Me}_{2} \mathrm{CO} / \mathrm{H}_{2} \mathrm{O}$ and $\mathrm{Me}_{2} \mathrm{CO}$ fractions from the screen were also analysed by NMR to identify the presence of interesting compounds of a more or less polar nature than those highlighted by the screen. This would also determine if the metabolites already identified had eluted 
into either of these two fractions, as knowledge of this could help optimise an isolation of the targeted compounds.

\subsection{Sponge-Screening Results}

During this study, 51 sponges were screened using the refined NMR based screening protocol described above (see table 2.1). All of the sponges were screened in conjunction with the National Institute of Water and Atmospheric research (NIWA). All organisms, including sponges, collected by NIWA as part of their marine natural products chemical bio-prospecting programme, are given a Marine Natural Products (MNP) number as a unique identifier. The sponges screened were collected from around the North Island of New Zealand, mainly from the Three Kings Islands and Northland, and also from around D'Urville Island on the northern tip of the South Island (see figure 1.1). Of the 51 sponges screened, 43 were not investigated further for a number of reasons. Many (29) did not appear to contain any compounds of particular interest, or any novel compounds present were in too low a concentration to allow for efficient isolation. Fourteen sponges were of moderate interest but were superseded by those of a higher rating. Eight sponges were selected for large-scale extraction. Unfortunately, the spectra generated from a large extract of one bore no resemblance to those of the original screen and so this sample was not further investigated. The remaining seven sponges are discussed below. 


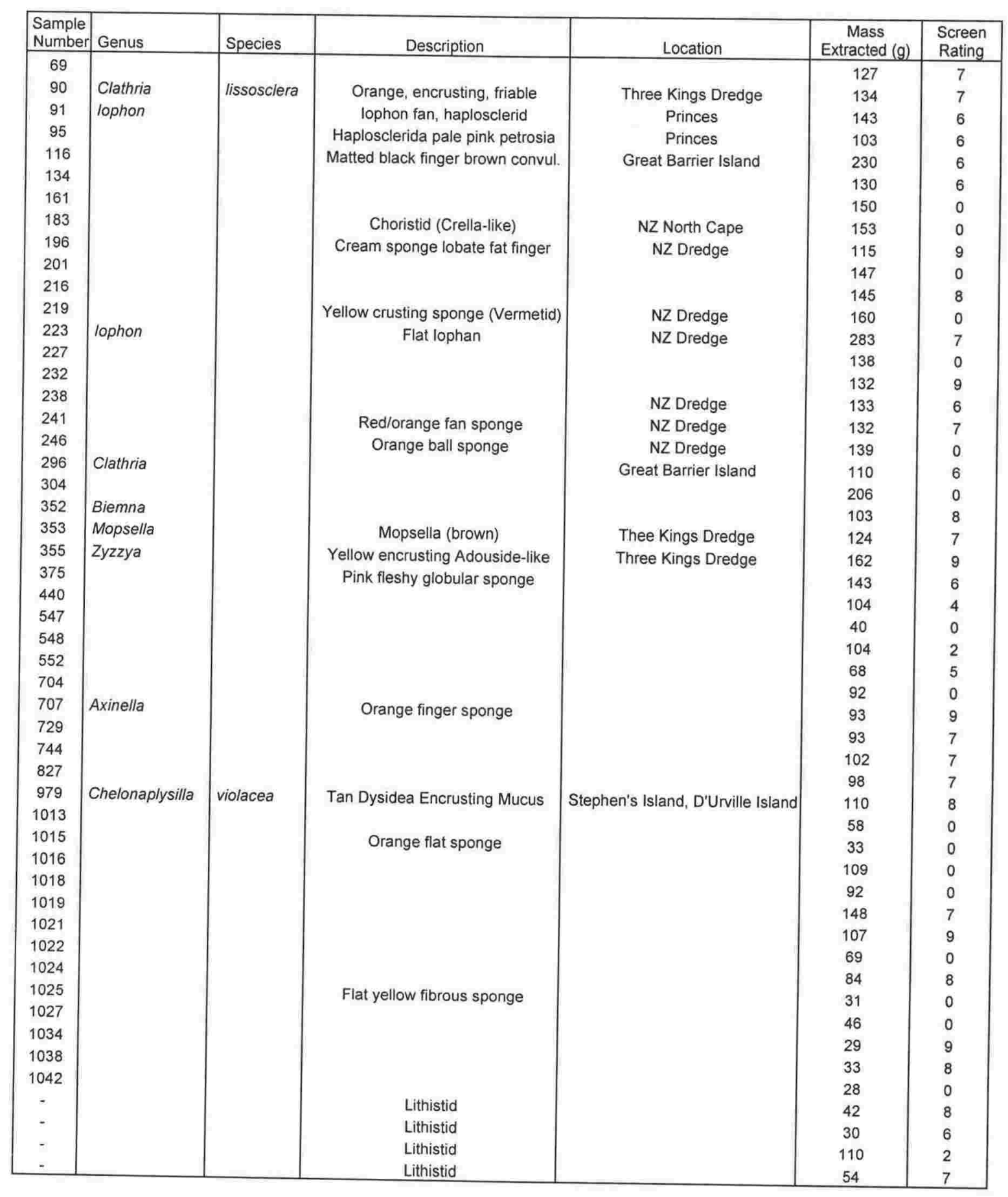

Table 2.1 Summary of the sponges screened during this study. 
An unidentified cream coloured lobate sponge that was collected by dredging near the Three Kings Islands. The initial screen indicated the presence of an aromatic compound from several deshielded HSQC correlations $\left(\delta_{C} 110-130, \delta_{H} 6.90-7.80\right)$. None of the aromatic resonances showed further COSY correlations to other parts of the spectrum. Several other weak correlations in the aliphatic region of the HSQC spectrum $\left(\delta_{\mathrm{C}} 30\right.$ $40, \delta_{\mathrm{H}} 1.00-2.10$ ) were also identified (see figure 2.1). An extract of a large sample of the sponge (426 g) was fractionated but unfortunately, the aromatic compound degraded before it could be purified.

\section{MNP0241}

An unidentified orange fan sponge collected by dredging from the northern tip of the North Island. Analysis of the screen indicated the presence of an olefin from two characteristic deshielded HSQC correlations $\left(\delta_{\mathrm{C}} 116\right.$ and 124, $\delta_{\mathrm{H}} 5.30$ and 5.60). Several oxygenated methines were also evident from other correlations in the HSQC spectrum $\left(\delta_{\mathrm{C}} 58-82, \delta_{\mathrm{H}} 3.50-4.20\right)$. These resonances showed significant couplings to resonances in other portions of the COSY spectrum (see figure 2.2). An extract of $779 \mathrm{~g}$ of the sponge was made and purification of the metabolite present attempted, however, the interesting compound degraded over time before it was pure enough to be characterised. A lack of more raw sponge material prevented further work to isolate the novel component observed in the original screen. 

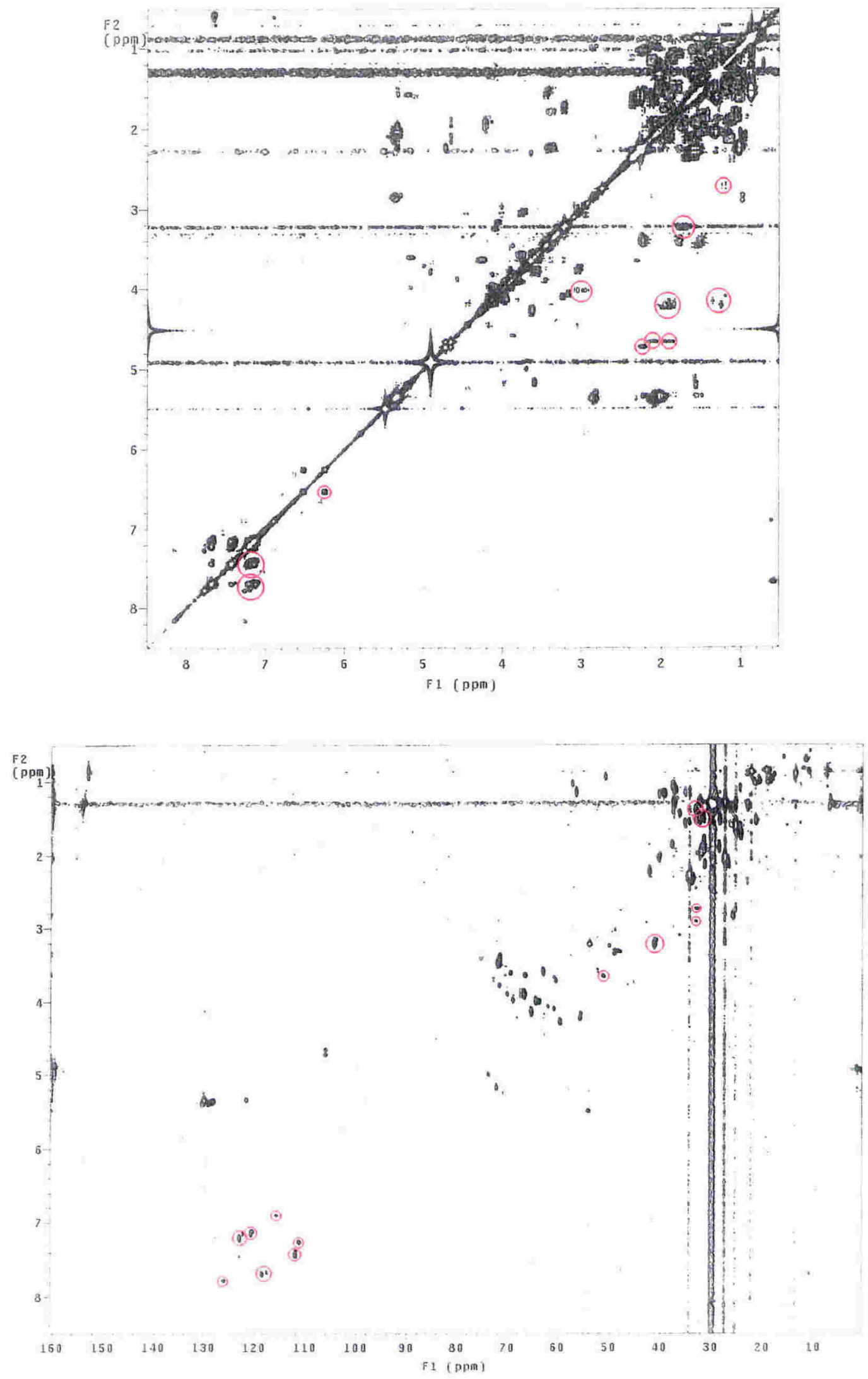

Figure 2.1 COSY and HSQC NMR screen of MNP0196

(Novel correlations circled in red). 

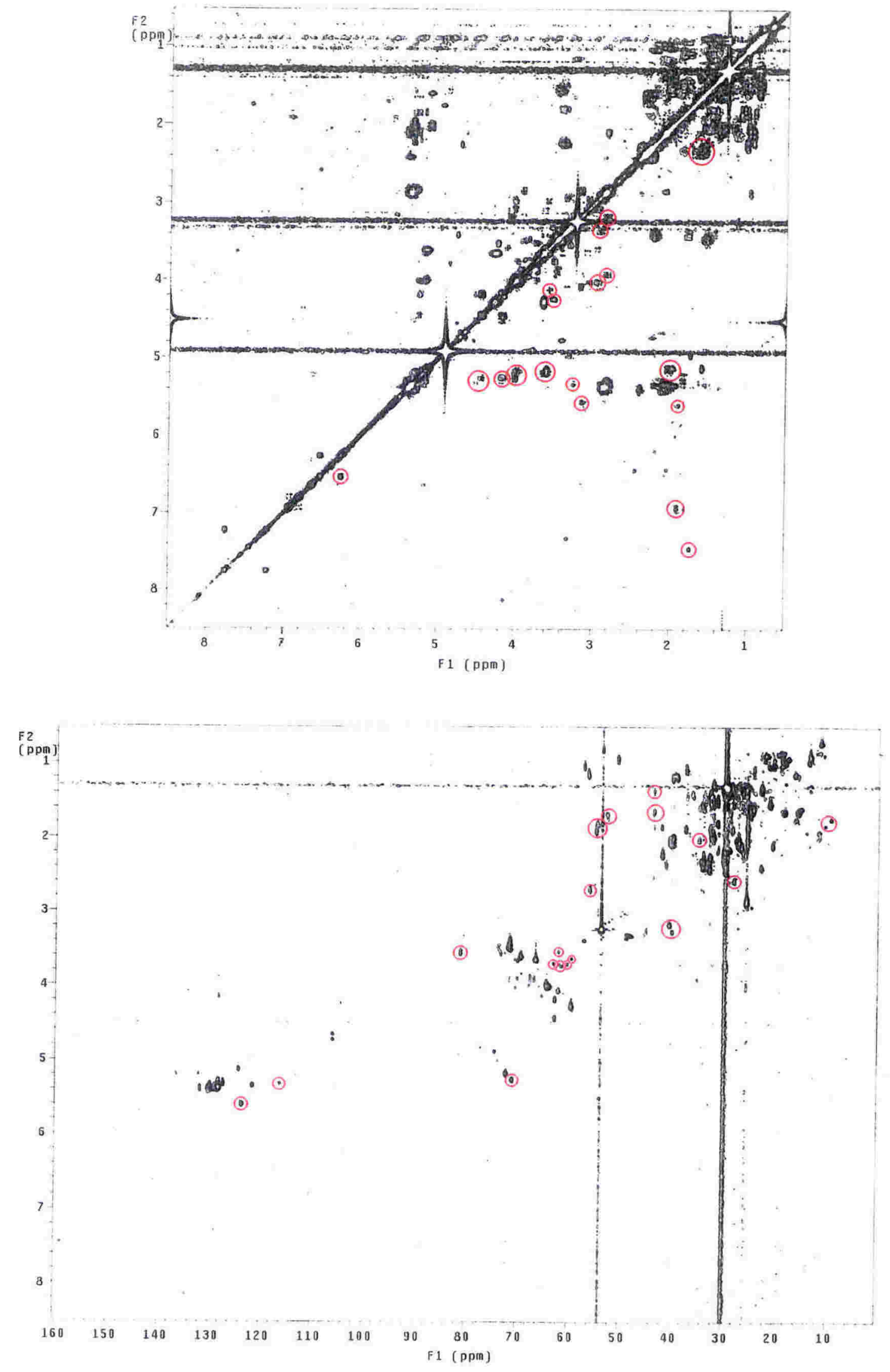

Figure 2.2 COSY and HSQC NMR screen of MNP0241. 
A sample of the sponge Biemna sp. collected from the northern part of the North Island. The sponge was described as massive with purple exterior and tan interior colouration. Analysis of the screen HSQC spectrum revealed interesting correlations from several oxygenated methines, possibly those of a carbohydrate $\left(\delta_{\mathrm{C}} 60-78, \delta_{\mathrm{H}} 3.00-4.00\right.$ and acetal/hemi-acetal $\left.\delta_{\mathrm{C}} 100, \delta_{\mathrm{H}} 4.85\right)$ as well as several ring junction methines $\left(\delta_{\mathrm{C}} 35-40, \delta_{\mathrm{H}} 1.00-1.50\right)$. This was supported by COSY correlations consistent with a carbohydrate moiety. Several of the carbohydrate resonances also coupled strongly to resonances in the aliphatic region $\left(\delta_{\mathrm{H}}\right.$ 1.50-2.10) of the COSY spectrum (see figure 2.3).

Extraction of $979 \mathrm{~g}$ of MNP0352 yielded the known steroid $5 \alpha, 8 \alpha$-epidioxy-6-ene-24- $R$ ethylcholesta-3 $\beta$-ol (7). ${ }^{65,66}$ The presence of a sphingolipid compound (8) was also suggested on the basis of characteristic deshielded NMR (oxymethine and amide) resonances observed. ${ }^{67-69}$ Isolation of this compound was stopped when it was deemed too difficult to separate the sphingolipid from any other lipid material in the sample. Compounds of this nature are difficult to follow in an NMR guided isolation as the methylene ${ }^{1} \mathrm{H}$ NMR envelope of the metabolites alkyl chain is indistinguishable from those of any fatty acids, triglycerides, or other long chain alkane derivatives present.

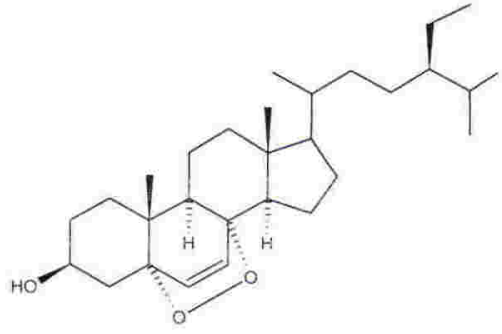

(7)<smiles>[R]OCC(NC([R3])=O)C([R])O</smiles>

(8) $\quad R_{1}=$ carbohydrate $\mathrm{R}_{2}=$ alkyl chain $\mathrm{R}_{3}=$ alkyl chain 

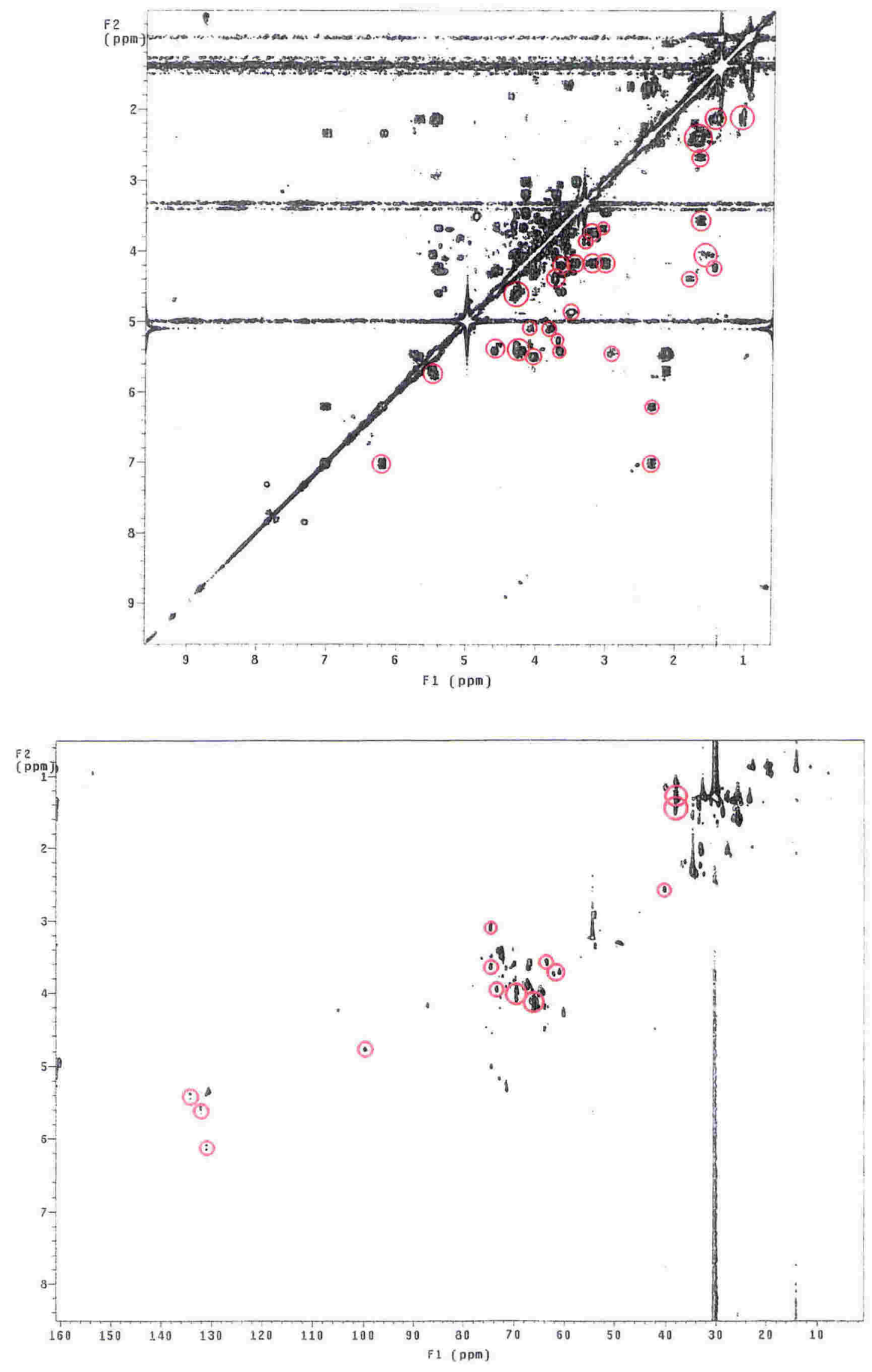

Figure 2.3 COSY and HSQC NMR screen of MNP0352. 


\section{MNP0355}

A sample of Zyzzya sp. dredged at the Three Kings Islands. Several notable HSQC correlations from the screen indicated the presence of several hetero-aromatic resonances $\left(\delta_{\mathrm{C}} 110-140, \delta_{\mathrm{H}} 6.80-8.40\right)$. Analysis of the strong COSY correlations from these aromatic resonances allowed construction of two individual spin systems. Several of the aromatic ${ }^{1} \mathrm{H}$ resonances showed further COSY correlations into the aliphatic region of the spectrum (see figure 2.5). Fractionation of an extract of a further $84 \mathrm{~g}$ of the sponge, in combination with the original screen of $162 \mathrm{~g}$, led to the isolation of a bis-indole compound containing three bromine atoms, similar in structure to the topsentin (9) or dragmicidin (10) families. ${ }^{70-74}$ Unfortunately, a suitable chemical formula could not be identified from analysis of the HRESIMS spectra, nor could the complete structure of the molecule be determined from the NMR data obtained, preventing the identification of the isolated metabolite.

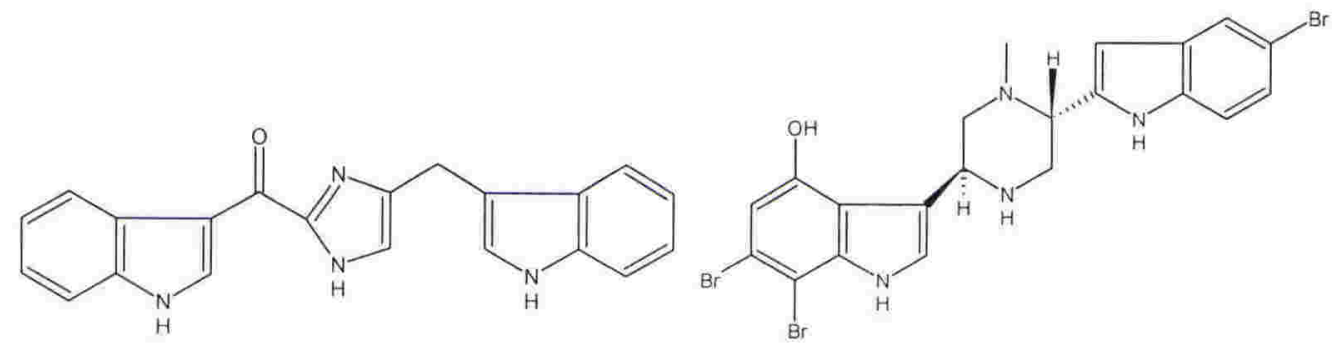

(9)

Two substructures identified for the brominated bisindole are shown below (see figure 2.4).
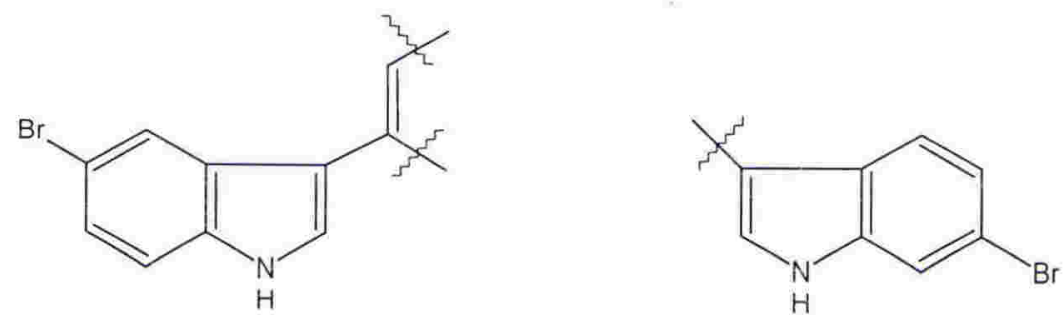

Figure 2.4 Substructures of a tribromobisindole compound isolated from Zyzzya sp. 

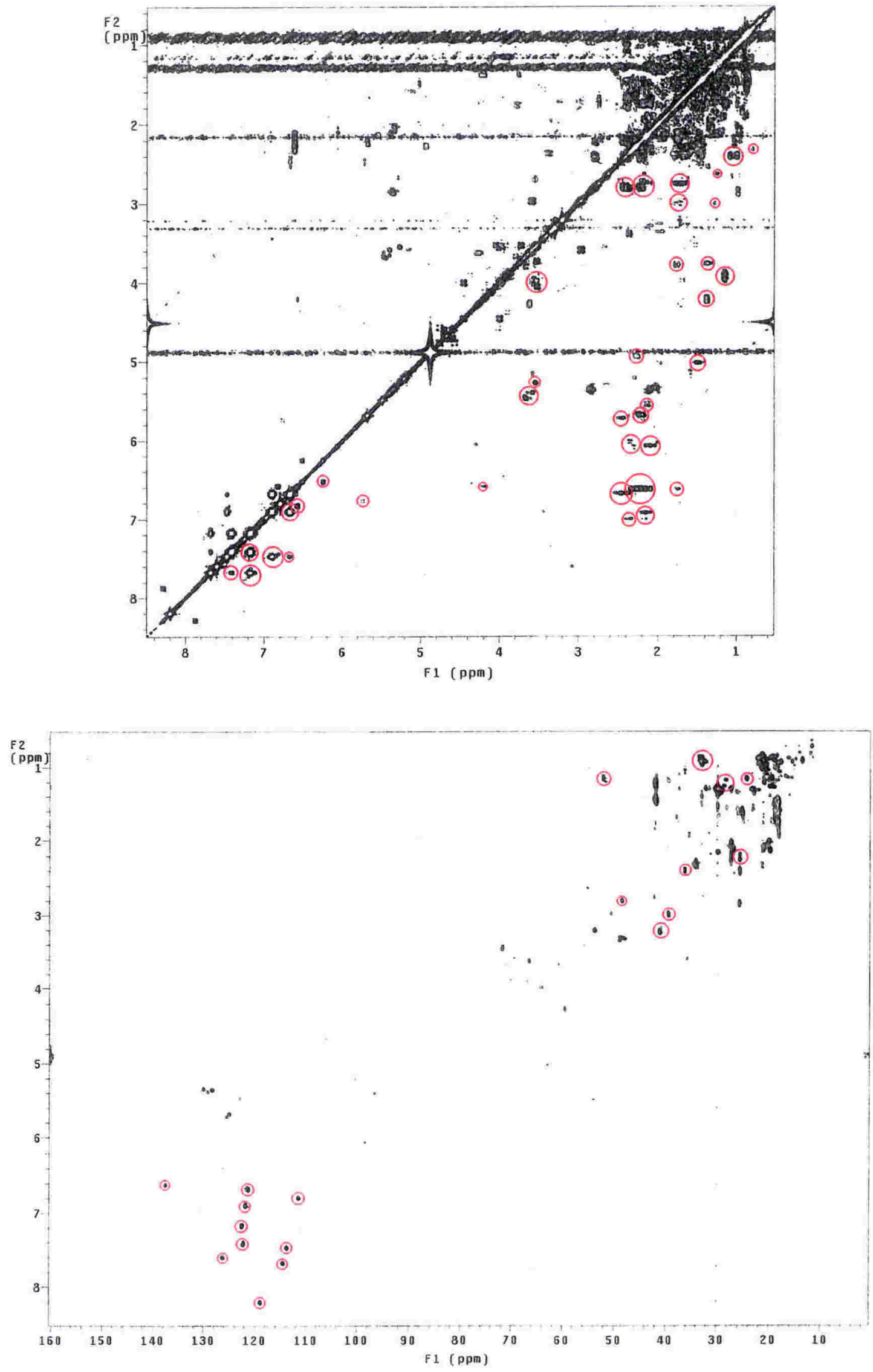

Figure 2.5 COSY and HSQC NMR screen of MNP0355. 
Bioassays carried out by NIWA on a crude extract of MNP0355 indicated strong anti-viral RNA activity, prompting further biological testing. A repeat assay on this crude extract, along with testing of semi-purified fractions of the isolated bisindole, and on further crude extracts of small samples of MNP0355, all returned negative results.

\section{MNP0707}

A sample of Axinella sp., collected by dredging from Spirits Bay, at the northern tip of the North Island. The initial screen of the sponge $(93 \mathrm{~g})$ was one of the most promising found using the revised screening protocol. The HSQC spectrum indicated the presence of a large variety of deshielded aromatic $\left(\delta_{\mathrm{C}} 105-135, \delta_{H} 6.20-7.60\right)$ and oxymethine $\left(\delta_{\mathrm{C}} 70-75, \delta_{\mathrm{H}} 3.70-4.10\right)$ resonances. Substantial numbers of couplings from these

${ }^{1} \mathrm{H}$ resonances were noted in the COSY spectrum (see figure 2.6).

Unfortunately, only a small amount of sponge $(59 \mathrm{~g})$ was available for extraction. The extract of this was combined with the original screen sample for further fractionation. Even when combined, this was less than optimal for an isolation procedure (ideally $>0.5 \mathrm{~kg}$ of sponge is extracted). Analysis of correlations in both the HSQC and COSY spectra of semi-purified fractions indicated the presence of a number of compounds belonging to the oroidin (11) family of compounds. ${ }^{75}$ The (10Z)- (12) and (10E)- (13) geometrical isomers of hymenialdisine were isolated from the sponge and their structures confirmed by comparison with published HRESIMS and NMR data. ${ }^{76,77}$ The NMR signatures of both $\mathbf{1 2}$ and $\mathbf{1 3}$ show a strong $p \mathrm{H}$ dependence, as they can exist in tautomeric forms depending on the degree of protonation that the metabolite exhibits. ${ }^{77}$ 

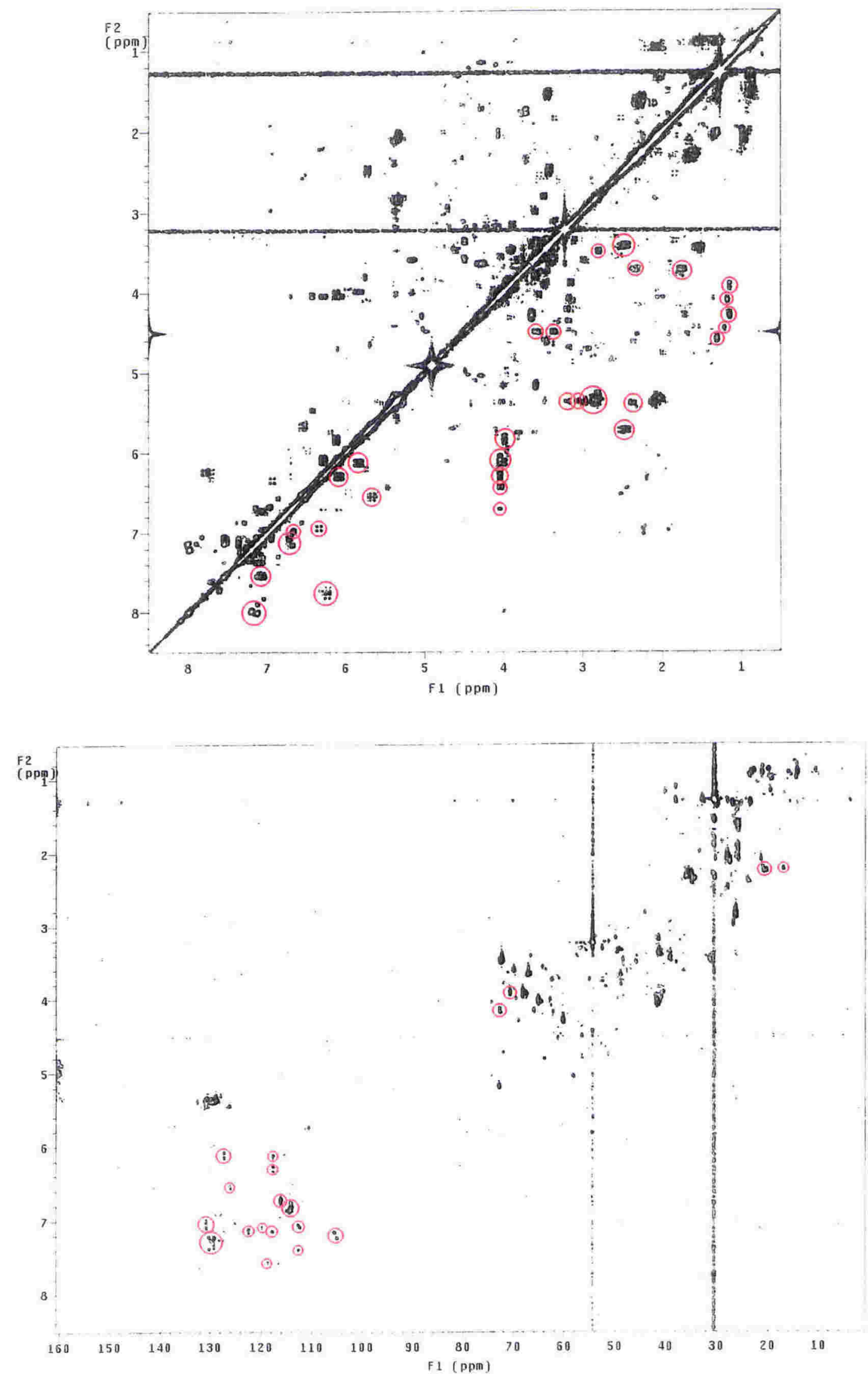

Figure 2.6 COSY and HSQC NMR screen of MNP0707. 
<smiles>NC/C=C/c1c[nH]c(NC(=O)c2cc(Br)c(Br)[nH]2)n1</smiles>

(11)

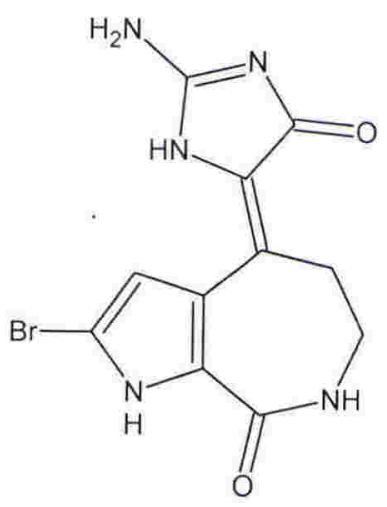

(12)

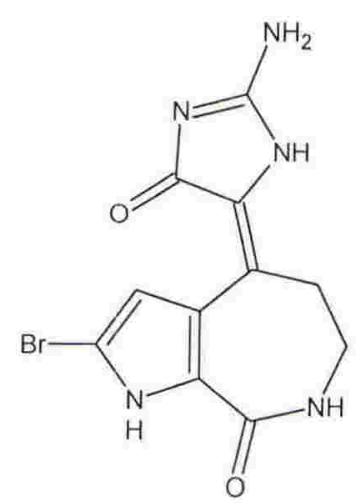

(13)

A further metabolite was also isolated with several NMR resonances similar to those observed for $\mathbf{1 2}$ and $\mathbf{1 3}$. Also, like $\mathbf{1 2}$ and $\mathbf{1 3}$, this compound suffered from a lack of COSY and HMBC correlations from which to assign its structure due to the highly proton deficient nature of the molecule. Compounds $\mathbf{1 2}$ and $\mathbf{1 3}$ are especially proton deficient in the conjugated heterocyclic portion of the molecules. As a consequence, the structure of the third metabolite could not be elucidated using the spectra obtained. Several attempts were made to produce crystals suitable for a single crystal x-ray structure analysis, as the identities of $\mathbf{1 2}, \mathbf{1 3}$, and other related metabolites had been determined in this way. ${ }^{76,78,79}$ Unfortunately none yielded crystals amenable to analysis by this method. 


\section{MNP0090}

A sample of the orange friable sponge Clathria lissosclera, collected at the Three Kinds Islands, was screened. Novel resonances observed in the HSQC spectrum included those of several oxygenated methines $\left(\delta_{\mathrm{C}} 62-80, \delta_{\mathrm{H}} 3.00-4.20\right)$ as well as several that appeared to be from the ring junction methines and the ring methylenes of a steroid $\left(\delta_{\mathrm{C}} 45-55, \delta_{\mathrm{H}} 0.90-1.50\right)$. Also observed were several weak deshielded correlations from a series of aromatic resonances $\left(\delta_{C} 115-135, \delta_{H} 6.60-8.30\right)$ (see figure 2.7). An extract of $610 \mathrm{~g}$ of the sponge was fractionated to yield two novel anti-inflammatory sterols (32) and (33). This work is detailed further in chapter three.

\section{MNP0979}

An extract of a tan encrusting sponge of the genus Chelonaplysilla, collected using SCUBA at Stephen's Island, Marlborough, gave the most interesting screen encountered during this study, with the largest number of novel correlations in both the HSQC and COSY spectra. Several correlations attributable to acetal or hemi-acetal resonances were present $\left(\delta_{\mathrm{C}} 100-110, \delta_{\mathrm{H}} 4.90-5.20\right)$, as were those of multiple oxymethines $\left(\delta_{\mathrm{C}} 70-75, \delta_{\mathrm{H}} 3.30-4.00\right)$. Also of interest were correlations relatively shielded in the carbon dimension $\left(\delta_{\mathrm{C}} 40-55\right)$ yet deshielded in the proton $\left(\delta_{\mathrm{H}} 4.00-4.20\right)$. Strong COSY correlations were found from all of the previously mentioned ${ }^{\mathrm{I}} \mathrm{H}$ resonances to all other parts of the spectrum (see figure 2.8). An extract of $182 \mathrm{~g}$ of the sponge was fractionated to yield several novel metabolites based upon the rearranged spongian diterpene skeleton (138-143). The isolation and structural elucidation of these compounds is dealt with in chapter four. 

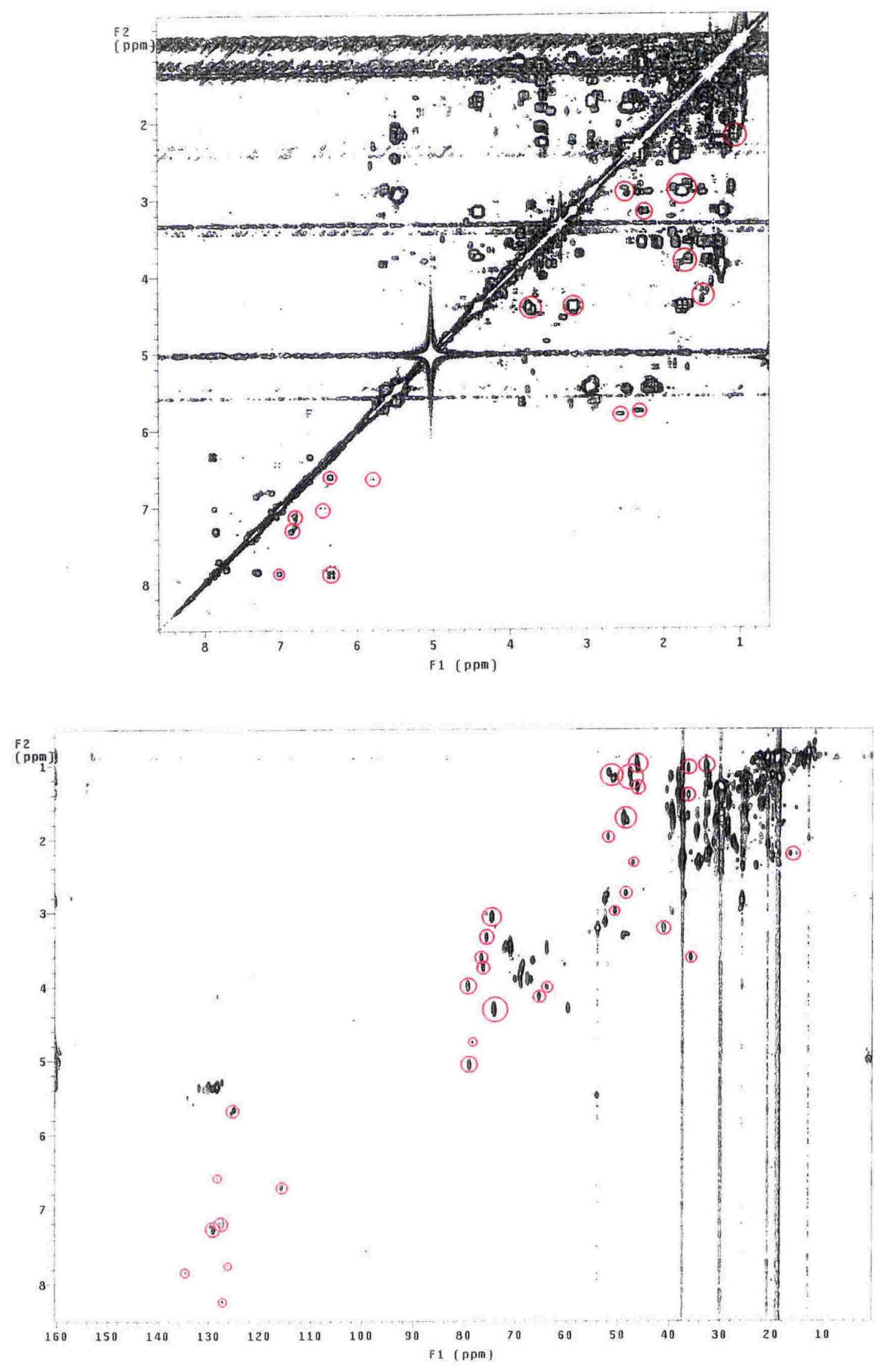

Figure 2.7 COSY and HSQC NMR screen of MNP0090. 

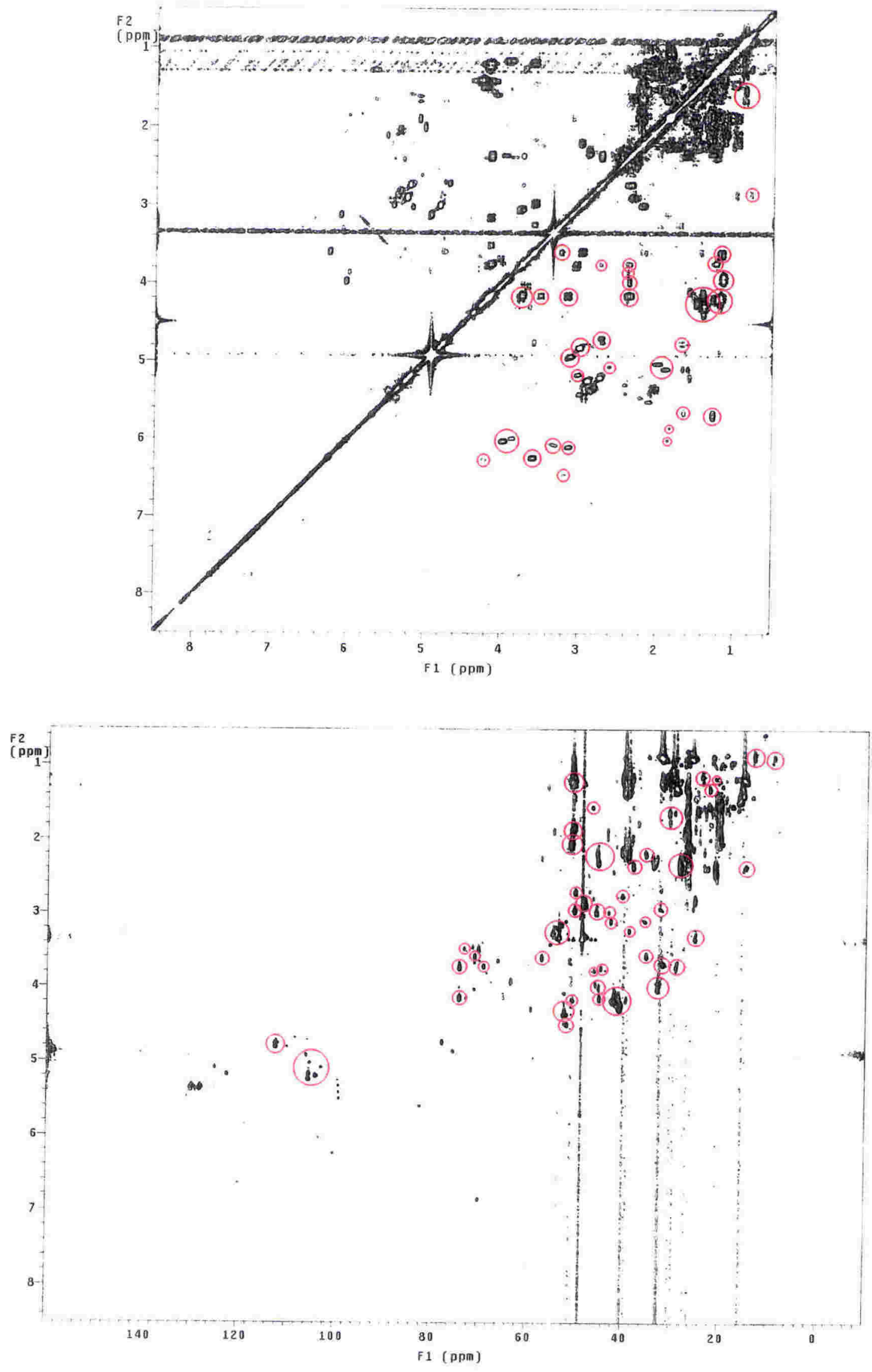

Figure 2.8 COSY and HSQC NMR screen of MNP0979. 
The isolation of several metabolites without significant deshielded ${ }^{1} \mathrm{H}$ resonances highlights the importance of using NMR experiments that will reveal signals that would otherwise be masked by the resonances of common primary metabolites. This has been achieved by using 2D COSY and HSQC spectra, and by developing masks of primary metabolite signals, which are used to readily identify the position of any other novel correlations present. The use of $\mathrm{CD}_{3} \mathrm{OD}$, rather than $\mathrm{CDCl}_{3}$, has led to the isolation of several metabolites of a more polar nature than those West isolated. Whether the majority of metabolites found using this revised screening method are more likely to be structurally novel and/or biologically active than those using West's original protocol remains to be seen.

Subsequent development of the screening protocol described here has included addition of an internal standard to the NMR sample to help quantify the approximate amounts of any novel compounds present, and also a refinement of the criteria used to select and prioritise sponges for large-scale extraction. Further work searching spectral databases, and potential use of other spectroscopic techniques including MS, will further enhance the protocol to be able to identify known compounds, and to identify the most promising sponge extracts for further investigation.

One important aspect of the refined NMR screening method used during this study is the relationship between a screen, the fractionation of the target compounds, and the guidance of an isolation procedure. Guidance of an isolation is obviously carried out using NMR after each step in isolation. Any resonances highlighted in the screen should be easily identified in subsequent fractions generated after various stages of purification. The purification procedures themselves represent the application of accepted, robust, 
methods for isolating compounds from crude extracts and semi-purified fractions. What is not immediately obvious with the current method is which sponges are worthy of further investigation. The crucial part of the screening process is to rapidly identify sponges containing interesting secondary metabolites, in order to select which samples are worthy of further detailed investigation. 


\section{Clathriols A and B: Novel polyoxygenated $14 \beta$ sterols}

\section{isolated from the sponge Clathria lissosclera}

\subsection{Steroid Biosynthesis}

Steroids, which can be regarded as very highly modified terpenes, are common metabolites found in all eukaryotic organisms. The steroid biosynthetic pathway has been extensively studied and can be summarised as follows. The triterpene precursor squalene (14), is produced by the unusual tail-to-tail coupling of two farnesyl pyrophosphate molecules. ${ }^{13,80}$ Squalene (14) is then enzymatically epoxidised by squalene oxidase at one of the two terminal alkenes. The epoxide is protonated, and then opened via nucleophilic attack by the olefin six carbons away. This yields a carbocation intermediate which undergoes a series of ring closures that in-turn, is followed by several 1,2-hydride and 1,2-methyl shifts to form the tetracyclic triterpene lanosterol (15) (see scheme 3.1). It is thought that both the cyclizations and the carbocation rearrangements are carried out in a concerted fashion and not as stepwise processes. ${ }^{13,80}$

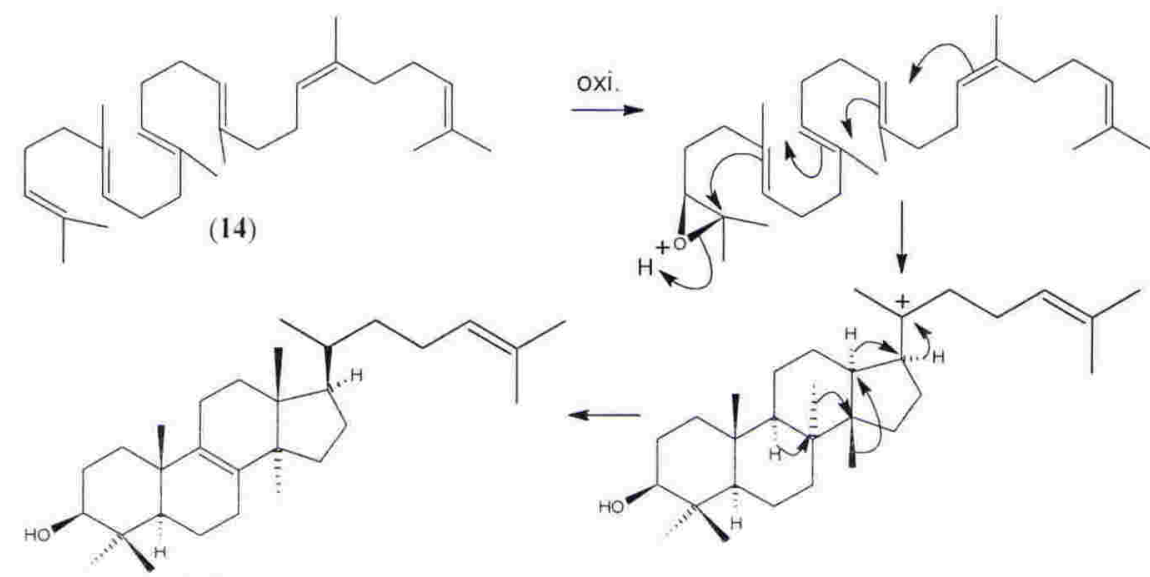

(15)

Scheme 3.1 Biosynthesis of lanosterol (15). ${ }^{13,80}$ 
Hydrogenation of the side chain of lanosterol (15) is followed by oxidation of one of three methyls to a hydroxymethyl and then to a formyl group. The formyl group is then lost as methanoic acid, to form a demethylated product. Similar demethylation occurs twice more. Isomerization of the $\Delta^{8,9}$ olefin to a $\Delta^{5,6}$ olefin finally forms the archetypical steroid cholesterol (16) (see scheme 3.2). ${ }^{13}$
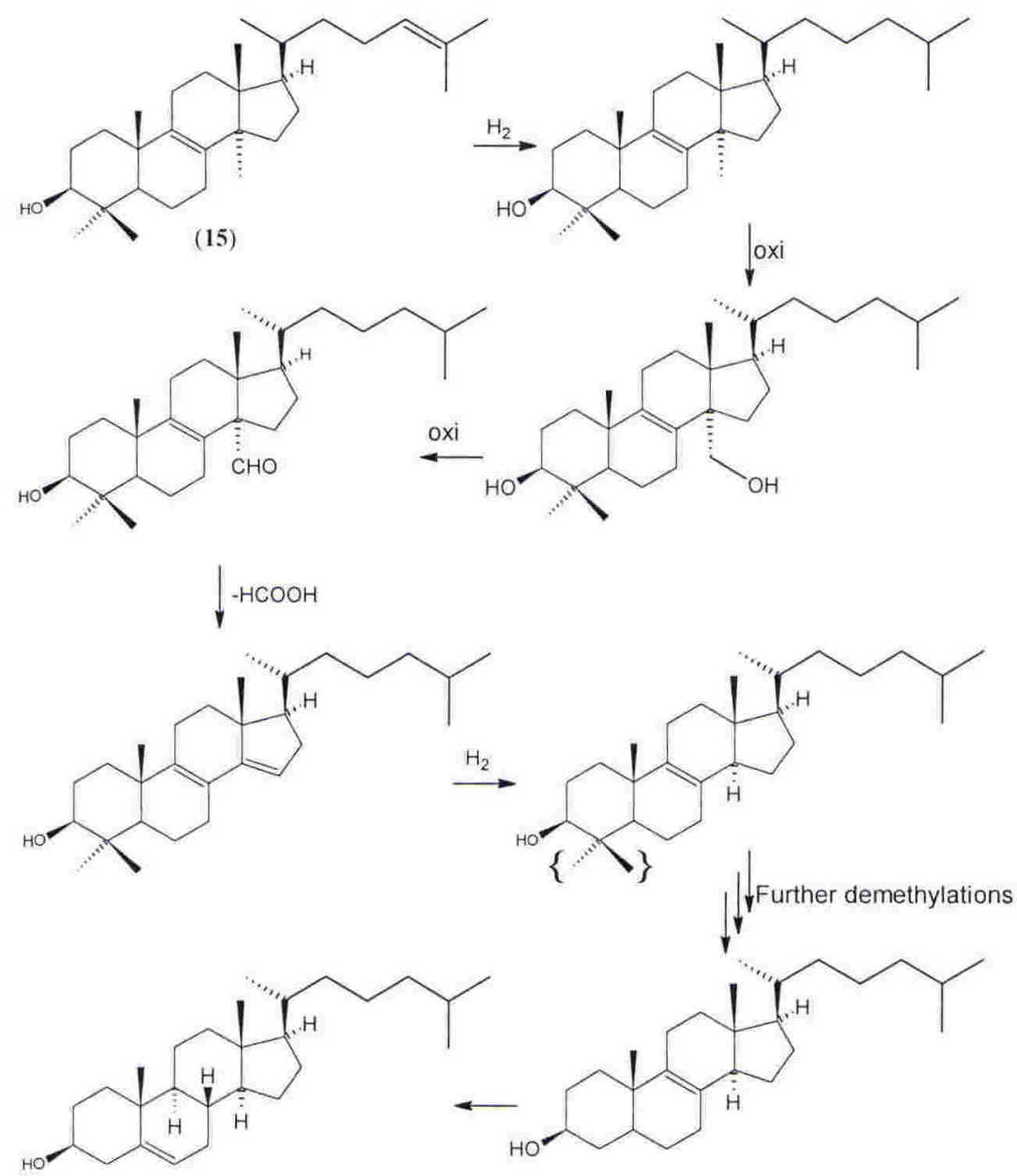

(16)

Scheme 3.2 Oxidative demethylation of lanosterol (15) to form cholesterol (16). ${ }^{13}$

Common derivatives of the basic steroid skeleton, exemplified by $\mathbf{1 6}$, include oxidation and alkylation of both the tetracyclic and side-chain portions of the molecule. Alkylation is believed to occur through stepwise methylation by the biological methyl-group donor, $S$-adenosylmethionine (17), in a reaction involving nucleophilic attack of an olefin upon the activated methyl of the sulfonium group of $\mathbf{1 7}$, followed by a 1,2-hydride shift, before loss of a proton to form a new double bond. Methyl and ethyl 
derivatives are commonly found, although examples of up to pentyl extensions are known (see scheme 3.3). ${ }^{81}$ Biological oxidation is generally carried out by enzymes of the cytochrome $P_{450}$ family, although other enzymatic pathways can also be used. ${ }^{13}$<smiles>CCCCCCCC(N)C(=O)O</smiles><smiles>CCC(C)=C(C)CCC(C)(C)C</smiles><smiles>CCCCCCCCCC[Si+](C)(C)CC(N)C(=O)O</smiles><smiles>CC(C)CCC(C)C(C)C</smiles><smiles>CC=C(C(C)C)C(C)CC(C)(C)C</smiles><smiles>CCC(C)C(CC)CC</smiles><smiles>CCC(/C=C/C(C)C)C(C)C</smiles>

Scheme 3.3 Alkylation of steroid side chains. ${ }^{13,81}$

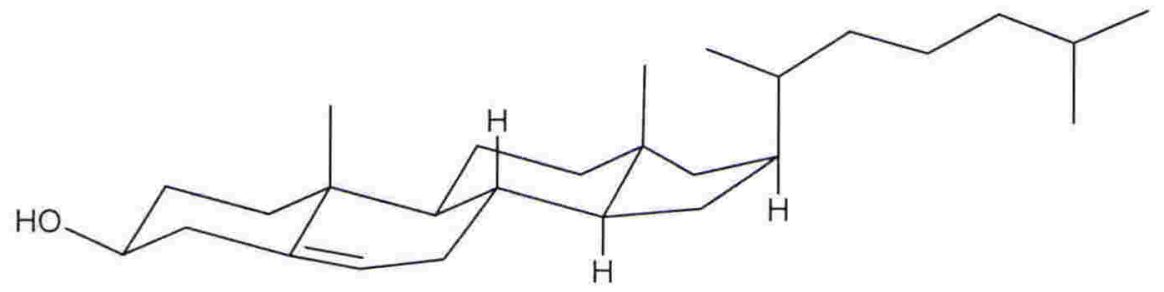

Figure 3.1 Stereochemistry of cholesterol $(\mathbf{1 6}){ }^{80}$

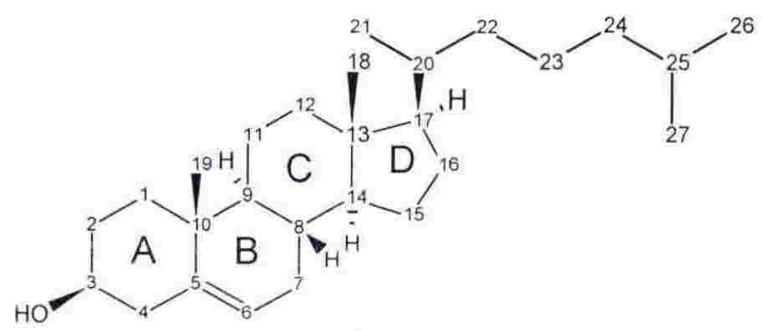

(16) 


\section{2}

\section{Sponge Sterols}

Following the pioneering research of Djerassi in the 1970 s, sponges have proven to be a rich source of interesting steroids. Many recent examples have exhibited both novel biological activities and structural diversity with unusual side chains and varied functionalities. ${ }^{81-83}$ Some researchers believe that sponges may provide the widest steroid diversity in the whole animal kingdom. ${ }^{82,84}$

Aragusterol A (18) was isolated from an Okinawan sponge, Xestospongia sp.. Sterol 18 exhibited very strong in vitro activity, inhibiting cell proliferation with sub-nanomolar range activities against a variety of mammalian cancer cell lines. It also exhibited potent in vivo activity against tumours in mice. ${ }^{85}$ Aragusterols B (19) and D (20) were later reported from the same sponge. Bioassays revealed that 19 showed reduced activity when compared to $\mathbf{1 8}$ whilst $\mathbf{2 0}$, surprisingly, showed no activity at all. ${ }^{86}$

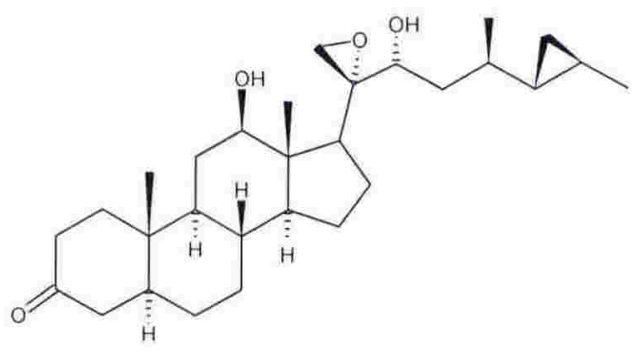

(18)

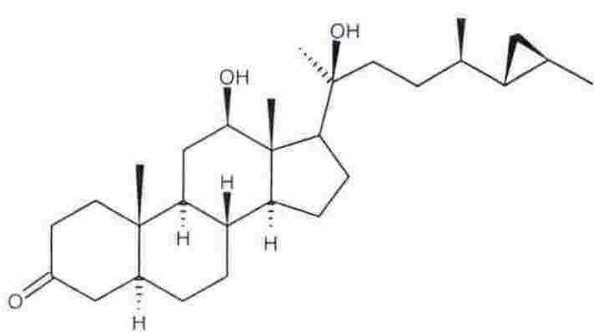

(19)

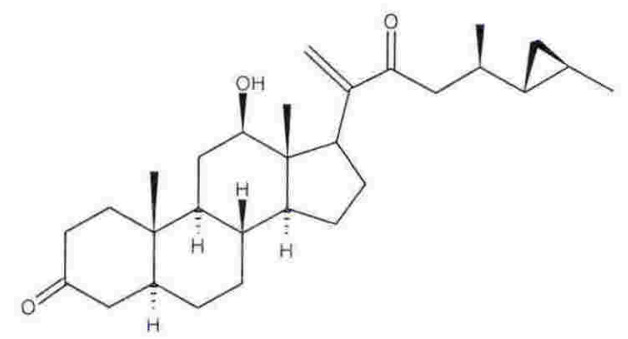

(20) 
Two novel cytotoxic sterols were isolated from the Tunisian sponge Dysidea incrustans. Incrustasterols A (21) and B (22) exhibited nanomolar range activity against a series of human tumour cell lines. ${ }^{87}$

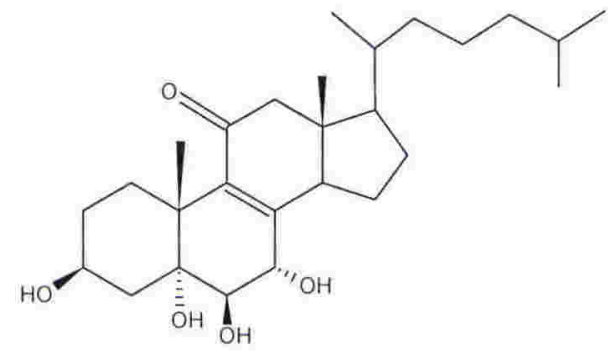

(21)

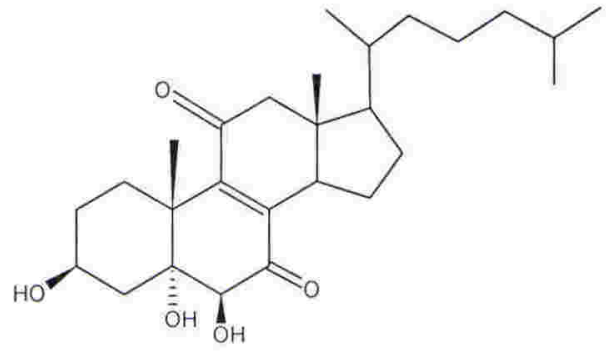

(22)

Agosterol A (23) was isolated from a sample of Spongia sp.. This sterol acetate possesses activity that reverses Multidrug Resistance (MDR) in tumour cells, a desirable effect for improving the efficacy of cancer chemotherapy agents. After treatment with 23, KB-C2 and KB-CV60 cancer cells showed a complete reversal of drug resistance to colchicine and vincristine respectively, making 23 a strong candidate for further clinical investigations. ${ }^{88,89}$

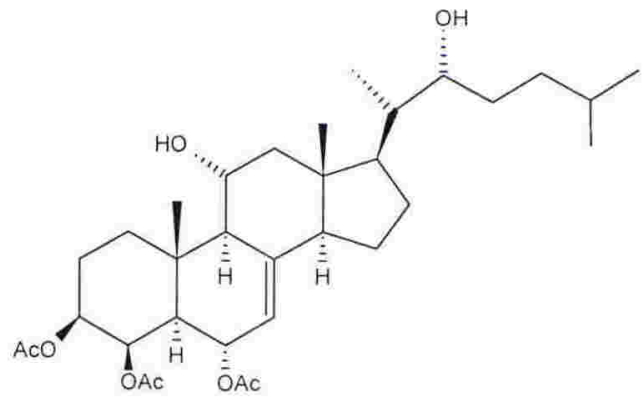

(23)

\section{$3.3 \quad 14 \beta$ Sterols}

In 1992, Andersen and Burgoyne isolated a unique sterol from the sponge Petrosia contignata. Contignasterol (24) is the first example of a naturally occurring sterol where rings $\mathrm{C}$ and $\mathrm{D}$ are fused in a cis fashion, leading $\mathrm{H}-14$ to be in the "unnatural" $\beta$ 
orientation. Equilibration studies on semi-synthetic 15-keto sterols have shown that $14 \beta$ sterols are more stable than their $14 \alpha$ epimers. ${ }^{90,91}$ Contignasterol (24) showed strong anti-inflammatory activity, inhibiting histamine release in rat peritoneal mast cells $\left(\mathrm{IC}_{50}=0.8 \mu \mathrm{M}\right)$. As well, 24 inhibited tracheal contractions in ovalbumin-sensitised guinea pig tracheal rings in a dose dependent manner, indicating its potential use as an anti-asthmatic agent. The absolute stereochemistry of $\mathbf{2 4}$ was recently published. ${ }^{92-95}$

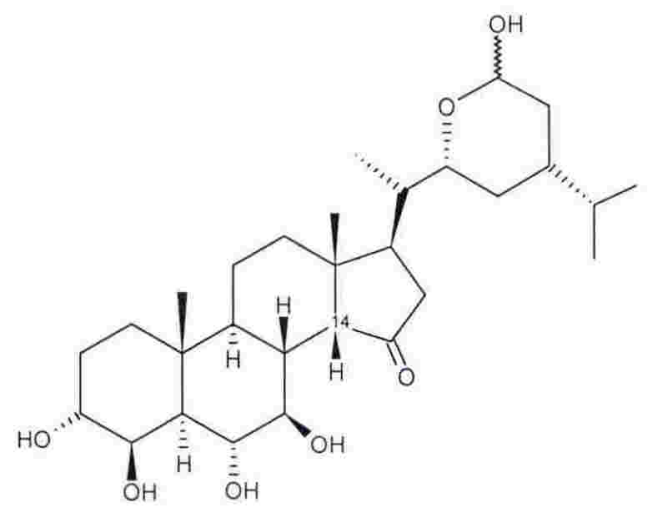

(24)

Shortly after the publication of $\mathbf{2 4}$, two more $14 \beta$ sterols were reported from the Okinawan sponge Xestospongia bergquista. Xestobergsterols A (25) and B (26) exhibited potent inhibition of histamine release in rat peritoneal mast cells $\left(\mathrm{IC}_{50}=0.05\right.$ and $0.10 \mu \mathrm{M}$ respectively). ${ }^{96}$ The structures of $\mathbf{2 5}$ and $\mathbf{2 6}$ were later revised, after they were reisolated along with xestobergsterol C (27) from an Okinawan Irincia sp.. This study also identified the absolute stereochemistry of $\mathbf{2 5}$ by the cd chiral exciton method. Cytotoxic activity was noted for both 25 and 27 against murine leukaemia cells. ${ }^{97}$ 


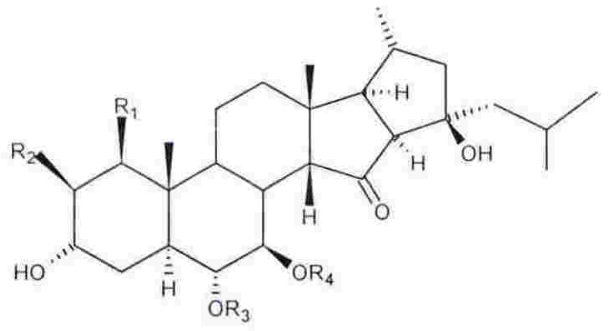

(25) $\mathrm{R}_{1}=\mathrm{H}, \mathrm{R}_{2}=\mathrm{OH}$

(26) $\mathrm{R}_{1}=\mathrm{R}_{2}=\mathrm{H}$

(27) $\mathrm{R}_{1}=\mathrm{R}_{2}=\mathrm{OH}$

A new $14 \beta$ sterol, haliclostanone sulfate (28), was isolated, along with the known metabolite halistanol sulfate (29), from a Malaysian Haliclona sp.. ${ }^{98}$

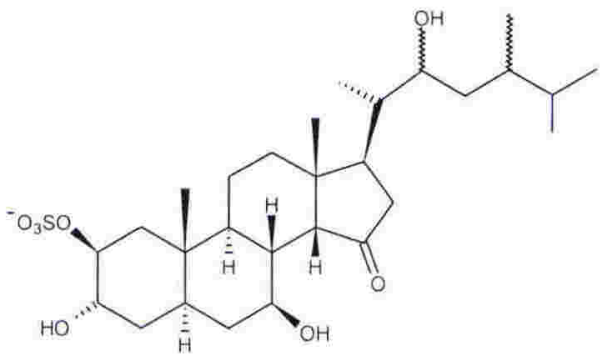

(28)

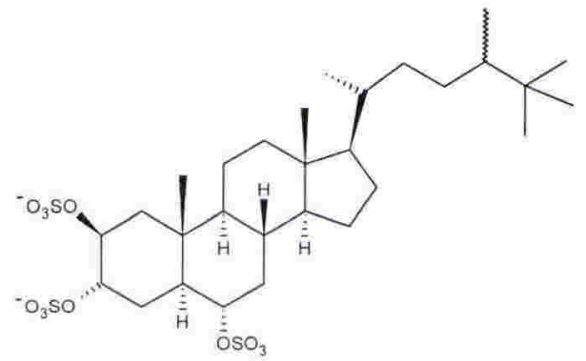

(29)

The $14 \alpha(30)$ and $14 \beta(31)$ epimers of the same sterol, tamosterone sulfate, were isolated from a Micronesian sponge of a new genus within the haplosclerid family Oceanapiidae. This is the first example of the co-isolation of both C-14 epimers of the same sponge sterol. ${ }^{99}$

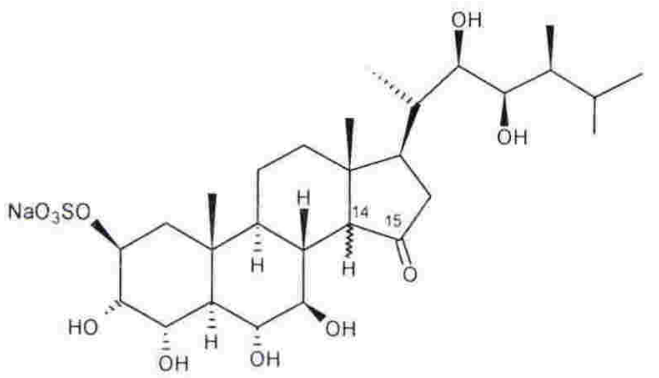

(30) $14 \alpha$

(31) $14 \beta$ 
All six reported naturally occurring $14 \beta$ steroids have been isolated from marine sponges although there are many examples of synthetic $14 \beta$ sterols. All naturally occurring $14 \beta$ sterols have a ketone at C-15, potentially offering an enol-stabilised carbanion route for the epimerisation of $\mathrm{C}-14$.

\subsection{Clathria lissosclera}

During the course of routine NMR based sponge screening, it was noted that an extract of the sponge Clathria lissosclera (MNP0090), dredged from $100 \mathrm{~m}$ at the Three Kings Islands, Northland, New Zealand (see figure 3.2), showed correlations indicative of several oxygenated methines in both the COSY and HSQC NMR spectra (see chapter two). A subsequent $\mathrm{MeOH}$ extract of $610 \mathrm{~g}$ of $C$. lissosclera, fractionated using repeated reversed-phase chromatography, yielded the novel sterol clathriol A (32) (26.2 mg). ${ }^{100}$ Further analysis of the side fractions generated during the isolation of $\mathbf{3 2}$ indicated the presence of several more related sterols. Further purification using both size-exclusion and repeated normal-phase chromatography, resulted in the isolation of a second novel sterol, clathriol B (1.3 mg) (33) (see scheme 3.3). ${ }^{101}$ 


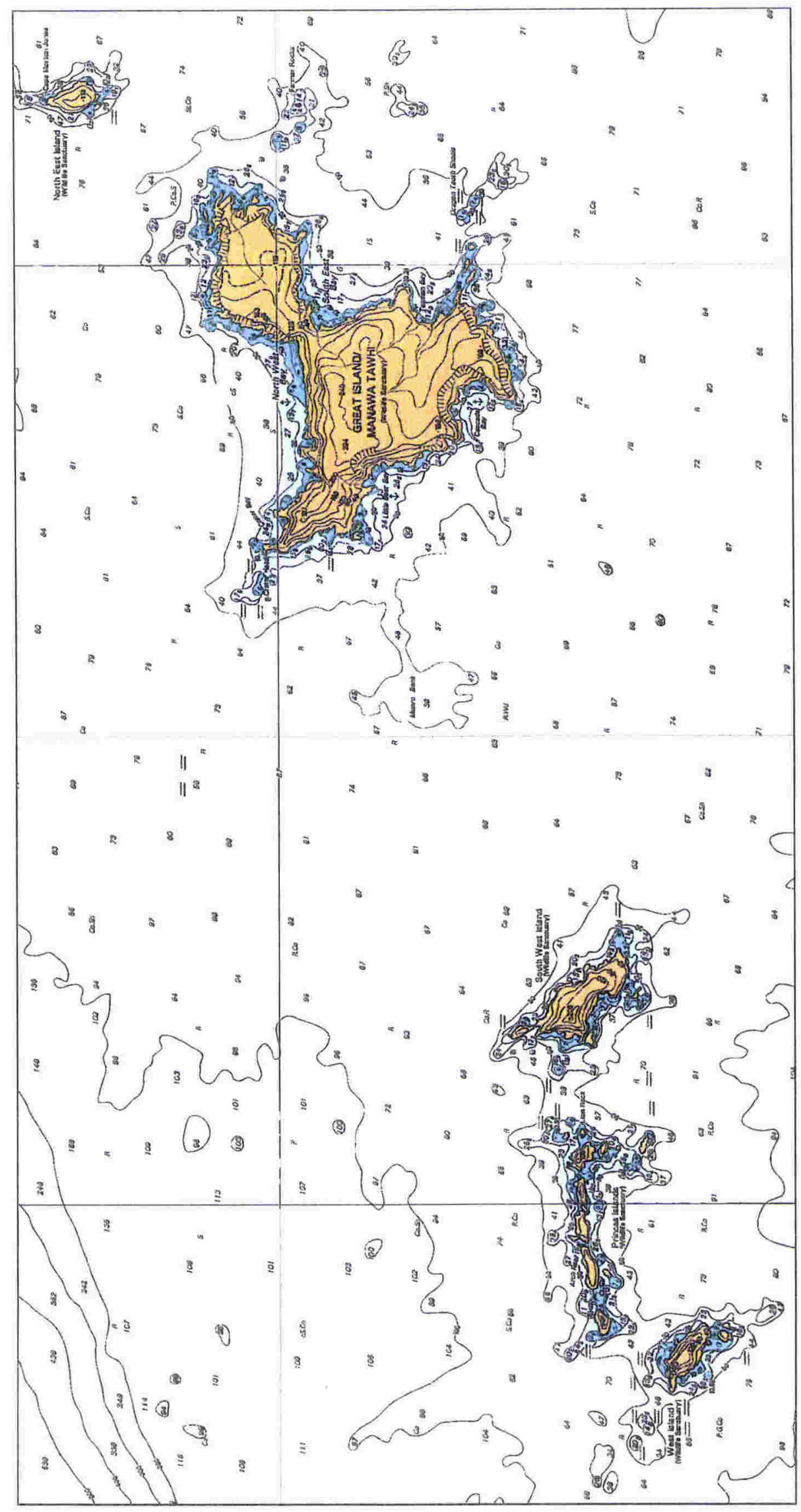

Figure 3.2 Hydrographic chart of the Three Kings Islands. (Courtesy of Land and Information New Zealand) 


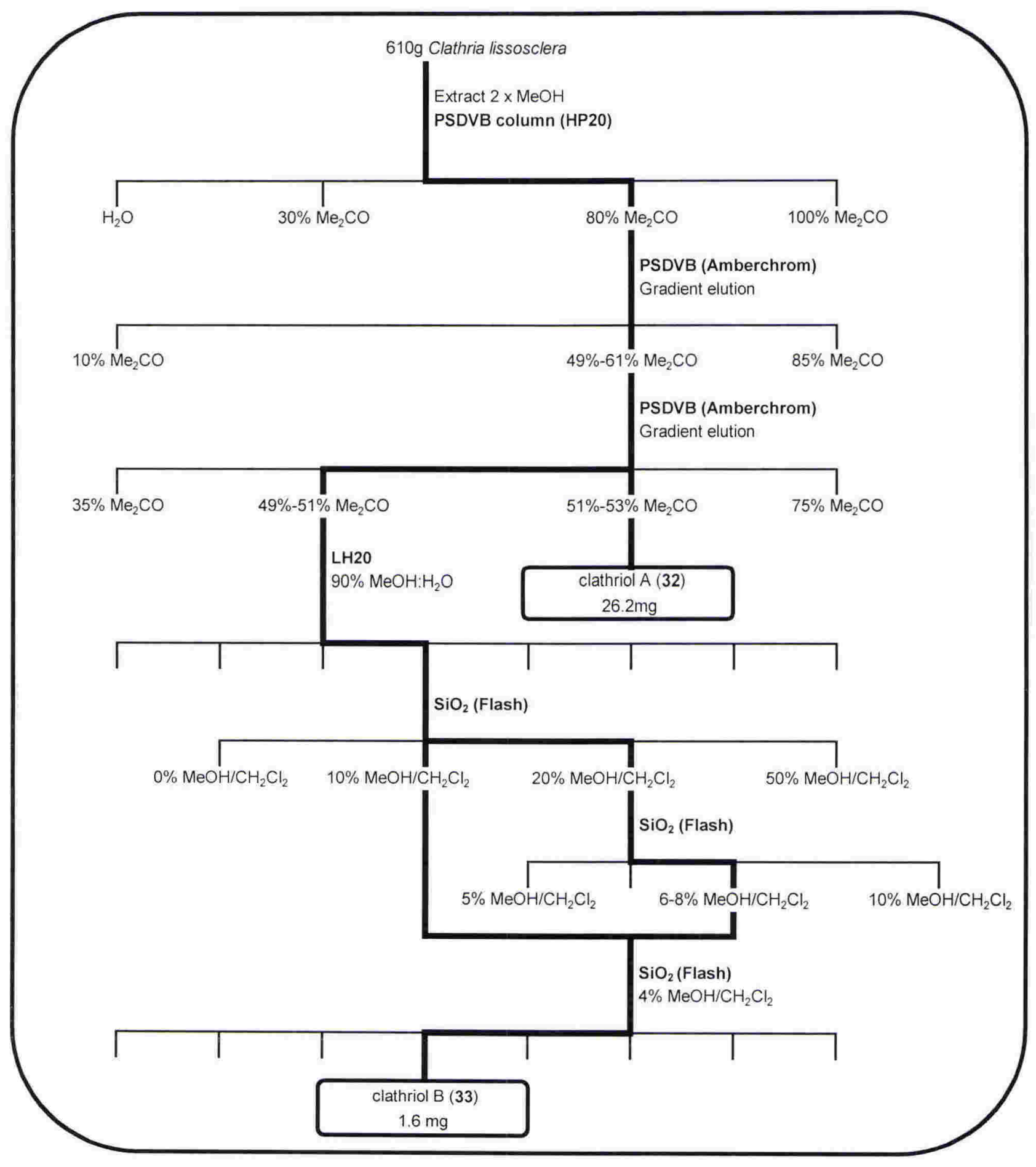

Scheme 3.4 The isolation of clathriol A (32)

and clathriol B (33) from Clathria lissosclera.

Clathria lissosclera Bergquist \& Fromont (Class Demospongiae, Order Poecilosclerida,

Family Microcionidae) is a thickly encrusting to massive, orange, friable sponge. The ectosomal skeleton is comprised of a sparse tangential or paratangential layer of 
subectosomal styles supporting a thin layer of isochelae. Megascleres are smooth styles (250 $\mu \mathrm{m}$ long) with microscleres being palmate isochelae (20 $\mu \mathrm{m}$ long) and, less frequently, toxas $(70 \mu \mathrm{m})$ (see figure 3.3$){ }^{23,102}$
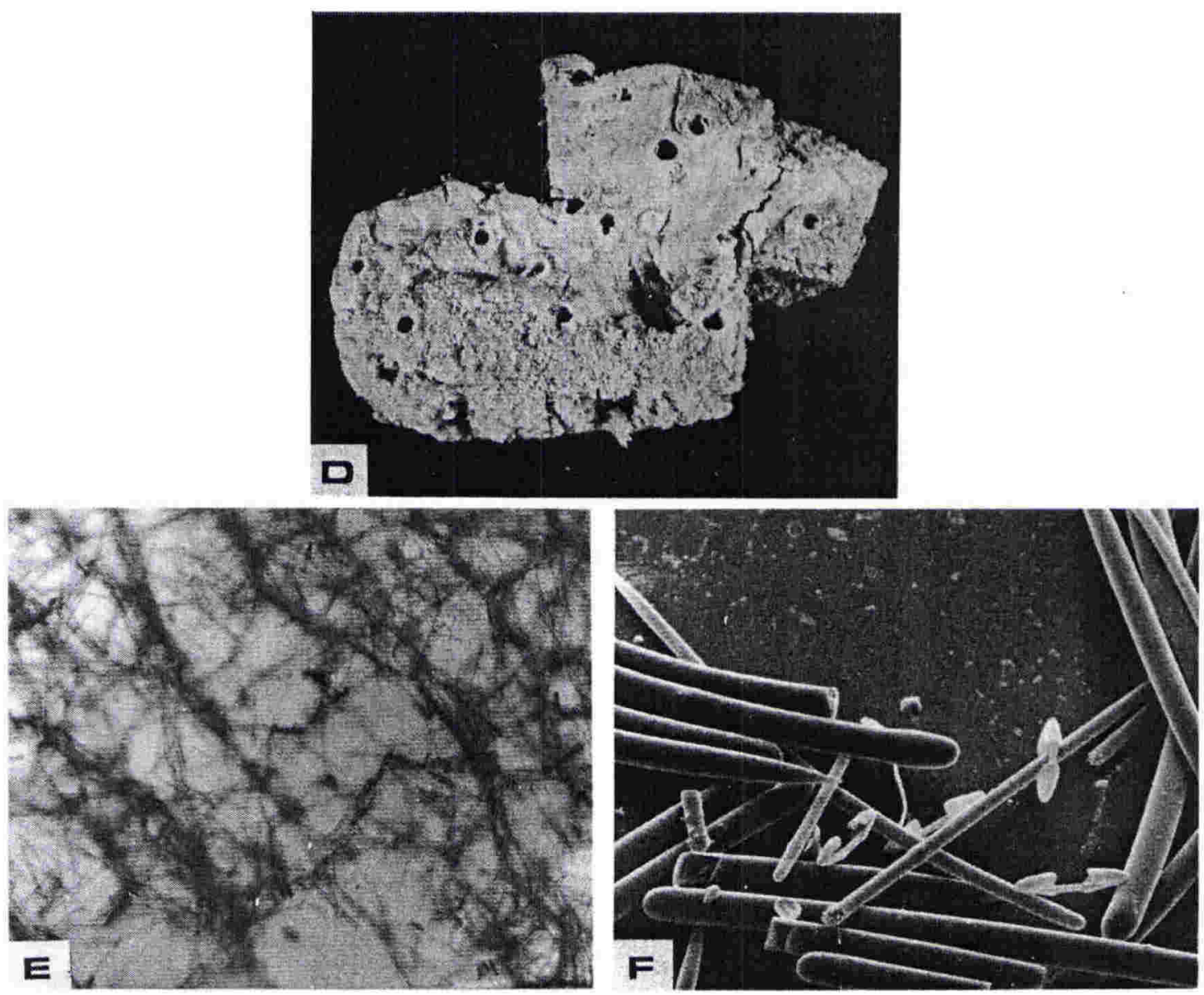

Figure 3.3 Physical structure of Clathria lissosclera.

(Courtesy of Professor P. Bergquist)

D: Surface view of Clathria lissosclera surface showing circular oscules. ${ }^{102}$

E: Irregular reticulate choanosomal skeleton $(\times 113) .^{102}$

F: Spicules $(x 650) .{ }^{102}$ 


\subsection{Clathriol A}

Observation of HRESIMS pseudo-molecular ions in both positive and negative ion modes enabled the molecular formula of clathriol A (32) $(26.2 \mathrm{mg})$ to be established as $\mathrm{C}_{29} \mathrm{H}_{50} \mathrm{O}_{5}\left(479.3754[\mathrm{M}+\mathrm{H}]^{+}, \Delta 3.7 \mathrm{ppm} ; 477.3582[\mathrm{M}-\mathrm{H}]^{-}, \Delta 1.5 \mathrm{ppm}\right)$, implying five degrees of unsaturation. Evidence of four exchangeable protons was obtained by running a negative ion mode HRESIMS of 32, using $\mathrm{D}_{2} \mathrm{O}$ and $\mathrm{CD}_{3} \mathrm{OD}$ as the injection solvent, where a pseudo-molecular ion of 481.3903 was observed, corresponding to the exchange of four deuteriums. The four exchangeable protons were assumed to be from hydroxyl functionalities as a strong $\mathrm{OH}$ stretching band $\left(3308 \mathrm{~cm}^{-1}\right)$ was observed in the IR spectrum. $\mathrm{A} C \mathrm{C}=\mathrm{O}$ stretching band indicative of a saturated ketone was also observed $\left(1729 \mathrm{~cm}^{-1}\right)$. Further evidence for a saturated ketone was obtained from a ${ }^{13} \mathrm{C} \mathrm{NMR}$ resonance at $221.9 \mathrm{ppm}$. All other 28 carbons and 46 hydrogens attached to carbon were observed in the ${ }^{13} \mathrm{C}$ and ${ }^{1} \mathrm{H}$ NMR spectra respectively. One-bond carbon-proton correlations were established by an HSQC experiment. Detailed analyses of the ${ }^{13} \mathrm{C}$ and DEPT NMR spectra gave evidence of a saturated carbonyl, four oxygenated methines $\left(\delta_{\mathrm{C}} 75.8,75.1,71.8,69.6\right)$, eight methines $\left(\delta_{\mathrm{C}} 53.4,52.649 .8,48.5,47.2,40.4,35.4\right.$, $29.4)$, eight methylenes $\left(\delta_{C} 39.0,38.2,38.1,33.5,33.0,31.5,25.8,22.8\right)$, six methyls $\left(\delta_{\mathrm{C}} 21.5,20.7,19.8,19.4,19.4,13.7\right)$ and two quaternary carbons $\left(\delta_{\mathrm{C}} 42.6,36.9\right)$. With no further evidence of double bonds, a tetracyclic structure was required to satisfy the remaining four degrees of unsaturation. It should be noted that severe resonance overlap in most of the NMR spectra, especially for the majority of the methylene ${ }^{1} \mathrm{H}$ resonances of 32, made the unequivocal assignment of the metabolite's structure a non-trivial exercise (see figure 3.4). 


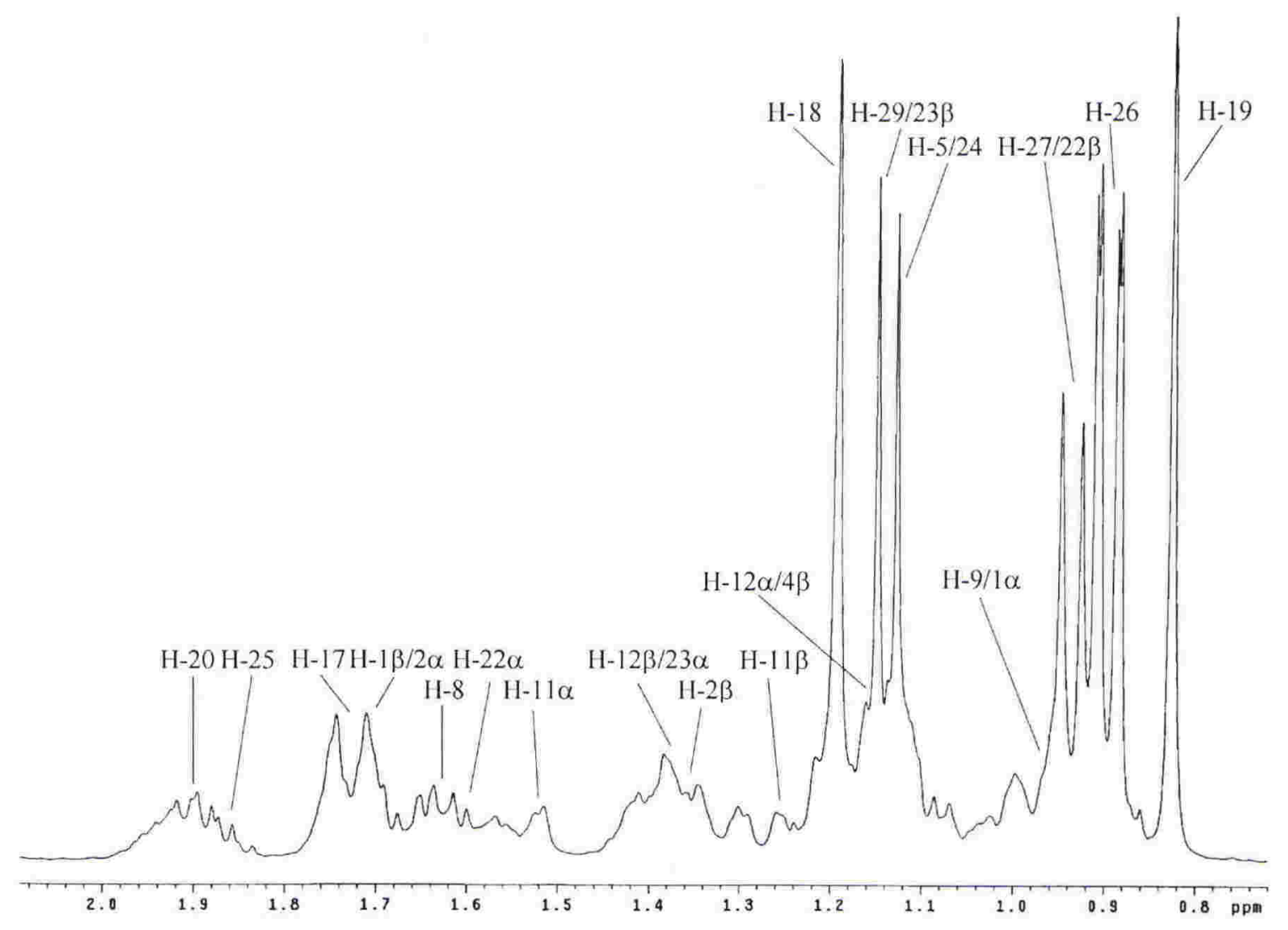

Figure 3.4 Shielded portion of the ${ }^{1}$ H NMR spectrum of clathriol A (32) indicating severe spectral overlap at $300 \mathrm{MHz}$.

Examination of correlations in both COSY and 1D-TOCSY experiments from the four well resolved oxymethine ${ }^{1} \mathrm{H}$ resonances between 4.5 and $3.0 \mathrm{ppm}$ allowed the establishment of two separate substructures. A linear fragment (fragment A) was established from the observation of COSY correlations from the ${ }^{1} \mathrm{H}$ resonances of a methylene $\left(\mathrm{C}-2: \delta_{\mathrm{C}} \quad 31.5, \quad \delta_{\mathrm{H}} \quad 1.71,1.34\right)$ to those of an oxymethine $\left(\mathrm{C}-3: \delta_{\mathrm{C}} 71.8, \delta_{\mathrm{H}} 3.47\right)$ and then to another methylene $\left(\mathrm{C}-4: \delta_{\mathrm{C}} 33.0, \delta_{\mathrm{H}} 2.15,1.16\right)$. Similarly, a linear sequence of five methine carbons was established on the basis of observed COSY cross peaks from the resonances of their attached protons [methine (C-5: $\left.\delta_{\mathrm{C}} 48.5, \delta_{\mathrm{H}} 1.10\right)$ to oxymethine $\left(\mathrm{C}-6: \delta_{\mathrm{C}} 75.1, \delta_{\mathrm{H}} 3.06\right)$, to oxymethine $\left(\mathrm{C}-7: \delta_{\mathrm{C}} 75.6, \delta_{\mathrm{H}} 4.30\right)$, to a methine $\left(\mathrm{C}-8: \delta_{\mathrm{C}} 40.4, \delta_{\mathrm{H}} 1.62\right)$ and finally to a methine $\left.\left(\mathrm{C}-14: \delta_{\mathrm{C}} 53.4, \delta_{\mathrm{H}} 2.84\right)\right]$ to give fragment $\mathrm{B}$. Although no direct linkage between fragments A and B could be determined through COSY correlations, the linkage was 
established by strong $1 \mathrm{D}$-TOCSY correlations, observed between the ${ }^{1} \mathrm{H}$ resonances of H-3 and H-6. This connection was confirmed by an HMBC correlation from the ${ }^{1} \mathrm{H}$ resonance of $\mathrm{H}-6$ to the ${ }^{13} \mathrm{C}$ resonance of $\mathrm{C}-4$, completing substructure I (see figure 3.5 ).

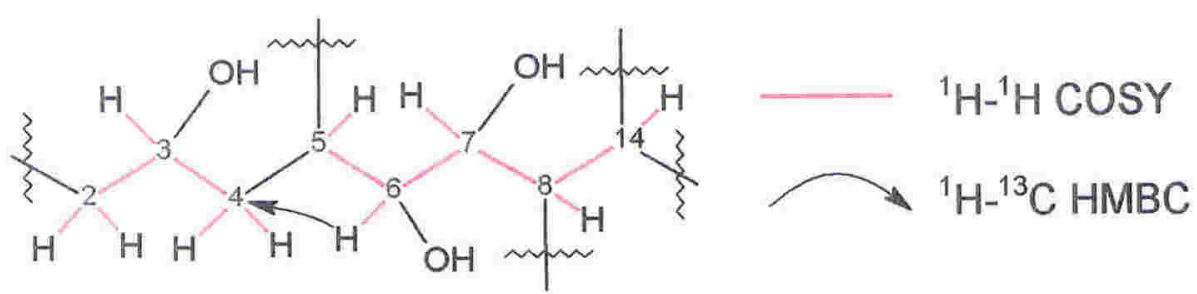

Figure 3.5 Selected COSY and HMBC correlations used to establish substructure I of clathriol A (32).

Substructure II was determined from COSY correlations observed between the ${ }^{1} \mathrm{H}$ resonances of an oxymethine $\left(\mathrm{C}-28: \delta_{\mathrm{C}} 69.8, \delta_{\mathrm{H}} 3.77\right)$ to those of a methyl $\left(\mathrm{C}-29: \delta_{\mathrm{C}} 20.7, \delta_{\mathrm{H}} 1.14\right)$ and a methine $\left(\mathrm{C}-24: \delta_{\mathrm{C}} 52.6, \delta_{\mathrm{H}} 1.08\right)$ (see figure 3.6$)$.

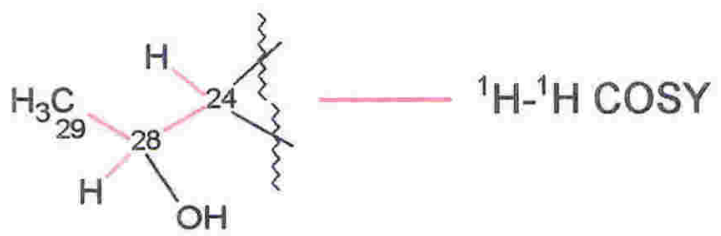

Figure 3.6 COSY correlations used to establish substructure II of clathriol A (32).

Strong long-range HMBC correlations were observed from the ${ }^{1} \mathrm{H}$ resonances of the five remaining methyls of 32 . Two secondary methyls $\left[\left(\mathrm{C}-26: \delta_{\mathrm{C}} 19.4, \delta_{\mathrm{H}} 0.88\right)\right.$ and (C-27: $\left.\left.\delta_{\mathrm{C}} 21.5, \delta_{\mathrm{H}} 0.91\right)\right]$ showed $\mathrm{HMBC}$ correlations to the ${ }^{13} \mathrm{C}$ resonances of each other, and also to those of a methine $\left(\mathrm{C}-25: \delta_{\mathrm{C}} 29.4, \delta_{\mathrm{H}} 1.86\right)$, establishing an isopropyl side chain. They also both correlated to the ${ }^{13} \mathrm{C}$ resonance of methine $\mathrm{C}-24$, thereby extending substructure II (see figure 3.7) 


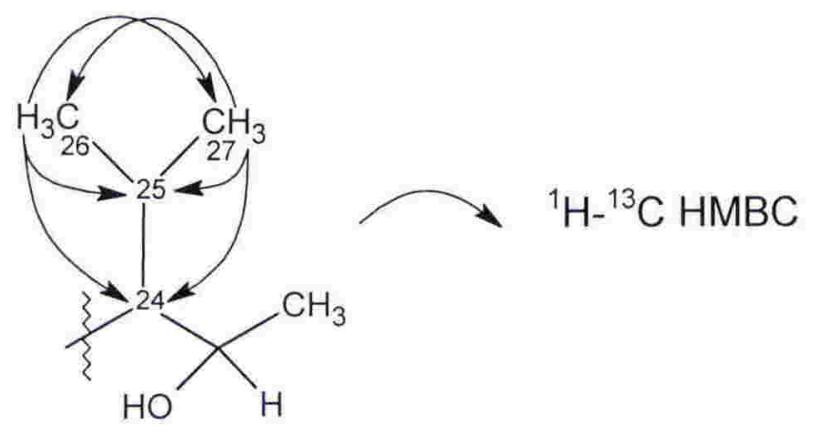

Figure 3.7 Selected HMBC correlations establishing the -3-(4-methylpentan-2-ol) side chain (substructure II) of clathriol A (32).

Detailed analysis of the HMBC spectrum revealed strong correlations from the ${ }^{1} \mathrm{H}$ resonance of a tertiary angular methyl $\left(\mathrm{C}-19: \delta_{\mathrm{C}} 13.7, \delta_{\mathrm{H}} 0.83\right)$ to the ${ }^{13} \mathrm{C}$ resonances of a methylene $\left(\mathrm{C}-1: \delta_{\mathrm{C}} 38.2, \delta_{\mathrm{H}} 1.71,0.93\right)$, a methine $\left(\mathrm{C}-9: \delta_{\mathrm{C}} 47.2, \delta_{\mathrm{H}} 0.93\right)$, a quaternary carbon $\left(\mathrm{C}-10: \delta_{\mathrm{C}} 36.9\right)$ and methine C-5 of substructure I. This allowed methyl $\mathrm{CH}_{3}-19$ to be connected to quaternary centre $\mathrm{C}-10$ and also established linkages from C-10 to C-1, C-5 and C-9 of substructure I (see figure 3.8).

The ${ }^{1} \mathrm{H}$ resonance of the other tertiary angular methyl $\left(\mathrm{C}-18: \delta_{\mathrm{C}} 19.4, \delta_{\mathrm{H}} 1.19\right)$ showed strong HMBC correlations to the ${ }^{13} \mathrm{C}$ resonances of a methylene $\left(\mathrm{C}-12: \delta_{\mathrm{C}} 39.0, \delta_{\mathrm{H}} 1.37,1.17\right)$, a quaternary centre $\left(\mathrm{C}-13: \delta_{\mathrm{C}} 42.6\right)$, a methine $\left(\mathrm{C}-17: \delta_{\mathrm{C}} 49.8, \delta_{\mathrm{H}} 1.72\right)$ and also methine $\mathrm{C}-14$ of substructure I. These observations allowed placement of $\mathrm{CH}_{3}-18$ at $\mathrm{C}-13$, established the linkages between $\mathrm{C}-13$ and $\mathrm{C}-12$, C-14, and C-17, and allowed for expansion of substructure I to include the two angular methyls (see figure 3.8). 


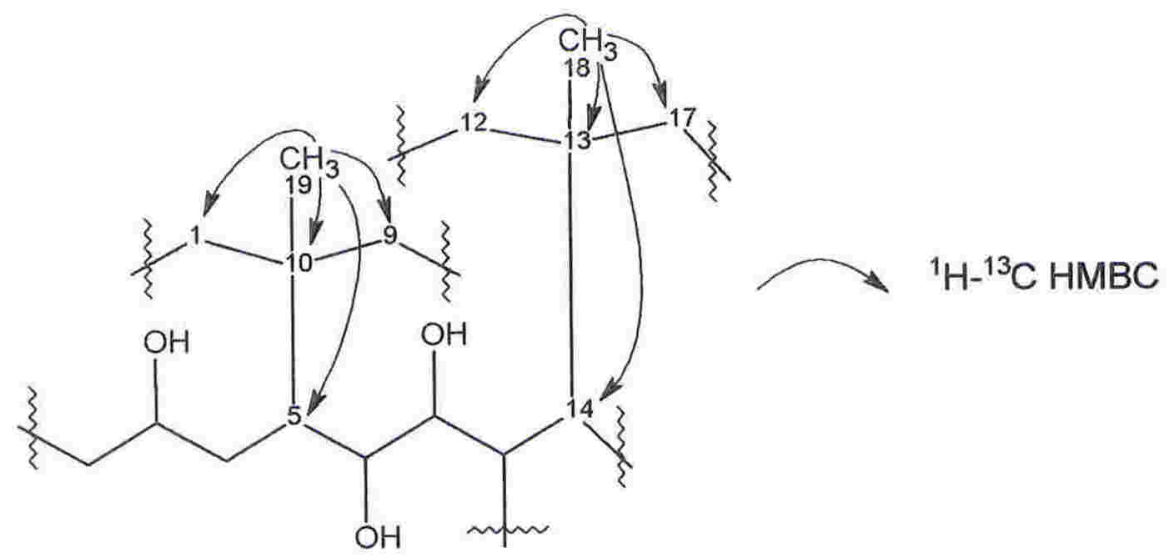

Figure 3.8 Selected $\mathrm{HMBC}$ correlations connecting $\mathrm{CH}_{3}-18$ and $\mathrm{CH}_{3}-19$ to substructure I of clathriol A (32).

The ${ }^{1} \mathrm{H}$ resonance of a secondary methyl $\left(\mathrm{C}-21: \delta_{\mathrm{C}} 19.8, \delta_{\mathrm{H}} 0.94\right)$ showed HMBC correlations to the ${ }^{13} \mathrm{C}$ resonances of a methylene $\left(\mathrm{C}-22: \delta_{\mathrm{C}} 33.5, \delta_{\mathrm{H}} 1.60,0.90\right)$ and two methines [C-17 and $\left.\left(\mathrm{C}-20: \delta_{\mathrm{C}} 35.4, \delta_{\mathrm{H}} 1.91\right)\right]$. A COSY correlation observed between the ${ }^{1} \mathrm{H}$ resonances of $\mathrm{H}-20$ and $\mathrm{CH}_{3}-21$ indicated the attachment of the secondary methyl on C-20 between C-17 and C-22 (see figure 3.9).

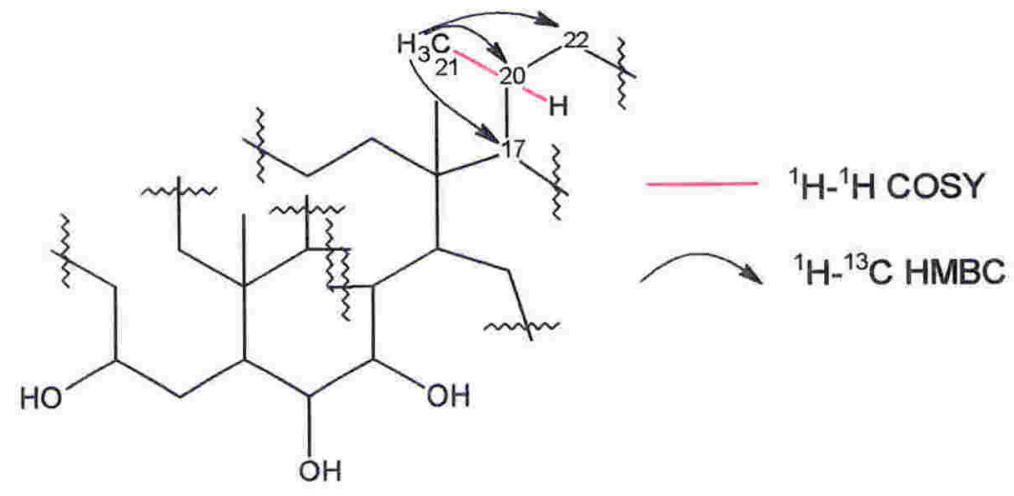

Figure 3.9 Selected HMBC correlations connecting $\mathrm{CH}_{3}-21$ to substructure I of clathriol A (32).

All of the previous observations had established the connectivities of substructure I (nineteen carbons) and substructure II (six carbons), leaving three methylenes and a ketone to be assembled into a tetracyclic structure as required by the remaining degrees of unsaturation. COSY cross-peaks could not be reliably used to identify correlations 
from the ${ }^{1} \mathrm{H}$ resonances of the remaining methylenes, due to the severe spectral overlap in the shielded region of the ${ }^{1} \mathrm{H}$ NMR spectrum (see figure 3.4). 1D-TOCSY, HMBC, and HSQC-TOCSY experiments were therefore used to assign the remaining connections within the molecule.

The presence of a six-membered ring (ring A of a steroid) was identified by selective excitation of the ${ }^{1} \mathrm{H}$ resonance of H-3 in a 1D-TOCSY experiment. Correlations to the ${ }^{1} \mathrm{H}$ resonances of both protons attached to methylenes $\mathrm{C}-2$ and $\mathrm{C}-1$ were revealed sequentially as the spin-lock mixing time was increased, thereby establishing the connection of C-3 to $\mathrm{C}-2$ and $\mathrm{C}-2$ to $\mathrm{C}-1$. This connectivity was confirmed by a series of HSQC-TOCSY experiments where a ${ }^{1} \mathrm{H}$ resonance of $\mathrm{C}-1\left(\delta_{\mathrm{C}} 38.2, \delta_{\mathrm{H}} 0.93\right)$, showed TOCSY correlations to a ${ }^{1} \mathrm{H}$ resonance of $\mathrm{C}-2\left(\delta_{\mathrm{H}} 1.34\right)$ and also of $\mathrm{C}-3\left(\delta_{\mathrm{H}} 3.47\right)$, thereby confirming the $\mathrm{C}-1 \mathrm{C}-2$ bond and reinforcing the establishment of ring $\mathrm{A}$ (see figure 3.10).

The presence of ring B was established from HSQC-TOCSY correlations from the ${ }^{1} \mathrm{H}$ resonance of $\mathrm{H}-7\left(\delta_{\mathrm{C}} 75.6, \delta_{\mathrm{H}} 4.30\right)$ to that of H-9 $\left(\delta_{\mathrm{H}} 0.93\right)$ via $\mathrm{H}-8\left(\delta_{\mathrm{H}} 1.62\right)$, as observed sequentially through experiments with increasing spin-lock mixing times. The connectivity of the final six-membered ring (ring C), was determined from further HSQC-TOCSY correlations between the ${ }^{1} \mathrm{H}$ resonance of $\mathrm{H}-9\left(\delta_{\mathrm{C}} 47.2, \delta_{\mathrm{H}} 0.93\right)$ and those of two methylenes $\left[\left(\mathrm{C}-11: \delta_{\mathrm{C}} 22.8, \delta_{\mathrm{H}} 1.52,1.25\right)\right.$ and $\left.\mathrm{C}-12\right]$, indicating the placement of C-11 between C-9 and C-12. The placement of C-11 was confirmed by the observation of an $\mathrm{HMBC}$ correlation from the ${ }^{1} \mathrm{H}$ resonance of $\mathrm{H}-8$ to the ${ }^{13} \mathrm{C}$ resonance of C-11, thereby completing the connectivity of rings A, B and C (see figure 3.10). 


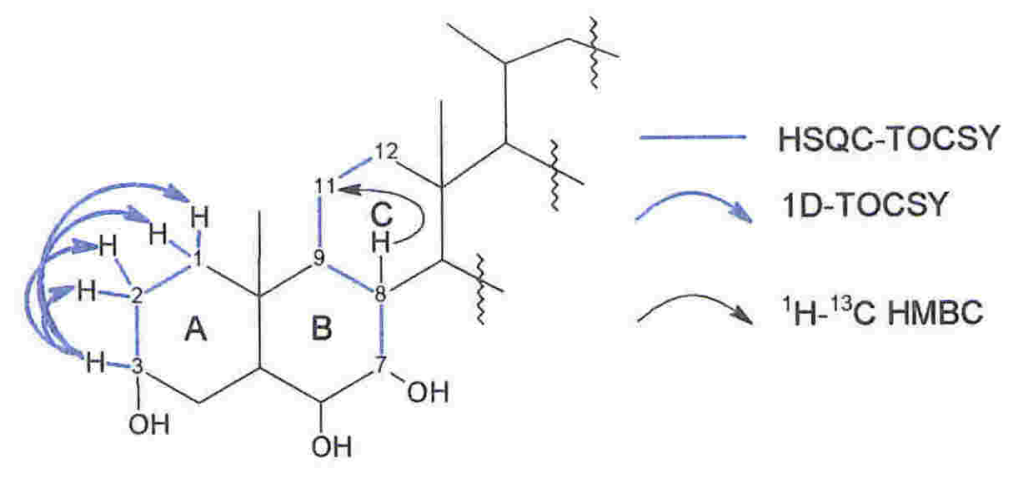

Figure 3.10 Selected correlations establishing the connectivity of rings $\mathrm{A}, \mathrm{B}$ and $\mathrm{C}$ of clathriol A (32).

Further analysis of HSQC-TOCSY correlations from the ${ }^{1} \mathrm{H}$ resonances of a methylene $\left(\mathrm{C}-23: \delta_{\mathrm{C}} 25.8, \delta_{\mathrm{H}} 1.36,1.14\right)$ to those of $\mathrm{H}-20\left(\delta_{\mathrm{H}} 1.91\right)$ and oxymethine $\mathrm{H}-28\left(\delta_{\mathrm{H}} 3.77\right)$ revealed connections from $\mathrm{C}-23$ to both $\mathrm{C}-22$ and $\mathrm{C}-24$. This observation was consistent with an $\mathrm{HMBC}$ correlation observed from the ${ }^{1} \mathrm{H}$ resonance of $\mathrm{H}-28$ to the ${ }^{13} \mathrm{C}$ resonance of $\mathrm{C}-23$, thereby linking substructure II with rings $\mathrm{A}, \mathrm{B}$ and $\mathrm{C}$ (see figure 3.11).

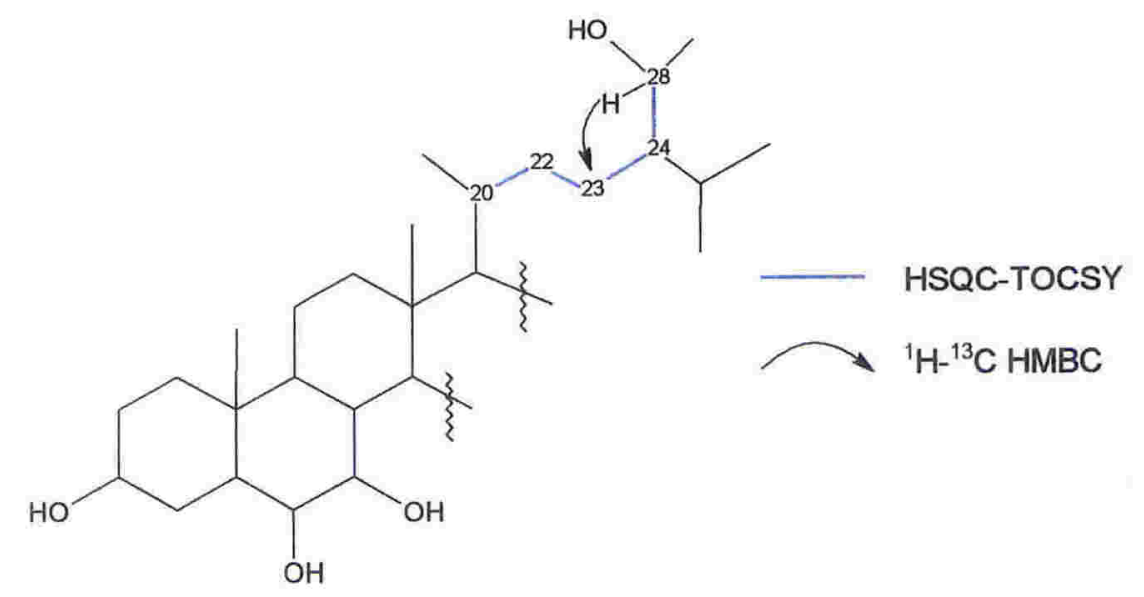

Figure 3.11 Selected correlations connecting rings

A, B and C with substructure II of clathriol A (32).

Connections to the remaining methylene $\left(\mathrm{C}-16: \delta_{\mathrm{C}} 38.1, \delta_{\mathrm{H}} 2.36,2.18\right)$ and ketone $\left(\mathrm{C}-15 ; \delta_{\mathrm{C}} 221.9\right)$ were assigned on the basis of observed HMBC correlations. HMBC correlations from the deshielded ${ }^{1} \mathrm{H}$ resonance of $\mathrm{H}-16 \beta\left(\delta_{\mathrm{H}} 2.36\right)$ to the ${ }^{13} \mathrm{C}$ resonances 
of $\mathrm{C}-13, \mathrm{C}-17$ and $\mathrm{C}-20$ revealed the attachment of $\mathrm{C}-16$ to C-17. Observation of HMBC correlations from the ${ }^{1} \mathrm{H}$ resonances of $\mathrm{H}-8, \mathrm{H}-14$ and $\mathrm{H}-16 \beta$ to the ${ }^{13} \mathrm{C}$ resonance of ketone $\mathrm{C}-15$ provided evidence for the last two carbon bonds, $\mathrm{C}-14$ C-15 and C-15 C-16. Further evidence for the placement of C-15 was provided by the deshielded chemical shifts of the ${ }^{1} \mathrm{H}$ resonances of $\mathrm{H}-14\left(\delta_{\mathrm{H}} 2.84\right), \mathrm{H}-16 \alpha\left(\delta_{\mathrm{H}} 2.18\right)$ and $\mathrm{H}-16 \beta\left(\delta_{\mathrm{H}} 2.36\right)$, and also by an HMBC correlation from the resonance of $\mathrm{H}-16 \beta$ to that of C-14. These correlations established the five-membered ring (ring D) and completed the steroidal carbon skeleton of clathriol A (32) (see figure 3.12).

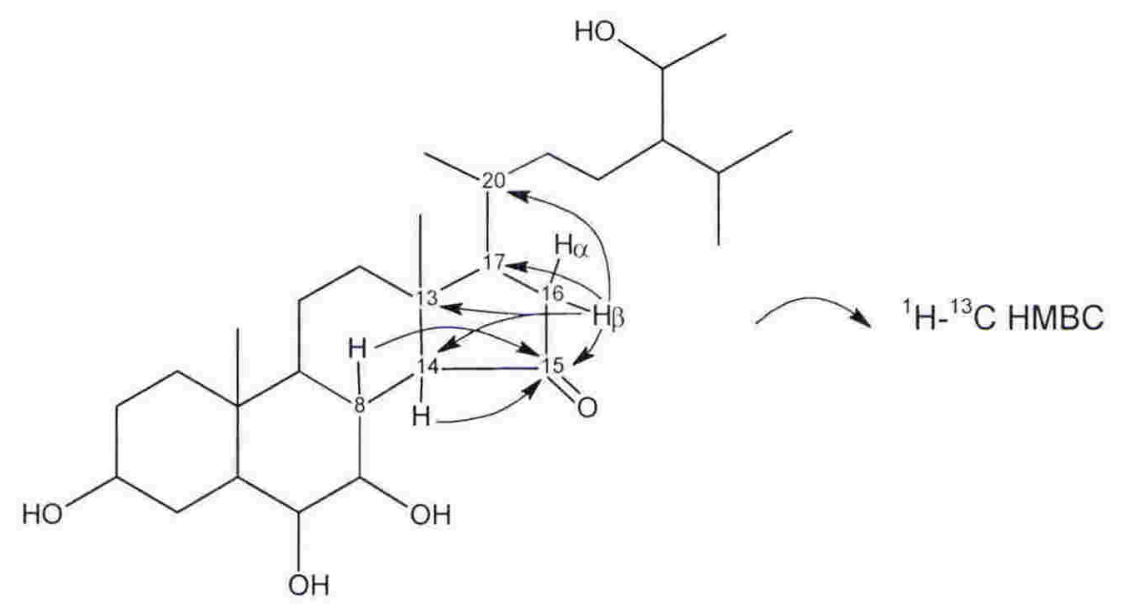

Figure 3.12 Establishment of the final connectivity of clathriol A (32).

The relative stereochemistry of ten of the thirteen stereogenic centres of clathriol A (32) were determined by a combination of analysis of NOE correlations, observed in both 1D-gNOESY and ROESY experiments, and by measurement of ${ }^{1} \mathrm{H}-{ }^{1} \mathrm{H}$ coupling constants, determined from ${ }^{1} \mathrm{H}$ NMR, ${ }^{1} \mathrm{H}-{ }^{1} \mathrm{H}$ homonuclear decoupling, and double quantum filtered COSY (DQF-COSY) experiments. Ring A was assigned as being in a chair conformation, based on the both large and small vicinal coupling constants of the protons attached to $\mathrm{C}-1, \mathrm{C}-3$ and $\mathrm{C}-4$. The hydroxyl attached to $\mathrm{C}-3$ was assigned as $\beta$ (equatorial), on the basis of the multiplicity of $\mathrm{H}-3$. Proton $\mathrm{H}-3$ was assigned as $\alpha$ (axial), as its resonance showed coupling constants of greater than $10 \mathrm{~Hz}$ to both $\mathrm{H}-2 \alpha$ and $\mathrm{H}-4 \alpha$ while also showing couplings of less than $5 \mathrm{~Hz}$ to $\mathrm{H}-2 \beta$ and $\mathrm{H}-4 \beta$. Strong 
NOE correlations from the ${ }^{1} \mathrm{H}$ resonance of $\mathrm{H}-3$ to those of both $\mathrm{H}-1 \alpha$ and $\mathrm{H}-5$ confirmed this assignment and also allowed placement of H-5 on the $\alpha$ (bottom) face of the molecule. The $\alpha$ orientation of $\mathrm{H}-5$ was consistent with the large coupling measured between its resonance and that of $\mathrm{H}-4 \beta$. Strong NOE correlations were also observed between the ${ }^{1} \mathrm{H}$ resonances of $\mathrm{H}-2 \beta, \mathrm{H}-4 \beta$ and those of $\mathrm{CH}_{3}-19$, revealing the $\beta$ (axial) orientation of angular methyl $\mathrm{CH}_{3}-19$, and also establishing the standard steroid trans A/B ring fusion (see figure 3.13).

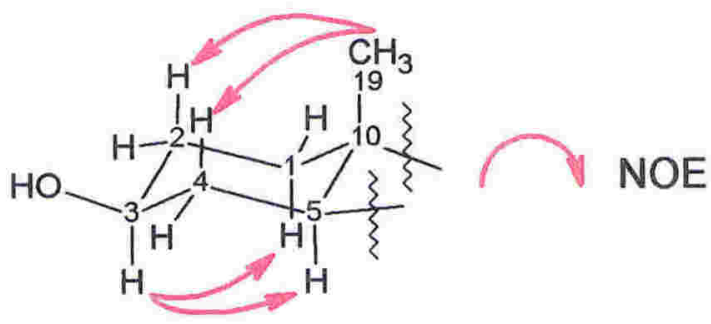

Figure 3.13 Observed NOEs establishing stereochemistry of ring A of clathriol A (32).

The ${ }^{1} \mathrm{H}$ resonance of $\mathrm{H}-6$ showed strong NOE correlations to the resonances of both $\mathrm{H}$ $4 \beta$ and $\mathrm{CH}_{3}-19$. This, together with its large 1,2-trans diaxial coupling to the resonance of $\mathrm{H}-5(10.5 \mathrm{~Hz})$, established $\mathrm{H}-6$ as being axial on the $\beta$ face of the molecule, and thereby assigned the hydroxyl attached to C- 6 as being equatorial below the plane of the molecule $(\alpha)$. Observation of strong NOEs from resonance H-8 to those of both H-6 and $\mathrm{CH}_{3}-19$ placed $\mathrm{H}-8$ on the $\beta$ face of the molecule as well. The resonance of $\mathrm{H}-7$ showed large vicinal couplings to those of both $\mathrm{H}-6(9 \mathrm{~Hz})$ and $\mathrm{H}-8(10.5 \mathrm{~Hz})$, consistent with the C-7 hydroxyl being equatorial ( $\beta$ ). Assignment of the $\alpha$ orientation of H-7 was supported by strong NOE correlations from the resonance of $\mathrm{H}-7$ to those of $\mathrm{H}-5$ and H-9 (see figure 3.14). 


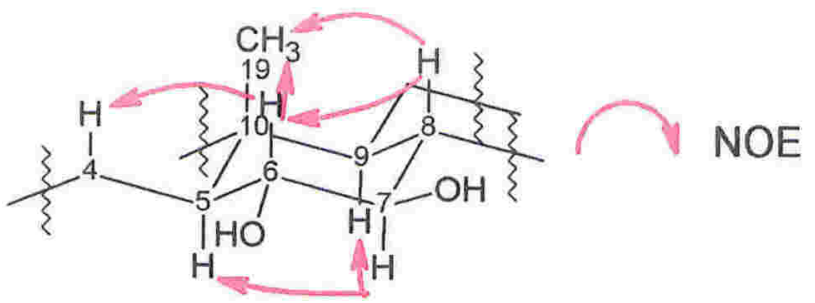

Figure 3.14 Observed NOEs establishing stereochemistry of ring B of clathriol A (32).

A large coupling observed between the resonances of H-8 and H-9, consistent with a 1,2-trans diaxial relationship, implied a trans $\mathrm{B} / \mathrm{C}$ ring fusion. Methyl $\mathrm{CH}_{3}-18$ was assigned on the $\beta$ side of the molecule on the basis of the strong NOE correlation observed between its ${ }^{1} \mathrm{H}$ resonance and that of $\mathrm{H}-8$. Further NOE correlations between the ${ }^{1} \mathrm{H}$ resonances of methyl $\mathrm{CH}_{3}-18$ and methine $\mathrm{C}-20$ also placed the side-chain of clathriol A (32) on the $\beta$ face of the molecule (see figure 3.15).

Almost all steroids possess a trans C/D ring fusion. Detailed analyses of NOE correlations in both 1D-gNOESY and ROESY experiments revealed that the resonance of $\mathrm{H}-14$ showed strong correlations to the ${ }^{\mathrm{I}} \mathrm{H}$ resonances of both $\mathrm{H}-8$ and $\mathrm{CH}_{3}-18$ on the $\beta$ face of the molecule. This observation was consistent with $\mathrm{H}-14$ being in the rare $\beta$ orientation, implying a cis $\mathrm{C} / \mathrm{D}$ ring fusion for the sterol. This was confirmed by the small vicinal coupling constant observed between the resonances of H-8 and H-14 (3 $\mathrm{Hz})$ and also the weak long-range $\mathrm{W}$ coupling $(<2 \mathrm{~Hz})$ between the resonance of $\mathrm{H}-14$ and those of $H-12 \beta$ and $H-16 \alpha$, as observed in the COSY spectrum (see figures 3.15 and 3.16). 


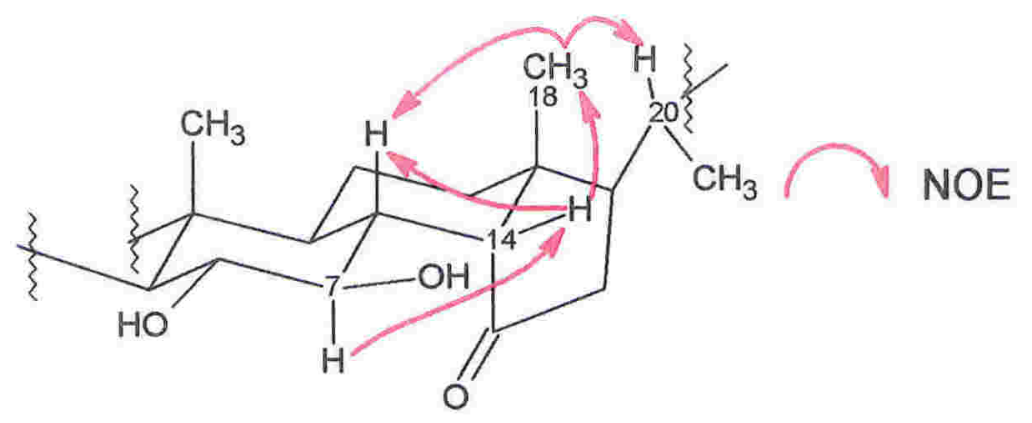

Figure 3.15 Observed NOEs establishing stereochemistry of C-14 and rings $\mathrm{C}$ and $\mathrm{D}$ of clathriol $\mathrm{A}(32)$.

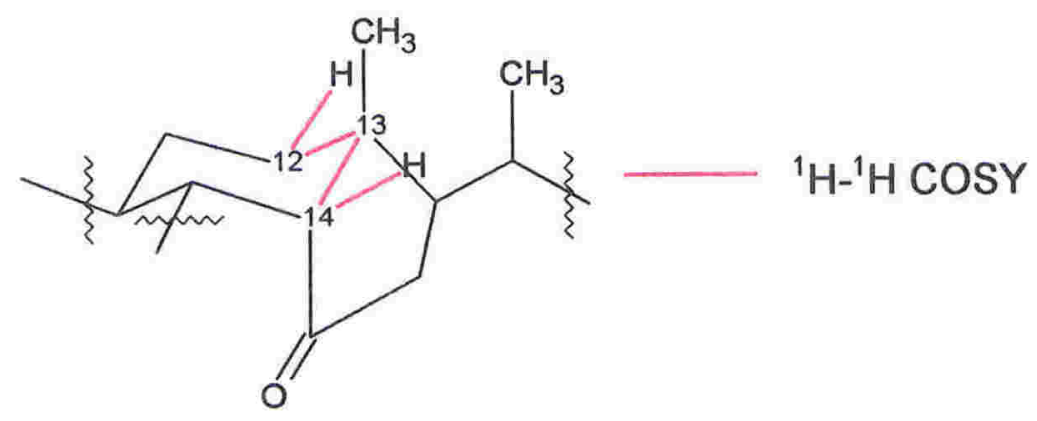

Figure 3.16 W-coupling observed in the COSY spectrum between $\mathrm{H}-14$ and $\mathrm{H}-12 \beta$ of clathriol A (32).

Assuming normal steroidal absolute configuration, the cyclic portion of the molecule can therefore be assigned as $3 S, 5 S, 6 R, 7 R, 8 R, 9 S, 10 R, 13 R, 14 R, 17 R$.

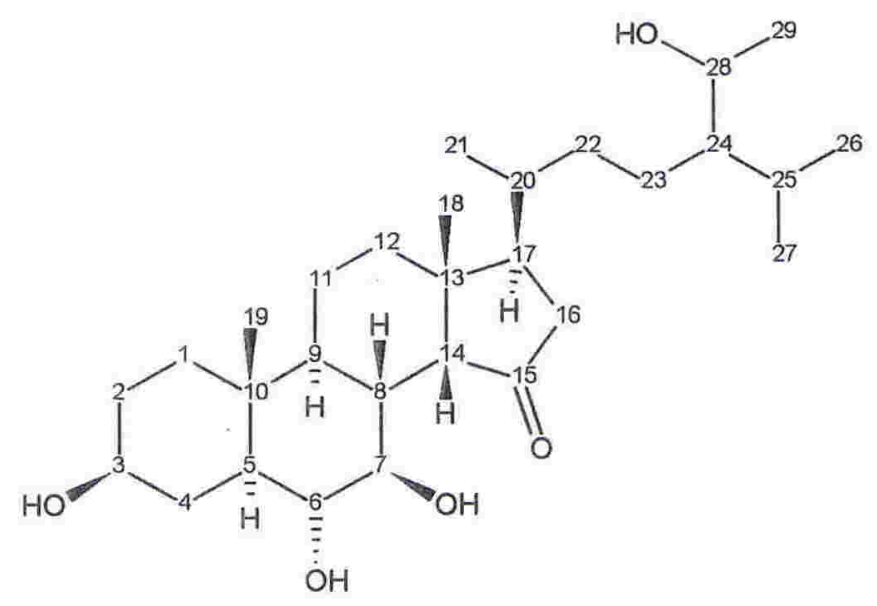

(32) 
Table 3.1. ${ }^{13} \mathrm{C}(75 \mathrm{MHz})$ and ${ }^{1} \mathrm{H}(300 \mathrm{MHz}) \mathrm{NMR}$ data $\left(\mathrm{CD}_{3} \mathrm{OD}\right)$ of clathriol $\mathrm{A}(\mathbf{3 2})$.

\begin{tabular}{|c|c|c|c|c|c|c|c|}
\hline \multirow[b]{2}{*}{ Position } & \multicolumn{2}{|c|}{${ }^{13} \mathrm{C}^{\mathrm{a}}$} & \multicolumn{2}{|c|}{${ }^{1} \mathrm{H}^{\mathrm{b}}$} & \multirow[b]{2}{*}{ COSY } & \multirow{2}{*}{$\begin{array}{l}\text { HMBC } \\
\text { (H to } \mathrm{C} \text { ) }\end{array}$} & \multirow[b]{2}{*}{ NOE } \\
\hline & $\delta(\mathrm{ppm})$ & mult & $\delta(\mathrm{ppm})$ & mult, J (Hz) & & & \\
\hline $1 \alpha$ & 38.2 & $\mathrm{CH}_{2}$ & 0.93 & $\mathrm{dd}(13.5,10)$ & $2 \alpha, 2 \beta$ & & 3 \\
\hline $1 \beta$ & & & 1.71 & $d(11)$ & $2 \alpha, 2 \beta$ & & \\
\hline $2 \alpha$ & 31.5 & $\mathrm{CH}_{2}$ & 1.71 & $\mathrm{~m}$ & $1 \alpha, 1 \beta, 3$ & & 3 \\
\hline $2 \beta$ & & & 1.34 & $\mathrm{~m}$ & $1 \alpha, 1 \beta, 3$ & & \\
\hline 3 & 71.8 & $\mathrm{CH}$ & 3.47 & dddd $(13.5,10,4.5,3)$ & $2 \alpha, 2 \beta, 4 \alpha, 4 \beta$ & 2,4 & $1 \alpha, 2 \alpha, 4 \alpha, 5$ \\
\hline $4 \alpha$ & 33.0 & $\mathrm{CH}_{2}$ & 2.15 & $\operatorname{brd}(11.5)$ & $3,5,6$ & & 3 \\
\hline $4 \beta$ & & & 1.16 & $\mathrm{~m}$ & 3,5 & & 6 \\
\hline 5 & 48.5 & $\mathrm{CH}$ & 1.10 & $\mathrm{~m}$ & $4 \alpha, 4 \beta, 6$ & & 3,7 \\
\hline 6 & 75.1 & $\mathrm{CH}$ & 3.06 & $\mathrm{dd}(10.5,9)$ & $4 \alpha, 5,7,8$ & $4,5,7,8$ & $4 \beta, 7,8,18$ \\
\hline 7 & 75.6 & $\mathrm{CH}$ & 4.30 & $\mathrm{dd}(10.5,9)$ & 6,8 & $5,6,8,14$ & $5,6,8,9,14$ \\
\hline 8 & 40.4 & $\mathrm{CH}$ & 1.62 & ddd $(12,10,5.3)$ & $6,7,8,12 \alpha, 12 \beta$ & 11,15 & $6,7,18,19$ \\
\hline 9 & 47.2 & $\mathrm{CH}$ & 0.93 & $\mathrm{~m}$ & $11 \beta$ & 7,8 & 7 \\
\hline 10 & 36.9 & $\mathrm{C}$ & & & & & \\
\hline $11 \alpha$ & 22.8 & $\mathrm{CH}_{2}$ & 1.52 & $\mathrm{~m}$ & & $9,12,13$ & \\
\hline $11 \beta$ & & & 1.25 & $\mathrm{~m}$ & $9,12 \alpha, 12 \beta$ & & 19 \\
\hline $12 \alpha$ & 39.0 & $\mathrm{CH}_{2}$ & 1.17 & $\mathrm{~m}$ & $8,11 \beta$ & & \\
\hline $12 \beta$ & & & 1.37 & $\mathrm{~m}$ & $8,11 \beta, 14,18$ & & \\
\hline 13 & 42.6 & $\mathrm{C}$ & & & & & \\
\hline 14 & 53.4 & $\mathrm{CH}$ & 2.84 & $\mathrm{dd}(3,2)$ & $8,12 \beta, 16 \alpha$ & $8,9,12,13,15,18$ & $7,8,18$ \\
\hline 15 & 221.9 & C & & & & & \\
\hline $16 \alpha$ & 38.1 & $\mathrm{CH}_{2}$ & 2.18 & $\mathrm{~m}$ & 14,17 & & $16 \beta$ \\
\hline $16 \beta$ & & & 2.36 & $\mathrm{dd}(20,10)$ & 17 & $13,14,15,17,20$ & $16 \alpha$ \\
\hline 17 & 49.8 & $\mathrm{CH}$ & 1.72 & $\mathrm{~m}$ & $16 \alpha, 16 \beta, 21$ & & 20 \\
\hline 18 & 19.4 & $\mathrm{CH}_{3}$ & 1.19 & s & 12 & $12,13,14,17$ & $6,8,20$ \\
\hline 19 & 13.7 & $\mathrm{CH}_{3}$ & 0.83 & s & & $1,5,9,10$ & $2 \beta, 4 \beta, 6,8,11 \beta$ \\
\hline 20 & 35.4 & $\mathrm{CH}$ & 1.91 & $\mathrm{~m}$ & & & $17,18,21$ \\
\hline 21 & 19.8 & $\mathrm{CH}_{3}$ & 0.94 & $d(6.5)$ & 17 & $17,20,22$ & 20 \\
\hline $22 \alpha$ & 33.5 & $\mathrm{CH}_{2}$ & 1.60 & $\mathrm{~m}$ & & & \\
\hline $22 \beta$ & & & 0.90 & $\mathrm{~m}$ & & & \\
\hline $23 \alpha$ & 25.8 & $\mathrm{CH}_{2}$ & 1.36 & $\mathrm{~m}$ & & & \\
\hline $23 \beta$ & & & 1.14 & $\mathrm{~m}$ & & & \\
\hline 24 & 52.6 & $\mathrm{CH}$ & 1.08 & $\mathrm{~m}$ & 28 & & \\
\hline 25 & 29.4 & $\mathrm{CH}$ & 1.86 & $\mathrm{~m}$ & & & 26,27 \\
\hline 26 & 19.4 & $\mathrm{CH}_{3}$ & 0.88 & $d(6.5)$ & & $24,25,27$ & 25 \\
\hline 27 & 21.5 & $\mathrm{CH}_{3}$ & 0.91 & $\mathrm{~d}(6.5)$ & & $24,25,26$ & 25 \\
\hline 28 & 69.6 & $\mathrm{CH}$ & 3.77 & quin (6) & 24,29 & $23,24,25,29$ & \\
\hline 29 & 20.7 & $\mathrm{CH}_{3}$ & 1.14 & $d(6.5)$ & 28 & 24,28 & \\
\hline
\end{tabular}

a Multiplicity determined from DEPT and HSQC experiments

b Carbon connectivity determined from an HSQC experiment, multiplicity determined from ${ }^{1} \mathrm{H}$,

1D-TOCSY and homo2DJ experiments. 


\subsection{Clathriol B}

Pseudo-molecular ions were observed for clathriol B (33) $(1.3 \mathrm{mg})$ in both the positive and negative ion HRESIMS modes, corresponding to the formula $\mathrm{C}_{28} \mathrm{H}_{46} \mathrm{O}_{5}(463.3423$ $\left.[\mathrm{M}+\mathrm{H}]^{+}, \Delta 1.0 \mathrm{ppm} ; 461.3298[\mathrm{M}-\mathrm{H}]^{-}, \Delta 4.7 \mathrm{ppm}\right)$, differing from that of clathriol A (32) by one carbon and four hydrogens, and implying six degrees of unsaturation. As with 32, all 28 carbons and 42 protons attached to carbon were observed in the ${ }^{13} \mathrm{C}$ and ${ }^{1} \mathrm{H}$ NMR spectra. One-bond proton-carbon correlations were established in an HSQC-DEPT experiment. Again, the presence of a saturated ketone was suggested by an IR $\mathrm{C}=\mathrm{O}$ stretch $\left(1729 \mathrm{~cm}^{-1}\right)$ and supported by a deshielded ${ }^{13} \mathrm{C}$ NMR resonance $\left(\delta_{\mathrm{C}} 222.3\right)$. The presence of a disubstituted carbon-carbon double bond was indicated by two deshielded ${ }^{1} \mathrm{H}$ resonances $\left(\delta_{\mathrm{H}} 5.63,5.26\right)$ that correlated to two ${ }^{13} \mathrm{C}$ resonances $\left(\delta_{\mathrm{C}} 134.6,130.3\right)$ in the HSQC-DEPT spectrum. As there was no further evidence of double bonds, a tetracyclic structure was required for $\mathbf{3 3}$ by the remaining degrees of unsaturation.

Detailed analysis of the COSY and 1D-TOCSY spectra allowed construction of two separate ${ }^{1} \mathrm{H}$ spin systems. The two systems were identified and constructed from the most prominent resonances in the ${ }^{1} \mathrm{H}$ NMR spectrum, those of an oxygenated methylene, two olefinic methines, and three oxygenated methines. The first spin system consisted of a linear sequence of carbons from a methylene $\left(C-1: \delta_{C} 36.8, \delta_{H} 0.94,1.69\right)$ and ending with a methine (C-14: $\left.\delta_{\mathrm{C}} 51.7, \delta_{\mathrm{H}} 2.74\right)$, as determined by COSY correlations between the resonances of their attached protons [methylene $\mathrm{C}-1$ to a methylene $\left(\mathrm{C}-2: \delta_{\mathrm{C}} 30.4, \delta_{\mathrm{H}} 1.36,1.77\right)$, oxymethine $\left(\mathrm{C}-3: \delta_{\mathrm{C}} 71.1, \delta_{\mathrm{H}} 3.54\right)$, methylene $\left(\mathrm{C}-4: \delta_{\mathrm{C}} 32.1, \delta_{\mathrm{H}} 1.24,2.24\right)$, methine $\left(\mathrm{C}-5: \delta_{\mathrm{C}} 47.0, \delta_{\mathrm{H}} 1.16\right)$, oxymethine 
$\left(\mathrm{C}-6: \delta_{\mathrm{C}} 73.5, \delta_{\mathrm{H}} \quad 3.16\right), \quad$ oxymethine $\quad\left(\mathrm{C}-7: \delta_{\mathrm{C}} \quad 74.5, \quad \delta_{\mathrm{H}} \quad 4.26\right)$, methine $\left(\mathrm{C}-8: \delta_{\mathrm{C}} 38.9, \delta_{\mathrm{H}} 1.58\right)$ and finally methine $\left.\mathrm{C}-14\right]$ (see figure 3.17$)$.

The branch from C-8 to C-12 was difficult to identify from COSY correlations due to the severe spectral overlap of various shielded ${ }^{1} \mathrm{H}$ resonances. A series of $1 \mathrm{D}-\mathrm{TOCSY}$ experiments were therefore carried out to establish this connection. Selective irradiation of the ${ }^{1} \mathrm{H}$ resonance of $\mathrm{H}-7\left(\delta_{\mathrm{H}} 4.26\right)$, using a variety of spin-lock mixing times, revealed correlations to two methines [H-8 $\left(\delta_{\mathrm{H}} 1.58\right)$ and $\left.\left(\mathrm{C}-9: \delta_{\mathrm{C}} 46.0, \delta_{\mathrm{H}} 0.89\right)\right]$, and two methylenes $\left[\left(\mathrm{C}-11: \delta_{\mathrm{C}} 21.3, \delta_{\mathrm{H}} 1.20,1.50\right)\right.$ and $\left.\left(\mathrm{C}-12: \delta_{\mathrm{C}} 36.5, \delta_{\mathrm{H}} 1.19,1.36\right)\right]$ in sequence, establishing the C-8 C-9, C-9 C-11, and C-11 C-12 linkages. A COSY correlation from the ${ }^{1} \mathrm{H}$ resonance of methine $\mathrm{H}-9$ to the deshielded proton $\mathrm{H}-11 \beta$, which was itself coupled to deshielded proton $\mathrm{H}-12 \beta$, confirmed these connections and completed spin system I. This spin system was entirely consistent with the structure established for clathriol A (32) (see figure 3.17).

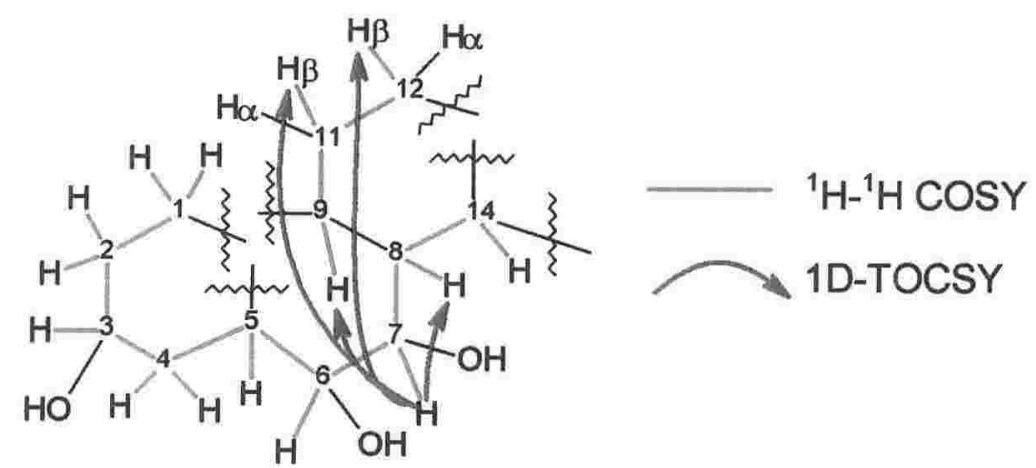

Figure 3.17 Selected COSY and 1D-TOCSY correlations establishing spin system I of clathriol B (33).

The second spin system from a methylene $\left(\mathrm{C}-16: \delta_{\mathrm{C}} 37.9, \delta_{\mathrm{H}} 2.17,2.40\right)$ to an oxymethylene (C-28: $\left.\delta_{\mathrm{C}} 64.7, \delta_{\mathrm{H}} 3.45,3.64\right)$ was established from analysis of observed COSY correlations between adjacent ${ }^{1} \mathrm{H}$ resonances [oxymethylene $\mathrm{H}-28$ to a methine $\left(\mathrm{C}-24: \delta_{\mathrm{C}} 52.3, \delta_{\mathrm{H}} 1.96\right)$, methine $\left(\mathrm{C}-25: \delta_{\mathrm{C}} 29.3, \delta_{\mathrm{H}} 1.58\right)$ to two methyls 
$\left(\mathrm{C}-26: \delta_{\mathrm{C}} 19.6, \delta_{\mathrm{H}} 0.81\right)$ and $\left(\mathrm{C}-27: \delta_{\mathrm{C}} 20.8, \delta_{\mathrm{H}} 0.86\right) ; \mathrm{H}-24$ to olefinic methine $\left(\mathrm{C}-23: \delta_{\mathrm{C}} 130.3, \delta_{\mathrm{H}}\right.$ 5.26), olefinic methine (C-22: $\left.\delta_{\mathrm{C}} 134.6, \delta_{\mathrm{H}} 5.63\right)$, methine $\left(\mathrm{C}-20: \delta_{\mathrm{C}} 34.9, \delta_{\mathrm{H}} 2.75\right)$ to both a methyl $\left(\mathrm{C}-21: \delta_{\mathrm{C}} 19.6, \delta_{\mathrm{H}} 0.99\right)$ and a methine (C-17: $\delta_{\mathrm{C}} 48.2, \delta_{\mathrm{H}} 1.76$ ), and finally $\mathrm{H}-17$ to methylene $\left.\mathrm{C}-16\right]$, completing spin system II (see figure 3.18).

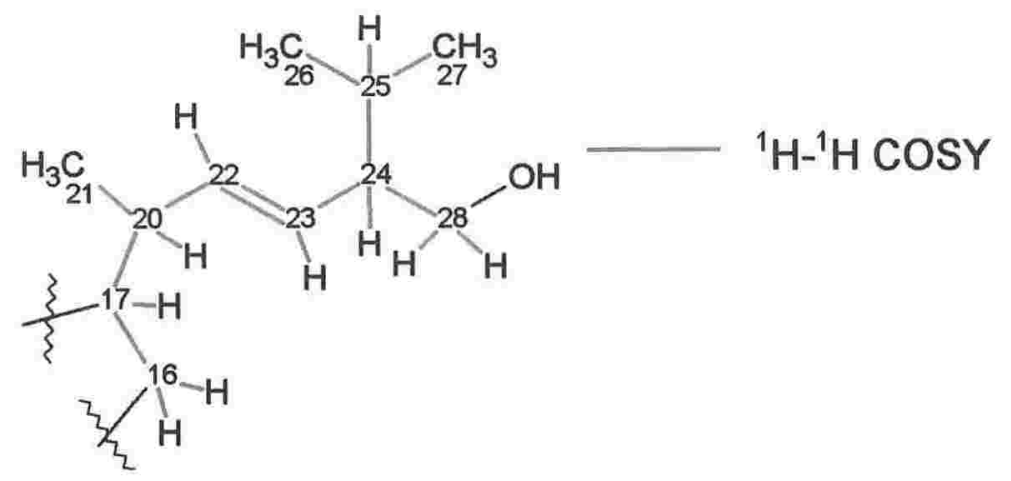

Figure 3.18 Selected COSY correlations establishing spin system II of clathriol B (33).

The final connectivity of the A, B, and C rings was established by analysis of the strong HMBC correlations observed from the ${ }^{1} \mathrm{H}$ resonances of two angular methyls $\left[\left(\mathrm{C}-18: \delta_{\mathrm{C}} 18.9, \delta_{\mathrm{H}} 1.21\right)\right.$ and $\left.\left(\mathrm{C}-19: \delta_{\mathrm{C}} 13.5, \delta_{\mathrm{H}} 0.80\right)\right]$. Strong correlations from $\mathrm{CH}_{3}-19$ to the ${ }^{13} \mathrm{C}$ resonances of a quaternary centre $\left(\mathrm{C}-10\right.$ : $\left.\delta_{\mathrm{C}} 36.0\right)$, methylene $\mathrm{C}-1$, and methines C-5 and C-9, connected ring A with ring B. A similar analysis from $\mathrm{CH}_{3}-18$ to the ${ }^{13} \mathrm{C}$ resonances of a quaternary centre $\left(\mathrm{C}-13: \delta_{C} 41.7\right)$, methines $\mathrm{C}-14$ and $\mathrm{C} 17$, and methylene C-12, completed ring C and connected spin systems I and II together (see figure 3.19). 


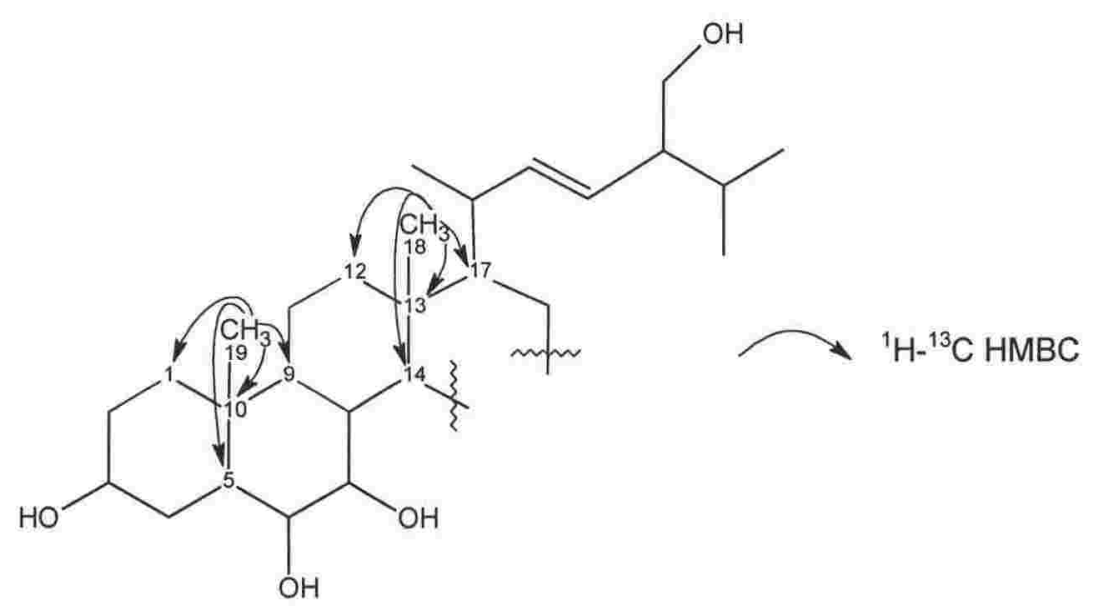

Figure 3.19 Selected HMBC correlations connecting spin systems I and II of clathriol B (33).

The only connections within the molecule remaining to be assigned were those to the ketone, which were finalised on the basis of observed HMBC correlations. Strong HMBC correlations were observed to a carbonyl ${ }^{13} \mathrm{C}$ resonance $\left(\mathrm{C}-15: \delta_{\mathrm{C}} 222.3\right)$ from the ${ }^{1} \mathrm{H}$ resonances of $\mathrm{H}-8, \mathrm{H}-14 \mathrm{H}-16 \alpha$ and $\mathrm{H}-16 \beta$. As with clathriol $\mathrm{A}(32)$, further evidence for the placement of ketone $\mathrm{C}-15$ was given by the deshielded chemical shifts of the adjacent protons $\mathrm{H}-14\left(\delta_{\mathrm{H}} 2.74\right), \mathrm{H}-16 \alpha\left(\delta_{\mathrm{H}} 2.40\right)$ and $\mathrm{H}-16 \beta\left(\delta_{\mathrm{H}} 2.17\right)$. Weak W-coupling between the resonances of $\mathrm{H}-14$ and $\mathrm{H}-16 \beta$, as observed in the COSY spectrum also helped to confirm this assignment. This completed ring D and finalised the tetracyclic structure of clathriol B (33) (see figure 3.20). 


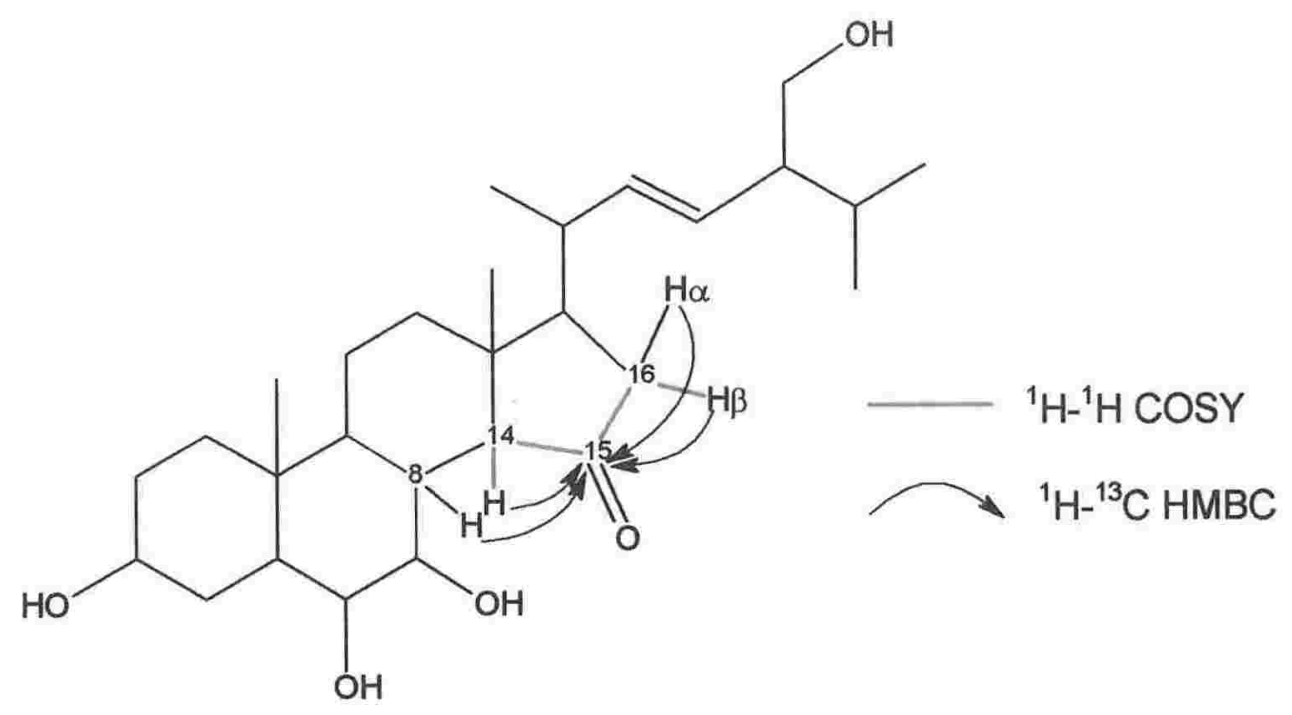

Figure 3.20 Selected COSY and HMBC correlations

establishing the final connectivity of clathriol B (33).

The relative stereochemistry of eleven of the twelve possible stereogenic centres of $\mathbf{3 3}$, as well as the geometry of the olefin, were determined from a combination of ${ }^{1} \mathrm{H}-{ }^{1} \mathrm{H}$ vicinal coupling constants and NOE measurements. The magnitudes of the ${ }^{1} \mathrm{H}-{ }^{1} \mathrm{H}$ vicinal coupling constants were measured in both the ${ }^{1} \mathrm{H}$ NMR spectrum and in several selective ${ }^{1} \mathrm{H}-{ }^{1} \mathrm{H}$ homonuclear-decoupling experiments. NOE correlations were detected in both 1D-gNOESY and ROESY experiments. The normal sterol conformation of rings A, B, and C, was confirmed by NOE correlations observed from the ${ }^{1} \mathrm{H}$ resonances of the two angular methyls $\mathrm{CH}_{3}-18$ and $\mathrm{CH}_{3}-19$ over the $\beta$ face of the molecule. In particular, 1,3-diaxial NOE correlations from the ${ }^{1} \mathrm{H}$ resonance of $\mathrm{CH}_{3}-19$ to those of $\mathrm{H}-2 \beta, \mathrm{H}-4 \beta$ and $\mathrm{H}-6$, established the chair conformations of rings $\mathrm{A}$ and $\mathrm{B}$, consistent with the standard steroid trans ring fusion. Similarly, strong NOE correlations from the ${ }^{1} \mathrm{H}$ resonance of $\mathrm{CH}_{3}-18$ to those of $\mathrm{H}-8, \mathrm{H}-22$ and $\mathrm{H}-23$, confirmed the chair conformation of ring $\mathrm{C}$ and also the placement of the side chain on the $\beta$ face of the molecule (see figures 3.21 and 3.22). 
The C-3 hydroxyl can be placed as equatorial on the basis of the observed coupling constants of the resonance of $\mathrm{H}-3$. The resonance of proton $\mathrm{H}-3$ shows two large couplings (greater than $10 \mathrm{~Hz}$ ) to those of $\mathrm{H}-2 \beta$ and $\mathrm{H}-4 \beta$, whilst showing two small couplings (less than $5 \mathrm{~Hz}$ ) to those of $\mathrm{H}-2 \alpha$ and $\mathrm{H}-4 \alpha$. This assignment supports the stereochemistry suggested by the observed NOE correlations from angular methyl $\mathrm{CH}_{3}-19$. This proposed assignment is further strengthened by observation of an NOE correlation from the resonance of H-3 to that of H-5 across the $\alpha$ face of $\mathbf{3 3}$ (see figure 3.21).

Strong NOE correlations from the resonance of $\mathrm{H}-6$ to those of both $\mathrm{H}-8$ and $\mathrm{CH}_{3}-19$, combined with the two large coupling constants measured between the resonances of H-6 and both H-5 and H-7, which are consistent with 1,2-trans diaxial relationships, allow placement of the C-6 hydroxyl as equatorial. The C-7 hydroxyl was also assigned as equatorial on the basis of the large coupling constants between the resonance of $\mathrm{H}-7$ $(>10 \mathrm{~Hz}$ ) and those of both $\mathrm{H}-6$ and $\mathrm{H}-8$, and also from the strong NOEs observed from the resonance of $\mathrm{H}-7$ to those of both $\mathrm{H}-5$ and to $\mathrm{H}-9$ across the $\alpha$ face of the molecule (see figure 3.21).

The unusual $\beta$ configuration of $\mathrm{H}-14$ was again suggested by the strong NOE observed between the resonances of $\mathrm{H}-8$ and $\mathrm{H}-14$. Weak long-range W-couplings were also observed between the resonances of $\mathrm{H}-14$ and both $\mathrm{H}-12 \beta$ and $\mathrm{H}-16 \beta$, confirming this assignment. A strong NOE was observed from the ${ }^{1} \mathrm{H}$ resonance of $\mathrm{CH}_{3}-18$ to a resonance at $2.74 \mathrm{ppm}$. This correlation, however, had to be ignored as both $\mathrm{H}-14$ and allylic proton $\mathrm{H}-20$ resonate at almost the same frequency $\left(\mathrm{H}-14: \delta_{\mathrm{H}}\right.$ 2.74; $\left.\mathrm{H}-20: \delta_{\mathrm{H}} 2.75\right)$ and both are in close spatial proximity to methyl $\mathrm{CH}_{3}-18$, therefore no valid inference could be made from this observation (see figure 3.21). 


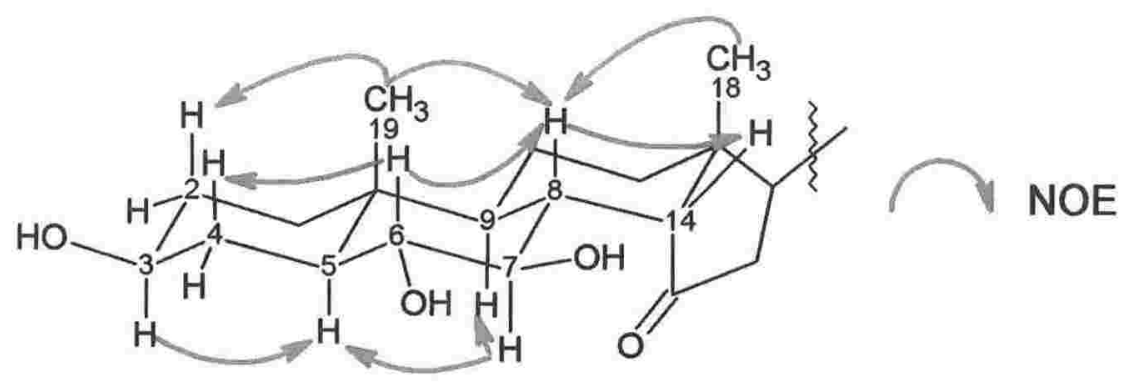

Figure 3.21 Selected NOE correlations establishing the relative stereochemistry of the tetracyclic portion of clathriol B (33).

Restricted rotation of the side chain, and therefore one dominant conformation, between C-17 and C-20 was indicated by the weak coupling observed between their attached protons. This was evidenced by the observation of a COSY cross peak between their ${ }^{1} \mathrm{H}$ resonances and yet the absence of a measurable coupling constant. These observations are indicative of a bond angle of approximately $90^{\circ}$ between $\mathrm{H}-17$ and $\mathrm{H}-20$. NOE correlations from the resonance of $\mathrm{H}-23$ to those of $\mathrm{CH}_{3}-18$ and $\mathrm{H}-16 \beta$, together with a correlation from the resonance of $\mathrm{H}-22$ also to that of $\mathrm{CH}_{3}-18$ clearly places the olefin of the side chain above the plane of ring $D$ on the $\beta$ face of the molecule in an anti-periplanar relationship to $\mathrm{H}-17$. The $R$ configuration of $\mathrm{C}-20$ is confirmed by an NOE correlation from the resonance of $\mathrm{H}-16 \beta$ to that of $\mathrm{CH}_{3}-21$ (see figure 3.22).

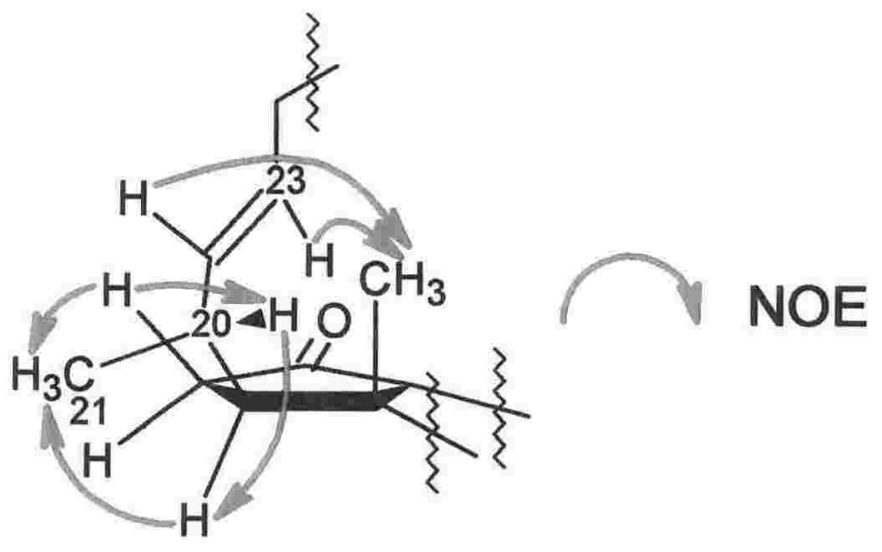

Figure 3.22 Selected NOE correlations establishing the relative stereochemistry of C-20 of clathriol B (33). 
Finally, the geometry of the olefin was assigned as $E$ on the basis of the large observed coupling constant measured between H-22 and H-23 (15.8 Hz). NOE correlations were observed from the resonance of $\mathrm{H}-22$ to that of $\mathrm{H}-20$, and from $\mathrm{H}-23$ to those of $\mathrm{H}-16 \beta$, $\mathrm{CH}_{3}-21$ and $\mathrm{H}-24$, which helped to confirm this proposal. Assuming normal steroid stereochemistry, these observations allow clathriol B (33) to be assigned as $3 S, 5 S, 6 R$, $7 R, 8 R, 9 S, 10 R, 13 R, 14 R, 17 R, 20 R, 22(E)$.

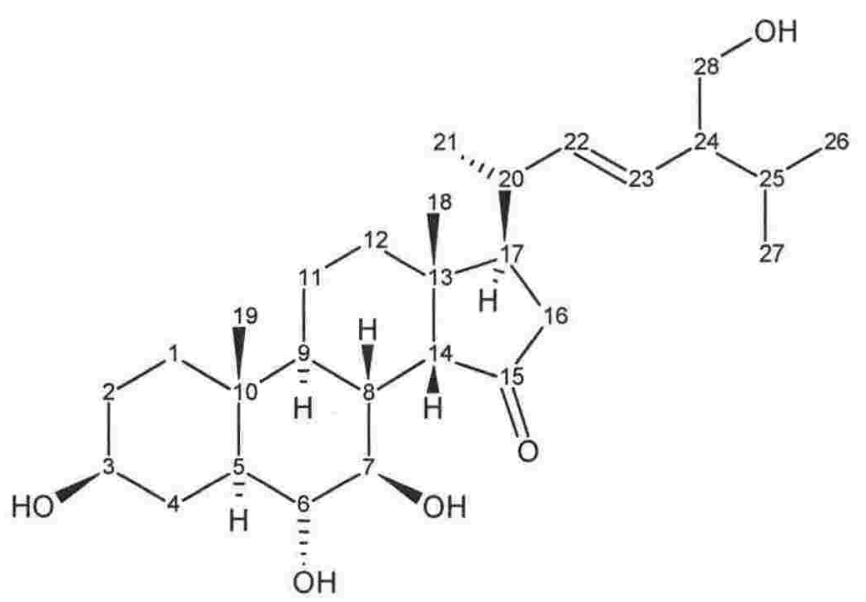

(33) 
Table $3.2{ }^{13} \mathrm{C}(75 \mathrm{MHz})$ and ${ }^{1} \mathrm{H}(300 \mathrm{MHz}) \mathrm{NMR}$ data $\left(\mathrm{CDCl}_{3}\right)$ of clathriol B (33).

\begin{tabular}{|c|c|c|c|c|c|c|c|}
\hline \multirow[b]{2}{*}{ Position } & \multicolumn{2}{|c|}{${ }^{13} C^{a}$} & \multicolumn{2}{|r|}{${ }^{1} \mathrm{H}^{\mathrm{b}}$} & \multicolumn{3}{|c|}{ HMBC } \\
\hline & $\delta(\mathrm{ppm})$ & mult & $\delta(\mathrm{ppm})$ & mult, $J(\mathrm{~Hz})$ & COSY & (H to $\mathrm{C})$ & NOE \\
\hline $1 \alpha$ & 36.8 & $\mathrm{CH}_{2}$ & 0.94 & $\mathrm{~m}$ & $1 \beta, 2 \alpha$ & & $1 \beta, 3$ \\
\hline$\beta$ & & & 1.69 & $\mathrm{~m}$ & $1 \alpha, 2 \alpha$ & & $1 \alpha$ \\
\hline $2 \beta$ & 30.4 & $\mathrm{CH}_{2}$ & 1.36 & $\mathrm{~m}$ & $1 \alpha, 1 \beta, 2 \beta, 3,4 \beta$ & & $2 \alpha, 19$ \\
\hline$\alpha$ & & & 1.77 & $\mathrm{~m}$ & $2 \alpha, 3$ & & $2 \beta, 6$ \\
\hline 3 & 71.1 & $\mathrm{CH}$ & 3.54 & dddd $(14.6,10.3,4.3,3.8)$ & $2 \alpha, 2 \beta, 4 \alpha, 4 \beta$ & & $1 \alpha, 2 \alpha, 4 \alpha, 4 \beta, 5$ \\
\hline $4 \beta$ & 32.1 & $\mathrm{CH}_{2}$ & 1.24 & $\mathrm{~m}$ & $3,4 \beta$ & 6 & $3,6,19$ \\
\hline$\alpha$ & & & 2.24 & $\mathrm{~m}$ & $3,4 \alpha, 5,2 \alpha$ & 6 & 3,5 \\
\hline 5 & 47.0 & $\mathrm{CH}$ & 1.16 & $\mathrm{~m}$ & $4 \beta, 6$ & & $3,6,7$ \\
\hline 6 & 73.5 & $\mathrm{CH}$ & 3.16 & $\mathrm{dd}(10.3,9.1)$ & 5,7 & & $4 \beta, 5,7,8,19$ \\
\hline 7 & 74.5 & $\mathrm{CH}$ & 4.26 & dd $(10.6,8.8)$ & 6,8 & 6 & $5,6,8,9,12 \alpha, 14$ \\
\hline 8 & 38.9 & $\mathrm{CH}$ & 1.58 & $\mathrm{dt}(11.8,4.2)$ & 7,14 & $7,9,11,15$ & $6,7,14,18$ \\
\hline 9 & 46.0 & $\mathrm{CH}$ & 0.89 & $\mathrm{~m}$ & $11 \beta$ & & 7 \\
\hline 10 & 36.0 & $\mathrm{C}$ & & & & & \\
\hline $11 \alpha$ & 21.3 & $\mathrm{CH}_{2}$ & 1.20 & $\mathrm{~m}$ & $11 \beta$ & & \\
\hline$\beta$ & & & 1.50 & $\mathrm{~m}$ & $9,11 \alpha, 12 \beta$ & & 18 \\
\hline $12 \alpha$ & 36.5 & $\mathrm{CH}_{2}$ & 1.19 & $\mathrm{~m}$ & $12 \beta$ & & 17 \\
\hline$\beta$ & & & 1.36 & $\mathrm{~m}$ & $11 \alpha, 12 \alpha, 14$ & & \\
\hline 13 & 41.7 & $\mathrm{C}$ & & & & & \\
\hline 14 & 51.7 & $\mathrm{CH}$ & 2.74 & $\mathrm{~m}$ & $8,12 \beta, 16 \alpha$ & $8,12,13,15$ & $7,8,18$ \\
\hline 15 & 222.3 & $\mathrm{C}$ & & & & & \\
\hline $16 \beta$ & 37.9 & $\mathrm{CH}_{2}$ & 2.17 & br d (19.9) & $16 \beta, 17,14$ & $13,15,20$ & $16 \alpha, 17,18,21,22,23$ \\
\hline$\alpha$ & & & 2.40 & dd $(19.9,9.3)$ & $16 \alpha, 17$ & 15,20 & $16 \beta, 21$ \\
\hline 17 & 48.2 & $\mathrm{CH}$ & 1.76 & $\mathrm{dd}(10.8,2.7)$ & $16 \alpha, 16 \beta, 20$ & & $12 \alpha, 21$ \\
\hline 18 & 18.9 & $\mathrm{CH}_{3}$ & 1.21 & $\mathrm{~s}$ & & $12,13,14,17$ & $8,11 \beta, 14,20,22$ \\
\hline 19 & 13.5 & $\mathrm{CH}_{3}$ & 0.80 & $\mathrm{~s}$ & & $1,5,9,10$ & $2 \beta, 4 \beta, 6$ \\
\hline 20 & 34.9 & $\mathrm{CH}$ & 2.75 & $\mathrm{~m}$ & $17,21,22$ & 17 & $16 \beta, 18,21,22$ \\
\hline 21 & 19.6 & $\mathrm{CH}_{3}$ & 0.99 & $\mathrm{~d}(7.0)$ & 20 & $17,20,22$ & $16 \beta, 17,20,23$ \\
\hline 22 & 134.6 & $\mathrm{CH}$ & 5.63 & $\mathrm{dd}(15.8,7.5)$ & 20,23 & $20,21,24$ & $16 \beta, 18,20,24$ \\
\hline 23 & 130.3 & $\mathrm{CH}$ & 5.26 & $\mathrm{dd}(15.3,8.0)$ & 22,24 & $20,24,28$ & $12 \alpha, 16 \beta, 21,24,26,28 \mathrm{a}$ \\
\hline 24 & 52.3 & $\mathrm{CH}$ & 1.96 & $\mathrm{~m}$ & $23,25,28 \mathrm{a}, 28 \mathrm{~b}$ & & 22 \\
\hline 25 & 29.3 & $\mathrm{CH}$ & 1.58 & $\mathrm{~m}$ & $24,26,27$ & 24,28 & 26,27 \\
\hline 26 & 19.6 & $\mathrm{CH}_{3}$ & 0.81 & $\mathrm{~d}(6.7)$ & 25 & $24,25,27$ & 25 \\
\hline 27 & 20.8 & $\mathrm{CH}_{3}$ & 0.86 & $\mathrm{~d}(6.7)$ & 25 & $24,25,26$ & 23,25 \\
\hline $28 \mathrm{a}$ & 64.7 & $\mathrm{CH}_{2}$ & 3.45 & $\mathrm{t}(10.4)$ & $24,28 b$ & 23,24 & $24,26,27$ \\
\hline $\mathrm{b}$ & & & 3.64 & dd $(10.6,4.1)$ & $24,28 \mathrm{a}$ & 23,24 & $24,25,27$ \\
\hline
\end{tabular}

a Multiplicity determined from an HSQC-DEPT (multiplicity edited HSQC) experiment

b Carbon connectivity determined from an HSQC-DEPT experiment, multiplicity determined from ${ }^{1} \mathrm{H}$, 1D-TOCSY and ${ }^{1} \mathrm{H}$ homonuclear decoupled experiments. 
All of the NMR spectral correlations observed for the tetracyclic portions of clathriol A (32) and for clathriol A (33) were in complete agreement with each other. The substitution of the tetracyclic portion is identical in both cases, therefore the consistency of the independently determined NMR data helped to confirm the proposed structures of both metabolites. Both assignments were also consistent with those of the $\mathrm{B}, \mathrm{C}$ and $\mathrm{D}$ rings of contignasterol (24) which shares the same functionality of these rings. ${ }^{92}$ The ${ }^{1} \mathrm{H}$ and ${ }^{13} \mathrm{C}$ NMR chemical shifts of the side chain of $\mathbf{3 3}$ are also consistent with that published previously for sterols with the same side chain. ${ }^{103,104}$

\subsection{Biological Activity}

As contignasterol (24), and both xestobergsterols A (25) and B (26), had exhibited potent anti-inflammatory activity, clathriol A (32) was submitted for both cytotoxicity and anti-inflammatory testing. ${ }^{90-94,96}$ It was found that $\mathbf{3 2}$ was not cytotoxic to any great degree in the assay used (HL-60). It was, however, found to possess moderate antiinflammatory activity as measured by the inhibition of superoxide production by human peripheral blood neutrophils, stimulated with either fMLP (N-formyl-methionineleucine-phenylalanine) or PMA (phorbol myristate acetate) with $\mathrm{IC}_{50}$ values of $33 \mu \mathrm{M}$ and $140 \mu \mathrm{M}$ respectively. ${ }^{105}$ Due to a paucity of material, clathriol B (33) was not submitted for cytotoxicity screening, although it returned values of $27 \mu \mathrm{M}$ and $130 \mu \mathrm{M}$ in the superoxide anti-inflammatory assay, showing remarkably similar potency to that of 32. Superoxide has been implicated as a cause of inflammation. In this assay, human neutrophils are stimulated with PMA or fMLP to produce superoxide. The cells are then exposed to the test compounds in order to assess whether they prevent inflammation as measured by production of superoxide. ${ }^{105}$ PMA and fMLP activate neutrophils at different points of the cellular anti-inflammation process therefore the different 
activities of $\mathbf{3 2}$ and $\mathbf{3 3}$ against activation by PMA or fMLP imply that they inhibit inflammation at different points of the cycle.

Literature describing the use of contignasterol (24) as an anti-inflammatory agent, suggests that hemi-acetal or alcohol functionality is needed at C-29 for full biological potency. It is also suggested that $\mathrm{CH}_{3}-21$ methyl or $\mathrm{C}-24$ isopropyl groups are not crucial for the biological activity of the molecules. ${ }^{106,107}$ Both $\mathbf{3 2}$ and $\mathbf{3 3}$ possess alcoholic functionality at $\mathrm{C}-28$, which is close to that portion of $\mathbf{2 4}$ that is required for activity. Minor variation of the side chain between $\mathbf{3 2}$ and $\mathbf{3 3}$ (i.e. presence of an olefin, methyl or ethyl substitution at C-24) has no noticeable effect on their anti-inflammatory activity. Similarly, the substitution of the B, C and D rings of $\mathbf{3 2}$ and $\mathbf{3 3}$ is the same as that of 24. This may suggest that this functionalisation of the rings is required for the anti-inflammatory activity noted for these three molecules.

\section{Derivatisations of Clathriol A (32)}

Several derivatisations of clathriol A (32) were attempted in order to confirm the structure of the molecule. Contignasterol (24) shares both similar substitution and the same stereochemistry of the B, C and D rings as $\mathbf{3 2}$ and clathriol B (33). Due to the slow epimerisation of the hemi-acetal side-chain, the structural assignment of $\mathbf{2 4}$ was carried out on its tetra-acetate derivative. In order to compare the structure of $\mathbf{2 4}$ with that of 32, the tetra-acetate derivative of clathriol A was prepared. The ${ }^{13} \mathrm{C}$ and ${ }^{1} \mathrm{H}$ resonances of clathriol tetra-acetate were assigned from correlations observed in the COSY, HSQC and HMBC spectra. Both the chemical shifts and coupling constants of H-8, H-9, H-16 $\alpha$ and H-16 $\beta$ in both tetra-acetates were essentially identical to those observed for the contignasterol tetra-acetate, confirming the structural assignment of $\mathbf{3 2}$. 
Two further reactions were carried out upon clathriol A (32). First, the base-induced epimerisation of $\mathrm{C}-14$ via an enolate ion was attempted by reacting 32 with $\mathrm{NaOMe}$. The reaction generated an intractable mixture of compounds, including some starting material. It is possible that alkoxide ions were formed with the four hydroxyl functionalities in competition to with the formation of the enolate ions, forming the mixture of compounds.

Second, the $\mathrm{NaBH}_{4}$ reduction of C-15 was attempted. Again, this gave a mixture of at least two compounds. The ${ }^{1} \mathrm{H}$ NMR spectrum of the mixture included at least two additional oxygenated methine resonances. This implies the formation of two C-15 reduction products that are epimers of each other. These would be formed due to a lack

of preference for the attack of the hydride nucleophile upon the two faces of the $\mathrm{sp}^{2}$ ketone centre.

\subsection{Other Secondary Metabolites Isolated From the Genus Clathria}

Metabolites from Clathria appear to be largely under-represented in the marine natural products literature with very few compounds reported from the genus. The only other compounds previously reported include four steroids, three carotenoids, two ceramides, thio-sugars, alkaloids, and polyolefinics.

Three novel 7-keto sterols were isolated from the Mediterranean sponge Clathria clathrus. Structures (34-36) are the first examples of steroids isolated from a natural source with the novel dienone oxidation pattern of ring B shown. ${ }^{108}$ 


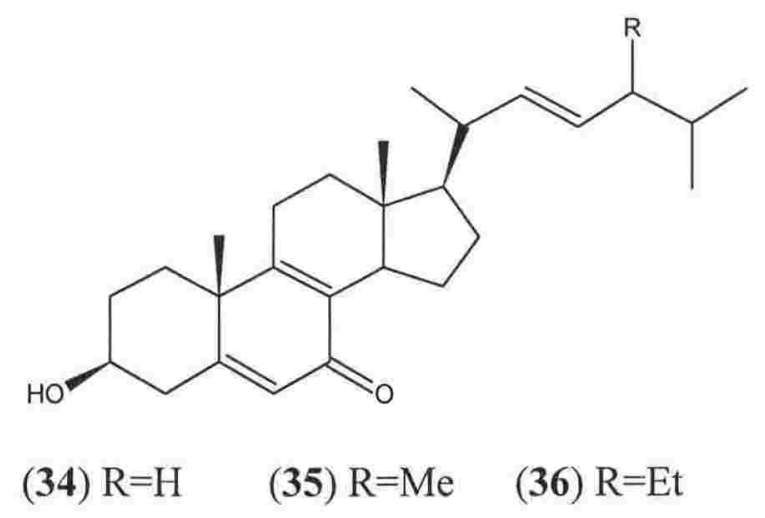

A novel sulfated sterol has recently been isolated from an undescribed Clathria species. Clathsterol (37) was isolated by bioactivity directed fractionation after the sponge's crude extract was shown to contain a strong inhibitor of human immunodeficiency virus type 1 reverse transcriptase (HIV-1 RT). ${ }^{109}$

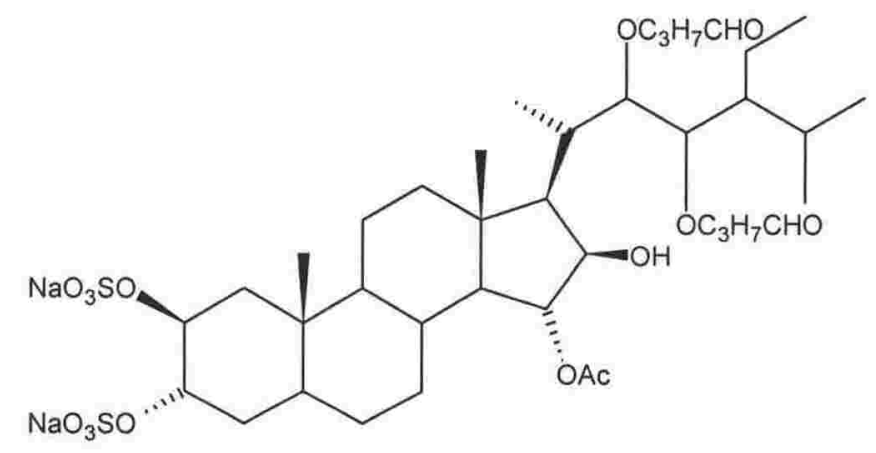

Tanaka et al have reported a series of related carotenoids (38-40) from the sponge

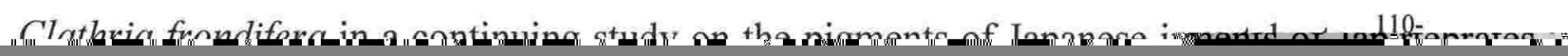


<smiles>[R]C=CC1=C(C)C(=O)C(O)=CC1(C)C</smiles>

(38)<smiles>[R]OC1CC(C)(C)C(/C=C/P)=C(C)C1=O</smiles>

(39)<smiles>[R]C(=O)CC(=O)C1(C)CC(O)CC1C</smiles>

(40)

$\mathrm{R}=$<smiles>CC(C)=C/C=C/C(C)=C/C=C/C=C/C=C/C=C/C=C/c1ccc(C)c(C)c1C</smiles>

Capon's group in Australia have isolated several novel compounds from different specimens of Clathria including 5-thio-D-mannose (41) from Clathria pyramida, which was the first reported isolation of a 5-thiosugar from a natural source. ${ }^{114}$ They also published clathrins A, B, and C (42-44) from an undescribed Clathria species. Although not biologically active, they are of interest as they are possible linking intermediates between the mixed terpenoid/shikimate and sesquiterpene/benzoid biosynthetic pathways. ${ }^{115}$ Finally, they have also published the structure of the novel anti-bacterial alkaloid, mirabilin G (45), from an undescribed Clathria sp.. ${ }^{116}$

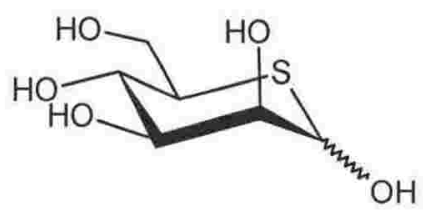

(41)<smiles>CC(=O)C1=CC(CC/C(C)=C/C=C/C(C)/C=C\CC(C)C)CCC1</smiles>

(42) 
<smiles>CC(/C=C/C(O)c1cccc(C)c1C)=C\C=C\C(C)C</smiles>

(43)<smiles>CC(/C=C/C(=O)c1cccc(C)c1C)=C\C=C\C(C)C</smiles>

(44)

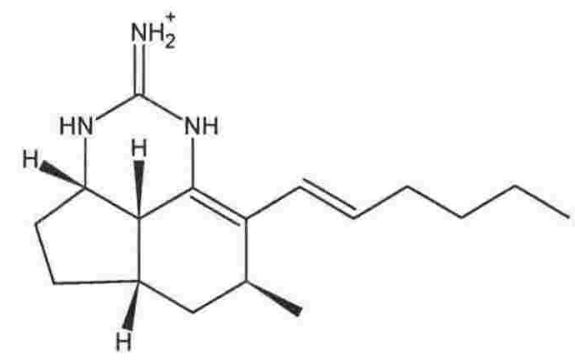

(45)

The clathrynamides A, B, and C (46-48) were isolated from an undescribed Clathria sp.. Amide $\mathbf{4 6}$ was shown to strongly inhibit the mitotic cell division of starfish eggs, and also inhibited the growth of human myeloid K-562 cells in vitro, whilst $\mathbf{4 7}$ and $\mathbf{4 8}$ were not particularly active in either assay. ${ }^{117}$

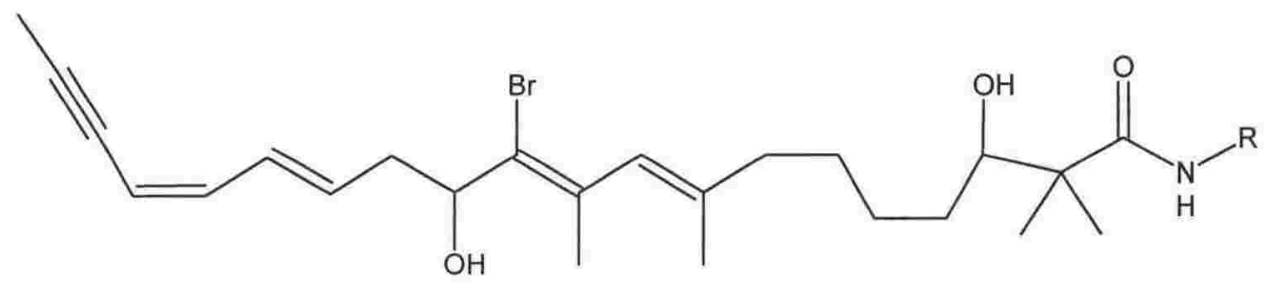

(46) $\mathrm{R}=\mathrm{H}$

(47) $\mathrm{R}=\mathrm{CH}\left(\mathrm{CH}_{3}\right) \mathrm{CH}_{2} \mathrm{CH}_{2} \mathrm{CH}(\mathrm{OH}) \mathrm{CH}_{3}$

(48) $\mathrm{R}=\mathrm{CH}\left(\mathrm{CH}_{3}\right) \mathrm{CH}_{2} \mathrm{CH}_{2} \mathrm{COCH}_{3}$

A series of novel ceramide homologues (49) and (50) were recently isolated from Clathria fasciulate collected in the South China Sea near Hainan Island. ${ }^{118}$ 


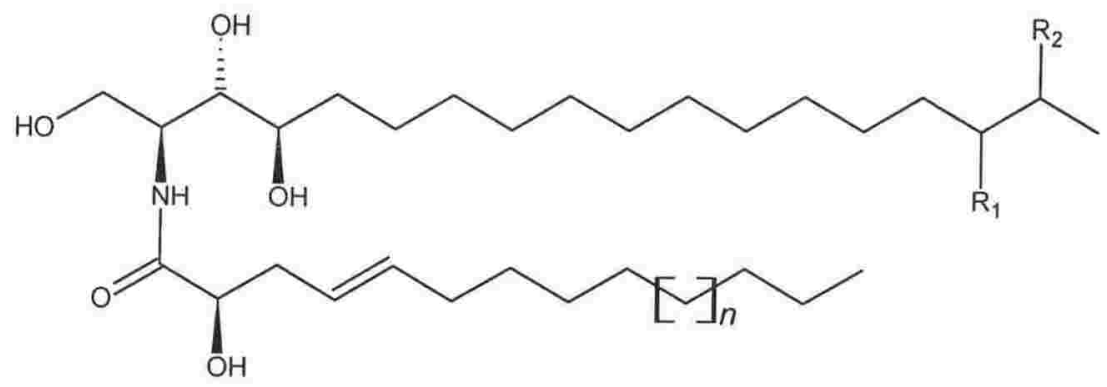

(49) $\mathrm{R}_{1}=\mathrm{CH}_{3}, \mathrm{R}_{2}=\mathrm{H}, n=10-13$

(50) $\mathrm{R}_{1}=\mathrm{H}, \mathrm{R}_{2}=\mathrm{CH}_{3}, n=10-13$

The massive orange Indo-Pacific sponge Clathria basilana yielded the inactive alkaloid clathryimine A (51), which was notable as it was the first quinolizinium metabolite isolated from a sponge. ${ }^{119}$<smiles></smiles>

(51)

Finally, the Palermo group has recently described three novel pteridine-type alkaloids, pseudoanchynazines A, B and C (52-54), from a deep sea Clathria sp., collected in the Southern Atlantic Ocean at a clam fishery. This group is taking advantage of aquaculture farms to gain access safely to sponges in areas that would normally be either too deep or too extreme an environ (low visibility, rough weather conditions, etc) to collect in. ${ }^{120}$ 


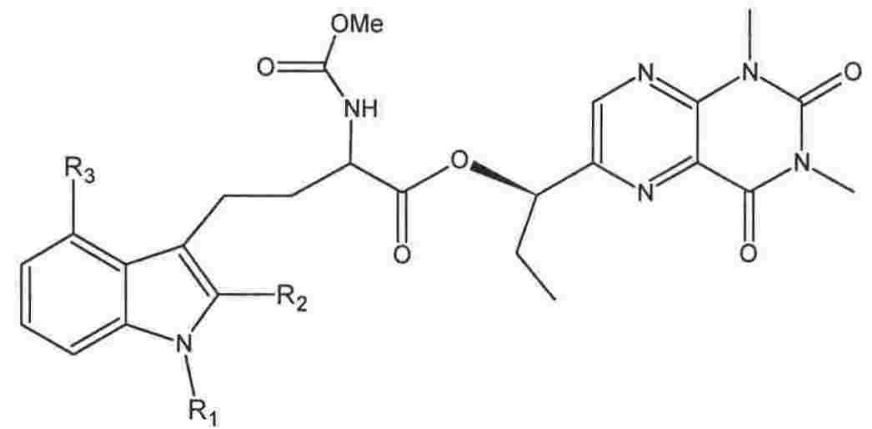

(52) $\mathrm{R}_{1}=\mathrm{R}_{3}=\mathrm{H}, \mathrm{R}_{2}=\mathrm{A}$

(53) $\mathrm{R}_{1}=\mathrm{A}, \mathrm{R}_{2}=\mathrm{R}_{3}=\mathrm{H}$

(54) $\mathrm{R}_{1}=\mathrm{R}_{2}=\mathrm{H}, \mathrm{R}_{3}=\mathrm{A}$

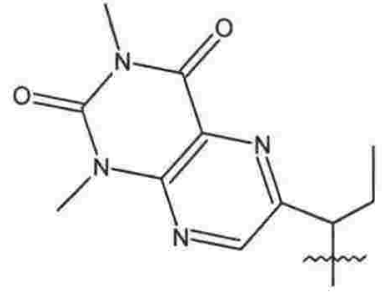

A 


\section{Chapter Four}

\section{Spongian Diterpene Constituents of the}

\section{Marine Sponge Chelonaplysilla violacea}

\subsection{Dictyoceratid and Dendroceratid Sponges}

Sponge taxonomists still debate the phylogenic placement of many sponges within various orders, families, and genera, a situation closely linked to the difficulty in positively identifying different specimens. ${ }^{21-23,34}$ In fact, there is even still debate at the phylum and class level of sponge taxonomy. ${ }^{23}$ Problems with identification may arise from either a historical taxonomic misplacement of an archetypical sponge, or from disagreement on which morphological characteristics (spicule composition, colour, shape, etc) to use for identification. ${ }^{21,22,34}$

Sponges of the Dictyoceratid and Dendroceratid orders exemplify this debate. These two orders contain sponges totally devoid of mineral spicules, as does the Verongid order. It is not surprising that sponges devoid of spicules are difficult to identify, as the presence of certain spicule types is the most commonly used taxonomic marker. Historically, sponges lacking inorganic spicules and with an obvious dendritic spongin skeleton and large sac-shaped choanocytes, were classified as Dendroceratid sponges, while others with anastomosing (netted) spongin skeletons were placed in the Dictyoceratid order. Members of the Verongida are quite distinct from those of either the Dictyoceratid or Dendroceratid orders, as their skeletons are comprised of pith containing collagen fibres, rather than fibres made from spongin. The histology of Verongid sponge cells is also quite different to that of the Dictyoceratid or

Dendroceratid orders. ${ }^{21,33,121}$ The difference between dendritic and anastomosing skeletons can be difficult to identify, however, and may have led to sponges currently 
classed as either Dendroceratida or Dictyoceratida, to be artificially separated. As techniques for the identification of sponges become more sophisticated, problems in identification may be eliminated and various sponge orders, families, and genera, may be taxonomically reassigned, created, or removed, on the basis of genetic, microbiological, or chemotaxonomic considerations. Interestingly, Bergquist has suggested that, contrary to accepted practice for identification of most other sponge orders, colour is often a diagnostic feature that can be used to differentiate between species of the Dictyoceratid and Dendroceratid orders. ${ }^{33}$

Bergquist has also suggested that sesquiterpene content can be used a chemotaxonomic marker between members of the two orders. ${ }^{23,121}$ Previous work on a New Zealand Dysidea sp. (Dictyoceratida) carried out within our laboratory has supported this proposal. ${ }^{122}$ During the current study, a series of spongian diterpenes were isolated from a New Zealand specimen of Chelonaplysilla violacea (Dendroceratida). It was therefore prudent to evaluate the available literature on any diterpenoids isolated from both the Dictyoceratid and Dendroceratid orders, to determine if this class of compounds are also of chemotaxonomic value. 


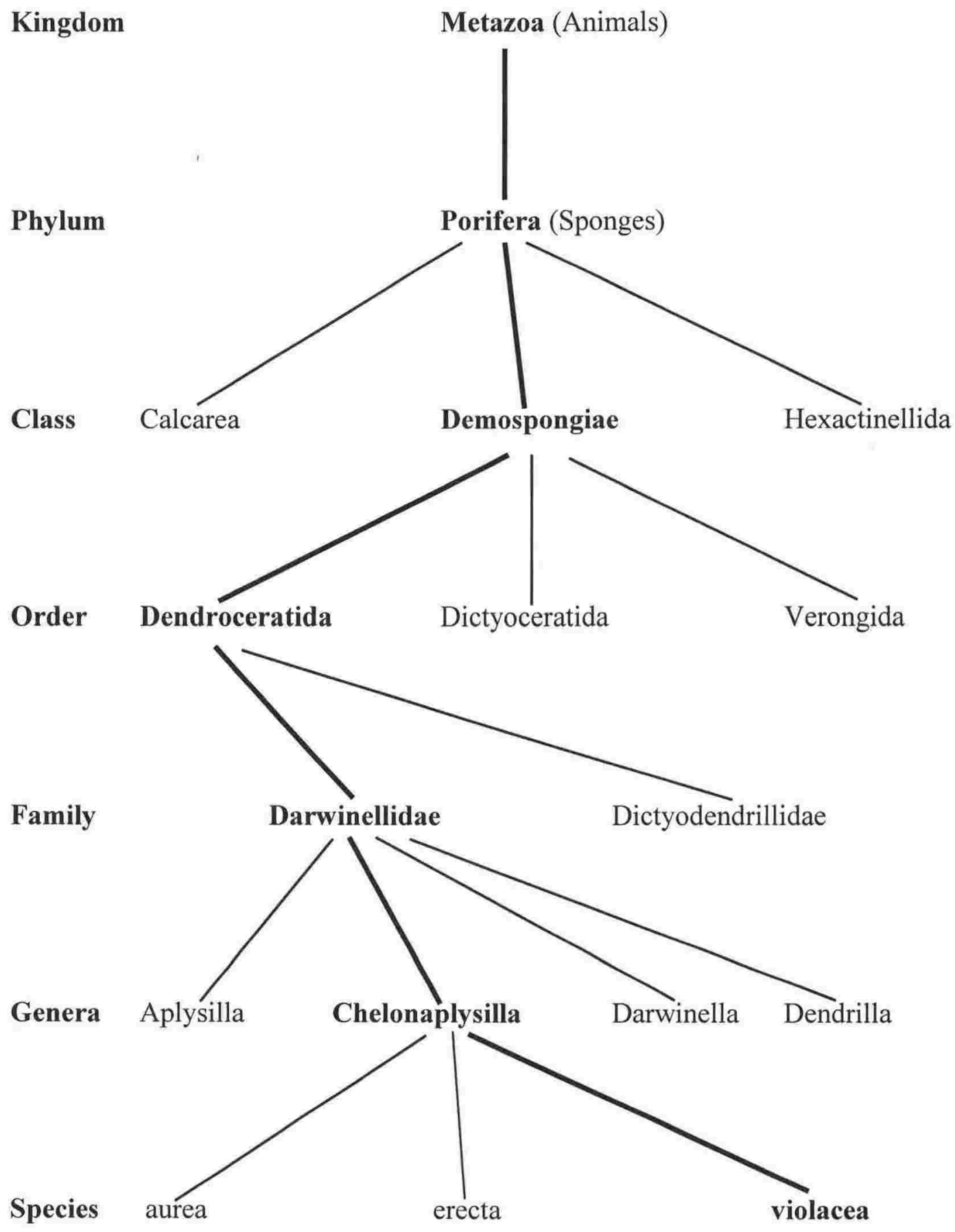

Scheme 4.1 Phylogenic relationship of Chelonaplysilla violacea. For simplicity, not all orders or species are shown. $^{21,23,34,121,123}$ 
A survey of the literature to the end of 2002, encompassing all diterpenes produced by both Dictyoceratid and Dendroceratid sponges, yielded well over 130 individual structures. The following review summarises the major carbon skeletal groups, with salient examples, rather than being a comprehensive listing of all the diterpenes isolated from the two orders.

\subsection{Miscellaneous Diterpenes}

Several varied classes of diterpenes have been isolated from Dictyoceratid and Dendroceratid sponges. Faulkner and Walker published the structures ambliols A (55) and B (56), ambliofuran (57), ambliolide (58) and dehydroambliol A (59), isolated from the sponge Dysidea amblia. The structure of $\mathbf{5 6}$ was later revised when it was reisolated with ambliol C (60). ${ }^{124,125}$

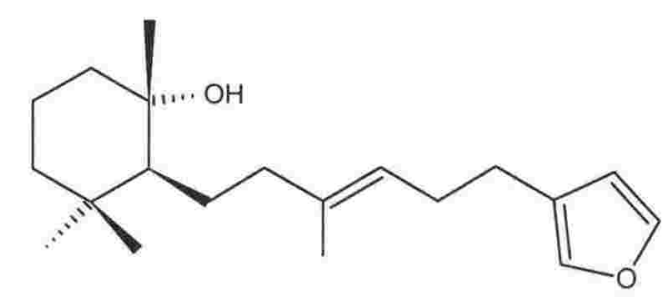

(55)

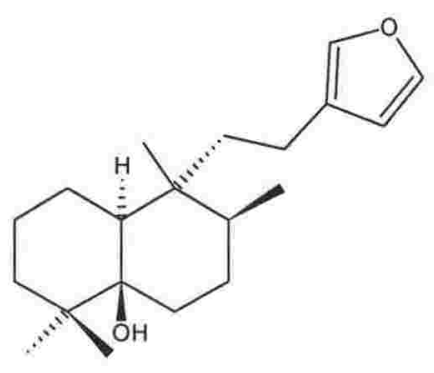

(56)

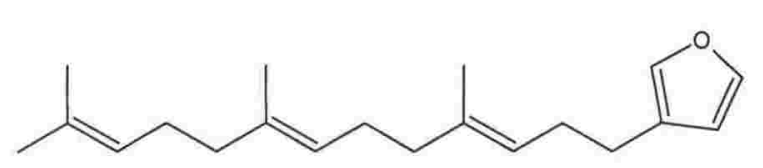

(57)

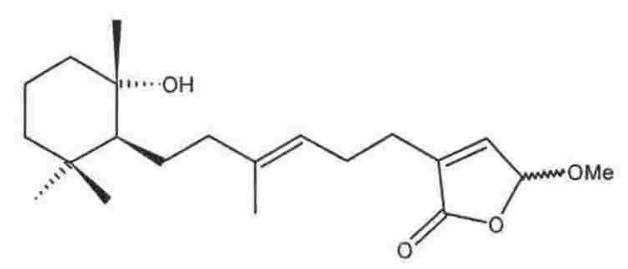

(58) 


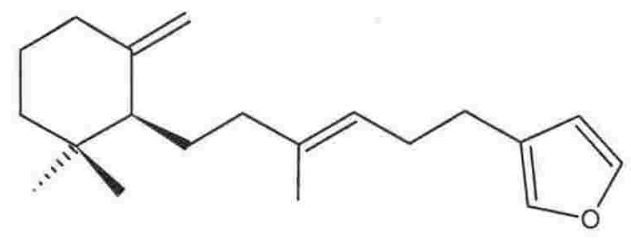

(59)

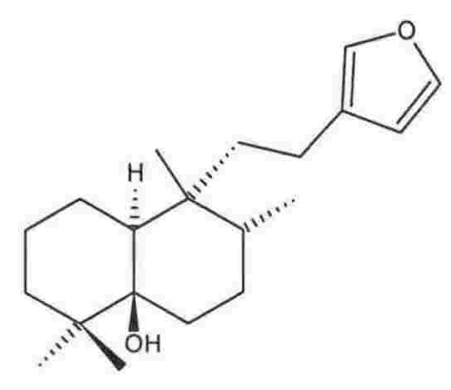

(60)

The sponge Dendrilla sp. collected from a lake in Palau yielded (61), along with several members of the dendrillolide family of compounds. Several structural revisions of the dendrillolides were suggested after further isolation of these metabolites from the predatory nudibranch Chromodoris macfarlandi ${ }^{27,126,127}$

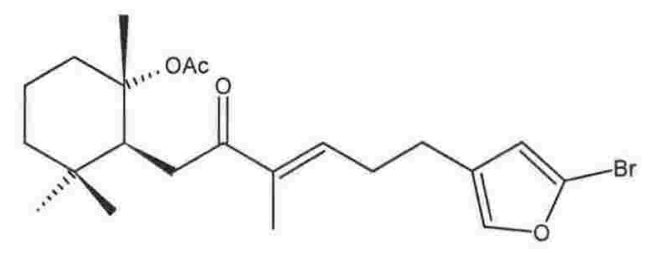

(61)

Three diterpenes with very different carbon skeletons were isolated from the Red Sea sponge Chelonaplysilla erecta. Chelodane (62), barekoxide (63) and zaatirin (64) were all biologically inactive in the assays used, although the initial extract from the sponge showed significant anti-tumour activity. ${ }^{128}$

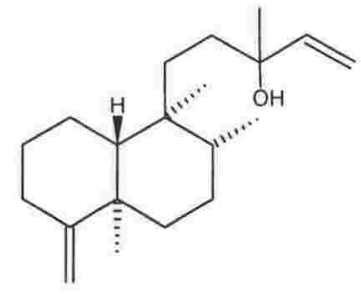

(62)

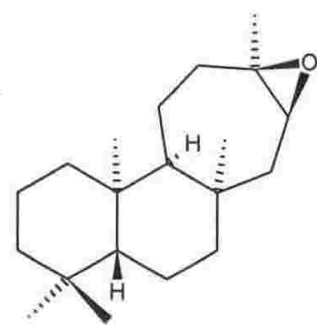

(63)

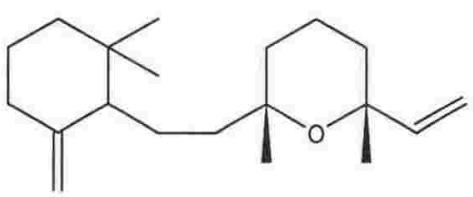

(64)

$\mathrm{Lu}$ and Faulkner isolated three new dolabellane diterpenes (65-67) and a macrolide (68) from a Dysidea sp. collected in Palau. Neither dolabellane diterpenes nor any 
macrolides had previously been isolated from any species of Dysidea, thus the authors felt that the four novel compounds were likely to be metabolic products from other organisms that the sponge had obtained from its environment. ${ }^{129}$

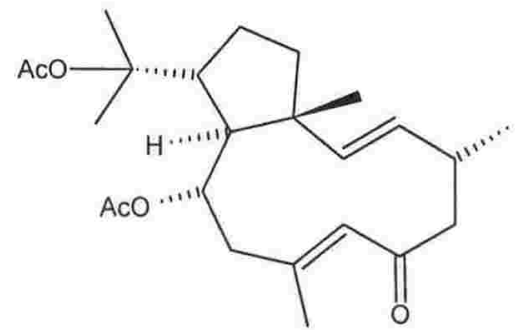

(65)

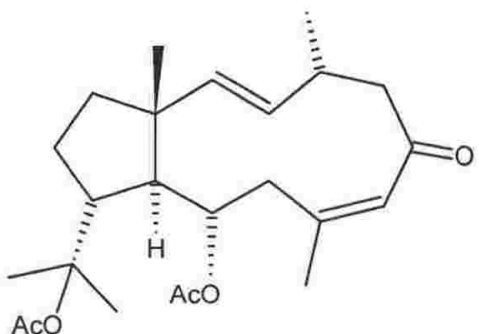

(66)

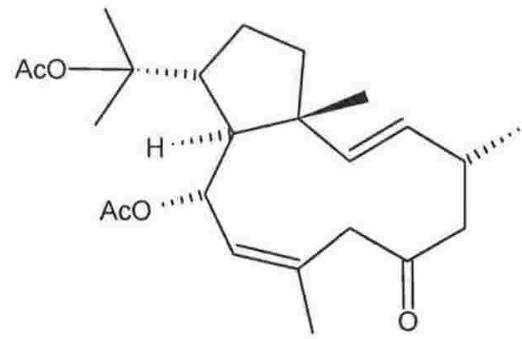

(67)<smiles>C=C(CCCC(O)CC(C)O)C[C@H](CC(=C)C[C@@H](O)C/C(C)=C/[C@H](O)CCCC(=O)O)OC(=O)CC</smiles>

(68)

\subsection{Spongian Diterpenes}

By far, the most common type of diterpene from either the Dictyoceratid or Dendroceratid orders, are those based upon the spongian carbon skeleton (69). This group of compounds is so named, as many of the early members of this class were isolated from the Dictyoceratid genus Spongia. ${ }^{130}$ The biochemistry of the spongian diterpenes is punctuated with many examples of highly oxygenated and oxidatively cleaved carbon skeletons. This is a quite different theme to that of many other classes of compounds found from sponges.

The first metabolite isolated in this large group was isoagatholactone (70), from the common Mediterranean bath sponge Spongia officinalis. The structure of $\mathbf{7 0}$ was 
confirmed by chemical correlation to the known compound grindelic acid, which also established the absolute stereochemistry of the natural product. ${ }^{131}$

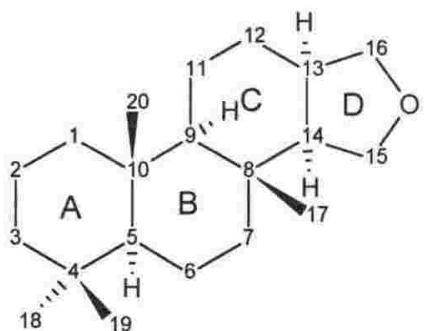

(69)

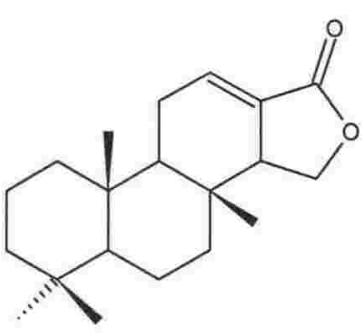

(70)

Three furanospongian diterpenes (71-73) were isolated from a Papua New Guinean Spongia officinalis bearing different levels of oxygenation at C-18. ${ }^{132}$ A sample of Hyatella intestinalis (Dictyoceratida) collected near Darwin, Northern Australia, yielded three related furanospongian diterpenes (74-76). ${ }^{133}$

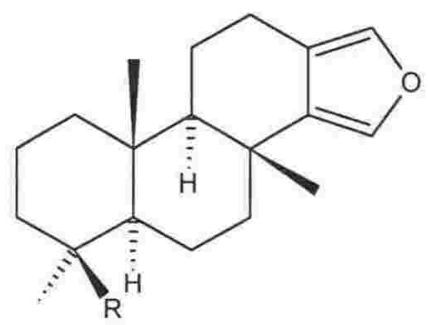

(71) $\mathrm{R}=\mathrm{CH}_{3}$

(72) $\mathrm{R}=\mathrm{CHO}$

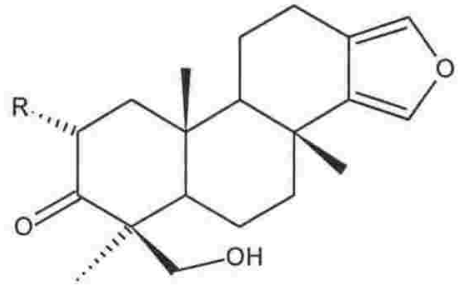

(74) $\mathrm{R}=\mathrm{H}$

(75) $\mathrm{R}=\mathrm{OH}$

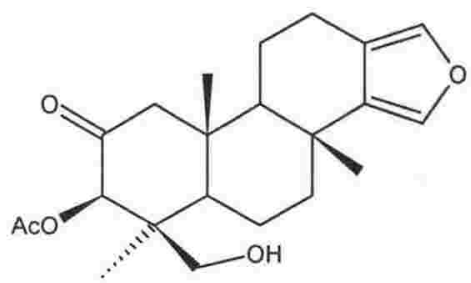

(76)

(73) $\mathrm{R}=\mathrm{COOH}$

Non-furanospongian diterpenes include those from the New Zealand Dendroceratid sponge Dictyodendrilla cavernosa that yielded (77-78). A previous study on a specimen of the same sponge had only given the novel furanosesquiterpenoid pallescenone. ${ }^{134}$ 


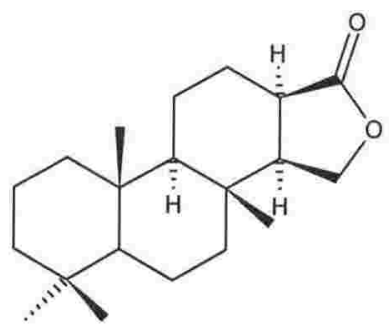

(77)

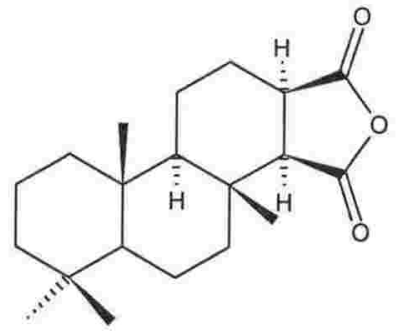

(78)

Six spongian diterpenes (79-84) were isolated from the sponge Spongia matamata. All possess carboxylic acid functionality at C-19 and some oxygenation of ring D. ${ }^{135}$

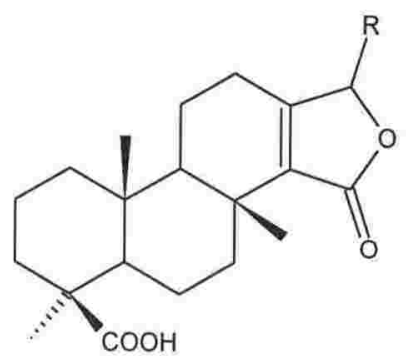

(79) $\mathrm{R}=\mathrm{H}$

(80) $\mathrm{R}=\alpha-\mathrm{OMe}$

(81) $\mathrm{R}=\beta-\mathrm{OMe}$

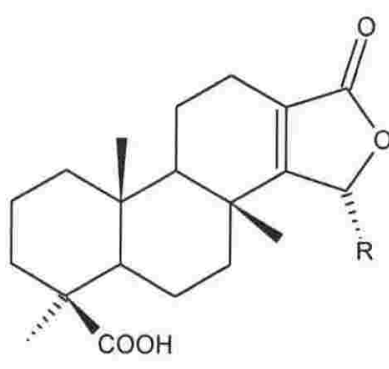

(82) $\mathrm{R}=\mathrm{H}$

(83) $\mathrm{R}=\mathrm{OMe}$

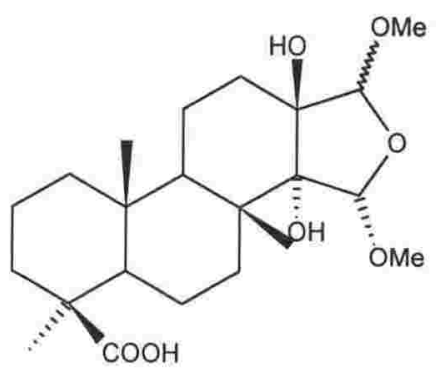

(84)

A common structural feature of many of the spongian diterpenes is an acetal linkage between $\mathrm{C}-15$ and C-17. Examples include (85-87) from the Caribbean Dendroceratid sponge Igernella notables, ${ }^{136}$ and both (88) and (89) from an Australian Darwinella sp. (Dendroceratida). ${ }^{137}$

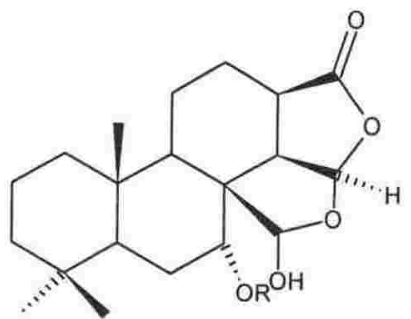

(85) $\mathrm{R}=\mathrm{H}$

(86) $\mathrm{R}=\mathrm{COCH}_{3}$

(87) $\mathrm{R}=\mathrm{COCH}_{2} \mathrm{CH}_{2} \mathrm{CH}_{3}$

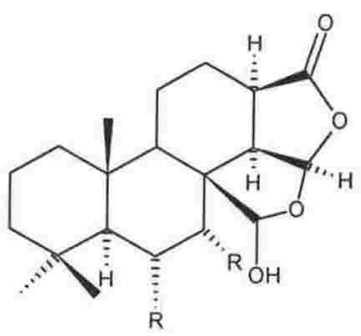

(88) $\mathrm{R}=\mathrm{H}$

(89) $\mathrm{R}=\mathrm{OAc}$ 
Various rearrangements of the basic spongian carbon skeleton are possible. One of the most common is that found in the shahamin group of compounds, where ring A has been expanded to a seven membered carbocycle with concomitant contraction of ring B. Shahamins A-E (90-94) were isolated from two different species of Dysidea sp. (Dictyoceratida). ${ }^{138}$

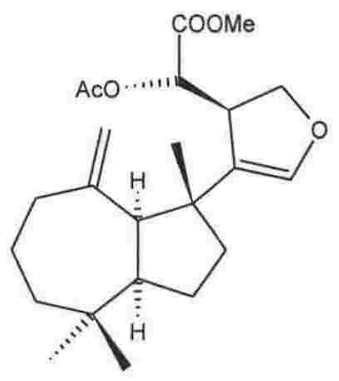

(90)

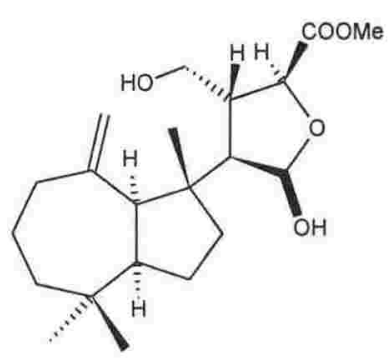

(91)

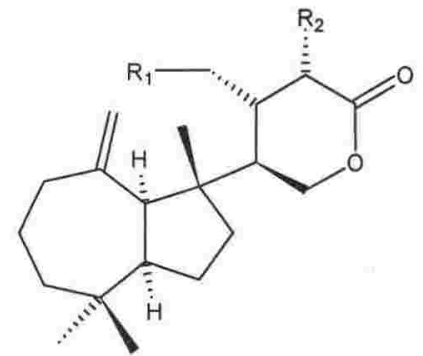

(92) $\mathrm{R}_{1}=\mathrm{OAc}, \mathrm{R}_{2}=\mathrm{OAc}$

(93) $\mathrm{R}_{1}=\mathrm{OAc}, \mathrm{R}_{2}=\mathrm{OH}$

(94) $\mathrm{R}_{1}=\mathrm{OH}, \mathrm{R}_{2}=\mathrm{OH}$

The related chelonaplysins A and B (95-96) were isolated from the Dendroceratid sponge Chelonaplysilla sp. collected at Pohnpei, Micronesia. Metabolite 96 exhibited moderate anti-microbial activity against the Gram-positive bacterium Bacillus subtilis. $^{139}$

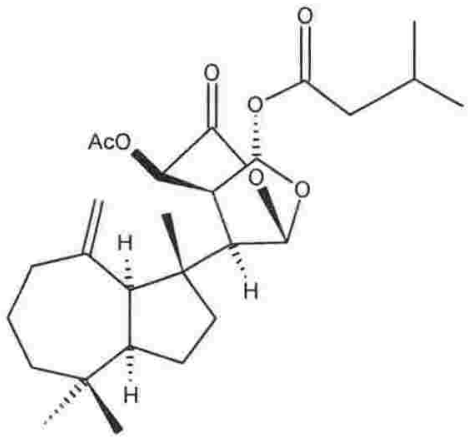

(95)

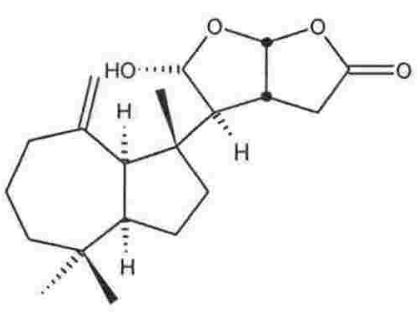

(96) 
Further members of the shahamin group of compounds, shahamins F-J (97-101), were isolated concurrently with those mentioned above, although they are based upon a different rearranged carbon skeleton. ${ }^{138}$

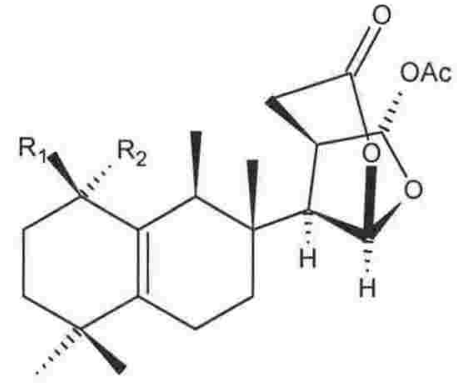

(97) $\mathrm{R}_{1}=\mathrm{H}, \mathrm{R}_{2}=\mathrm{H}$

(98) $\mathrm{R}_{1}=\mathrm{H}, \mathrm{R}_{2}=\mathrm{OH}$

(99) $\mathrm{R}_{1}=\mathrm{OH}, \mathrm{R}_{2}=\mathrm{H}$

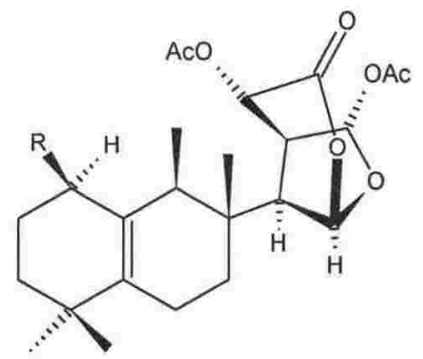

$(100) \mathrm{R}=\mathrm{H}$

$(101) \mathrm{R}=\mathrm{OH}$

There is only one example of a compound where ring A has been contracted. Norditerpene (102) was isolated from a sample of the Mediterranean sponge Spongionella gracilis (Dendroceratida). ${ }^{140}$

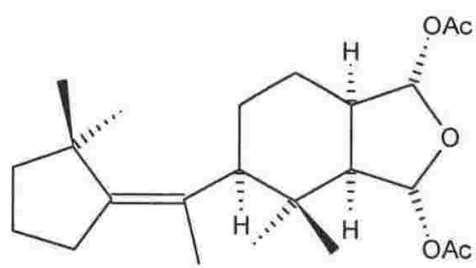

(102)

Rearrangement of ring B gives the norrisolide family of compounds, including the three novel diterpenes (103-105) isolated from a Red Sea Dysidea sp.. One of the new compounds, 103 , showed some cytotoxic activity. ${ }^{141}$ In the same class of compounds is chelonaplysin C (106) isolated from the same collection of the Micronesian Chelonaplysilla $\mathrm{sp}$. mentioned previously. ${ }^{139}$ 


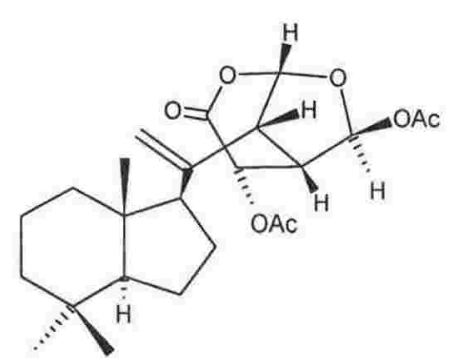

(103)

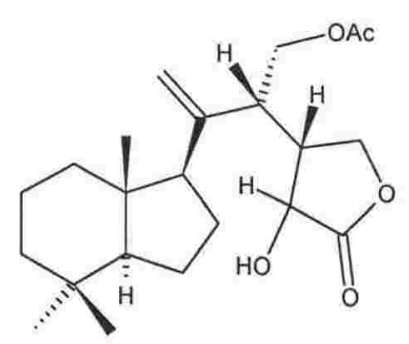

(105)

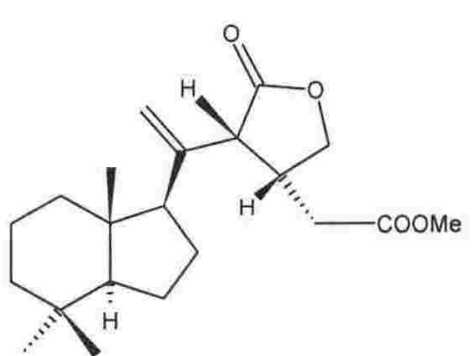

(104)

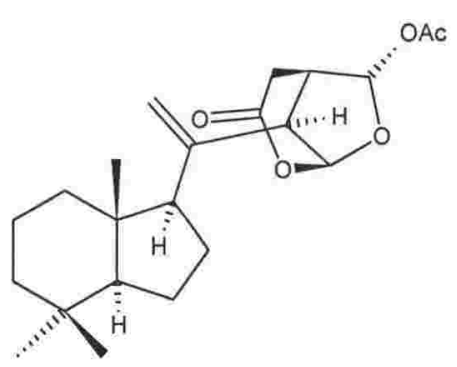

(106)

Baeyer-Villiger type oxidation of ring A also allows for an expansion to occur, forming a lactone functionality exemplified by spongialactone (107), isolated from the Red Sea sponge Spongia arabica. ${ }^{142}$ Collections of a Great Barrier Reef sponge Spongia sp. yielded a second ring A lactone diterpene (108). Compound $\mathbf{1 0 8}$ exhibited marginal cytotoxicity against P388 murine leukaemia cells. ${ }^{143}$

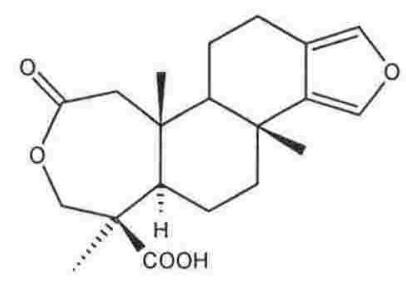

(107)

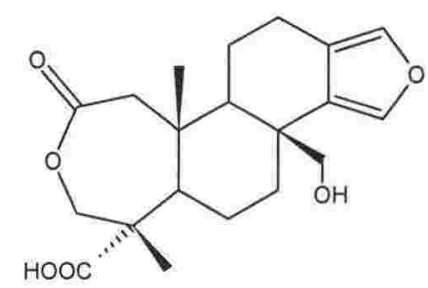

(108)

Oxidative degradation of ring $\mathrm{D}$ is noted in several rearranged spongian diterpenes. A collection of Darwinella oxeata yielded structures (109-110). Both metabolites possess a lactone linkage between $\mathrm{C}-15$ and $\mathrm{C}-17$, and ring $\mathrm{D}$ has been lost. ${ }^{144}$ 


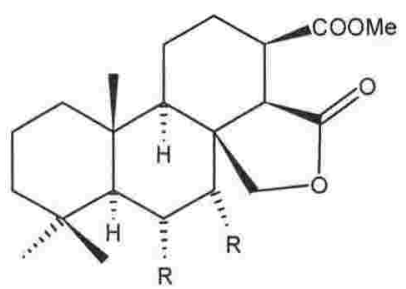

(109) $\mathrm{R}=\mathrm{H}$

(110) $\mathrm{R}=\mathrm{OH}$

Similarly, Hambley et al isolated four norditerpenes (111-114) from a sample of Aplysilla pallida (Dendroceratida). ${ }^{145}$ In another study, diterpene (115) was reported from the Dictyoceratid sponge Coscinoderma mathewsi, which was collected at Pohnpei, Micronesia. Aldehyde $\mathbf{1 1 5}$ is a possible decarbonylation product of (116). ${ }^{146}$

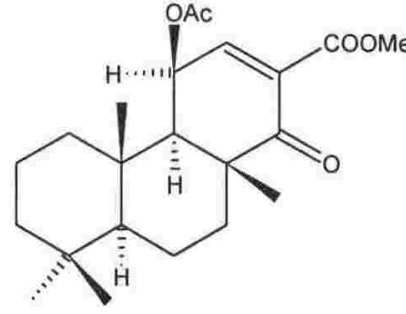

(111)

(114)

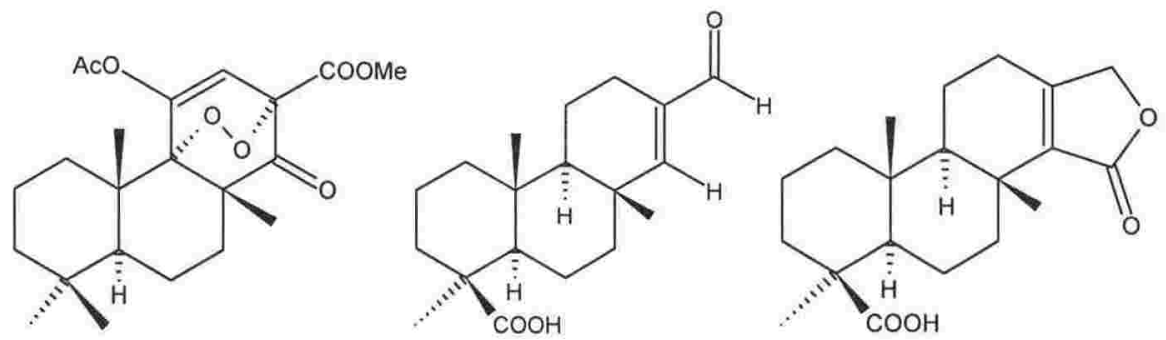

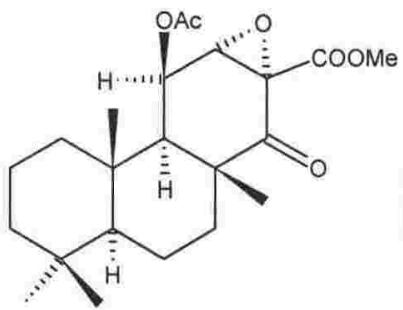

(112)

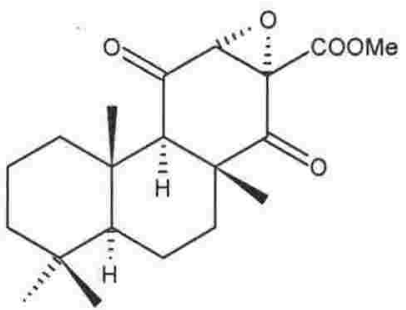

(113)

(115)

(116)

The final class of spongian diterpenes are those where methyl C-17 has undergone a 1,2-migration and the $\mathrm{C}-5$ to $\mathrm{C}-6$ bond has been cleaved to open ring $\mathrm{B}$. This common feature is often found in conjunction with some of the structural motifs noted previously. Aplysulphurin (117), from a New Zealand Aplysilla sulphurea, is the first example of an aromatic spongian diterpene. ${ }^{147}$ Mayol, Piccialli and Sica isolated the related compounds gracilins A (118) and B (119), from a Mediterranean Spongionella 
gracilis (Dictyoceratida). Gracilin B (119) was the first example of a bis-norditerpene from a marine sponge. The same group achieved the isolation of four more gracilin-type diterpenes (120-123) over subsequent years. ${ }^{140,148-150}$<smiles>CO[C@H]1O[C@@H]2OC(=O)[C@H](C)c3c([C@]4(C)CCCC(C)(C)C4)ccc1c32</smiles>

(117)

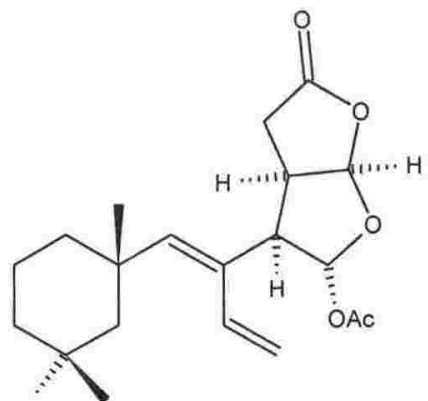

(120)<smiles>C/C=C1\C([C@]2(C)CCCC(C)(C)C2)=CC[C@H]2[C@@H](OC(C)=O)O[C@@H](OC(C)=O)[C@@H]12</smiles>

(118)

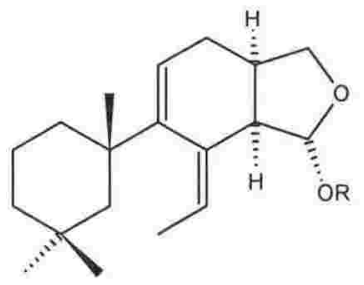

(121) $\mathrm{R}=\mathrm{Ac}$

(122) $\mathrm{R}=\mathrm{H}$<smiles>CO[C@H]1O[C@H]2O[C@@H]3OC(=O)/C(=C\C=C4CCCC(C)(C)C4)[C@H]3[C@H]2[C@H]1OC</smiles>

(119)

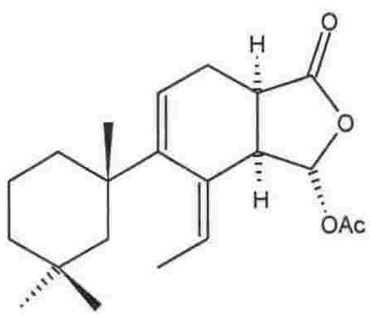

(123)

A further group of related metabolites, (124-126), were isolated from a sample of New Zealand Darwinella oxeata (Dendroceratida), as well as from several individuals of the morphologically similar sponge Dendrilla rosea. This collection was the same as that which contained 109-110. ${ }^{144}$<smiles>CO[C@H]1O[C@H]2OC(=O)[C@H](C)C3=C(C4(C)CCCC(C)(C)C4)CC[C@H]1[C@@H]32</smiles>

(124)<smiles>CO[C@H]1O[C@H]2OC(=O)[C@@H](C)C3=C2[C@@H]1CC[C@H]3C1(C)CCCC(C)(C)C1</smiles>

(125)<smiles>CC(=O)O[C@H]1O[C@H]2OC(=O)C=C3C2C1CC[C@H]3C1(C)CCCC(C)(C)C1</smiles>

(126) 
The anti-bacterial diterpenes 9,11-dihydrogracilin A (127) and membranolide (128) were isolated from the Antarctic Dendroceratid sponge Dendrilla membranosa. The authors suggested that $\mathbf{1 2 7}$ and $\mathbf{1 2 8}$ are defensive metabolites as this particular sponge had never been observed being predated upon and yet it is devoid of any spicules. ${ }^{151}$

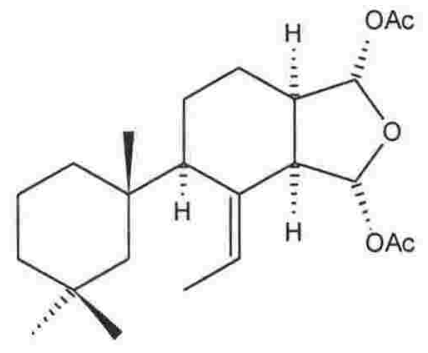

(127)

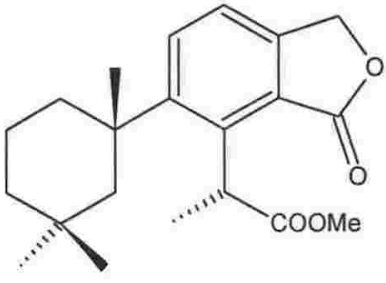

(128)

Three rearranged spongian diterpenes (129-131) were reported from the Dendroceratid sponge Aplysilla tango, collected in New South Wales, Australia. ${ }^{152}$

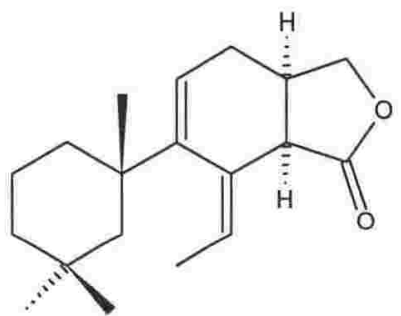

(129)

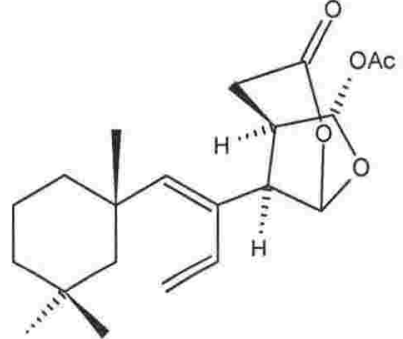

(130)

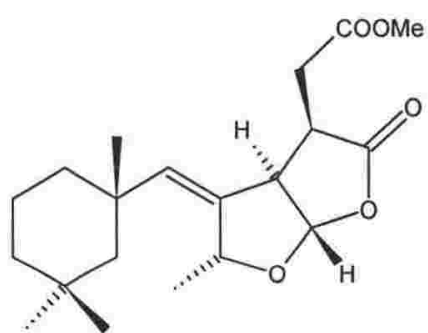

(131)

Finally, several rearranged diterpenes from a Canadian Aplysilla glacialis were reported during a study of the bioactive metabolites of nudibranchs, carried out to determine whether the compounds isolated from nudibranchs were from dietary sources, or were de novo synthesised by the organism itself. As well as isolating metabolites (132-135) from $A$. glacialis, several related rearranged spongian diterpenes were obtained from the predatory nudibranch Chromodoris luteomarginata, implying a dietary source for the isolated natural products. Another diterpene of the marginatane class (136) was also isolated from the sponge. ${ }^{28}$ 


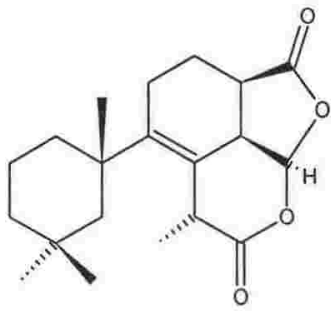

(132)

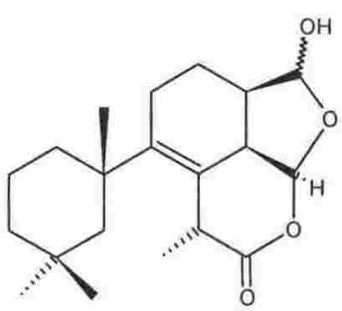

(133)

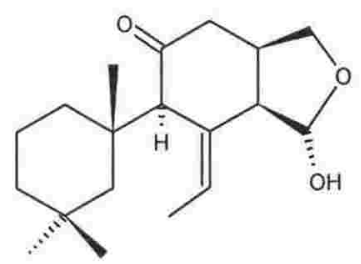

(134)

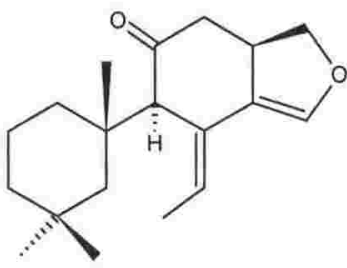

(135)<smiles>CC1(C)CCC[C@]2(C)C1CC[C@]1(C)c3occc3C(=O)CC12</smiles>

(136)

There are many other spongian diterpenes reported in the literature. Some have been isolated from nudibranchs and are considered to be of dietary origin whilst others are synthetic products. Many of the natural products have not been examined for biological activity due to a lack of material available for testing. Recent surveys have therefore focussed on testing both natural and synthetic spongian diterpenes in a variety of assays to determine uses of these compounds. ${ }^{153-156}$

\subsection{Taxonomic Considerations}

The literature survey indicates that spongian diterpenes are found from members of both the Dictyoceratid and Dendroceratid orders. Diterpenoid content cannot therefore be reliably used as a taxonomic marker for differentiating between families or genera of these two orders, as they are currently defined. Also, various researchers have noted that some species of sponge that cannot be differentiated by classic taxonomic methods may have very different chemical constituents. Chemotaxonomic criteria may therefore be difficult to establish for families or genera of the Dendroceratid and Dictyoceratid orders. ${ }^{125,134,157}$ Bergquist, however, has suggested that Dendroceratid sponges produce 
only diterpenes, therefore the presence of other classes of terpene would clearly identify members of the Dictyoceratida. ${ }^{23}$

\subsection{Biogenesis of Spongian Diterpenes and Spongian Derived Skeletons}

The basic tetracyclic spongian skeleton is generated by the cyclization of the diterpene isoprenoid precursor, geranylgeraniol (137), which is formed by head-to-tail condensations of four isoprene units. In this particular biosynthetic pathway, $\mathbf{1 3 7}$ is in an all-trans folded conformation prior to cyclization as shown in scheme $4.2 .^{158}$

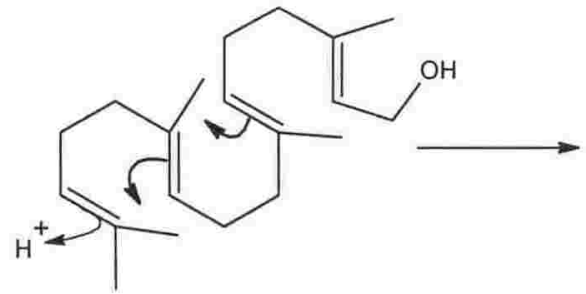

(137)

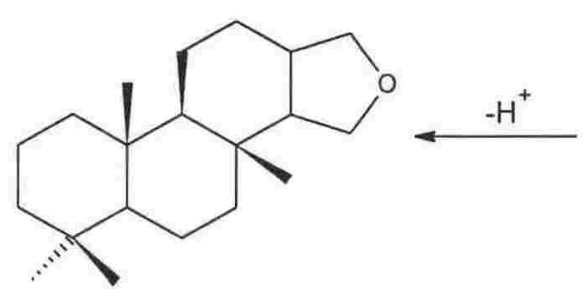

Scheme 4.2 Biogenesis of the basic spongian diterpene skeleton. ${ }^{158}$
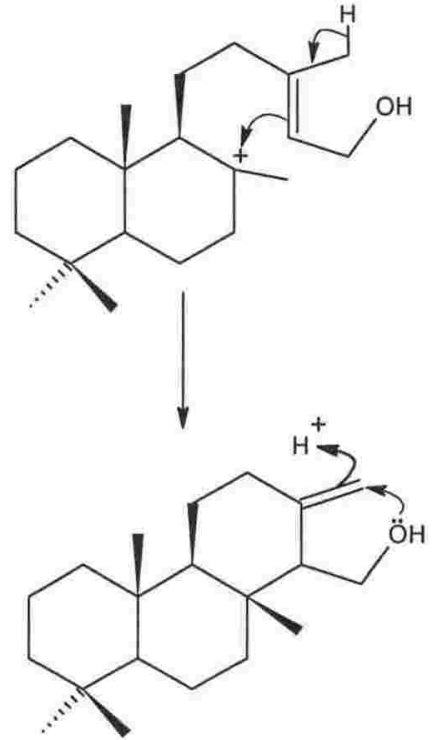

The biosynthesis of the shahamin class of compounds follows two linked pathways. The first forms the seven-member ring shahamins A-E (90-94). Oxidative cleavage of the C-9 C-11 bond places an electronegative leaving group at C-9. A 1,2-hydride shift, followed by a ring expansion with concomitant loss of the leaving group, gives the final carbon skeleton. Functionlisation of ring $\mathrm{D}$ then forms the individual shahamin compounds as indicated in scheme 4.3. The formation of shahamins F-J (97-101) starts in a similar way with the oxidative cleavage of the C-9 C-11 bond. Elimination of H-5 
followed by a 1,2-methyl shift from $\mathrm{C}-10$ to $\mathrm{C}-9$ generates the carbon skeleton, followed by functionalisation of ring $\mathrm{D}$ to again form the various compounds (see scheme 4.4). ${ }^{138}$

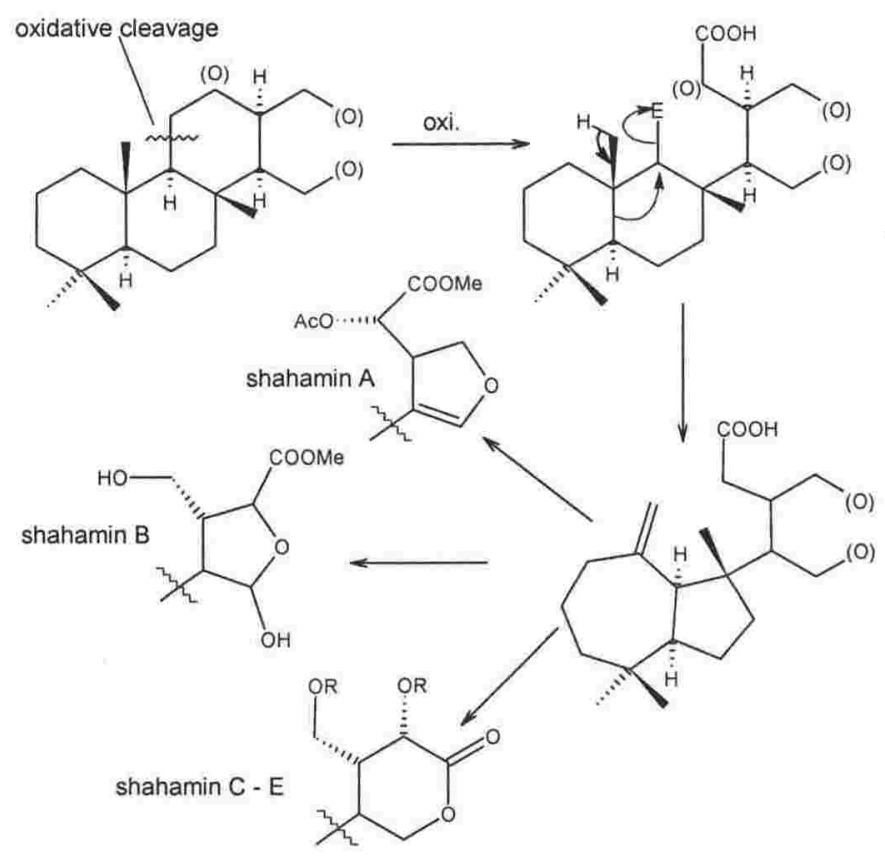

Scheme 4.3 Proposed biogenesis of shahamins A-E (90-94). ${ }^{138}$

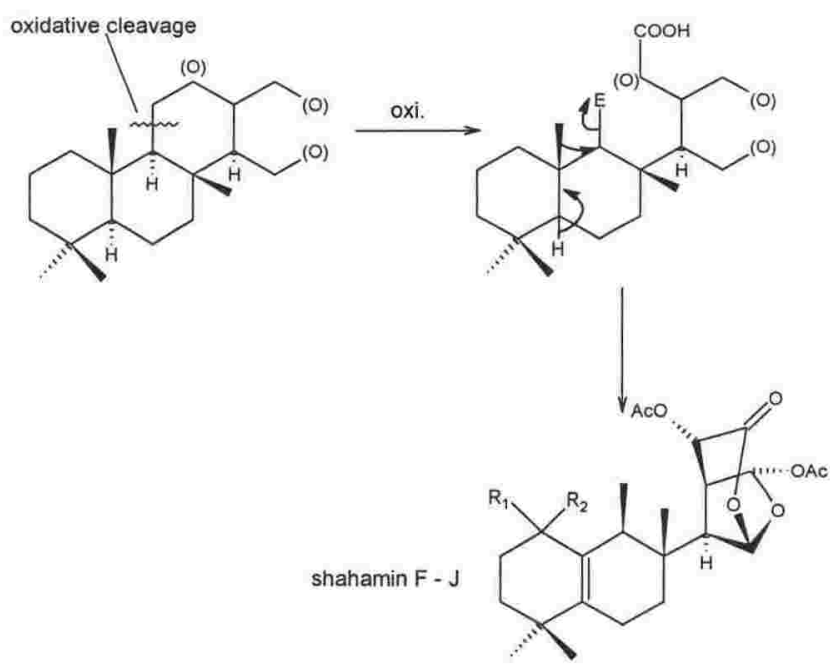

Scheme 4.4 Proposed biogenesis of shahamins F-J (97-101). ${ }^{138}$

Ring contracted metabolite (102) is proposed to be generated via the mechanism described in scheme 4.5. The opening of a 5,6-epoxide allows a bond shift from C-1 C-10 to C-1 C-5 to form a contracted spiro-moiety. The C-10 carbocation then 
undergoes nucleophilic attack. Cleavage of the C-6 C-7 bond, followed by oxidation of C-6 and hydrogenation at C-7, would generate a molecule that could decarboxylate to generate $\mathbf{1 0 2} .^{140}$
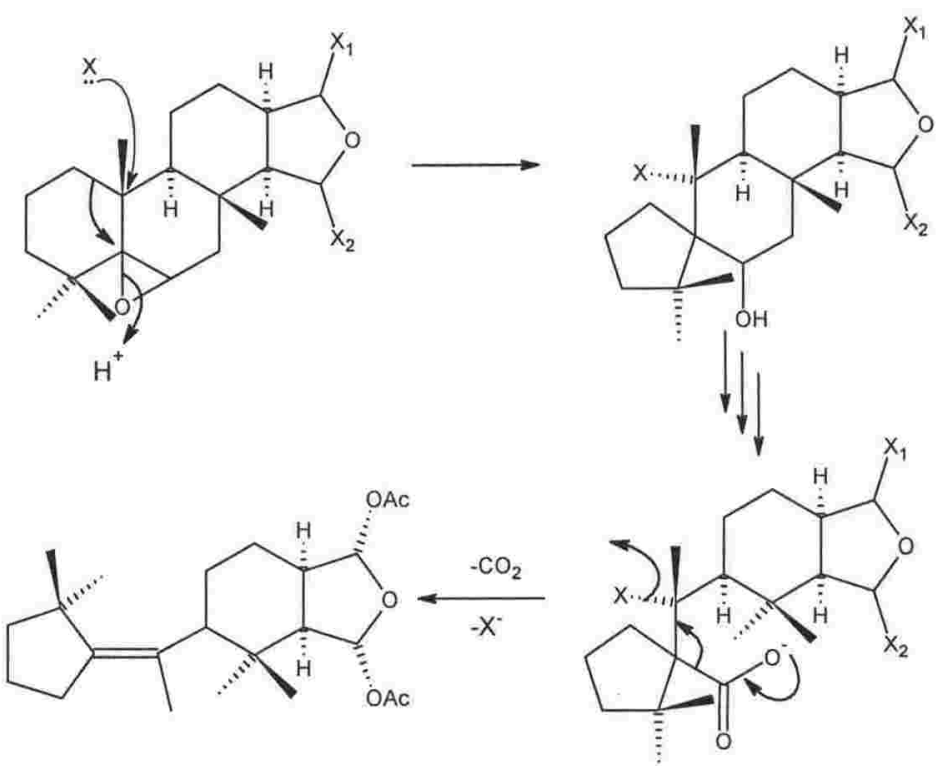

Scheme 4.5 Proposed biogenesis of (102). ${ }^{140}$

Members of the norrisolide class (103-105) are biosynthesised as described in scheme 4.6, which starts with the same initial step as that of the shahamins. Initially, the C-9 C-11 bond is cleaved, which is followed by the migration of the C-7 C-8 bond to form the five membered ring $\mathrm{B}$, followed by elimination to yield the norrisolides. ${ }^{126}$
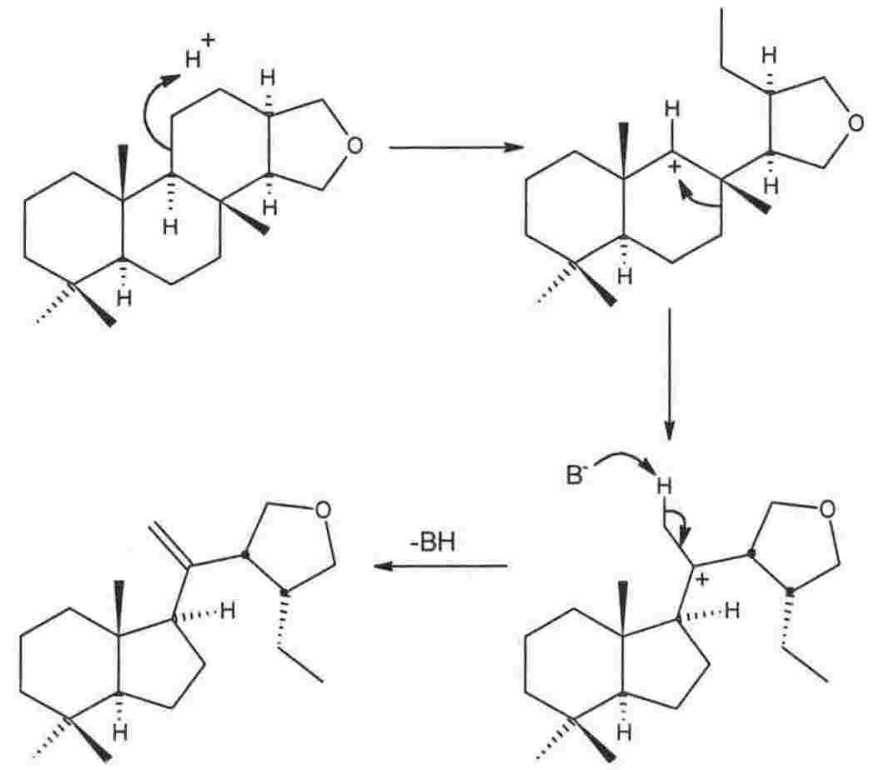

Scheme 4.6 Proposed biogenesis of the norrisolide compounds (103-105). ${ }^{126}$ 
There are many different compounds reported with ring B opened; all presumably are generated via a similar biosynthetic pathway. Oxidation to form a C-6 C-7 epoxide is followed by 1,2-hydride and 1,2-methyl shifts. Oxidative cleavage of the C-5 C-6 bond forms a carboxylic acid which can undergo decarboxylation to form the precursor of gracilin A (118) and other related compounds, which are generated by various derivatisations of ring $\mathrm{D}$ as in scheme 4.7. Isomerization of the diene precursor to $\mathbf{1 1 8}$ or epoxidation followed by reformation of the diene gives an allylic alcohol that can oxidatively ring open to form $\mathbf{1 2 0}$ and related compounds, shown in scheme $4.8 .^{150,152}$<smiles>[X]C1C[C@H]2C(O)O[C@@H](O)[C@]2(C)[C@@H]2CC[C@H]3C(C)(C)CCC[C@]3(C)[C@H]12</smiles>

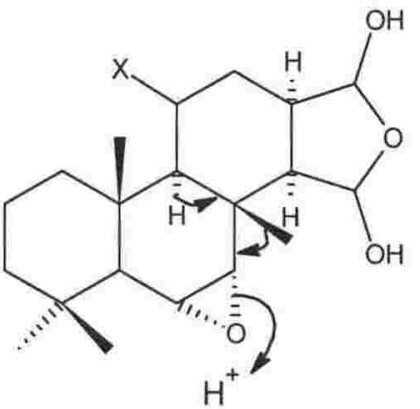

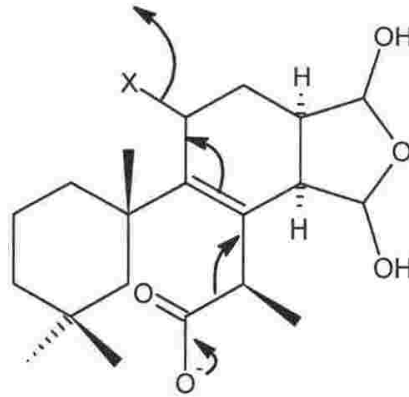<smiles>[CH][Co]</smiles><smiles>[X]C1C[C@H]2C(O)O[C@@H](O)[C@H]2[C@H]2C1[C@@]1(C)CCCC(C)(C)[C@@H]1[C@@H](O)[C@@H]2C</smiles><smiles>[Y]C1C[C@@H]2C(O)O[C@@H](O)[C@H]2/C(=C/C)C1[C@]1(C)CCCC(C)(C)C1</smiles><smiles>CC=C1C=C[C@@H]2C(OC(C)=O)OC(OC(C)=O)C2CC=C1[C@]1(C)CCCC(C)(C)C1</smiles>

Scheme 4.7 Proposed biogenesis of gracilin A (118) and related compounds. ${ }^{150,152}$ 


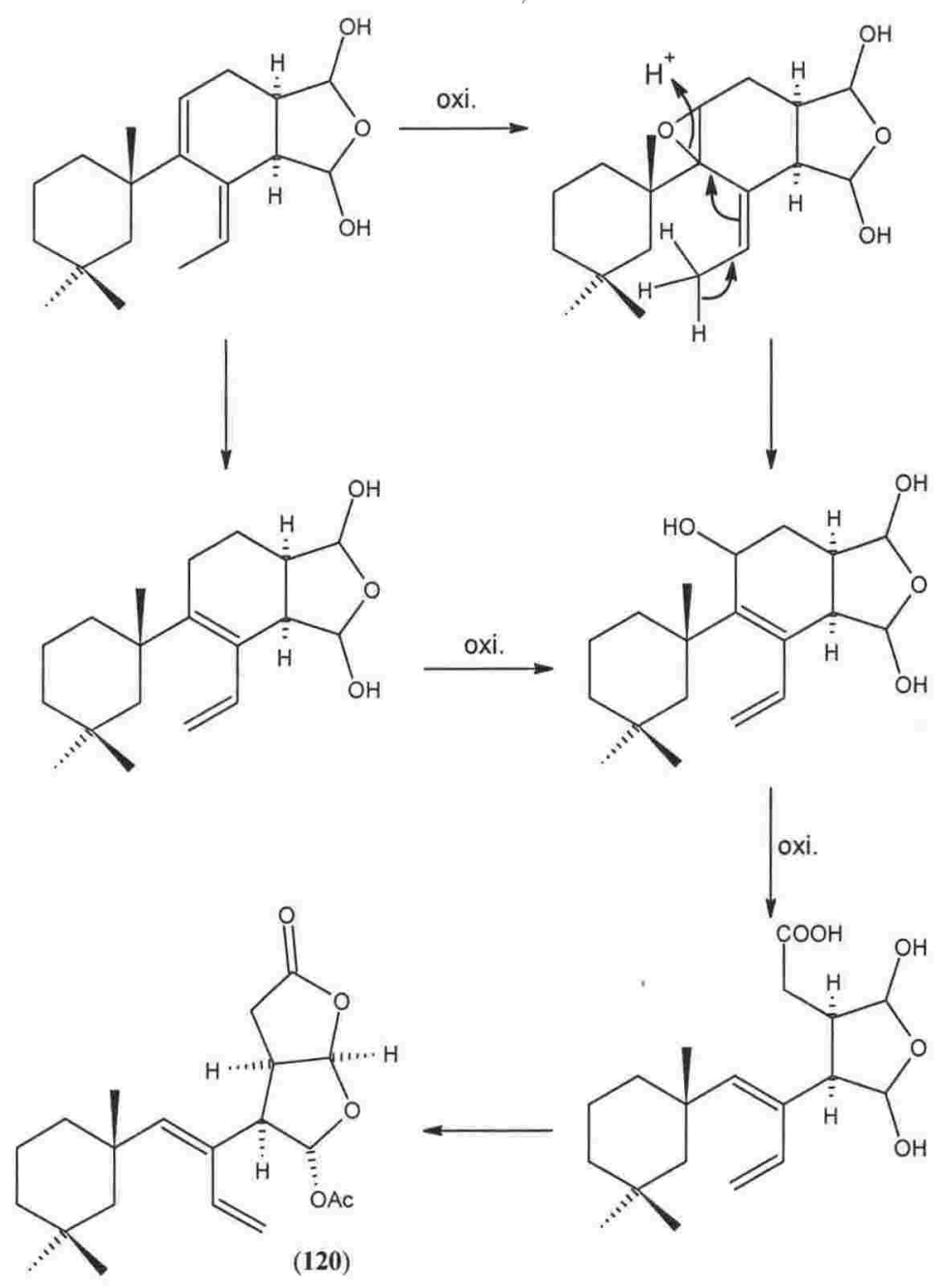

Scheme 4.8 Proposed biogenesis of (120) and related compounds. ${ }^{150,152}$

\subsection{Novel Spongian Diterpenes from Chelonaplysilla violacea}

A sample of Chelonaplysilla violacea (MNP0979) was collected using SCUBA at Stephen's Island, Marlborough Sounds, at the northern tip of the South Island of New Zealand (see figure 4.1). The organism was initially described in the field as a tan, encrusting sponge of the genus Dysidea (Dictyoceratida). Chelonaplysilla violacea (Class Demospongiae, Order Dendroceratida, Family Darwinellidae) is described as having a dendritic skeleton composed of smooth, erect, fibres, rising from a basal plate of spongin. Fibres are concentrically laminar with a very dense outer bark and a diffuse pith interior. The sponge is usually found in shaded areas such as caves and archways, 
encrusting on boulders in sheets $2-3 \mathrm{~mm}$ thick. The colour of the sponge is described as dark purple throughout, with white surface patterning caused by organised sand reticulation. ${ }^{23,121}$

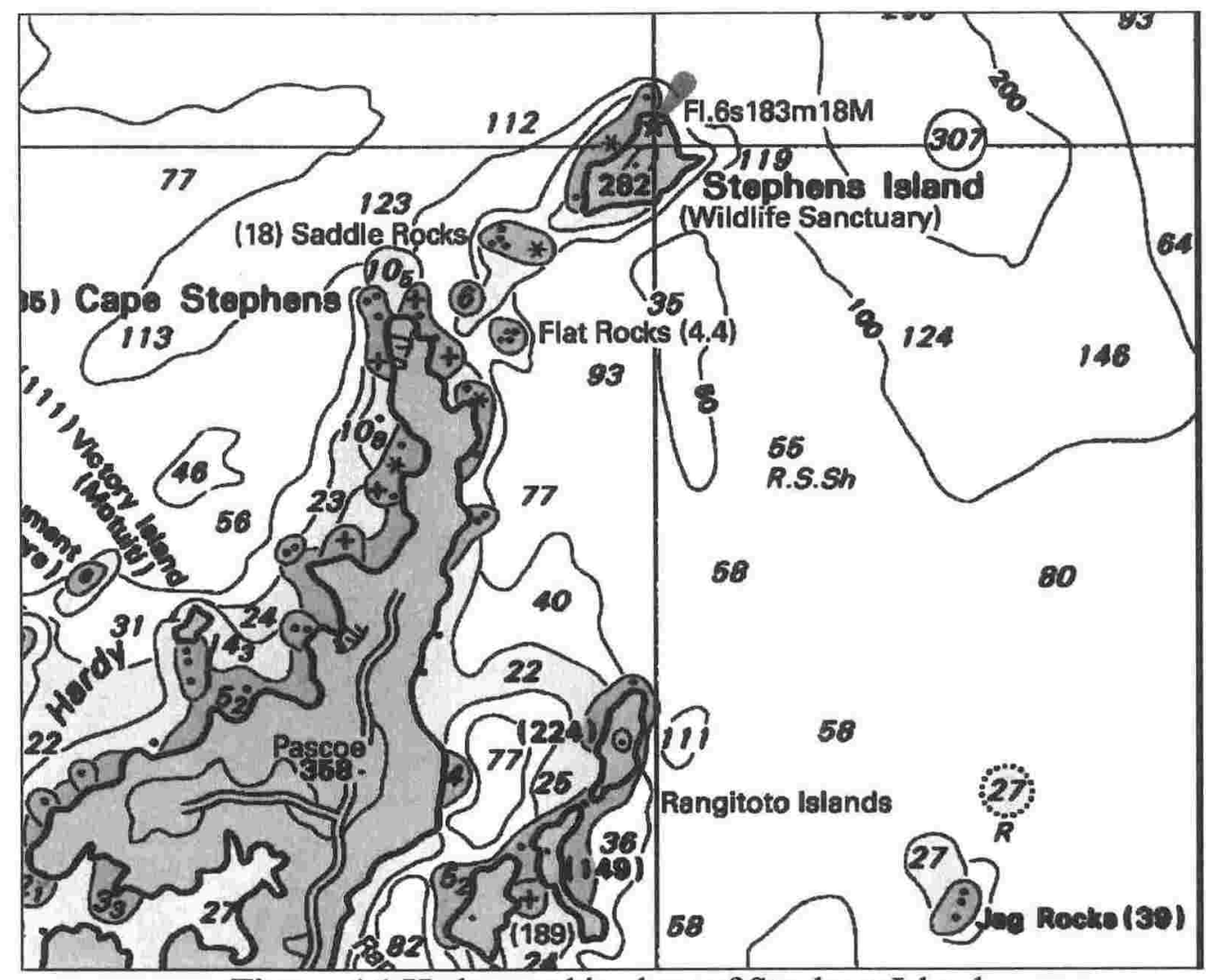

Figure 4.1 Hydrographic chart of Stephens Island.

(Courtesy of Land and Information New Zealand)

Initial NMR based screening of $110 \mathrm{~g}$ of the sponge revealed many interesting resonances, including those of multiple acetal/hemi-acetal functionalities, several oxygenated methines, and also those of several methines with shielded carbon and deshielded proton correlations, as indicated in the HSQC spectrum (see figure 2.8). An extract of a further $182 \mathrm{~g}$ was combined with the material generated from the screen. This combined extract was fractionated using normal- and reversed-phase solid supports, size-exclusion gel-permeation chromatography and finally, reversed-phase HPLC, to yield ten spongian diterpenes. Two of the compounds were the known metabolites tetrahydroaplysulphurin-1 (124) (1.8 mg) and cadlinolide B (133) (1.4 mg), based upon comparison with published MS and NMR data. ${ }^{28,144,159}$ Two of the 
remaining eight metabolites could not be identified on the basis of the NMR and MS data obtained. The last six metabolites isolated constituted novel structures and are dealt with below. Two of the six novel compounds share the same carbon skeleton as cadlinolides A (132) and B (133) and are therefore reported as cadlinolides C and D. ${ }^{28}$ The remaining four compounds possess different carbon skeletons to those previously reported and are named based upon the Maori name for Stephen's Island (Takapourewa).

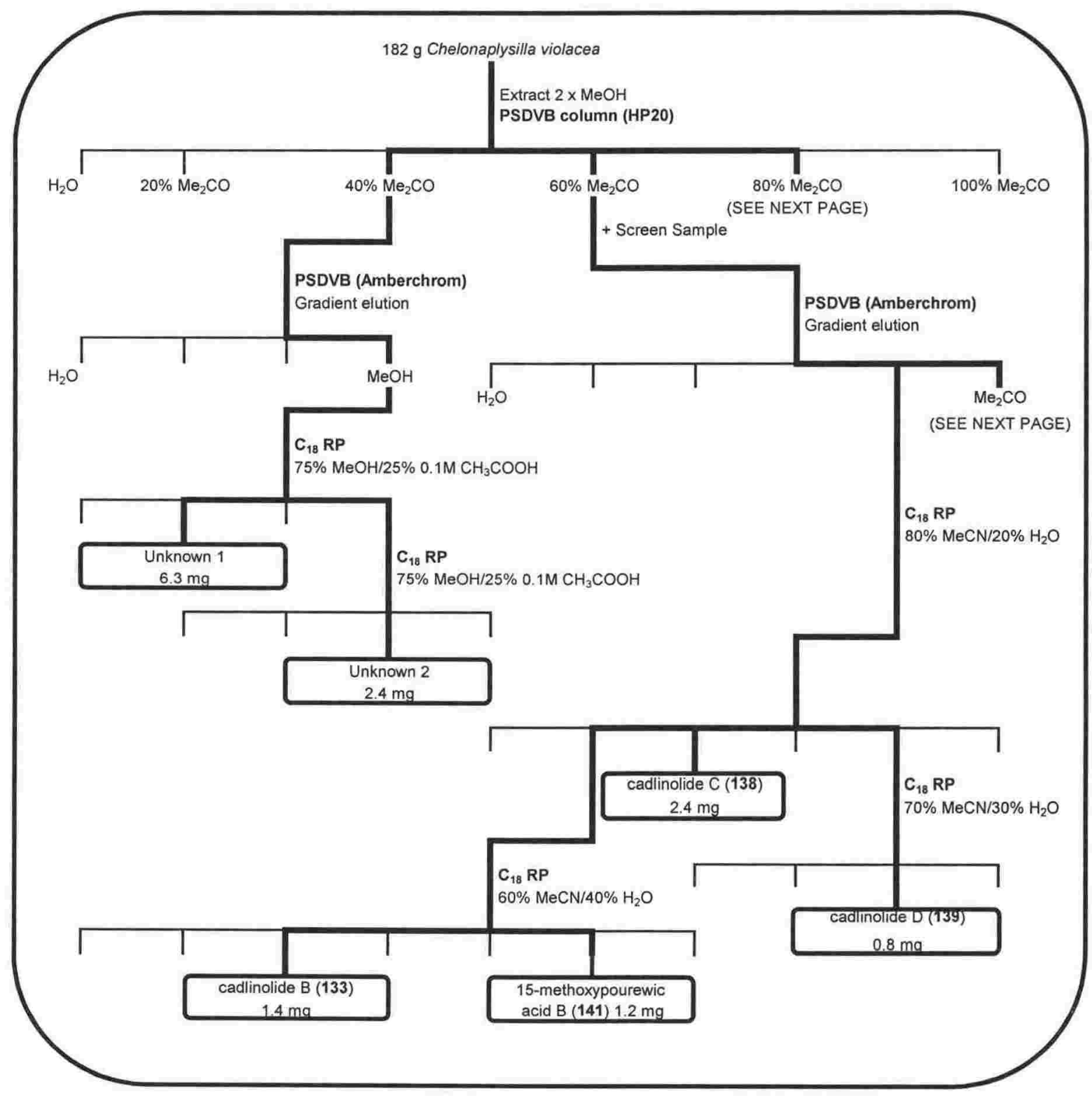

Scheme 4.9 Isolation scheme for spongian diterpenes from Chelonaplysilla violacea sp. (part 1). 


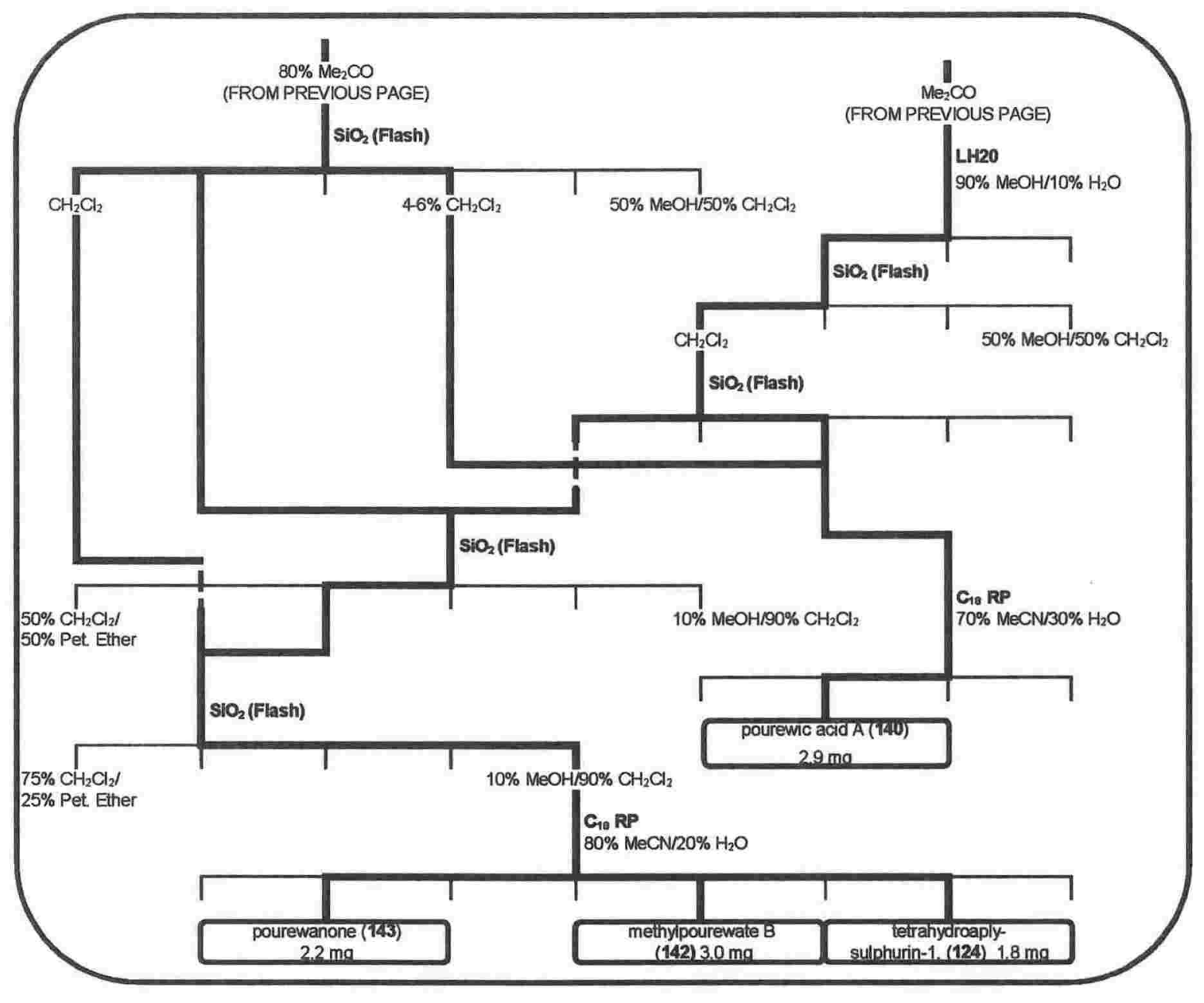

Scheme 4.10 Isolation scheme for spongian diterpenes

from Chelonaplysilla violacea $\mathrm{sp}$. (part 2).
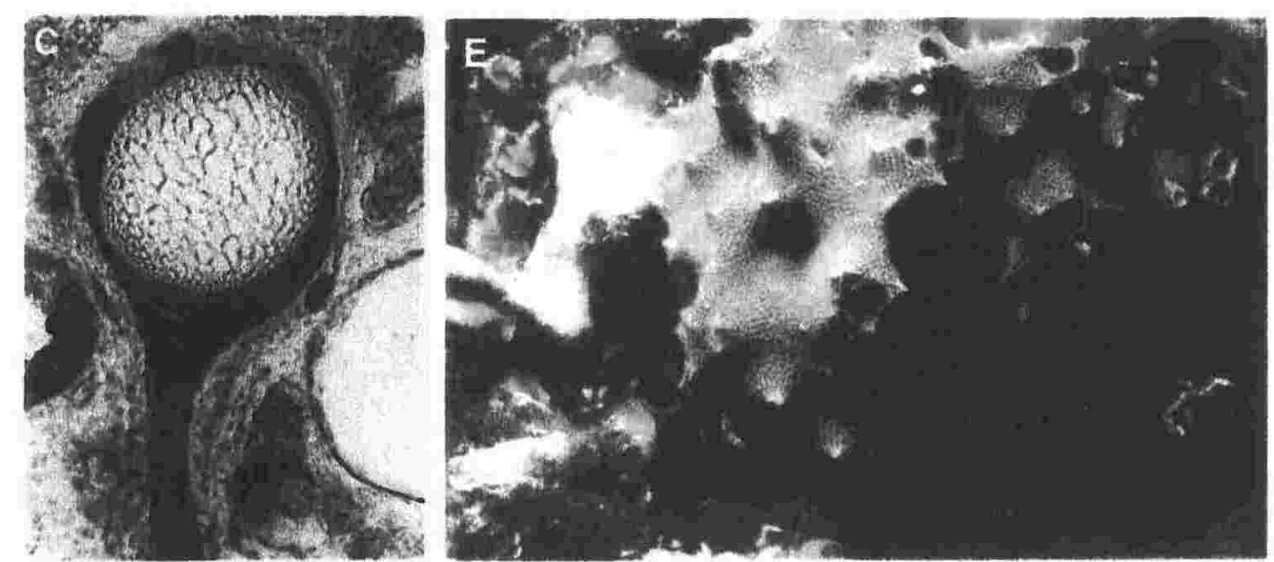

Figure 4.2 Skeleton and appearance of Chelonaplysilla violacea.

(Courtesy of Professor P. Bergquist)

C: Chelonaplysilla violacea, section of fibre to show marked vesicular pith $(x \quad 120){ }^{121}$

E: Chelonaplysilla violacea. ${ }^{121}$ 
A single intense pseudo-molecular ion was observed for cadlinolide C (138) (2.4 mg) in the positive ion HRESIMS mode establishing a molecular formula of $\mathrm{C}_{20} \mathrm{H}_{30} \mathrm{O}_{3}$ $\left(319.2260[\mathrm{M}+\mathrm{H}]^{+}, \Delta 2.6 \mathrm{ppm}\right)$ and requiring six degrees of unsaturation. Only one significant band was identified in the IR spectrum, that of a $\mathrm{C}=\mathrm{O}$ stretch $\left(1739 \mathrm{~cm}^{-1}\right)$. All 20 carbon and all 30 proton resonances were observed in the ${ }^{13} \mathrm{C}$ and ${ }^{1} \mathrm{H}$ NMR spectra, and one-bond proton-carbon correlations were detected in an HSQC-DEPT experiment. Prominent signals in the ${ }^{13} \mathrm{C}$ spectrum included resonances of a carbonyl $\left(\delta_{\mathrm{C}} 172.5\right)$, an olefin $\left(\delta_{\mathrm{C}} 147.0,122.9\right)$, an acetal $\left(\delta_{\mathrm{C}} 103.5\right)$, and an oxygenated methylene $\left(\delta_{\mathrm{C}} 73.1\right)$. The olefin was assigned as tetrasubstituted from the lack of any HSQC-DEPT correlations to these ${ }^{13} \mathrm{C}$ resonances. With no other evidence of multiple bonds, the remaining degrees of unsaturation required cadlinolide $\mathrm{C}$ (138) to have a tetracyclic structure.

Initial analysis of the correlations from various proton resonances in both the COSY and HMBC spectra, allowed construction of several substructures. In particular, a linear fragment of three methylenes was connected on the basis of COSY correlations between the resonances of their attached protons $\left[\left(\mathrm{C}-1: \delta_{\mathrm{C}} 40.0, \delta_{\mathrm{H}} 1.95,1.13\right)\right.$ to $\left(\mathrm{C}-2: \delta_{\mathrm{C}} 20.6, \delta_{\mathrm{H}} 1.51\right)$ to $\left.\left(\mathrm{C}-3: \delta_{\mathrm{C}} 39.7, \delta_{\mathrm{H}} 1.32,1.19\right)\right]$. Strong HMBC correlations were observed from the ${ }^{1} \mathrm{H}$ resonances of two methyl singlets [(C-18: $\left.\delta_{\mathrm{C}} 27.6, \delta_{\mathrm{H}} 0.76\right)$, $\left.\left(\mathrm{C}-19: \delta_{\mathrm{C}} 32.9, \delta_{\mathrm{H}} 0.90\right)\right]$ to the ${ }^{13} \mathrm{C}$ resonances of each other, two methylenes [C-3 and $\left.\left(\mathrm{C}-5: \delta_{\mathrm{C}} 51.2, \delta_{\mathrm{H}} 1.80,1.26\right)\right]$ and a quaternary centre $\left(\mathrm{C}-4: \delta_{\mathrm{C}} 31.9\right)$, establishing the link of C-3 to gem-dimethyl quaternary centre C-4. Finally, the ${ }^{1} \mathrm{H}$ resonance of a third methyl singlet (C-20: $\left.\delta_{\mathrm{C}} 31.9, \delta_{\mathrm{H}} 1.13\right)$ showed HMBC correlations to two methylenes 
[C-1 and C-5] and to a quaternary carbon (C-10: $\delta_{C}$ 39.6), establishing a 1,3,3-trimethylcyclohexyl moiety (see figure 4.3).

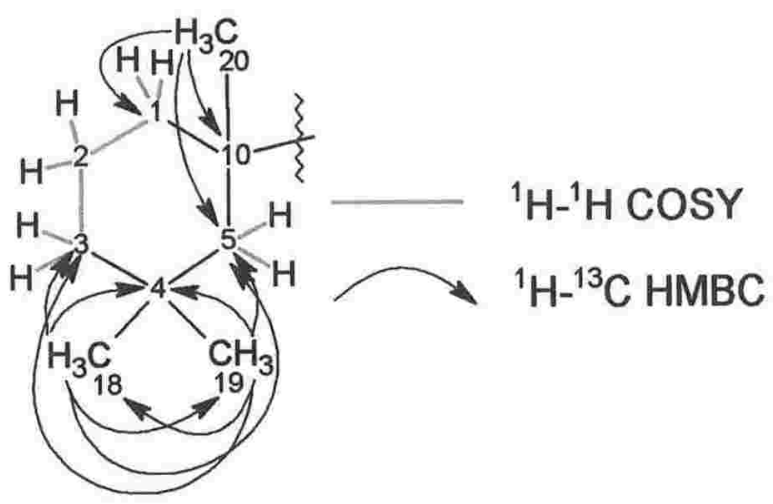

Figure 4.3 Establishment of 1,3,3-trimethylcyclohexyl substructure of cadlinolide C (138).

A second substructure was constructed from observed COSY cross-peaks between the ${ }^{1} \mathrm{H}$ resonances of several methylenes and methines. These observations allowed connection of a linear chain consisting of a pair of methylenes [(C-11: $\delta_{C}$ 27.0, $\left.\left.\delta_{\mathrm{H}} 2.48,1.93\right),\left(\mathrm{C}-12: \delta_{\mathrm{C}} 25.6, \delta_{\mathrm{H}} 1.95,0.92\right)\right]$ that were adjacent to two methines [(C-13: $\left.\left.\delta_{\mathrm{C}} 37.6, \delta_{\mathrm{H}} 2.65\right),\left(\mathrm{C}-14: \delta_{\mathrm{C}} 41.7, \delta_{\mathrm{H}} 3.01\right)\right]$. The resonance of H-13 showed further correlations to an oxymethylene pair $\left(\mathrm{C}-16: \delta_{\mathrm{C}} 73.1, \delta_{\mathrm{H}} 4.18,3.58\right)$, while $\mathrm{H}-14$ showed a significant cross-peak to an acetal ${ }^{1} \mathrm{H}$ resonance $\left(\mathrm{C}-15: \delta_{\mathrm{C}} 103.5, \delta_{\mathrm{H}} 6.01\right)$. HMBC correlations from the ${ }^{1} \mathrm{H}$ resonance of $\mathrm{H}-15$ to $\mathrm{C}-16$, and from the deshielded ${ }^{1} \mathrm{H}$ resonance of $\mathrm{C}-16$ to $\mathrm{C}-15$, established the connectivity of a tetrahydrofuran ring. This was confirmed by observation of long-range W-coupling between $\mathrm{H}-15$ and the shielded ${ }^{1} \mathrm{H}$ resonance of oxymethylene $\mathrm{C}-16$ (see figure 4.4).

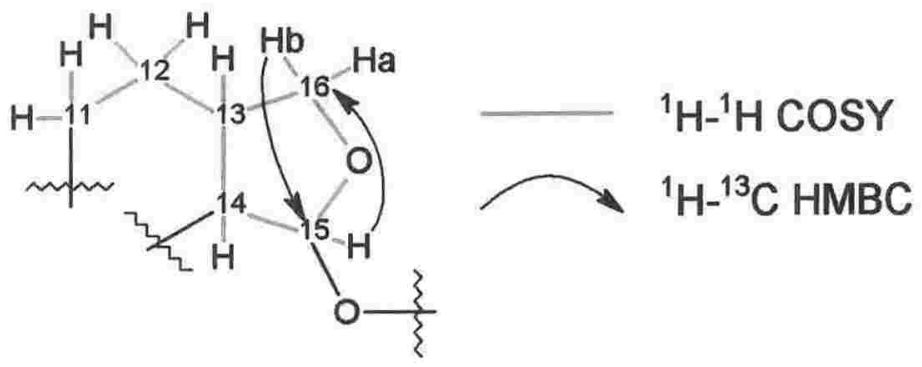

Figure 4.4 Establishment of the tetrahydrofuran substructure of cadlinolide C (138). 
A strong COSY correlation was observed between the ${ }^{1} \mathrm{H}$ resonances of a methyl (C-6: $\left.\delta_{\mathrm{C}} 14.4, \delta_{\mathrm{H}} 1.40\right)$ and a methine $\left(\mathrm{C}-7: \delta_{\mathrm{C}} 40.7, \delta_{\mathrm{H}} 4.18\right)$. HMBC correlations were observed from the ${ }^{1} \mathrm{H}$ resonances of both these centres to the ${ }^{13} \mathrm{C}$ resonances of each other, and also to that of the carbonyl (C-17: $\left.\delta_{\mathrm{c}} 172.5\right)$ to establish a third fragment (see figure 4.5).

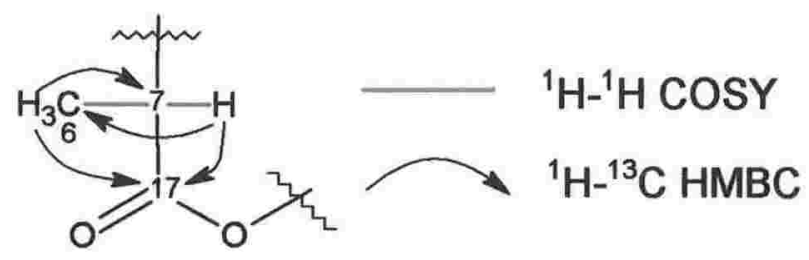

Figure 4.5 Establishment of the side chain of cadlinolide C (138).

The previous observations had accounted for 18 of the 20 carbons of 138, leaving a tetrasubstituted olefin to be assigned. The placement of the olefin, and therefore the final connectivity of the molecule, was established from analysis of several key COSY and $\mathrm{HMBC}$ correlations. HMBC correlations were observed between the ${ }^{1} \mathrm{H}$ resonances of methyl C- 6 and methine $\mathrm{C}-7$ to the ${ }^{13} \mathrm{C}$ resonance of a disubstituted olefinic carbon (C-8: $\delta_{\mathrm{C}}$ 122.9). The resonance of $\mathrm{H}-7$ also showed $\mathrm{HMBC}$ correlations to the ${ }^{13} \mathrm{C}$ resonances of the second disubstituted olefinic carbon (C-9: $\left.\delta_{\mathrm{C}} 147.0\right)$ and to $\mathrm{C}-14$, which established the linkage of the side chain to the olefin at C-8 between C-9 and C-14 and connected the side chain with the tetrahydrofuran substructure. A strong HMBC correlation was observed from the ${ }^{1} \mathrm{H}$ resonance of $\mathrm{C}-20$ to the ${ }^{13} \mathrm{C}$ resonance of C-9, allowing connection of the 1,3,3-trimethylcyclohexyl ring to the olefin. The deshielded ${ }^{1} \mathrm{H}$ resonance $\mathrm{H}-11 \mathrm{~b}$ showed a correlation to the ${ }^{13} \mathrm{C}$ resonance of quaternary centre $\mathrm{C}-10$, which helped to confirm the linkage between the olefin and the 1,3,3-trimethylcyclohexyl ring. A homo-allylic coupling was observed between $\mathrm{H}-11$ a and H-14 in the COSY spectrum that confirmed the placement of the olefin within a sixmembered ring. Finally, although no HMBC correlations were observed between the 
resonances of $\mathrm{H}-15$ and $\mathrm{C}-17$, a lactone linkage could be deduced by the final required degree of unsaturation, and also by the absence of any alcohol functionality within the molecule, as evidenced by the lack of an $\mathrm{OH}$ stretch in the IR spectrum. The lactone linkage is supported by the deshielded chemical shift of $\mathrm{H}-15$, and also the ${ }^{13} \mathrm{C}$ chemical shift of carbonyl C-17 which is similar to that of tetrahydroaplysulphurin-1 (124), cadlinolide A (132) and cadlinolide B (133) (see figure 4.6). ${ }^{28}$

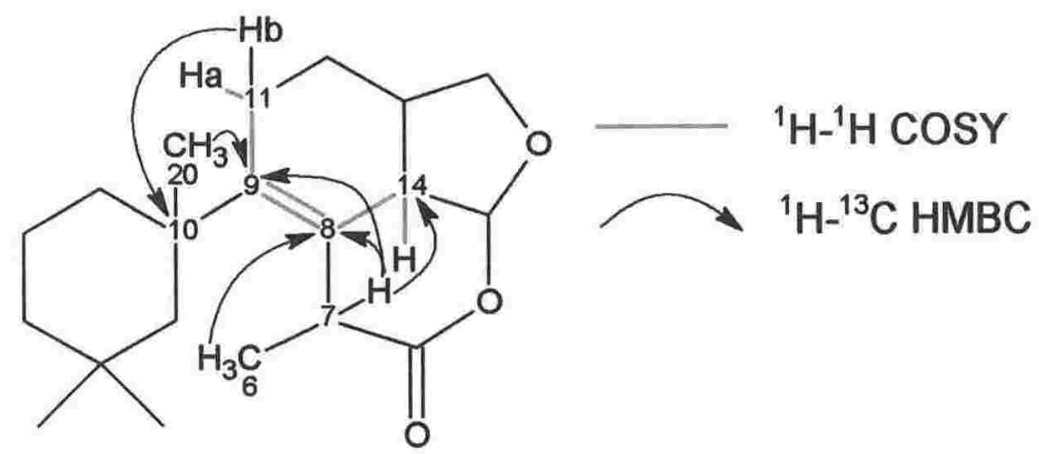

Figure 4.6 Establishment of final connectivity of cadlinolide C (138).

The relative stereochemistry of 138 was established from vicinal ${ }^{1} \mathrm{H}-{ }^{1} \mathrm{H}$ homonuclear coupling constants, measured in the ${ }^{1} \mathrm{H}$ NMR and ${ }^{1} \mathrm{H}$ decoupled NMR experiments, and from NOE correlations established from a ROESY experiment. A small coupling was measured between $\mathrm{H}-14$ and H-15, suggesting a small dihedral angle between these two protons. An NOE correlation was observed between $\mathrm{H}-13$ and $\mathrm{H}-14$, implying that they are on the same face of the molecule (see figure 4.7). H-15 also showed a strong NOE correlation to $\mathrm{CH}_{3}-6$, implying that $\mathrm{C}-6$ is on the same face as protons $\mathrm{H}-14$ and $\mathrm{H}-15$. NOE correlations were observed between $\mathrm{CH}_{3}-6$ and $\mathrm{H}-5 \mathrm{~b}$, implying that $\mathrm{H}-5 \mathrm{~b}$ is also on the same face as methyl C-6. NOE correlations were observed from $\mathrm{H}-7$ to $\mathrm{H}-5 \mathrm{a}, \mathrm{H}-$ $5 \mathrm{~b}$ and also to $\mathrm{CH}_{3}-20$, supporting this assignment (see figure 4.8). These observations allowed the relative stereochemistry of cadlinolide $\mathrm{C}(\mathbf{1 3 8})$ to be assigned as $7 R^{*}, 10 S^{*}$, $13 S^{*}, 14 S^{*}, 15 S^{*}$, which was consistent with the stereochemistry determined for tetrahydroaplysulphurin-1 (124), which had been solved by x-ray diffraction. ${ }^{28,144,159}$ 


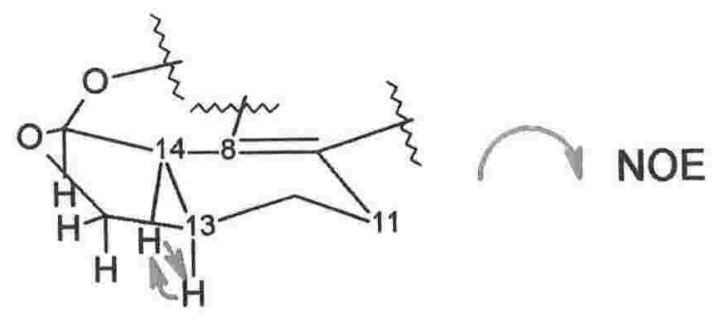

Figure 4.7 NOE correlations and stereochemistry of tetrahydrofuran ring of cadlinolide $\mathrm{C}$ (138)

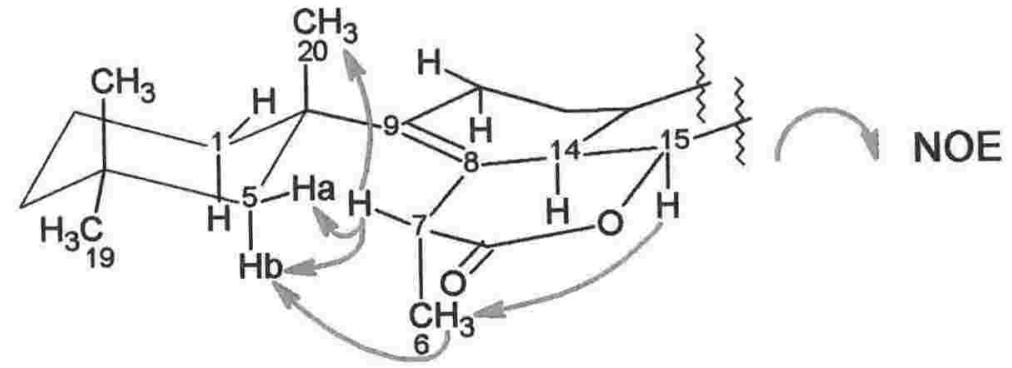

Figure 4.8 Selected NOE correlations establishing the stereochemistry of the 1,3,3-trimethylcyclohexyl and lactone rings of cadlinolide C (138). 
Table 4.1 ${ }^{13} \mathrm{C}(75 \mathrm{MHz})$ and ${ }^{1} \mathrm{H}(300 \mathrm{MHz})$ NMR Data $\left(\mathrm{CDCl}_{3}\right)$ of cadlinolide $\mathrm{C}(\mathbf{1 3 8})$.

\begin{tabular}{|c|c|c|c|c|c|c|c|}
\hline \multirow[b]{2}{*}{ Position } & \multicolumn{2}{|c|}{${ }^{13} \mathrm{C}^{\mathrm{a}}$} & \multicolumn{2}{|r|}{${ }^{\prime} \mathrm{H}^{\mathrm{b}}$} & \multicolumn{3}{|c|}{ HMBC } \\
\hline & $\delta(\mathrm{ppm})$ & mult & $\delta(\mathrm{ppm})$ & mult, $J(\mathrm{~Hz})$ & COSY & ( $\mathrm{H}$ to $\mathrm{C}$ ) & $\mathrm{NOE}$ \\
\hline la & 40.0 & $\mathrm{CH}_{2}$ & 1.13 & $\mathrm{~m}$ & $1 b$ & 3 & \\
\hline $\mathrm{b}$ & & & 1.95 & $\mathrm{~m}$ & $1 \mathrm{a}, 2$ & & \\
\hline 2 & 20.6 & $\mathrm{CH}_{2}$ & 1.51 & $\mathrm{~m}, 2 \mathrm{H}$ & $1 b, 3 a, 3 b$ & $3,4,10$ & \\
\hline $3 a$ & 39.7 & $\mathrm{CH}_{2}$ & 1.19 & $\mathrm{~m}$ & 2,18 & & $5 b$ \\
\hline $\mathrm{b}$ & & & 1.32 & $\mathrm{~m}$ & $2,5 b$ & & \\
\hline 4 & 31.9 & $\mathrm{C}$ & & & & & \\
\hline $5 \mathrm{a}$ & 51.2 & $\mathrm{CH}_{2}$ & 1.26 & $\mathrm{~d}(13.7)$ & $5 b$ & $9,10,18,19,20$ & 7 \\
\hline$b$ & & & 1.80 & $\mathrm{dt}(13.7,1.7)$ & $5 a, 3 b$ & $1,4,10$ & $3 a, 6,7$ \\
\hline 6 & 14.4 & $\mathrm{CH}_{3}$ & 1.40 & $\mathrm{~d}(7.3)$ & 7 & $7,8,17$ & $5 \mathrm{~b} 15$ \\
\hline 7 & 40.7 & $\mathrm{CH}$ & 4.18 & $\mathrm{q}(7.6)$ & 6 & $6,8,9,14,17$ & $5 \mathrm{a}, 5 \mathrm{~b}, 20$ \\
\hline 8 & 122.9 & $\mathrm{C}$ & & & & & \\
\hline 9 & 147.0 & C & & & & & \\
\hline 10 & 39.6 & $\mathrm{C}$ & & & & & \\
\hline $11 \mathrm{a}$ & 27.0 & $\mathrm{CH}_{2}$ & 1.93 & $\mathrm{~m}$ & $11 \mathrm{~b}, 12 \mathrm{a}, 14$ & & $1 \mathrm{lb}$ \\
\hline b & & & 2.48 & $\mathrm{dt}(16.1,4.4)$ & $11 \mathrm{a}, 12 \mathrm{a}, 12 \mathrm{~b}$ & $8,9,10,12,13$ & $11 \mathrm{a}$ \\
\hline $12 \mathrm{a}$ & 25.6 & $\mathrm{CH}_{2}$ & 0.92 & $\mathrm{~m}$ & $11 \mathrm{a}, 11 \mathrm{~b}, 12 \mathrm{~b}, 13$ & & $12 \mathrm{~b}$ \\
\hline $\mathrm{b}$ & & & 1.95 & $\mathrm{~m}$ & $11 \mathrm{~b}, 12 \mathrm{a}, 13$ & $11,13,14$ & $12 \mathrm{a}$ \\
\hline 13 & 37.6 & $\mathrm{CH}$ & 2.65 & $\mathrm{~m}$ & $12 \mathrm{a}, 12 \mathrm{~b}, 14,16 \mathrm{a}, 16 \mathrm{~b}$ & & 14 \\
\hline 14 & 41.7 & $\mathrm{CH}$ & 3.01 & ddd $(13.4,5.6,2.2)$ & $13,15,16 \mathrm{~b}, 11 \mathrm{a}$ & 8,9 & 13 \\
\hline 15 & 103.5 & $\mathrm{CH}$ & 6.01 & $\mathrm{~d}(5.9)$ & $14,16 \mathrm{a}$ & $13,14,16$ & 6 \\
\hline $16 \mathrm{a}$ & 73.1 & $\mathrm{CH}_{2}$ & 3.58 & $\mathrm{dd}(10.0,8.8)$ & $13,16 b, 15$ & & \\
\hline $\mathrm{b}$ & & & 4.18 & $\mathrm{t}(8.2)$ & $13,16 \mathrm{a}, 14$ & $13,14,15$ & \\
\hline 17 & 172.5 & $\mathrm{C}$ & & & & & \\
\hline $18^{\mathrm{c}}$ & 27.6 & $\mathrm{CH}_{3}$ & 0.76 & s & $3 a, 19$ & $3,4,5,19$ & \\
\hline $19^{\mathrm{c}}$ & 32.9 & $\mathrm{CH}_{3}$ & 0.90 & s & 18 & $3,4,5,18$ & \\
\hline 20 & 31.9 & $\mathrm{CH}_{3}$ & 1.13 & s & & $1,5,9,10$ & 7 \\
\hline
\end{tabular}

a Multiplicity determined from HSQC-DEPT (multiplicity edited HSQC) experiment.

b Carbon connectivity determined from an HSQC-DEPT experiment, multiplicity determined from ${ }^{1} \mathrm{H}$ and ${ }^{1} \mathrm{H}$ homonuclear decoupled experiments.

c Interchangeable.

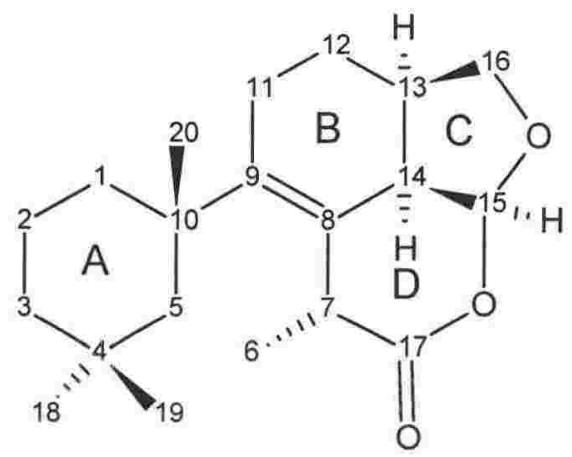

(138) 
The molecular formula of cadlinolide D (139) $(0.8 \mathrm{mg})$ was determined as $\mathrm{C}_{21} \mathrm{H}_{32} \mathrm{O}_{4}$ from observation of several pseudo-molecular ions in the positive ion HRESIMS mode $\left(349.2399[\mathrm{M}+\mathrm{H}]^{+}, \Delta 7.5 \mathrm{ppm} ; 371.2201[\mathrm{M}+\mathrm{Na}]^{+}, \Delta 2.1 \mathrm{ppm} ; 719.4483[2 \mathrm{M}+\mathrm{Na}]^{+}, \Delta\right.$ $2.5 \mathrm{ppm}$ ), requiring six degrees of unsaturation. Due to the small amount of 139 isolated, a ${ }^{13} \mathrm{C}$ NMR spectrum could not be obtained therefore all 21 observed ${ }^{13} \mathrm{C}$ chemical shifts referred to below, were observed as correlations in the HSQC-DEPT or HMBC spectra. All $32{ }^{1} \mathrm{H}$ resonances were observed in the ${ }^{1} \mathrm{H}$ NMR spectrum. As with cadlinolide $\mathrm{C}$ (138), only one significant peak was observed in the IR spectrum $(C=O$ stretch, $\left.1744 \mathrm{~cm}^{-1}\right)$. Notable ${ }^{13} \mathrm{C}$ resonances included those of a carbonyl $\left(\delta_{\mathrm{C}} 171.8\right)$, an olefin $\left(\delta_{C} 145.7,122.2\right)$ and two acetals $\left(\delta_{C} 109.2,102.2\right)$. Again, the olefin was assigned as tetrasubstituted as no HSQC-DEPT correlations were observed to these two ${ }^{13} \mathrm{C}$ resonances. After accounting for the olefin and the carbonyl, the remaining degrees of unsaturation of 139 required a tetracyclic structure.

A significant feature of the ${ }^{1} \mathrm{H}$ NMR spectrum was the 3:1 ratio observed between several closely related resonances, implying the presence of two similar compounds (diastereomers). Several unsuccessful attempts were made to separate these compounds; therefore the following structural elucidation was performed on a mixture of diastereomers in a 3:1 ratio. Cadlinolide B (133) had previously been reported as a similar inseparable pair of diastereomers. ${ }^{28}$

As with cadlinolide C (138), a 1,3,3-trimethylcyclohexyl ring could be established. A strong COSY correlation was observed between the ${ }^{1} \mathrm{H}$ resonances of two methylenes $\left[\left(\mathrm{C}-1: \delta_{\mathrm{C}} 39.2, \delta_{\mathrm{H}} 1.92,1.13\right),\left(\mathrm{C}-2: \delta_{\mathrm{C}} 20.9, \delta_{\mathrm{H}} 1.51\right)\right]$. Specific correlations between the ${ }^{1} \mathrm{H}$ resonances of methylenes $\mathrm{C}-2$ and $\mathrm{C}-3$ could not be unequivocally distinguished in 
the COSY spectrum due to some spectral overlap. A 1D-TOCSY correlation, however, was observed between the ${ }^{1} \mathrm{H}$ resonances of $\mathrm{C}-2$ and those of $\mathrm{C}-1$, and also to those of another methylene $\left(\mathrm{C}-3: \delta_{\mathrm{C}} 39.5, \delta_{\mathrm{H}} 1.35,1.20\right)$, which established the connectivity of the three methylene chain $\mathrm{C}-1$ to $\mathrm{C}-3$. As with cadlinolide $\mathrm{C}$ (138), strong HMBC correlations were observed from the ${ }^{1} \mathrm{H}$ resonances of two singlet methyls [(C-18: $\left.\left.\delta_{\mathrm{C}} 28.1, \delta_{\mathrm{H}} 0.76\right),\left(\mathrm{C}-19: \delta_{\mathrm{C}} 32.9, \delta_{\mathrm{H}} 0.90\right)\right]$ to the ${ }^{13} \mathrm{C}$ resonances of each other, a pair of methylenes [C-3 and $\left.\left(\mathrm{C}-5: \delta_{\mathrm{C}} 51.0, \delta_{\mathrm{H}} 1.78,1.25\right)\right]$ and a quaternary carbon (C-4: $\delta_{\mathrm{C}} 31.9$ ), establishing the connection of the methylene chain $\mathrm{C}-1$ to $\mathrm{C}-3$ with the gem-dimethyl quaternary carbon $\mathrm{C}-4$. The ${ }^{1} \mathrm{H}$ resonance of a third methyl singlet (C-20: $\left.\delta_{\mathrm{C}} 31.2, \delta_{\mathrm{H}} 1.13\right)$ showed $\mathrm{HMBC}$ correlations to the ${ }^{13} \mathrm{C}$ resonances of two methylenes [C-1 and C-5], and a quaternary carbon (C-10: $\left.\delta_{\mathrm{C}} 40,1\right)$, thereby completing the 1,3,3-trimethylcyclohexyl substructure (see figure 4.9).

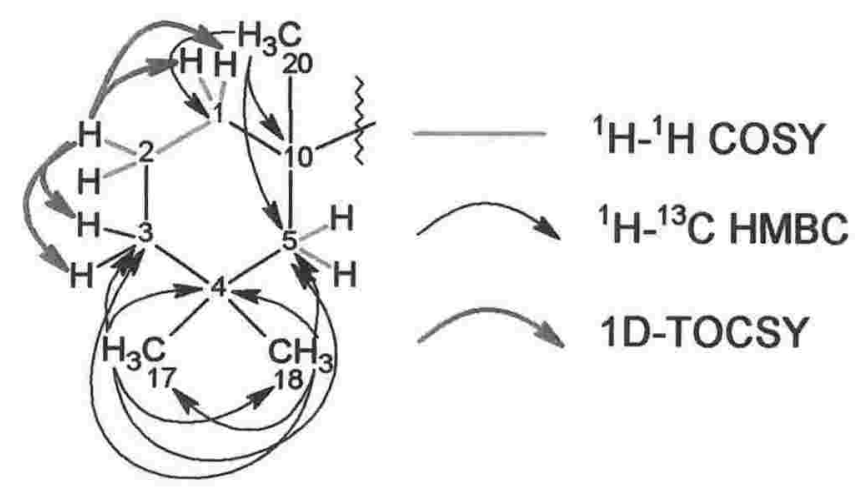

Figure 4.9 Establishment of 1,3,3-trimethylcyclohexyl substructure of cadlinolide $\mathrm{D}$ (139).

A chain of four carbons was connected on the basis of observed COSY cross-peaks between their attached ${ }^{1} \mathrm{H}$ resonances [methylene $\left(\mathrm{C}-11: \delta_{\mathrm{C}} 25.8, \delta_{\mathrm{H}} 2.35,2.07\right)$ to methylene $\left(\mathrm{C}-12: \delta_{\mathrm{C}} 24.7, \delta_{\mathrm{H}} 1.88,1.19\right)$ to methine $\left(\mathrm{C}-13: \delta_{\mathrm{C}} 30.0, \delta_{\mathrm{H}} 2.40\right)$ to methine (C-14: $\left.\left.\delta_{\mathrm{C}} 38.9, \delta_{\mathrm{H}} 3.17\right)\right]$. The resonance of $\mathrm{H}-13$ showed a further coupling to an acetal proton $\left(\mathrm{C}-16\right.$ : $\left.\delta_{\mathrm{C}} 109.2, \delta_{\mathrm{H}} 4.92\right)$ while $\mathrm{H}-14$ showed a correlation to the second acetal ${ }^{1} \mathrm{H}$ resonance $\left(\mathrm{C}-15: \delta_{\mathrm{C}} 102.2, \delta_{\mathrm{H}} 5.97\right)$. The lack of a hydroxyl functionality within the 
molecule, as evidenced by the lack of an $\mathrm{OH}$ stretching band in the $\mathbb{R}$ spectrum, coupled with the chemical shifts of both methines $\mathrm{C}-15$ and $\mathrm{C}-16$, suggest that $\mathrm{C}-15$ and C-16 are connected via an ether linkage, which would form a tetrahydrofuran ring similar to that found in cadlinolide C (138). Unfortunately, no $\mathrm{HMBC}$ correlations were observed between methines $\mathrm{C}-15$ and $\mathrm{C}-16$ to help confirm this linkage (see figure 4.10).

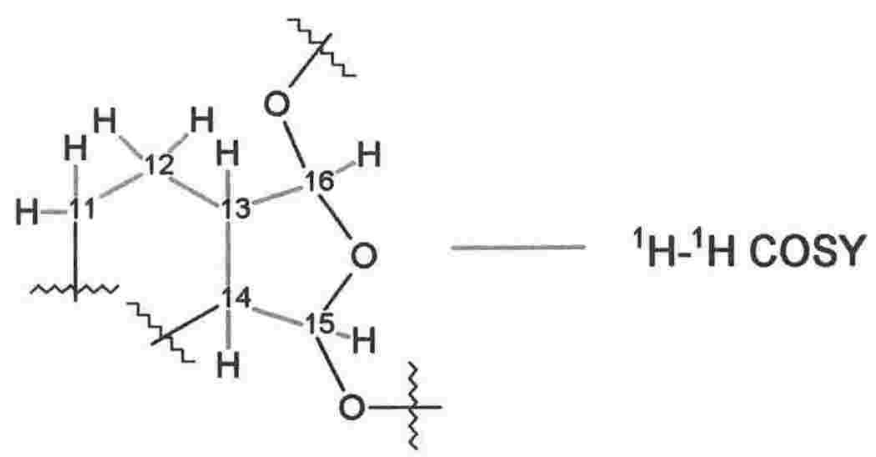

Figure 4.10 Establishment of the tetrahydrofuran ring of cadlinolide D (139).

As with 138, an intense COSY correlation was observed between the ${ }^{1} \mathrm{H}$ resonances of a methyl $\left(\mathrm{C}-6: \delta_{\mathrm{C}} 14.8, \delta_{\mathrm{H}} 1.40\right)$ and a methine $\left(\mathrm{C}-7: \delta_{\mathrm{C}} 41.2, \delta_{\mathrm{H}} 4.19\right)$. The ${ }^{1} \mathrm{H}$ resonances of both showed strong $\mathrm{HMBC}$ correlations to the ${ }^{13} \mathrm{C}$ resonance of the other, and also to that of a carbonyl (C-17: $\left.\delta_{\mathrm{C}} 171.8\right)$ to complete a third fragment (see figure 4.5 ).

The analysis detailed above had assigned 18 of the 21 carbon centres of 139 , leaving an oxygenated methyl and a tetrasubstituted olefin to be assigned. As with cadlinolide C (138), several key HMBC and COSY correlations were used to identify the placement of these functionalities, and to complete the final structure of the molecule. The ${ }^{1} \mathrm{H}$ resonances of $\mathrm{C}-6$ and $\mathrm{C}-7$ each showed an $\mathrm{HMBC}$ correlation to the ${ }^{13} \mathrm{C}$ resonance of an olefinic carbon $\left(\mathrm{C}-8\right.$ : $\left.\delta_{\mathrm{C}} 122.2\right)$ while $\mathrm{H}-7$ also showed an HMBC correlation to C-14, which together imply the attachment of the side chain at C-8. Similar to 138 , the ${ }^{1} \mathrm{H}$ resonance of $\mathrm{C}-20$ showed a strong correlation to the ${ }^{13} \mathrm{C}$ resonance of the second disubstituted olefinic carbon $\left(\mathrm{C}-9\right.$ : $\delta_{\mathrm{C}}$ 145.7), establishing the link between C-9 and 
C-10 and thereby connecting the 1,3,3-trimethylcyclohexyl moiety to the olefin. A homo-allylic coupling was observed in the COSY spectrum between H-11a and H-14, helping to confirm the olefinic linkage between C-8 and C-9 within a six-membered ring. The ${ }^{1} \mathrm{H}$ resonance of an oxygenated methyl $\left(\mathrm{C}-21: \delta_{\mathrm{C}} 56.2, \delta_{\mathrm{H}} 3.45\right)$ showed a strong $\mathrm{HMBC}$ correlation to $\mathrm{C}-16$, establishing the methyl ether attachment at, and the acetal nature of, $\mathrm{C}-16$. This was confirmed by observation of an HMBC correlation from the resonance of $\mathrm{H}-16$ to the resonance of $\mathrm{C}-21$. As with cadlinolide $\mathrm{C}$ (138), no direct $\mathrm{HMBC}$ correlation from $\mathrm{H}-15$ to $\mathrm{C}-17$ was observed to confirm a lactone linkage although this was implied by the remaining degree of unsaturation required by the molecular formula. The magnitudes of the ${ }^{1} \mathrm{H}$ resonance of $\mathrm{H}-15$ and the ${ }^{13} \mathrm{C}$ resonance of $\mathrm{C}-17$ and the absence of an $\mathrm{OH}$ stretching band in the $\mathbb{R}$ spectrum, were all consistent with a lactone linkage. Finally, the largest difference in ${ }^{1} \mathrm{H}$ chemical shifts noted between the major and minor epimers of 139 occurs at methine $\mathrm{H}-16$, implying that this is the epimeric position of the molecule, which is the same diastereomeric position as reported for cadlinolide B (133) (see figure 4.11). ${ }^{28}$

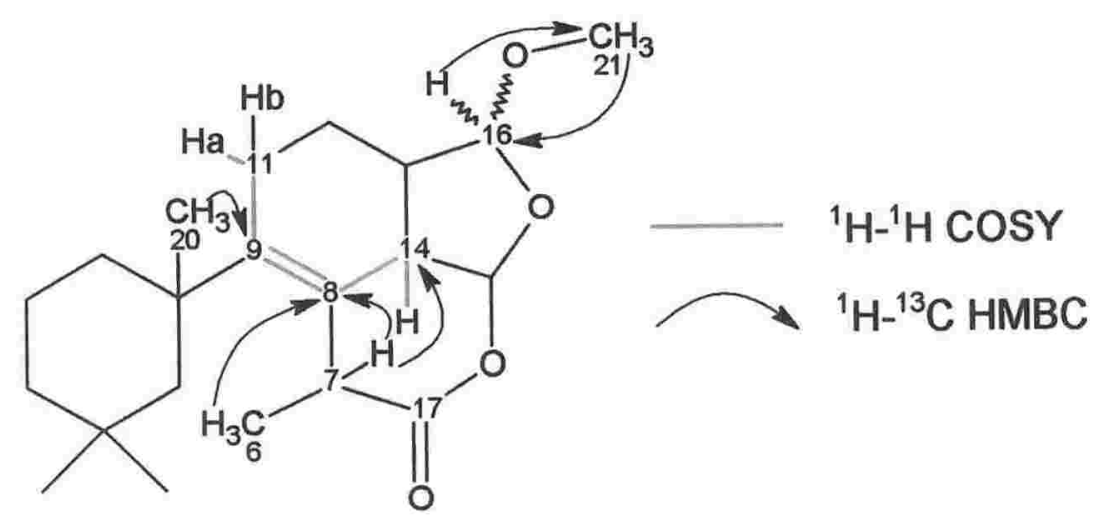

Figure 4.11 Establishment of final connectivity of cadlinolide D (139).

The relative stereochemistry of 139 was established by measurement of ${ }^{1} \mathrm{H}-{ }^{1} \mathrm{H}$ vicinal coupling constants, determined from the ${ }^{1} \mathrm{H}$ NMR spectrum, and also from NOE correlations observed in a ROESY experiment. The magnitude of the coupling constant between $\mathrm{H}-14$ and $\mathrm{H}-15$ is small, suggesting a small vicinal angle between these 
protons. An NOE correlation was observed between $\mathrm{H}-13$ and $\mathrm{H}-14$, indicating that these protons are on the same face of the molecule. NOE correlations were observed from $\mathrm{CH}_{3}-6$ to $\mathrm{H}-5 \mathrm{~b}, \mathrm{H}-14$ and $\mathrm{H}-15$ implying that methyl $\mathrm{C}-6$ and $\mathrm{H}-5 \mathrm{~b}$ are on the same face of the molecule as $\mathrm{H}-14$ and $\mathrm{H}-15$. This was supported by NOE correlations detected between $\mathrm{H}-7$ and both $\mathrm{H}-5 \mathrm{~b}$ and $\mathrm{CH}_{3}-20$ (see figure 4.12).

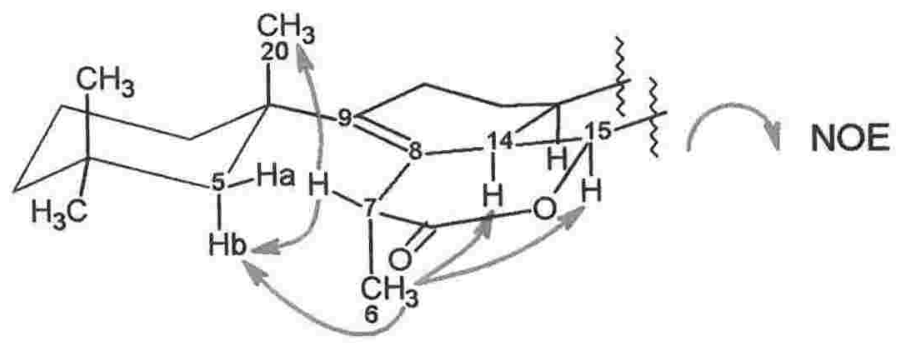

Figure 4.12 Selected NOE correlations establishing the stereochemistry of the 1,3,3trimethylcyclohexyl and lactone rings of cadlinolide D (139).

Small coupling constants are measured between $\mathrm{H}-13$ and $\mathrm{H}-16$ for both the major and minor epimers. The major epimer, however, shows a very small coupling constant $(3.2$ $\mathrm{Hz}$ ) which may be indicative of a dihedral angle of $\sim 90^{\circ}$ between these two protons, implying that the methoxy group of the major epimer is in pseudo-axial orientation (see figure 4.13).

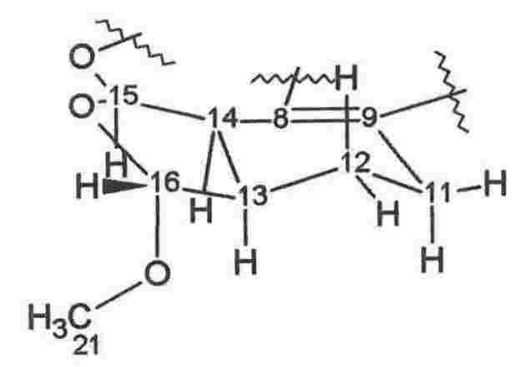

Figure 4.13 Proposed stereochemistry of tetrahydrofuran ring of the major epimer of cadlinolide D (139).

Based on the previous observations, the relative stereochemistry of the major epimer of cadlinolide D (139) can be assigned as $7 R^{*}, 10 S^{*}, 13 R^{*}, 14 S^{*}, 15 S^{*}$, consistent with the relative stereochemistry of cadlinolides $\mathrm{A}, \mathrm{B}$, and $\mathrm{C}(132,133,138){ }^{28}$ 
Table $4.2{ }^{13} \mathrm{C}(75 \mathrm{MHz})$ and ${ }^{1} \mathrm{H}(300 \mathrm{MHz})$ NMR Data $\left(\mathrm{CDCl}_{3}\right)$ of cadlinolide D (139).

\begin{tabular}{|c|c|c|c|c|c|c|c|c|}
\hline \multirow[b]{2}{*}{ Position } & \multicolumn{2}{|c|}{${ }^{13} \mathrm{C}^{\mathrm{a}}$} & \multicolumn{2}{|c|}{${ }^{\mathrm{t}} \mathrm{H}^{\mathrm{b}}$} & \multirow[b]{2}{*}{ COSY } & \multirow{2}{*}{$\begin{array}{l}\text { HMBC } \\
\text { (H to } \mathrm{C} \text { ) }\end{array}$} & \multirow[b]{2}{*}{ TOCSY } & \multirow[b]{2}{*}{ NOE } \\
\hline & $\delta(\mathrm{ppm})$ & mult & $\delta(\mathrm{ppm})$ & mult, $J(\mathrm{~Hz})$ & & & & \\
\hline 1a & 39.2 & $\mathrm{CH}_{2}$ & 1.13 & $\mathrm{~m}$ & $1 \mathrm{~b}, 2$ & & & $1 \mathrm{~b}$ \\
\hline $\mathrm{b}$ & & & 1.92 & $\mathrm{~m}$ & $1 \mathrm{a}, 2$ & & & la \\
\hline 2 & 20.9 & $\mathrm{CH}_{2}$ & 1.51 & $\mathrm{~m}, 2 \mathrm{H}$ & $1 \mathrm{a}, 1 \mathrm{~b}$ & & $3 a, 3 b$ & \\
\hline $3 a$ & 39.5 & $\mathrm{CH}_{2}$ & 1.20 & $\mathrm{~m}$ & & & & \\
\hline $\mathrm{b}$ & & & 1.35 & $\mathrm{~m}$ & & & & \\
\hline 4 & 31.9 & $\mathrm{C}$ & & & & & & \\
\hline $5 \mathrm{a}$ & 51.0 & $\mathrm{CH}_{2}$ & 1.25 & $\mathrm{~m}$ & $5 b$ & $4,7,9,10$ & & $5 \mathrm{~b}$ \\
\hline $\mathrm{b}$ & & & 1.78 & $\mathrm{~m}$ & $5 \mathrm{a}$ & & & $5 a, 6,18,19$ \\
\hline 6 & 14.8 & $\mathrm{CH}_{3}$ & 1.40 & $\mathrm{~d}(7.6)$ & 7 & $7,8,17$ & & $5 b, 14,15$ \\
\hline 7 & 41.2 & $\mathrm{CH}$ & 4.19 & $\mathrm{q}(7.4)$ & 6 & $6,8,14,17$ & & $5 \mathrm{~b}, 20$ \\
\hline 8 & 122.2 & $\mathrm{C}$ & & & & & & \\
\hline 9 & 145.7 & $\mathrm{C}$ & & & & & & \\
\hline 10 & 40.1 & $\mathrm{C}$ & & & & & & \\
\hline $11 \mathrm{a}$ & 25.8 & $\mathrm{CH}_{2}$ & 2.07 & $\mathrm{~m}$ & $11 \mathrm{~b}, 12 \mathrm{a}, 12 \mathrm{~b}, 14$ & & & $11 \mathrm{~b}$ \\
\hline $\mathrm{b}$ & & & 2.35 & $\mathrm{~m}$ & $11 \mathrm{a}, 12 \mathrm{a}, 12 \mathrm{~b}$ & & & $11 \mathrm{a}$ \\
\hline $12 \mathrm{a}$ & 24.7 & $\mathrm{CH}_{2}$ & 1.19 & $\mathrm{~m}$ & $11 \mathrm{a}, 11 \mathrm{~b}, 13$ & & & $12 \mathrm{~b}$ \\
\hline $\mathrm{b}$ & & & 1.88 & $\mathrm{~m}$ & $11 \mathrm{a}, 11 \mathrm{~b}, 13$ & & & $12 \mathrm{a}$ \\
\hline 13 & 30.0 & $\mathrm{CH}$ & 2.40 & $\mathrm{~m}$ & $11 \mathrm{a}, 11 \mathrm{~b}, 14,16$ & & & 14,16 \\
\hline 14 & 38.9 & $\mathrm{CH}$ & 3.17 & brt $(7.1)$ & $13,15,11 \mathrm{a}$ & & & $6,13,15$ \\
\hline 15 & 102.2 & $\mathrm{CH}$ & 5.97 & $\mathrm{~d}(6.3)$ & 14 & 14 & & 6,14 \\
\hline 16 & 109.2 & $\mathrm{CH}$ & 4.92 & $\mathrm{~d}(3.2)$ & 13 & & & 13,21 \\
\hline 17 & 171.8 & $\mathrm{C}$ & & & & & & \\
\hline $18^{\mathrm{C}}$ & 28.1 & $\mathrm{CH}_{3}$ & 0.76 & $\mathrm{~s}$ & $3 b, 19$ & $3,4,5,19$ & & $5 b, 19$ \\
\hline $19^{\mathrm{c}}$ & 32.9 & $\mathrm{CH}_{3}$ & 0.90 & s & 18 & $3,4,5,18$ & & $5 b, 18$ \\
\hline 20 & 31.2 & $\mathrm{CH}_{3}$ & 1.13 & s & & $1,5,9,10$ & & \\
\hline 21 & 56.2 & $\mathrm{CH}_{3}$ & 3.45 & $\mathrm{~s}$ & & 16 & & 16 \\
\hline
\end{tabular}

a Determined from HSQC-DEPT (multiplicity edited HSQC) and HMBC experiments.

b Carbon connectivity determined from an HSQC-DEPT experiment, multiplicity determined from ${ }^{1} \mathrm{H}$ experiment.

c Interchangeable.

* Data is for the major epimer

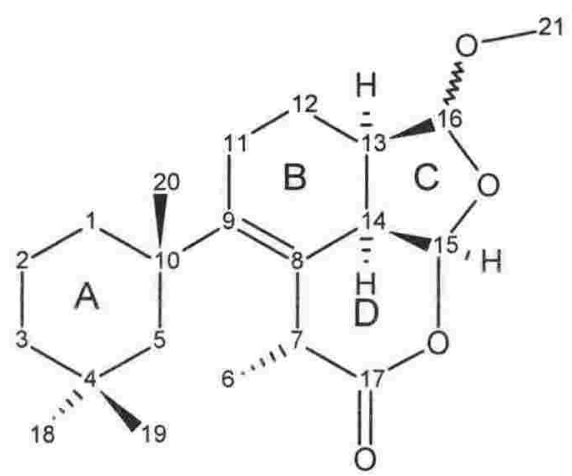

(139) 
The molecular formula of pourewic acid $\mathrm{A}(\mathbf{1 4 0})(2.9 \mathrm{mg})$ was established as $\mathrm{C}_{21} \mathrm{H}_{34} \mathrm{O}_{4}$ by observation of a pseudo-molecular ion in negative ion mode HRESIMS (349.2401 $\left.[\mathrm{M}-\mathrm{H}]^{-}, \Delta 4.8 \mathrm{ppm}\right)$, implying five degrees of unsaturation. All 21 carbons and 33 of the 34 protons of $\mathbf{1 4 0}$ were observed in the ${ }^{13} \mathrm{C}$ and ${ }^{1} \mathrm{H}$ NMR spectra respectively. Two prominent stretching bands (OH stretch, $3392 \mathrm{~cm}^{-1}, \mathrm{C}=\mathrm{O}$ stretch $1704 \mathrm{~cm}^{-1}$ ) were observed in the IR spectrum. The presence of a carbonyl was confirmed by a ${ }^{13} \mathrm{C}$ resonance observed $\left(\delta_{\mathrm{C}} 178.8\right)$. A tetrasubstituted olefin was implied by the two deshielded ${ }^{13} \mathrm{C}$ NMR resonances observed $\left(\delta_{\mathrm{C}} 144.2,127.9\right)$, combined with the absence of any HSQC-DEPT correlations to these resonances. With no other evidence of double bonds, the remaining degrees of unsaturation required a tricyclic structure for $\mathbf{1 4 0 .}$

As with cadlinolides C (138) and D (139), analysis of COSY and HMBC correlations from various ${ }^{1} \mathrm{H}$ resonances of $\mathbf{1 4 0}$ allowed several substructures to be constructed. In particular, close examination of the spectra allowed the rapid establishment of a 1,3,3-trimethylcylohexyl ring, in a similar manner to that of $\mathbf{1 3 8}$ [(C-1: $\delta_{\mathrm{C}} 39.1$, $\left.\delta_{\mathrm{H}} 2.13,1.21\right),\left(\mathrm{C}-2: \delta_{\mathrm{C}} 20.1, \delta_{\mathrm{H}} 1.85,1.49\right),\left(\mathrm{C}-3: \delta_{\mathrm{C}} 40.0, \delta_{\mathrm{H}} 1.33,1.18\right),\left(\mathrm{C}-4: \delta_{\mathrm{C}} 31.6\right)$, $\left(\mathrm{C}-5: \delta_{\mathrm{C}} 50.8, \delta_{\mathrm{H}} 1.76,0.96\right),\left(\mathrm{C}-10: \delta_{\mathrm{C}} 41.5\right),\left(\mathrm{C}-18: \delta_{\mathrm{C}} 26.6, \delta_{\mathrm{H}} 0.87\right),\left(\mathrm{C}-19: \delta_{\mathrm{C}} 33.1\right.$, $\left.\delta_{\mathrm{H}} 0.84\right)$ and $\left.\left(\mathrm{C}-20: \delta_{\mathrm{C}} 31.6, \delta_{\mathrm{H}} 1.03\right)\right]$ (see table 4.3 and figure 4.3$)$.

A similar series of COSY and HMBC correlations as those observed for $\mathbf{1 3 8}$ allowed a second substructure to be established. In particular, a sequence of two methylene pairs, two methines, an oxymethylene and an acetal centre $\left[\left(\mathrm{C}-11: \delta_{\mathrm{C}} 27.6, \delta_{\mathrm{H}} 2.22,1.91\right)\right.$, $\left(\mathrm{C}-12: \delta_{\mathrm{C}} 30.6, \delta_{\mathrm{H}} 1.63,1.24\right),\left(\mathrm{C}-13: \delta_{\mathrm{C}} 37.8, \delta_{\mathrm{H}} 2.30\right),\left(\mathrm{C}-14: \delta_{\mathrm{C}} 48.8, \delta_{\mathrm{H}} 2.71\right),(\mathrm{C}-15:$ $\left.\left.\delta_{\mathrm{C}} 111.0, \delta_{\mathrm{H}} 4.75\right),\left(\mathrm{C}-16: \delta_{\mathrm{C}} 74.7, \delta_{\mathrm{H}} 4.03,3.76\right)\right]$ was connected from observed COSY 
correlations between their various ${ }^{1} \mathrm{H}$ resonances. A strong $\mathrm{HMBC}$ correlation from $\mathrm{H}-15$ to $\mathrm{C}-16$, and a similar correlation from $\mathrm{H}-16$ a to $\mathrm{C}-15$, established the connectivity of the tetrahydrofuran ring. This was confirmed by observation of W-coupling between $\mathrm{H}-15$ and $\mathrm{H}-16 \mathrm{~b}$ as evidenced in the COSY spectrum (see figure 4.4).

Similarly, a strong COSY correlation between the ${ }^{1} \mathrm{H}$ resonances of a methyl (C-6: $\left.\delta_{\mathrm{C}} 18.8, \delta_{\mathrm{H}} 1.21\right)$ and of a methine $\left(\mathrm{C}-7: \delta_{\mathrm{C}} 41.9, \delta_{\mathrm{H}} 4.22\right)$ was observed. Both ${ }^{1} \mathrm{H}$ resonances also showed strong $\mathrm{HMBC}$ correlations to the ${ }^{13} \mathrm{C}$ resonances of each other, and to that of a carbonyl $\left(\mathrm{C}-17: \delta_{\mathrm{C}} 178.8\right)$ to establish a third fragment (see figure 4.5).

The final connectivity of pourewic acid A (140) was established by close examination of its HMBC spectrum. A methyl singlet (C-21: $\left.\delta_{\mathrm{C}} 54.9, \delta_{\mathrm{H}} 3.17\right)$ showed a strong HMBC correlation to C-15, while a similar correlation from $\mathrm{H}-15$ back to $\mathrm{C}-21$, established a methyl ether substituent on the $\mathrm{C}-15$ acetal centre. The ${ }^{1} \mathrm{H}$ resonances of $\mathrm{CH}_{3}-6$ and $\mathrm{H}-7$ both showed an intense HMBC correlation to an olefinic carbon (C-8: $\delta_{\mathrm{C}} 127.8$ ) while $\mathrm{H}-7$ also showed strong correlations to both the second non-protonated olefinic carbon (C-9: $\left.\delta_{\mathrm{C}} 144.2\right)$ and to methine $\mathrm{C}-14$, indicating the attachment of the side chain at C-8 between C-9 and C-14. Again, a strong HMBC correlation was observed from $\mathrm{CH}_{3}-20$ to $\mathrm{C}-9$, confirming the linkage of the 1,3,3-trimethylcyclohexyl moiety to olefin carbon C-9. Observation of HMBC correlations from the deshielded resonance $\mathrm{H}-11 \mathrm{~b}$ to $\mathrm{C}-8, \mathrm{C}-9$, and $\mathrm{C}-10$, confirmed the connection of the olefin within a six-membered ring and established the final connectivity of $\mathbf{1 4 0}$. As all the degrees of unsaturation for the molecule had been accounted for, carbonyl C-17 must be part of a carboxylic acid, which was consistent with the $\mathrm{OH}$ stretch noted in the IR spectrum (see figure 4.14). The presence of the carboxylic acid was confirmed by the preparation of the methyl ester of the natural product by reacting it with $\mathrm{CH}_{2} \mathrm{~N}_{2}$. The chemical shifts of 
the methyl ester derivative of $\mathbf{1 4 0}$ compare favourably with those of C-17 methyl ester metabolite membranolide (128). ${ }^{151}$ Pourewic acid A (140) is the first example of a rearranged spongian diterpene with a free carboxylic acid at $\mathrm{C}-17$. The biochemical significance of this will be discussed later.

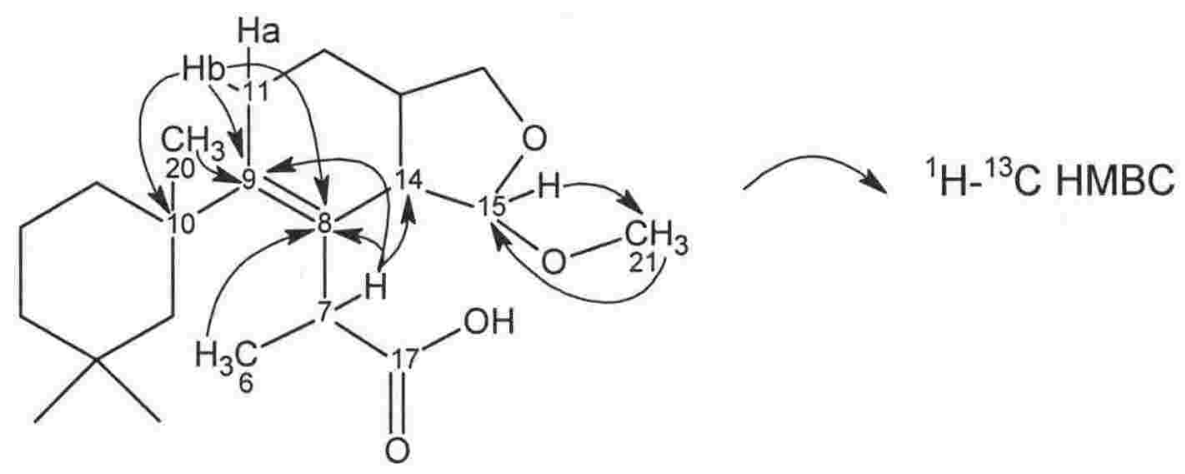

Figure 4.14 Selected HMBC correlations establishing the final connectivity of pourewic acid A (140).

The relative stereochemistry of $\mathbf{1 4 0}$ was established by analysis of vicinal homonuclear coupling constants and by observation of NOE correlations observed in both 1D-gNOESY and ROESY experiments. A small coupling constant was measured between $\mathrm{H}-14$ and $\mathrm{H}-15$, consistent with a small dihedral angle between these protons. NOE correlations were observed from $\mathrm{H}-14$ to $\mathrm{H}-13$ and $\mathrm{H}-16 \mathrm{~b}$, implying they are on the same face of the molecule. A strong NOE correlation was observed between H-16a and $\mathrm{H}-12 \mathrm{~b}$ indicating their spatial proximity. A 1,3-diaxial NOE was observed between $\mathrm{H}-13$ and $\mathrm{H}-11 \mathrm{a}$ helping to confirm these assignments. These observations were consistent with the stereochemistry shown in figure 4.15. NOE correlations were observed between $\mathrm{CH}_{3}-6$ and $\mathrm{H}-14$, placing methyl C-6 on the same face as H-14. NOE correlations between $\mathrm{CH}_{3}-6$ and $\mathrm{CH}_{3}-20, \mathrm{H}-7$ and $\mathrm{H}-1 \mathrm{~b}$, and from $\mathrm{H}-5 \mathrm{a}$ to $\mathrm{H}-11 \mathrm{~b}$, were consistent with this placement and allowed the relative stereochemistry of C-7 and C-10 to be established. All these observations allowed the relative stereochemistry of pourewic acid $\mathrm{A}(\mathbf{1 4 0})$ to be assigned as $7 R^{*}, 10 S^{*}, 13 S^{*}, 14 S^{*}, 15 R^{*}$ (see figure 4.16). 


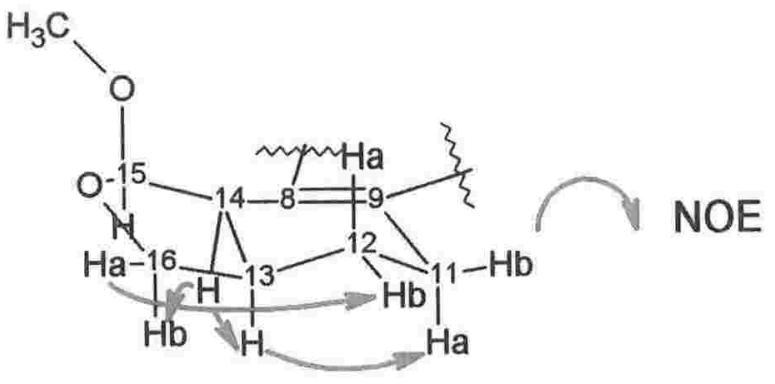

Figure 4.15 Selected NOE correlations establishing the stereochemistry of the tetrahydrofuran ring of pourewic acid A (140).

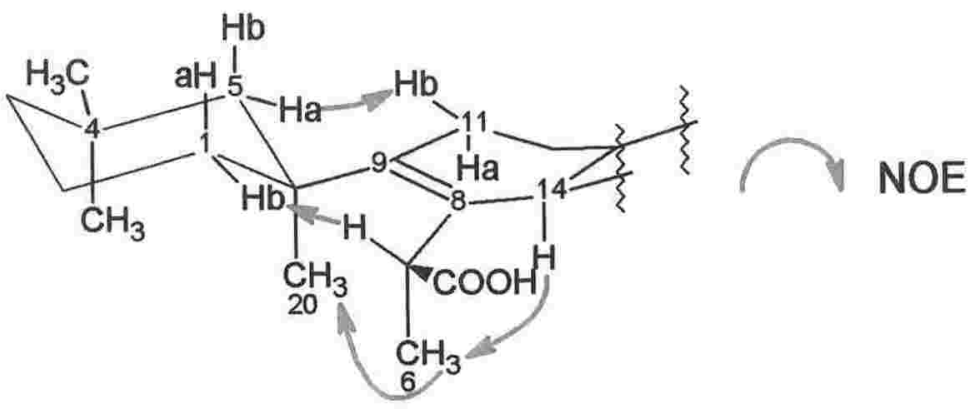

Figure 4.16 Selected NOE correlations establishing the stereochemistry of the side chain and the 1,3,3-trimethylcyclohexyl ring of pourewic acid A (140). 
Table $4.3{ }^{13} \mathrm{C}(75 \mathrm{MHz})$ and ${ }^{1} \mathrm{H}(300 \mathrm{MHz})$ NMR Data $\left(\mathrm{CDCl}_{3}\right)$ of pourewic acid A (140).

\begin{tabular}{|c|c|c|c|c|c|c|c|}
\hline \multirow[b]{2}{*}{ Position } & \multicolumn{2}{|c|}{${ }^{13} \mathrm{C}^{\mathrm{a}}$} & \multicolumn{2}{|r|}{${ }^{1} \mathrm{H}^{\mathrm{b}}$} & \multicolumn{3}{|c|}{ HMBC } \\
\hline & $\delta(\mathrm{ppm})$ & mult & $\delta(\mathrm{ppm})$ & mult, $J(\mathrm{~Hz})$ & COSY & (H to $\mathrm{C})$ & NOE \\
\hline la & 39.1 & $\mathrm{CH}_{2}$ & 1.21 & $\mathrm{~m}$ & $\mathrm{Ib}, 2 \mathrm{a}, 3 \mathrm{a}$ & & \\
\hline $\mathrm{b}$ & & & 2.13 & $\mathrm{~m}$ & $1 \mathrm{a}, 2 \mathrm{a}, 2 \mathrm{~b}$ & & 7 \\
\hline $2 \mathrm{~b}$ & 20.1 & $\mathrm{CH}_{2}$ & 1.49 & $\mathrm{dt}(13.2,3.4)$ & $1 a, 1 b, 2 a, 3 a, 3 b$ & & $1 \mathrm{a}, 1 \mathrm{~b}, 2 \mathrm{a}, 3 \mathrm{a}, 17,20$ \\
\hline a & & & 1.85 & $\mathrm{~m}$ & $1 b, 2 b, 3 a, 3 b$ & & \\
\hline $3 \mathrm{a}$ & 40.0 & $\mathrm{CH}_{2}$ & 1.18 & $\mathrm{t}(3.2)$ & $2 \mathrm{~b}, 3 \mathrm{~b}, 1 \mathrm{a}, 18$ & & \\
\hline $\mathrm{b}$ & & & 1.33 & $\mathrm{~m}$ & $2 \mathrm{~b}, 3 \mathrm{a}$ & & \\
\hline 4 & 31.6 & $\mathrm{C}$ & & & & & \\
\hline $5 a$ & 50.8 & $\mathrm{CH}_{2}$ & 0.96 & $\mathrm{~d}(13.9)$ & $5 \mathrm{~b}$ & & $11 \mathrm{~b}$ \\
\hline b & & & 1.76 & $\mathrm{~m}$ & $5 a$ & & \\
\hline 6 & 18.8 & $\mathrm{CH}_{3}$ & 1.21 & $\mathrm{~d}(6.8)$ & 7 & $7,8,9,17$ & $\mathrm{lb}, 7,12 \mathrm{~b}, 13,14,18,20$ \\
\hline 7 & 41.9 & $\mathrm{CH}$ & 4.22 & $\mathrm{q}(7.1)$ & 6 & $6,8,9,14,17$ & $1 \mathrm{~b}, 2 \mathrm{~b}, 6,20$ \\
\hline 8 & 127.9 & $\mathrm{C}$ & & & & & \\
\hline 9 & 144.2 & C & & & & & \\
\hline 10 & 41.5 & $\mathrm{C}$ & & & & & \\
\hline $11 \mathrm{a}(\mathrm{ax})$ & 27.6 & $\mathrm{CH}_{2}$ & 1.91 & dd $(11.7,3.7)$ & $11 \mathrm{~b}, 12 \mathrm{a}, 12 \mathrm{~b}$ & $8,9,12,13$ & \\
\hline $\mathrm{b}(\mathrm{eq})$ & & & 2.22 & $\mathrm{t}(4.3)$ & $11 a, 12 a, 12 b$ & $8,9,10,12,13$ & $5 \mathrm{a}$ \\
\hline $12 \mathrm{a}(\mathrm{ax})$ & 30.6 & $\mathrm{CH}_{2}$ & 1.24 & $\mathrm{~m}$ & $11 \mathrm{a}, 11 \mathrm{~b}, 12 \mathrm{~b}, 13$ & & \\
\hline$b(e q)$ & & & 1.63 & $\mathrm{dq}(11.5,3.7)$ & $11 \mathrm{a}, 11 \mathrm{~b}, 12 \mathrm{a}, 13$ & $9,11,13,14,16$ & $11 \mathrm{a}, 11 \mathrm{~b}, 12 \mathrm{a}, 13,15,16 \mathrm{a}$ \\
\hline 13 & 37.8 & $\mathrm{CH}$ & 2.30 & $\mathrm{~m}$ & $12 a, 12 b, 14,16 a, 16 b$ & & $11 \mathrm{a}, 12 \mathrm{a}, 12 \mathrm{~b}, 14,16 \mathrm{a}, 16 \mathrm{~b}$ \\
\hline 14 & 48.8 & $\mathrm{CH}$ & 2.71 & $\mathrm{dd}(8.0,2.7)$ & $13,15,16 \mathrm{a}$ & $7,8,9,12,13,15$ & $6,13,15,16 a, 16 b$ \\
\hline 15 & 111.0 & $\mathrm{CH}$ & 4.75 & $\mathrm{~d}(2.9)$ & $14,16 \mathrm{~b}$ & $8,13,14,16,21$ & $11 \mathrm{a}, 12 \mathrm{~b}, 14,18,21$ \\
\hline $16 a(e q)$ & 74.7 & $\mathrm{CH}_{2}$ & 3.76 & $\mathrm{~d}(8.5)$ & $13,16 b, 14$ & 15 & $12 b, 13,14,16 b$ \\
\hline $\mathrm{b}(\mathrm{ax})$ & & & 4.03 & $\mathrm{dd}(8.3,5.1)$ & $13,16 \mathrm{a}, 15$ & & $13,14,16 \mathrm{a}, 21$ \\
\hline 17 & 178.8 & $\mathrm{C}$ & & & & & \\
\hline $18^{\mathrm{c}}$ & 26.6 & $\mathrm{CH}_{3}$ & 0.87 & $s$ & $3 a$ & $3,4,5,19$ & $2 a, 3 b, 15$ \\
\hline $19^{\mathrm{c}}$ & 33.1 & $\mathrm{CH}_{3}$ & 0.84 & s & & $3,4,5,18$ & $2 a, 3 a$ \\
\hline 20 & 30.7 & $\mathrm{CH}_{3}$ & 1.03 & $\mathrm{~s}$ & & $1,5,9,10$ & $1 \mathrm{a}, 2 \mathrm{a}, 7,16 \mathrm{a}$ \\
\hline 21 & 54.9 & $\mathrm{CH}_{3}$ & 3.17 & $\mathrm{~s}$ & & 15 & 15 \\
\hline
\end{tabular}

a Multiplicity determined from HSQC-DEPT (multiplicity edited HSQC) experiment.

b Carbon connectivity determined from an HSQC-DEPT experiment, multiplicity determined from ${ }^{1} \mathrm{H}$ and ${ }^{1} \mathrm{H}$ homonuclear decoupled experiments.

c Interchangeable.

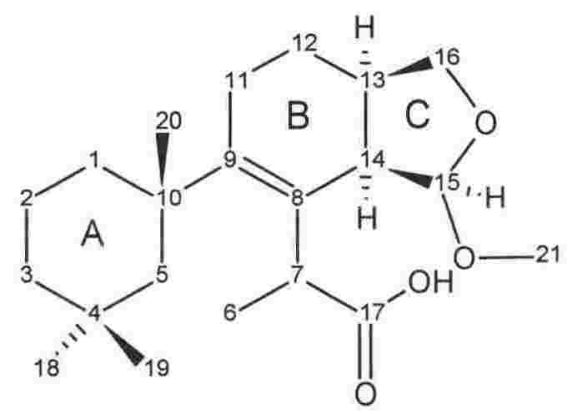


The molecular formula of 15-methoxypourewic acid B (141) (1.2 mg) was difficult to establish, as an obvious pseudo-molecular ion was not readily identifiable in the HRESIMS spectrum. Two peaks were detected in the negative ion mode differing by three $\mathrm{Da}$, the former being the expected $[\mathrm{M}-\mathrm{H}]^{-}$peak $\left(363.2194[\mathrm{M}-\mathrm{H}]^{-}\right)$while the latter peak corresponded to a pseudo-molecular ion with three deuteriums exchanged (366.2337 $\left.[\mathrm{M}(-3 \mathrm{H}+3 \mathrm{D})-\mathrm{H}]^{-}\right)$, caused by the exchange of a $\mathrm{CD}_{3} \mathrm{O}$ - group for a normal $\mathrm{CH}_{3} \mathrm{O}$ - group during the screening process, where $\mathrm{CD}_{3} \mathrm{OD}$ had been used as the NMR solvent (see figure 4.17). A corresponding deuterium exchanged peak was also observed in the positive ion mode $\left(368.2504[\mathrm{M}(-3 \mathrm{H}+3 \mathrm{D})+\mathrm{H}]^{+}\right)$. 15-Methoxypourewic acid B (141) is probably therefore an artefact of isolation.

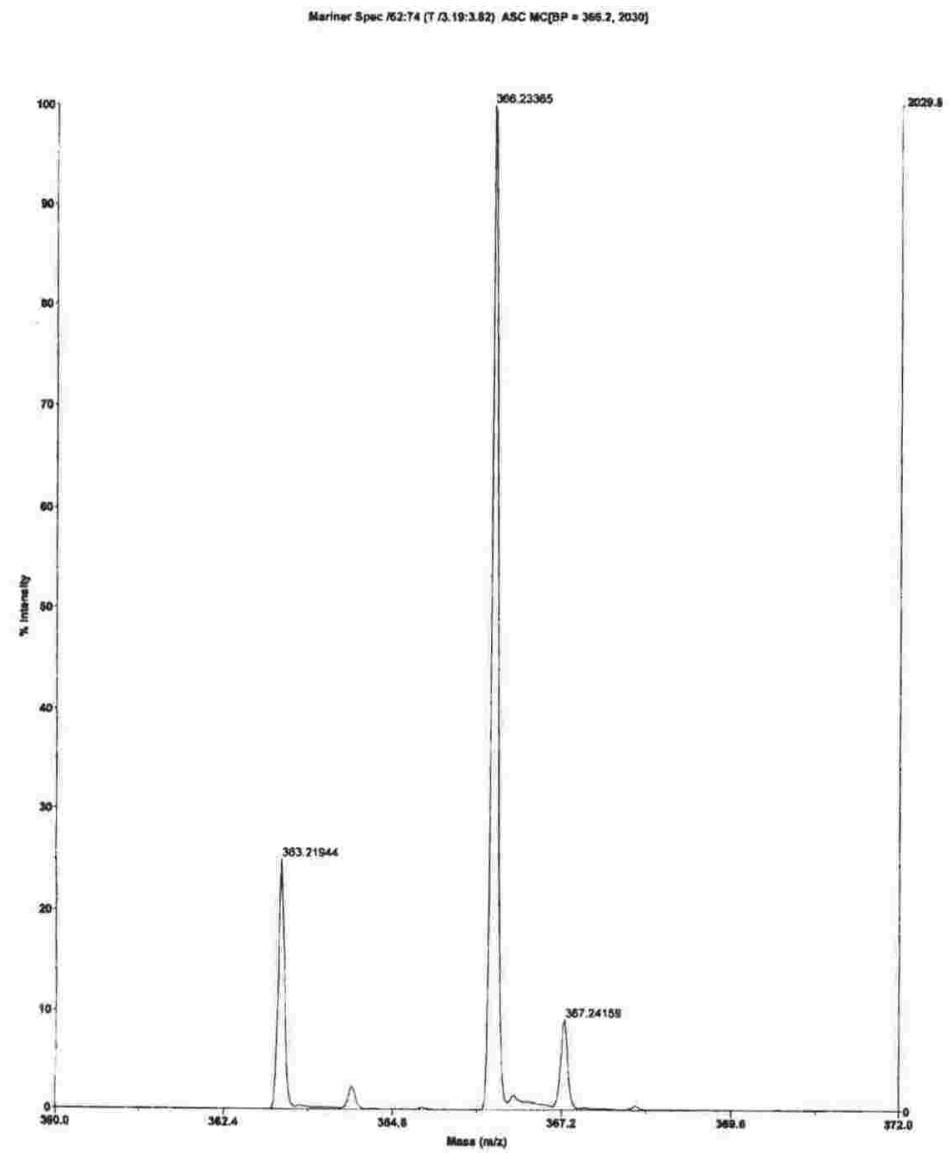

Figure 4.17 Negative ion HRESIMS spectrum of 15-methoxypourewic acid B (141). 
The molecular formula of $\mathbf{1 4 1}$ was finally established as $\mathrm{C}_{21} \mathrm{H}_{32} \mathrm{O}_{5}\left(363.2194[\mathrm{M}-\mathrm{H}]^{-}\right.$, $\Delta 4.8 \mathrm{ppm} ; 366.2337[\mathrm{M}(-3 \mathrm{H}+3 \mathrm{D})-\mathrm{H}]^{-}, \Delta 5.5 \mathrm{ppm} ; 368.2504[\mathrm{M}(-3 \mathrm{H}+3 \mathrm{D})+\mathrm{H}]^{+}$, $\Delta 2.5 \mathrm{ppm}$ ), requiring six degrees of unsaturation. Unfortunately, the compound degraded before a ${ }^{13} \mathrm{C}$ NMR spectrum could be acquired, therefore all $21{ }^{13} \mathrm{C}$ resonances of 141 were observed as correlations in either the HSQC-DEPT or HMBC spectra. All 31 protons attached to carbon were observed in the ${ }^{1} \mathrm{H}$ NMR spectrum. The IR spectrum contained characteristic stretching bands for two carbonyls $\left(1780\right.$ and $\left.1707 \mathrm{~cm}^{-1}\right)$, and also an $\mathrm{OH}$ stretching band $\left(3347 \mathrm{~cm}^{-1}\right)$. The frequency of one of the $\mathrm{C}=\mathrm{O}$ stretches $\left(1780 \mathrm{~cm}^{-1}\right)$ was consistent with a $\gamma$-lactone. ${ }^{28}$ The presence of two carbonyls was confirmed by observation of $\mathrm{HMBC}$ correlations from several ${ }^{1} \mathrm{H}$ resonances to two deshielded ${ }^{13} \mathrm{C}$ resonances $\left(\delta_{\mathrm{C}} 179.2,176.4\right)$. Consistent with cadlinolide $\mathrm{C}(\mathbf{1 3 8})$, a tetrasubstituted olefin was determined from the observation of HMBC correlations to two deshielded ${ }^{13} \mathrm{C}$ resonances $\left(\delta_{\mathrm{C}} 145.8,127.3\right)$ that did not show any correlations in the HSQC-DEPT spectrum. With no further evidence of double bonds, the remaining degrees of unsaturation required $\mathbf{1 4 1}$ to have a tricyclic structure.

In a similar manner to cadlinolide D (139), some spectral overlap in the ${ }^{1} \mathrm{H}$ NMR spectrum precluded the unequivocal assignment of a 1,3,3-trimethylcyclohexyl ring using only COSY and HMBC correlations. As a consequence, 1D-TOCSY correlations were needed to confirm the connectivity of this ring. Analysis of these spectra allowed establishment of a 1,3,3-trimethylcyclohexyl ring [(C-1: $\left.\delta_{\mathrm{C}} 38.8, \delta_{\mathrm{H}} 2.24,1.32\right),(\mathrm{C}-2$ : $\left.\delta_{\mathrm{C}} 19.8, \delta_{\mathrm{H}} 1.59\right),\left(\mathrm{C}-3: \delta_{\mathrm{C}} 39.7, \delta_{\mathrm{H}} 1.40,1.24\right),\left(\mathrm{C}-4: \delta_{\mathrm{C}} 31.4\right),\left(\mathrm{C}-5: \delta_{\mathrm{C}} 50.3\right.$, $\left.\delta_{\mathrm{H}} 1.80,1.07\right),\left(\mathrm{C}-10: \delta_{\mathrm{C}} 41.6\right),\left(\mathrm{C}-18: \delta_{\mathrm{C}} 26.9, \delta_{\mathrm{H}} 0.91\right),\left(\mathrm{C}-19: \delta_{\mathrm{C}} 32.4, \delta_{\mathrm{H}} 0.92\right)$ and $\left.\left(\mathrm{C}-20: \delta_{\mathrm{C}} 30.1, \delta_{\mathrm{H}} 1.13\right)\right]$ (see table 4.4 and figure 4.9 ). 
COSY correlations between the ${ }^{1} \mathrm{H}$ resonances of various methylenes and methines were used to establish the second substructure of 141. In particular, a four-carbon chain consisting of two methylenes $\left[\left(\mathrm{C}-11: \delta_{\mathrm{C}} 26.3, \delta_{\mathrm{H}} 2.31,1.63\right)\right.$ and $\left(\mathrm{C}-12: \delta_{\mathrm{C}} 26.5\right.$, $\left.\left.\delta_{\mathrm{H}} 2.02,1.41\right)\right]$ adjacent to a pair of methines $\left[\left(\mathrm{C}-13: \delta_{\mathrm{C}} 40.3, \delta_{\mathrm{H}} 2.91\right)\right.$ and $(\mathrm{C}-14$ : $\left.\left.\delta_{\mathrm{C}} 43.7, \delta_{\mathrm{H}} 3.09\right)\right]$ was determined from observed COSY cross-peaks. Proton H-14 in turn correlated to an acetal centre $\left(\mathrm{C}-15: \delta_{\mathrm{C}} 110.3, \delta_{\mathrm{H}} 5.22\right)$. The ${ }^{1} \mathrm{H}$ resonance of acetal C-15 showed a strong HMBC correlation to a lactone carbonyl (C-16: $\left.\delta_{\mathrm{C}} 179.2\right)$ to finalise a $\gamma$-lactone ring. The deshielded chemical shift of $\mathrm{H}-13$, and the absence of further COSY correlations from it, is consistent with other $\gamma$-lactone rings (see figure 4.18$)^{28,137,140}$

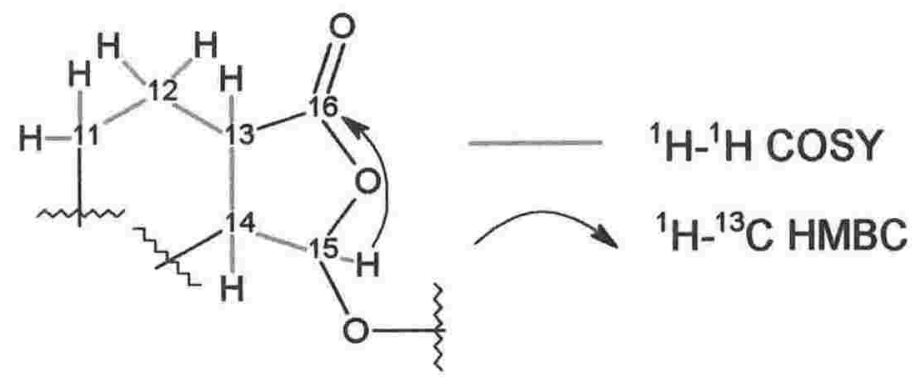

Figure 4.18 Establishment of $\gamma$-lactone substructure of 15-methoxypourewic acid B (141).

Finally, a strong COSY correlation was observed between a methyl (C-6: $\delta_{\mathrm{C}} 16.3$, $\left.\delta_{\mathrm{H}} 1.27\right)$ and a methine $\left(\mathrm{C}-7: \delta_{\mathrm{C}} 41.4, \delta_{\mathrm{H}} 4.32\right)$. Both $\mathrm{CH}_{3}-6$ and $\mathrm{H}-7$ also showed strong HMBC correlations to the ${ }^{13} \mathrm{C}$ resonances of each other, and also to that of the remaining carbonyl (C-17: $\left.\delta_{\mathrm{C}} 176.4\right)$ to establish the third substructure (see figure 4.5).

Again, the final connectivity of 141 was established on the basis of several key observed HMBC correlations to the two olefinic carbons. In particular, $\mathrm{CH}_{3}-6$ and $\mathrm{H}-7$ both correlated to an olefinic carbon (C-8: $\left.\delta_{C} 127.3\right)$ while $\mathrm{H}-7$ also correlated to $\mathrm{C}-14$, again indicating the attachment of the side chain at $\mathrm{C}-8$. The ${ }^{1} \mathrm{H}$ resonance of $\mathrm{C}-20$ 
showed a strong HMBC correlation to the remaining olefinic carbon (C-9: $\delta_{\mathrm{C}} 145.8$ ), establishing the connection of the 1,3,3-trimethylcyclohexyl ring to the olefin at C-9, whilst $\mathrm{H}-14$ correlated to carbon $\mathrm{C}-8$, helping to confirm the placement of the alkene. An HMBC correlation was observed from a methyl singlet $\left(\mathrm{C}-21: \delta_{\mathrm{C}} 56.7, \delta_{\mathrm{H}} 3.27\right)$ to C-15, establishing the methyl ether attachment at, and the acetal nature of, C-15 (see figure 4.19). The ${ }^{1} \mathrm{H}$ integration of methyl $\mathrm{C}-21$ was initially the cause of some concern, as it integrated for only two proton equivalents. This was due to a mixture of deuterated and non-deuterated molecules, as evidenced by the mass spectrum shown in figure 4.17. As all the degrees of unsaturation had been accounted for, $\mathrm{C}-17$ must be a carboxylic acid, consistent with the observation of an $\mathrm{OH}$ stretching band in the IR spectrum.

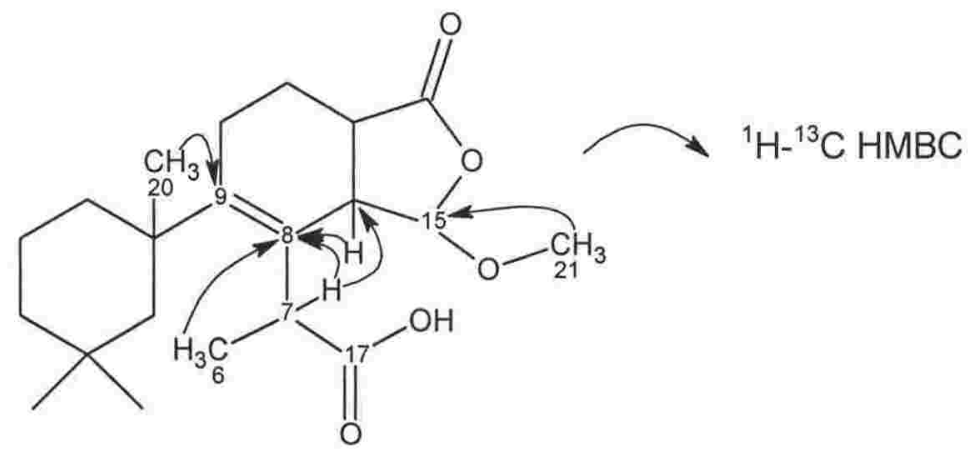

Figure 4.19 Establishment of the final connectivity of 15-methoxypourewic acid B (141).

The relative stereochemistry of 15-methoxypourewic acid B (141) was established on the basis of ${ }^{1} \mathrm{H}-{ }^{1} \mathrm{H}$ vicinal coupling constants and from NOE correlations observed in a ROESY experiment. A small dihedral angle between H-14 and H-15 was suggested by the small coupling constant observed between their ${ }^{1} \mathrm{H}$ resonances. An NOE correlation was observed between $\mathrm{H}-13$ and $\mathrm{H}-14$, indicating that these protons are on the same face of the molecule. $\mathrm{H}-14$ also correlated to the ${ }^{1} \mathrm{H}$ resonance of methyl C-6, implying that C-6 is on the same face as H-13, H-14, and H-15. This was supported by NOE correlations observed, from $\mathrm{H}-7$ to both $\mathrm{H}-1 \mathrm{~b}$ and $\mathrm{CH}_{3}-20$, and also from $\mathrm{H}-11 \mathrm{~b}$ to $\mathrm{H}-5 \mathrm{~b}$ 
and $\mathrm{CH}_{3}-20$ (see figure 4.20). These observations allowed the relative stereochemistry of 15-methoxypourewic acid $\mathrm{B}(141)$ to be assigned as $7 R^{*}, 10 S^{*}, 13 R^{*}, 14 R^{*}, 15 \mathrm{R}^{*}$.

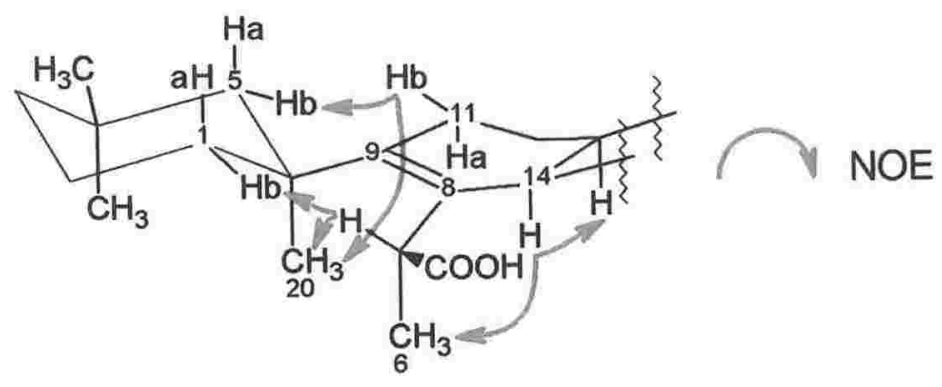

Figure 4.20 Establishment of the stereochemistry of 15-methoxypourewic acid B (141) 
Table 4.4 ${ }^{13} \mathrm{C}(75 \mathrm{MHz})$ and ${ }^{1} \mathrm{H}(300 \mathrm{MHz})$ NMR Data $\left(\mathrm{CDCl}_{3}+\mathrm{C}_{5} \mathrm{H}_{5} \mathrm{~N}\right)$ of $15-$ methoxypourewic acid B (141).

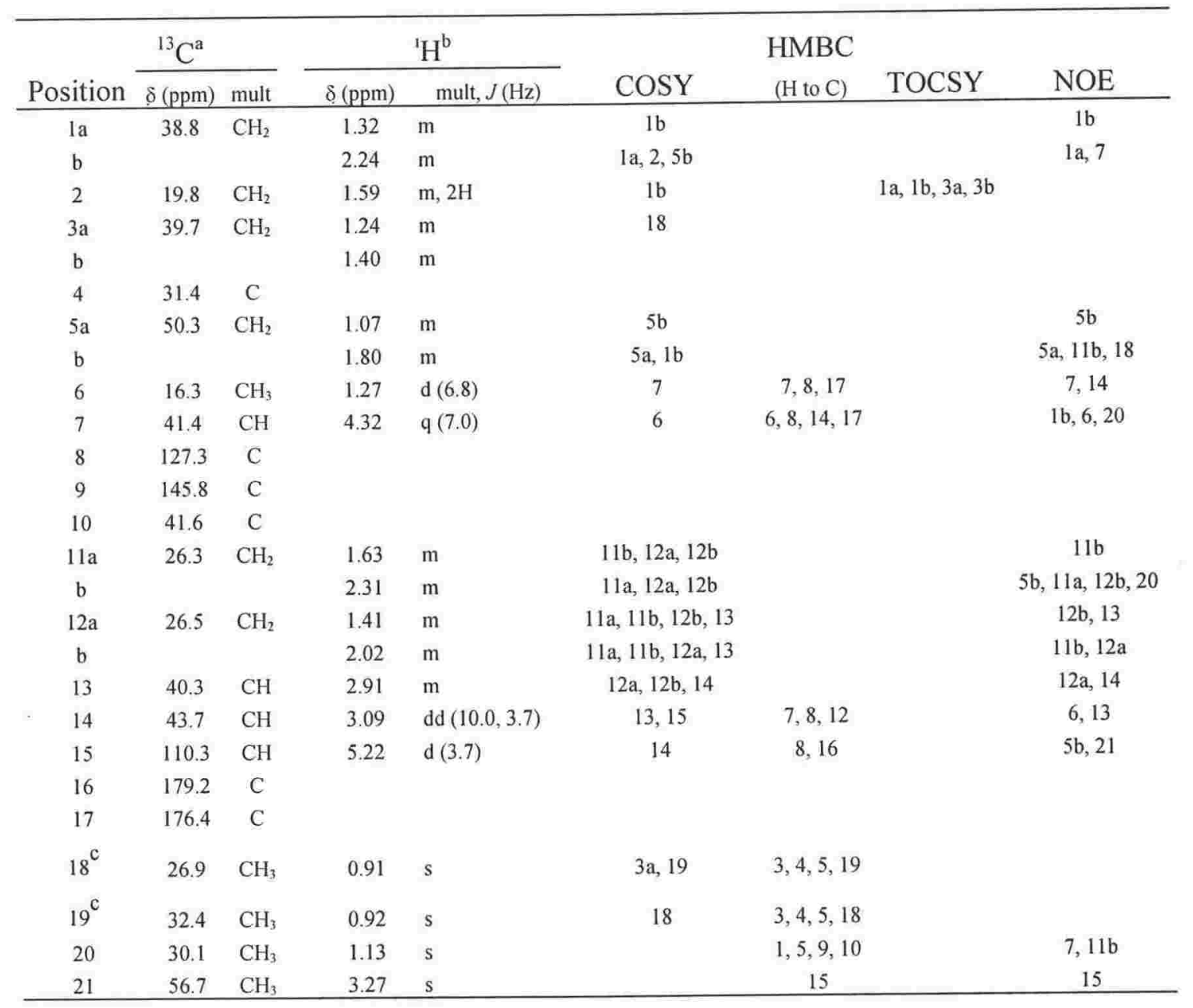

a Determined from HSQC-DEPT (multiplicity edited HSQC) and HMBC experiments.

b Carbon connectivity determined from an HSQC-DEPT experiment, multiplicity determined from ${ }^{1} \mathrm{H}$ experiment.

c Interchangeable.

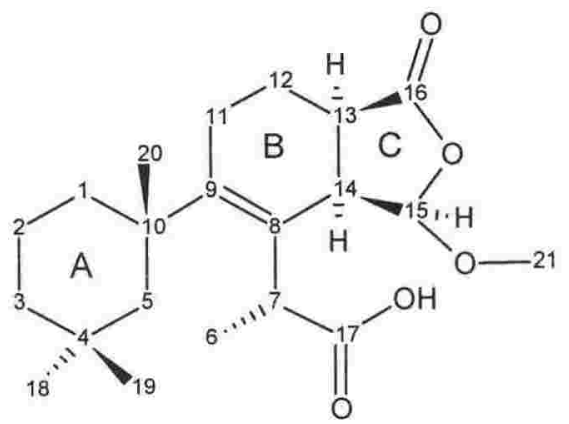

(141) 
The molecular formula of methylpourewate B (142) $(3.0 \mathrm{mg})$ was established as $\mathrm{C}_{21} \mathrm{H}_{32} \mathrm{O}_{5}$ from observation of intense pseudo-molecular ions in both the negative and positive ion HRESIMS modes $\left(365.2335[\mathrm{M}+\mathrm{H}]^{+}, \Delta 3.5 \mathrm{ppm} ; 363.2185[\mathrm{M}-\mathrm{H}]^{-}, \Delta 5.3\right.$ ppm), implying six degrees of unsaturation. All 21 carbons and 31 protons attached to carbon were observed in the ${ }^{13} \mathrm{C}$ and ${ }^{1} \mathrm{H}$ NMR spectra respectively. Prominent peaks in the IR spectrum included an $\mathrm{OH}$ stretch $\left(3325 \mathrm{~cm}^{-1}\right)$ and two $\mathrm{C}=\mathrm{O}$ stretches $(1762,1735$ $\left.\mathrm{cm}^{-1}\right)$. Significant ${ }^{13} \mathrm{C}$ NMR signals included two carbonyl $\left(\delta_{\mathrm{C}} 178.9,175.3\right)$, one hemiacetal $\left(\delta_{C} 103.8\right)$ and two tetrasubstituted olefinic $\left(\delta_{C} 147.2,127.3\right)$ resonances. With no other evidence of multiple bonds, the remaining degrees of unsaturation required $\mathbf{1 4 2}$ to have a tricyclic structure.

The presence of a 1,3,3-trimethylcyclohexyl in $\mathbf{1 4 2}$ was established based upon a similar analysis of the COSY and HMBC spectra as that performed for cadlinolide C (138) $\left[\left(\mathrm{C}-1: \delta_{\mathrm{C}} 39.0, \delta_{\mathrm{H}} 2.18,1.29\right),\left(\mathrm{C}-2: \delta_{\mathrm{C}} 19.8, \delta_{\mathrm{H}} 1.83,1.51\right),\left(\mathrm{C}-3: \delta_{\mathrm{C}} 39.9\right.\right.$, $\left.\delta_{\mathrm{H}} 1.36,1.21\right),\left(\mathrm{C}-4: \delta_{\mathrm{C}} 31.6\right),\left(\mathrm{C}-5: \delta_{\mathrm{C}} 50.6, \delta_{\mathrm{H}} 1.77,1.00\right),\left(\mathrm{C}-10: \delta_{\mathrm{C}} 42.1\right),(\mathrm{C}-18:$ $\left.\left.\delta_{\mathrm{C}} 26.8, \delta_{\mathrm{H}} 0.85\right),\left(\mathrm{C}-19: \delta_{\mathrm{C}} 32.9, \delta_{\mathrm{H}} 0.87\right),\left(\mathrm{C}-20: \delta_{\mathrm{C}} 30.6, \delta_{\mathrm{H}} 1.05\right)\right]$ (see table 4.5 and figure 4.3).

COSY correlations were used to establish a linear chain of three carbons $[(\mathrm{C}-11$ : $\left.\delta_{\mathrm{C}} 26.8, \delta_{\mathrm{H}} 2.34,1.47\right)$ to methylene $\left(\mathrm{C}-12: \delta_{\mathrm{C}} 27.5, \delta_{\mathrm{H}} 2.09,1.30\right)$ to methine $(\mathrm{C}-13$ : $\left.\left.\delta_{\mathrm{C}} 41.2, \delta_{\mathrm{H}} 2.97\right)\right]$. Initially, the resonance of $\mathrm{H}-13$ overlapped with that of another methine proton at the same frequency. Addition of a small amount of $\mathrm{D}_{5}-\mathrm{C}_{5} \mathrm{H}_{5} \mathrm{~N}$ caused these two overlapping resonances to spread apart. It was found that the two resonances coupled to each other, as evidenced by a resolved COSY cross-peak between them, 
revealing the connection between $\mathrm{C}-13$ and a second methine $\left(\mathrm{C}-14: \delta_{\mathrm{C}} 45.8, \delta_{\mathrm{H}} 2.97\right)$.

A COSY cross-peak was also observed between $\mathrm{H}-14$ and a hemi-acetal resonance (C-15: $\left.\delta_{\mathrm{C}} 103.8, \delta_{\mathrm{H}} 5.41\right)$. Proton $\mathrm{H}-15$ showed HMBC correlations to $\mathrm{C}-14$ and also to that of a $\gamma$-lactone carbonyl [(C-16: $\left.\left.\delta_{\mathrm{C}} 178.9\right)\right]$. As with 141, the deshielded chemical shift of, and the absence of any other couplings from, H-13 supports the proposal of a $\gamma$-lactone ring (see figure 4.18).

Finally, a strong COSY correlation was observed between the ${ }^{1} \mathrm{H}$ resonances of a methyl (C-6: $\left.\delta_{\mathrm{C}} 16.6, \delta_{\mathrm{H}} 1.21\right)$ and a methine $\left(\mathrm{C}-7: \delta_{\mathrm{C}} 41.5, \delta_{\mathrm{H}} 4.30\right)$. Both $\mathrm{CH}_{3}-6$ and $\mathrm{H}-7$ showed strong $\mathrm{HMBC}$ correlations to the ${ }^{13} \mathrm{C}$ resonances of each other and also to that of a carbonyl $\left(\mathrm{C}-17\right.$ : $\left.\delta_{\mathrm{C}} 175.3\right)$. A strong $\mathrm{HMBC}$ correlation was also observed from an oxygenated methyl $\left(\mathrm{C}-21: \delta_{\mathrm{C}} 52.1, \delta_{\mathrm{H}} 3.71\right)$ to $\mathrm{C}-17$, implying a methyl-ester functionality (see figure 4.21). The chemical shifts observed are comparable to those noted for the methyl ester derivative of pourewic acid A (140), and for membranolide (128). ${ }^{151}$

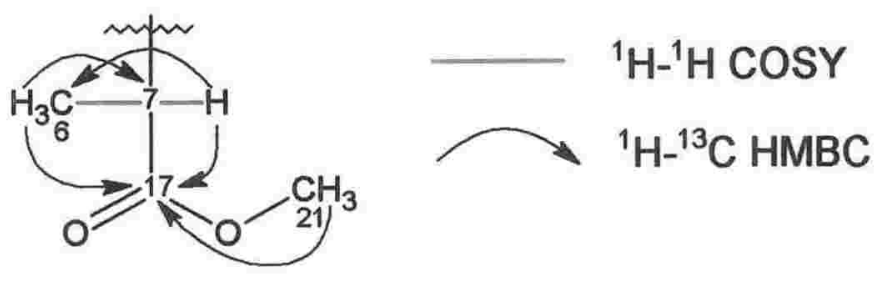

Figure 4.21 Establishment of the side chain of methylpourewate B (142).

All of the correlations already mentioned had accounted for all the carbons in the molecule except two substituted olefinic centres [(C-8: $\left.\delta_{\mathrm{C}} 127.3\right)$, (C-9: $\left.\left.\delta_{\mathrm{C}} 147.2\right)\right]$. As before, the placement of the olefin and the final connectivity of the molecule were established from key observed COSY and HMBC correlations. Strong HMBC correlations from $\mathrm{H}-7$ to $\mathrm{C}-8, \mathrm{C}-9$, and $\mathrm{C}-14$, and from $\mathrm{CH}_{3}-6$ to $\mathrm{C}-8$, established the connection of the side chain at C-8 between C-9 and C-14. As with cadlinolide C (138), 
$\mathrm{CH}_{3}-20$ also showed a strong correlation to C-9, confirming the attachment of the 1,3,3-trimethylcyclohexyl moiety at C-9, and established the final connectivity of the molecule. The connection of the olefin within a six-membered ring was confirmed by a homo-allylic coupling between $\mathrm{H}-11 \mathrm{~b}$ and $\mathrm{H}-14$ as observed in the COSY spectrum (see figure 4.22).

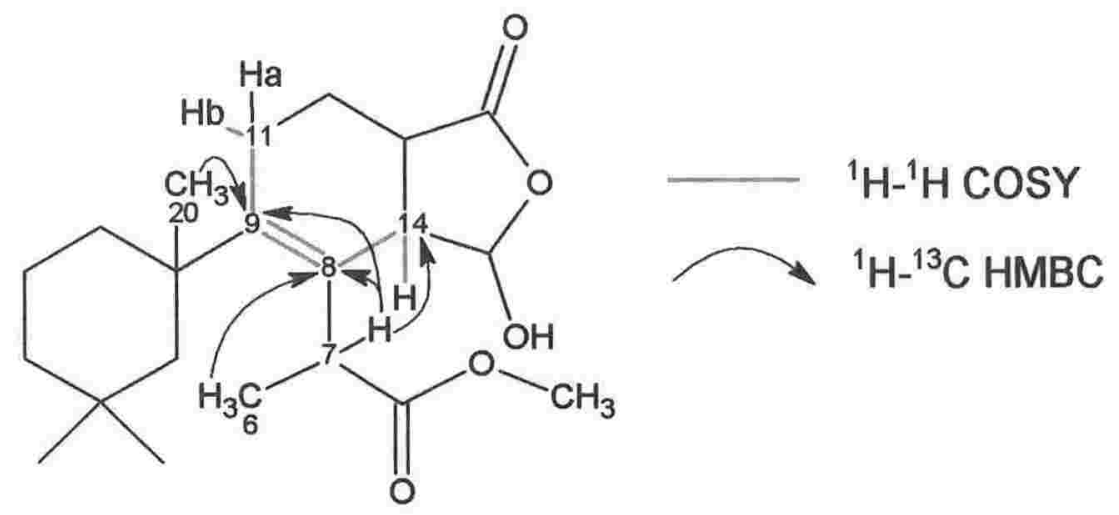

Figure 4.22 Selected correlations establishing the final connectivity of methylpourewate B (142).

The stereochemistry of methylpourewate B (142) was determined from both ${ }^{1} \mathrm{H}-{ }^{1} \mathrm{H}$ vicinal coupling constants, measured in the ${ }^{1} \mathrm{H}$ NMR spectrum, and NOE correlations, detected in a ROESY experiment. As with $\mathbf{1 3 8}, \mathrm{H}-15$ shows a small coupling to H-14, consistent with a small dihedral angle between these protons. Due to overlap of their close chemical shifts, no reliable NOE could be detected between $\mathrm{H}-13$ and $\mathrm{H}-14$. The coupling constant between these two protons, however, is similar in magnitude to those measured for 138 , therefore it is assumed that the stereochemical relationship between the two protons is the same. Several of the NOE correlations used to determine the relative stereochemistry of $\mathbf{1 3 8}$ were absent in $\mathbf{1 4 2}$, precluding a similar stereochemical analysis. Although none were observed between $\mathrm{H}-5 \mathrm{a}$ and $\mathrm{H}-11 \mathrm{~b}, \mathrm{NOE}$ correlations were observed between $\mathrm{CH}_{3}-6$ and both $\mathrm{H}-14$ and $\mathrm{CH}_{3}-20$, and also between $\mathrm{H}-7$ and both $\mathrm{H}-1 \mathrm{~b}$ and $\mathrm{CH}_{3}-20$, in a similar manner to that observed for 138 , therefore the stereochemistry for C-7 and C-10 is assumed to be the same. These observations and 
assumptions allow the relative stereochemistry of methylpourewate B (142) to be tentatively assigned as: $7 R^{*}, 10 S^{*}, 13 R^{*}, 14 S^{*}, 15 R^{*}$ (see figure 4.23 ).

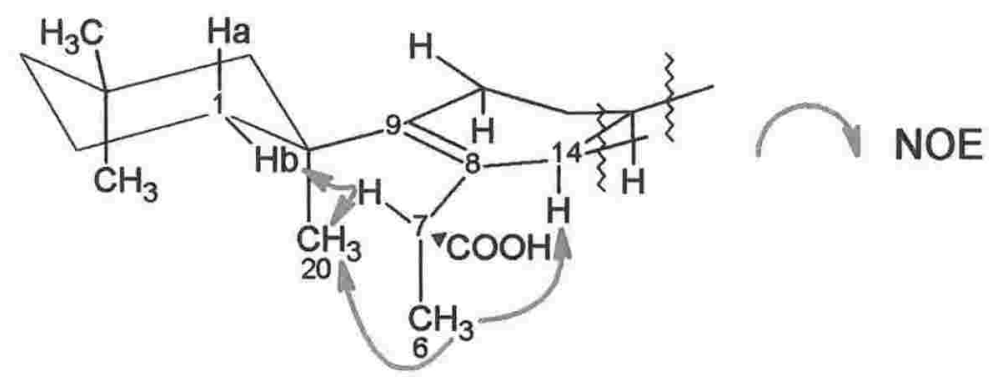

Figure 4.23 NOE correlations establishing the stereochemistry of methylpourewate B (142) 
Table $4.5{ }^{13} \mathrm{C}(75 \mathrm{MHz})$ and ${ }^{1} \mathrm{H}(300 \mathrm{MHz})$ NMR Data $\left(\mathrm{CDCl}_{3}\right)$ of methylpourewate $\mathrm{B}(\mathbf{1 4 2})$.

\begin{tabular}{|c|c|c|c|c|c|c|c|}
\hline \multirow[b]{2}{*}{ Position } & \multicolumn{2}{|c|}{${ }^{13} \mathrm{C}^{\mathrm{a}}$} & \multicolumn{2}{|r|}{${ }^{1} \mathrm{H}^{\mathrm{b}}$} & \multirow{2}{*}{\multicolumn{2}{|c|}{$\begin{array}{c}\text { HMBC } \\
\text { (H to } \mathrm{C} \text { ) }\end{array}$}} & \multirow[b]{2}{*}{ NOE } \\
\hline & $\delta(\mathrm{ppm})$ & mult & $\delta(\mathrm{ppm})$ & mult, $J(\mathrm{~Hz})$ & & & \\
\hline la & 39.0 & $\mathrm{CH}_{2}$ & 1.29 & $\mathrm{~m}$ & $1 \mathrm{~b}$ & & \\
\hline $\mathrm{b}$ & & & 2.18 & $\mathrm{~m}$ & $1 \mathrm{a}, 2 \mathrm{a}, 2 \mathrm{~b}$ & & $1 \mathrm{~b}, 3 \mathrm{a}, 7$ \\
\hline $2 b$ & 19.8 & $\mathrm{CH}_{2}$ & 1.51 & $\mathrm{~m}$ & $1 \mathrm{~b}, 2 \mathrm{a}, 3 \mathrm{a}$ & & $11 \mathrm{~b}, 12 \mathrm{~b}$ \\
\hline $\mathrm{a}$ & & & 1,83 & $\mathrm{~m}$ & $1 b, 2 b, 3 a, 3 b$ & & $11 \mathrm{~b}$ \\
\hline $3 a$ & 39.9 & $\mathrm{CH}_{2}$ & 1.36 & $\mathrm{~m}$ & $2 \mathrm{a}, 2 \mathrm{~b}$ & & \\
\hline $\mathrm{b}$ & & & 1.21 & $\mathrm{~m}$ & $2 a, 18$ & $1,4,5$ & $5 a$ \\
\hline 4 & 31.6 & $\mathrm{C}$ & & & & & \\
\hline $5 \mathrm{a}$ & 50.6 & $\mathrm{CH}_{2}$ & 1.00 & $\mathrm{~m}$ & $5 b$ & $1,4,18$ & $3 \mathrm{~b}, 18$ \\
\hline $\mathrm{b}$ & & & 1.77 & $\mathrm{~m}$ & $5 \mathrm{a}$ & & \\
\hline 6 & 16.6 & $\mathrm{CH}_{3}$ & 1.21 & $\mathrm{~d}(6.8)$ & 7 & $7,8,17$ & 14,20 \\
\hline 7 & 41.5 & $\mathrm{CH}$ & 4.30 & $\mathrm{q}(7.1)$ & 6 & $6,8,9,14,17$ & $1 \mathrm{~b}, 20$ \\
\hline 8 & 127.3 & $\mathrm{C}$ & & & & & \\
\hline 9 & 147.2 & C & & & & & \\
\hline 10 & 42.1 & $\mathrm{C}$ & & & & & \\
\hline 11a (ax) & 26.8 & $\mathrm{CH}_{2}$ & 1.47 & $\mathrm{~m}$ & $11 \mathrm{~b}, 12 \mathrm{a}, 12 \mathrm{~b}$ & & $11 b, 12 b$ \\
\hline b (eq) & & & 2.34 & $\mathrm{dt}(15.9,2.9)$ & $11 \mathrm{a}, 12 \mathrm{a}, 12 \mathrm{~b}, 14$ & 12 & $2 a, 2 b, 11 a, 12 b, 20$ \\
\hline $12 \mathrm{a}(\mathrm{ax})$ & 27.5 & $\mathrm{CH}_{2}$ & 1.30 & $\mathrm{~m}$ & $11 \mathrm{a}, 11 \mathrm{~b}, 13$ & & $12 \mathrm{~b}$ \\
\hline $\mathrm{b}(\mathrm{eq})$ & & & 2.09 & $\mathrm{~m}$ & $11 \mathrm{a}, 11 \mathrm{~b}, 13$ & & $2 \mathrm{~b}, 11 \mathrm{a}, 11 \mathrm{~b}, 12 \mathrm{a}$ \\
\hline 13 & 41.2 & $\mathrm{CH}$ & 2.97 & $q(8.6)$ & 14 & & \\
\hline 14 & 45.8 & $\mathrm{CH}$ & 2.97 & $\mathrm{dd}(10.0,3.9)$ & $12 \mathrm{a}, 12 \mathrm{~b}, 11 \mathrm{~b}, 13,15$ & $8,13,15$ & 6 \\
\hline 15 & 103.8 & $\mathrm{CH}$ & 5.41 & $\mathrm{~d}(3.3)$ & 14 & 14,16 & \\
\hline 16 & 178.9 & $\mathrm{C}$ & & & & & \\
\hline 17 & 175.3 & $\mathrm{C}$ & & & & & \\
\hline $18^{\mathrm{c}}$ & 26.8 & $\mathrm{CH}_{3}$ & 0.85 & s & $3 b, 18$ & $3,4,5,19$ & \\
\hline $19^{\mathrm{c}}$ & 32.9 & $\mathrm{CH}_{3}$ & 0.87 & s & 18 & $3,4,5,18$ & \\
\hline 20 & 30.6 & $\mathrm{CH}_{3}$ & 1.05 & s & & $1,5,9,10$ & $6,11 b$ \\
\hline 21 & 52.1 & $\mathrm{CH}_{3}$ & 3.71 & s & & 17 & \\
\hline
\end{tabular}

a Multiplicity determined from HSQC-DEPT (multiplicity edited HSQC) experiment.

b Carbon connectivity determined from an HSQC-DEPT experiment, multiplicity determined from ${ }^{1} \mathrm{H}$ experiment.

c Interchangeable.

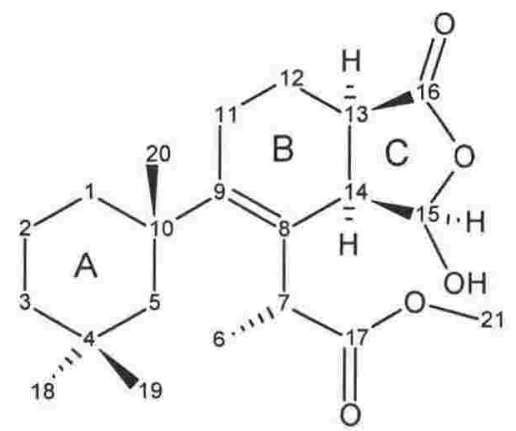

(142) 
Strong pseudo-molecular ions were observed for pourewanone (143) (2.2 $\mathrm{mg}$ ) in both positive and negative ion HRESIMS experiments, establishing a molecular formula of $\mathrm{C}_{20} \mathrm{H}_{30} \mathrm{O}_{5}\left(351.2160[\mathrm{M}+\mathrm{H}]^{+}, \Delta 1.8 \mathrm{ppm}, 718.4527\left[2 \mathrm{M}+\mathrm{NH}_{4}\right]^{+}, \Delta 0.3 \mathrm{ppm}, 349.2019\right.$ $[\mathrm{M}-\mathrm{H}] ; \Delta 0.5 \mathrm{ppm}$ ) and requiring six degrees of unsaturation. All 20 carbons and 29 protons attached to carbon were observed in the ${ }^{13} \mathrm{C}$ and ${ }^{1} \mathrm{H}$ NMR spectra. Notable features of the IR spectrum included an $\mathrm{OH}$ stretch $\left(3335 \mathrm{~cm}^{-1}\right)$, and both a broad and a narrow $\mathrm{C}=\mathrm{O}$ stretch $\left(1725,1665 \mathrm{~cm}^{-1}\right.$ respectively). The band at $1665 \mathrm{~cm}^{-1}$ was suitable for an $\alpha, \beta$-unsaturated carbonyl. ${ }^{160}$ A single peak was noted in the UV spectrum $\left(\lambda_{\max }=248 \mathrm{~nm}, \varepsilon_{\max }=14,425\right)$, characteristic of a $\alpha, \beta, \beta$-saturated-enone system. ${ }^{160}$ The ${ }^{13} \mathrm{C}$ NMR spectrum of $\mathbf{1 4 3}$ was quite different from any of the other diterpenes isolated during this study. Notable ${ }^{13} \mathrm{C}$ resonances included those of three carbonyls $\left(\delta_{\mathrm{C}} 198.1\right.$, $177.2,161.0)$, a polarised olefin $\left(\delta_{C} 167.3,135.6\right)$, and an oxygenated methylene $\left(\delta_{C}\right.$ 62.9). The ${ }^{13} \mathrm{C}$ resonance at $161.0 \mathrm{ppm}$ was assigned as that of a carbonyl, and not as part of a polarised olefin, by the following reasoning. Attachment of a proton at 8.06 ppm was determined from a correlation in the HSQC-DEPT spectrum. The ${ }^{1} J_{\mathrm{C}, \mathrm{H}}$ coupling constant between these resonances was found to be $225 \mathrm{~Hz}$ when measured in a ${ }^{1} \mathrm{H}$ coupled HSQC-DEPT experiment. A ${ }^{1} J_{\mathrm{C}, \mathrm{H}}$ coupling constant of this magnitude is diagnostic of formate functionality, which was also consistent with both the ${ }^{1} \mathrm{H}$ and ${ }^{13} \mathrm{C}$ chemical shifts. ${ }^{161-164}$ Initially, observation of an HSQC-DEPT correlation between the ${ }^{1} \mathrm{H}$ and ${ }^{13} \mathrm{C}$ resonances of the formate was difficult, as the signal was weak due to the inappropriate ${ }^{1} J_{\mathrm{C}, \mathrm{H}}$ value $(140 \mathrm{~Hz})$ used when running the experiment. Once this value had been optimised $\left({ }^{1} J_{\mathrm{C}, \mathrm{H}}=190 \mathrm{~Hz}\right)$, the strength of the formate HSQC-DEPT correlation was enhanced, allowing for better detection. 
As with all the previous related metabolites, careful analysis of the COSY and HMBC spectra allowed the rapid assignment of a 1,3,3-trimethylcyclohexyl moiety [(C-1: $\left.\delta_{\mathrm{C}} 38.3, \delta_{\mathrm{H}} 1.96,1.29\right),\left(\mathrm{C}-2: \delta_{\mathrm{C}} 20.5, \delta_{\mathrm{H}} 1.61\right),\left(\mathrm{C}-3: \delta_{\mathrm{C}} 39.5, \delta_{\mathrm{H}} 1.28\right),\left(\mathrm{C}-4: \delta_{\mathrm{C}} 31.8\right)$, $\left(\mathrm{C}-5: \delta_{\mathrm{C}} 49.6, \delta_{\mathrm{H}} 1.84,1.38\right),\left(\mathrm{C}-10: \delta_{\mathrm{C}} 41.0\right),\left(\mathrm{C}-18: \delta_{\mathrm{C}} 29.0, \delta_{\mathrm{H}} 0.83\right),\left(\mathrm{C}-19: \delta_{\mathrm{C}} 31.4\right.$, $\left.\left.\delta_{\mathrm{H}} 0.95\right),\left(\mathrm{C}-20: \delta_{\mathrm{C}} 28.8, \delta_{\mathrm{H}} 1.28\right)\right]$ for $\mathbf{1 4 3}$ (see table 4.6 and figure 4.3 ).

A linear chain of three methylenes and a methine was established on the basis of observed COSY correlations. The protons of a methylene (C-11: $\left.\delta_{\mathrm{C}} 27.7, \delta_{\mathrm{H}} 2.72,2.63\right)$ were strongly coupled to the protons of another methylene (C-12: $\delta_{C} 25.1$, $\left.\delta_{\mathrm{H}} 2.11,1.74\right)$. These in turn showed strong COSY correlations to a methine (C-13: $\left.\delta_{\mathrm{C}} 44.7, \delta_{\mathrm{H}} 2.56\right)$, which was coupled to an oxygenated methylene pair (C-15: $\delta_{\mathrm{C}} 62.9$, $\delta_{\mathrm{H}} 4.49,4.26$ ) (see figure 4.24$)$.

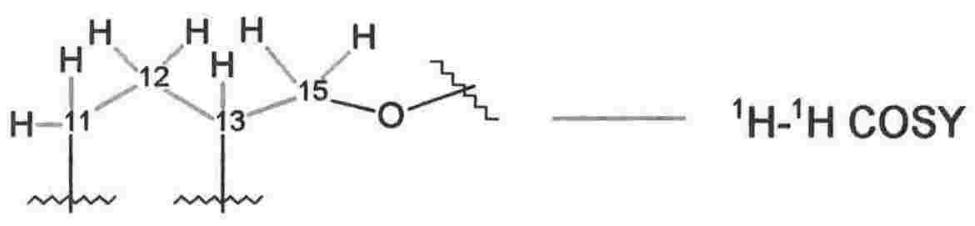

Figure 4.24 Establishment of the methylene chain substructure of pourewanone (143).

The final COSY correlations observed were between a methyl doublet (C-6: $\delta_{\mathrm{C}} 15.0$, $\left.\delta_{\mathrm{H}} 1.37\right)$ and a methine $\left(\mathrm{C}-7: \delta_{\mathrm{C}} 39.0, \delta_{\mathrm{H}} 3.84\right)$. As before, both $\mathrm{CH}_{3}-6$ and $\mathrm{H}-7$ showed $\mathrm{HMBC}$ correlations to the ${ }^{13} \mathrm{C}$ resonances of each other, and to that of a carbonyl (C-17: $\delta_{\mathrm{C}}$ 177.1) (see figure 4.5).

The COSY and HMBC correlations detailed above had accounted for 16 of the 20 carbons of 143 , leaving a polarised olefin, a carbonyl, and a formate, to be assigned to define the final connectivity of the metabolite. The final connectivity of the molecule 
was achieved after analysis of several key $\mathrm{HMBC}$ correlations. Both $\mathrm{CH}_{3}-6$ and $\mathrm{H}-7$ showed strong HMBC correlations to the two polarised olefinic carbons [(C-9: $\left.\delta_{C} 167.3\right)$ and $\left.\left(\mathrm{C}-8: \delta_{\mathrm{C}} 135.6\right)\right]$, indicating the attachment of the side chain at C-8. As previously, $\mathrm{CH}_{3}-20$ showed a strong correlation to $\mathrm{C}-9$, linking the 1,3,3-trimethylcyclohexyl ring with the substituted olefin at $\mathrm{C}-9$. The ${ }^{1} \mathrm{H}$ resonances of methine $\mathrm{C}-7$, and methylenes C-13 and C-15, all showed strong HMBC correlations to a ketone carbonyl (C-14: $\left.\delta_{\mathrm{C}} 198.1\right)$, establishing the position of the carbonyl within the enone system suggested by the UV spectrum. Finally, the ${ }^{1} \mathrm{H}$ resonances of $\mathrm{C}-15$ correlated to the formate carbonyl $\left(\mathrm{C}-16: \delta_{\mathrm{C}} 161.0, \delta_{\mathrm{H}} 8.08\right)$, the ${ }^{1} \mathrm{H}$ resonance of which correlated back to $\mathrm{C}-15$ (see figure 4.25 ) to establish the attachment of the formate at $\mathrm{C}-15$. This is, to the best of the author's knowledge, the first formate fully characterised from the marine environment.

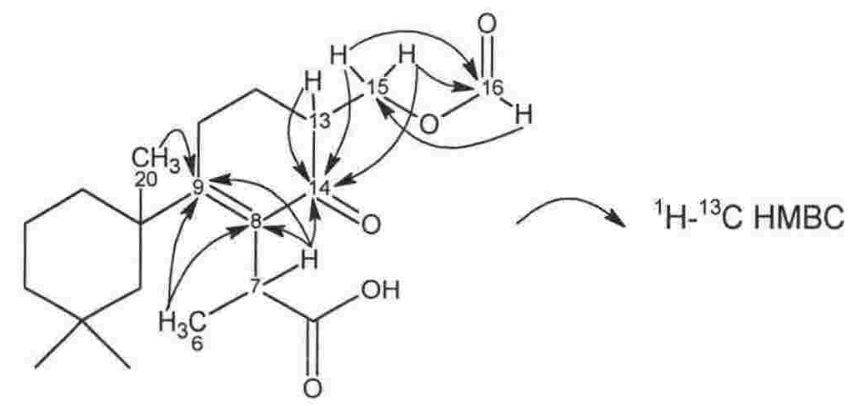

Figure 4.25 Establishment of the final connectivity of pourewanone (143).

The obvious proximity of ketone C-14 to the tetrasubstituted olefin of $\mathbf{1 4 3}$ is the cause of both the UV chromophore and the polarisation of the double bond. The proximity of the enone system also accounts for the change in resonant frequency of $\mathrm{H}-7$ from that found in the other diterpenes isolated in this study. As the three stereogenic centres of 143 are quite isolated from each other, no obvious conclusions can be drawn from any NOE correlations observed. The stereochemistry of C-7, C-10 and C-13 is assumed to be the same as the other metabolites isolated, however, and therefore the three 
stereogenic centres of pourewanone $(\mathbf{1 4 3})$ are tentatively assigned as $7 R^{*}, 10 S^{*}, 13 S^{*}$ consistent with all other metabolites isolated during this study. 
Table 4.6 ${ }^{13} \mathrm{C}(100 \mathrm{MHz})$ and ${ }^{1} \mathrm{H}(300 \mathrm{MHz})$ NMR Data $\left(\mathrm{CDCl}_{3}\right)$ of pourewanone (143).

\begin{tabular}{|c|c|c|c|c|c|c|}
\hline \multirow[b]{2}{*}{ Position } & \multicolumn{2}{|c|}{${ }^{13} C^{a}$} & \multicolumn{2}{|c|}{${ }^{\mathrm{t}} \mathrm{H}^{\mathrm{b}}$} & \multirow[b]{2}{*}{ COSY } & \multirow{2}{*}{$\begin{array}{c}\text { HMBC } \\
\text { (H to C) }\end{array}$} \\
\hline & $\delta(\mathrm{ppm})$ & mult & $\delta(\mathrm{ppm})$ & mult, $J(\mathrm{~Hz})$ & & \\
\hline la & 38.3 & $\mathrm{CH}_{2}$ & 1.29 & $\mathrm{~m}$ & $1 b, 2$ & \\
\hline $\mathrm{b}$ & & & 1.96 & $\mathrm{~m}$ & 1a, 2 & \\
\hline 2 & 20.5 & $\mathrm{CH}_{2}$ & 1.61 & $\mathrm{~m} .2 \mathrm{H}$ & $1 \mathrm{a}, 1 \mathrm{~b}, 3$ & 4,10 \\
\hline 3 & 39.5 & $\mathrm{CH}_{2}$ & 1.28 & $\mathrm{~m}, 2 \mathrm{H}$ & 2 & 18 \\
\hline 4 & 31.8 & $\mathrm{C}$ & & & & \\
\hline $5 \mathrm{a}$ & 49.6 & $\mathrm{CH}_{2}$ & 1.38 & $\mathrm{~m}$ & $5 b, 18$ & 10,18 \\
\hline$b$ & & & 1.84 & $\mathrm{~m}$ & $5 a$ & 10 \\
\hline 6 & 15.0 & $\mathrm{CH}_{3}$ & 1.37 & $\mathrm{~d}(6.8)$ & 7 & $7,8,9,17$ \\
\hline 7 & 39.0 & $\mathrm{CH}$ & 3.84 & $q(6.8)$ & 6 & $6,8,9,14,17$ \\
\hline 8 & 135.6 & $\mathrm{C}$ & & & & \\
\hline 9 & 167.3 & C & & & & \\
\hline 10 & 41.0 & $\mathrm{C}$ & & & & \\
\hline $11 \mathrm{a}$ & 27.7 & $\mathrm{CH}_{2}$ & 2.63 & $\mathrm{dd}(10.3,4.6)$ & $11 \mathrm{~b}, 12 \mathrm{a}, 12 \mathrm{~b}$ & 8 \\
\hline $\mathrm{b}$ & & & 2.72 & $\mathrm{t}(3.9)$ & $11 \mathrm{a}, 12 \mathrm{a}, 12 \mathrm{~b}$ & \\
\hline $12 \mathrm{a}$ & 25.1 & $\mathrm{CH}_{2}$ & 1.74 & $\mathrm{~m}$ & $11 \mathrm{a}, 11 \mathrm{~b}, 12 \mathrm{~b}, 13$ & 13 \\
\hline $\mathrm{b}$ & & & 2.11 & $\mathrm{~m}$ & $11 \mathrm{a}, 11 \mathrm{~b}, 12 \mathrm{a}, 13$ & 13 \\
\hline 13 & 44.7 & $\mathrm{CH}$ & 2.56 & $\mathrm{~m}$ & $12 \mathrm{a}, 12 \mathrm{~b}, 15 \mathrm{a}, 15 \mathrm{~b}$ & 14 \\
\hline 14 & 198.1 & $\mathrm{C}$ & & & & \\
\hline $15 \mathrm{a}$ & 62.9 & $\mathrm{CH}_{2}$ & 4.26 & $\mathrm{dd}(11.5,7.1)$ & $13,15 b, 16$ & $12,13,14,16$ \\
\hline $\mathrm{b}$ & & & 4.49 & $\mathrm{dd}(11.2,4.1)$ & $13,15 \mathrm{a}, 16$ & $12,13,14,16$ \\
\hline 16 & 161.0 & $\mathrm{COH}$ & 8.08 & $\mathrm{~s}$ & $15 \mathrm{a}, 15 \mathrm{~b}$ & 15 \\
\hline 17 & 177.1 & C & & & & \\
\hline $18^{\mathrm{c}}$ & 29.0 & $\mathrm{CH}_{3}$ & 0.83 & $\mathrm{~s}$ & 5a, 19 & $3,4,5,19$ \\
\hline $19^{\mathrm{c}}$ & 31.4 & $\mathrm{CH}_{3}$ & 0.95 & s & 18 & $3,4,5,18$ \\
\hline 20 & 28.8 & $\mathrm{CH}_{3}$ & 1.28 & $s$ & & $1,5,9,10$ \\
\hline
\end{tabular}

a Multiplicity determined from HSQC-DEPT (multiplicity edited HSQC) experiment.

b Carbon connectivity determined from an HSQC-DEPT experiment, multiplicity determined from ${ }^{1} \mathrm{H}$ experiment.

c Interchangeable.

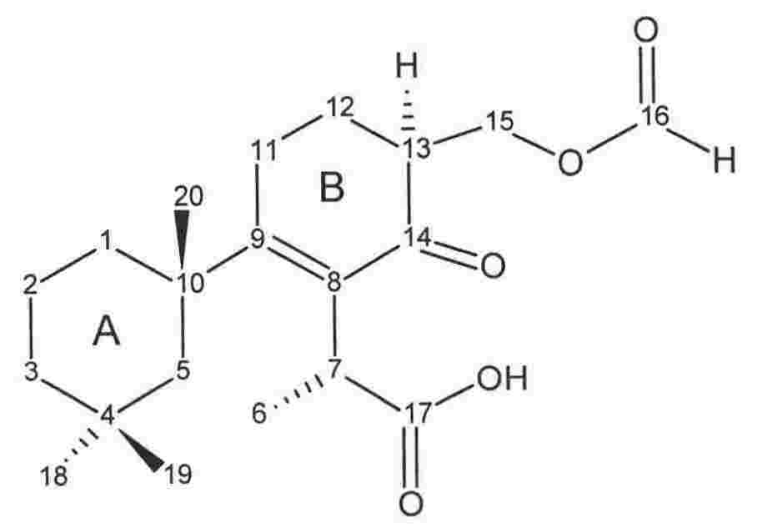

(143) 
Several of the spongian diterpenes isolated as part of this study are of biogenetic significance. They are either suggested intermediates in previously proposed biosynthetic pathways, or they represent a new carbon skeleton that is formed via a new mechanism. Each group of metabolites will be dealt with in turn.

Cadlinolides A-D $(\mathbf{1 3 2}-133, \mathbf{1 3 8}-139)$ are structurally related to the gracilin-class of compounds (118-123) and are therefore presumably formed via a similar mechanism to that shown in scheme 4.7. ${ }^{140,148-150}$ Key steps of the biogenesis are the 1,2-hydride and 1,2-methyl shifts, followed by the opening of the epoxide ring to form an alcohol. The C-5 C-6 bond is then oxidatively cleaved to form a carboxylic acid. Instead of undergoing a decarboxylation as proposed for the gracilins, however, the C-17 carboxylic acid would undergo a lactonisation with a C-15 alcohol. The four members of the class are formed by various oxidations and functionalisations of the five-membered heterocyclic ring. This mechanism is summarised in scheme 4.11. 

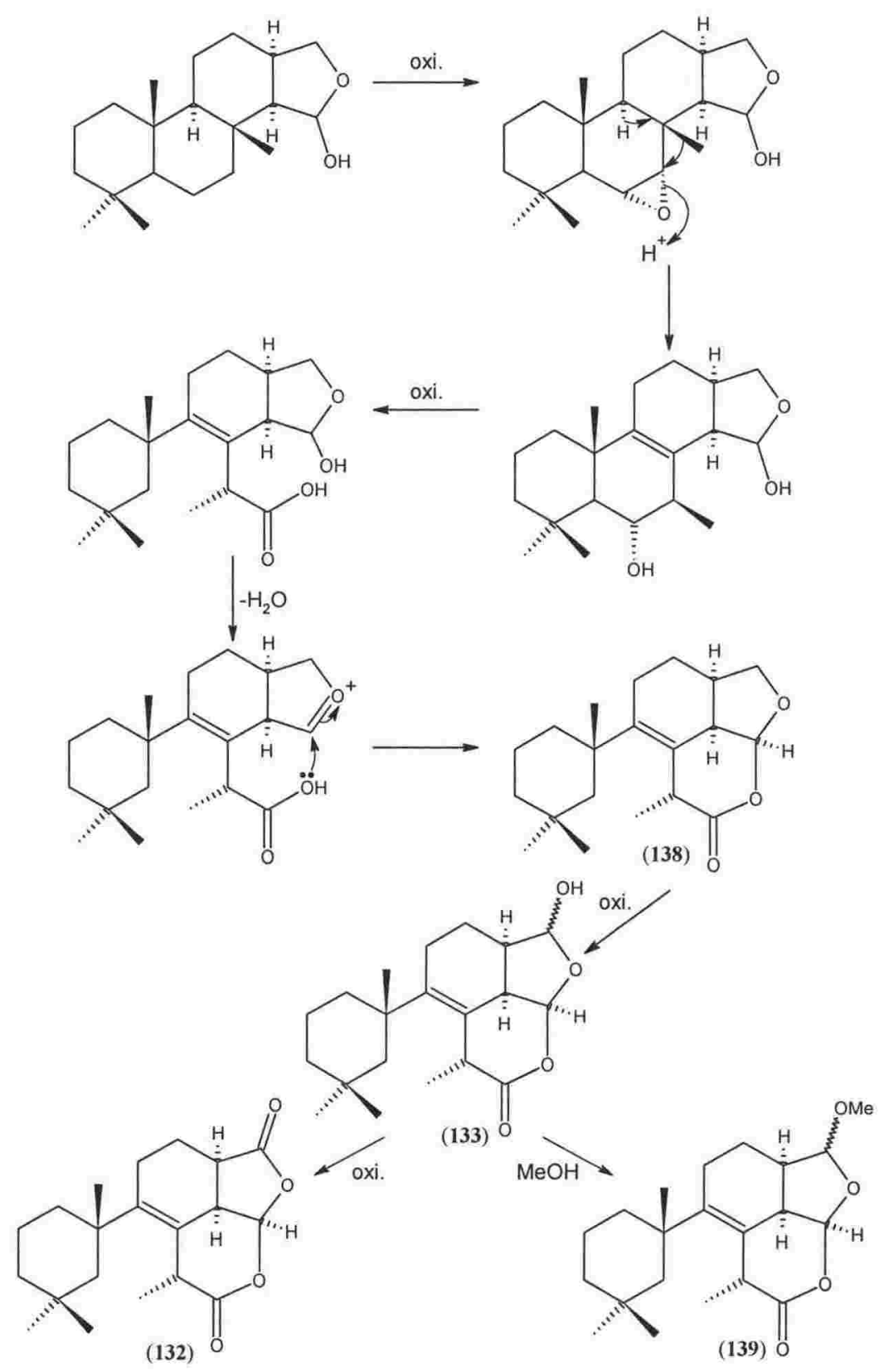

Scheme 4.11 Proposed biogenesis of cadlinolides A-D (132-133, 138-139). Based upon the proposed biogenesis of the gracilins. ${ }^{150,152}$

Pourewic acid A (140), 15-methoxypourewic acid B (141) and methylpourewate B (142) are also closely related to the gracilin family of compounds (118-123). ${ }^{140,148-150}$ The biosynthesis of the gracilin compounds involves the oxidative opening of ring B to give various carboxylic acids that decarboxylate to give compounds $\mathbf{1 1 8 - 1 2 3}{ }^{150,152}$ The isolation of 140-142 helps to confirm this biosynthetic pathway, as they were predicted intermediates of these mechanisms. It is unlikely that traditional purification method 
using silica gel would have allowed isolation of these compounds, as irreversible binding of the metabolites to the chromatographic substrate may occur. In the biosynthetic pathway proposed for the formation of $\mathbf{1 1 8 - 1 2 3}$, a leaving group $(\mathrm{X})$ that takes part in the decarboxylation reaction has been thought to be present on the original ring $\mathrm{C}$ throughout the mechanism. As none of the compounds isolated during this study have any functionality on ring $\mathrm{C}$, it would appear more likely that some oxidation of ring $\mathrm{C}$ takes place after the oxidative cleavage of ring $\mathrm{B}$, to help facilitate the decarboxylation suggested. A suggested biosynthesis of compounds $\mathbf{1 4 0 - 1 4 2}$ is detailed in scheme 4.12 . 


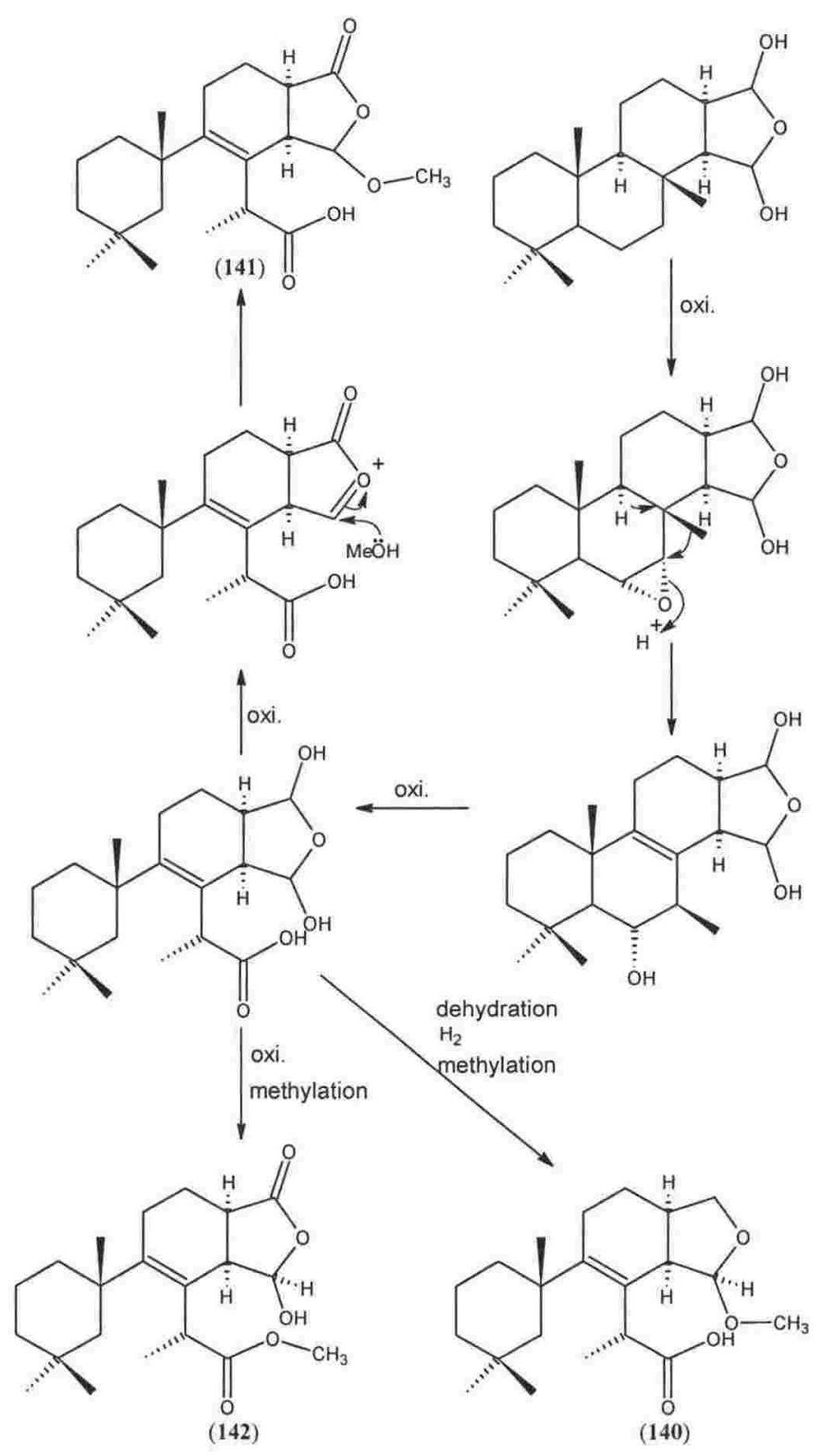

Scheme 4.12 Proposed biogenesis of pourewic acid A (140), 15-methoxypourewic acid B (141) and methylpourewate B (142). Based upon the proposed biogenesis of the gracilins. ${ }^{150,152}$

Pourewanone (143) is the first representative of the new pourewanane carbon skeleton. It is proposed that the oxidative opening of ring B occurs as for the cadlinolide and pourewic acid classes. Loss of $\mathrm{H}_{2} \mathrm{O}$ leads to the formation of a $\Delta^{14,15}$ olefin that then undergoes an oxidative cleavage to form formate 143. This pathway is described in scheme 4.13. Alternatively, a furanospongian diterpene could undergo oxidative cleavage of the $\mathrm{C}-14 \mathrm{C}-15$ bond followed by hydrogenation of the $\mathrm{C}-13 \mathrm{C}-16$ bond (or 
hydrogenation of $\mathrm{C}-13 \mathrm{C}-16$ and then cleavage). This is summarised in scheme 4.14. No furano-diterpenes and at least one $\mathrm{C}-15 \mathrm{OH}$ diterpene were isolated from Chelonaplysilla violacea during this study, therefore the mechanism shown in scheme 4.13 would appear more likely. As mentioned, to the best of the author's knowledge, pourewanone is the first example of a formate from the marine environment.

The oxidative cleavage of the double bond proposed is similar to the synthetic cleavage of an olefin by $\mathrm{O}_{3}{ }^{80}$ Such cleavages have been proposed for the biosynthesis of retinal and vitamin $A_{1}$ from $\beta$-carotene. ${ }^{13,80}$ More recently, similar cleavages have been proposed for the biosynthesis of the degraded pregnanes muricenones A and B (144, 145), and for the aromatic alkaloid cyclomegistine (146) (see schemes 4.15 and 4.16). ${ }^{165,166}$ Both these biosyntheses have been supported by synthetic studies. ${ }^{166,167}$<smiles>[R]C[C@@H]1[C@@H]2CC[C@@]3(C)[C@@H](CC[C@@H]3C=C)[C@@H]2CCC(=O)[C@]1(C[R])CCC(=O)OC</smiles><smiles>COC(=O)[C@@]1(OC)C2(OC)c3c(c(=O)c4ccccc4n3C)[C@@]21OC</smiles> 

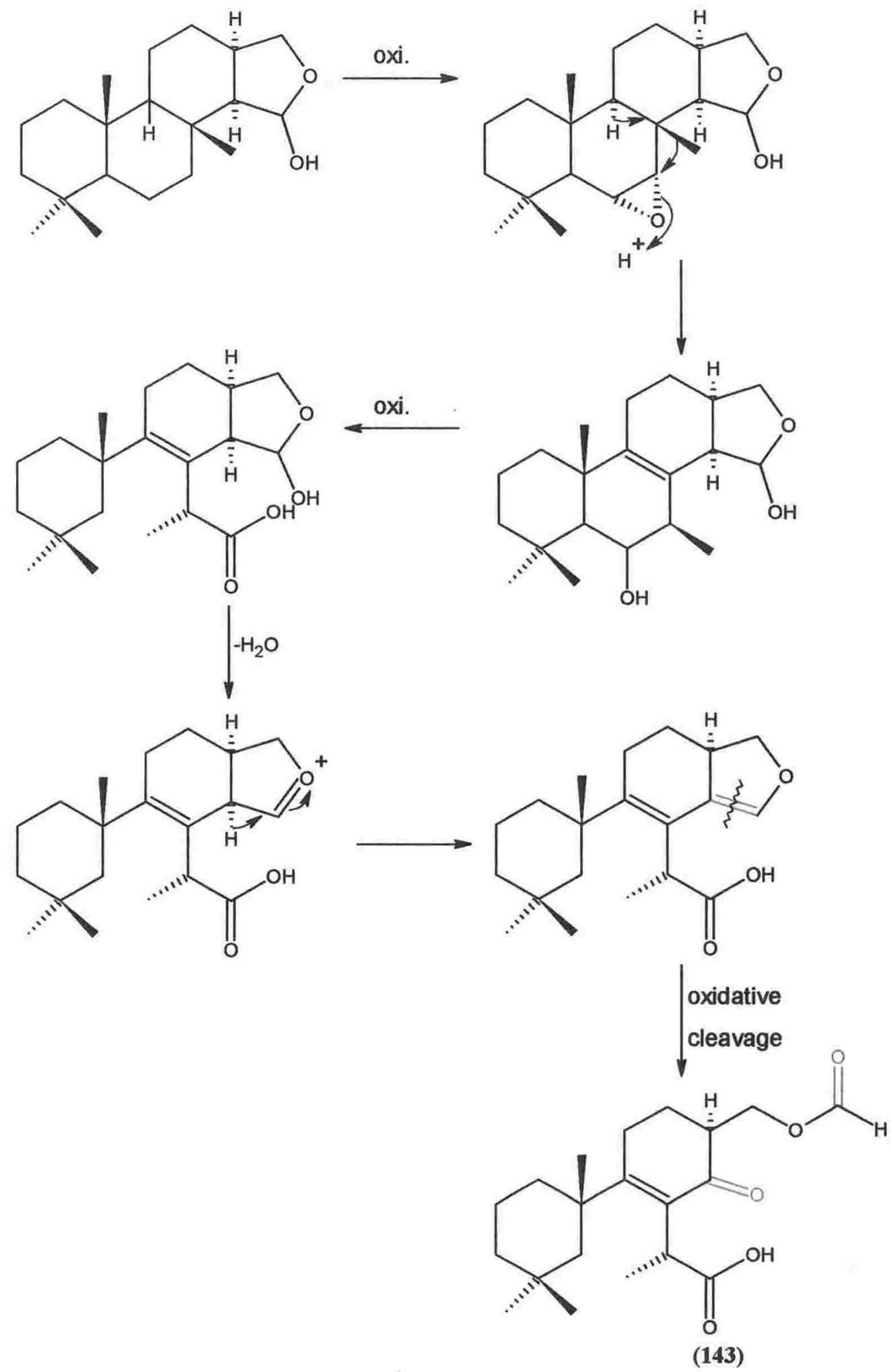

Scheme 4.13 Proposed biogenesis of pourewanone (143). 

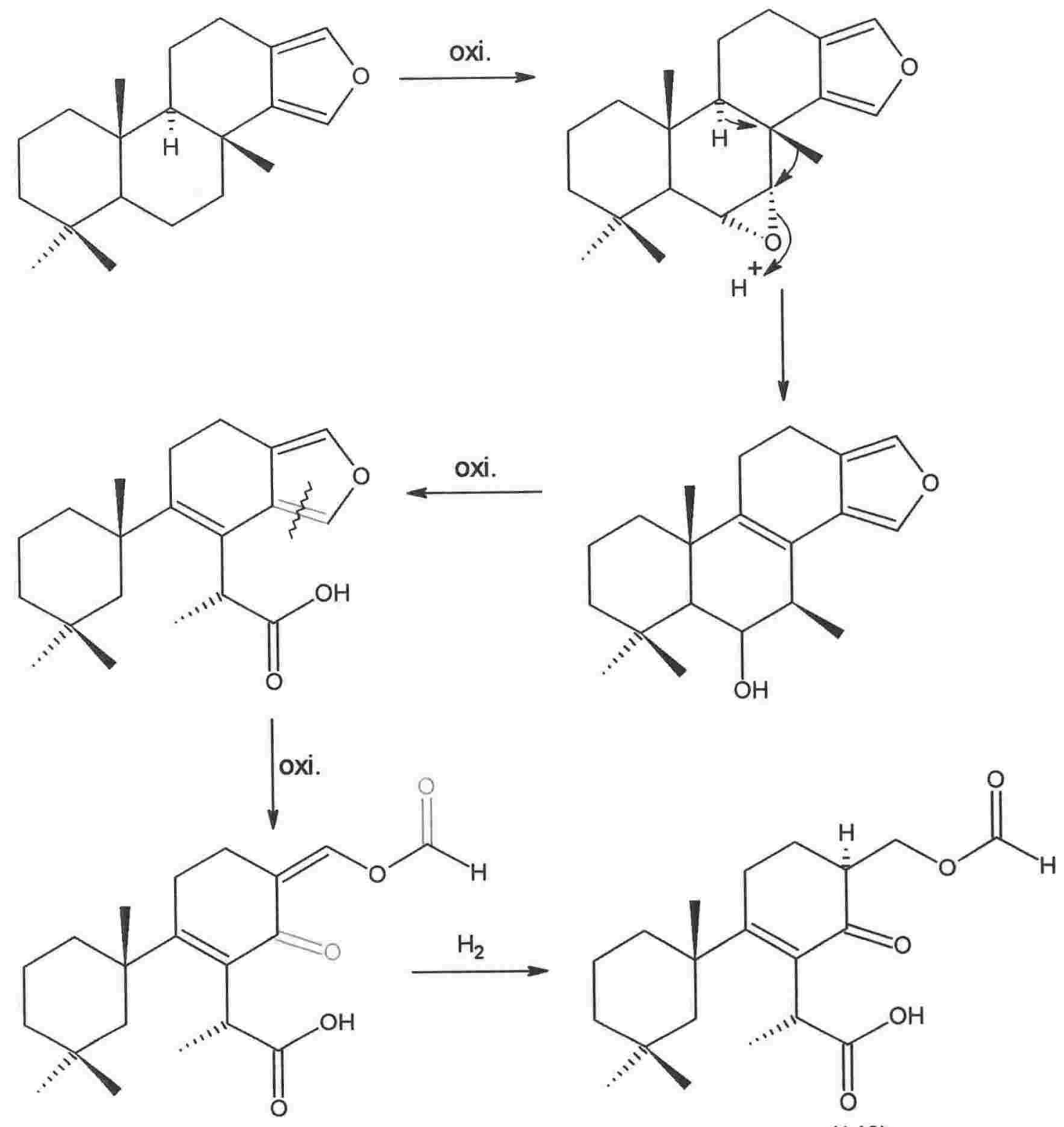

(143)

Scheme 4.14 Alternative biogenesis of pourewanone (143). 


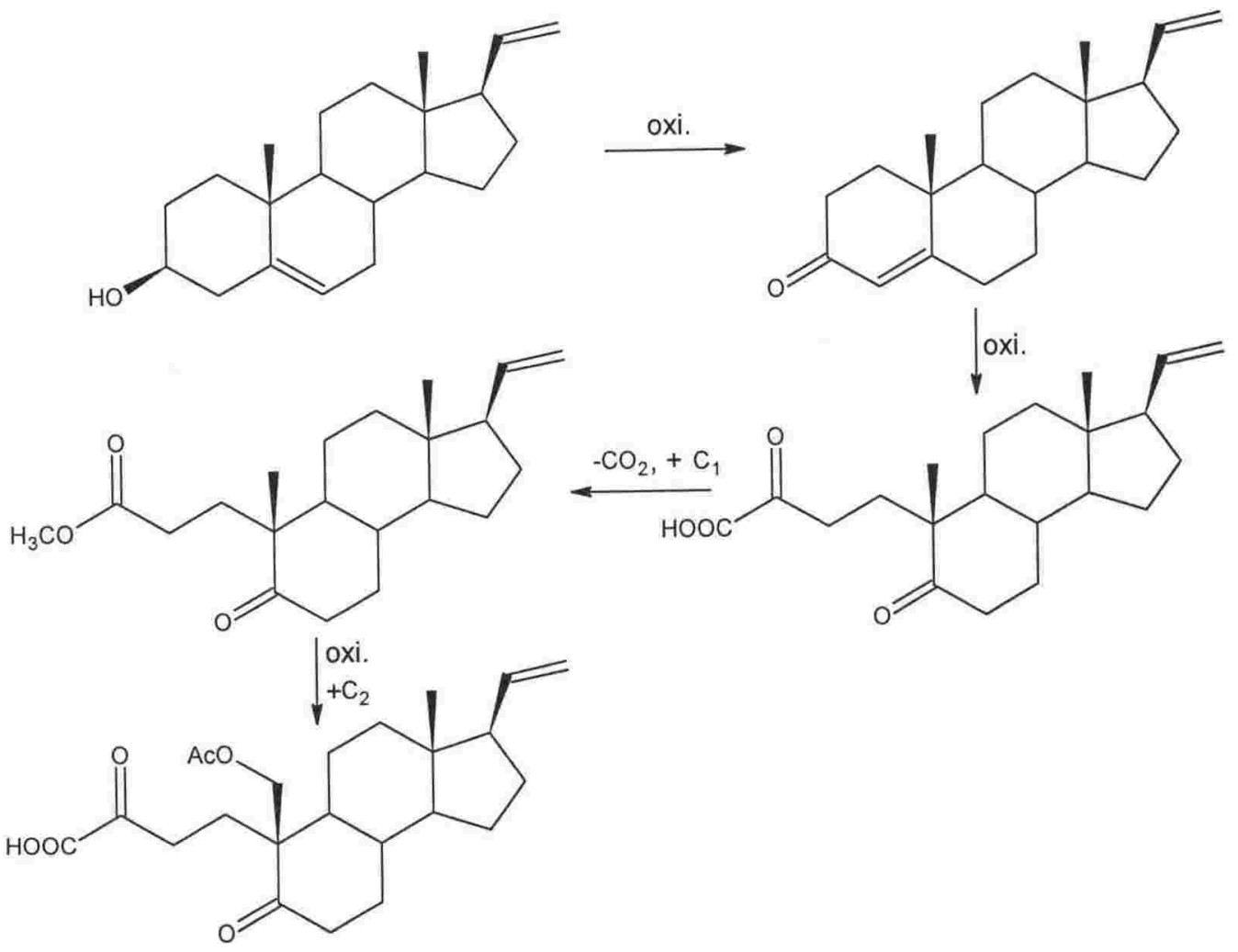

Scheme 4.15 Proposed biogenesis of muricenones A and B $(\mathbf{1 4 4}, \mathbf{1 4 5}){ }^{166}$

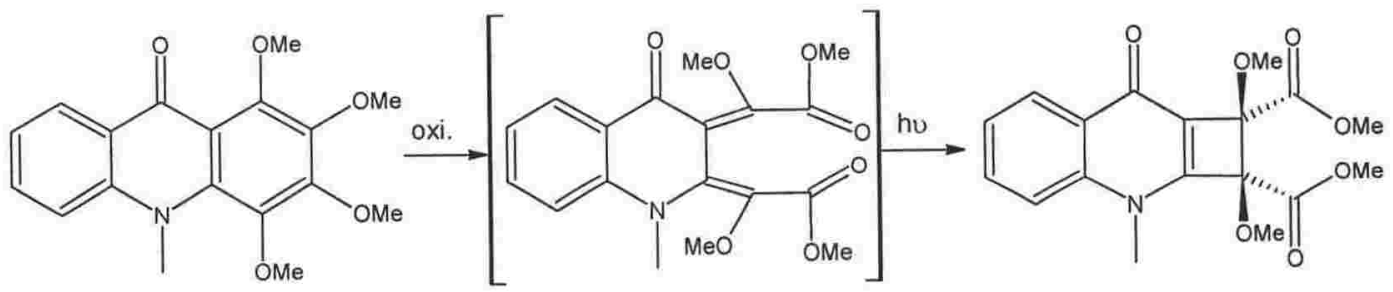

Scheme 4.16 Proposed biogenesis of cyclomegistine (146). ${ }^{165}$

\subsection{Biological Activity of the Isolated Spongian Diterpenes}

Due to the small amounts of the metabolites isolated, only a few could be submitted for biological evaluation. Cadlinolide C (138), pourewic acid A (140), and methylpourewate B (142) were all submitted for evaluation in both cytotoxicity and anti-inflammatory assays. None of the three metabolites showed any cytotoxic activity against the HL-60 leukaemia cell line (maximum concentration $10 \mu \mathrm{M}$ ). The 
compounds did show moderate anti-inflammatory activity as measured by inhibition of superoxide production by human peripheral blood neutrophils stimulated with either fMLP or PMA. ${ }^{105}$ The results are summarised in table 4.7. Finally, 140 was also submitted for anti-microbial activity and was shown to be inactive.

\begin{tabular}{|l|c|c|}
\cline { 2 - 3 } \multicolumn{1}{c|}{} & \multicolumn{2}{c|}{ IC $_{50}$} \\
\cline { 2 - 3 } \multicolumn{1}{c|}{} & PMA & fMLP \\
\hline cadlinolide C (138) & $13 \mu \mathrm{M}$ & $13 \mu \mathrm{M}$ \\
pourewic acid A (140) & $77 \mu \mathrm{M}$ & $74 \mu \mathrm{M}$ \\
methylpourewate B (142) & $58 \mu \mathrm{M}$ & $58 \mu \mathrm{M}$ \\
\hline
\end{tabular}

Table 4.7 Anti-inflammatory activity of several isolated spongian diterpenes.

It is unfortunate that a paucity of material prevented more of the diterpenes being assayed for anti-inflammatory activity, as this prevents a true structure-activity relationship to be suggested. Cadlinolide $\mathrm{C}(\mathbf{1 3 8})$, which contains a $\mathrm{C}-15 \mathrm{C}-17$ lactone ring, is the most active of all, whilst pourewic acid A (140) and methylpourewate B (142), which do not have this lactone linkage, show comparable and reduced activity.

\subsection{Other metabolites from the Genus Chelonaplysilla}

Other than the diterpenes mentioned during the literature survey, only one other publication regarding metabolites from the genus Chelonaplysilla could be found. The anti-microbial aromatic alkaloids (147-150) were isolated from the polar fractions of an extract of the same sponge that yielded several diterpenes. It was felt by the authors that the plethora of diterpenes found in various species of Dendroceratid sponges had meant that other researchers had overlooked the more polar, and generally more biologically active, nitrogenous compounds that the sponges produce. It was also suggested that these compounds may more important components of the animal's defensive mechanism. ${ }^{168}$ 


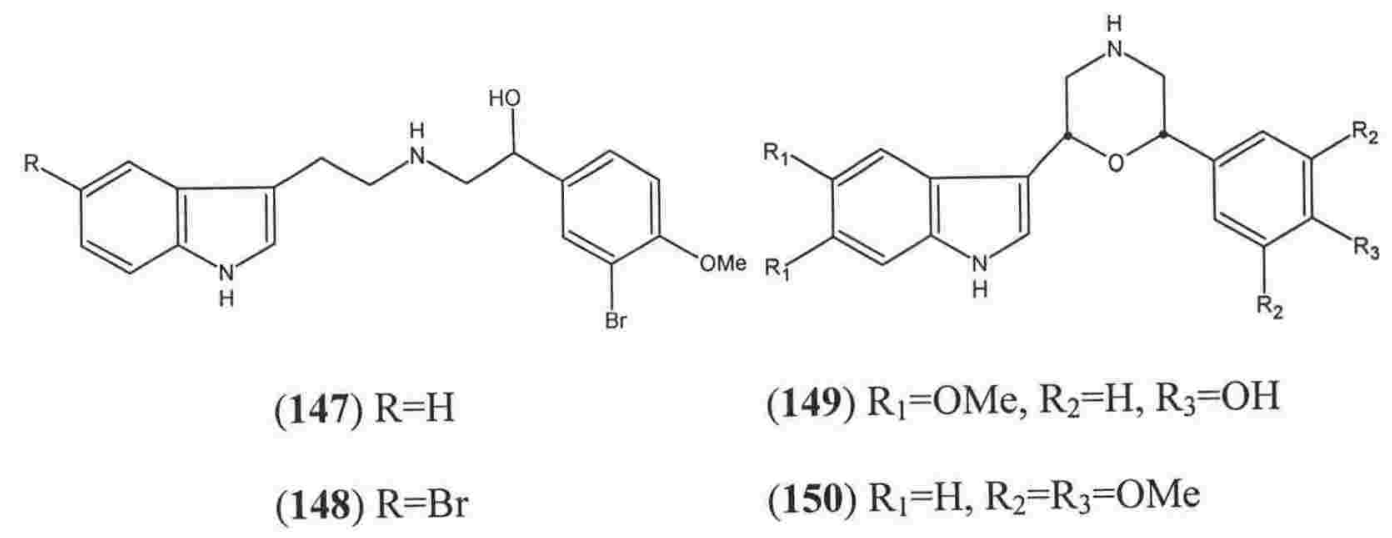

The use of NMR during this study, for the screening of sponges and the guidance of isolation procedures, coupled with the use of reversed-phase stationary supports, has resulted in the isolation of several nitrogenous compounds (see chapter two). It is unlikely that polar nitrogenous compounds were present in the sample of Chelonaplysilla violacea that yielded diterpenes 138-143. Alkaloids such as these would have easily been noted during the screening process. 


\section{Isolation of a Marine Toxin Produced by the Dinoflagellate Karenia brevisulcata.}

\subsection{Dinoflagellate Toxin Classes}

The presence of a dinoflagellate toxin in the environment is often not recognised until cases of human intoxication from eating contaminated seafood, usually shellfish, are reported. This is because many shellfish species can bioaccumulate toxins in their various tissues without causing harm to the animal itself. The toxin is only noticed when higher predators consume the seafood and become sick. ${ }^{10,39,42}$ The toxic symptoms elicited in humans are therefore often used to classify the broad categories of dinoflagellate toxin. The four main classes of dinoflagellate toxin are Paralytic Shellfish Poisoning (PSP), Neurotoxic Shellfish Poisoning (NSP), Amnesic Shellfish Poisoning (ASP) and Diarrhetic Shellfish Poisoning (DSP). There is also a collection of other unique toxins that do not fall under these categories. . $^{10,39,42,169-177}$

\subsection{Paralytic Shellfish Poisoning (PSP)}

PSP is caused by the alkaloids saxitoxin (151), neosaxitoxin (152), and their homologues. Both the saxitoxins and neosaxitoxins are produced by marine and freshwater dinoflagellates, including various species of Alexandrium, Karenia and Pyrodinium. ${ }^{171,172,178-183}$ 


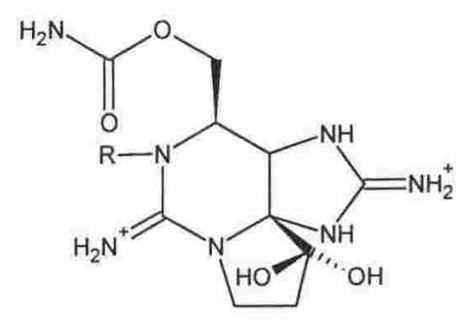

(151) $\mathrm{R}=\mathrm{H} \quad(152) \mathrm{R}=\mathrm{OH}$

The mode of action of both the saxitoxins and neosaxitoxins is the same; both classes block the sodium channels of excitable nerve membranes which inhibits the propagation of action potentials (voltages), thereby causing paralysis of muscles. Other major symptoms in humans include hypersensitivity of the mouth and skin, ataxia, muscle weakness, and a sensation of floating. PSP is often fatal due to paralysis of the muscles controlling the lungs of victims. ${ }^{184}$

\subsection{Neurotoxic Shellfish Poisoning (NSP)}

NSP is caused by the brevetoxins, a class of polyether toxins produced by Karenia brevis (syn. Gymnodinium breve) and other members of the same genus. It is blooms of $K$. brevis that have led to the commonly used phrase "Red Tide", due to the characteristic colour of large blooms of the dinoflagellate (see figure 1.3). One of the first records of a Red Tide is found in the Bible in Exodus 7 20-21: “... and the water changed into blood. The fish died and the river stank...". 6 The brevetoxin family include brevetoxins A (153), B (154) and B2 (155). ${ }^{169,170,185}$

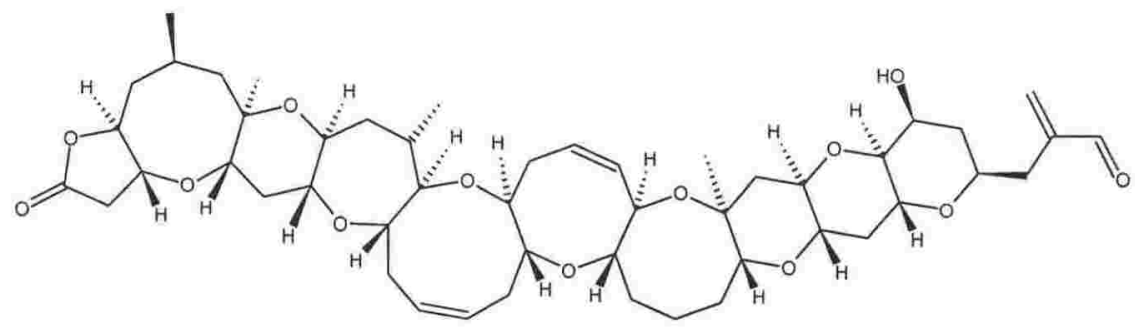

(153) 

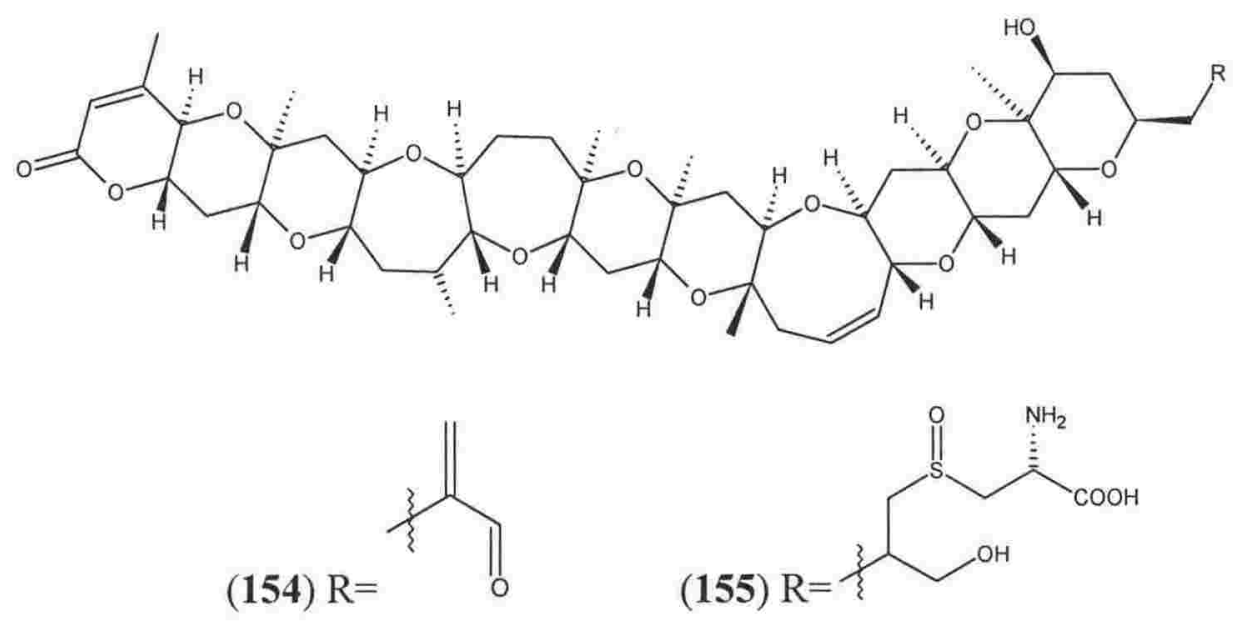

The mode of action of the brevetoxins is quite different to that of the saxitoxins, even though both classes affect the sodium channels of cell membranes. This is because brevetoxins bind at a different site on the channel than the PSP toxins. The brevetoxins cause sodium channels to stay open longer, by stabilising both the pre-open and open states of the channel, allowing greater sodium ion flow into the cell. This increased ion concentration causes neuronal membrane depolarisation, which stops nerve signal propagation. ${ }^{42}$ NSP can cause a wide variety of symptoms, including a reversal of thermoreception, loss of coordination, headache, and general malaise. ${ }^{42,184,186}$

\subsection{Diarrhetic Shellfish Poisoning (DSP)}

DSP is caused by several different organisms and covers a wide variety of symptoms. The main causative agent is okadaic acid (156), which has been isolated from many sources. Okadaic acid (156) was originally isolated from the sponges Halichondria okadai and H. melandocia. One major producing dinoflagellate is Prorocentrum lima although other genera, including Dinophysis, have been found to produce $\mathbf{1 5 6}$ and other congeners. ${ }^{175,176,187}$ 


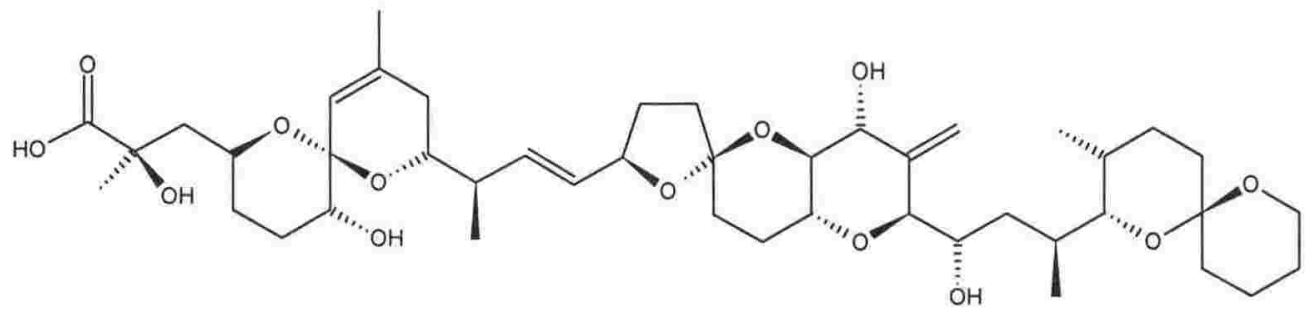

Okadaic acid (156) is a potent tumour promoter due to its ability to greatly inhibit the serine/threonine protein phosphatases PP1 and PP2A, which allows a build-up of phosphorylated proteins in cells. This mode of action is similar to that of the wellknown tumorigenic phorbol esters, which activate protein kinases. Increased protein phosphorylation leads to continuous "secondary messenger" production, which includes excess fluid excretion from gut cells causing diarrhoea. Other symptoms of DSP include gastrointestinal disorders such as nausea and abdominal pain. ${ }^{184}$

\subsection{Amnesic Shellfish Poisoning (ASP)}

ASP is caused by domoic acid (157) which was first isolated from the macro-alga Chondria armata. Domoic acid (157) was later identified as also being produced by members of the diatom genus Pseudonitzschia. Like PSP, cases of ASP have been fatal. ${ }^{173,174,188,189}$

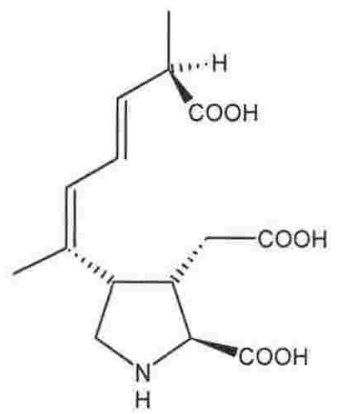

(157)

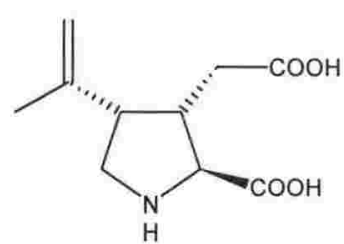

(158) 
The mode of action of $\mathbf{1 5 7}$ is similar to that of kainic acid (158). Both metabolites are excitatory amino acid agonists to glutamate, a neurotransmitter in the central nervous system. ${ }^{173,174,189}$ Both are thought to bind to kainate-sensitive glutamate receptors on nerves. This causes a depolarisation effect, followed by an influx of calcium ions into the cell that causes eventual cell death. Some domoic acid extracts may be more toxic than others due to possible synergy between domoate and other excitatory amino acids such as glutamate. Symptoms of ASP intoxication include vomiting and diarrhoea, followed by short-term memory loss, confusion, disorientation, and possible coma. ${ }^{184}$

\subsection{Other Dinoflagellate Toxins}

There are many other toxins produced by dinoflagellates that do not fall into the four main symptom classes, many of which are still problematic for the seafood industry. These toxins include ciguatoxin (159), the agent that causes ciguatera seafood poisoning (CSP), found after the consumption of many species of tropical fish. ${ }^{42}$ The earliest report of ciguatera poisoning is believed to have been recorded in China by Ch'en T'sang-chi sometime during the T'ang dynasty (618-907 A.D.) who claimed that the yellowtail is "...a large poisonous fish fatally toxic to man." 190

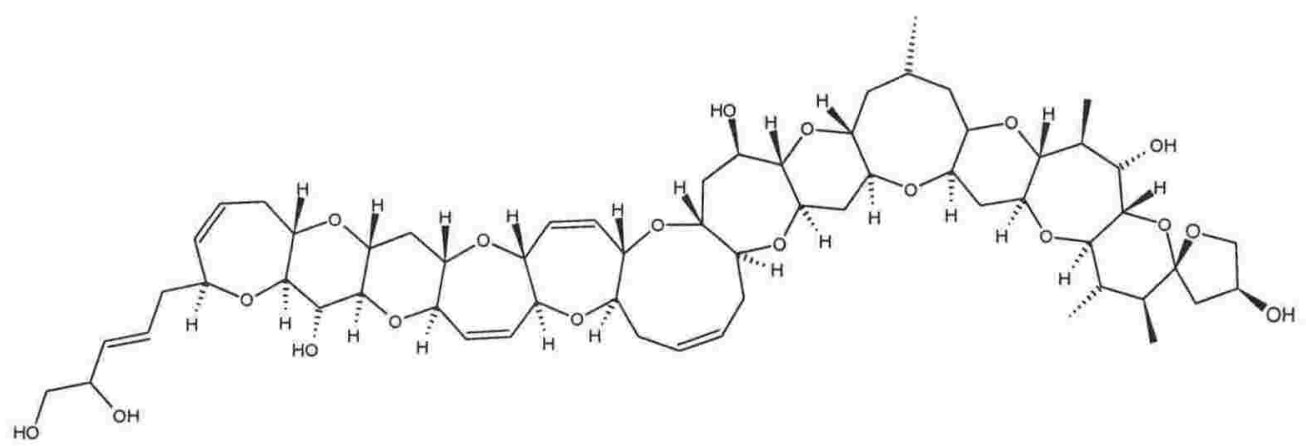

(159)

Ciguatoxin (159) is produced by the epiphytic dinoflagellate, Gambierdiscus toxicus, which is ingested by fish and introduced into the food chain. ${ }^{191-193}$ The symptoms of 
ciguatera are similar to that of NSP and include reversal of thermoreception, joint pain, low blood pressure, nausea, vomiting, and diarrhoea. Studies have shown that $\mathbf{1 5 9}$ binds to the same voltage-sensitive sodium channel as the brevetoxins, hence the similarity in observed symptoms. ${ }^{42}$ Although other congeners contribute, the major toxic component of ciguatera is $159 .^{42}$

A second class of toxin is produced by G. toxicus which is also of great interest. Maitotoxin (160) is a unique natural product as it is the largest, non-biopolymer, natural product ever isolated $\left(\mathrm{C}_{164} \mathrm{H}_{256} \mathrm{O}_{68} \mathrm{~S}_{2} \mathrm{Na}_{2}, 3422 \mathrm{Da}\right)$, and it may also be the most potent non-proteinaceous toxin known $\left(\mathrm{LD}_{50} \sim 50 \mathrm{ng} / \mathrm{kg}\right.$ intraperitoneal [IP] versus mice). ${ }^{42,194-}$ 196 Studies to determine the mode of action of $\mathbf{1 6 0}$ are still currently being carried out. $^{42,194-196}$

Several other toxins have until recently been included in the DSP class, as they are extracted in a similar manner and are often found in conjunction with okadaic acid (156), although they do not show any structural similarity to $\mathbf{1 5 6}$ nor do they appear to possess the same mode of action. These toxins include the pectenotoxins, such as pectenotoxin-1 (161) from Dinophysis fortii and D. acuminata, and the yessotoxin class, exemplified by yessotoxin (162), from Protoceratium reticulatum. ${ }^{197-200}$ It is now recognised that these metabolites are not true DSP toxins. ${ }^{201}$

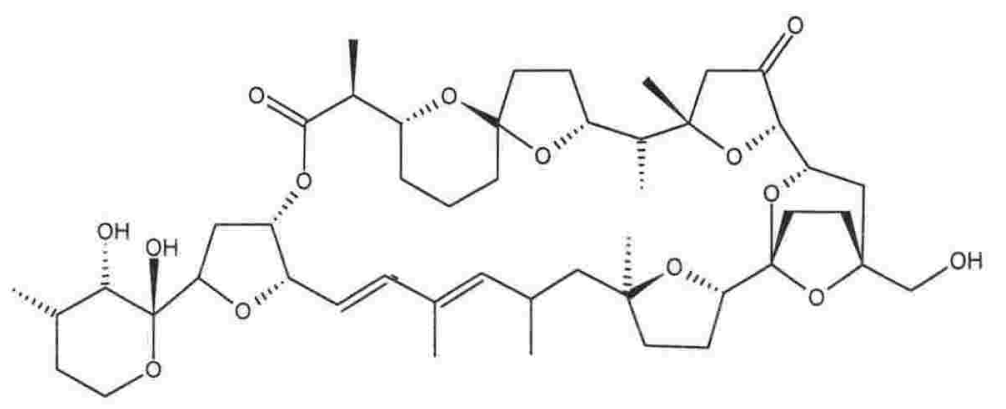




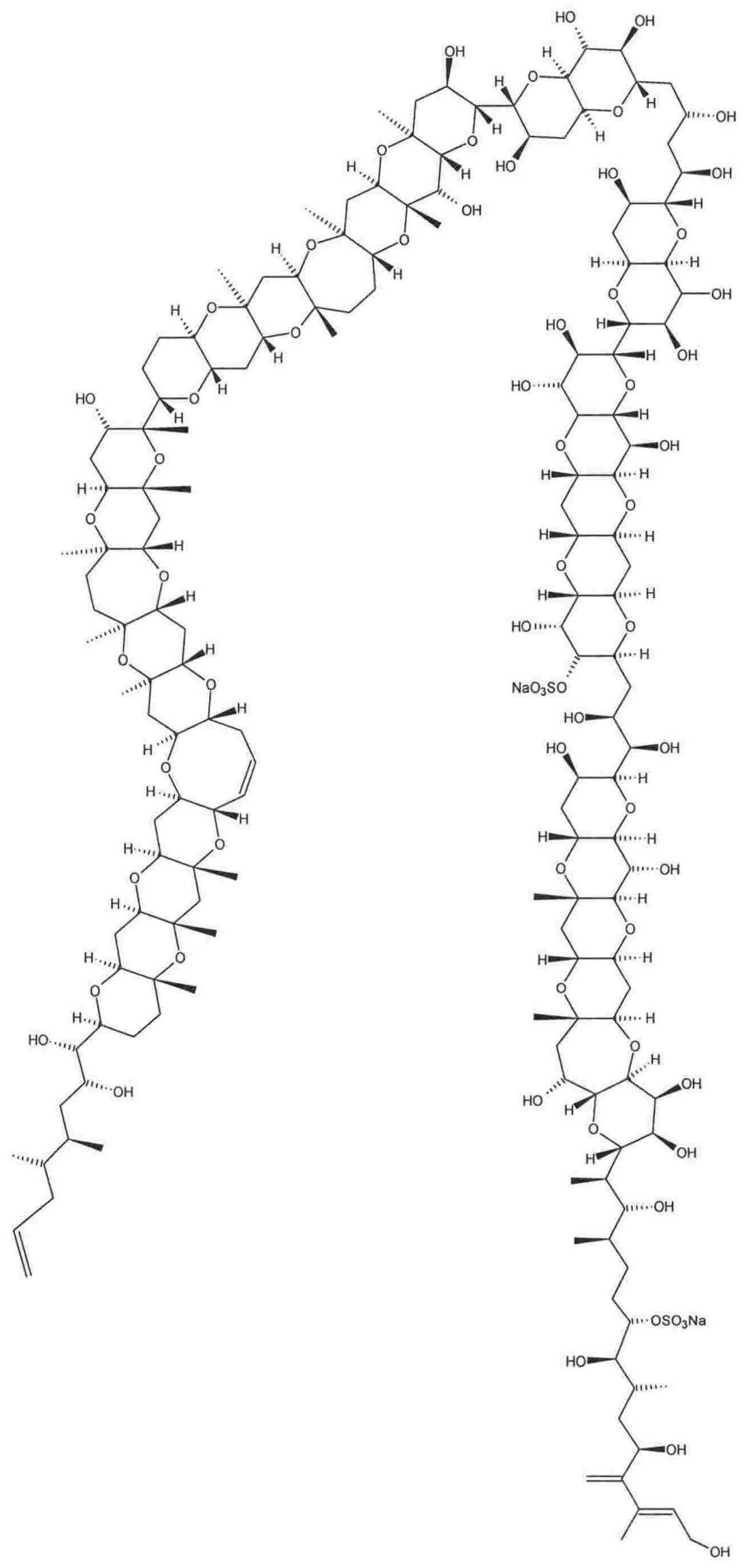

(160) 


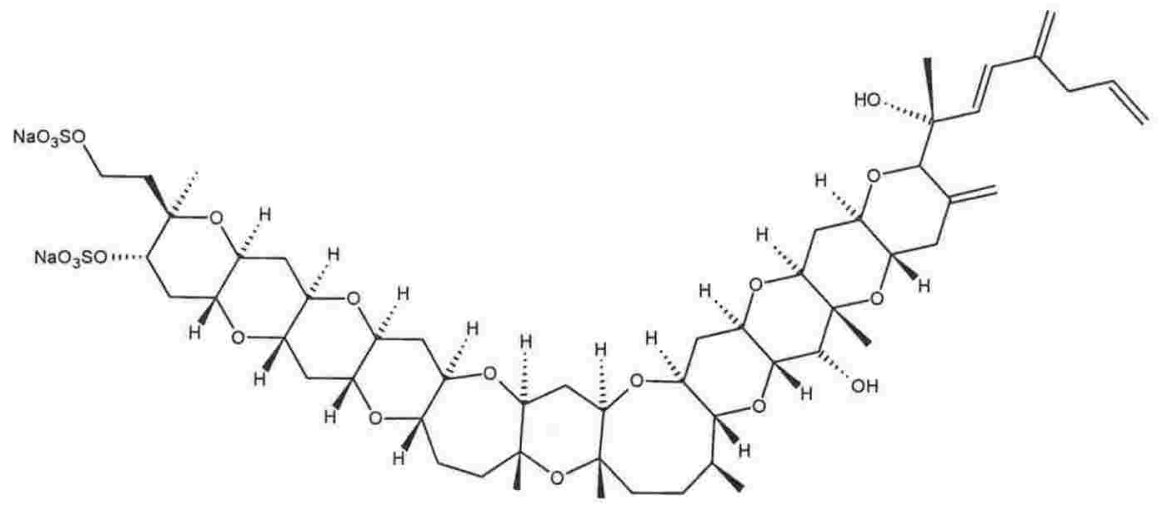

(162)

\section{$5.7 \quad$ Structural Considerations}

It can be seen from the previous examples that there are two common structural features of these natural products. First, most are highly oxygenated and they often contain other rare functionalities (hydrates, imines, spiro centres etc). Second, the fused polycyclicether carbon backbone is present in almost each toxin class and yet the modes of action and toxic symptoms elicited in humans are all very different.

The basic polyether structure has been proposed by Shimizu to be formed by a cascade ring closure of a polyepoxide compound. Shimizu proposed this mechanism for the biosynthesis of brevetoxin B (154). ${ }^{39}$ The isolation of hemibrevetoxin B (163), the exocyclic olefin portion of $\mathbf{1 5 4}$, would suggest that the cascade begins at that side of the molecule. ${ }^{202}$ The suggested biosynthesis begins with a hydride shift, followed by the cascade opening of the cis expoxides. The other polycyclic-ether toxins could presumably be produced via a similar mechanism (see scheme 5.1). ${ }^{39}$

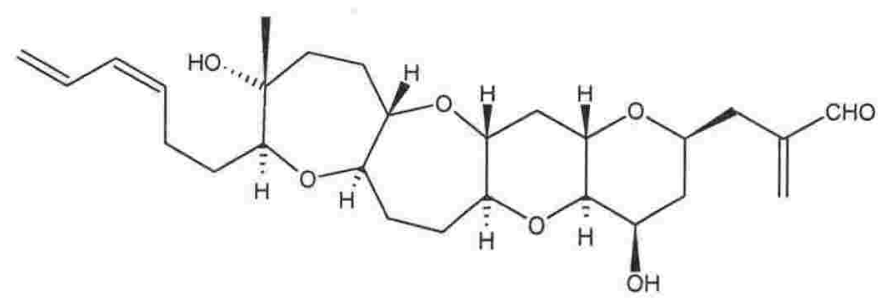




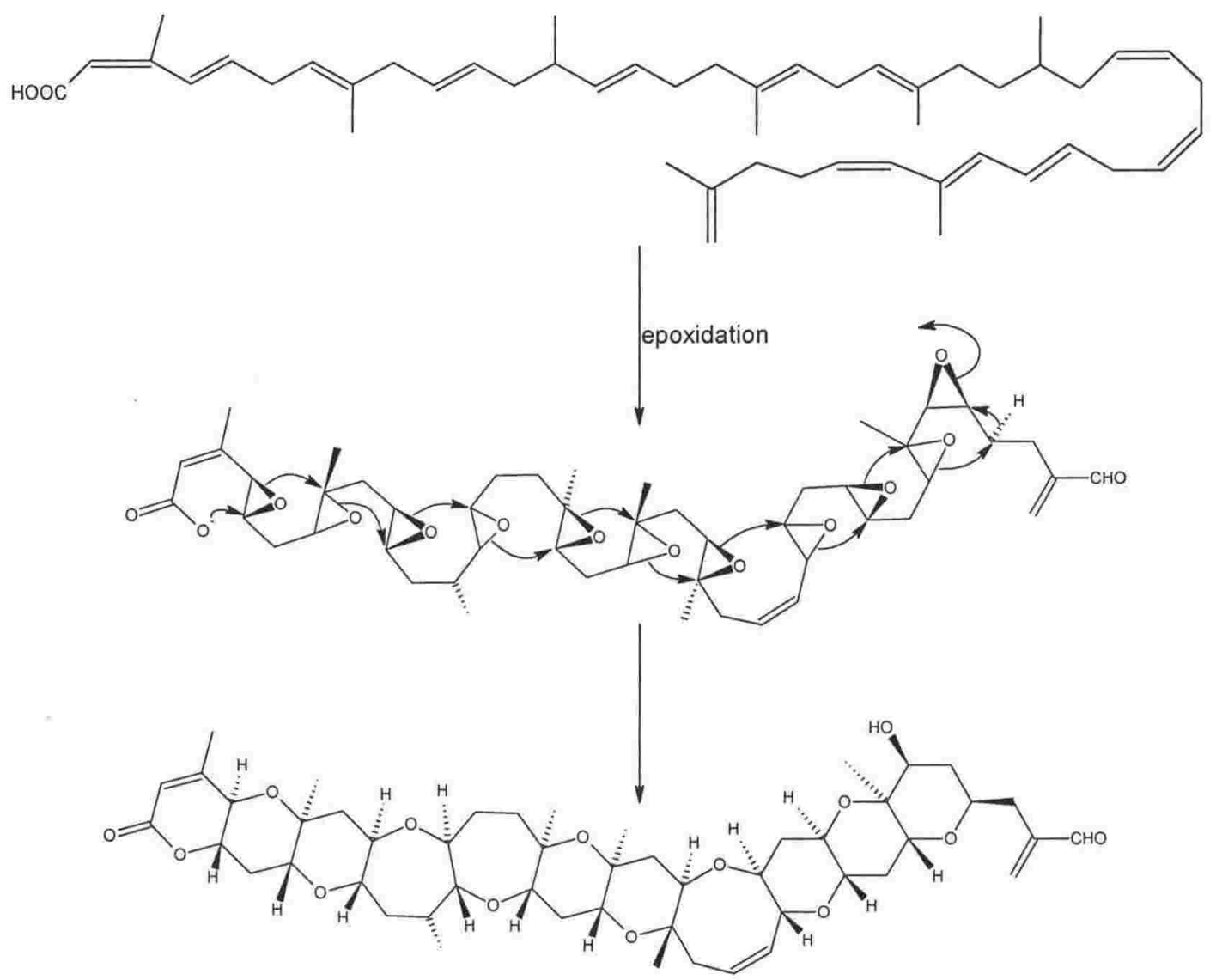

Scheme 5.1 Proposed biosynthesis of brevetoxin B (154). ${ }^{39}$

\subsection{Dinoflagellate Toxins and New Zealand}

Shellfish harvesting forms an integral part of the seafood industry in New Zealand, with both economic and cultural significance. In 1998, fisheries exports were worth \$NZ 1,236.8 million to the national economy, with shellfish accounting for \$NZ 273.9 million of that total. ${ }^{203}$ Any long-term closure of shellfish harvesting because of a toxic dinoflagellate bloom could have a serious impact on the New Zealand economy.

In January 1993, the entire coastline of New Zealand was closed to all shellfish harvesting after cases of human intoxication were reported. The intoxications were found to be caused by NSP toxins produced during a large algal bloom in late 1992. The dinoflagellates present during the bloom were Alexandrium minutum and 
Karenia brevis, reaching peak levels of $1.17 \times 10^{5}$ and $4.1 \times 10^{3}$ cells $/ \mathrm{L}$ respectively. ${ }^{204,205}$ The effect of this closure was the loss of several million dollars to New Zealand's economy and over one thousand jobs during the first three months of 1993. It was fortuitous that the shellfish industry had reserved large stocks of noncontaminated shellfish prior to Christmas 1992, and that the closure occurred while most workers were on summer holidays. By the end of the financial year, the industry had made almost a full recovery and most workers had been reinstated in their jobs. ${ }^{206}$ The results of the 1993 closure were not too serious for the New Zealand shellfish industry, although that was due more to good fortune than good planning or management. The New Zealand authorities have since instigated a continuous shellfishmonitoring programme in order to better detect and respond to such situations, should they arise again in the future. It is therefore important that new toxins are identified, and methods to detect and quantify any new toxins are established, in order to protect the shellfish industry and its consumers.

\subsection{Karenia brevisulcata}

In the summer of $1997 / 1998$, a bloom of a previously undescribed toxic dinoflagellate species formed in the waters off the East Coast of the North Island of New Zealand, corresponding to an unusually warm and still spring period. ${ }^{44}$ The bloom spread south along the eastern coastline, and then across the bottom of the North Island along the southern Wairarapa Coast and into Wellington Harbour (see figure 5.1). The dinoflagellate was isolated and named Gymnodinium brevisulcatum (etymology: Latin brevis $=$ short, sulcatum $=$ groove $).{ }^{43}$ The concentration of $G$. brevisulcatum peaked at $3.3 \times 10^{7}$ cells/L in Wellington on March $13^{\text {th }}, 1998 .^{44}$ Several toxic dinoflagellate genera including Gymnodinium, have recently been taxonomically reclassified and the dinoflagellate is now known as Karenia brevisulcata, a species closely related to the 
known toxic dinoflagellates $K$. brevis and $K$. mikimotoi (see figure 5.2 and scheme 5.2$)^{43-45,207,208}$

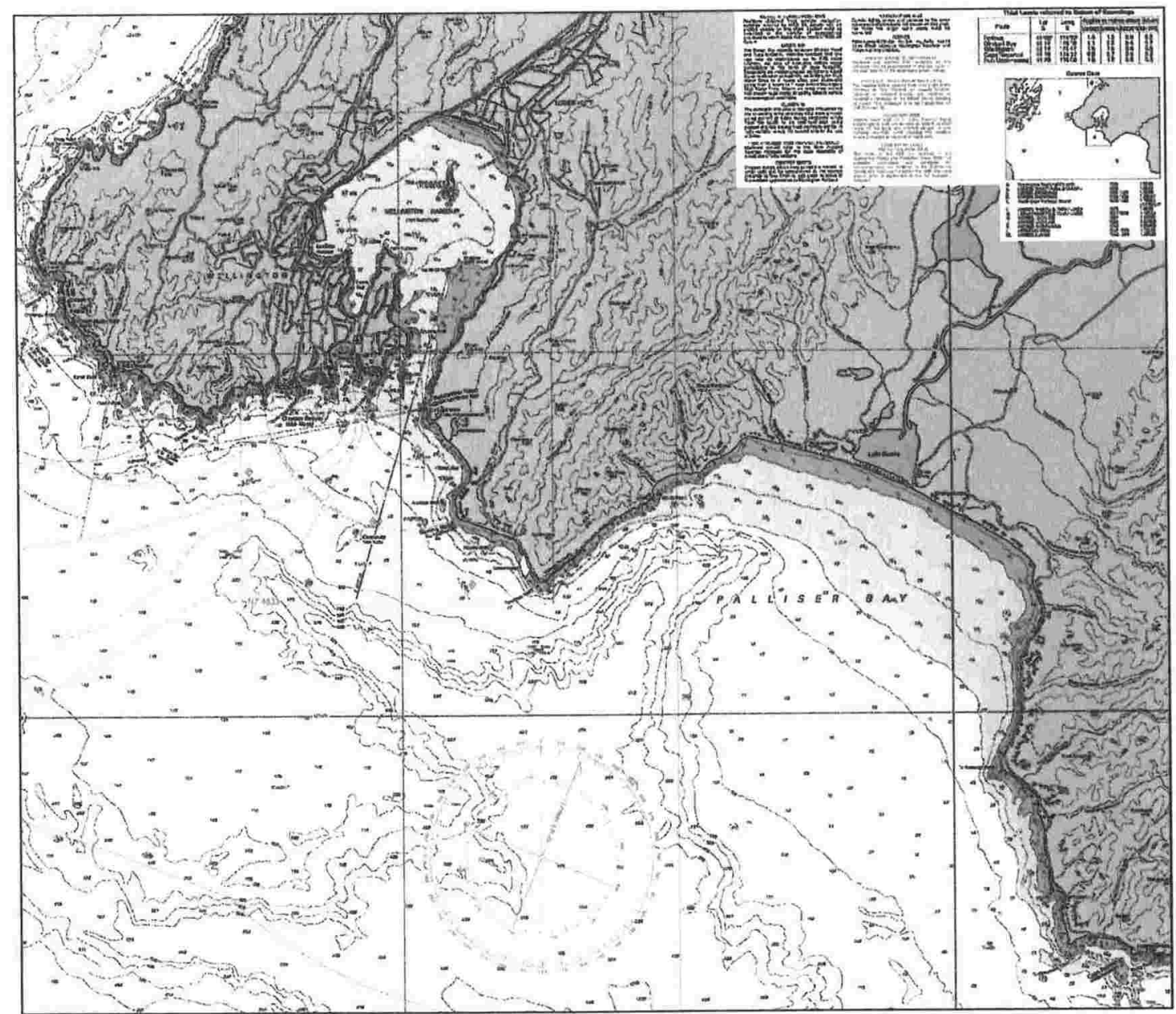

Figure 5.1 Hydrographic chart of Wellington Harbour and southern Wairarapa Coast.

(Courtesy of Land and Information New Zealand)

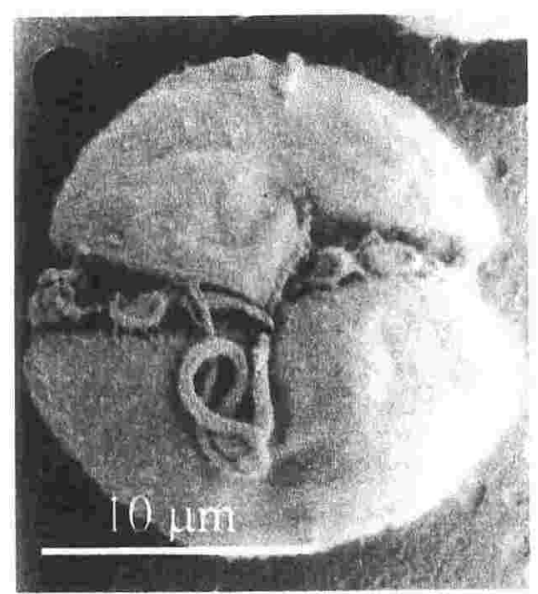

Figure 5.2 SEM image of Karenia brevisulcata.

(Courtesy of Dr H. Chang, NIWA, 2003). 
The most notable effects of the bloom were due to the extreme biological activity exhibited by the dinoflagellate. Almost all the fish, plant, and invertebrate species within Wellington Harbour died. Most of the species not killed by the dinoflagellate stopped eating and died of starvation instead. The biological activity of the presumed toxin was quite unlike anything previously reported. $43,44,207,208$

Over 150 cases of human respiratory distress were noted during the bloom, with symptoms including a dry cough, severe sore throat, inflamed and puffy eyes, dermatitis, feverish influenza-like symptoms, headache, nausea, and stomach cramps. The cases of respiratory distress were thought to be due to aerosol effects from sea spray containing a toxin and/or live toxic algal cells. ${ }^{44}$

Samples of $K$. brevisulcata were acquired, and cultures of the dinoflagellate grown. It was decided to attempt the isolation of the causative toxin(s) from artificially grown cultures of $K$. brevisulcata, in order to determine the identity of the toxin. If the toxin proved to be a novel compound, a secondary goal was to establish detection methods for the metabolite that could be integrated into the current national shellfish-monitoring programme. 
Kingdom

Plantae (Plants)

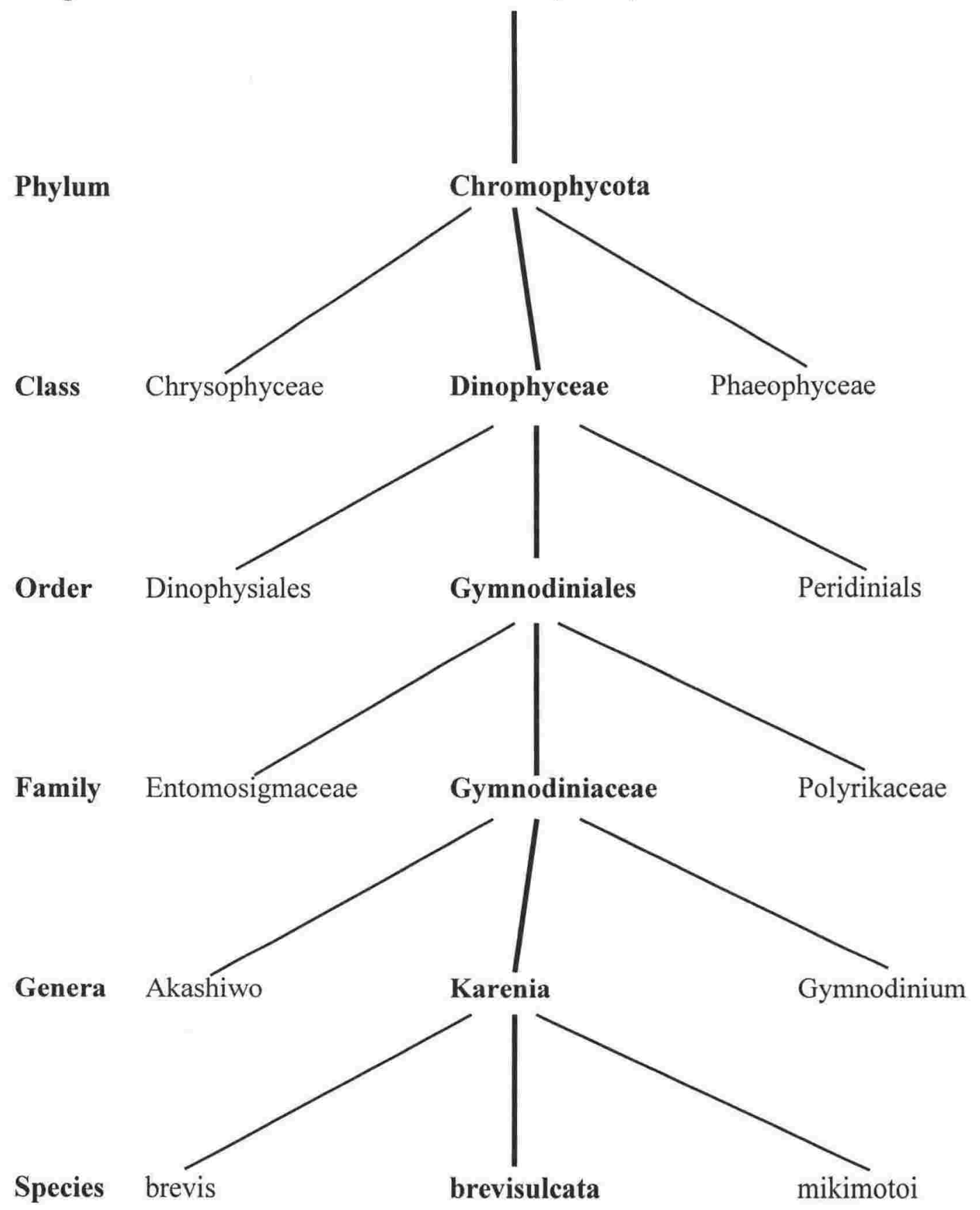

Scheme 5.2 Phylogenic relationship of Karenia brevisulcata. For simplicity, not all classes, orders, families, genera or species are shown. ${ }^{35,43,45}$ 
One of the key issues relating to the isolation of the Karenia brevisulcata Toxin (KBT) was the method used to direct isolation. Unlike the NMR based screening method described in chapter two, no chemical signature appropriate to guide an isolation procedure was evident for KBT. Rather, as any toxin is obviously biologically active, bioassays would be more appropriate to guide an isolation of KBT. After identification, other methods including ELISA (Enzyme Linked Immuno-Sorbent Assay), LC-MS or NMR, may be established for quantitatively identifying toxin levels in a variety of samples. The major requirements of a bioassay to be used were fast turnaround time, accuracy of results, and a need to be quantitative. The last requirement was of supreme importance. If the assay used to detect a toxin is only qualitative, and the assay is very sensitive or the toxin highly potent, then recovery of only a small amount of toxin may still trigger a positive response in the assay, even if most of the active material had been lost. Loss of the active material may occur through irreversible binding to the column substrate or by degradation of the molecule. A quantitative assay, which can accurately calculate the total recovery of the toxin, is therefore needed.

The first assay used to monitor the isolation of KBT from cultures of $K$. brevisulcata measured the death of another dinoflagellate species following exposure to KBT. The number of cells killed and the time to death were used to estimate the amount of KBT. This assay was used to assess the toxicity of various cultures of $K$. brevisulcata, and was also initially used to direct the attempted isolation of KBT. One $300 \mathrm{~L}$ culture of $K$. brevisulcata was semi-purified through two fractionation steps using direction by this assay. The resulting fractions from this batch were used for various biological trials, including some stability tests (see below). Unfortunately, the assay was slow and difficult to use as it relied on observing the time taken for the test organisms to die, 
which could take a considerable time as samples could only be processed in batches of five. The time taken to analyse many fractions generated from a purification step would be prohibitive. Determining when the cells died was in itself a subjective matter as it relied on personal experience and knowledge of the test organism. This assay was dropped as the primary means of monitoring the isolation of KBT.

The assay used subsequently to direct isolation was a murine neuroblastoma (N2A) assay. This assay is an MTT (3-(4,5-dimethylthiazol-2-yl)-2,5-diphenyltetrazolium) based assay, originally developed for detecting the NSP or PSP toxin classes. ${ }^{209,210} \mathrm{It}$ has been found, during the course of this study, that KBT has a distinctive and reproducible activity that varies in a linear fashion with dilution in the N2A assay, making it a quantitative system for measuring the amount of KBT.

In order to accurately quantify the amount of toxicity that a sample contains, a standard must be used as a control against which the unknown samples are measured. To do this, a measurement of arbitrary toxicity units (TUs) is used. One TU was defined as the minimum amount of a specific sample of KBT (the "Gold Standard") required to affect the viability of the $\mathrm{N} 2 \mathrm{~A}$ cells in the assay system. A standard aliquot of the gold standard is added to a control well during each quantification, in order to calibrate the results obtained from the N2A assay system by checking that the response of the N2A cells is the same each time. Each sample is measured against the response of the cells to the gold standard, and is quantified in terms of TUs without knowing any of the physical characteristics of KBT (in theory, similar to determining molar concentration without knowledge of molecular mass). It is therefore possible to note how many TUs are loaded onto a column at any step, and then measure the recovery afterwards (see figure 5.3). 

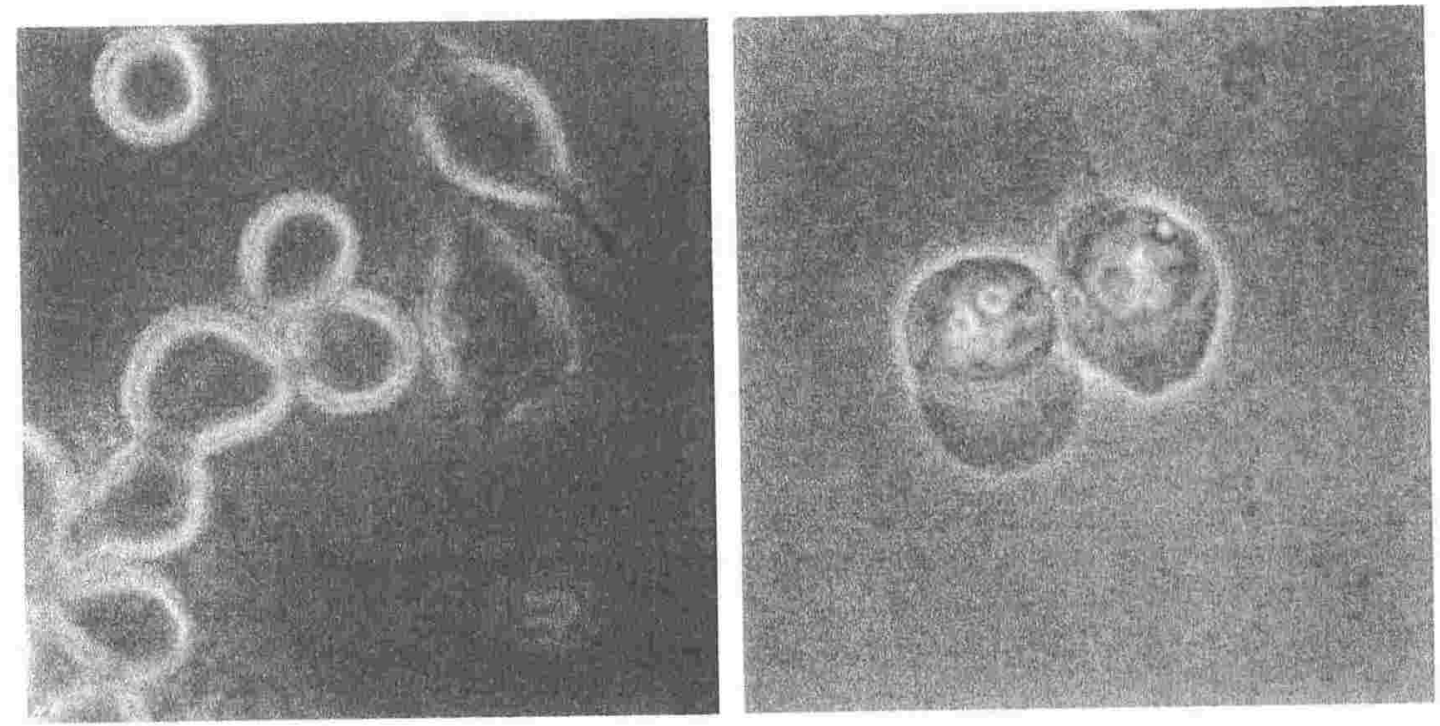

Figure 5.3 N2A cells (left) untreated, (right) treated with KBT.

(Courtesy of Dr P. Truman, ESR, 2003)

\subsection{Culture Harvesting}

Once an appropriate assay for guiding the isolation was in place, the main objective was to quickly and quantitatively fractionate and purify KBT. The initial step of extracting the maximum amount of toxin from the cell culture was of major importance, as this would limit the maximum yield from any harvest. Previous work carried out in our laboratory had proven the use of PSDVB in the initial extraction of dinoflagellate toxins from culture media. ${ }^{64}$ It was assumed that most dinoflagellates would secrete any toxins produced into the surrounding media, in order to deter predation. Any secreted toxins passed through a column of PSDVB should absorb to the stationary phase. The PSDVB could then be eluted to achieve basic fractionation. The algal cells could also be collected (by filtration or centrifugation) and extracted as well. An extract of the cells could be cyclic loaded and eluted, with any toxic fractions combined with those generated from the column that the media had been passed though. Normal chromatographic methods could then be applied to isolate the toxin itself. ${ }^{64}$ 
In this way, several batches of $K$. brevisulcata, from 20-320 L culture media, were harvested throughout the course of this study, each time yielding an extractable toxin that performed consistently in all the chromatographic separations and assay systems used. It was noted early on, however, that the amount of toxicity yielded varied greatly from batch to batch. This could be, in part, due to differences in the timing of harvest in relation to the life cycle of $K$. brevisulcata, a facet that has not yet been explored fully. The time of harvesting, however, was not felt to be a major factor. More likely, the dinoflagellate itself was not secreting the same amount of toxin in each batch due to differences in culture conditions. Batches grown with dense cell concentrations, indicating good conditions for growth, may not yield the most toxin, as the culture may not be stressed enough to respond by secreting as much toxin.

In order to maximise the yield of KBT from each batch, the dinoflagellate cells were lysed to release all toxin into the surrounding media. This was achieved by adding $\mathrm{Me}_{2} \mathrm{CO}$ until a concentration of $10 \% \mathrm{Me}_{2} \mathrm{CO} / 90 \%$ media was reached. The change in polarity caused all the dinoflagellate cells to lyse, thereby releasing any KBT contained within the cell itself. By harvesting in this way, the toxic yield from $\sim 320 \mathrm{~L}$ $K$. brevisulcata was increased from $150 \times 10^{6} \mathrm{TU}$ to $2,500 \times 10^{6} \mathrm{TU}(\sim 16$ fold increase in toxicity). In a subsequent experiment, addition of freshwater to decrease the salinity of the culture media has successfully lysed the cells of a wild sample of Karenia sp. in a similar manner. ${ }^{211}$ This may in future be a cheaper and more environmentally safe method to maximise the yield of various toxins from batches of cultured dinoflagellates.

\subsection{Stability Tests}

With the availability of a quantitative assay, stability tests were carried out on aliquots of semi-purified KBT. Each sample was exposed to varying amounts of either acid or 
base over time. The activity of each fraction was then quantified by the N2A assay. It was found that the level of activity was largely unaffected by exposure to acid, but was highly sensitive to even small amounts of base (exposure to $p \mathrm{H} 9$ for several hours showed considerable loss of activity). ${ }^{212}$ This work was of extreme importance, as it gave clear guidelines on the potential use of acidic or basic buffers as solvents in the isolation of KBT.

It was also shown that $\mathrm{KBT}$ remained stable in $\mathrm{H}_{2} \mathrm{O} / \mathrm{MeOH}$ or $\mathrm{H}_{2} \mathrm{O} / \mathrm{Me}_{2} \mathrm{CO}$ mixtures at $4^{\circ} \mathrm{C}$ with no noticeable loss of activity. ${ }^{212}$ Finally, initial tests using the dinoflagellate bioassay indicated that KBT is thermally unstable as most of the activity was lost after heating to $100^{\circ} \mathrm{C}$. Nonetheless, after prolonged exposure to boiling $\mathrm{H}_{2} \mathrm{O}$ for 30 minutes, some toxic activity was still noticeable. ${ }^{207}$

\subsection{Isolation}

The isolation of KBT from cultures of $K$. brevisulcata is problematic. Two cultures of KBT have been used to supply material for the purification procedure. The first was without, and the second with, lysing of the algal cells prior to harvest. The first batch, containing less toxic material, has been used as a pilot sample for experimental trials of various isolation methods. Any purification steps that were shown to be effective were performed upon the second sample that contained the larger amount of KBT.

The initial process remained the stepped elution of the HP-20 PSDVB column that the toxic culture media had been passed through. Initially, the column was washed with $\mathrm{H}_{2} \mathrm{O}$ and eluted with five different solvent mixtures $(20,40,60,80$, $100 \% \mathrm{Me}_{2} \mathrm{CO} / \mathrm{H}_{2} \mathrm{O}$ ), with the bulk of the toxicity concentrated in the $60 \% \mathrm{Me}_{2} \mathrm{CO} / \mathrm{H}_{2} \mathrm{O}$ fraction, although detectable amounts of toxicity were noted in both the $40 \%$ and 
$80 \% \mathrm{Me}_{2} \mathrm{CO} / \mathrm{H}_{2} \mathrm{O}$ elutions. For subsequent batches, the elution profile was changed to maximise yield of toxin while reducing the number of fractions collected $\left(\mathrm{H}_{2} \mathrm{O}\right.$ wash, $\left.30,70,100 \% \mathrm{Me}_{2} \mathrm{CO} / \mathrm{H}_{2} \mathrm{O}\right)$.

The next step was to cyclic load the toxic fraction onto a small amount of HP-20S or Amberchrom that was then transferred onto a larger column of PSDVB as a slurry for gradient MPLC elution. The toxin streaked from the column (eg. KBT eluted between 53-83\% $\mathrm{Me}_{2} \mathrm{CO} / \mathrm{H}_{2} \mathrm{O}$ from the HP-20S MPLC column) each time this step was used. This was assumed to be due to either overloading of the column or, more likely, from a mixture of closely related toxic compounds eluting separately.

As a trial separation, aliquots of KBT were run on a PRP-1 (PSDVB) analytical HPLC column using a gradient from $40-70 \% \mathrm{Me}_{2} \mathrm{CO} / \mathrm{H}_{2} \mathrm{O}, p \mathrm{H} 5$, or $p \mathrm{H} 8$ buffer, in order to test the effect of acidic or basic buffers on the elution profile. Surprisingly, it was found that under neutral conditions, two peaks of activity were detected while under both acidic and basic conditions, only one peak was observed. Two repeat injections under neutral conditions confirmed the original response. A fraction collected from each peak of activity was re-injected under neutral conditions. In both cases, only one peak eluted with the same retention time as previously, indicating that the toxicity was not from a mixture of tautomers in equilibrium with each other. This was the first evidence suggesting the presence of more than one toxic compound from cultures of $K$. brevisulcata.

Many other chromatographic separations were attempted with the pilot KBT sample. Column substrates used included further PSDVB (Amberchrom), LH-20 size-exclusion gel, silica gel, and diol. Streaking of the activity was observed with most of the substrates used. Diol was the only substrate that showed any particular promise as a 
suitable column packing material. Initial trials using silica gel had shown that KBT almost irreversibly retained on the column and would only elute in very polar solvent (10-20\% MeOH/EtOAc), which was too polar for adequate separation. Furthermore, only $60 \%$ of the toxicity was recovered from the silica column. Diol is much more stable to polar solvents such as $\mathrm{MeOH}$ and EtOAc, and polar molecules will generally retain less to the substrate, therefore generally eluting earlier than as compared to silica gel. Results from an initial trial using an aliquot of KBT on a small diol column showed that all the toxicity loaded eluted from the column in the $25 \% \mathrm{MeOH} / 75 \% \mathrm{EtOAc}$ fraction, which although no earlier than with silica, was still well within the chromatographic range of the packing material. On the basis of the small-scale diol trial, a larger separation using the pilot sample was performed under flash chromatographic conditions. The sample was loaded in $1 \% \mathrm{EtOAc} / 99 \% \mathrm{CH}_{2} \mathrm{Cl}_{2}$ and eluted with mixtures of $\mathrm{EtOAc} / \mathrm{MeOH}$. Bioassay testing of the fractions generated revealed that four peaks of activity had eluted from the column, the first being the largest in terms of total activity eluted (see figure 5.4). Each step in an isolation procedure generates a large number of samples. In each diol separation, either every fraction or every second fraction had to be quantified. As a result, a substantial number of assays were performed to identify the elution profile of KBT at each stage.

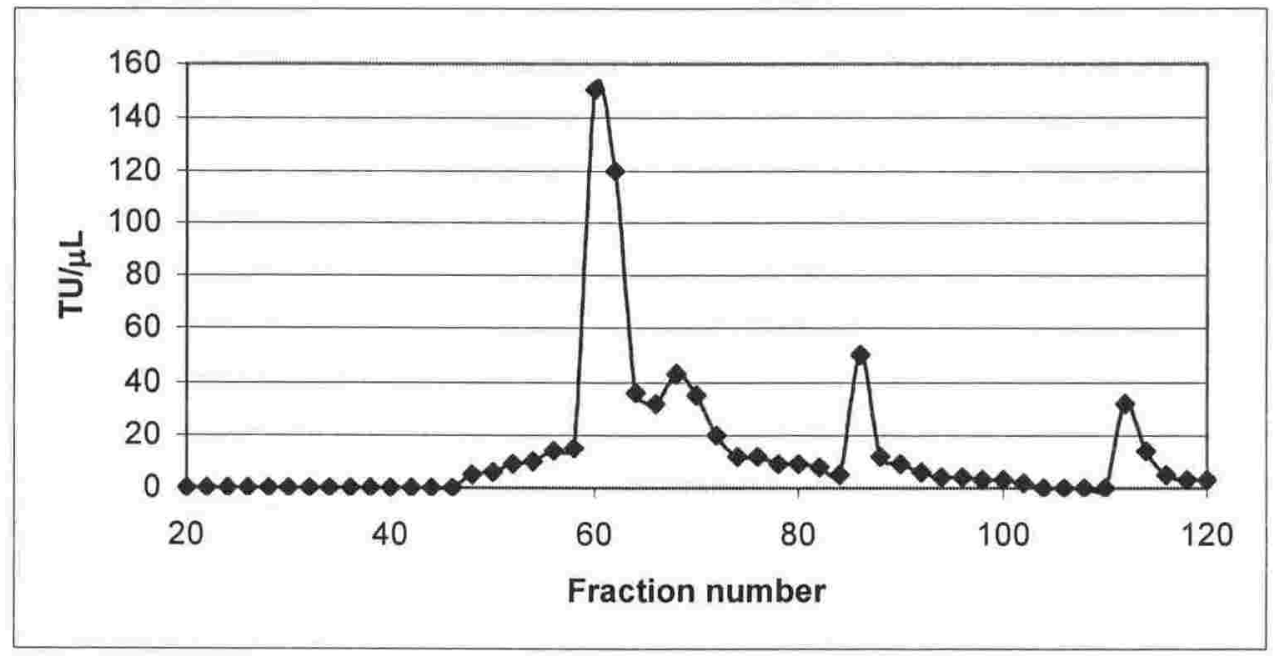

Figure 5.4 Elution profile of KBT after running the pilot sample on a diol column. 
As the pilot KBT sample had been successfully chromatographed using diol stationary support, the major sample of KBT was processed in a similar manner. It had been noted at each stage of the isolation procedure that KBT was only sparingly soluble in all solvents tried, except for $\mathrm{MeOH}$ in which it was partially soluble. Even with $\mathrm{MeOH}$, large volumes were required to dissolve most of the sample. The column was therefore loaded as a suspension of this sample, rather than as a solution. Again, four major peaks of activity were eluted from this diol column.

As before, most of the fractions combined from this fractionation step still showed a large degree of insolubility in most solvents. Unfortunately, the amount of $\mathrm{MeOH}$ required to dissolve the major sample of KBT prohibited the use of HPLC as a method for final purification. As diol is chemically stable to $\mathrm{MeOH}$, it was decided to dissolve the $\mathrm{KBT}$ fully in $\mathrm{MeOH}$ and then cyclic load the solution onto a small amount of diol by diluting with $\mathrm{CH}_{2} \mathrm{Cl}_{2}$. Once loaded, the diol was transferred as a slurry onto a larger MPLC diol column and run under a gradient elution profile from $10-50 \% \mathrm{MeOH} /$ EtOAc. During the flash diol column runs, several of the later peaks of activity eluted when a change in solvent had occurred. This may imply that the peaks of activity were not from several different toxins, but were due to the same toxin streaking off the column, "pulse eluting" with each new solvent front. This theory was strengthened by the observation of the toxicity streaking from the diol MPLC column, where a long tail was noted during elution rather than several peaks. This was similar to what had been observed when earlier MPLC runs using HP-20S had been carried out. 


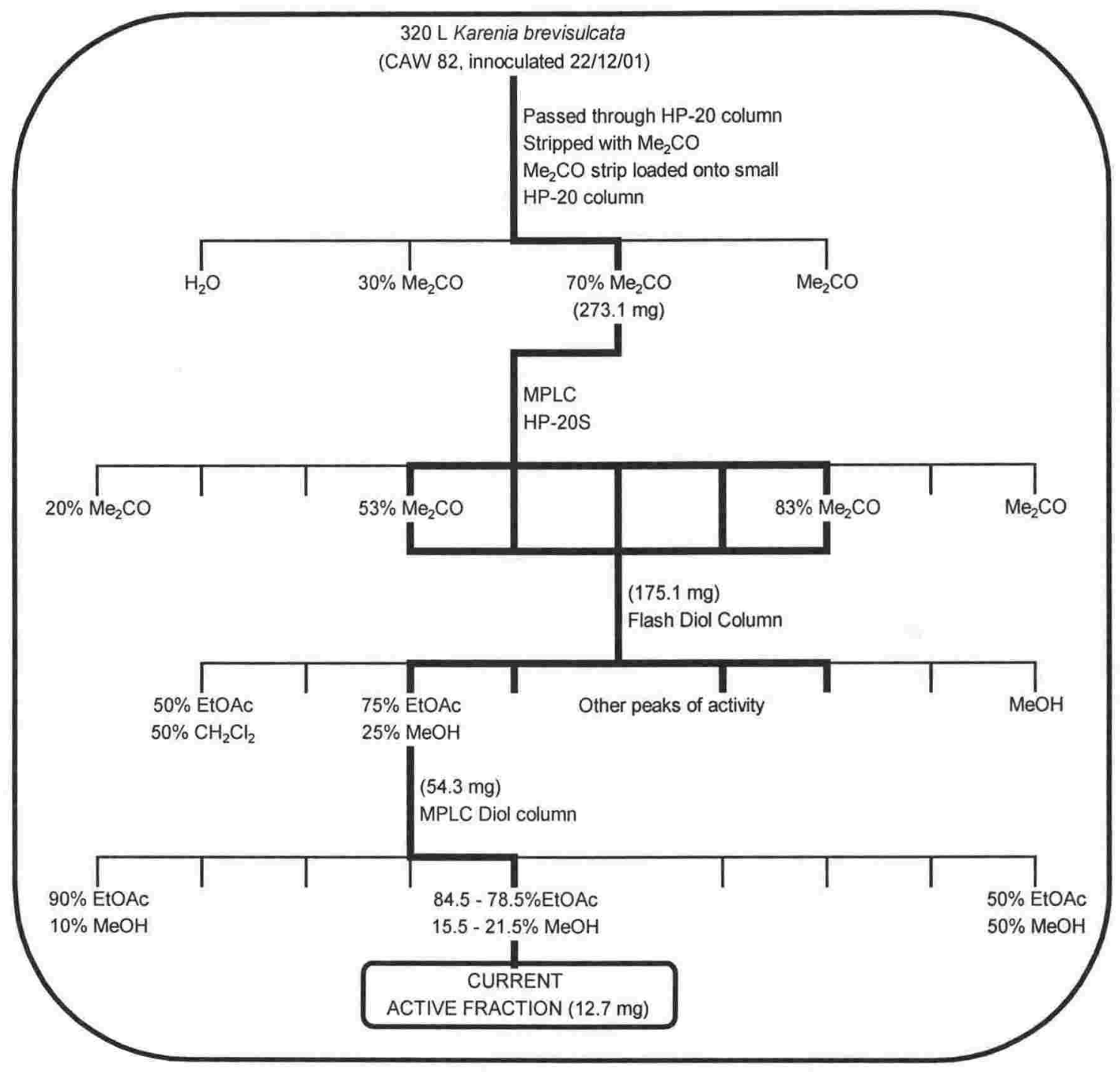

Scheme 5.3 Optimised large-scale isolation scheme for KBT.

Several samples were generated by combining fractions from running the major KBT fraction on the diol MPLC column. Each sample was analysed by NMR. Again, each sample was largely insoluble in the $\mathrm{CD}_{3} \mathrm{OD}$ NMR solvent. As a trial, the samples were concentrated to dryness and re-dissolved in $\mathrm{D}_{6}$-DMSO. Each sample was fully soluble in this solvent. It is thought that KBT becomes less soluble in most organic solvents as it becomes more pure, a problem that has been noted previously in dinoflagellate toxin research. ${ }^{51,213-215}$ This lack of solubility may account for the poor separation and streaking of KBT from the chromatographic substrates used, as any metabolite needs to be fully dissolved in order to achieve maximum chromatographic performance. Unfortunately, DMSO is not a particularly good solvent for chromatography as it is 
viscous, difficult to remove under reduced pressure, and its ability to pass through the skin, possibly carrying with it any dissolved toxic material, makes it a hazardous solvent to use in the isolation of natural toxins. It has been suggested that KBT may be a large molecule as this sometimes correlates with lack of solubility except in DMSO. ${ }^{216}$

Finally, aliquots of KBT were run through amino or CBA (carboxylic acid) ion exchange columns to test whether they would be useful for the final purification of the toxin. It was found that KBT irreversibly binds to the amino substrate, suggesting some kind of acidic functionality (carboxylic or sulfamic acid, phenol etc) in the molecule. KBT was not retained by CBA, suggesting that no basic functionality is present in the molecule. The presence of an acidic and absence of basic functionality may be supported by the observation that KBT is highly sensitive to the presence of bases, and not affected by acids, as noted during the stability tests.

The use of $\mathrm{D}_{6}$-DMSO to dissolve the KBT sample for NMR analysis has highlighted several ${ }^{1} \mathrm{H}$ resonances, the intensities of which appear to wax and wane in a manner similar to the biological activity, as measured with the N2A assay. These resonances are of similar size, as measured by peak integration, and may therefore be from the same molecule. There are also several other resonances that do not follow the biological activity noted in the N2A assay, and whose signal intensity is an order of magnitude larger than that of many of the other resonances noted. This certainly implies that the sample is not yet pure.

\subsection{Spectral Analysis of KBT}

Each fraction generated throughout the isolation using both the pilot and large samples of KBT, was tested using in-house NMR and MS facilities. HRESIMS was attempted 
using both positive and negative ion modes. Many signals were observed in each sample in both modes, although none appeared to follow the biological activity measured in the N2A assay. As noted above, KBT shows a marked lack of solubility in all the solvents trialled except DMSO. This may have hampered opportunities during the early stages of isolation to identify possible NMR signals attributable to KBT. It may also have precluded analysis by HRESIMS, as sufficient KBT may not have been dissolved to allow for detection. In addition, DMSO is not useful as a solvent for analysis by HRESIMS as it can suppress analyte signal. ${ }^{217}$

Extensive analysis of the NMR spectral data of the most pure sample of KBT, collected at Victoria University, proved to be inadequate for a structural analysis. In particular, the $300 \mathrm{MHz}$ spectrometer available did not show enough sensitivity or resolution to be able to generate adequate spectra of KBT. Several NMR experiments were therefore carried out at the University of Canterbury using a $500 \mathrm{MHz}$ spectrometer. The use of a higher-field magnet gave better sensitivity and resolution than obtained previously, allowing for identification of several substructures of KBT. Extensive analysis of the ${ }^{1} \mathrm{H}, \mathrm{COSY}$, HSQC, and TOCSY spectra obtained at $500 \mathrm{MHz}$ revealed at least 97 individual ${ }^{1} \mathrm{H}$ resonances possibly attributable to $\mathrm{KBT}$, falling between 0.67 and $6.48 \mathrm{ppm}$. Of these 97 resonances, 90 correlated to $81{ }^{13} \mathrm{C}$ resonances between 8.5 and 129.2 ppm in an HSQC experiment. Three exchangeable ${ }^{1} \mathrm{H}$ resonances were noted from COSY correlations, observed when the sample was dissolved in $\mathrm{D}_{6}$-DMSO. These resonances are assigned to exchangeable protons as there is no correlation to these resonances in the HSQC spectrum, and the COSY correlations are absent when run using $\mathrm{CD}_{3} \mathrm{OD}$. Finally, it is assumed that all six ${ }^{1} \mathrm{H}$ resonances between 5.88 and 6.04 ppm are attached to carbon, even though four show no direct correlations in the HSQC spectrum (see table 5.1). It should be noted that any analysis of the NMR spectra must be viewed with some caution, as the sample used for the NMR experiments is of an 
unknown purity (there is at least one major impurity in the sample). The sample may also contain several congeners or homologues of a toxin in vary concentrations, and the toxin may be a significantly large molecule, therefore any correlations observed in the TOCSY or COSY spectra may be due to overlapping resonances of different spin systems within the same molecule, or even between different molecules altogether. 


\begin{tabular}{|c|c|c|c|c|}
\hline${ }^{13} \mathrm{C}(\mathrm{ppm})$ & ${ }^{1} \mathrm{H}(\mathrm{ppm})$ & ${ }^{13} \mathrm{C}(\mathrm{ppm})$ & \multicolumn{2}{|c|}{${ }^{1} \mathrm{H}(\mathrm{ppm})$} \\
\hline$?$ & 6.04 & 65.89 & 3.74 & \\
\hline$?$ & 5.99 & 65.63 & 3.79 & \\
\hline$?$ & 5.95 & 65.35 & 3.85 & \\
\hline$?$ & 5.88 & 63.56 & 4.01 & \\
\hline 129.17 & 5.96 & 63.41 & 3.74 & \\
\hline 129.10 & 5.89 & 63.18 & 3.62 & \\
\hline 93.58 & 6.48 & 56.08 & 1.68 & \\
\hline 87.45 & 4.63 & 45.55 & 1.35 & \\
\hline 87.03 & 4.68 & 45.09 & 2.00 & \\
\hline 83.76 & 5.29 & 43.12 & 1.29 & \\
\hline 83.75 & 5.33 & 41.44 & 1.35 & \\
\hline 83.63 & 5.37 & 40.49 & 1.52 & 1.98 \\
\hline 82.18 & 3.23 & 38.28 & 1.46 & 1.76 \\
\hline 81.06 & 3.10 & 38.07 & 1.54 & 1.58 \\
\hline 79.38 & 3.01 & 34.86 & 1.36 & 1.73 \\
\hline 78.51 & 3.33 & 31.35 & 0.67 & 2.16 \\
\hline 78.51 & 3.18 & 31.34 & 1.77 & \\
\hline 78.46 & 3.25 & 31.33 & 1.30 & 2.18 \\
\hline 78.30 & 3.04 & 29.71 & 1.31 & \\
\hline 77.82 & 4.20 & 28.67 & 1.24 & 2.03 \\
\hline 77.54 & 4.07 & 26.99 & 1.31 & 1.76 \\
\hline 77.44 & 4.04 & 21.60 & 1.29 & \\
\hline 76.24 & 3.84 & 21.60 & 1.25 & \\
\hline 76.06 & 3.10 & 21.40 & 1.04 & \\
\hline 75.88 & 2.97 & 21.25 & 1.07 & \\
\hline 75.83 & 2.85 & 21.03 & 1.13 & \\
\hline 75.35 & 3.27 & 20.69 & 1.05 & \\
\hline 74.49 & 3.59 & 20.63 & 1.33 & \\
\hline 73.41 & 3.60 & 20.54 & 1.37 & \\
\hline 73.13 & 4.31 & 20.39 & 1.25 & \\
\hline 72.72 & 3.34 & 20.01 & 1.14 & \\
\hline 72.69 & 3.29 & 19.28 & 1.35 & \\
\hline 71.35 & 3.41 & 18.28 & 1.13 & \\
\hline 71.19 & 3.73 & 16.39 & 1.08 & \\
\hline 71.18 & 3.15 & 15.28 & 1.08 & \\
\hline 70.97 & 3.31 & 14.86 & 1.15 & \\
\hline 70.63 & 3.83 & 14.31 & 1.17 & \\
\hline 70.36 & 3.41 & 14.16 & 1.02 & \\
\hline 70.29 & 3.09 & 9.57 & 0.74 & \\
\hline 70.13 & 3.29 & 8.88 & 1.63 & \\
\hline 70.12 & 4.24 & 8.54 & 0.73 & 1.64 \\
\hline 69.16 & 3.42 & - & $4.91(\mathrm{OH})$ & \\
\hline 69.01 & 3.40 & - & $4.84(\mathrm{OH})$ & \\
\hline 68.75 & 3.78 & - & $4.77(\mathrm{OH})$ & \\
\hline
\end{tabular}

Table 5.1 Observed ${ }^{1} \mathrm{H}$ and ${ }^{13} \mathrm{C}$ NMR resonances of KBT (500 MHz, $\mathrm{D}_{6}$-DMSO).

To help in the identification of KBT, a database of over 1480 individual ${ }^{1} \mathrm{H}-{ }^{13} \mathrm{C}$ onebond NMR correlations was established, containing data from 30 reported algal toxins 
including okadaic acid (156), azaspiracid, two pectenotoxins, several brevetoxins, two ciguatoxins, yessotoxin (162), various saxitoxins, a prymnesin, several amphidinolides and others, as well as the zooanthid toxin palytoxin (see figure 5.5). ${ }^{51,176,178,180,185,187,195,197,214,218-234}$ The most important observation from analysis of the database is that KBT is not one of these compounds. For instance, it is unlikely that KBT is related to the prymnesins or colopsinol $A$ as these structures contain carbohydrate residues whilst KBT shows no evidence of any anomeric acetal or hemiacetal centres. ${ }^{214,215,227}$ Similarly, KBT is unlikely to be a saxitoxin derivative as it obviously contains olefins, a functionality not seen in this class of toxins. ${ }^{178,180,225}$ Finally, many common dinoflagellate toxins, including yessotoxin (162), the brevetoxins, amphidinolides and others, contain exocyclic olefins; there is no evidence that KBT possesses this structural feature. ${ }^{197,221,222,228-230}$ The presence or absence of several other functional classes precludes KBT as being one of the structures of the other common algal toxins, the NMR data of which are contained within the database. This analysis does not preclude KBT being a congener or homologue of one of these compounds or classes.

As well as being used to establish that KBT is not a common dinoflagellate toxin, the database was also used to identify chemical environments that may give rise to the various observed correlations in the COSY, TOCSY, and HSQC spectra of KBT. Many of the correlations identified in the HSQC spectrum of KBT are indicative of oxygenated methines, the majority of which are consistent with polycyclic-ether functionality. From a chemotaxonomic perspective, this is entirely consistent with the many toxins already isolated from dinoflagellates. 


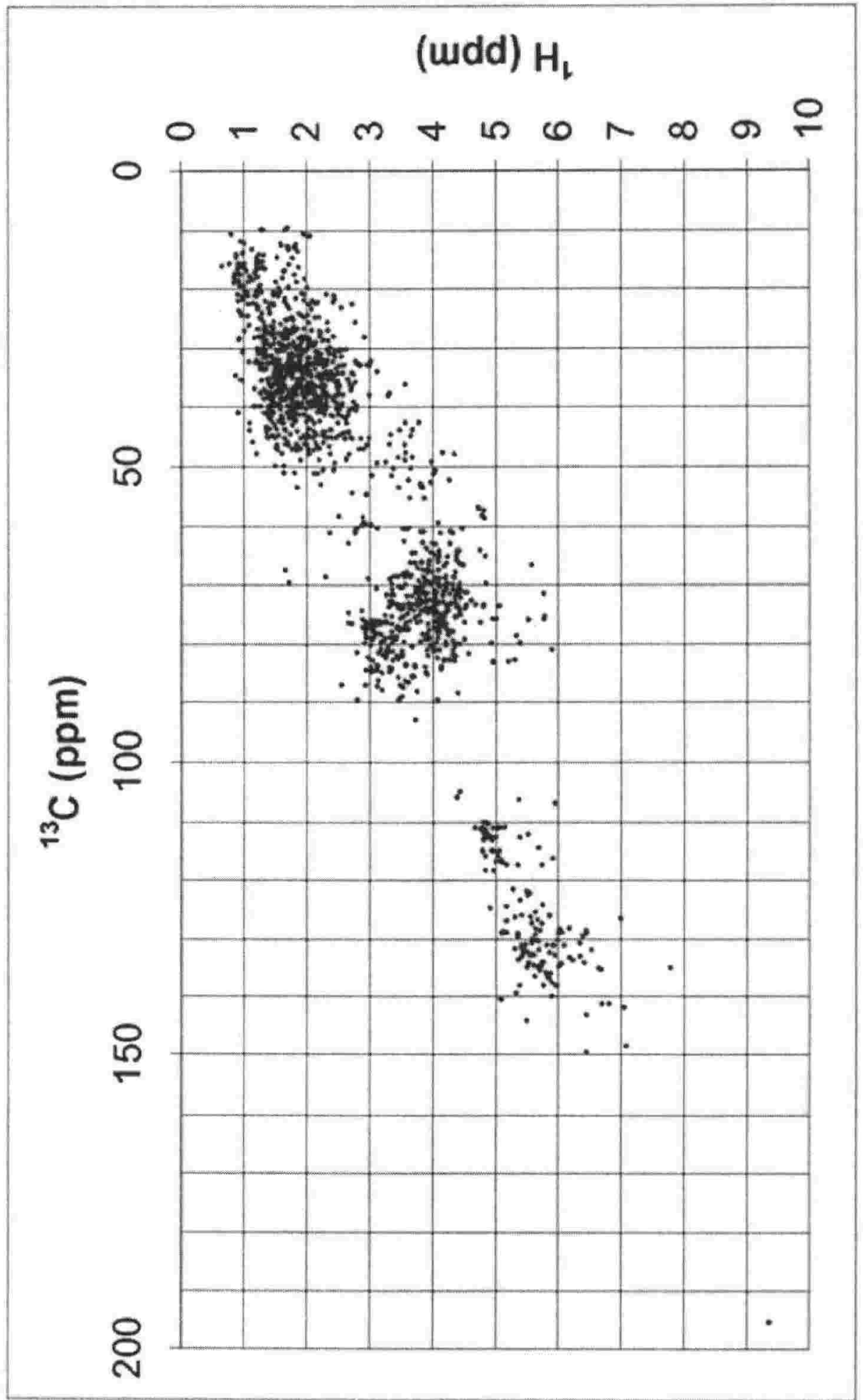

Figure $5.5{ }^{1} \mathrm{H}^{13} \mathrm{C}$ one-bond correlations of common dinoflagellate toxins. 
Detailed analysis of the COSY, TOCSY and HSQC correlations of KBT have allowed some possible substructures to be constructed. There are six ${ }^{1} \mathrm{H}$ resonances near $6 \mathrm{ppm}$, coupled together in three pairs of disubstituted olefins as evidenced by COSY correlations. The chemical shifts of these ${ }^{1} \mathrm{H}$ resonances are indicative of non-polarised olefins, as each pair of resonances do not differ significantly from each other. The shifts, however, are de-shielded from what would be expected for an isolated olefin in an aliphatic chain and are, in fact, more consistent with the resonances of a conjugated diene system, or possibly an olefin within a small ring system. Conjugated diene systems are precedented, as both the prymnesins and palytoxin (164) contain this functionality. In particular, 164 has two pairs of conjugated dienes, one of which has chemical shifts similar to those observed in KBT (see figure 5.6). ${ }^{195}$ Palytoxin (164) is not a valid candidate structure for KBT, however, as several other prominent NMR signals from it are absent in KBT. For example, 164 contains 15 olefinic ${ }^{1} \mathrm{H}-{ }^{13} \mathrm{C}$ correlations, including one with a ${ }^{1} \mathrm{H}$ resonance at $\delta_{\mathrm{H}} 7.79$ which is significantly less shielded than any observed in $\mathrm{KBT} .^{231}$

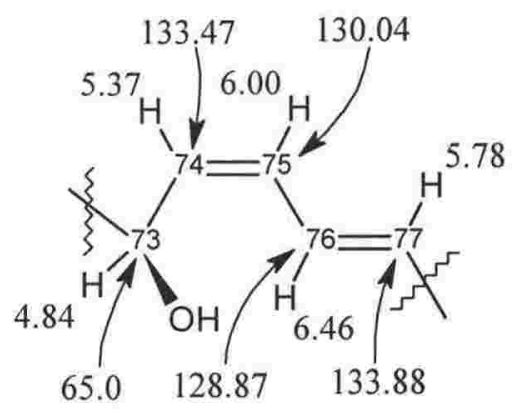

Figure 5.6 Selected NMR chemical shifts of palytoxin (164). ${ }^{231}$

Furthermore, analysis of observed COSY cross-peaks indicates a connection between two olefinic protons $\left(\delta_{\mathrm{H}} 5.96, \mathrm{~d} 4.9 \mathrm{~Hz} ; \delta_{\mathrm{H}} 6.04, \mathrm{~d}, 5.9 \mathrm{~Hz}\right)$, each connected to one of two oxygenated methines $\left(\delta_{\mathrm{C}} 87.45, \delta_{\mathrm{H}} 4.63 ; \delta_{\mathrm{C}} 83.76, \delta_{\mathrm{H}} 5.29\right)$. Each olefinic resonance shows only one coupling, to the other, in the ${ }^{1} \mathrm{H}$ NMR spectrum (evidenced 
by the multiplicity of the ${ }^{1} \mathrm{H}$ resonances), implying a dihedral angle of approximately $90^{\circ}$ between the olefinic and oxygenated methine protons. Both the oxygenated methine ${ }^{1} \mathrm{H}$ resonances show a COSY correlation to each other. Based upon these observations, it appears that this system may be a 1,4-disubstituted $\Delta^{2,3}$-dihydrofuran ring, although there is no precedence for this functionality in the natural products literature. The de-shielded chemical shift of the ${ }^{1} \mathrm{H}$ resonance at $5.29 \mathrm{ppm}$ may be explained by the proximity of another oxygenated methine $\left(\delta_{\mathrm{C}} 78.46, \delta_{\mathrm{H}} 3.25\right)$ to which it is coupled, as indicated by cross-peaks in both the COSY and TOCSY spectra. The ${ }^{1} \mathrm{H}$ resonance at $4.63 \mathrm{ppm}$ is coupled further to another methine proton $\left(\delta_{\mathrm{H}} 1.29\right)$. There is severe spectral overlap in the ${ }^{1} \mathrm{H}$ spectrum at this frequency that precludes extension of this spin system, even though there are several other COSY and TOCSY correlations observed from this resonant frequency (see figure 5.7).

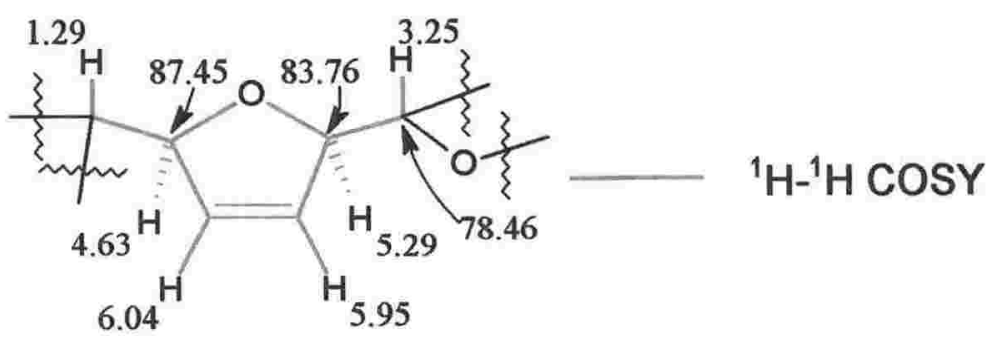

Figure 5.7 Proposed NMR chemical shifts of a 1,4-disubstituted $\Delta^{2,3}$-dihydrofuran ring in KBT.

A similar system that may also be considered for these resonances is a substituted 1,2dioxene ring, formed by the Diels-Alder addition of singlet $\mathrm{O}_{2}$ to a diene to form a peroxide. This type of functionality is precedented in the natural products literature, although the ${ }^{1} \mathrm{H}$ and ${ }^{13} \mathrm{C}$ chemical shifts of the resonances from these molecules do not compare favourably with those observed for KBT (see figure 5.8). ${ }^{235}$ 


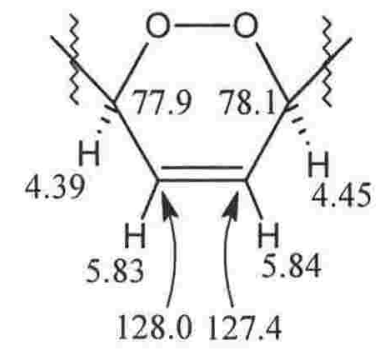

Figure 5.8 NMR chemical shifts of a 1,2-dioxene ring. ${ }^{235}$

The closest match for the chemical shifts of the observed resonances of KBT comes from the ciguatoxin congeners CTX-3C (165) and C-CTX-1 (166). ${ }^{232,236}$ Both 165 and 166 have the same structure from rings B to J. In particular, ring D consists of an olefinic seven-membered oxygenated heterocycle. The chemical shifts of the olefin, and the oxygenated methines adjacent to the olefin, are similar to those observed in KBT (see figure 5.9). ${ }^{232,236}$ It is unlikely, however, that KBT contains a ring similar to this, as the COSY correlation observed between the two oxygenated methine ${ }^{1} \mathrm{H}$ resonances of KBT is stronger (implying a stronger coupling) than what would be expected for a purely homo-allylic coupling that would give rise to such a cross-peak in the ciguatoxin derivatives. Also, the coupling constants of the olefinic protons measured in KBT (5.9 $\mathrm{Hz}, 4.9 \mathrm{~Hz}$ ) differ substantially from those measured in CTX-3C (165) (both $13 \mathrm{~Hz}$ ) or C-CTX-1 (166) $(13 \mathrm{~Hz}, 7 \mathrm{~Hz})$, indicating a different conformation of these protons from those of KBT (see figure 5.9). ${ }^{232,236}$

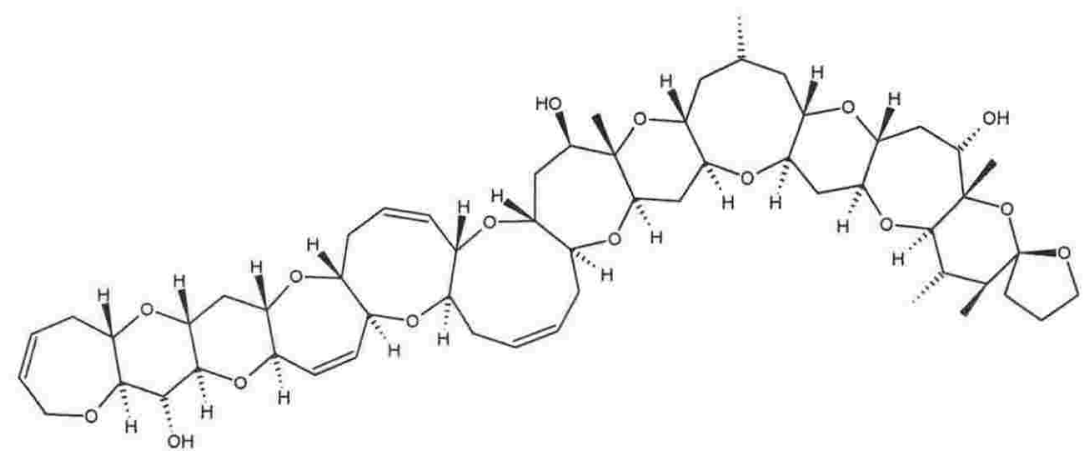

(165) 


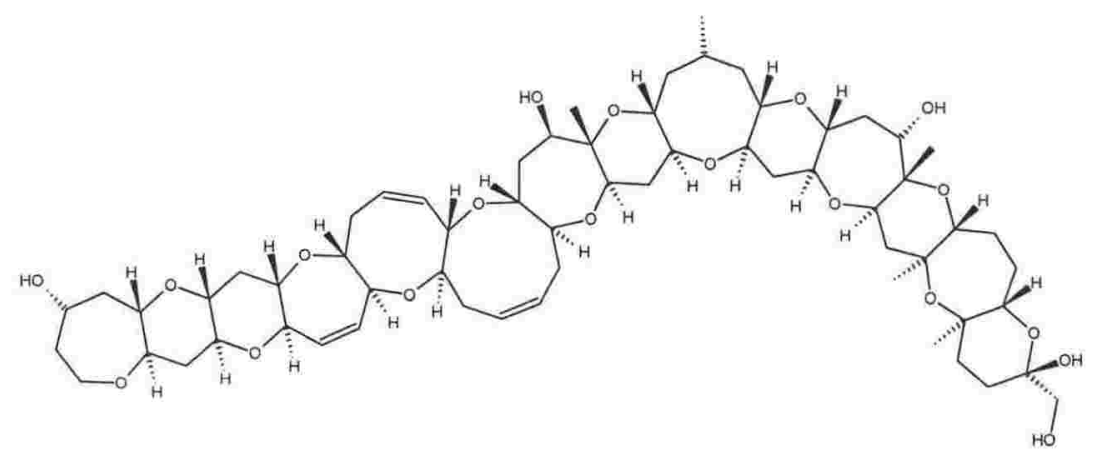

(166)

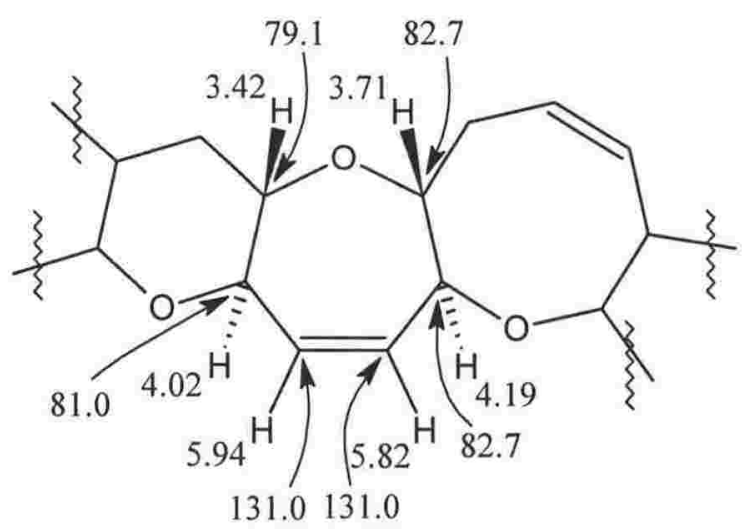

Figure 5.9 Selected NMR chemical shifts of C-CTX-1 (166). ${ }^{232}$

A second series of correlations was noted in the COSY and TOCSY spectra. Three exchangeable ${ }^{1} \mathrm{H}$ resonances $\left(\delta_{\mathrm{H}} 4.91,4.84,4.77\right)$ all appear to show strong correlations to a methine proton $\left(\delta_{\mathrm{H}} 4.00\right)$. Although an ortho-acid functionality $\left(\mathrm{R}-\mathrm{C}(\mathrm{OH})_{3}\right)$ is not without precedence in organic chemistry, there is no precedent for such a functional group in the natural products literature. It is also unlikely that such a functional group would show such strong ${ }^{4} J_{\mathrm{H}, \mathrm{H}}$ correlations to a proton on an adjacent carbon. It is therefore much more likely that the three exchangeable ${ }^{1} \mathrm{H}$ resonances each correlate to a different methine proton, all of which resonate at the same frequency $\left(\delta_{\mathrm{H}} 4.00\right)$. This may be indicative of three repeating structural units within KBT, or may suggest that the sample is a mixture of three or more congeners. Finally, several other isolated substructures have been identified through COSY correlations between their attached ${ }^{1} \mathrm{H}$ resonances. All these substructures are detailed below (see figure 5.10). 

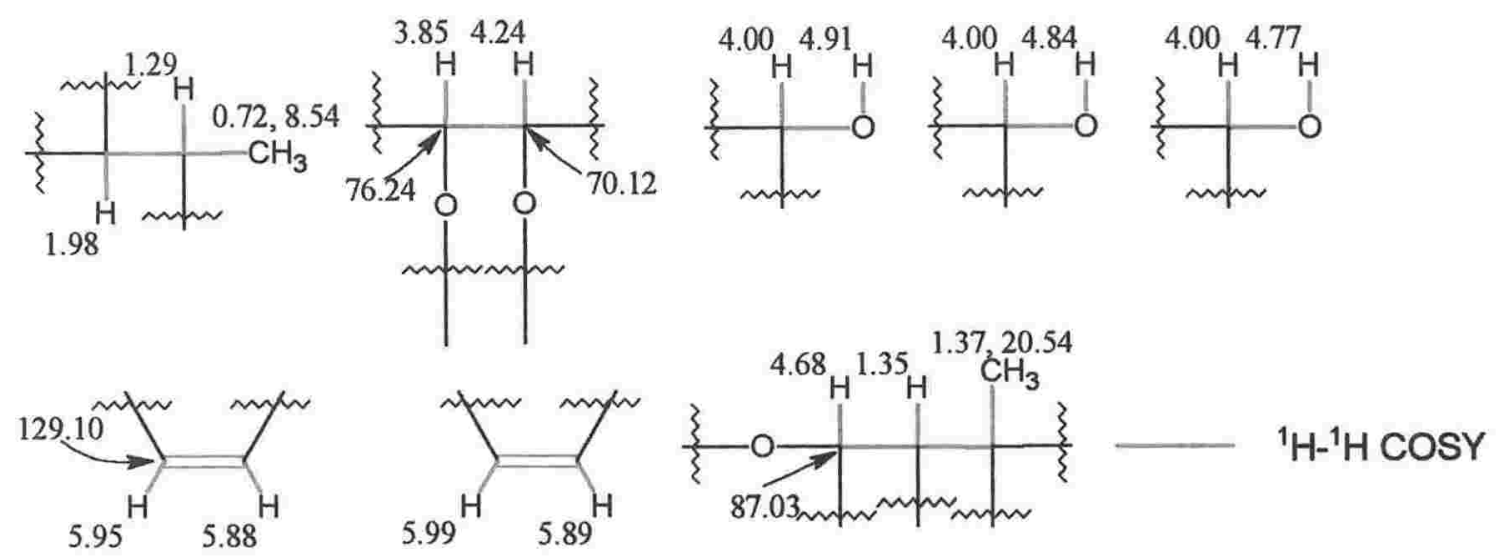

Figure 5.10 Possible substructures and NMR chemical shifts of KBT.

\subsection{Biological Activity of KBT}

KBT is a toxin and therefore has potent biological activity. Initially, after harvesting of the culture media by lysing of the cells and elution of the HP-20 column, the toxic sample of KBT had an N2A activity of $9.2 \times 10^{6} \mathrm{TU} / \mathrm{mg}$. The most pure active fraction has an N2A activity of $2.7 \times 10^{6} \mathrm{TU} / \mathrm{mg}$. Analysis of a semi-purified sample of KBT from $100 \mathrm{~L}$ of $K$. brevisulcata has shown that the toxin has an $\mathrm{LD}_{50} \sim 500 \mathrm{TU} / \mathrm{mouse}$ when injected $\mathbb{I P} .^{237}$ The most pure sample at the current level of activity therefore has as an $\mathrm{LD}_{50} \sim 200 \mathrm{ng} /$ mouse IP (i.e. $\mathrm{LD}_{50} \sim 10 \mu \mathrm{g} / \mathrm{kg} \mathbb{I P}, \mathrm{KBT}$ can kill $500020 \mathrm{~g}$ mice per mg).

The symptoms of KBT in mice are most pronounced at high levels of exposure. At IP doses of 50,000 $\mathrm{TU}$, toxic effects were noted soon after injection. The mice became hunched and lethargic, and respiration slowed with cyanosis becoming apparent. Death occurred approximately five hours after dosing. At doses between 5,000 and 20,000 TU, the mice were unaffected for several hours with symptoms only becoming apparent later; the animals died between ten and thirteen hours after dosing. When dosed with between 500 and 2,000 TU, the mice showed no symptoms for 24 hours. After this time, similar signs of toxicity were noted as before. No recovery was made after the 
symptoms became apparent. The mice dosed at these lower levels became progressively more lethargic and were destroyed on humanitarian grounds. ${ }^{237}$

Histological studies were carried out on mice killed by KBT. Mice injected with between $400,000-800,000$ TU show significant necrosis of various tissues. KBT appears to be a nephrotoxin (damaging kidney tissue) although the major target appears to be striated muscle tissue, including the diaphragm and, more importantly, the heart. Heart muscle, unlike skeletal muscle, is not able to regenerate after injury. KBT could therefore cause irreparable damage to the heart, with repeated exposure of small doses of KBT possibly showing cumulative damage to the organ. ${ }^{237}$ At this stage, it is unknown whether KBT can be absorbed through the skin or lungs. Absorption of KBT through the respiratory tract would be of particular concern, as people were affected by breathing salt spray/live cells during the original $K$. brevisulcata bloom. ${ }^{44,207}$ If KBT does act in this way then people originally affected may have already suffered irreparable heart damage. This type of biological activity is unique amongst dinoflagellate toxins. In the future, another bloom of $K$ brevisulcata could pose a major health risk to any person exposed. If the toxin is absorbed through the respiratory tract, then people exposed to sea spray would be at risk of heart damage. Workers in the shellfish industry would also be exposed.

It should be noted that mice were orally dosed with up to 2,000,000 TU each of KBT with no obvious sign of toxicity. There is therefore some barrier to absorption of KBT in the gut. This may or may not imply that there would be barriers to adsorption through the skin or lungs. ${ }^{237}$

The harvesting of $320 \mathrm{~L} \mathrm{~K}$. brevisulcata culture yielded $2,500 \times 10^{6} \mathrm{TU}$. The cell concentration of this culture peaked at 7,060,000 cells/L $\left(\sim 2,260 \times 10^{6}\right.$ total cells in the 
culture) prior to harvesting. ${ }^{238}$ This implies that the toxic production is $\sim 1.1 \mathrm{TU} /$ cell. The cell concentration of $K$. brevisulcata peaked in Wellington Harbour at $33 \times 10^{6}$ cells/L on March $13^{\text {th }} 1998$, implying that each L of seawater contained $~ 36.3 \times 10^{6} \mathrm{TU}$ or enough KBT to kill 72,600 mice via IP injection. ${ }^{44,237}$

\subsection{Further Work on KBT}

The final isolation and structural elucidation of KBT remains incomplete. Dinoflagellate toxins are often large and complex metabolites that require sophisticated instrumental techniques to identify. ${ }^{196,239-243}$ Several reported dinoflagellate toxins constitute the largest natural products isolated, and some have suggested that investigation of these "middle weight" (intermediate between small secondary metabolites and biopolymers consisting of proteins, lipids and oligosaccharides) metabolites will become a separate area of research within natural products chemistry. ${ }^{196}$ Molecules of this size pose substantial challenges in both isolation and structural elucidation, and several have taken many years to identify. ${ }^{5,196,231,244-246}$ Unfortunately, New Zealand does not possess adequate NMR and MS facilities capable of successfully identifying the more complex dinoflagellate toxins, be they novel or previously reported. It is therefore likely that the current sample of KBT will be sent to an overseas research group to complete the isolation and structural elucidation studies. Once this work is completed, and if the toxin proves to be novel, then methods may be developed to help in the detection of this metabolite that may be integrated into standard shellfish-monitoring programs. It is also likely that several congeners of KBT are produced by $K$. brevisulcata and once one has been identified, strategies for the detection, isolation, and elucidation of these related compounds can be established. The establishment of chemical methods to test for KBT is of major importance in light of the extremely potent and possibly irreparable damage that the toxin can cause in mammals. Chemical methods of detection would also be 
instrumental in helping isolate more KBT to carry out further studies on the biological activity of the molecule, and in determining the mode of action of the observed activity. Finally, other uses of the toxin may be found. For example, several dinoflagellate toxins have been instrumental in elucidating the mechanisms by which several biological processes work. $^{42}$ It may be that the novel biological activity of KBT makes this metabolite useful for research, or as a component in other general technologies. 


\section{Chapter Six}

\section{Concluding Remarks}

The isolation of novel secondary metabolites from marine organisms relies on natural products chemists selecting a suitable organism to investigate, using a robust method by which to guide them through an isolation procedure, and by applying various separation techniques to purify the target molecules. These three concepts are all intimately linked. In the course of this study, natural products from two different phyla were examined, each requiring the use of contrasting approaches. Various species of marine sponge

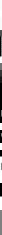


isolated during this study do not possess functionality that would be observable in West's original protocol; the majority of the ${ }^{1} \mathrm{H}$ resonances of these molecules are masked in the ${ }^{1} \mathrm{H}$ NMR spectrum by other resonances from common primary metabolites.

Of the eight compounds that West isolated using the original protocol, one exhibited anti-inflammatory activity whilst the remaining seven were inactive in the assays used. $^{50}$ Of the novel compounds isolated during this study that were submitted for bioassay, all exhibited moderate anti-inflammatory activity. The isolation of these compounds validates the use of reversed-phase stationary supports for the initial prescreen fractionation, and also the use of $\mathrm{CD}_{3} \mathrm{OD}$, rather than $\mathrm{CDCl}_{3}$, as the $\mathrm{NMR}$ solvent of choice in the screening process. Reversed-phase supports are better suited than silica gel for isolating mid-polarity molecules, which will often be biologically active, therefore they are more applicable for a pre-screen purification. Although $\mathrm{CD}_{3} \mathrm{OD}$ is much more expensive than $\mathrm{CDCl}_{3}$, it is also more polar and therefore its use as the NMR solvent in the screening process increases the likelihood of dissolving the various intermediate polarity compounds present in a semi-purified extract.

The use of reversed-phase solid supports has also been instrumental in the isolation of several spongian diterpenes of a more polar nature than those that have been previously reported. Several of these compounds represent likely intermediates in proposed biogenetic sequences, strengthening the suggested pathways. It is doubtful that these metabolites would have been isolated using traditional silica gel based isolation techniques.

The attempted isolation of $\mathrm{KBT}$ has been a long and difficult project. The isolation process has been complicated at various stages by lack of quantitative assays, the time 
taken to receive assay results, and the insolubility of the toxin itself. However, a semipurified toxin has been isolated which does not appear to be a common, previously reported, metabolite. The potent biological activity and possible unique structure of this molecule make it an important candidate for further investigation.

The biological (bioassay) and chemical (spectral) approaches to organism selection and isolation guidance have both inherent strengths and weaknesses. Neither is better or worse than the other. The NMR based system allows for all testing to be carried out inhouse and is also useful for the possible dereplication of known metabolites. It suffers, however, in being a relatively insensitive technique requiring milligram quantities of material, and there is no guarantee that molecules isolated using this strategy will be biologically active. Conversely, bioassays will often need to be performed off-site and may slow an isolation procedure dramatically. There is also limited possibility of structure dereplication of known compounds. The advantages of a bioassay system are that it is much more sensitive than an NMR based method and the molecules targeted will always be biologically active. Both methods have been utilised successfully in this study. 


\section{Chapter Seven}

\section{Experimental}

\section{1}

\section{General Methods}

Unless otherwise noted, all NMR spectra were recorded using a Varian Unity-Inova 300 spectrometer operating at $300 \mathrm{MHz}$ for ${ }^{1} \mathrm{H}$ and $75 \mathrm{MHz}$ for ${ }^{13} \mathrm{C}$. The ${ }^{13} \mathrm{C}$ NMR spectra of clathriol B (33), cadlinolide C (138) and methylpourewate B (142) were recorded using a Brüker Avance 300 spectrometer operating at $75 \mathrm{MHz}$. The ${ }^{13} \mathrm{C}$ NMR spectrum of pourewanone (143) was recorded using a Brüker Avance 400 spectrometer operating at $100 \mathrm{MHz}$, while the ${ }^{1} \mathrm{H}, \mathrm{COSY}$, TOCSY and HSQC spectra of KBT were recorded using a Varian Unity-Inova 500 spectrometer operating at $500 \mathrm{MHz}$ for ${ }^{1} \mathrm{H}$ and $125 \mathrm{MHz}$ for ${ }^{13} \mathrm{C}$. All chemical shifts ( $\delta$ ) were referenced to the residual solvent peak. ${ }^{247}$ HRESIMS were obtained using a PE Biosystem Mariner 5158 TOF mass spectrometer. Infrared spectra were recorded using a Biorad FTS-7 spectrometer or a Brüker Tensor 27 spectrometer to $\pm 2 \mathrm{~cm}^{-1}$. UV/vis spectra were recorded using a HewlettPackard 8452A diode array spectrometer or a Varian Cary 100 spectrometer to $\pm 1 \mathrm{~nm}$. Optical rotations were recorded using a Perkin-Elmer 241 polarimeter.

MPLC and HPLC were performed using a Rainin Dynamax SD-200 HPLC system coupled to a Rainin UV-1 detector. All solvents for MPLC either were analytical reagent grade or glass distilled before use. Solvents for HPLC were all analytical reagent grade. $\mathrm{H}_{2} \mathrm{O}$ for MPLC and HPLC was glass distilled and deionised using a MilliQ system. All solvent mixtures are reported as \% vol/vol. Reversed-phase HPLC was performed using either a Hamilton PRP-1 PSDVB column or a Phenomenex Prodigy ODS column [analytical $(0.46 \times 25 \mathrm{~cm})$ or semi-preparative $(1.0 \times 25 \mathrm{~cm})]$. 
TLC analyses were performed using Merck Kieselgel (Alufoilen) $60 \quad \mathrm{~F}_{254}$ (normal-phase) or Macherey-Nagel (Alugram) RP-18 W/UV 254 (reversed-phase). TLC plates were visualised by (1) fluorescence quenching under UV light ( $\lambda=254 \mathrm{~nm})$, (2) spraying with $50 \% \mathrm{MeOH} / \mathrm{H}_{2} \mathrm{SO}_{4}$ and then heating, or (3) spraying with $50 \% \mathrm{MeOH} / \mathrm{H}_{2} \mathrm{SO}_{4}$, dipping in $1 \%$ vanillin/EtOH, and then heating. Molecular size-exclusion chromatography was performed using Sephadex LH-20. Reversed-phase chromatography on PSDVB was performed using HP-20 (Mitsubishi), HP-20S (Supelco), or Amberchrom CG-161M (Tosohaas). Kieselgel 60 (230-400 mesh ASTM) or International Sorbent Technologies Diol was used for normal-phase chromatography.

\subsection{Revised Sponge-Screening Protocol}

Extraction and Cyclic Loading: The sample of sponge ( 100 g) was cut into small cubes ( $2 \mathrm{~cm}$ per side) and was extracted twice with $\mathrm{MeOH}(2 \times 250 \mathrm{~mL})$ for $12 \mathrm{~h}$. The second extract was passed through an HP-20 column $(2.5 \times 13 \mathrm{~cm})$ pre-equilibrated with $50 \% \mathrm{MeOH} / \mathrm{H}_{2} \mathrm{O}(300 \mathrm{~mL})$. The first extract was then passed through the same column and its eluent was combined with that from the second extract. The combined eluants were diluted with $\mathrm{H}_{2} \mathrm{O}(1 \mathrm{~L})$ and were passed back through the same column. Finally, the eluent was diluted further with $\mathrm{H}_{2} \mathrm{O}(2 \mathrm{~L})$ and was passed again through the same column.

Elution and Backloading: The column was then washed with $\mathrm{H}_{2} \mathrm{O}(150 \mathrm{~mL})$ and was eluted with $150 \mathrm{~mL}$ fractions of (1) $30 \% \mathrm{Me}_{2} \mathrm{CO} / \mathrm{H}_{2} \mathrm{O}$, (2) $75 \% \mathrm{Me}_{2} \mathrm{CO} / \mathrm{H}_{2} \mathrm{O}$ and (3) $\mathrm{Me}_{2} \mathrm{CO}$. Fraction (2) was diluted with $\mathrm{H}_{2} \mathrm{O}(150 \mathrm{~mL})$ and was passed through an HP-20 column $(1.8 \times 15 \mathrm{~cm})$ which had been pre-equilibrated with $35 \% \mathrm{Me}_{2} \mathrm{CO} / \mathrm{H}_{2} \mathrm{O}$ (150 mL). The eluent was further diluted with $\mathrm{H}_{2} \mathrm{O}(200 \mathrm{~mL})$ and was then passed again 
through the same column. This column was washed with $\mathrm{H}_{2} \mathrm{O}(150 \mathrm{~mL})$ and then eluted with $\mathrm{Me}_{2} \mathrm{CO}(150 \mathrm{~mL})$.

NMR: The $\mathrm{Me}_{2} \mathrm{CO}$ fraction was evaporated to dryness and was then analysed by ${ }^{1} \mathrm{H}$, COSY, and HSQC NMR (CD $\left.{ }_{3} \mathrm{OD}, 750 \mu \mathrm{L}\right)$.

${ }^{1}$ H NMR: $\quad$ Spectrum narrowed -0.5 to $15.0 \mathrm{ppm}$

32 scans

Time 0 h $02 \mathrm{~min}$

COSY NMR: $\quad$ Spectrum narrowed 0.5 to $8.5 \mathrm{ppm}$

d1 relaxation $=2 \mathrm{sec}$

1 scan

512 increments per scan

Time $0 \mathrm{~h} 21 \mathrm{~min}$

HSQC NMR: $\quad$ Spectrum narrowed 0.5 to $8.5 \mathrm{ppm}$ in ${ }^{1} \mathrm{H}$ dimension

Spectrum narrowed 1 to $161 \mathrm{ppm}$ in ${ }^{13} \mathrm{C}$ dimension

d1 relaxation $=1.5 \mathrm{sec}$

8 scans

512 increments per scan

Time 4 h 08 min

7.3 Sponges and Amounts Screened Using the Above Protocol that were

Found to Contain Interesting Secondary Metabolites

MNP0090 Clathria lissosclera (134 g)

MNP0196 (115 g)

MNP0241 (132 g)

MNP0352 Biemna sp. (103 g)

MNP0355 Zyzzya sp. (162 g) 
MNP0707 Axinella sp. (93 g)

MNP0979 Chelonaplysilla violacea $(110 \mathrm{~g})$

Isolation of $5 \alpha, 8 \alpha$-epidioxy-6-ene-24- $R$-ethylcholesta-3 $\beta$-ol (7) and a

Sphingolipid from Biemna sp. (MNP0352)

MNP0352 (971 g) was extracted with MeOH $(2 \times 2 \mathrm{~L})$. The two extracts were cyclic loaded to $25 \% \mathrm{MeOH} / \mathrm{H}_{2} \mathrm{O}$ onto a large column of $\mathrm{HP}-20(6.3 \times 15 \mathrm{~cm})$. The column was washed with $\mathrm{H}_{2} \mathrm{O} \quad\left(\begin{array}{ll}1 & \mathrm{~L}\end{array}\right)$ and then eluted with $1.5 \mathrm{~L}$ fractions of (1) $30 \% \mathrm{Me}_{2} \mathrm{CO} / \mathrm{H}_{2} \mathrm{O}$, (2) $50 \% \quad \mathrm{Me}_{2} \mathrm{CO} / \mathrm{H}_{2} \mathrm{O}$, (3) $70 \% \quad \mathrm{Me}_{2} \mathrm{CO} / \mathrm{H}_{2} \mathrm{O}$, (4) $80 \% \mathrm{Me}_{2} \mathrm{CO} / \mathrm{H}_{2} \mathrm{O}$, and (5) $\mathrm{Me}_{2} \mathrm{CO}$. Fractions (1) and (2) were combined and cyclic loaded onto a small amount of HP-20S $(\sim 20 \mathrm{~mL})$ which was transferred onto an HP-20S MPLC column $(2.5 \times 45 \mathrm{~cm})$ as a slurry. This column was eluted using a gradient profile from $0-100 \% \mathrm{Me}_{2} \mathrm{CO} / \mathrm{H}_{2} \mathrm{O}$. The fractions from $60-80 \% \mathrm{Me}_{2} \mathrm{CO} / \mathrm{H}_{2} \mathrm{O}$ were combined, on the basis of TLC chars, and then chromatographed on a Sephadex LH-20 column $(1.8 \times 90 \mathrm{~cm})$ using $90 \% \mathrm{MeOH} / \mathrm{H}_{2} \mathrm{O}$ as the eluting solvent. Fractions with similar TLC chars were combined to yield an impure sample of a sphingolipid (11.8 mg).

Fraction (5) (493 mg) from the initial HP-20 column was loaded onto a silica gel column $(2.5 \times 20 \mathrm{~cm})$ using petroleum ether and was then chromatographed using a stepped elution profile with $150 \mathrm{~mL}$ portions of (1) petroleum ether, (2) $50 \% \mathrm{CH}_{2} \mathrm{Cl}_{2}$ /petroleum ether, (3) $\mathrm{CH}_{2} \mathrm{Cl}_{2}$, (4) $5 \% \quad \mathrm{EtOAc} / \mathrm{CH}_{2} \mathrm{Cl}_{2}$, (5) $10 \% \mathrm{EtOAc} / \mathrm{CH}_{2} \mathrm{Cl}_{2}$, (6) $25 \% \quad \mathrm{EtOAc} / \mathrm{CH}_{2} \mathrm{Cl}_{2}$, (7) $50 \% \mathrm{EtOAc} / \mathrm{CH}_{2} \mathrm{Cl}_{2}$, and (8) EtOAc. Several fractions eluted with portion (7) were combined (72.1 mg) on the basis of TLC chars. The combined fraction was loaded onto a second silica gel column $(2.5 \times 10 \mathrm{~cm})$ in petroleum ether. The column was eluted using a stepped elution profile 
with (1) $10 \% \mathrm{Et}_{2} \mathrm{O}$ /petroleum ether $(50 \mathrm{~mL}),(2) 20 \% \mathrm{Et}_{2} \mathrm{O} /$ petroleum ether $(50 \mathrm{~mL})$, (3) $50 \% \mathrm{Et}_{2} \mathrm{O} /$ petroleum ether $(100 \mathrm{~mL})$, and (4) $\mathrm{Et}_{2} \mathrm{O}(150 \mathrm{~mL})$. The fractions eluted with $\mathrm{Et}_{2} \mathrm{O}$ were combined $(31.9 \mathrm{mg})$ and then chromatographed on a Sephadex LH-20 column $(1.8 \times 90 \mathrm{~cm})$ using $50 \% \mathrm{MeOH} / \mathrm{CH}_{2} \mathrm{Cl}_{2}$ as the eluting solvent. Several fractions that had similar TLC chars were combined to give $5 \alpha, 8 \alpha$-epidioxy-6-ene-24- $R$ ethylcholesta-3 $\beta$-ol (7) (23.8 mg).

Sphingolipid: Pale yellow solid; NMR data, ${ }^{1} \mathrm{H}$ NMR (D $\left.-\mathrm{DMSO}, 300 \mathrm{MHz}\right) \delta 5.74$, $4.59,4.01,3.94,3.91,3.86,3.71,3.17,2.96,2.91,2.57,2.30,1.51,1.23,0.87,0.85$ ${ }^{13} \mathrm{C}$ NMR (D6-DMSO, $\left.75 \mathrm{MHz}\right) \delta 98.3,74.1,73.4,73.1,71.8,69.0,68.3,67.1,65.7$, $62.2,59.9,55.0,54.3,44.8,36.3,34.8,33.5,31.2,26.4,24.5,23.2,22.0,13.8$; HRESIMS, obsd. $m / z 555.8756$.

5 $\alpha, 8 \alpha$-epidioxy-6-ene-24- $\boldsymbol{R}$-ethylcholesta-3 $\beta$-ol (7): Pale yellow solid; all spectroscopic data were in agreement with those previously reported. ${ }^{65,66}$

\subsection{Isolation of a Tribromobisindole Compound from Zyzzya sp. (MNP0355)}

MNP0355 (82 g) was extracted with $\mathrm{MeOH}(3 \times 400 \mathrm{~mL})$. The three extracts were cyclic loaded onto an HP-20 column $(2.5 \times 13 \mathrm{~cm})$. The column was washed with $\mathrm{H}_{2} \mathrm{O}$ (200 mL) and then eluted with $200 \mathrm{~mL}$ portions of (1) $15 \% \mathrm{Me}_{2} \mathrm{CO} / \mathrm{H}_{2} \mathrm{O}$, (2) $75 \% \mathrm{Me}_{2} \mathrm{CO} / \mathrm{H}_{2} \mathrm{O}$, and (3) $\mathrm{Me}_{2} \mathrm{CO}$. Fraction (2) was combined with the screen sample (extracted from $162 \mathrm{~g}$ of sponge). This combined sample was cyclic loaded onto Amberchrom (1 mL) which was transferred onto an Amberchrom MPLC column $(1.1 \times 25 \mathrm{~cm})$ as a slurry. This column was eluted using a gradient profile from 
$10-100 \% \mathrm{Me}_{2} \mathrm{CO} / \mathrm{H}_{2} \mathrm{O}$. Fractions collected from $50-63 \% \mathrm{Me}_{2} \mathrm{CO} / \mathrm{H}_{2} \mathrm{O}$ were combined on the basis of TLC chars to give a tribromobisindole compound (31.6 mg).

Tribromobisindole: Pale yellow solid; NMR data, ${ }^{1} \mathrm{H}$ NMR $\left(\mathrm{D}_{6}\right.$-DMSO + TFA, 300 MHz) $\delta 11.60$ (br s), 10.70 (br s), 8.00 (s), 7.70 (d, $1.6 \mathrm{~Hz}), 7.68$ (d, $2.3 \mathrm{~Hz}), 7.50$ (d, $1.8 \mathrm{~Hz}), 7.34$ (d, 8.5 Hz), 7.17 (dd, 8.1, 1.4 Hz), 6.92 (dd 8.5, $1.8 \mathrm{~Hz}), 6.73$ (s), 6.59 (d, 8.5 Hz); ${ }^{13} \mathrm{C}$ NMR $\left(\mathrm{D}_{6}\right.$-DMSO + TFA, $\left.75 \mathrm{MHz}\right) \delta 153.8,141.1,137.4,130.3,126.2$, 122.3, 121.7, 121.4, 120.7, 115.8, 113.9, 113.3, 111.0, 108.3, 104.1; HRESIMS, obsd. $m / z \quad 749.9301: 751.9287: 753.9327: 755.9298$ in a $1: 4: 4: 1$ ratio, (indicating three $\mathrm{Br}$ atoms).

Isolation of (10Z)- and (10E)-hymenialdisine (12) and (13), and a related compound from Axinella sp. (MNP0707)

A sample of MNP0707 (93.4 g) was extracted with $\mathrm{MeOH}(2 \times 400 \mathrm{~mL})$. The two extracts were cyclic loaded onto an HP-20 column $(2.5 \times 13 \mathrm{~cm})$. The column was washed with $\mathrm{H}_{2} \mathrm{O}\left(200 \mathrm{~mL}\right.$ ) then eluted with $200 \mathrm{~mL}$ fractions of (1) $30 \% \mathrm{Me}_{2} \mathrm{CO} / \mathrm{H}_{2} \mathrm{O}$, (2) $75 \% \mathrm{Me}_{2} \mathrm{CO} / \mathrm{H}_{2} \mathrm{O}$, and (3) $\mathrm{Me}_{2} \mathrm{CO}$. Fraction (1) (159 mg) was cyclic loaded onto Amberchrom $(6 \mathrm{~mL})$ which was transferred onto an MPLC Amberchrom column $(1.1 \times 25 \mathrm{~cm})$ as a slurry. This column was eluted using a gradient profile from $0-100 \%$ $\mathrm{MeOH} / \mathrm{H}_{2} \mathrm{O}$. The fractions eluted from $68-78 \% \mathrm{MeOH} / \mathrm{H}_{2} \mathrm{O}$ were combined $(88.0 \mathrm{mg}$ ) on the basis of TLC chars. This sample was chromatographed on an LH-20 column (1.8 x $90 \mathrm{~cm}$ ) using $90 \% \mathrm{MeOH} / 9.5 \% \mathrm{H}_{2} \mathrm{O} / 0.5 \%$ TFA as the eluting solvent. Fractions from this column were combined on the basis of TLC chars for NMR analysis. One such fraction ( $62 \mathrm{mg}$ ) was dissolved in $\mathrm{D}_{6}$-DMSO. The NMR solvent that was not transferred to the NMR tube slowly evaporated to leave crystals of (10E)-hymenialdisine (13) (11.1 mg). The solvent that was transferred into the NMR tube was removed after analysis 
and was filtered through a $0.45 \mu \mathrm{m}$ PTFE filter. The filter was washed with $50 \%$ $\mathrm{MeOH} / 45 \% \mathrm{H}_{2} \mathrm{O} / 5 \% \mathrm{AcOH}$. The filtrate was then left to slowly evaporate to give (10Z)-hymenialdisine (12) (4.1 mg).

An extract $(2 \times 300 \mathrm{~mL})$ of a second sample of MNP0707 (59 g) was cyclic loaded onto a small column of HP-20 $(2.5 \times 7 \mathrm{~mL})$. This column was washed with $\mathrm{H}_{2} \mathrm{O}(200 \mathrm{~mL})$ and then eluted with $200 \mathrm{~mL}$ fractions of (1) $20 \% \mathrm{Me}_{2} \mathrm{CO} / \mathrm{H}_{2} \mathrm{O}$, (2) $40 \% \mathrm{Me}_{2} \mathrm{CO} / \mathrm{H}_{2} \mathrm{O}$, (3) $60 \% \mathrm{Me}_{2} \mathrm{CO} / \mathrm{H}_{2} \mathrm{O}$, (4) $80 \% \mathrm{Me}_{2} \mathrm{CO} / \mathrm{H}_{2} \mathrm{O}$, and (5) $\mathrm{Me}_{2} \mathrm{CO}$. Fraction (2) (64.7 mg) was combined on the basis of TLC chars with another fraction $(7.4 \mathrm{mg})$ from the original Amberchrom MPLC separation. This combined sample was chromatographed on an LH-20 column $(1.8 \times 90 \mathrm{~cm})$ using $90 \% \mathrm{MeOH} / \mathrm{H}_{2} \mathrm{O}$ as the eluting solvent. Several fractions from this separation were combined $(3.8 \mathrm{mg})$ on the basis of TLC chars. These fractions were then combined with another sample, with a similar TLC char, from the Amberchrom separation (19.3 mg). This combined sample was chromatographed on a further LH-20 column $(1.8 \times 90 \mathrm{~cm})$ using $90 \% \mathrm{MeOH} / \mathrm{CH}_{2} \mathrm{Cl}_{2}$ as the eluting solvent. This yielded a brominated alkaloid compound ( $8.6 \mathrm{mg})$, related to 12 and 13 , which could not be identified.

(10Z)-Hymenialdisine (12): Pale yellow solid; all spectroscopic data were in agreement with those previously reported. ${ }^{76}$

(10E)-Hymenialdisine (13): Pale yellow solid; all spectroscopic data were in agreement with those previously reported. ${ }^{77}$

Related compound: Pale yellow solid; NMR data, ${ }^{1} \mathrm{H}$ NMR (D $\left.-\mathrm{DMSO}, 300 \mathrm{MHz}\right)$ $\delta 11.60,7.96,7.11,7.00,6.99,6.93,6.80,6.71,5.42,1.88,1.23 ;{ }^{13} \mathrm{C}$ NMR $\left(\mathrm{D}_{6}\right.$-DMSO, 
$75 \mathrm{MHz}) \delta 172.2,156.5,138.4,134.6,131.5,118.6,110.4,104.0,95.5,28.9,21.3$;

HRESIMS, obsd. $\mathrm{m} / \mathrm{z}$ 278.0028:280.0019 in a 1:1 ratio, (indicating one $\mathrm{Br}$ atom).

7.7 Isolation of Clathriol A (32) and Clathriol B (33) from Clathria
lissosclera (MNP0090)

MNP0090 (610 g) was extracted with MeOH $(2 \times 1.5 \mathrm{~L})$. The two extracts were cyclic loaded onto an HP-20 column $(2.5 \times 20 \mathrm{~cm})$. The column was washed with $\mathrm{H}_{2} \mathrm{O}$ $(300 \mathrm{~mL})$ and eluted with $300 \mathrm{~mL}$ fractions of (1) $40 \% \mathrm{Me}_{2} \mathrm{CO} / \mathrm{H}_{2} \mathrm{O}$, (2) $80 \% \mathrm{Me}_{2} \mathrm{CO} / \mathrm{H}_{2} \mathrm{O}$, and (3) $\mathrm{Me}_{2} \mathrm{CO}$. Fraction (2) (452 mg) was cyclic loaded onto Amberchrom $(10 \mathrm{~mL})$ which was transferred onto an Amberchrom MPLC column $(1.1 \times 25 \mathrm{~cm})$ as a slurry. The column was eluted using a gradient profile from $0-100 \%$ $\mathrm{Me}_{2} \mathrm{CO} / \mathrm{H}_{2} \mathrm{O}$. The $49-61 \% \mathrm{Me}_{2} \mathrm{CO} / \mathrm{H}_{2} \mathrm{O}$ fractions were combined on the basis of TLC chars, and concentrated to dryness to give a pale yellow solid $(117 \mathrm{mg})$ that was re-chromatographed in a similar manner, using a slower gradient. The 51-53\% $\mathrm{Me}_{2} \mathrm{CO} / \mathrm{H}_{2} \mathrm{O}$ fractions were combined on the basis of TLC chars to give clathriol A (32) (26.2 mg). The $48-50 \% \mathrm{Me}_{2} \mathrm{CO} / \mathrm{H}_{2} \mathrm{O}$ fractions were combined on the basis of TLC chars to give a mixture of sterols $(17.1 \mathrm{mg})$. Repeated normal-phased chromatography on silica gel $\left(0-10 \% \mathrm{MeOH} / \mathrm{CH}_{2} \mathrm{Cl}_{2}\right)$ yielded clathriol B (33) $(1.3 \mathrm{mg})$.

Clathriol A (32): White solid; $[\alpha]^{20}{ }_{\mathrm{D}}+22.6^{\circ}($ c. $1.4, \mathrm{MeOH})$; IR $(\mathrm{KBr}) v_{\max } 3410,2958$, 2872, 1734, 1584, $1055 \mathrm{~cm}^{-1}$; NMR data see Table 3.1; HRESIMS, obsd. $m / z 479.4754$ $[\mathrm{M}+\mathrm{H}]^{+}, \quad 477.3582[\mathrm{M}-\mathrm{H}]^{-}, \quad \mathrm{C}_{29} \mathrm{H}_{50} \mathrm{O}_{5}$ requires $479.4736 \Delta 3.7 \mathrm{ppm}, 477.3575$ $\Delta 1.5 \mathrm{ppm}$ 
Clathriol B (33): White solid; $[\alpha]^{20}{ }_{\mathrm{D}}-29.4^{\circ}\left(\right.$ c. $\left.0.71, \mathrm{CH}_{2} \mathrm{Cl}_{2}\right)$; IR $(\mathrm{KBr}) v_{\max } 3376$, 2928, 1728, 1602, 1461, 1206, 1153, $1056 \mathrm{~cm}^{-1}$; NMR data see Table 3.2; HRESIMS, obsd. $m / z 463.3423[\mathrm{M}+\mathrm{H}]^{+}, 461.3298[\mathrm{M}-\mathrm{H}]^{-}, \mathrm{C}_{28} \mathrm{H}_{46} \mathrm{O}_{5}$ requires $463.3418 \Delta 1.0 \mathrm{ppm}$, $461.3273 \Delta 4.7 \mathrm{ppm}$.

7.8

\section{Per-acetylation of Clathriol A (32)}

Acetic anhydride $(0.5 \mathrm{~mL})$ was added to clathriol A (32) $(3.0 \mathrm{mg})$ dissolved in pyridine $(0.5 \mathrm{~mL})$. The reaction mixture was stirred at room temperature for $17 \mathrm{~h}$. The mixture was cyclic loaded onto a small glass column prepacked with Amberchrom resin (1 mL). The column was washed with $\mathrm{H}_{2} \mathrm{O}(20 \mathrm{~mL})$ and then eluted with (1) $\mathrm{MeOH}(5 \mathrm{~mL})$ and (2) $\mathrm{Me}_{2} \mathrm{CO}(5 \mathrm{~mL})$. Fraction (2) was concentrated to yield clathriol tetra-acetate $(2.4$ $\mathrm{mg})$.

Clathriol tetra-acetate: White amorphous powder; IR ( $\mathrm{KBr}$ film) $v_{\max } 2938,2875$, $1736,1368,1247,1031 \mathrm{~cm}^{-1} ;{ }^{1} \mathrm{H}$ NMR data $\left(\mathrm{CDCl}_{3}, 300 \mathrm{MHz}\right) \delta 5.86(1 \mathrm{H}, \mathrm{dd}, 10.5,9$ Hz, H-7), 4.99 (1H quin, 6 Hz, H-28), 4.81 (1H, dd, 10.5, 9 Hz, H-6), 4.61 (1H, dddd, 13.5, 10, 4.5, 3 Hz, H-3), 2.32 (1H, dd, 3, 2 Hz, H-14), 2.30 (1H, dd, 20, 10 Hz, H-16ß), $2.17(1 \mathrm{H}, \mathrm{m}, \mathrm{H}-16 \alpha), 2.02(3 \mathrm{H}, \mathrm{s}, \mathrm{C}=\mathrm{O}), 2.01(6 \mathrm{H}, \mathrm{s}, 2 \times \mathrm{C}=\mathrm{O}), 1.94(3 \mathrm{H}, \mathrm{s}, \mathrm{C}=\mathrm{O}), 1.88$

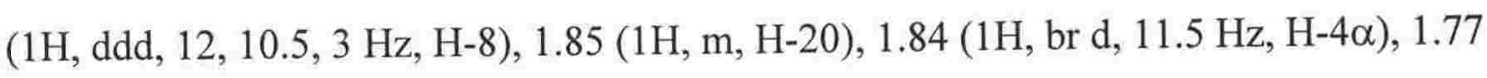
$(1 \mathrm{H}, \mathrm{m}, \mathrm{H}-2 \alpha), 1.72(1 \mathrm{H}, 11 \mathrm{~Hz}, \mathrm{H}-1 \beta), 1.69$ (1H, m, H-17), 1.51 (1H, m, H-5), 1.50

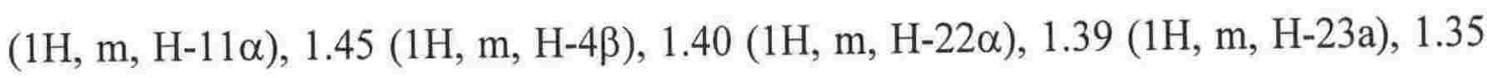

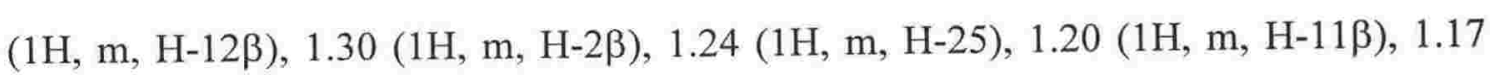
(1H, m, H-24), 1.16 (1H, m, H-9), 1.15 (3H, d, $\left.6.5 \mathrm{~Hz}, \mathrm{CH}_{3}-29\right), 1.14$ (1H, m, H-12 $\alpha$ ), $1.12\left(3 \mathrm{H}, \mathrm{s}, \mathrm{CH}_{3}-18\right), 1.12$ (1H, m, H-23b), 1.03 (1H, dd 13.5, $\left.10 \mathrm{~Hz}, \mathrm{H}-1 \alpha\right), 0.94(1 \mathrm{H}$, m, H-22b), 0.90 (3H, s, $\left.\mathrm{CH}_{3}-19\right), 0.90\left(3 \mathrm{H}, \mathrm{d}, 6.5, \mathrm{CH}_{3}-21\right), 0.87\left(3 \mathrm{H}, \mathrm{d}, 6.5 \mathrm{~Hz}, \mathrm{CH}_{3}-\right.$ 
26), $0.87\left(3 \mathrm{H}, \mathrm{d}, 6.5 \mathrm{~Hz}, \mathrm{CH}_{3} 27\right) ;{ }^{13} \mathrm{C} \mathrm{NMR}$ data $\left(\mathrm{CDCl}_{3}, 75 \mathrm{MHz}\right) \delta 217.8(\mathrm{C}-15)$, $171.0\left(\mathrm{C}=\mathrm{OCH}_{3}\right), 170.1\left(2 \times \mathrm{C}=\mathrm{OCH}_{3}\right), 169.9\left(\mathrm{C}=\mathrm{OCH}_{3}\right), 73.9(\mathrm{C}-7), 73.9(\mathrm{C}-6), 72.4$ (C-3), 71.3 (C-28), 51.2 (C-14), 48.6 (C-24), 47.6 (C-17), 44.6 (C-5), 44.6 (C-9), 41.0 (C-13), 37.1 (C-12), 36.5 (C-8), 36.5 (C-16), 35.8 (C-1), 33.6 (C-20), 31.3 (C-22), 29.2 (C-25), $27.8(\mathrm{C}-2), 26.4(\mathrm{C}-4), 23.1\left(2 \times \mathrm{CH}_{3} \mathrm{C}=\mathrm{O}\right), 22.7\left(\mathrm{CH}_{3}=\mathrm{O}\right), 22.4\left(\mathrm{CH}_{3}=\mathrm{O}\right), 21.2$ (C-11), 21.0 (C-27), 18.9 (C-21), 18.7 (C-26), 18.6 (C-18), 17.0 (C-29), 12.7 (C-19); HRESIMS, obsd. $m / z 645.4006[\mathrm{M}-\mathrm{H}]{ }^{-}, \mathrm{C}_{37} \mathrm{H}_{57} \mathrm{O}_{9}$ requires $645.3997 \Delta 1.4 \mathrm{ppm}$.

A catalytic amount of hexane washed $\mathrm{Na}$ metal was added to clathriol $\mathrm{A}(\mathbf{3 2})(3.5 \mathrm{mg})$ dissolved in $\mathrm{MeOH}(1 \mathrm{~mL})$. After refluxing for $6: 45 \mathrm{~h}$, the reaction was quenched by addition of $\mathrm{H}_{2} \mathrm{O}(1 \mathrm{~mL})$. The reaction mixture was cyclic loaded onto Amberchrom resin $(1 \mathrm{~mL})$ that was then washed with $\mathrm{H}_{2} \mathrm{O}(3 \mathrm{~mL})$. The column was stripped with $\mathrm{Me}_{2} \mathrm{CO}(3 \mathrm{~mL})$ which was evaporated to dryness to give a mixture of products $(4.6 \mathrm{mg})$.

$\mathrm{NaBH}_{4}(2.5 \mathrm{mg})$ was dissolved in $\mathrm{MeOH}(1 \mathrm{~mL}, 65 \mathrm{nM})$. This solution was added to clathriol A (32) (2.0 mg). The solution was stirred at room temperature for 5:40 h after which the reaction was quenched by addition of $\mathrm{H}_{2} \mathrm{O}(1 \mathrm{~mL})$. The reaction mixture was cyclic loaded onto a column of Amberchrom resin $(1 \mathrm{~mL})$ that was then washed with $\mathrm{H}_{2} \mathrm{O}(3 \mathrm{~mL})$. The column was then stripped with $\mathrm{Me}_{2} \mathrm{CO}(3 \mathrm{~mL})$ which was evaporated to dryness to give a mixture of products $(2.2 \mathrm{mg})$. 
(MNP0979)

MNP0979 (182 g) was extracted with MeOH $(2 \times 400 \mathrm{~mL})$. Both extracts were cyclic loaded onto a column of HP-20 $(2.5 \times 10 \mathrm{~cm})$. The column was then washed with $\mathrm{H}_{2} \mathrm{O}$ $(300 \mathrm{~mL})$ and eluted with $300 \mathrm{~mL}$ portions of (1) $20 \% \mathrm{Me}_{2} \mathrm{CO} / \mathrm{H}_{2} \mathrm{O}$, (2) $40 \% \mathrm{Me}_{2} \mathrm{CO} / \mathrm{H}_{2} \mathrm{O}$, (3) $60 \% \mathrm{Me}_{2} \mathrm{CO} / \mathrm{H}_{2} \mathrm{O}$, (4) $80 \% \mathrm{Me}_{2} \mathrm{CO} / \mathrm{H}_{2} \mathrm{O}$ and (5) $\mathrm{Me}_{2} \mathrm{CO}$. Fraction (2) (176 mg) was cyclic loaded onto HP-20S (5 mL). The loaded stationary phase was transferred onto an HP-20S MPLC column $(2.5 \times 45 \mathrm{~cm})$ as a slurry. The MPLC column was eluted using a gradient elution profile from $10-100 \% \mathrm{MeOH} / \mathrm{H}_{2} \mathrm{O}$. The fractions eluting from the column in $\mathrm{MeOH}$ were combined and chromatographed isocratically on a Phenomenex Prodigy ODS semi-preparative HPLC column (75\% $\mathrm{MeOH} / 25 \% \quad 0.1 \mathrm{~mol} / \mathrm{L} \mathrm{AcOH}, 4.75 \mathrm{~mL} / \mathrm{min}$ flow-rate, $\lambda=230 \mathrm{~nm}$ ). The first unidentified diterpene $(6.3 \mathrm{mg}$ ) eluted with a retention time of $7.16 \mathrm{~min}$. A compound with a retention time of $17.00 \mathrm{~min}$ was reinjected isocratically $(67 \% \mathrm{MeOH} / 33 \%$ $0.1 \mathrm{~mol} / \mathrm{L} \mathrm{AcOH}, 4.75 \mathrm{~mL} / \mathrm{min}$ flow-rate, $\lambda=230 \mathrm{~nm}$ ) on the same HPLC column. The second unidentified diterpene $(2.4 \mathrm{mg})$ eluted with a retention time of $52.90 \mathrm{~min}$.

Fraction (3) from the HP-20 column was combined with the screen sample (197 mg). This sample was cyclic loaded onto HP-20S $(10 \mathrm{~mL})$ which was transferred onto a large HP-20S MPLC column $(2.5 \times 45 \mathrm{~cm})$ as a slurry. This column was eluted using a gradient profile from $10-100 \% \mathrm{Me}_{2} \mathrm{CO} / \mathrm{H}_{2} \mathrm{O}$. The fractions that eluted from the column in $\mathrm{Me}_{2} \mathrm{CO}$ were combined into two samples, based upon TLC chars. The first (40.7 mg) was chromatographed isocratically on a Phenomenex Prodigy semi-preparative ODS column $\left(80 \% \mathrm{MeCN} / \mathrm{H}_{2} \mathrm{O}, 4.50 \mathrm{~mL} / \mathrm{min}\right.$ flow-rate, $\left.\lambda=230 \mathrm{~nm}\right)$. Cadlinolide $\mathrm{C}$ (138) $(2.4$ $\mathrm{mg}$ ) eluted with a retention time of $12.23 \mathrm{~min}$. Two other fractions (retention times of $7.41(4.2 \mathrm{mg})$ and $13.02(1.4 \mathrm{mg}) \mathrm{min}$ respectively) were reinjected. The first was 
chromatographed using the same column $\left(60 \% \mathrm{MeCN} / \mathrm{H}_{2} \mathrm{O}, 4.50 \mathrm{~mL} / \mathrm{min}\right.$ flow-rate, $\lambda=230 \mathrm{~nm})$. Cadlinolide B (133) $(1.4 \mathrm{mg})$ eluted with a retention time of $17.42 \mathrm{~min}$ while 15-methoxypourewic acid B (141) $(1.2 \mathrm{mg})$ eluted with a retention time of $19.91 \mathrm{~min}$. The fraction collected with a retention time of $13.02 \mathrm{~min}$ from the $80 \%$ $\mathrm{MeCN} / \mathrm{H}_{2} \mathrm{O}$ separation was reinjected on the same column $\left(70 \% \mathrm{MeCN} / \mathrm{H}_{2} \mathrm{O}\right.$, $4.50 \mathrm{~mL} / \mathrm{min}$ flow-rate, $\lambda=230 \mathrm{~nm})$. Cadlinolide D (139) $(0.8 \mathrm{mg})$ eluted with a retention time of $24.62 \mathrm{~min}$.

The second sample from the combination of the $\mathrm{Me}_{2} \mathrm{CO}$ fractions from the HP-20S MPLC column $(27.9 \mathrm{mg})$ was chromatographed on an LH-20 column $(1.8 \times 90 \mathrm{~cm})$ using $90 \% \mathrm{MeOH} / \mathrm{H}_{2} \mathrm{O}$ as the eluting solvent. Fractions generated by this separation were combined on the basis of TLC chars. One such sample (20.9 mg) was chromatographed on a silica gel column $(1.6 \times 7.5 \mathrm{~cm})$ using $100 \mathrm{~mL}$ portions of (1) $\mathrm{CH}_{2} \mathrm{Cl}_{2}$, (2) $2 \% \mathrm{MeOH} / \mathrm{CH}_{2} \mathrm{Cl}_{2}$, (3) $3 \% \mathrm{MeOH} / \mathrm{CH}_{2} \mathrm{Cl}_{2}$, (4) $4 \% \mathrm{MeOH} / \mathrm{CH}_{2} \mathrm{Cl}_{2}$, (5) $5 \% \mathrm{MeOH} / \mathrm{CH}_{2} \mathrm{Cl}_{2}$, (6) $10 \% \mathrm{MeOH} / \mathrm{CH}_{2} \mathrm{Cl}_{2}$, and (7) $50 \% \mathrm{MeOH} / \mathrm{CH}_{2} \mathrm{Cl}_{2}$. The fractions eluted using portions (1) and (2) were combined $(10.4 \mathrm{mg})$ on the basis of TLC chars.

Fraction (4) from the original HP-20 column $\left(80 \% \mathrm{Me}_{2} \mathrm{CO} / \mathrm{H}_{2} \mathrm{O}\right)(227.3 \mathrm{mg})$ was chromatographed on a silica gel column $(2.5 \times 14 \mathrm{~cm})$ using $250 \mathrm{~mL}$ portions of (1) $\mathrm{CH}_{2} \mathrm{Cl}_{2}$, (2) $2 \% \mathrm{MeOH} / \mathrm{CH}_{2} \mathrm{Cl}_{2}$, (3) $4 \% \mathrm{MeOH} / \mathrm{CH}_{2} \mathrm{Cl}_{2}$, (4) $6 \% \mathrm{MeOH} / \mathrm{CH}_{2} \mathrm{Cl}_{2}$, (5) $10 \% \mathrm{MeOH} / \mathrm{CH}_{2} \mathrm{Cl}_{2}$, and (6) $50 \% \mathrm{MeOH} / \mathrm{CH}_{2} \mathrm{Cl}_{2}$. All the fractions collected when eluting with portions (1), (2) and (3) were combined (80.7 mg) on the basis of TLC chars. This sample was combined with the sample from the silica gel column of the second $\mathrm{Me}_{2} \mathrm{CO}$ fraction generated from the HP-20S MPLC separation. This combined sample was chromatographed on a further silica gel column using $200 \mathrm{~mL}$ portions of (1) $50 \% \quad \mathrm{CH}_{2} \mathrm{Cl}_{2}$ /petroleum ether, (2) $75 \% \mathrm{CH}_{2} \mathrm{Cl}_{2}$ /petroleum ether, (3) $\mathrm{CH}_{2} \mathrm{Cl}_{2}$, 
(4) $1 \% \mathrm{MeOH} / \mathrm{CH}_{2} \mathrm{Cl}_{2}$,

$(5)$

$2 \% \quad \mathrm{MeOH} / \mathrm{CH}_{2} \mathrm{Cl}_{2}$,

(6) $3 \% \quad \mathrm{MeOH} / \mathrm{CH}_{2} \mathrm{Cl}_{2}$,

(7) $4 \% \mathrm{MeOH} / \mathrm{CH}_{2} \mathrm{Cl}_{2}$, and (8) $10 \% \mathrm{MeOH} / \mathrm{CH}_{2} \mathrm{Cl}_{2}$. Several fractions eluted using portion (5) were combined (48.4 mg) on the basis of TLC chars and were chromatographed on a further silica gel column $(2.5 \times 10 \mathrm{~cm})$ using $400 \mathrm{~mL}$ portions of (1) $75 \% \mathrm{CH}_{2} \mathrm{Cl}_{2}$ /petroleum ether, (2) $10 \% \mathrm{CH}_{2} \mathrm{Cl}_{2} /$ petroleum ether, (3) $\mathrm{CH}_{2} \mathrm{Cl}_{2}$, (4) $1 \%$ $\mathrm{MeOH} / \mathrm{CH}_{2} \mathrm{Cl}_{2}$, (5) $2 \% \mathrm{MeOH} / \mathrm{CH}_{2} \mathrm{Cl}_{2}$, (6) $3 \% \mathrm{MeOH} / \mathrm{CH}_{2} \mathrm{Cl}_{2}$ and $200 \mathrm{~mL}$ portions of (7) $4 \% \mathrm{MeOH} / \mathrm{CH}_{2} \mathrm{Cl}_{2}$ and (8) $10 \% \mathrm{MeOH} / \mathrm{CH}_{2} \mathrm{Cl}_{2}$. The fractions generated by eluting with portions (5) to (8) were combined $(24.5 \mathrm{mg})$ on the basis of TLC chars, and were chromatographed isocratically on the same Phenomenex semi-preparative column as before $\left(80 \% \mathrm{MeCN} / \mathrm{H}_{2} \mathrm{O}, 4.50 \mathrm{~mL} / \mathrm{min}, \lambda=230 \mathrm{~nm}\right)$. Pourewanone (143) (2.2 mg), methylpourewate B (142) (3.0 mg) and tetrahydroaplysulphurin-1 (124) (1.8 mg) eluted with retention times of $5.61,8.11$, and 10.98 min respectively.

A sample was generated by the combination of fractions, with similar TLC chars, from one of the previous silica gel column separations. The $80 \% \mathrm{Me}_{2} \mathrm{CO} / \mathrm{H}_{2} \mathrm{O}$ fraction from the HP-20 column had been combined with the second sample eluted with $100 \% \mathrm{Me}_{2} \mathrm{CO}$ from the HP-20S MPLC column. This combined sample had already been chromatographed twice on silica gel. Those fractions eluted using $3 \%$ and $4 \% \mathrm{MeOH} / \mathrm{CH}_{2} \mathrm{Cl}_{2}$ from the second separation were combined $(10.0 \mathrm{mg})$ and were chromatographed on the same Phenomenex semi-preparative column $\left(70 \% \mathrm{MeCN} / \mathrm{H}_{2} \mathrm{O}\right.$, $4.50 \mathrm{~mL} / \mathrm{min}, \lambda=230 \mathrm{~nm})$. Pourewic acid A (140) $(2.9 \mathrm{mg})$ eluted with a retention time of $19.69 \mathrm{~min}$.

Tetrahydroaplysulphurin-1 (124): White solid; all spectroscopic data were in agreement with those previously reported. ${ }^{28,144,159}$ 
Cadlinolide B (133): White solid; all spectroscopic data were in agreement with those previously reported. $^{28}$

Cadlinolide C (138): Pale yellow solid; $[\alpha]^{20}{ }_{\mathrm{D}}+27.3^{\circ}$ (c. $0.68, \mathrm{CH}_{2} \mathrm{Cl}_{2}$ ); IR (film) $v_{\max } 2945,1739,1455,1228,1026 \mathrm{~cm}^{-1}$; NMR data see Table 4.1; HRESIMS, obsd. $m / z 319.2260[\mathrm{M}+\mathrm{H}]^{+}, \mathrm{C}_{20} \mathrm{H}_{30} \mathrm{O}_{3}$ requires $319.2268, \Delta 2.6 \mathrm{ppm}$.

Cadlinolide D (139): Off-white solid; $[\alpha]^{20}{ }_{D}$ Na. as diastereomeric mixture; IR (film) $v_{\max } 2946,1744,1456,1206,1028 \mathrm{~cm}^{-1}$; NMR data see Table 4.2; HRESIMS, obsd. $m / z 349.2399[\mathrm{M}+\mathrm{H}]^{+}, 371.2201[\mathrm{M}+\mathrm{Na}]^{+}, 791.4483[2 \mathrm{M}+\mathrm{Na}]^{+}, \mathrm{C}_{21} \mathrm{H}_{32} \mathrm{O}_{4}$ requires $349.2373, \Delta 7.5 \mathrm{ppm}, 371.2193, \Delta 2.1 \mathrm{ppm}, 719.4501, \Delta 2.5 \mathrm{ppm}$.

Pourewic acid A (140): White solid; $[\alpha]^{20}-10.8^{\circ}$ (c. $0.86, \mathrm{CH}_{2} \mathrm{Cl}_{2}$ ); IR (film) $v_{\max } 3392,2927,1704,1455,1221,1096,1060 \mathrm{~cm}^{-1}$; NMR data see Table 4.3; HRESIMS, obsd. $m / z$ 349.2401 [M-H] ${ }^{-}, \mathrm{C}_{21} \mathrm{H}_{34} \mathrm{O}_{4}$ requires $349.2384, \Delta 4.8 \mathrm{ppm}$.

15-methoxypourewic acid B (141): Pale yellow solid; $[\alpha]^{20}-368.6^{\circ}\left(\right.$ c. $\left.0.23, \mathrm{CH}_{2} \mathrm{Cl}_{2}\right)$; IR (film) $v_{\max } 3347,2927,1778,1707,1456,1204 \mathrm{~cm}^{-1}$; NMR data see Table 4.4; HRESIMS, obsd. $m / z \quad 363.2194 \quad[\mathrm{M}-\mathrm{H}]^{-}, \quad 366.2337 \quad[\mathrm{M}(-3 \mathrm{H}+3 \mathrm{D})-\mathrm{H}]^{-}, \quad 368.2504$ $[\mathrm{M}(-3 \mathrm{H}+3 \mathrm{D})+\mathrm{H}]^{+}, \quad \mathrm{C}_{21} \mathrm{H}_{32} \mathrm{O}_{5}$ requires $363.2177 \Delta 4.8 \mathrm{ppm}, 366.2365 \Delta 7.9 \mathrm{ppm}$, $368.2504 \Delta 3.7 \mathrm{ppm}$.

Methylpourewate B (142): Pale yellow solid; $[\alpha]^{20}{ }_{\mathrm{D}}-81.8^{\circ}$ (c. $0.70, \mathrm{CH}_{2} \mathrm{Cl}_{2}$ ); IR (film) $v_{\max } 2947,1762,1735,1455,1206,1124 \mathrm{~cm}^{-1}$; NMR data see Table 4.5; HRESIMS, obsd. $m / z 365.2335[\mathrm{M}+\mathrm{H}]^{+}, 363.2185$ [M-H]', $\mathrm{C}_{21} \mathrm{H}_{32} \mathrm{O}_{5}$ requires 365.2323 $\Delta 3.5 \mathrm{ppm}, 363.2166 \Delta 5.3 \mathrm{ppm}$. 
Pourewanone (143): White solid; $[\alpha]^{20}{ }_{\mathrm{D}}+22.6^{\circ}$ (c. $0.35, \mathrm{CH}_{2} \mathrm{Cl}_{2}$ ); UV $\left(\mathrm{CH}_{2} \mathrm{Cl}_{2}\right.$ ) $\lambda_{\max } 248 \mathrm{~nm}(\varepsilon 14,425)$; IR (film) $v_{\max } 3335,2929,1725,1665,1456,1366,1307,1186$ $\mathrm{cm}^{-1}$; NMR data see Table 4.6; HRESIMS, obsd. $\mathrm{m} / z$ 351.2160 [M+H] $]^{+}, 349.2019$ $[\mathrm{M}-\mathrm{H}]^{-}, \mathrm{C}_{20} \mathrm{H}_{30} \mathrm{O}_{5}$ requires $351.2166 \Delta 1.8 \mathrm{ppm}, 349.2021 \Delta 0.5 \mathrm{ppm}$.

\subsection{2}

\section{Methylation of Pourewic Acid A (140)}

Diazald ( $N$-methyl- $N$-nitroso-p-toluenesulfonamide) $(1 \mathrm{~g})$ was reacted with $\mathrm{KOH}$ ( $5 \mathrm{~g}$ dissolved in $18 \mathrm{~mL} \mathrm{44 \%} \mathrm{H}_{2} \mathrm{O} / \mathrm{EtOH}$ ) to generate $\mathrm{CH}_{2} \mathrm{~N}_{2}$, which was dissolved in $\mathrm{Et}_{2} \mathrm{O}$. A solution of pourewic acid A (140) $\left(0.8 \mathrm{mg}\right.$ dissolved in $\left.1 \mathrm{~mL} \mathrm{CH}_{2} \mathrm{Cl}_{2}\right)$ was treated with excess $\mathrm{CH}_{2} \mathrm{~N}_{2}$ for $4 \mathrm{~h}$. The solvent was removed under vacuum to yield a mixture of compounds. The mixture was dissolved in $50 \% \mathrm{CH}_{2} \mathrm{Cl}_{2} / \mathrm{MeOH}(1 \mathrm{~mL})$ which was passed through an amino column $(0.5 \times 1.5 \mathrm{~cm})$ that had been pre-equilibrated with $50 \% \mathrm{CH}_{2} \mathrm{Cl}_{2} / \mathrm{MeOH}(5 \mathrm{~mL})$. The column was washed with $50 \% \mathrm{CH}_{2} \mathrm{Cl}_{2} / \mathrm{MeOH}(4 \mathrm{~mL})$ which was collected together with the eluent of loading. The column was then eluted with $5 \% \mathrm{AcOH} / 47.5 \% \mathrm{CH}_{2} \mathrm{Cl}_{2} / 47.5 \% \mathrm{MeOH}(5 \mathrm{~mL})$. The sample containing the eluent of loading and the column washings was evaporated to dryness under reduced pressure to yield methyl pourewate A $(0.7 \mathrm{mg})$.

Methyl pourewate A: White amorphous powder; IR (film) $v_{\max } 2928,1732,1455$, 1366, 1096, 909, $732 \mathrm{~cm}^{-1} ;{ }^{1} \mathrm{H}$ NMR data $\left(\mathrm{CDCl}_{3}, 300 \mathrm{MHz}\right) \delta 4.62(1 \mathrm{H}, \mathrm{d}, 2.4 \mathrm{~Hz}$, H-15), 4.18 (1H, q, 4.4 Hz, H-7), 4.02 (1H, dd, 8.5, $6.4 \mathrm{~Hz}, \mathrm{H}-16 \mathrm{~b}), 3.76$ (1H, dd, 8.8, 3.4 Hz, H-16a), 3.67 (3H, s, methyl ester H-22), 3.24 (3H, s, methoxy H-21), 2.65 (1H, dd, 8.1, $2.4 \mathrm{~Hz}, \mathrm{H}-14), 2.34$ (1H, q, $7.6 \mathrm{~Hz}, \mathrm{H}-13), 2.20$ (1H, m, H-11b), $2.18(1 \mathrm{H}$, m, H-1b), 1.94 (1H, m, H-2a), 1.86 (1H, m, H-11a), 1.84 (1H, m, H-5b), 1.65 (1H, m, H-12b), 1.49 (1H, m, H-2b), 1.38 (1H, m, H-3b), 1.26 (1H, m, H-1a), 1.24 (1H, m, 
H-12a), 1.20 (3H, d 6.8 Hz, H-6), 1.18 (1H, m, H-3a) 1.02 (3H, s, H-20), 0.97 (1H, m, H-5a), 0.92 (3H, s, H-18), 0.88 (3H, s, H-19); ${ }^{13} \mathrm{C} \mathrm{NMR} \mathrm{data}\left(\mathrm{CDCl}_{3}, 75 \mathrm{MHz}\right) \delta 174.7$ (C-17), 143.9 (C-9), 128.2 (C-8), 110.8 (C-15), 74.9 (C-16), 54.6 (C-21), 51.7 (C-22), 50.9 (C-5), 49.0 (C-14), 42.0 (C-7), 41.6 (C-10), 40.1 (C-3), 39.2 (C-1), 38.0 (C-13), 33.3 (C-19), 31.6 (C-4), 31.2 (C-12), 30.9 (C-20), 27.7 (C-11), 26.2 (C-18), 20.0 (C-2), 16.1 (C-6); HRESIMS, obsd. $\mathrm{m} / z 333.2417$ [M-OMe] ${ }^{+}, \mathrm{C}_{21} \mathrm{H}_{33} \mathrm{O}_{3}$ requires 333.2424 $\Delta 2.1 \mathrm{ppm}$.

\subsection{Basic Harvesting of Karenia brevisulcata}

337 L of $K$. brevisulcata (Cawthron organism number CAWD 82) was filtered through glass fibre filters ( $3 \times$ large pre-filter tubes, $6 \times$ filter pads) to isolate the algal cells. The culture filtrate was passed through a column of HP-20 $(5.2 \times 36 \mathrm{~cm})$. This column was washed with $\mathrm{H}_{2} \mathrm{O}(1.5 \mathrm{~L})$ and then eluted with $\mathrm{Me}_{2} \mathrm{CO}(2.1 \mathrm{~L})$. The filters retaining the cells of $K$. brevisulcata were also extracted (pre-filters: $3 \times 3.5 \mathrm{~L} \mathrm{MeOH}$, filter pads: $3 \times 800 \mathrm{~mL}$ ). Each sample was analysed by N2A assay. The majority of the activity was concentrated in the cell extracts. The extracts from both the pre-filters and the filter pads were cyclic loaded onto individual HP-20 columns $(2.5 \times 20 \mathrm{~cm})$. Each column was washed with $\mathrm{H}_{2} \mathrm{O}(300 \mathrm{~mL})$ and then eluted with $300 \mathrm{~mL}$ portions of (1) $20 \% \mathrm{Me}_{2} \mathrm{CO} / \mathrm{H}_{2} \mathrm{O}$, (2) $40 \% \quad \mathrm{Me}_{2} \mathrm{CO} / \mathrm{H}_{2} \mathrm{O}$,

(3) $60 \% \quad \mathrm{Me}_{2} \mathrm{CO} / \mathrm{H}_{2} \mathrm{O}$, (4) $80 \% \mathrm{Me}_{2} \mathrm{CO} / \mathrm{H}_{2} \mathrm{O}$, and (5) $\mathrm{Me}_{2} \mathrm{CO}$. In both cases, fraction (3) showed significant N2A activity. The $\mathrm{Me}_{2} \mathrm{CO}$ elution of the HP-20 column showed no significant activity. The two $60 \% \mathrm{Me}_{2} \mathrm{CO} / \mathrm{H}_{2} \mathrm{O}$ fractions were combined and gave a total activity of $150 \times 10^{6}$ TU. (Note: This combined $60 \% \mathrm{Me}_{2} \mathrm{CO} / \mathrm{H}_{2} \mathrm{O}$ sample was sub-sampled and submitted for N2A assay. The sub-sample was serial diluted until no activity was noted. The addition of $1 \mu \mathrm{L}$ of this sub-sample was then defined as the effect of one TU). 
$\mathrm{Me}_{2} \mathrm{CO}(32 \mathrm{~L})$ was added to a $320 \mathrm{~L}$ culture of $\mathrm{K}$. brevisulcata (Cawthron organism number CAWD 82), which was allowed to stand for $12 \mathrm{~h}$. The culture was then filtered through glass fibre filters as before. The culture filtrate was passed through a column of $\mathrm{HP}-20(5.2 \times 36 \mathrm{~cm})$, which was washed with $\mathrm{H}_{2} \mathrm{O}(1.5 \mathrm{~L})$, and then eluted with $\mathrm{Me}_{2} \mathrm{CO}$ (2.1 L). The $\mathrm{Me}_{2} \mathrm{CO}$ elution was cyclic loaded onto a smaller column of HP-20 $(2.5 \times 17 \mathrm{~cm})$. This column was washed with $\mathrm{H}_{2} \mathrm{O}(250 \mathrm{~mL})$ and eluted with $250 \mathrm{~mL}$ portions of (1) $30 \% \mathrm{Me}_{2} \mathrm{CO} / \mathrm{H}_{2} \mathrm{O}$ (2) $70 \% \mathrm{Me}_{2} \mathrm{CO} / \mathrm{H}_{2} \mathrm{O}$, and (3) $\mathrm{Me}_{2} \mathrm{CO}$. A sub-sample of fraction (2) was taken for N2A assay. Fraction (2) was quantified as containing $2,500 \times 10^{6} \mathrm{TU}$.

\subsection{Optimised Isolation of KBT}

A culture of $K$. brevisulcata (Cawthron organism number CAWD 82) was harvested using the improved protocol, to generate a fraction $(273 \mathrm{mg})$ containing $2,500 \times 10^{6} \mathrm{TU}$ $\left(9.2 \times 10^{6} \mathrm{TU} / \mathrm{mg}\right)$ (see above). This fraction was cyclic loaded onto $15 \mathrm{~mL}$ of HP-20S which was transferred as a slurry onto a large HP-20S MPLC column $(2.5 \times 45 \mathrm{~cm})$. The HP-20S MPLC column was eluted using a gradient profile from $20-100 \% \mathrm{Me}_{2} \mathrm{CO}$, collecting 100 fractions. Every second fraction was sub-sampled and tested using the $\mathrm{N} 2 \mathrm{~A}$ assay. On the basis of the N2A assay results forty fractions, from $53-83 \%$ $\mathrm{Me}_{2} \mathrm{CO} / \mathrm{H}_{2} \mathrm{O}$, were combined $\left(175 \mathrm{mg}, 77.8 \times 10^{6} \mathrm{TU}, 0.44 \times 10^{6} \mathrm{TU} / \mathrm{mg}\right)$. This sample was dissolved in $50 \% \mathrm{EtOAc} / \mathrm{CH}_{2} \mathrm{Cl}_{2}(2 \mathrm{~mL})$ and was chromatographed on a diol column $(2.2 \times 20 \mathrm{~cm})$ using $500 \mathrm{~mL}$ portions of (1) $5 \% \mathrm{MeOH} / \mathrm{EtOAc}$, (2) $10 \% \mathrm{MeOH} / \mathrm{EtOAc}$, (3) $25 \% \mathrm{MeOH} / \mathrm{EtOAc}$, (4) $50 \% \mathrm{MeOH} / \mathrm{EtOAc}$ and (5) $\mathrm{MeOH}$. Each of the 121 fractions was sub-sampled for N2A assay. On the basis of the N2A assay results, 16 fractions collected when eluting with portion (3) were combined 
(54.3 mg, cumulative toxicity $41.9 \times 10^{6} \mathrm{TU}, 0.76 \times 10^{6} \mathrm{TU} / \mathrm{mg}$ ) (see figure 7.1$)$. This sample was dissolved in $25 \% \mathrm{MeOH} / \mathrm{EtOAc}(2 \mathrm{~mL})$. Half was cyclic loaded onto diol (1 $\mathrm{mL}$ ) by diluting with $\mathrm{CH}_{2} \mathrm{Cl}_{2}$ until a final concentration of $5.5 \% \mathrm{MeOH} / 16.6 \%$ EtOAc/77.9\% $\mathrm{CH}_{2} \mathrm{Cl}_{2}$ was achieved. This was transferred onto an MPLC diol column $(1.1 \times 25 \mathrm{~cm})$ as a slurry. The MPLC diol column was eluted using a gradient profile from $10-50 \% \mathrm{MeOH} /$ EtOAc. During the course of the diol separation, 105 fractions were collected. Every second tube was sub-sampled for N2A assay. Initial results indicated adequate separation, so the second half of the sample from the first diol column was cyclic loaded onto diol and processed in a similar manner (see figure 7.2). Twelve fractions from 15.5-21.5\% MeOH/EtOAc were combined (12.7 mg, $\left.25 \times 10^{6} \mathrm{TU}, 2.0 \times 10^{6} \mathrm{TU} / \mathrm{mg}\right)$ to generate the most pure sample of KBT. Side fractions from this separation were used to test the behaviour of KBT on amino and CBA ion exchange columns.

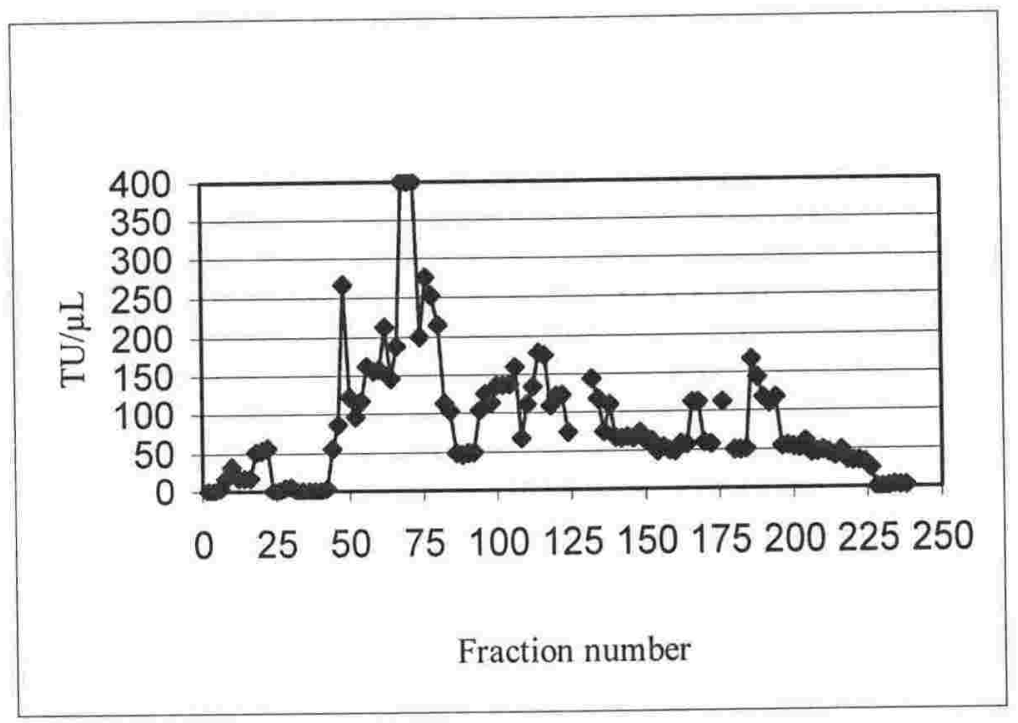

Figure 7.1 Elution profile from the large diol column. 


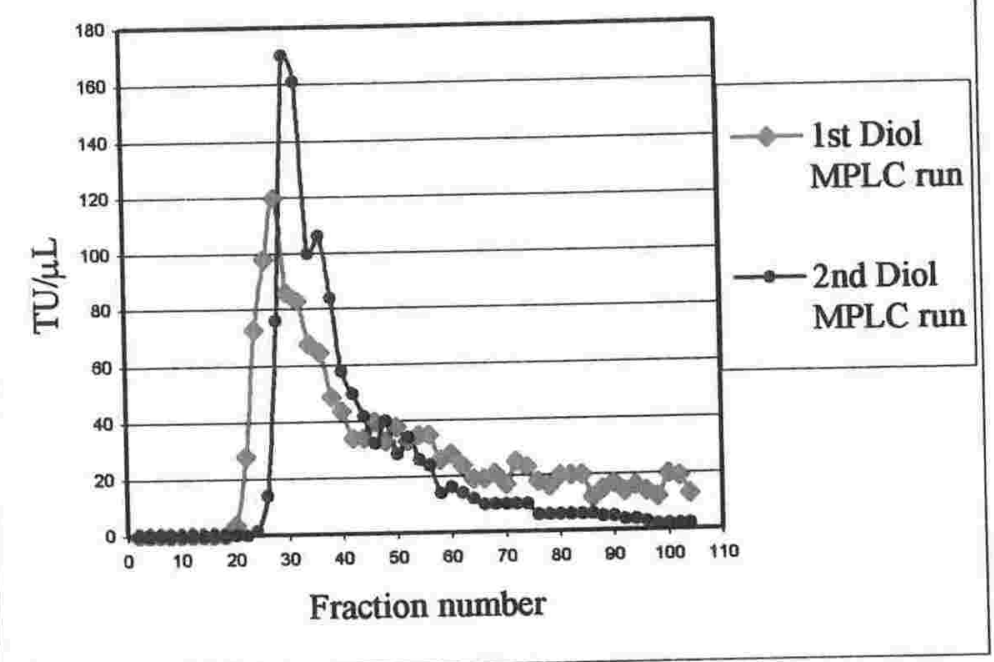

Figure 7.2 Comparison of toxicity recovered from the diol MPLC separations.

Five sub-samples $\left(250 \times 10^{3} \mathrm{TU}\right.$ each) were exposed to various acidic and basic conditions $(5 \% \mathrm{HCl}, \mathrm{HCOOH}, p \mathrm{H} 8$ buffer, $p \mathrm{H} 9$ buffer, and one neutral control) for 12h. Each sample was then cyclic loaded onto $1 \mathrm{~mL}$ Amberchrom, washed with $\mathrm{H}_{2} \mathrm{O}$ (10 mL), and eluted with $\mathrm{Me}_{2} \mathrm{CO}(5 \mathrm{~mL})$. Each sample was submitted for N2A assay. The acidic, neutral, and $p \mathrm{H} 8$ samples were all of comparable activity whilst the $p \mathrm{H} 9$ sample showed substantial loss of activity $\left(125 \times 10^{3} \mathrm{TU}\right)$.

\subsection{7}

\section{Effect of Acid or Base on Elution from PSDVB}

Three injections of KBT (15,000 TU) were performed on an analytical PRP-1 PSDVB column using a gradient elution profile from 40-70\% $\mathrm{Me}_{2} \mathrm{CO} / \mathrm{aqueous}$ solvent ( $1 \mathrm{~mL} / \mathrm{min}$ ) over a period of 15 minutes, collecting 30 fractions per run. Neutral, acidic ( $p \mathrm{H} 5$ buffer), or basic ( $p \mathrm{H} 8$ buffer) were used as the aqueous solvent component of the system. Buffers were prepared by adding $\mathrm{AcOH}$ or $\mathrm{NH}_{3}$ to a $0.2 \mathrm{M}$ solution of $\mathrm{NH}_{4} \mathrm{OAc}$ 
until the desired $\mathrm{pH}$ was obtained. Each fraction collected was submitted for N2A assay. As the neutral injection had resulted in two separate peaks of activity, two more injections were performed under identical conditions which confirmed the original observation (see figure 7.3). Two samples from the original neutral injection were concentrated and reinjected under the same conditions (see figure 7.4).

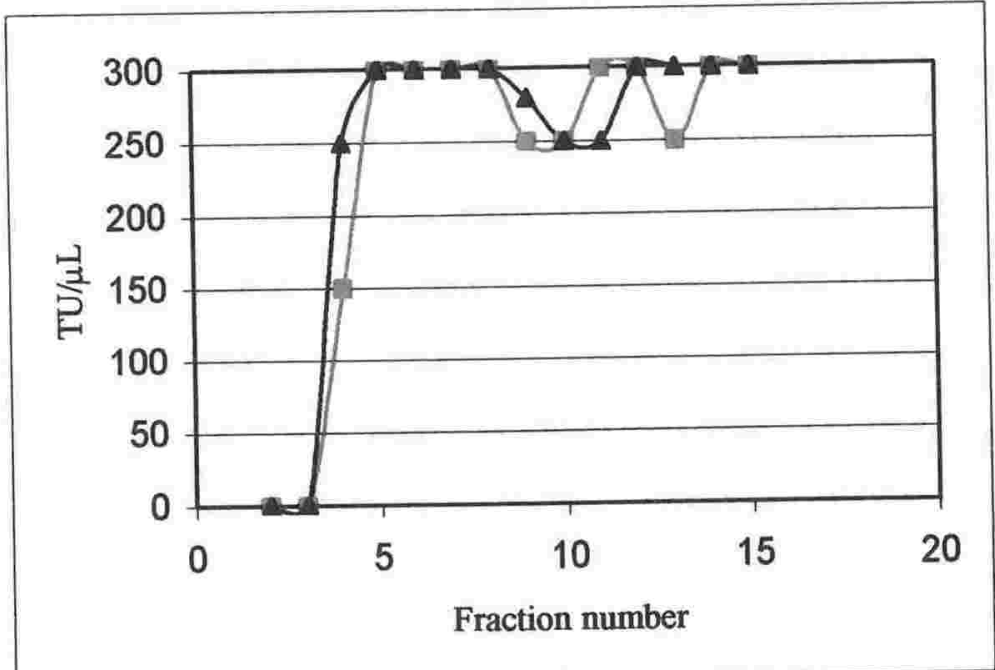

Figure 7.3 Graph of two repeat injections of KBT under neutral conditions using PSDVB.

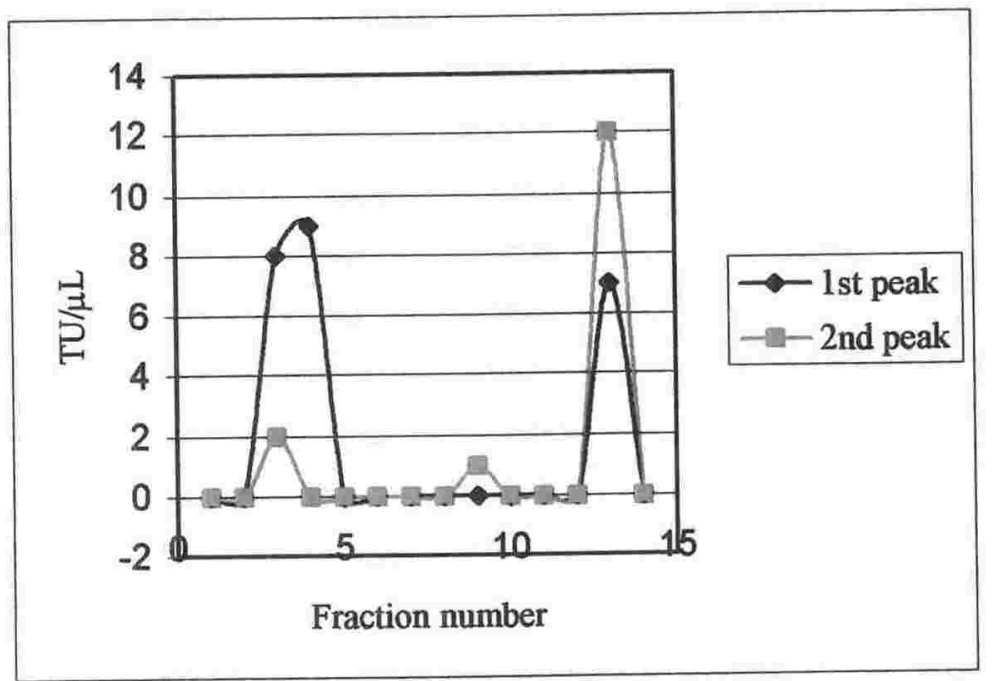

Figure 7.4 Graph of the re-injection of a fraction from each peak of activity under neutral conditions using PSDVB. 
An unquantified semi-purified sample of KBT (22.7 mg) (a side fraction combined from the two diol MPLC separations) was dissolved in DMSO $(0.3 \mathrm{~mL})$, to which $\mathrm{MeOH}$ $(2.7 \mathrm{~mL})$ was added. The resulting solution was passed through an amino column $(0.5 \mathrm{x}$ $2.0 \mathrm{~cm}$ ) that had been pre-equilibrated with $\mathrm{MeOH}(5 \mathrm{~mL})$. The column was washed with $\mathrm{MeOH}(2 \mathrm{~mL})$ which was collected together with the eluent of loading. The column was then eluted with $5 \mathrm{~mL}$ portions of (1) $2 \% \mathrm{AcOH} / \mathrm{MeOH}$, (2) DMSO, (3) $5 \% \mathrm{AcOH} / \mathrm{DMSO}$, (4) $5 \% \mathrm{HCOOH} / \mathrm{DMSO}$, and (5) $5 \% \mathrm{NH}_{3} / \mathrm{DMSO}$. None of the samples exhibited any significant cytotoxicity in the N2A assay, indicating that the KBT had irreversibly bound to the column substrate.

\subsection{9}

\section{Use of the CBA Ion Exchange Column}

An unquantified semi-purified sample of KBT $(7.9 \mathrm{mg}$ ) (a side fraction combined from the two diol MPLC separations) was dissolved in DMSO $(0.3 \mathrm{~mL})$, to which $\mathrm{MeOH}$ $(2.7 \mathrm{~mL})$ was added. The resulting solution was passed through a CBA column $(0.5 \times 2.0 \mathrm{~cm})$ that had been pre-equilibrated with $\mathrm{MeOH}(5 \mathrm{~mL})$. The column was then washed with $\mathrm{MeOH}(2 \mathrm{~mL})$ which was collected together with the eluent of loading. The column was then eluted with $5 \mathrm{~mL}$ portions of (1) $2 \% \mathrm{NH}_{3} / \mathrm{MeOH}$, (2) DMSO, and (3) $5 \% \mathrm{NH}_{3} / \mathrm{DMSO}$. All three samples were tested using the $\mathrm{N} 2 \mathrm{~A}$ assay with all the toxicity concentrated in fraction (1) $\left(8.7 \mathrm{mg}, 12.5 \times 10^{6} \mathrm{TU}, 1.44 \times 10^{6} \mathrm{TU} / \mathrm{mg}\right)$. 
NMR Spectra of Clathriol A (32)

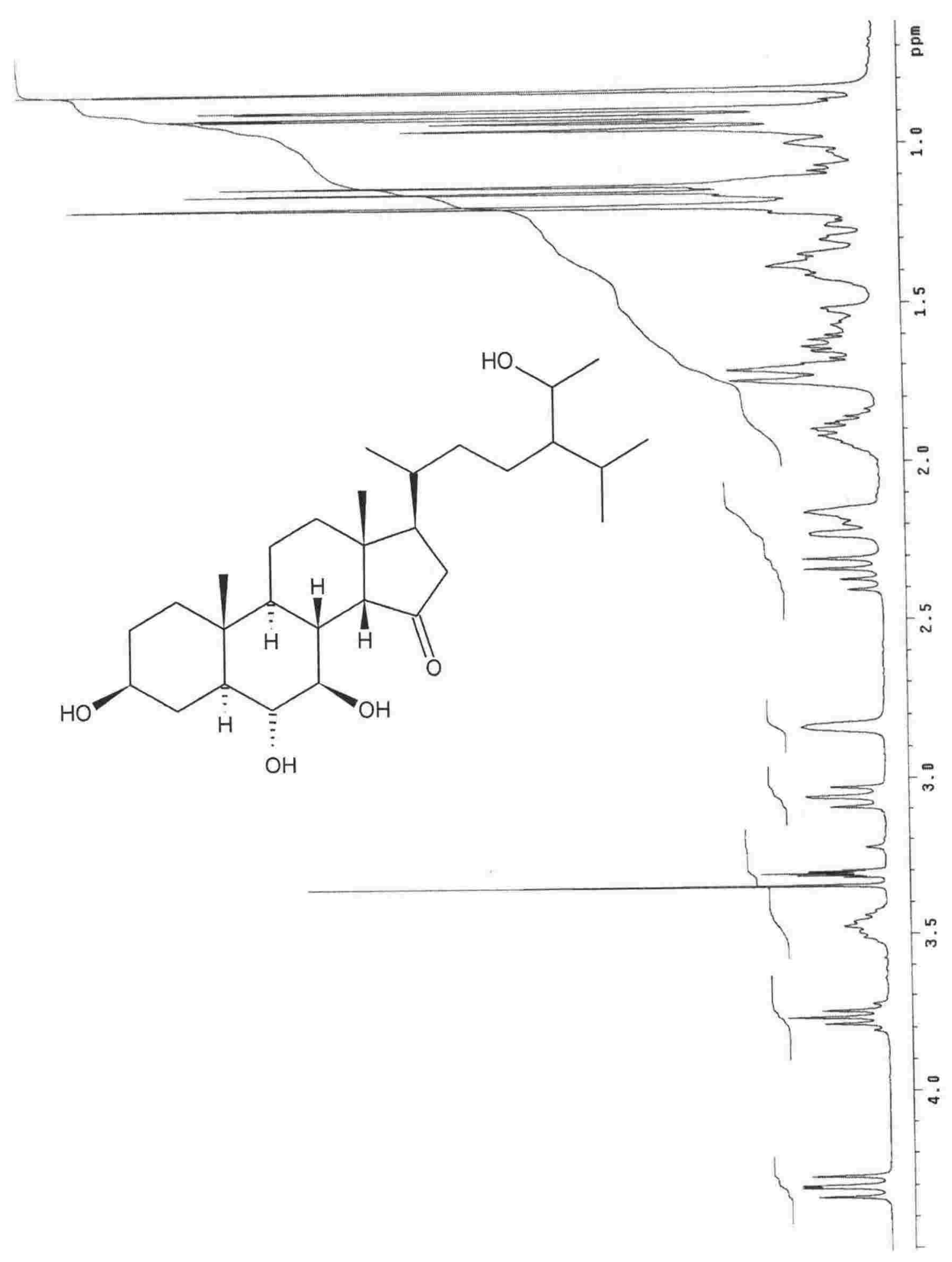

${ }^{1} \mathrm{H}$ spectrum of clathriol A (32) (300 MHz, $\mathrm{CD}_{3} \mathrm{OD}$ ) 


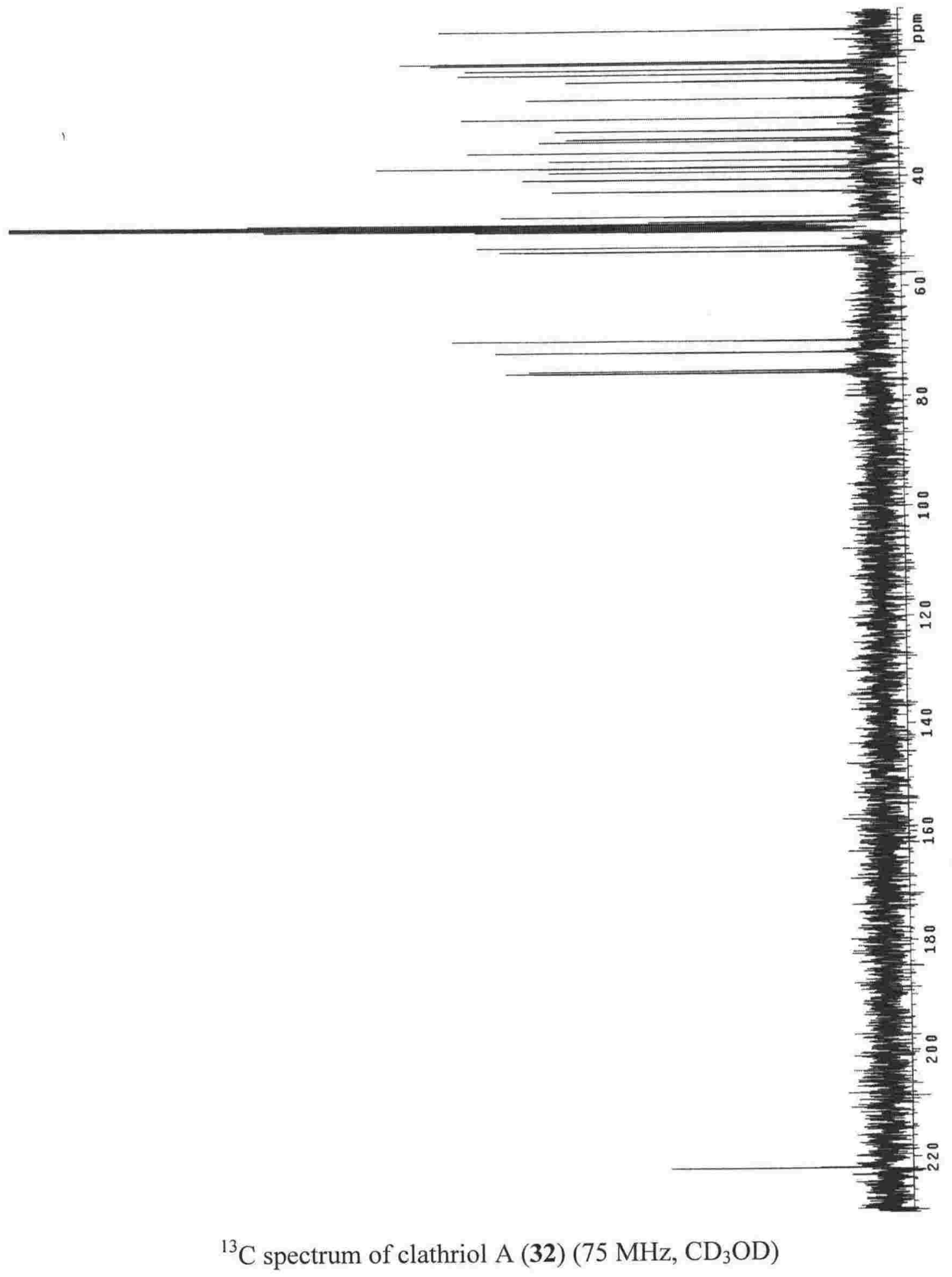




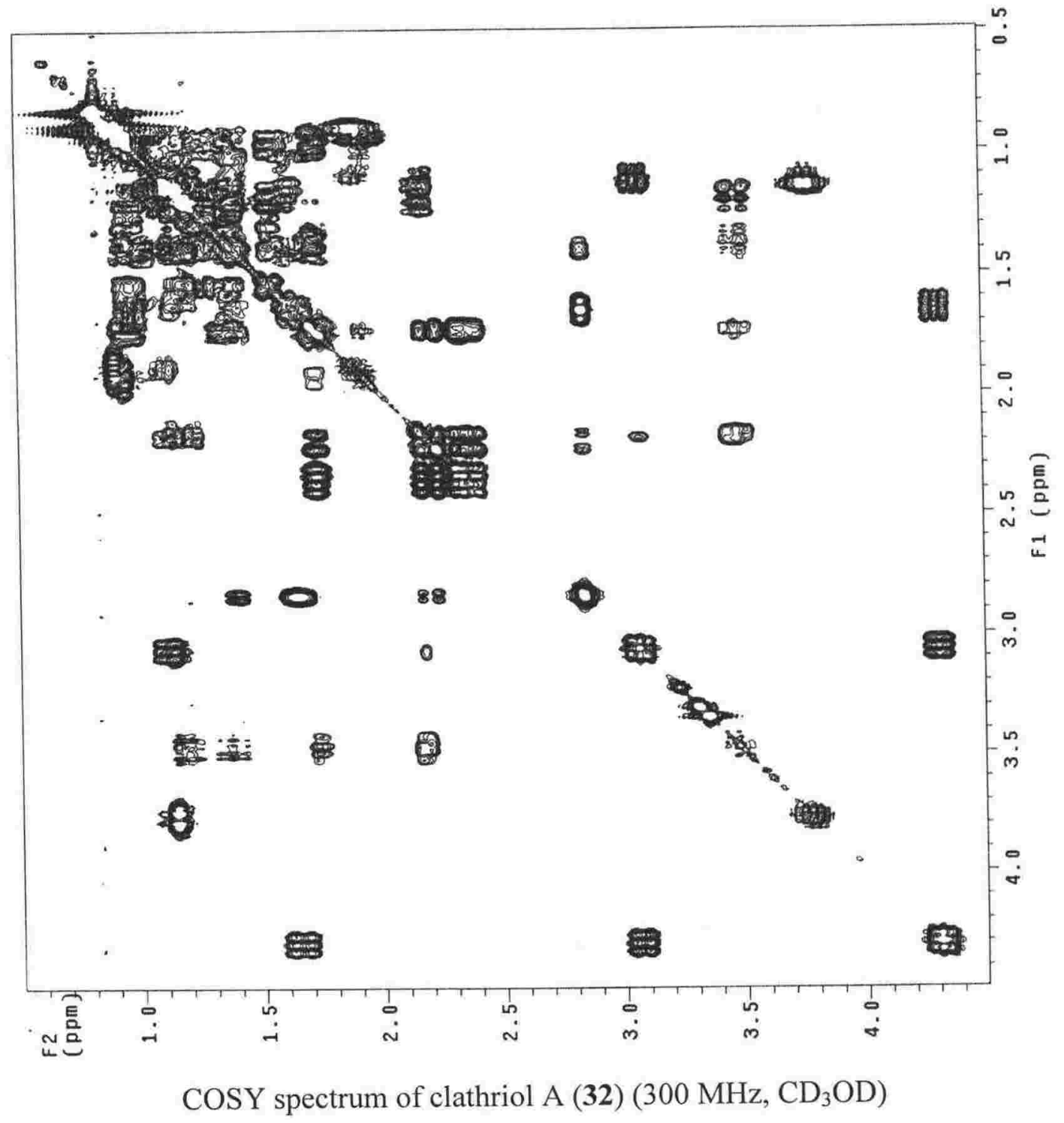




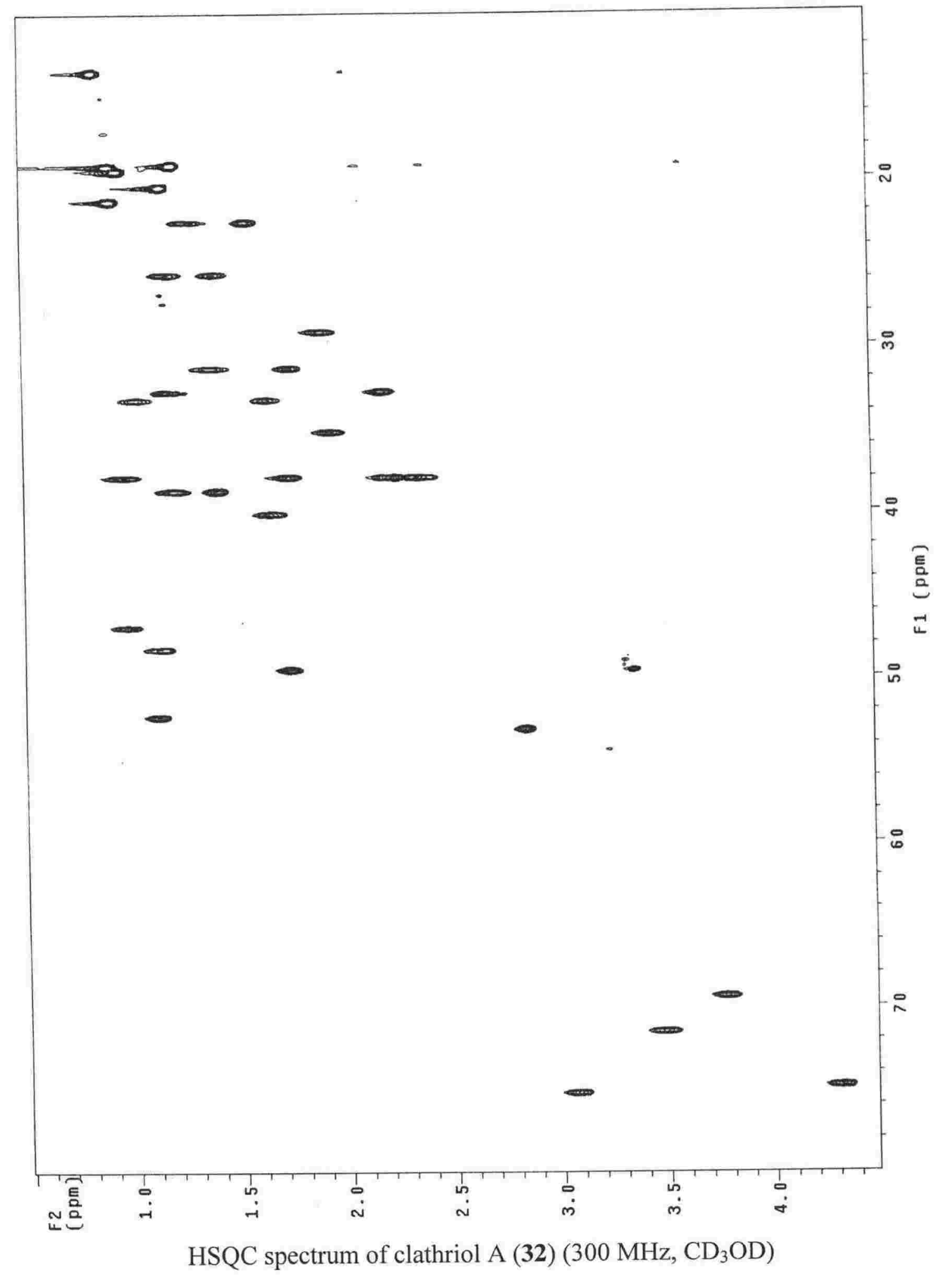




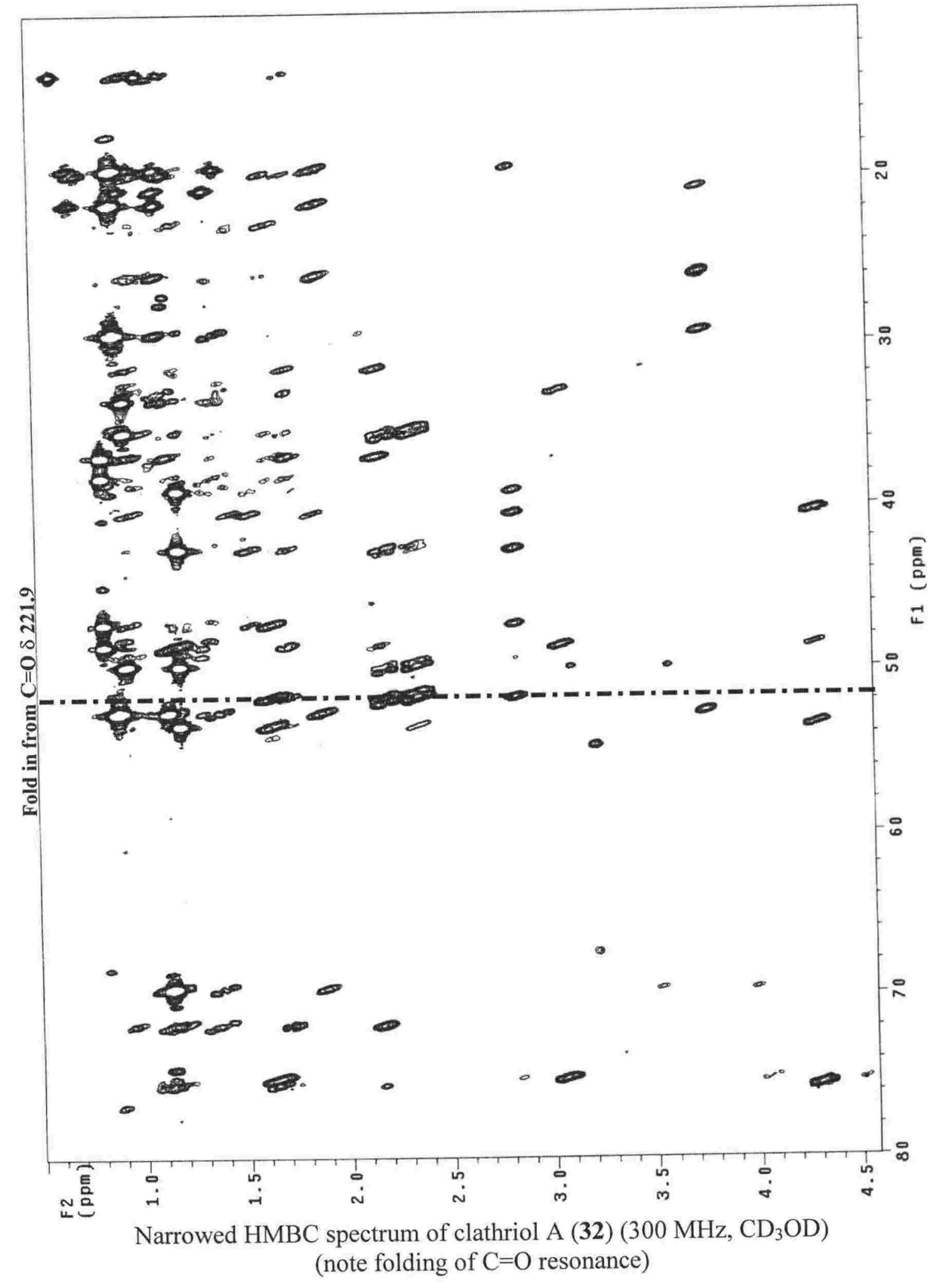




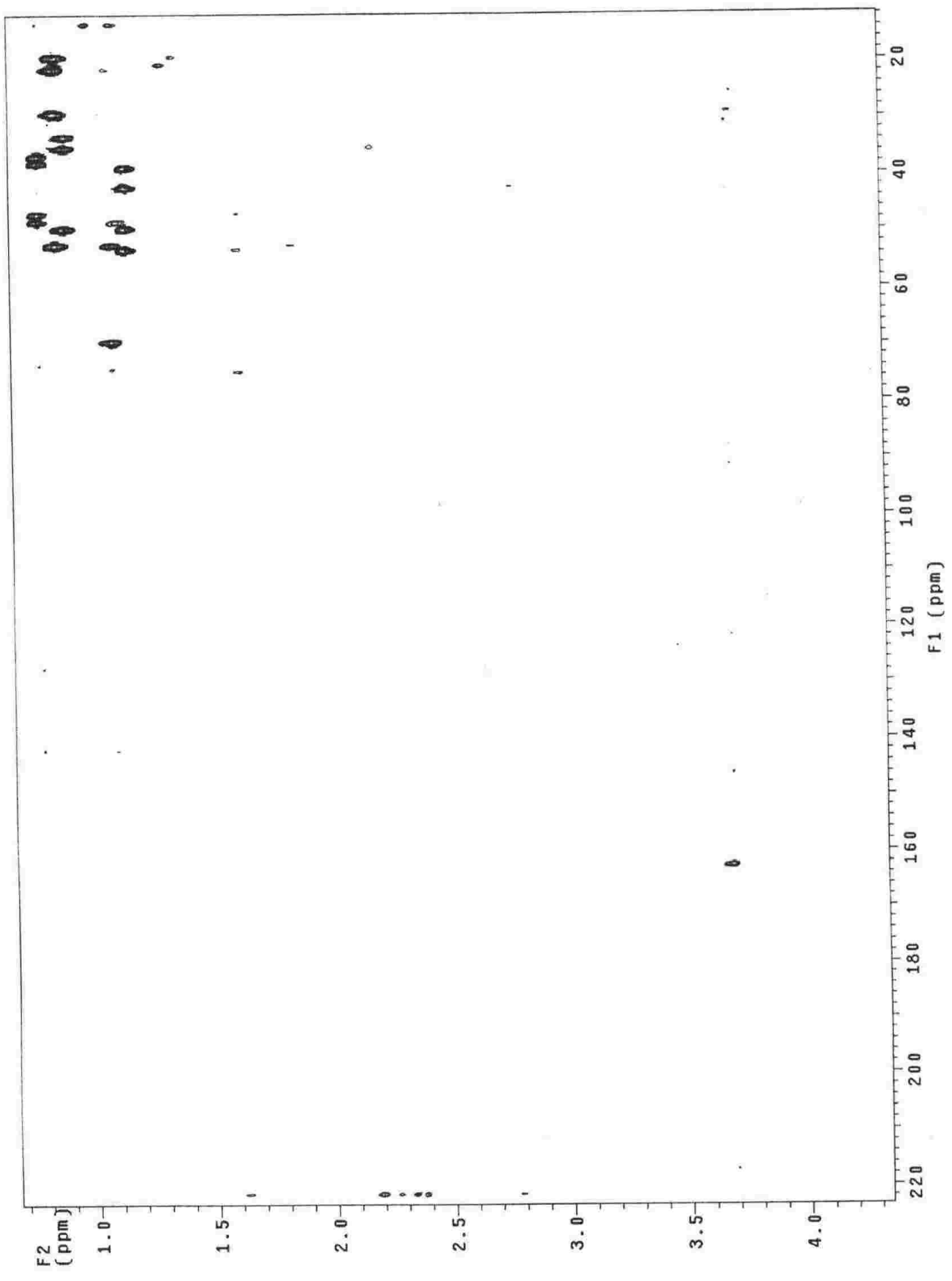

Full HMBC spectrum of clathriol A (32) (300 MHz, $\mathrm{CD}_{3} \mathrm{OD}$ ) 


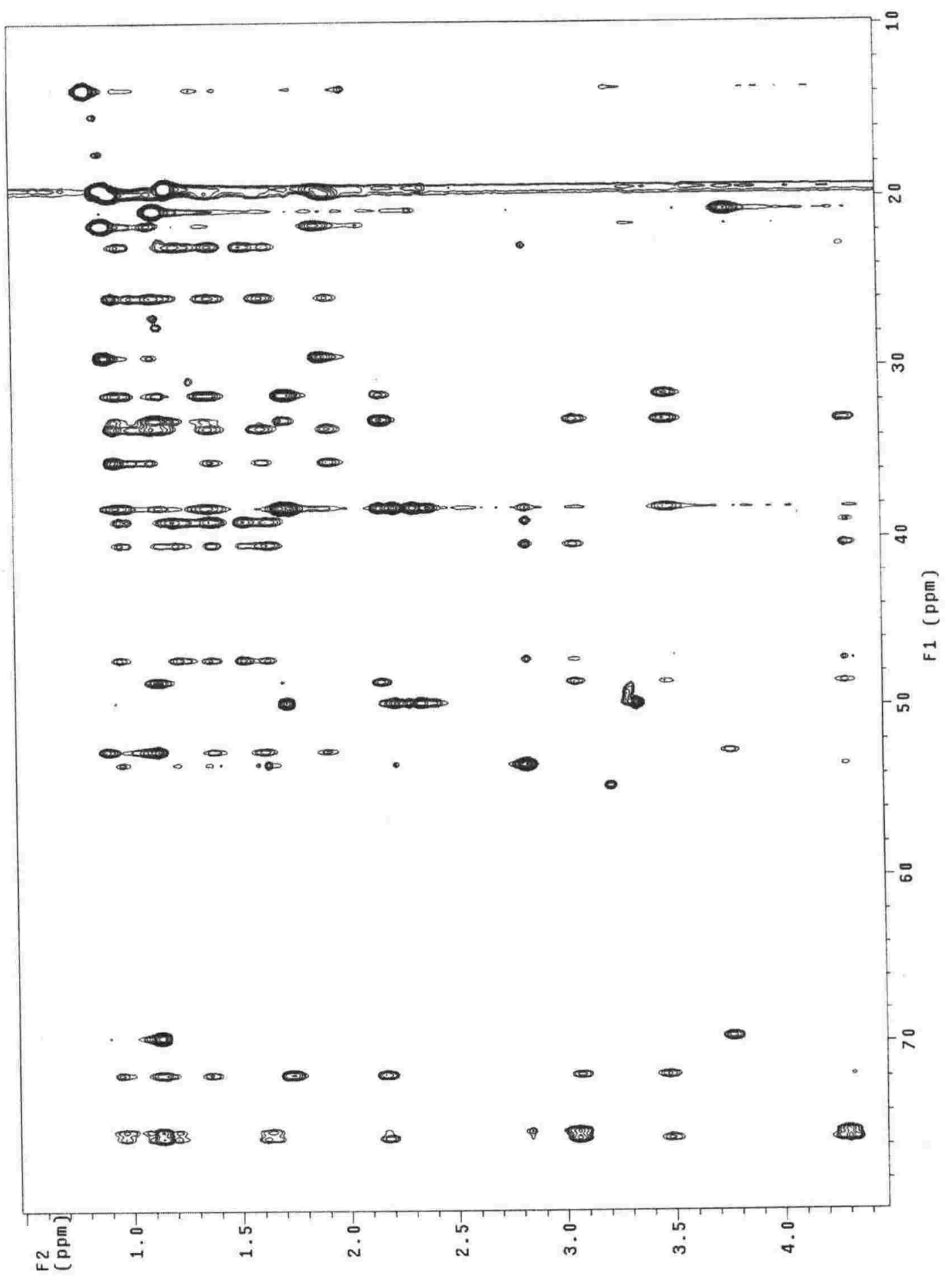

HSQC-TOCSY (80 ms mixing time) spectrum of clathriol A (32) (300 MHz, $\mathrm{CD}_{3} \mathrm{OD}$ ) 


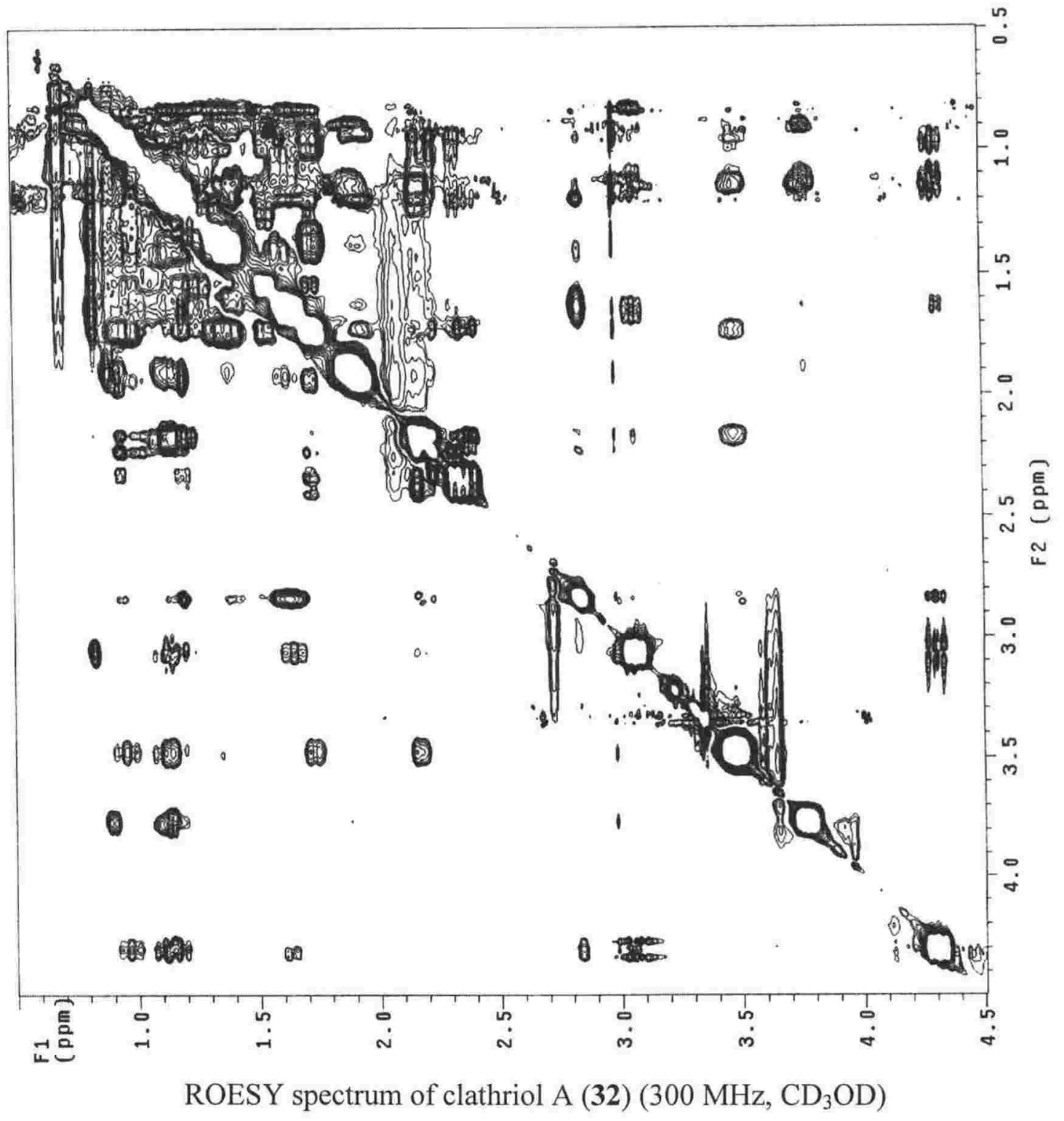


Appendix Two

NMR Spectra of Clathriol B (33)

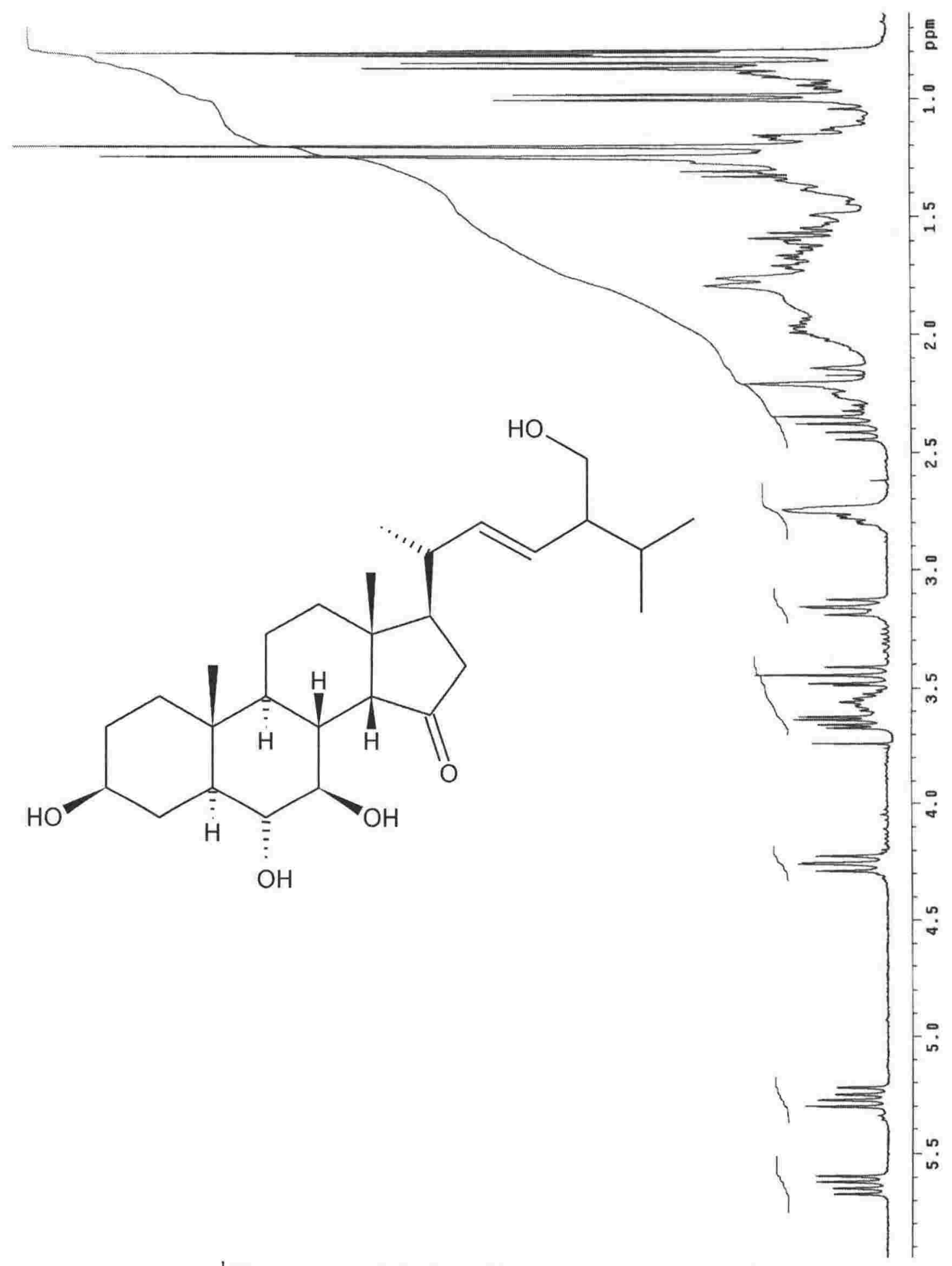

${ }^{\mathrm{I}} \mathrm{H}$ spectrum of clathriol B (32) (300 MHz, $\mathrm{CDCl}_{3}$ ) 


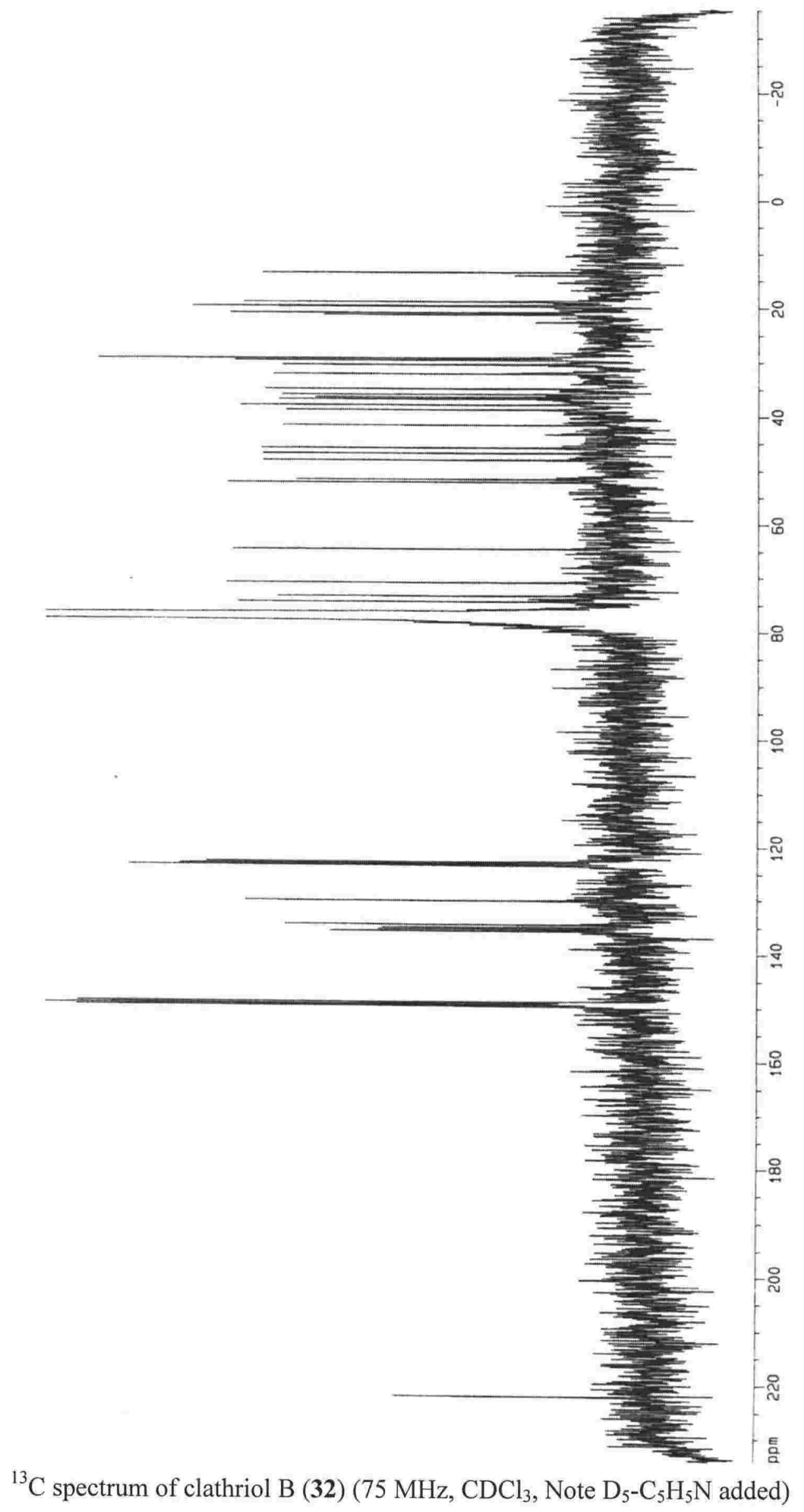




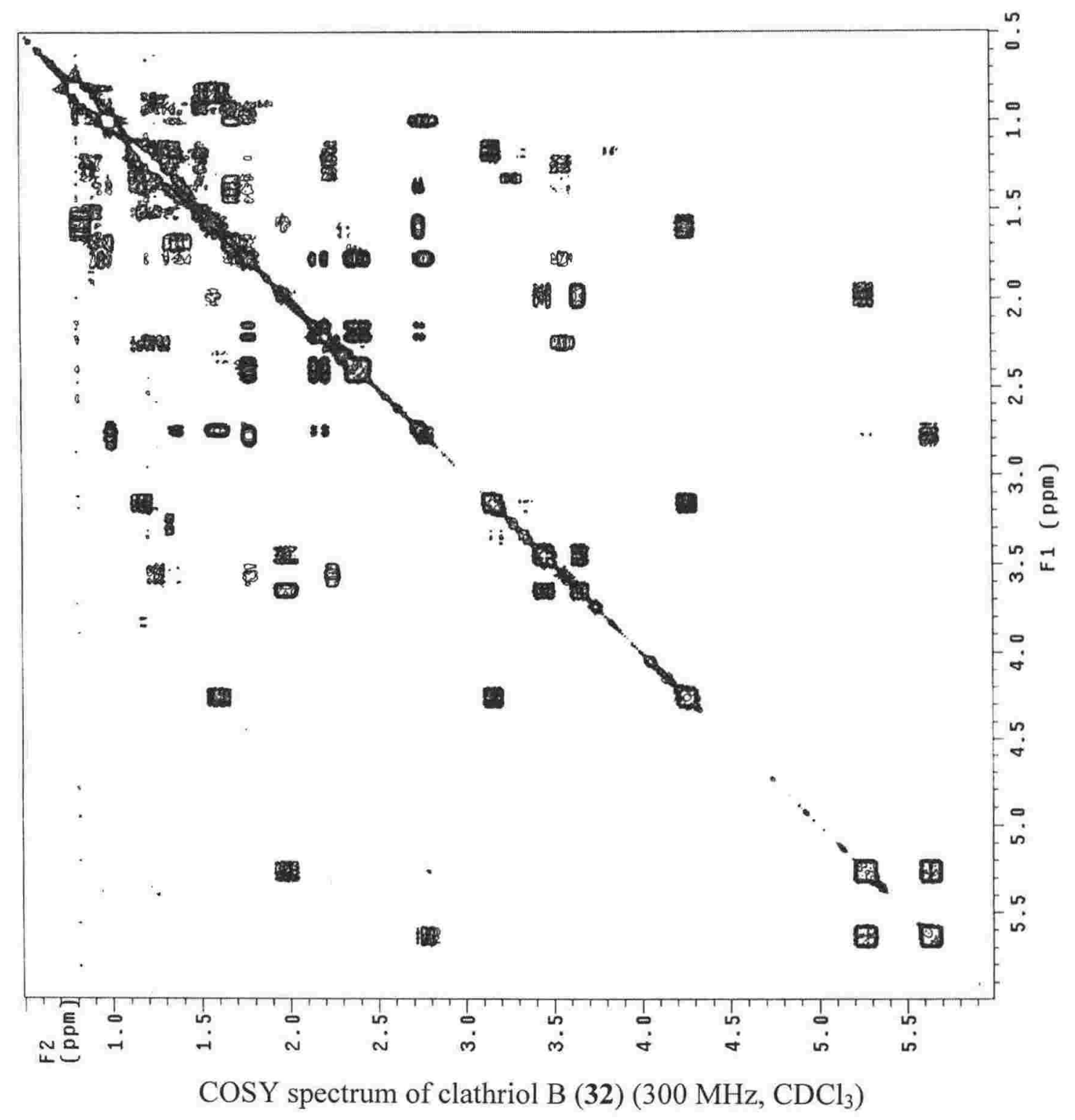




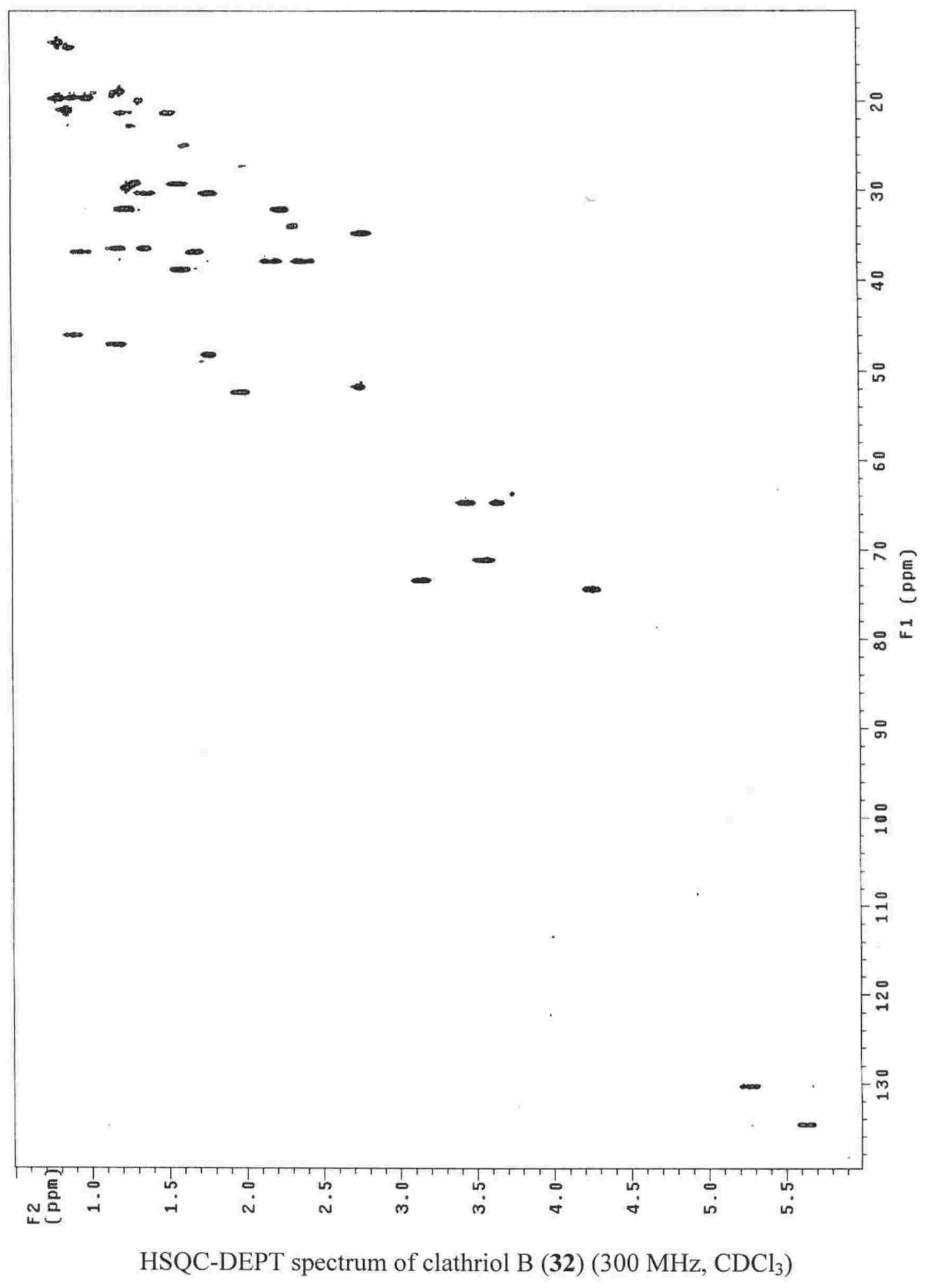




\section{NMR Spectra of Cadlinolide C (138)}

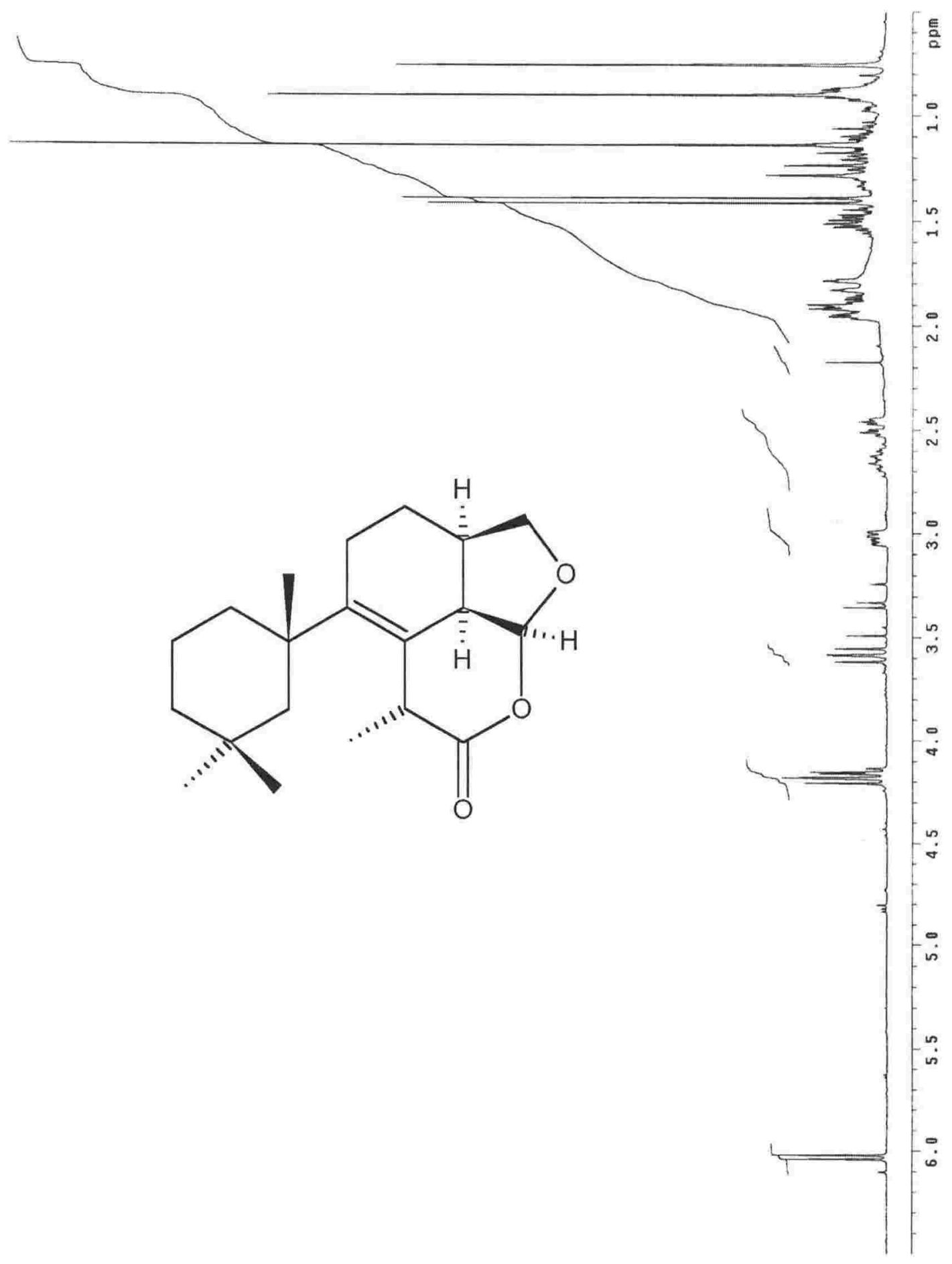

${ }^{1} \mathrm{H}$ spectrum of cadlinolide $\mathrm{C}(\mathbf{1 3 8})\left(300 \mathrm{MHz}, \mathrm{CDCl}_{3}\right)$ 


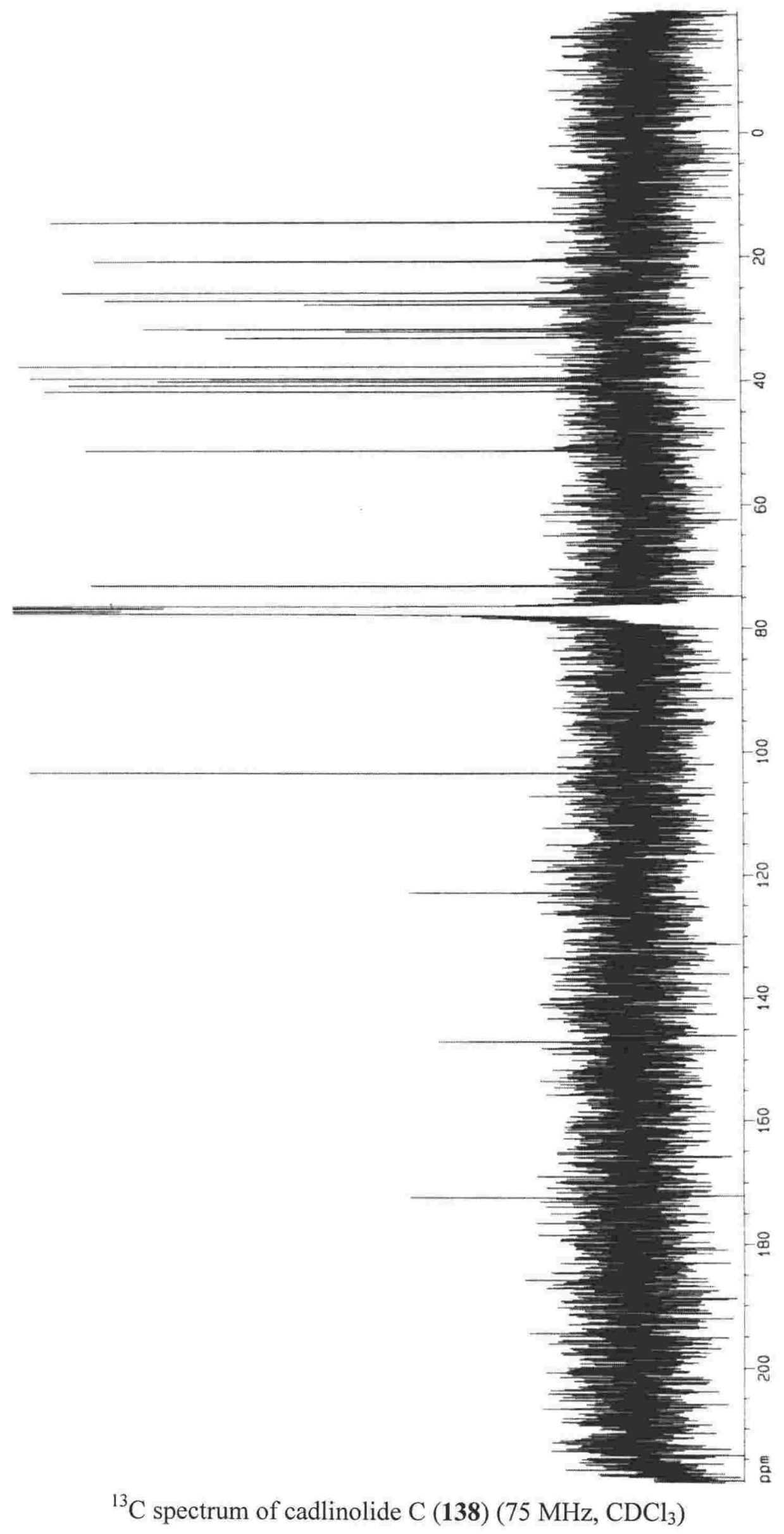




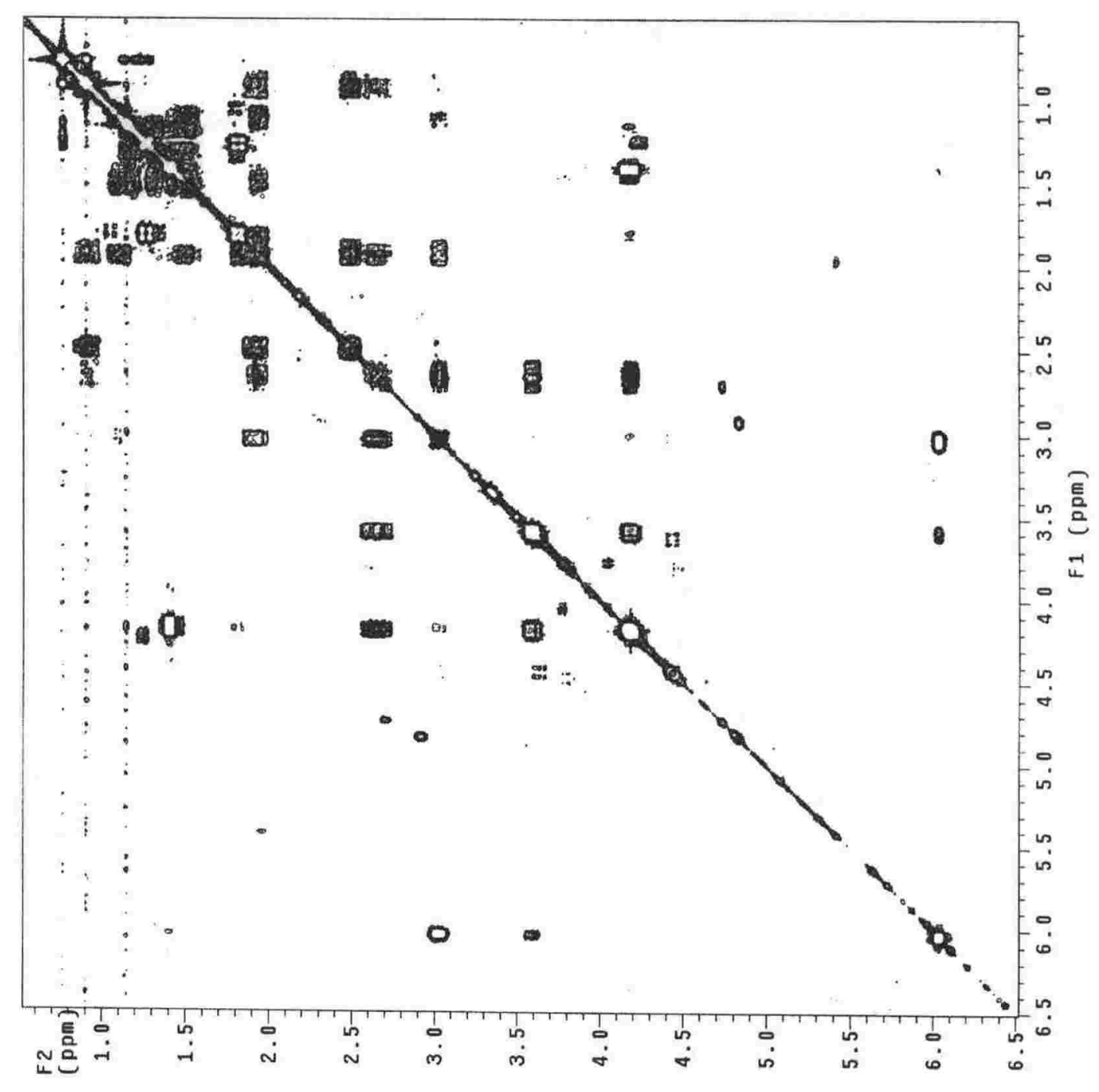

COSY spectrum of cadlinolide $\mathrm{C}(\mathbf{1 3 8})\left(300 \mathrm{MHz}, \mathrm{CDCl}_{3}\right)$ 


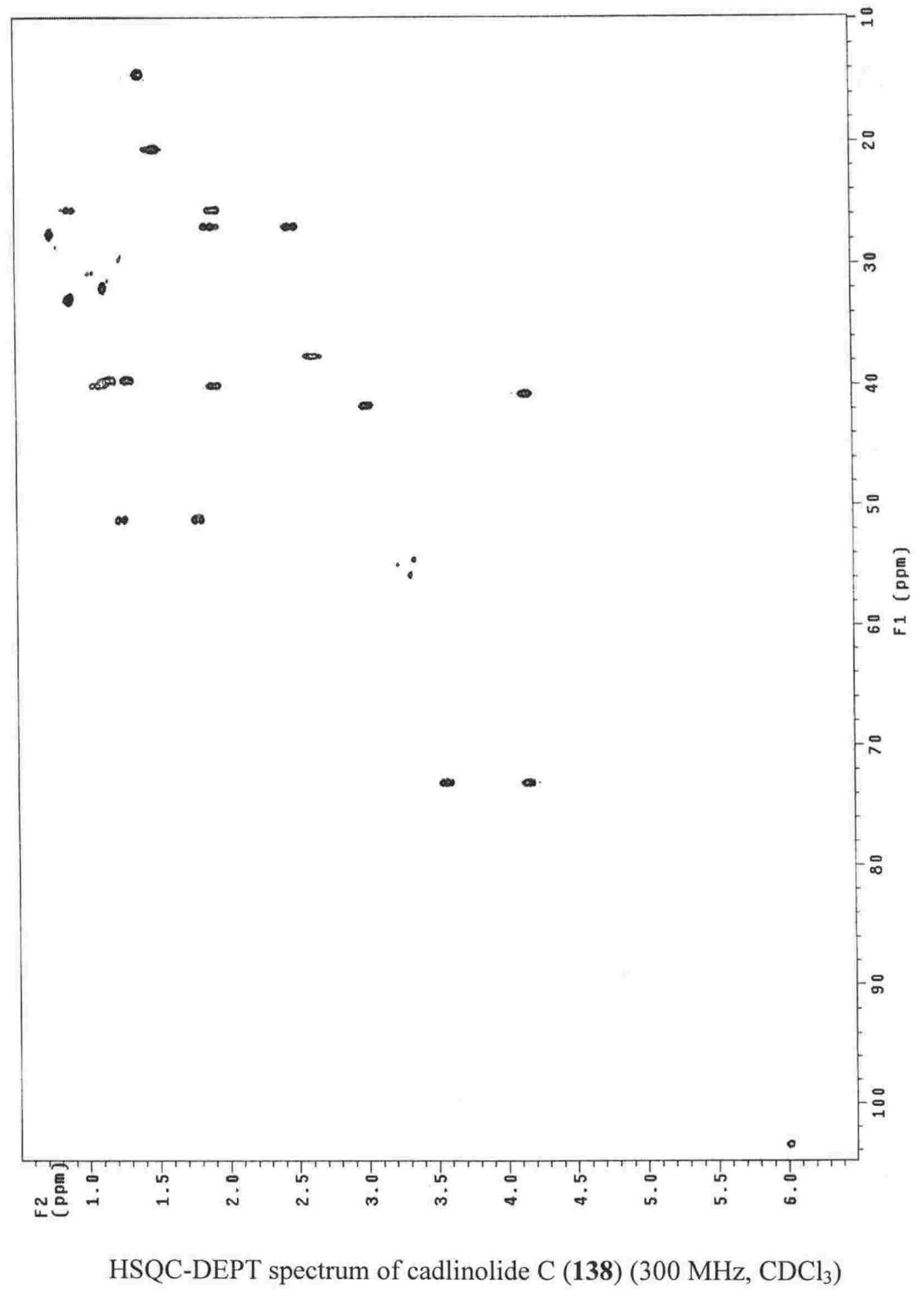




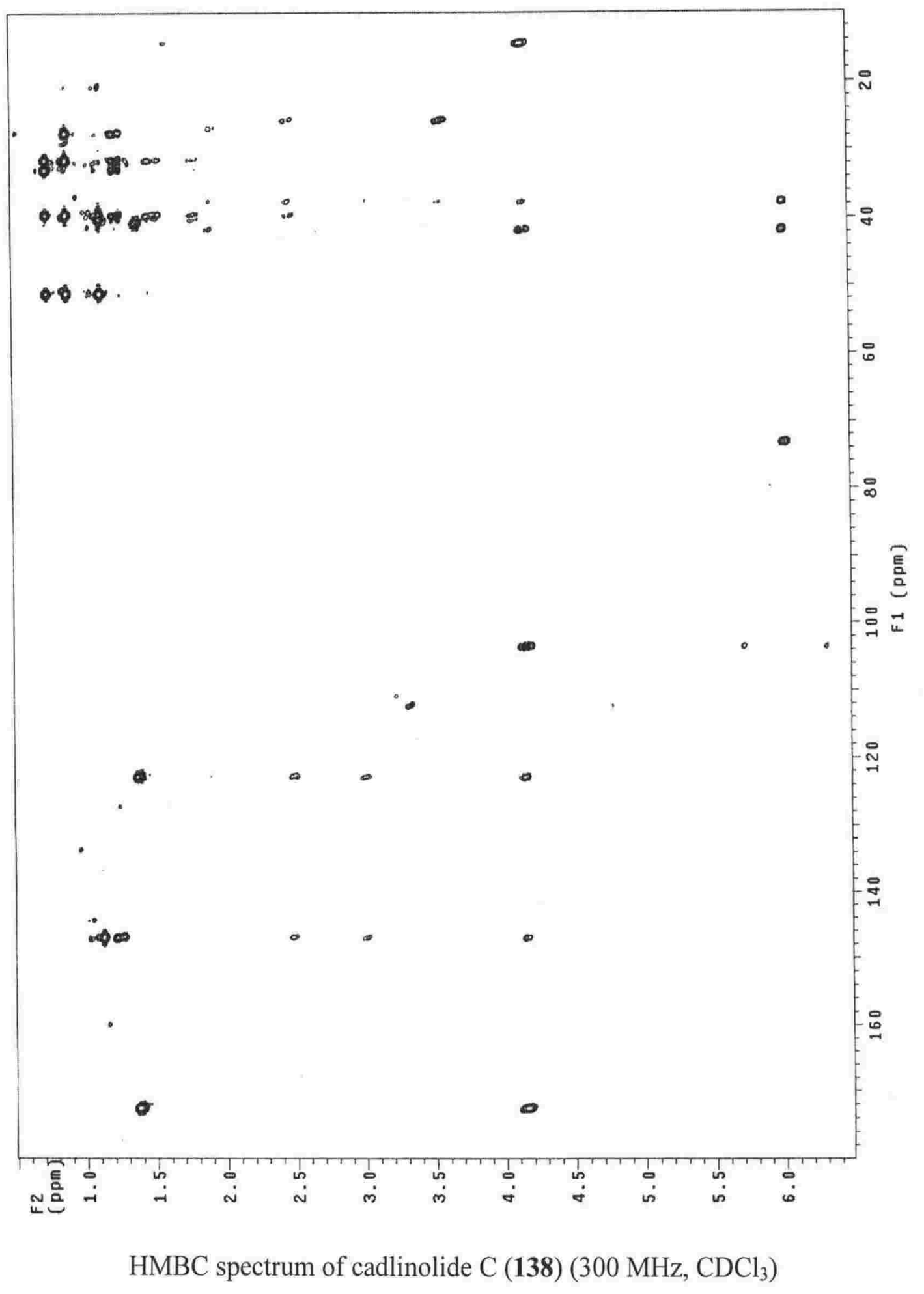




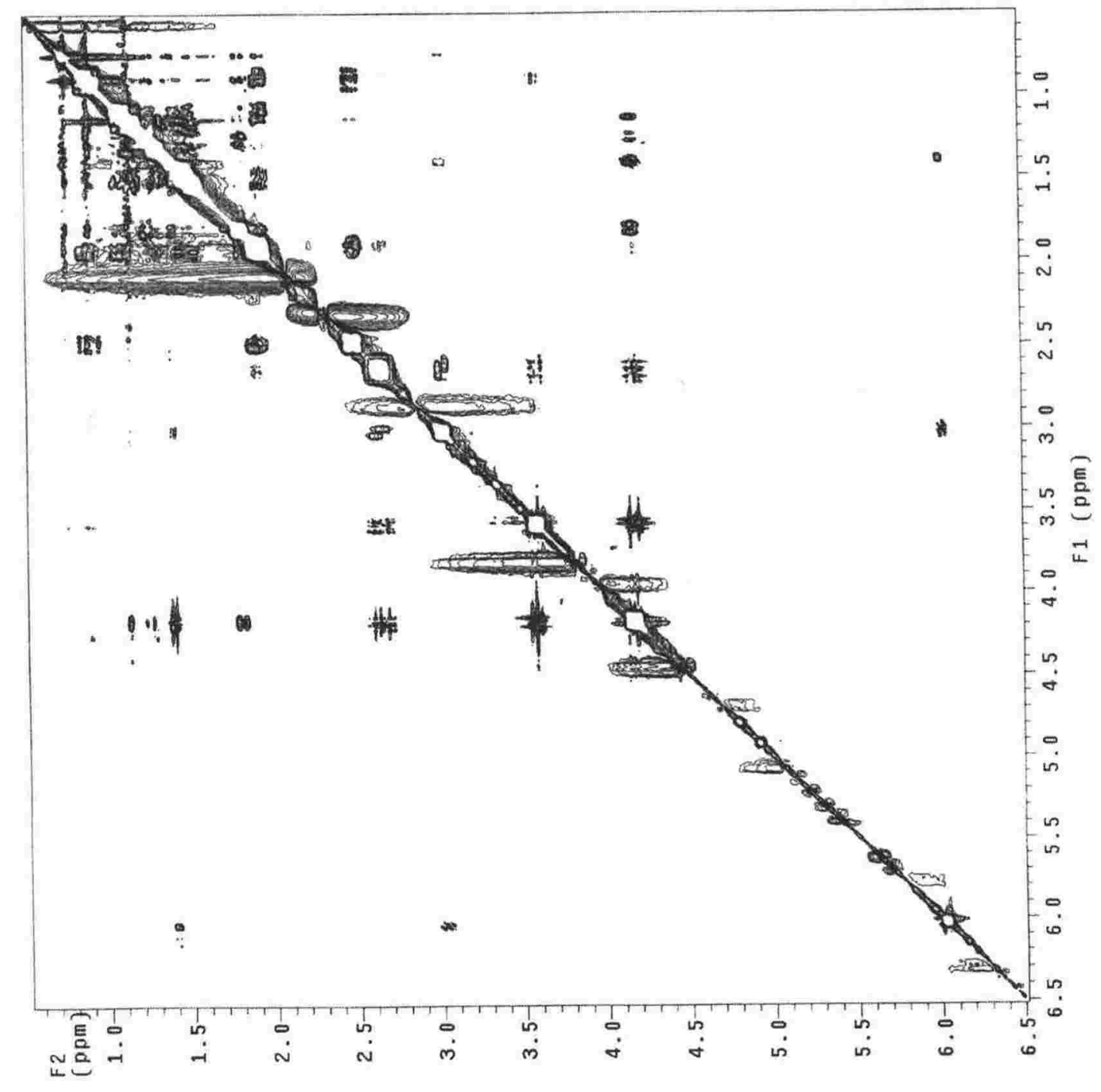

ROESY spectrum of cadlinolide C (138) $\left(300 \mathrm{MHz}, \mathrm{CDCl}_{3}\right)$ 
Appendix Four

NMR Spectra of Cadlinolide D (139)

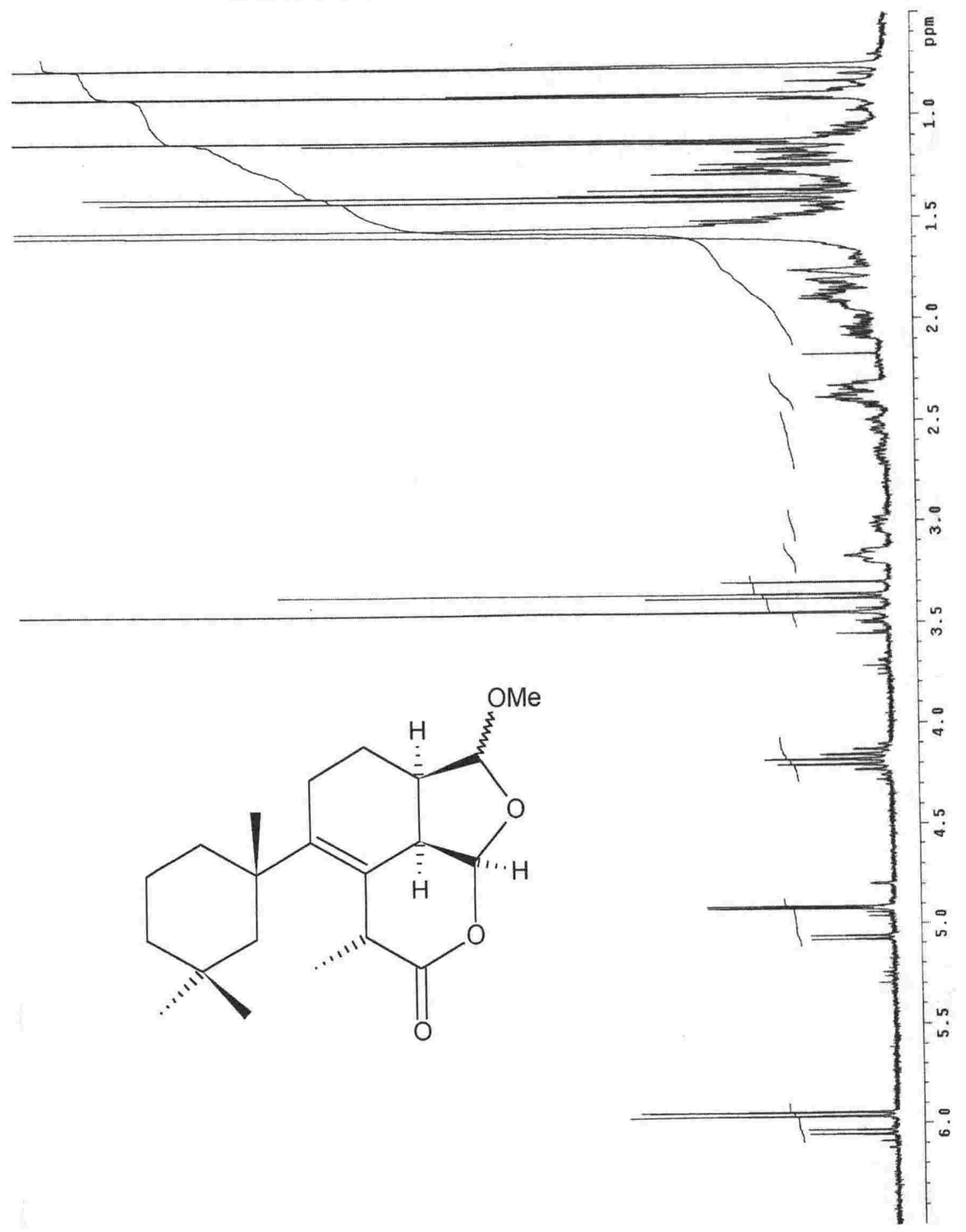

${ }^{1} \mathrm{H}$ spectrum of cadlinolide $\mathrm{D}$ (139) (300 $\mathrm{MHz}, \mathrm{CDCl}_{3}$ ) 


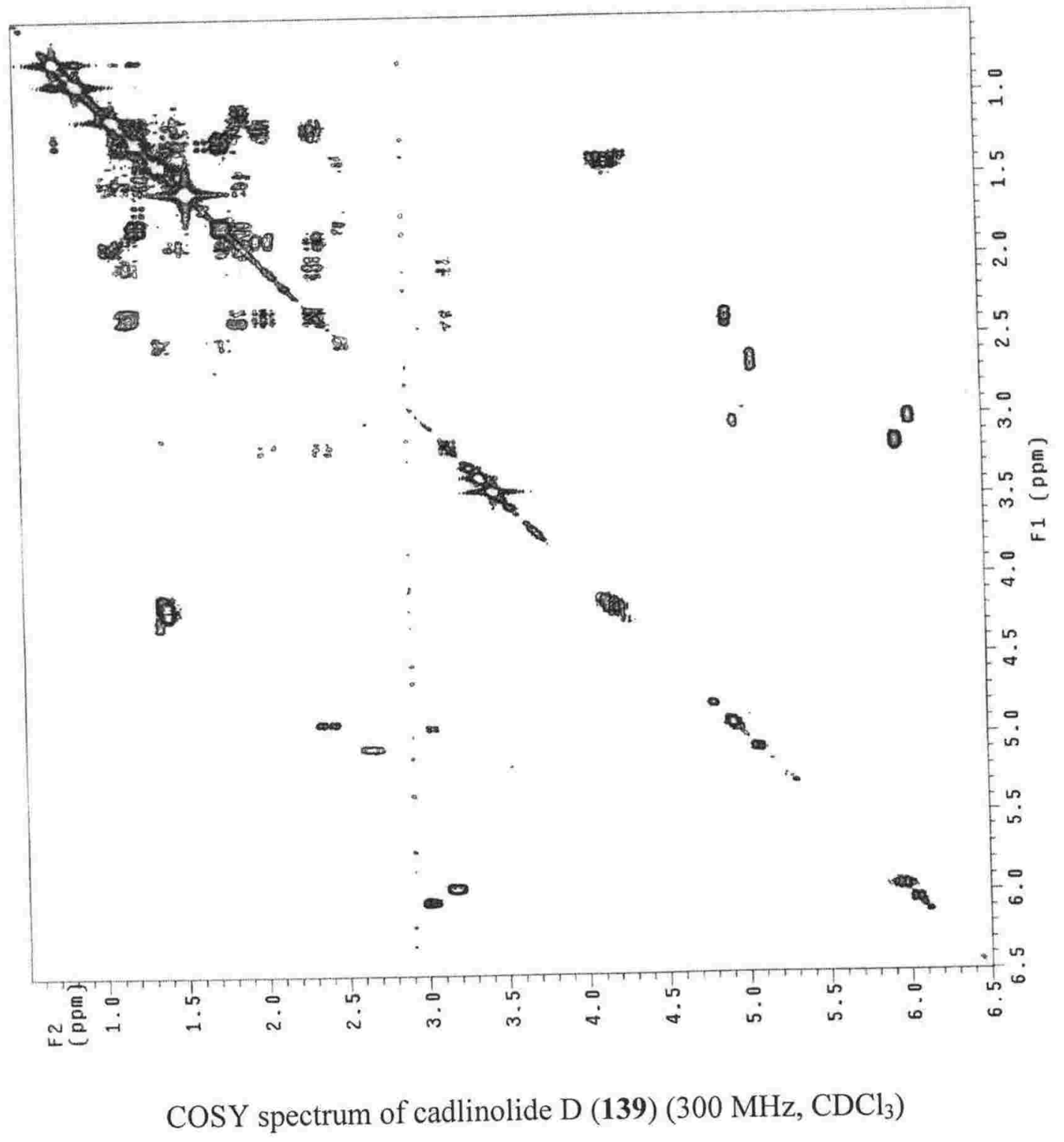


Appendix Five

\section{NMR Spectra of Pourewic Acid A (140)}

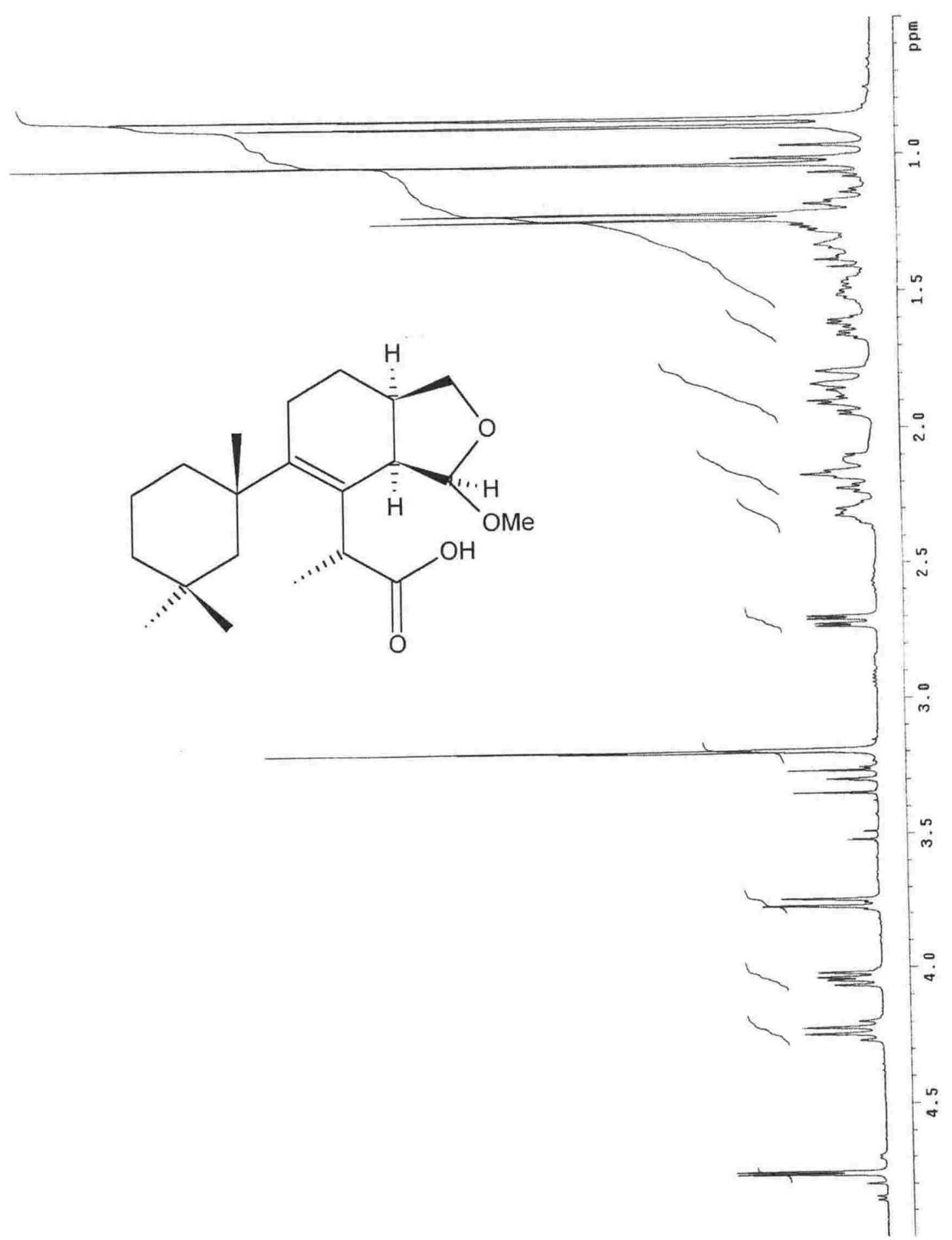

${ }^{1} \mathrm{H}$ spectrum of pourewic acid $\mathrm{A}(\mathbf{1 4 0})\left(300 \mathrm{MHz}, \mathrm{CDCl}_{3}\right)$ 


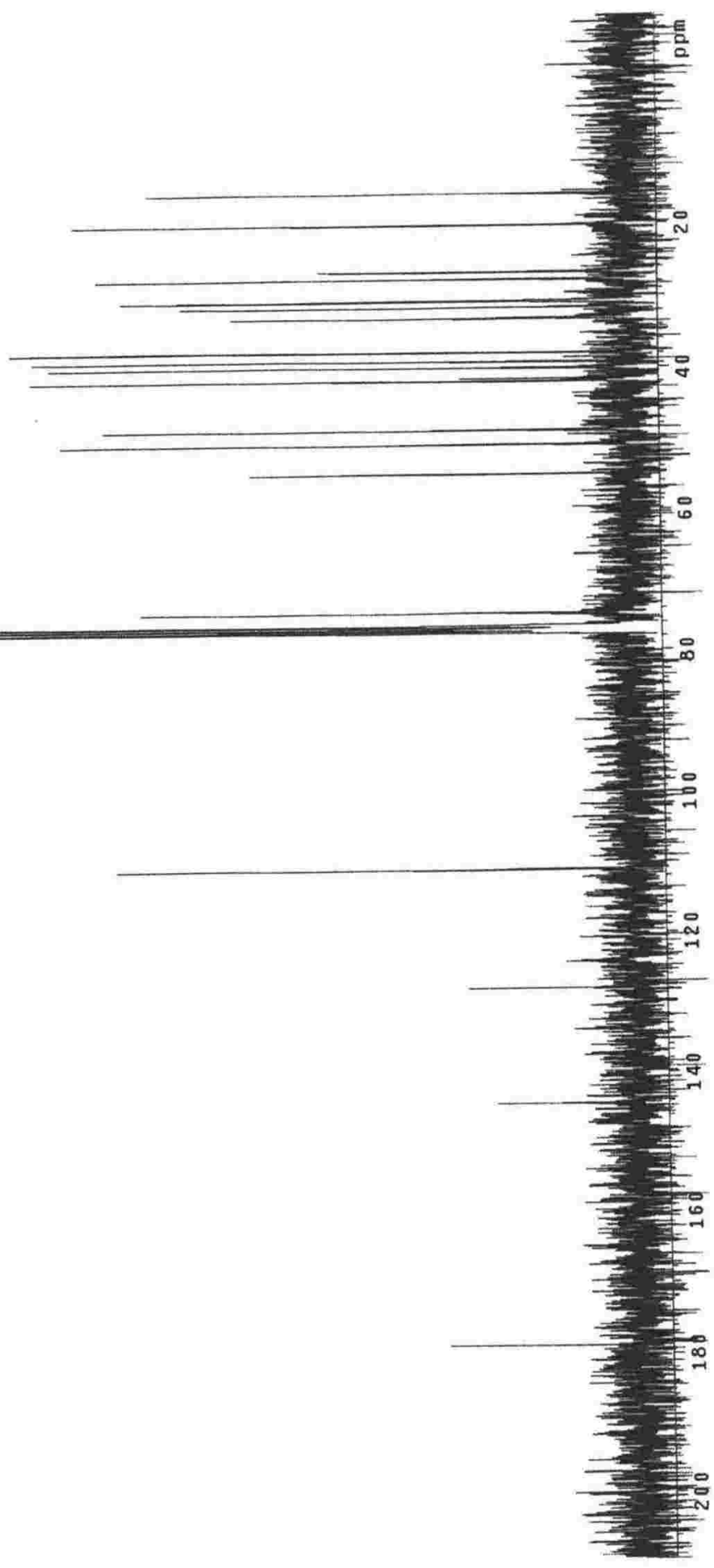

${ }^{13} \mathrm{C}$ spectrum of pourewic acid $\mathrm{A}(\mathbf{1 4 0})\left(75 \mathrm{MHz}, \mathrm{CDCl}_{3}\right)$ 


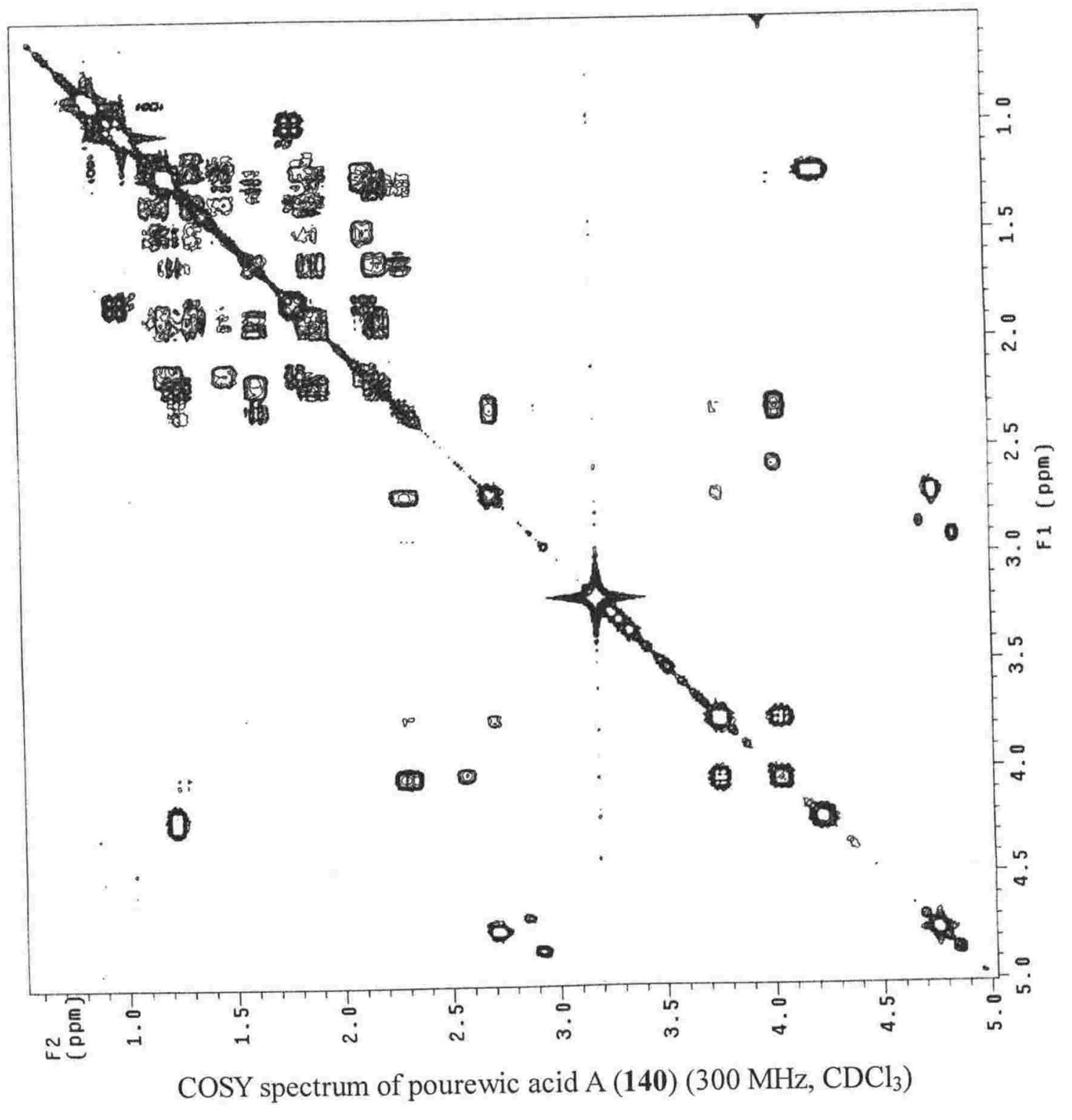




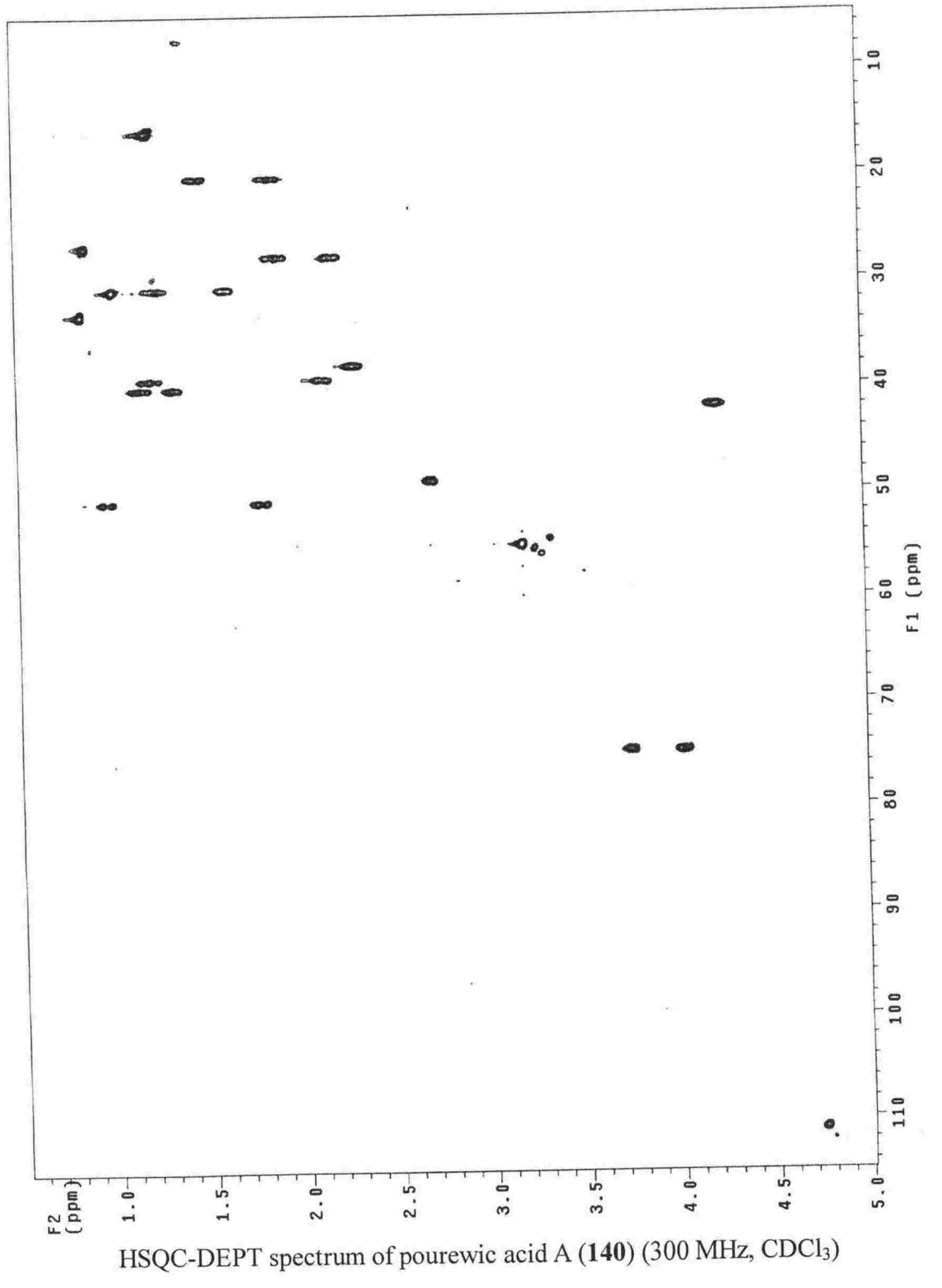




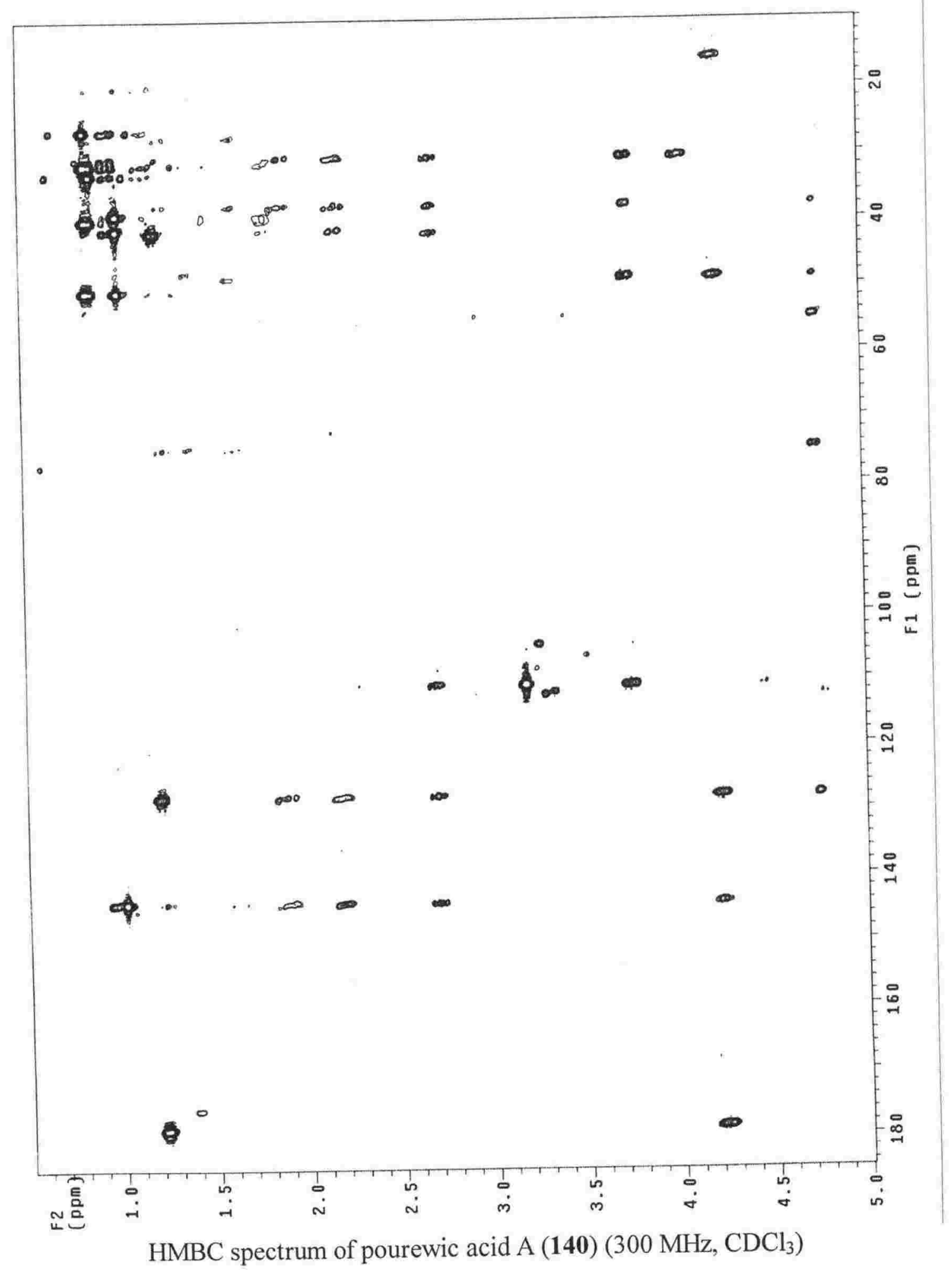




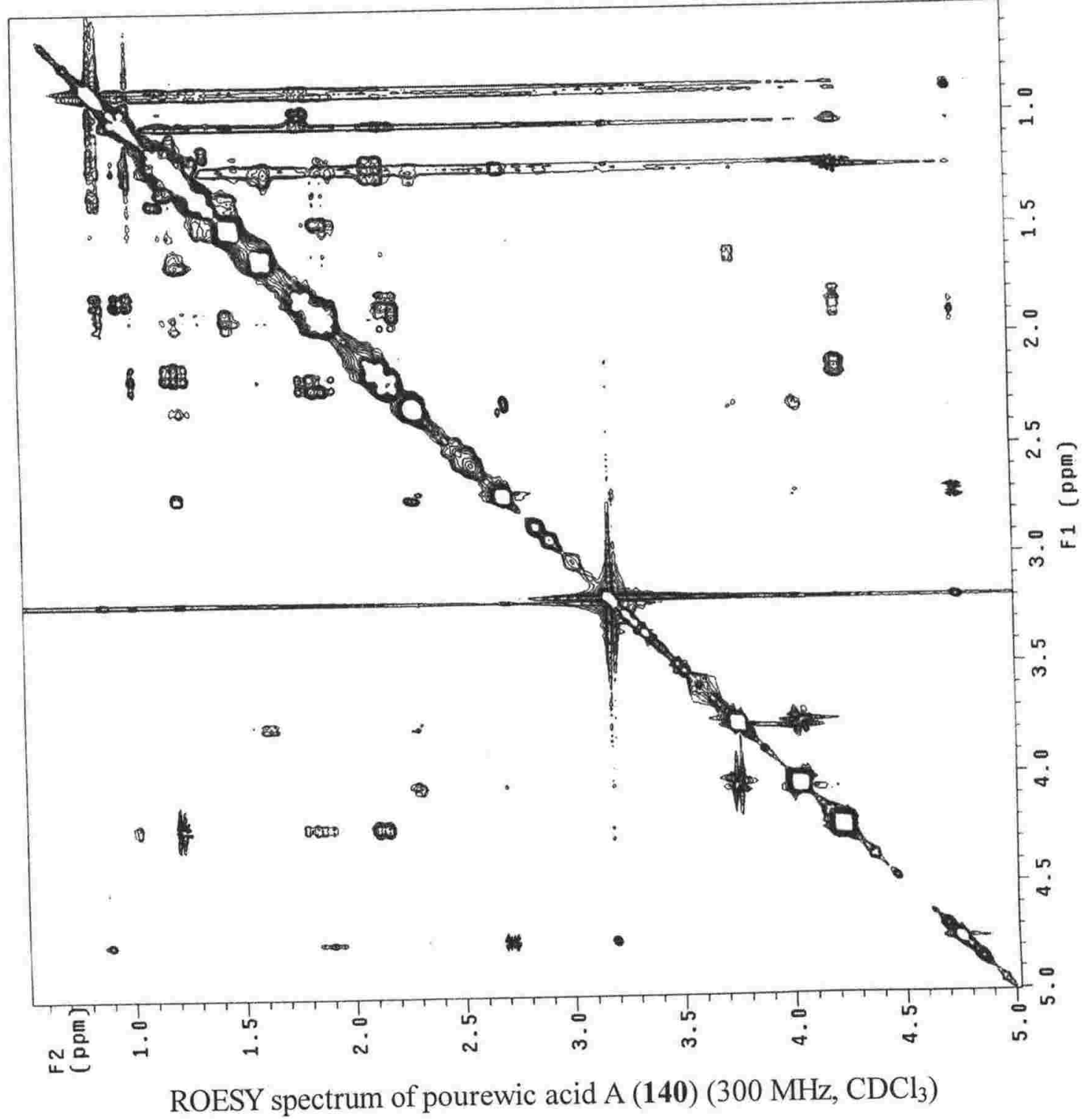


Appendix Six

\section{NMR Spectra of 15-Methoxypourewic Acid B (141)}

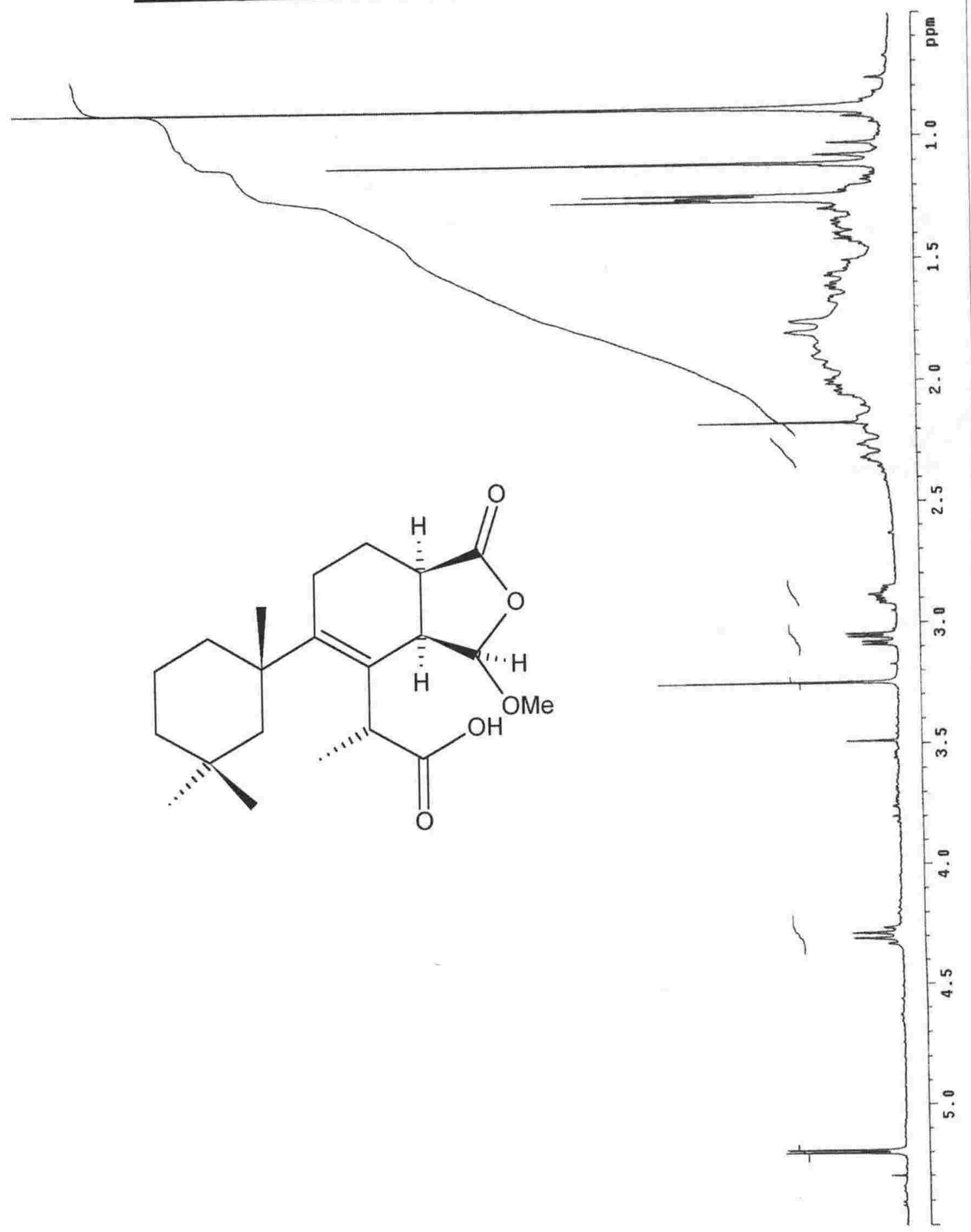

${ }^{1} \mathrm{H}$ spectrum of 15 -methoxypourewic acid $\mathrm{B}(\mathbf{1 4 1})\left(300 \mathrm{MHz}, \mathrm{CDCl}_{3}\right)$ 


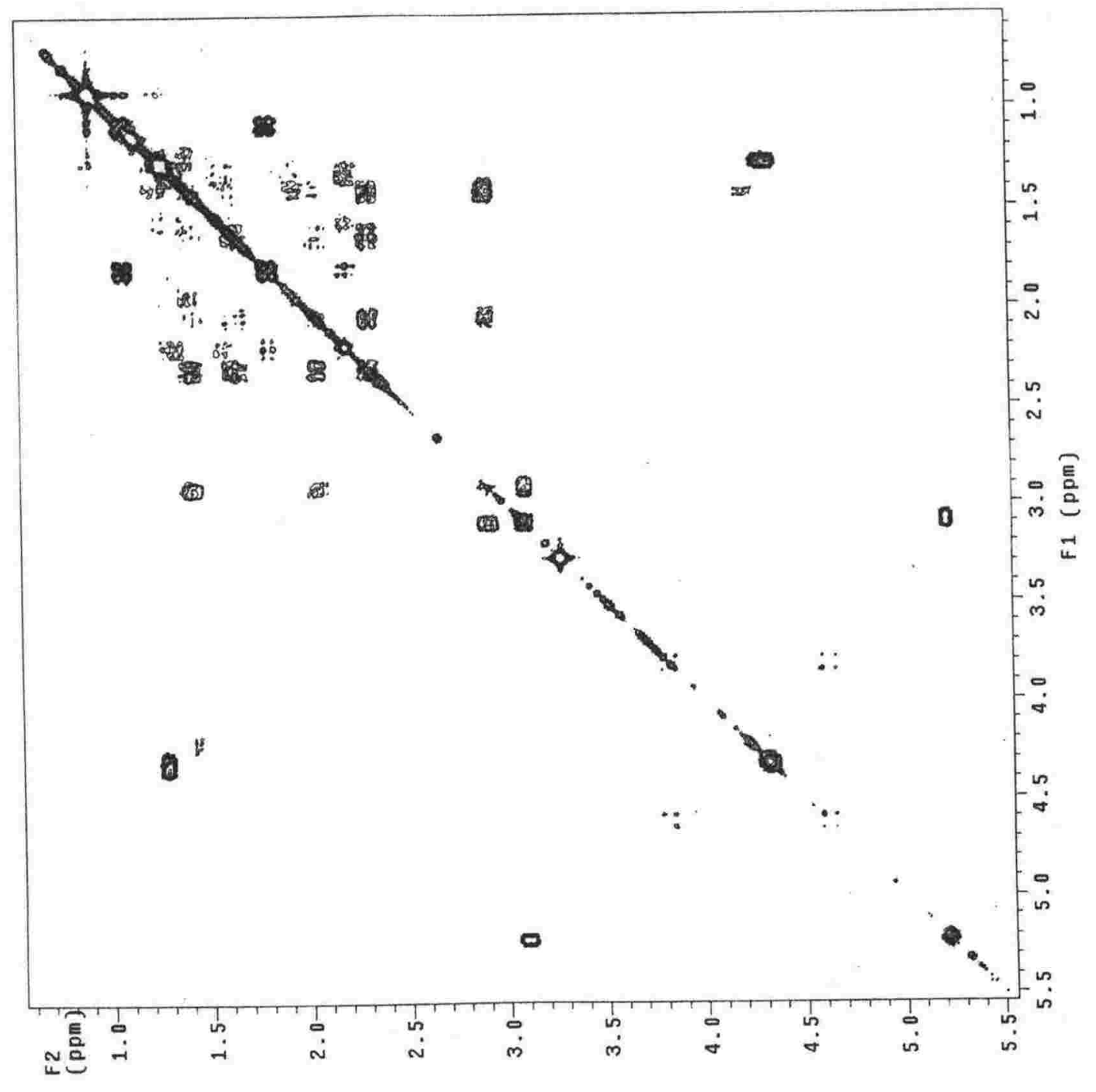

COSY spectrum of 15-methoxypourewic acid B (141) (300 MHz, $\left.\mathrm{CDCl}_{3}\right)$ 
NMR Spectra of Methylpourewate B (142)

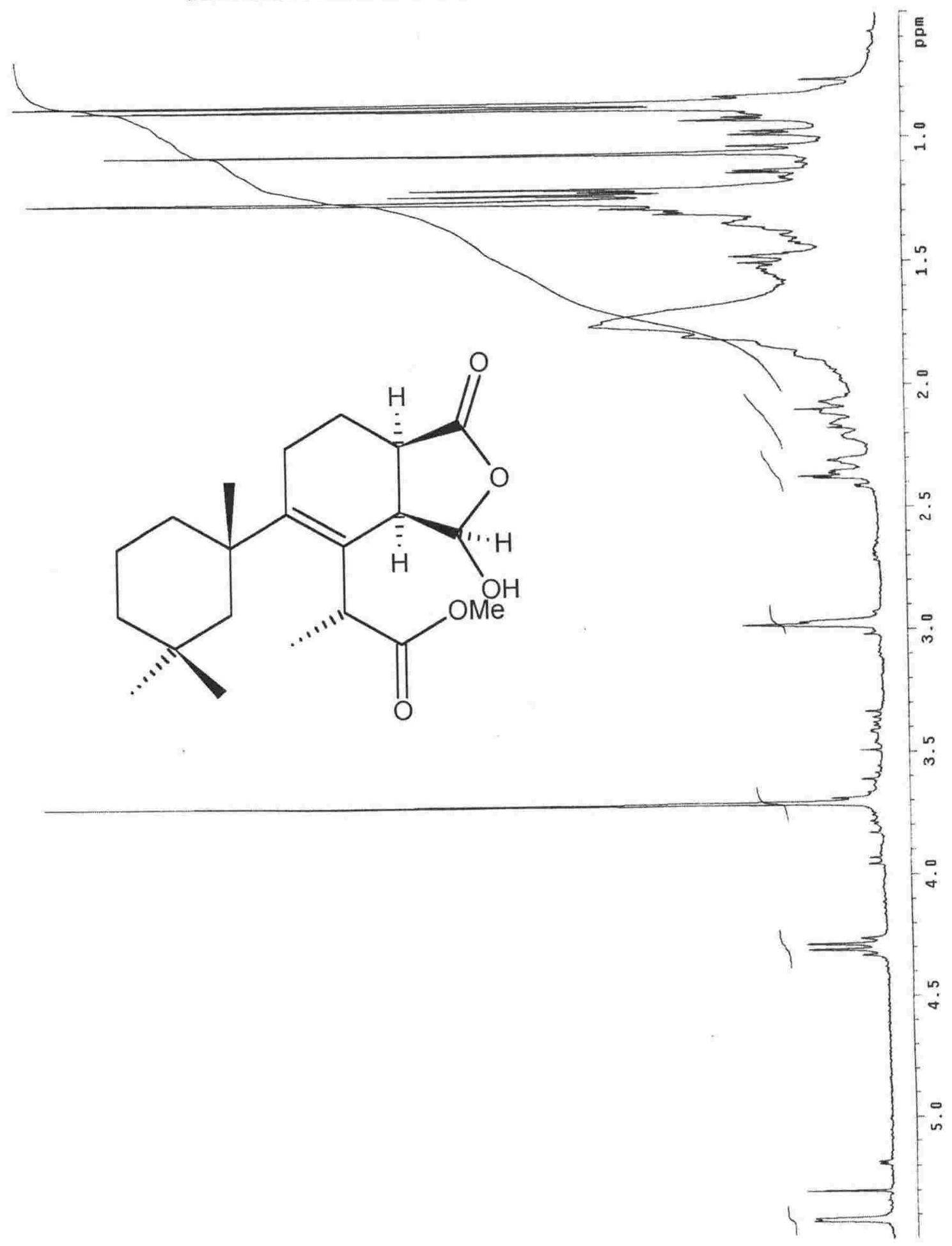

${ }^{1} \mathrm{H}$ spectrum of methylpourewate $\mathrm{B}(\mathbf{1 4 2})\left(300 \mathrm{MHz}, \mathrm{CDCl}_{3}\right)$ 


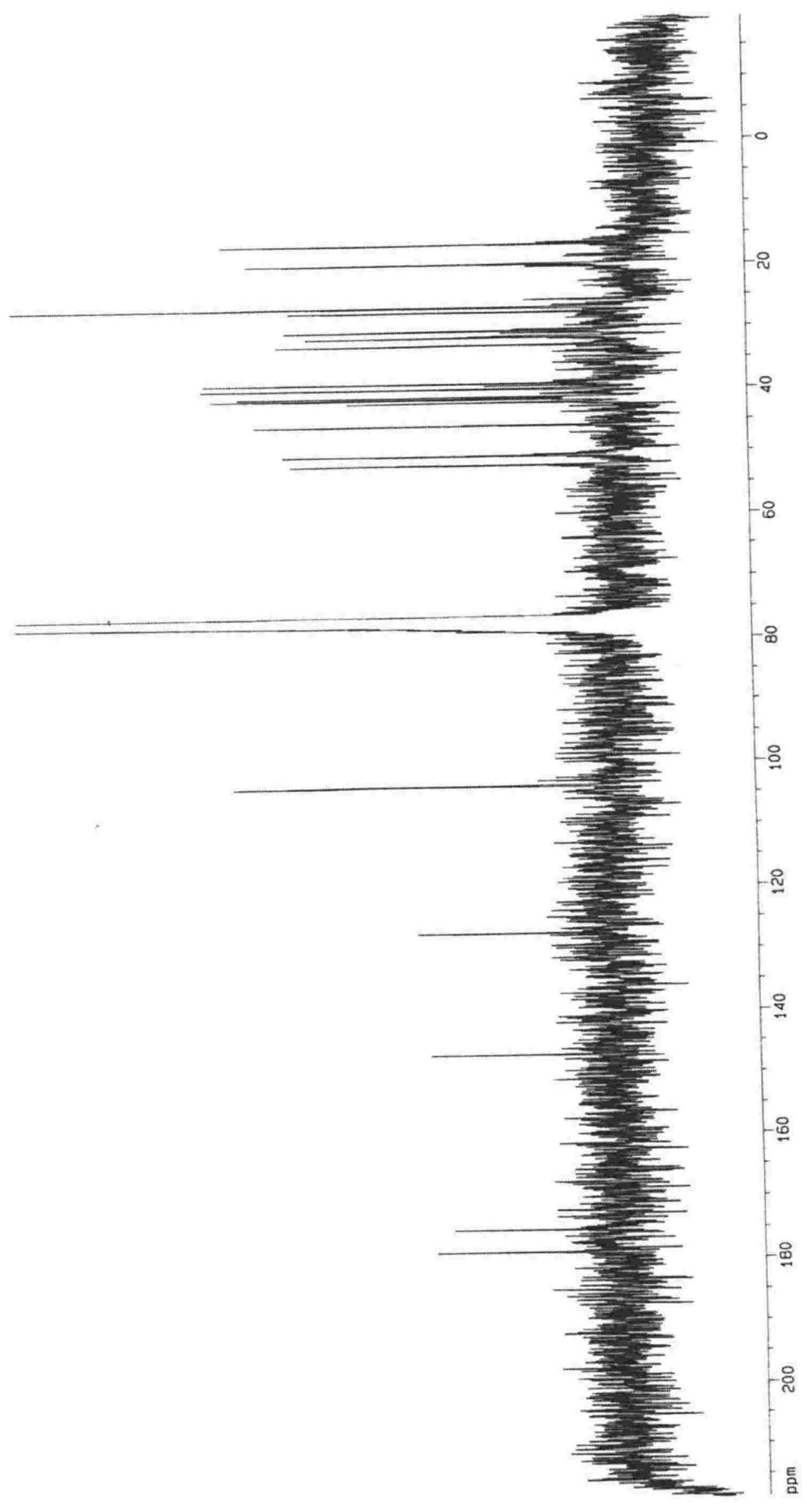

${ }^{13} \mathrm{C}$ spectrum of methylpourewate $\mathrm{B}(\mathbf{1 4 2})\left(75 \mathrm{MHz}, \mathrm{CDCl}_{3}\right)$ 


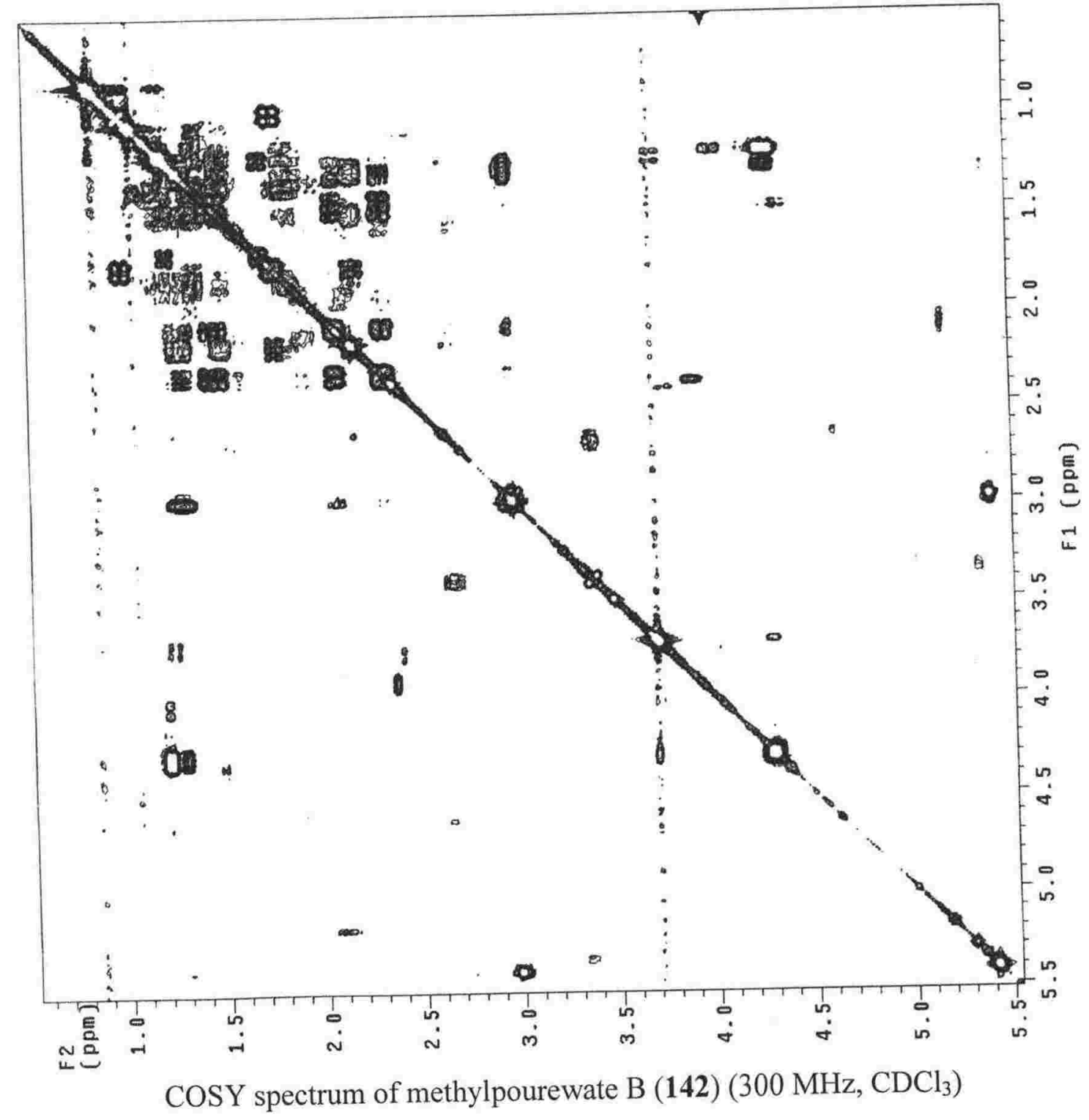


Appendix Eight

NMR Spectra of Pourewanone (143)

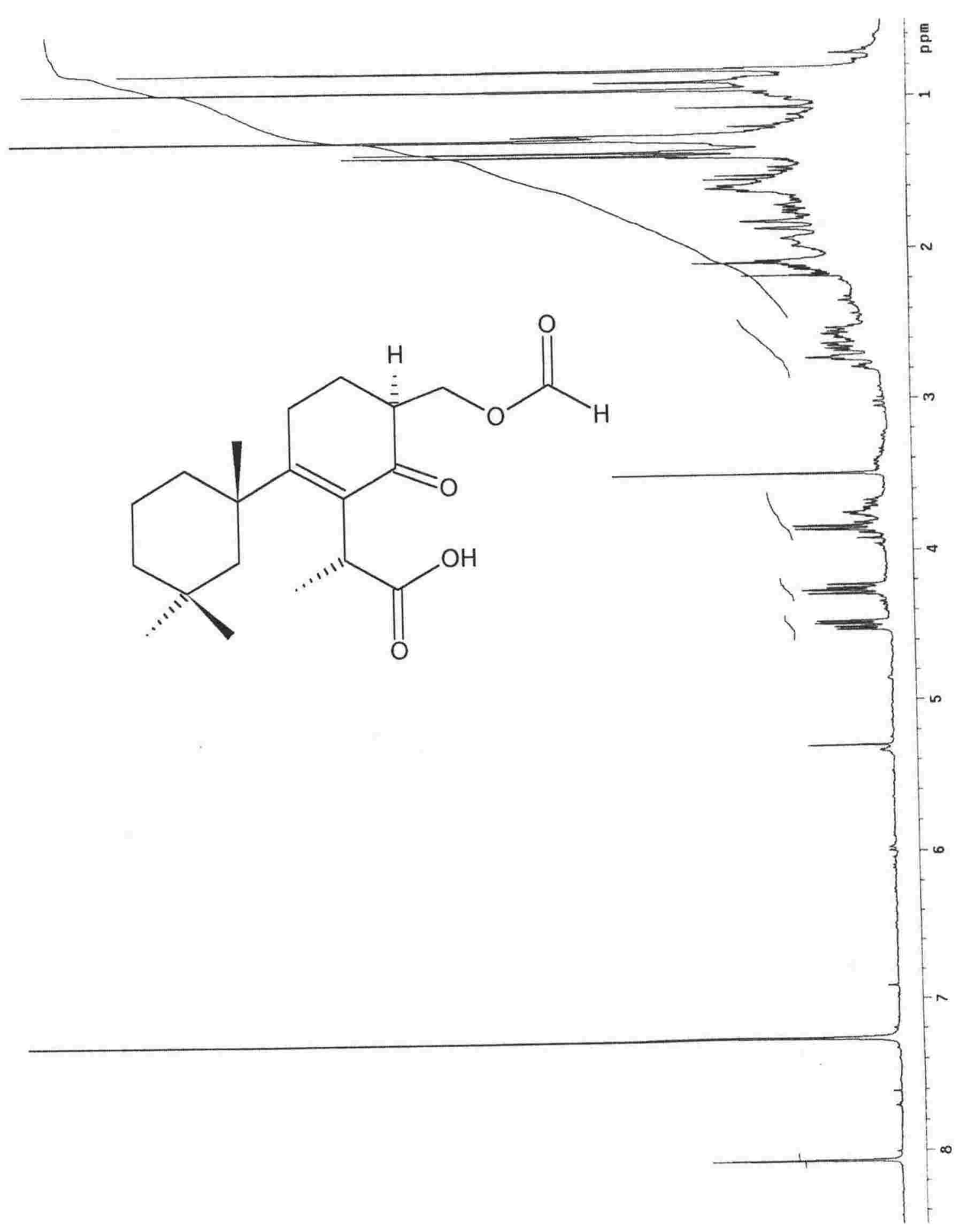

${ }^{1} \mathrm{H}$ spectrum of pourewanone (143) $\left(300 \mathrm{MHz}, \mathrm{CDCl}_{3}\right)$ 


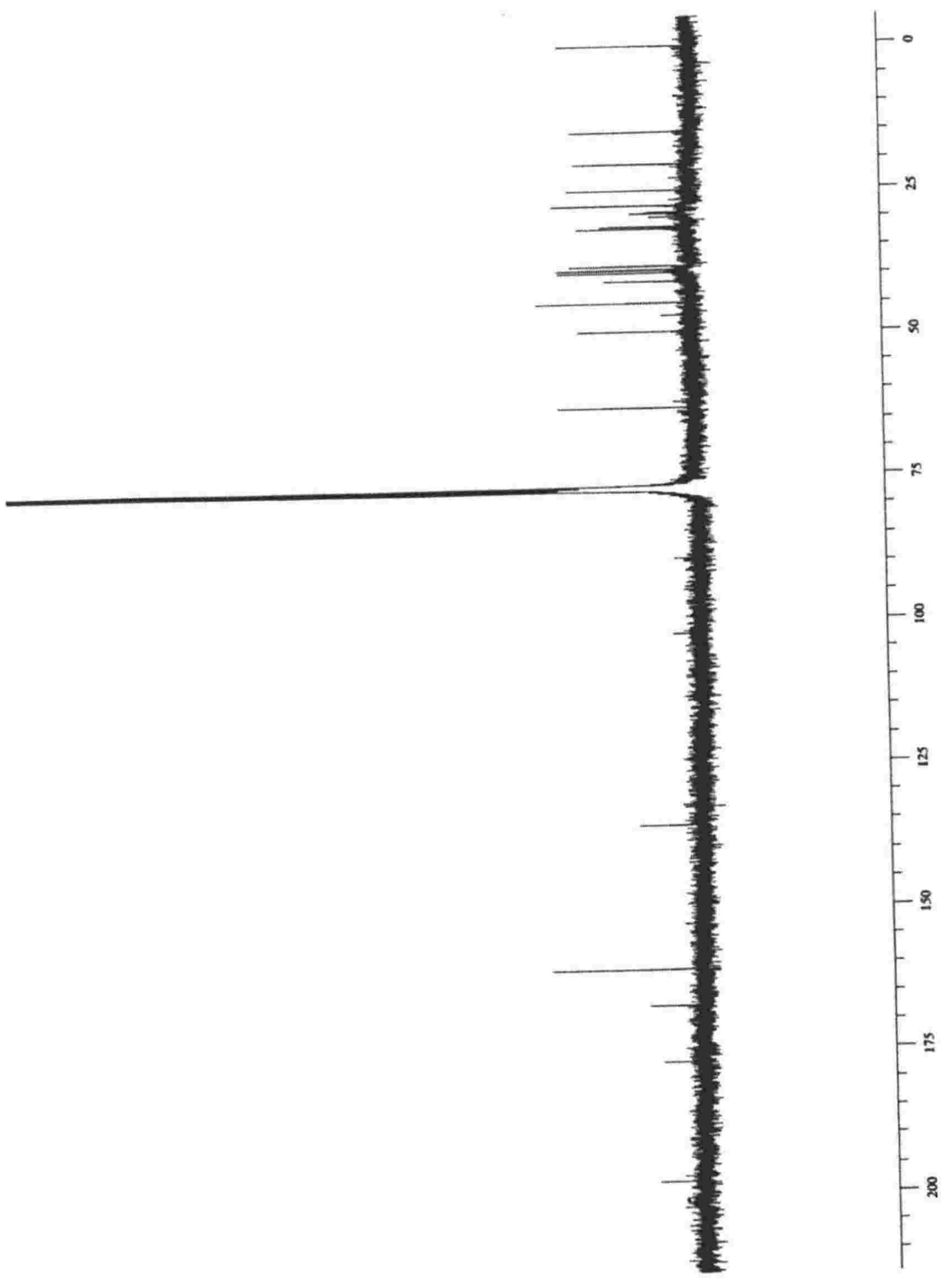

${ }^{13} \mathrm{C}$ spectrum of pourewanone $(143)\left(100 \mathrm{MHz}, \mathrm{CDCl}_{3}\right)$ 


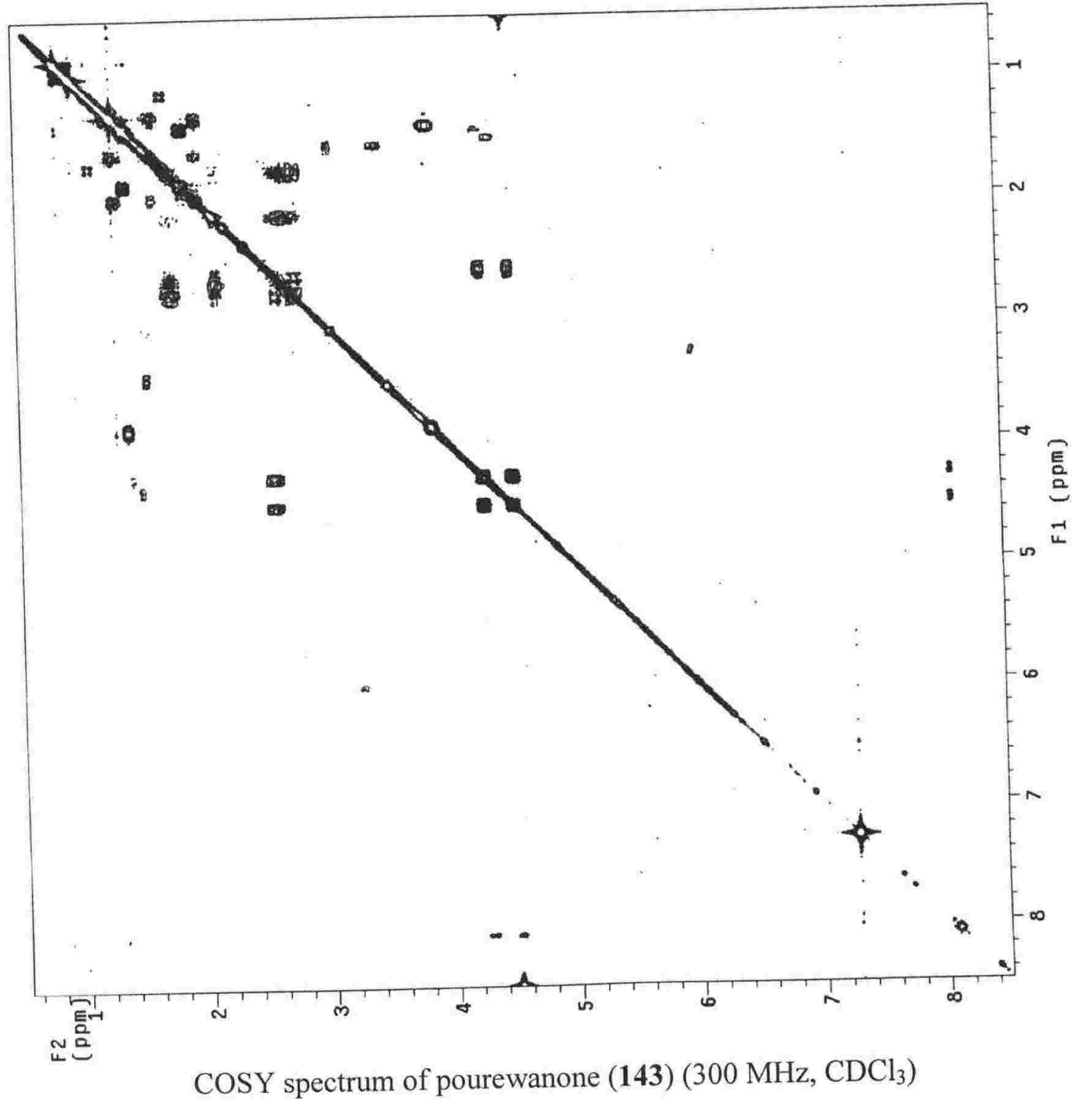




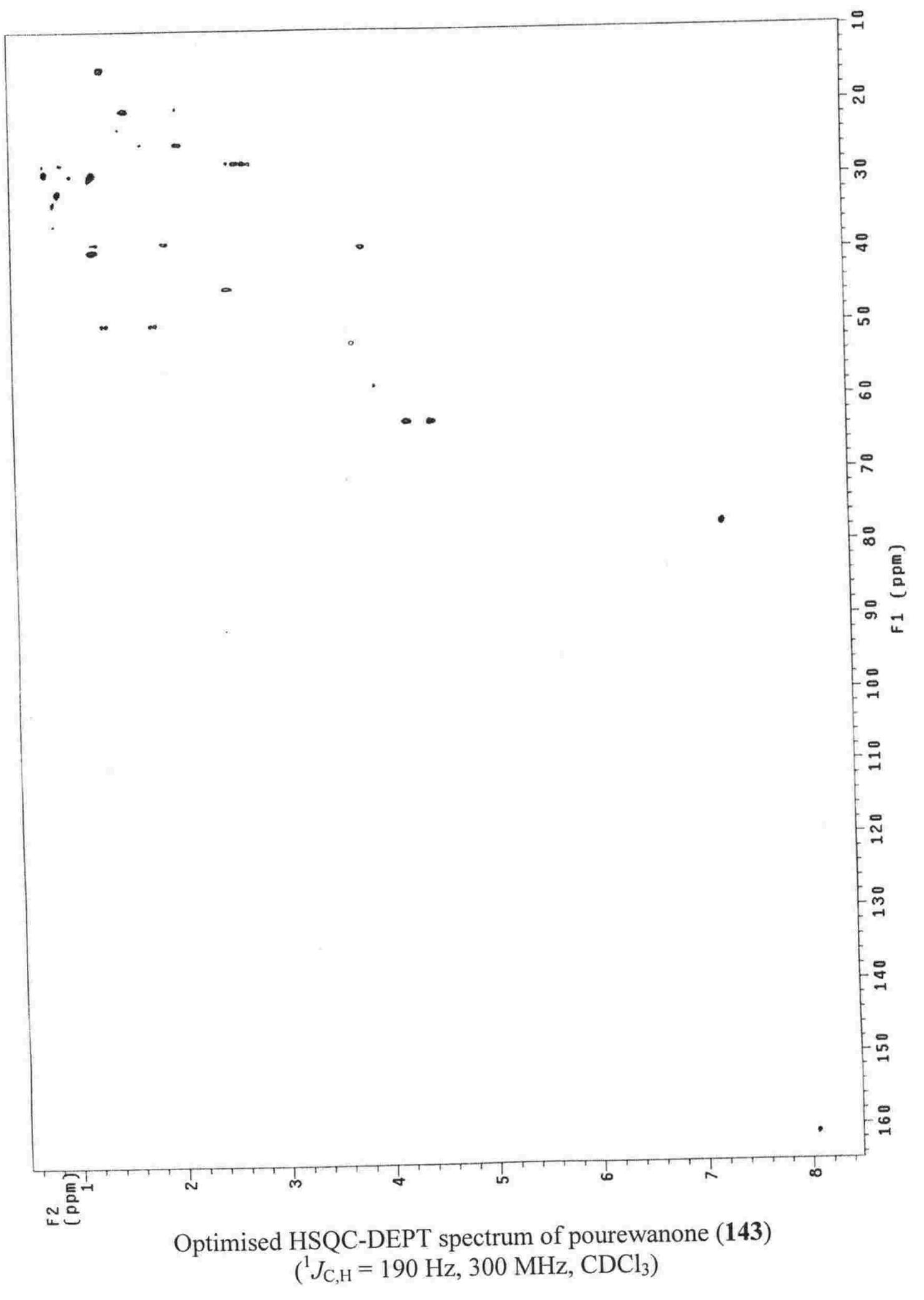




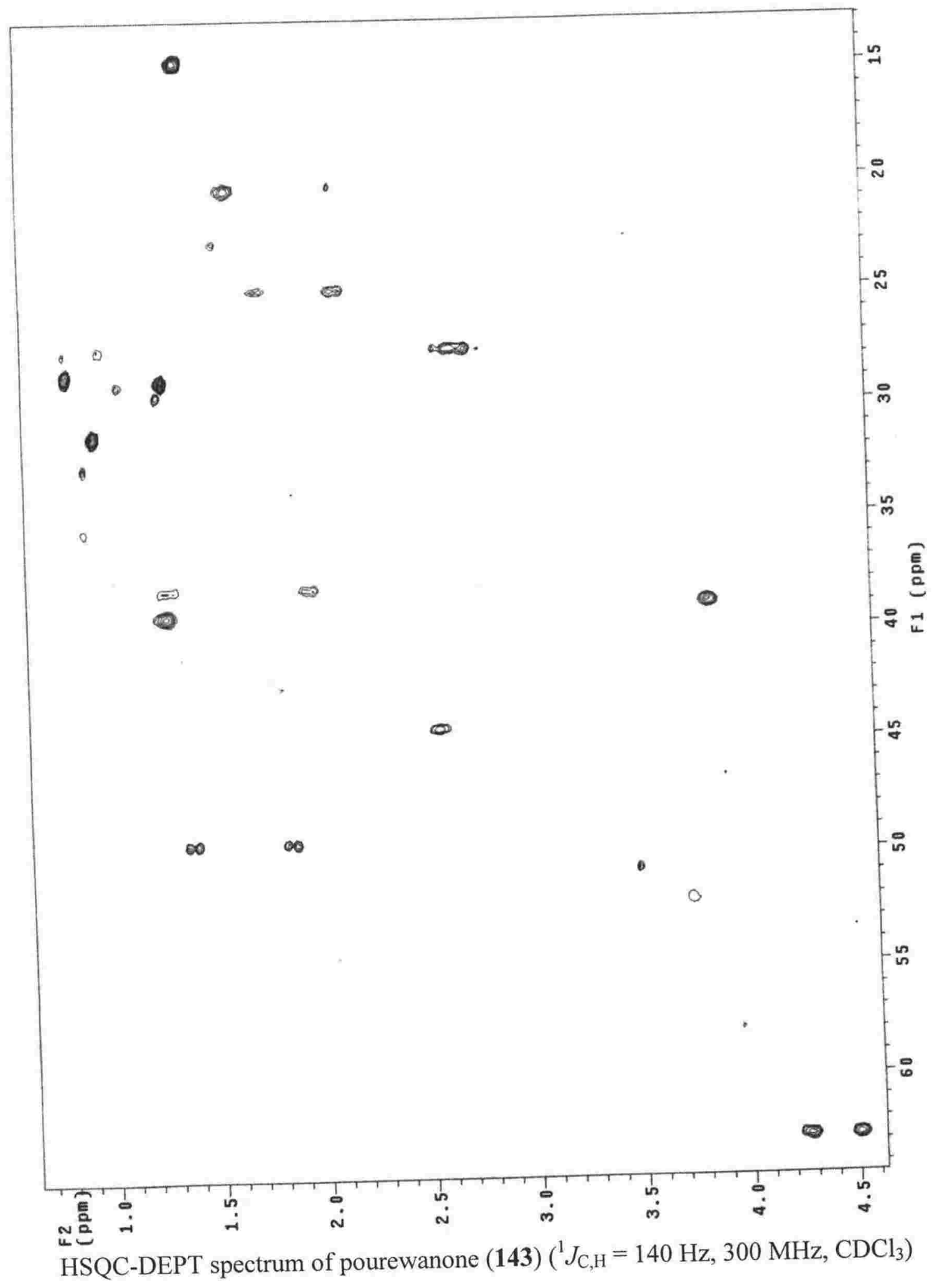




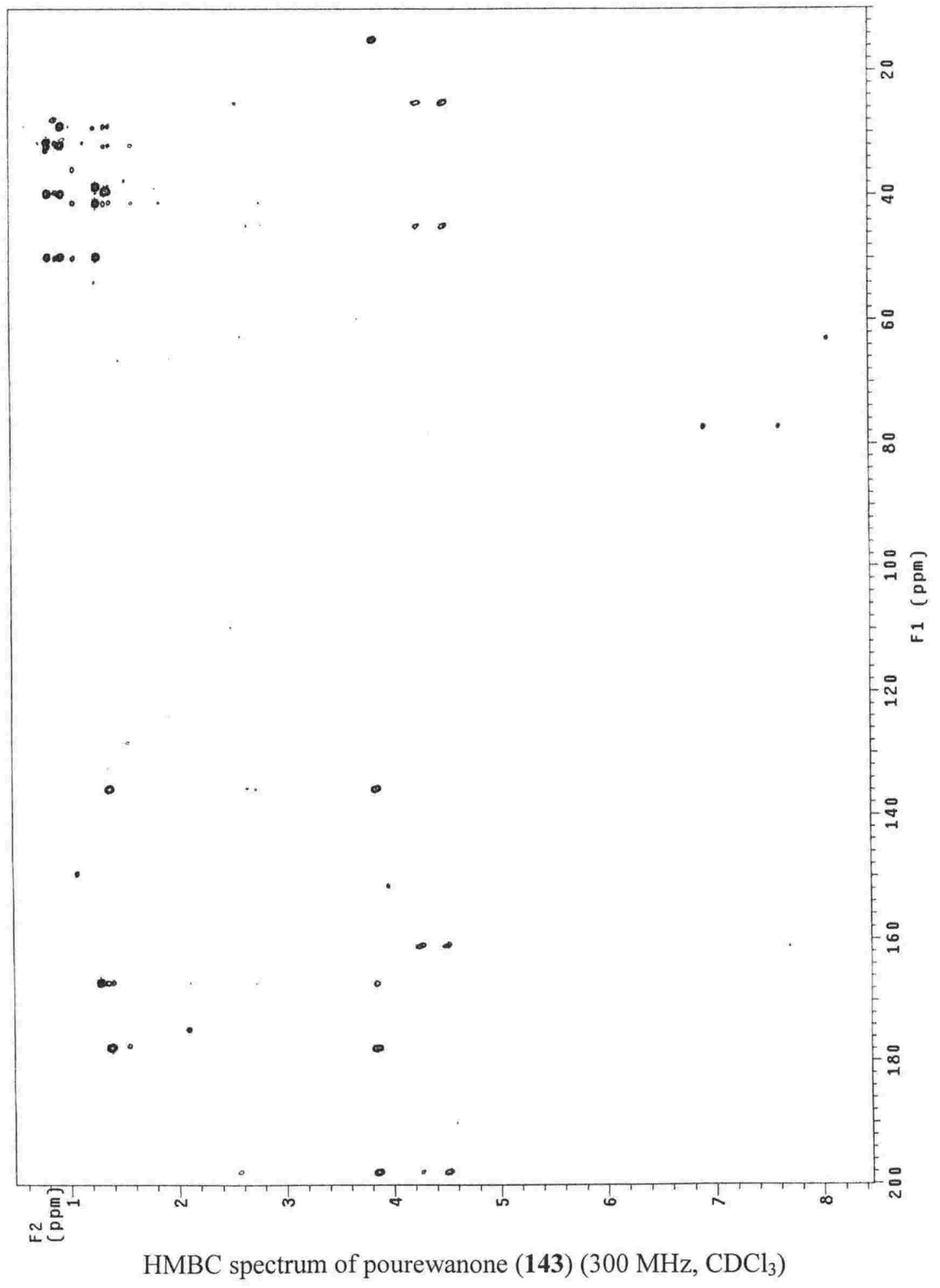




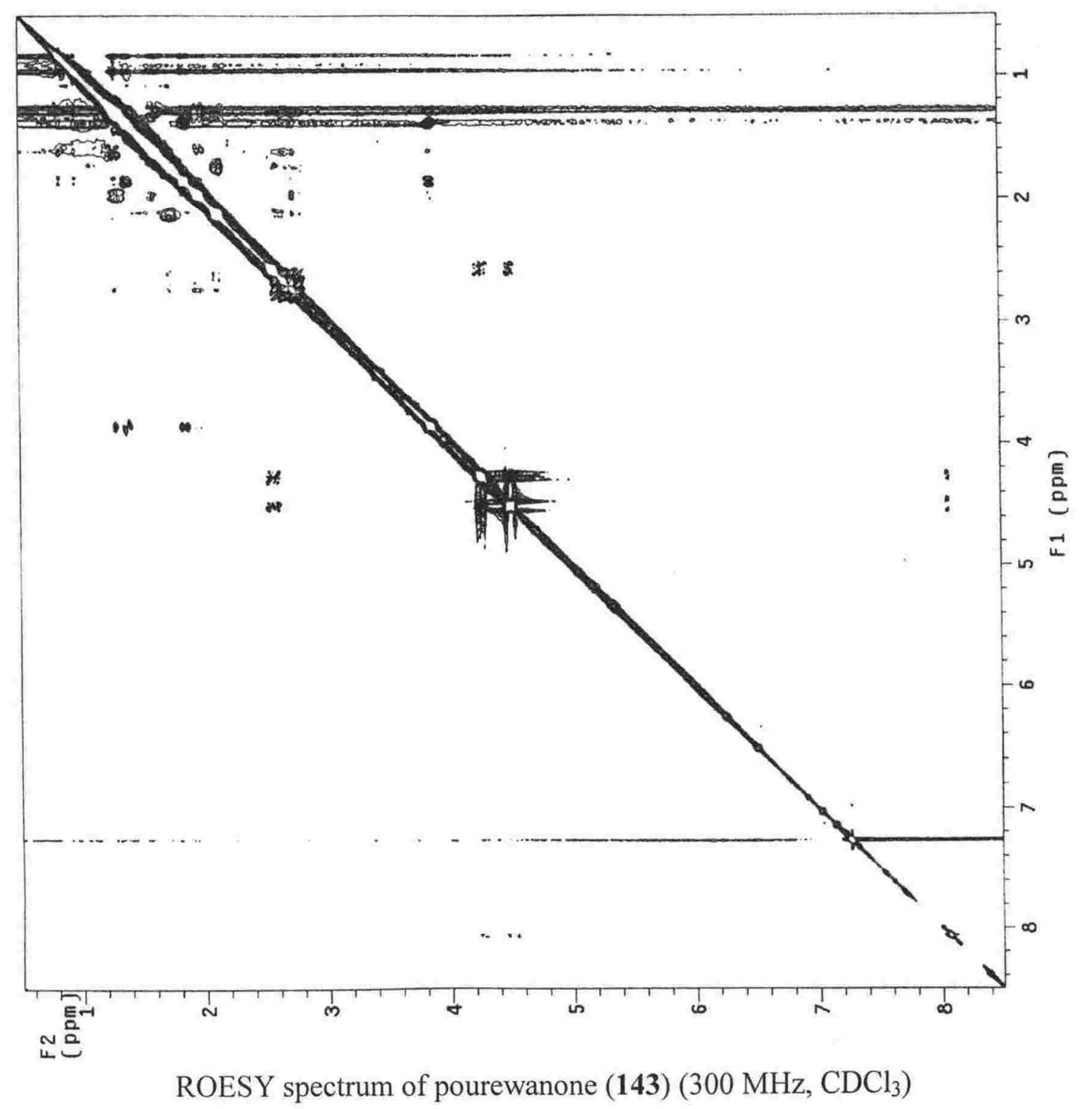




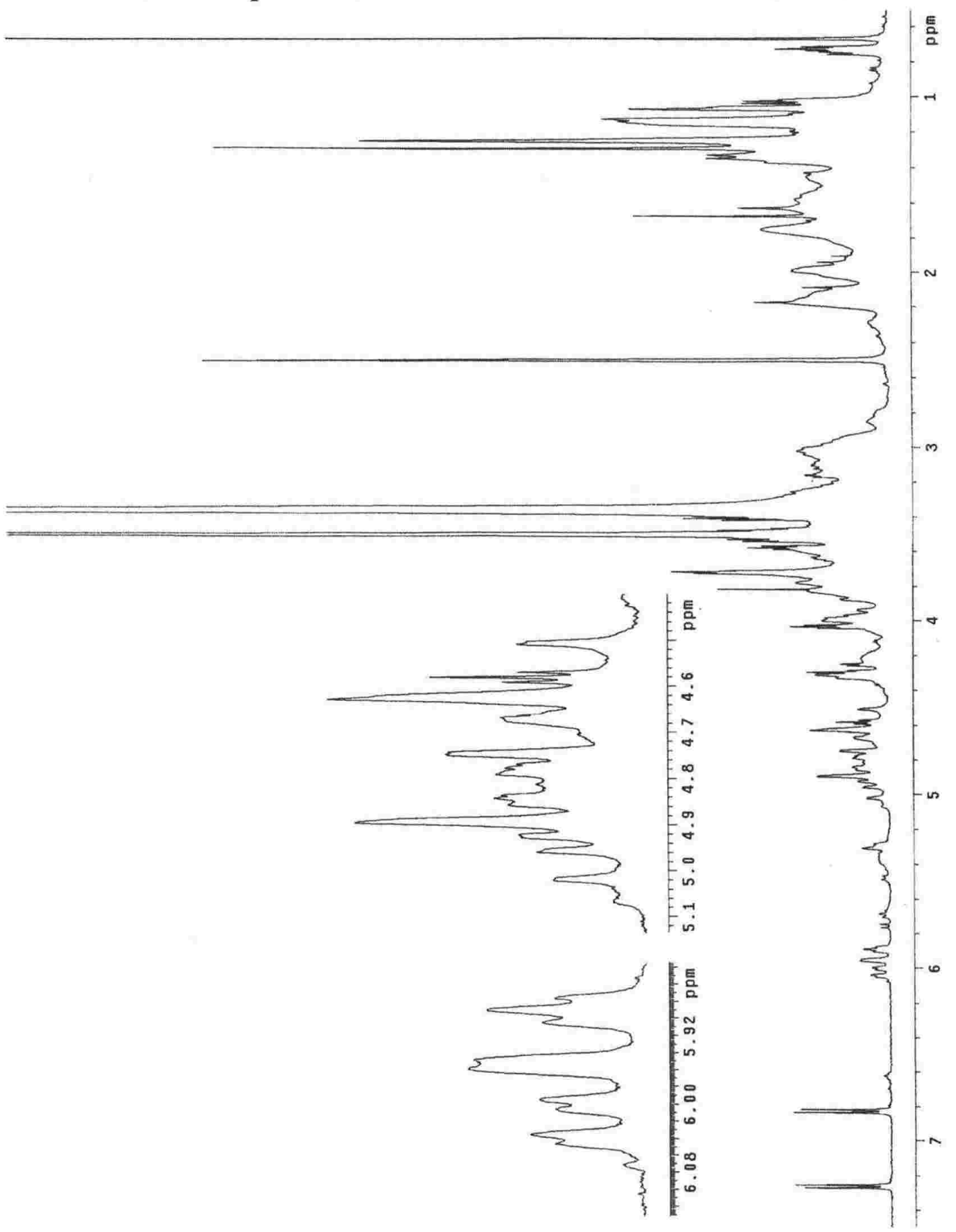

${ }^{\mathrm{I}} \mathrm{H}$ spectrum of $\mathrm{KBT}$ (500 MHz, $\mathrm{D}_{6}$-DMSO) 


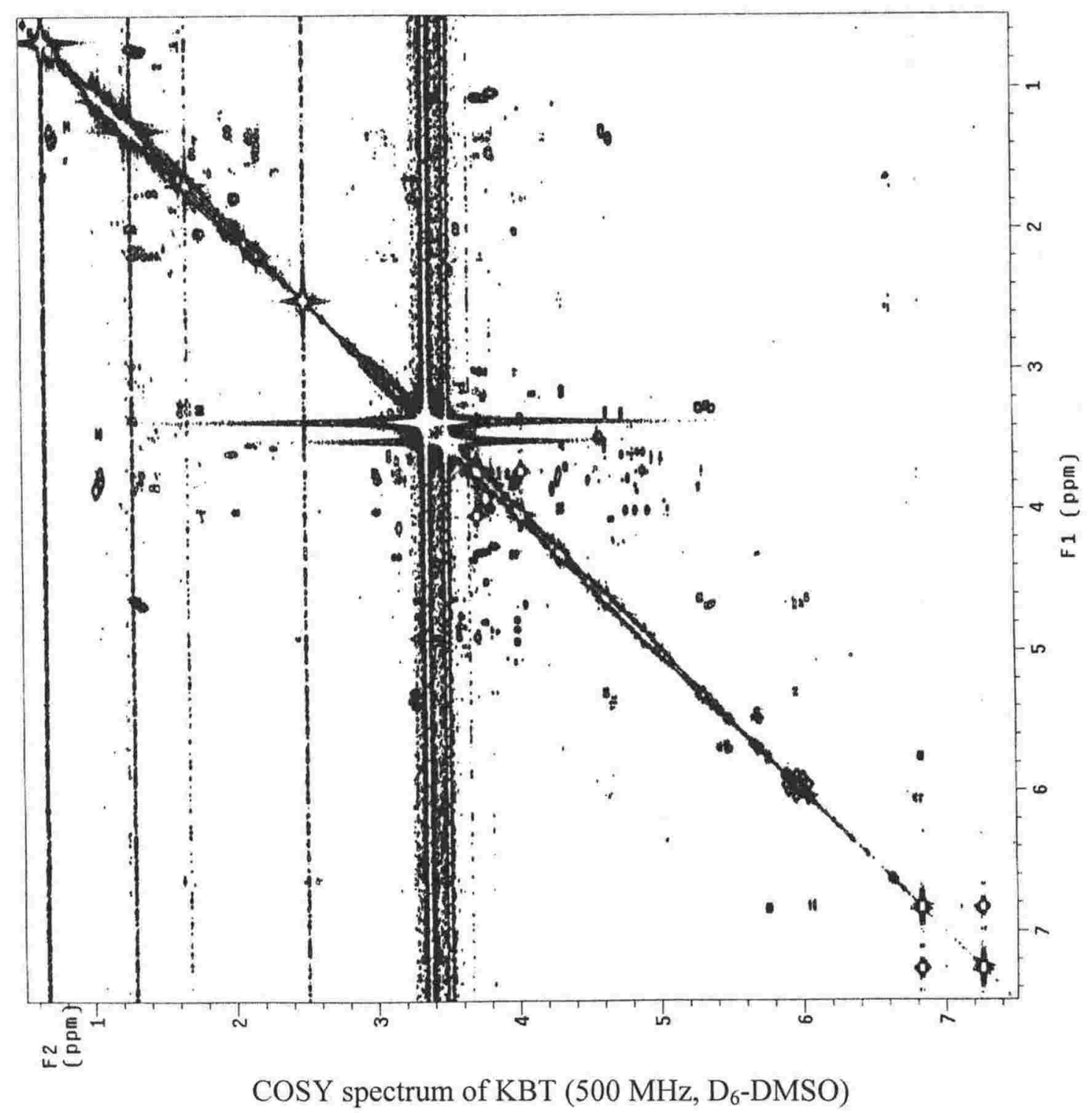




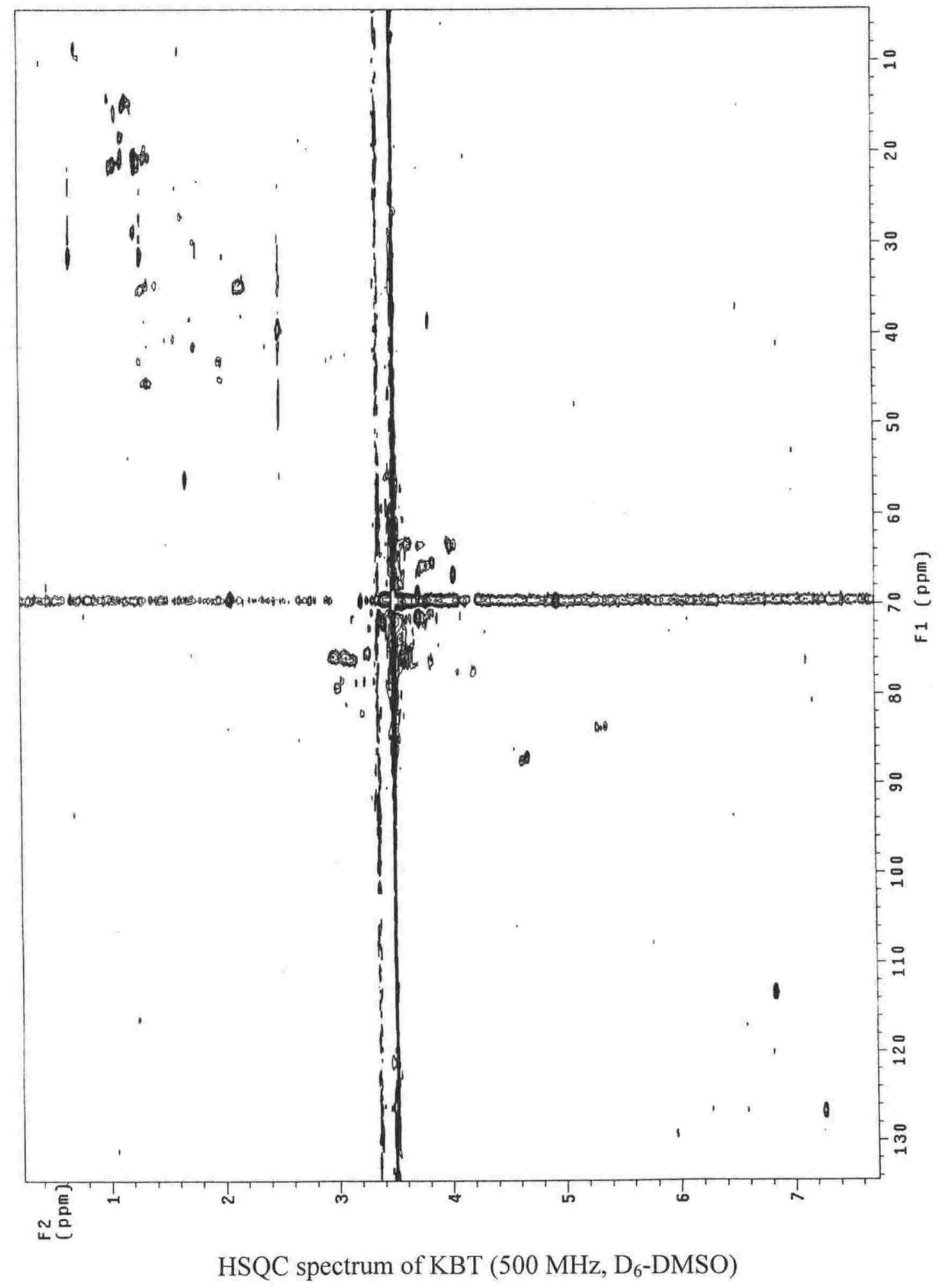




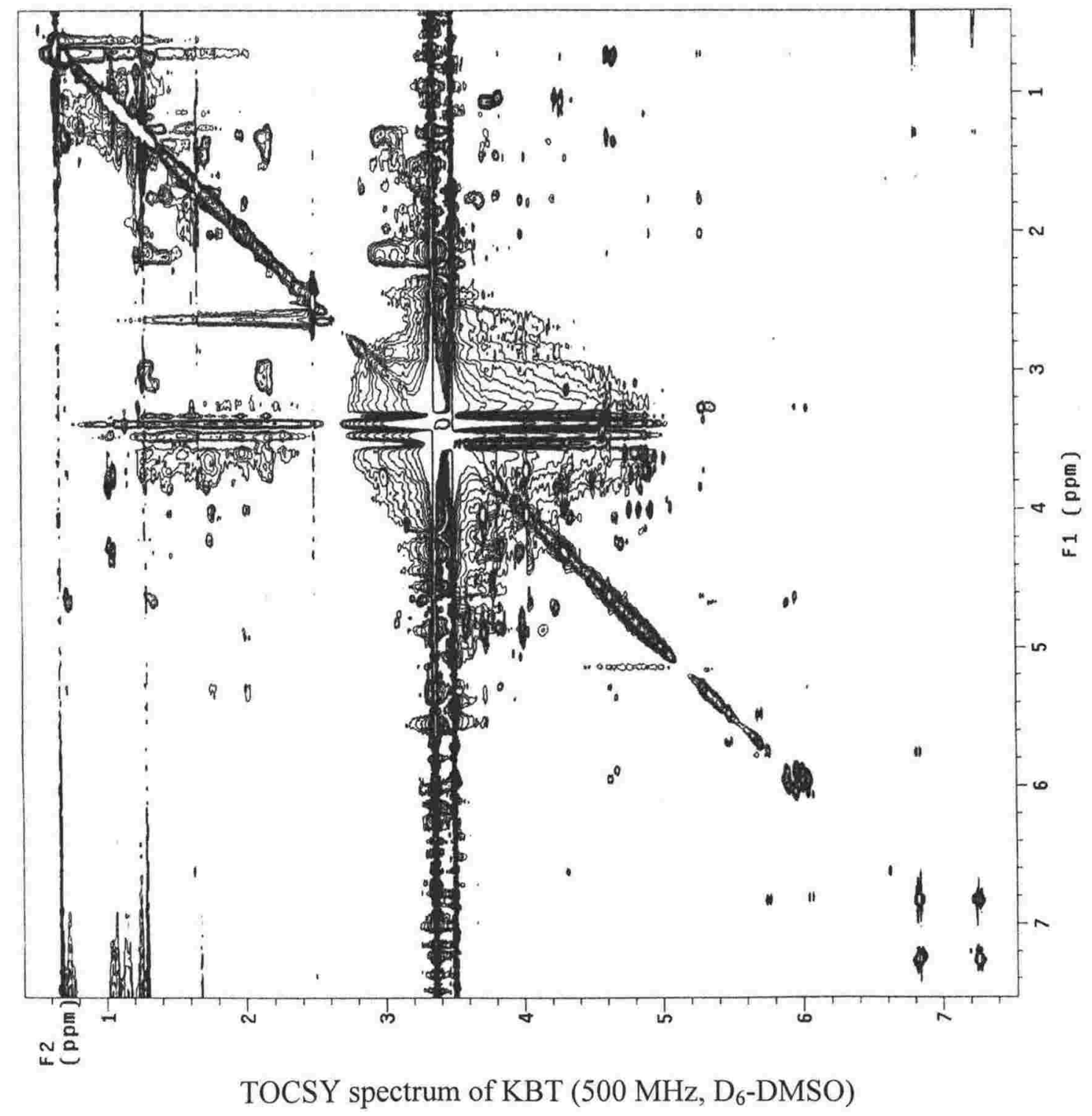




\section{References}

1. Cragg, G. M.; Boyd, M. R.; Khanna, R.; Kneller, R.; Mays, T. D.; Mazan Kate, D.; Newman, D. J.; Sausville, E. A. Pure Appl. Chem. 1999, 71, 1619 and references contained therein.

2. Hendrickson, J. B. The Molecules of Nature. In The Organic Chemistry Monograph Series; W. A. Benjamin, Inc.: New York, 1965.

3. Cobb, C.; Goldthwaite, H. Creations of Fire: Chemistry's Lively History from Alchemy to the Atomic Age.; Plenum Press: New York, 1995.

4. Halstead, B. W. Poisonous and Venomous Marine Animals of the World; United States Government Printing Office: Washington D.C., 1965; Vol. 1.

5. Moore, R. E; Scheuer, P. J. Science 1971, 172, 495.

6. Scheuer, P. J. Acc. Chem. Res. 1977, 10, 33.

7. Martin, L.; http://www.mtsinai.org/pulmonary/books/scuba/sectiona.htm; As at $26 / 08 / 02$.

8. Blunt, J. W.; Copp, B. R.; Munro, M. H. G.; Northcote, P. T.; Prinsep, M. R. Nat. Prod. Rep. 2003, 20, 1.

9. Proksch, P.; Ebel, R.; Edrada, R. A.; Schupp, P.; Lin, W. H.; Sudarsono; Wray, V.; Steube, K. Pure Appl. Chem. 2003, 75, 345.

10. Scheuer, P. J. Chemistry of Marine Natural Products. In Marine Natural Products; Academic Press, Inc.: New York and London, 1973; Vol. 1.

11. Zubay, G. L. Biochemistry, Fourth ed; Wm C. Brown: Melbourne, 1998.

12. Pawlik, J. R. Chem. Rev. 1993, 93, 1911.

13. Mann, J. Chemical Aspects of Biosynthesis. In Oxford Chemistry Primers; Oxford University Press: New York, 1994; Vol. 20.

14. Scheuer, P. J. Marine Natural Products: Chemical and Biological Perspectives. In Marine Natural Products; Academic Press, Inc.: New York and London, 1978; Vol. 2.

15. Cragg, G. M.; Newman, D. J.; Snader, K. M. J. Nat. Prod. 1997, 60, 52.

16. Quinn, R. J.; de Almeida Leone, P.; Guymer, G.; Hooper, J. N. A. Pure Appl. Chem. 2002, 74, 519.

17. Pettit, G. R. Pure Appl. Chem. 1994, 66, 2271.

18. Fleming, C. A. Tuatara 1949, 11, 72. 
19. Fleming, C. A. New Zealand Journal of Forestry 1977, 22, 249.

20. Fleming, C. A. The Geological History of New Zealand and its Life.; Auckland University Press, 1979.

21. Bergquist, P. R. Sponges; Hutchinson \& Co.: London, 1978.

22. Hooper, J. N. A.; Wiedenmayer, F. Porifera. In Zoological Catalogue of Australia; CSIRO: Melbourne, 1994; Vol. 12.

23. Hooper, J. N. A.; van Soest, R. W. M.; (Eds) Systema Porifera: A guide to the classification of sponges, , 1 ed.; Kluwer Academic/Plenum: New York, 2002.

24. Pritchard, K.; Ward, V. Marine Sponges: Forty-Six Sponges of Northern New Zealand; University of Auckland Marine Laboratory: Auckland, 1984; pp. 149.

25. Faulkner, D. J.; Unson, M. D.; Bewley, C. A. Pure Appl. Chem. 1994, 66, 1983.

26. Faulkner, D. J.; Molinski, T. F.; Andersen, R. J.; Dumdei, E. J.; de Silva, E. D. Comp. Biochem. Physiol. 1990, 97C, 233.

27. Molinski, T. F.; Faulkner, D. J.; Cun-heng, H.; Van Duyne, G. D.; Clardy, J. J. Org. Chem. 1986, 51, 4564.

28. Tischler, M.; Andersen, R. J.; Choudhary, M. I.; Clardy, J. J. Org. Chem. $1991,56,42$.

29. Hirota, H.; Okino, T.; Yoshimura, E.; Fusetani, N. Tetrahedron 1998, 54, 13971.

30. Dumdei, E. J.; Kubanek, J.; Coleman, J. E.; Pika, J.; Andersen, R. J.; Steiner, J. R.; Clardy, J. Can. J. Chem. 1997, 75, 773.

31. Simpson, J. S.; Garson, M. J.; Hooper, J. N. A.; Cline, E. I.; Angerhofer, C. K. Aust. J. Chem. 1997, 56, 1123.

32. de Jesus, R. P.; Faulkner, D. J. J. Nat. Prod. 2003, 66, 671.

33. Bergquist, P. R.; Wells, R. J. Chemotaxonomy of the Porifera: The Development and Current Status of the Field. In Marine Natural Products: Chemical and Biological Perspectives; Scheuer, P. J. Ed.; Academic Press: New York, 1983; Vol. 5.

34. Hartman, W. D.; Bergquist, P. R. Porifera. In Synopsis and Classification of Living Organisms; Parker, S. P. Ed.; McGraw-Hill: New York, 1982; Vol. 1; pp. 640 .

35. Kristiansen, J.; Loeblish III, A. R. Dinophyceae. In Synopsis and Classification of Living Organisms; Parker, S. P. Ed.; McGraw-Hill: New York, 1982; Vol. 1; pp. 640. 
36. Corliss, J. O. Protozoa. In Synopsis and Classification of Living Organisms; Parker, S. P. Ed.; McGraw-Hill: New York, 1982; Vol. 1; pp. 640.

37. Allaby, M.; Allaby, R.; Kent, M.; Sainsbury, D.; Whitmore, T. C. The Concise Oxford Dictionary of Botany; Oxford University Press: Oxford, 1992.

38. Dodge, J. D. Prog. Protozool. 1965, 264.

39. Shimizu, Y. Chem. Rev. 1993, 93, 1685.

40. Baretta-Bekker, J. G.; Duursma, E. K.; Kuipers, B. R. E. Encyclopaedia of Marine Sciences; Springer-Verlag: Berlin, 1992.

41. Personal Communication; Chang, F. H.; NIWA; September; 2002.

42. Yasumoto, T.; Murata, M. Chem. Rev. 1993, 93, 1897.

43. Chang, F. H. Phycologia 1999, 38, 377.

44. Chang, F. H.; Chriswell, S. M.; Uddstrom, M. J. Phycologia 2001, 40, 215.

45. Daugjberg, N.; Hansen, G.; Larsen, J.; Moestrup, O. Phycologia 2000, 39, 302.

46. Wagner, H.; Bladt, S.; Zgainski, E. M. Plant Drug Analysis; Springer-Verlag: Berlin, 1984.

47. Mestel, R. Discover 1999, March, 70.

48. Moore, R. E. Pure Appl. Chem. 1982, 54, 1919.

49. Faulkner, D. J. Academic chemistry and the discovery of bioactive marine natural products. In Marine Biotechnology: Pharmaceutical and Bioactive Natural Products; Attaway, D. H.; Zaborsky, O. R. Eds.; Plenum Press: New York, 1993; Vol. 1; pp. 500.

50. West, L. M. The Isolation of Secondary Metabolites from New Zealand Marine Sponges., Victoria University of Wellington 2001.

51. Satake, M.; Shoji, M.; Oshima, Y.; Naoki, H.; Fujita, T.; Yasumoto, T. Tetrahedron Lett. 2002, 43, 5829.

52. Kobayashi, J. i.; Cheng, J.-f.; Ohta, T.; Nozoe, S.; Ohizumi, Y.; Sasaki, T. J. Org. Chem. 1990, 55, 3666.

53. Umeyama, A.; Adachi, K.; Ito, S.; Arihara, S. J. Nat. Prod. 2000, 63, 1175.

54. Blunt, J. W.; Calder, V. L.; Fenwick, G. D.; Lake, R. J.; McCombs, J. D.; Munro, M. H. G.; Perry, N. B. J. Nat. Prod. 1987, 50, 290.

55. Still, W. C.; Kahn, M.; Mitra, A. J. Org. Chem. 1978, 43, 2923.

56. Coll, J. C.; Bowden, B. F. J. Nat. Prod. 1986, 49, 934. 
57. Shaksher, Z. M.; Seitz, W. R. Anal. Chem. 1989, 61, 590.

58. Evans, M. B.; Dale, A. D.; Little, C. J. Chromatographica 1980, 13, 5.

59. West, L. M.; Northcote, P. T.; Battershill, C. N. J. Org. Chem. 2000, 65, 445.

60. Northcote, P. T.; Blunt, J. W.; Munro, M. H. G. Tetrahedron Lett. 1991, 32, 6411 .

61. Perry, N. B.; Blunt, J. W.; Munro, M. H. G. J. Am. Chem. Soc. 1988, 110, 4850 .

62. West, L. M. Honours Project Report; Victoria University of Wellington: Wellington, 1996.

63. van Liedekerke, B. M.; Nelis, H. J.; Lambert, W. E.; de Leenheer, A. P. Anal. Chem. 1989, 61, 728 .

64. Keyzers, R. A. Isolation of Yessotoxin from contaminated green lipped mussels, Perna canaculus, and from cultures of the algae Protoceratium reticulatum.; Victoria University of Wellington: Wellington, 1998; pp. 43.

65. Anjaneyulu, A. S. R.; Sagar, K. S. J. Chem. Research (S) 1995, 142.

66. Gauvin, A.; Smadja, J.; Aknin, M.; Faure, R.; Gaydou, E. M. Can. J. Chem. 2000, 78, 986 .

67. Makita, A.; Taniguchi, N. Glycosphingolipids. In Glycolipids; Wiegandt, H. Ed.; Elsevier: Amsterdam, 1985; Vol. 10; pp. 314.

68. Nagle, D. G.; McClatchey, W. C.; Gerwick, W. H. J. Nat. Prod. 1992, 55, 1013.

69. Makarieva, T. N.; Denisenko, V. A.; Stonik, V. A.; Milgrom, Y. M.; Rashkes, Y. V. Tetrahedron Lett. 1989, 30, 6581.

70. Bartik, K.; Braekman, J. C.; Daloze, D.; Stoller, C.; Huysecom, J.; Vandevyver, G.; Ottinger, R. Can. J. Chem. 1987, 65, 2118.

71. Casapullo, A.; Bifulco, G.; Bruno, I.; Riccio, R. J. Nat. Prod. 2000, 63, 447.

72. Cutignano, A.; Bifulco, G.; Bruno, I.; Casapullo, A.; Gomez-Paloma, L.; Riccio, R. Tetrahedron 2000, 56, 3743.

73. Kohmoto, S.; Kashman, Y.; McConnell, O. J.; Rinehart Jnr, K. L.; Wright, A.; Koehn, F. J. Org. Chem. 1988, 53, 3116.

74. Tsuji, S.; Rinehart Jnr, K. L.; Gunasekera, S. P.; Kashman, Y.; Cross, S. C.; Lui, M. S.; Pomponi, S.; Diaz, M. C. J. Org. Chem. 1988, 53, 5446.

75. Forenza, S.; Minale, L.; Riccio, R. J. Chem. Soc. Chem. Commun. 1971, 1129. 
76. Cimino, G.; de Rosa, S.; de Stefano, S.; Mazzarella, L.; Puliti, R.; Sodano, G. Tetrahedron Lett. 1982, 23, 767.

77. Williams, D. H.; Faulkner, D. J. Nat. Prod. Lett. 1996, 9, 57.

78. Walker, R. P.; Faulkner, D. J.; van Engen, D.; Clardy, J. J. Am. Chem. Soc. 1981, 103, 6772.

79. Pettit, G. R.; Herald, C. L.; Leet, J. E.; Gupta, R.; Schaufelberger, D. E.; Bates, R. B.; Clewlow, P. J.; Doubek, D. L.; Manfredi, K. P.; Rutzler, K.; Schmidt, J. M.; Tackett, L. P.; Ward, F. B.; Bruck, M.; Camou, F. Can. J. Chem. 1990, 68, 1621.

80. McMurry, J. Organic Chemistry, 4 ed; Brooks/Cole: Pacific Grove, 1996.

81. Giner, J.-L. Chem. Rev. 1993, 93, 1735.

82. Djerassi, C.; Silva, C. J. Acc. Chem. Res. 1991, 24, 371.

83. Faulkner, D. J. Nat. Prod. Rep. 1993, 10, 497 and previous papers in this series.

84. Sheikh, Y. M.; Djerassi, C. Tetrahedron 1974, 30, 4095.

85. Iguchi, K.; Fujita, M.; Nagoka, H.; Mitome, H.; Yamada, Y. Tetrahedron Lett. 1993, 34, 6277.

86. Iguchi, K.; Shimura, H.; Taira, S.; Yokoo, C.; Keita, M.; Yamada, Y. J. Org. Chem. 1994, 59, 7499.

87. Casapullo, A.; Minale, L.; Zollo, F. Tetrahedron Lett. 1995, 36, 2669.

88. Aoki, S.; Yoshioka, Y.; Miyamoto, Y.; Higuchi, K.; Setiawan, A.; Murakami, N.; Chen, Z.-S.; Sumizawa, T.; Akiyama, S.-i.; Kobayashi, M. Tetrahedron Lett. 1998, 39, 6303.

89. Aoki, S.; Setiawan, A.; Yoshioka, Y.; Higuchi, K.; Fudetani, R.; Chen, Z.-S.; Sumizawa, T.; Akiyama, S.-i.; Kobayashi, M. Tetrahedron 1999, 55, 13965.

90. Allinger, N. L.; Hermann, R. B.; Djerassi, C. J. Org. Chem. 1960, 25, 922.

91. Kamano, Y.; Kumon, S.; Arai, T.; Komatsu, M. Chem. Pharm. Bull. 1973, 21, 1960.

92. Burgoyne, D. L.; Andersen, R. J.; Aleen, T. M. J. Org. Chem. 1992, 57, 525.

93. Takei, M.; Burgoyne, D. L.; Andersen, R. J. J. Pharm. Sci. 1994, 83, 1234.

94. Bramley, A. M.; Langlands, J. M.; Jones, A. K.; Li, Y.; Burgoyne, D. L.; Andersen, R. J.; Salari, H. Brit. J. Pharma. 1995, 115, 1433.

95. Yang, L.; Andersen, R. J. J. Nat. Prod. 2002, 65, 1924. 
96. Shoji, N.; Umeyama, A.; Shin, K.; Takeda, K.; Arihara, S.; Kobayashi, J. i.; Takei, M. J. Org. Chem. 1992, 57, 2996.

97. Kobayashi, J. i.; Shinonaga, H.; Shigemori, H.; Umeyama, A.; Shoji, N.; Arihara, S. J. Nat. Prod. 1995, 58, 312.

98. Sperry, S.; Crews, P. J. Nat. Prod. 1997, 60, 29.

99. Fu, X.; Ferreira, M. L. G.; Schmitz, F. J.; Kelly, M. J. Org. Chem. 1999, 64, 6706.

100. Keyzers, R. A.; Northcote, P. T.; Webb, V. J. Nat. Prod. 2002, 65, 598.

101. Keyzers, R. A.; Northcote, P. T.; Berridge, M. V. Aust. J. Chem. 2003, 56, 279.

102. Bergquist, P. R.; Fromont, P. J. The Marine Fauna of New Zealand: Porifera, Demospongiae, Part 4 (Poecilosclerida). In New Zealand Oceanographic Institute Memoir; New Zealand Oceanographic Institute: Wellington, 1988; Vol. 96.

103. Riccio, R.; Finamore, E.; Santaniello, M.; Zollo, F. J. Org. Chem. 1990, 55, 2548.

104. D'Auria, M. V.; Riccio, R.; Uriarte, E.; Minale, L.; Tanaka, J.; Higa, T. J. Org. Chem. 1989, 54, 234.

105. Tan, A. S.; Berridge, M. V. J. Immunological Methods 2000, 59.

106. Andersen, R. J.; Allen, T. M.; Burgoyne, D. L. Contignasterol, and related 3ahydroxy-6a-hydroxy-7b-hydroxy-15-keto-14b steroids useful as antiinflammatory and anti-thrombosis agents. In United States Patent Office; University of British Columbia, University of Alberta: United States of America, 1996.

107. Andersen, R. J.; Allen, T. M.; Burgoyne, D. L. Contignasterol compounds and pharmaceutical compositions comprising the same. In United States Patent Office; University of British Columbia, University of Alberta: United States of America, 1997.

108. Aiello, A.; Ciminiello, P.; Fattorusso, E.; Magno, S. Steroids 1988, 52, 533.

109. Rudi, A.; Yosief, T.; Loya, S.; Hizi, A.; Schleyer, M.; Kashman, Y. J. Nat. Prod. 2001, 64, 1451.

110. Tanaka, Y.; Katayama, T. Bulletin of the Japanese Society of Scientific Fisheries. 1976, 42, 801.

111. Tanaka, Y.; Fujita, Y.; Katayama, T. Bulletin of the Japanese Society of Scientific Fisheries. 1977, 42, 767. 
112. Tanaka, Y.; Soejima, T.; Katayama, T. Bulletin of the Japanese Society of Scientific Fisheries. 1978, 44, 1283.

113. Tanaka, Y.; Akase, S.-I.; Yamada, S. Fisheries Science 2001, 67, 378.

114. Capon, R. J.; MacLeod, J. K. J. Chem. Soc. Chem. Commun. 1987, 1200.

115. Capon, R. J.; Miller, M.; Rooney, F. J. Nat. Prod. 2000, 63, 821.

116. Capon, R. J.; Miller, M.; Rooney, F. J. Nat. Prod. 2001, 64, 643.

117. Ohta, S.; Okada, H.; Kobayashi, H.; Oclarit, J. M.; Ikegami, S. Tetrahedron Lett 1993, 34, 5935.

118. Xiao, D.-j.; Deng, S.-s.; Zeng, L.-m. Acta Scientiarium Naturalium Universitatis Sunyatseni 2002, 41, 111.

119. Sperry, S.; Crews, P. Tetrahedron Lett 1996, 37, 2389.

120. Zuleta, I. A.; Vitelli, M. L.; Baggio, R.; Garland, M. T.; Seldes, A. M.; Palermo, J. A. Tetrahedron 2002, 58, 4481.

121. Bergquist, P. R. The Marine Fauna of New Zealand: Porifera, Demospongiae, Part 5 (Dendroceratida and Halisarcida). In New Zealand Oceanographic Institute Memoir; NIWA: Wellington, 1996; Vol. 107.

122. Fake, A. D. Sesquiterpenoids from the New Zealand Marine Sponge Dysidea $s p$., Victoria University of Wellington 1999.

123. Dawson, E. W. The Marine Fauna of New Zealand: Index to the Fauna: 2. Porifera. In New Zealand Oceanographic Institute Memoir; NIWA: Wellington, 1993; Vol. 100.

124. Walker, R. P.; Faulkner, D. J. J. Org. Chem. 1981, 46, 1098.

125. Walker, R. P.; Rosser, R. M.; Faulkner, D. J.; Bass, L. S.; Cun-heng, H.; Clardy, J. J. Org. Chem. 1984, 49, 5160.

126. Sullivan, B.; Faulkner, D. J. J. Org. Chem. 1984, 49, 3204.

127. Bobzin, S. C.; Faulkner, D. J. J. Org. Chem. 1989, 54, 5727.

128. Rudi, A.; Kashman, Y. J. Nat. Prod. 1992, 55, 1408.

129. Lu, Q.; Faulkner, D. J. J. Nat. Prod. 1998, 61, 1096.

130. Kazlauskas, R.; Murphy, P. T.; Wells, R. J.; Noack, K.; Oberhansli, W. E.; Schonholzer, P. Aust. J. Chem. 1979, 32, 867.

131. Cimino, G.; de Rosa, D.; de Stefano, S.; Minale, L. Tetrahedron 1974, 30, 645. 
132. Capelle, N.; Braekman, J. C.; Daloze, D.; Tursch, B. Bull. Soc. Chim. Belg. 1980, 89, 399 .

133. Cambie, R. C.; Craw, P. A.; Stone, M. J.; Bergquist, P. R. J. Nat. Prod. 1988, $51,293$.

134. Kernan, M. R.; Cambie, R. C.; Bergquist, P. R. J. Nat. Prod. 1990, 53, 724.

135. Li, C.-J.; Schmitz, F. J.; Kelly-Borges, M. J. Nat. Prod. 1999, 62, 287.

136. Schmitz, F. J.; Chang, J. S.; Hossain, M. B.; van der Helm, D. J. Org. Chem. $1985,50,2862$.

137. Karuso, P.; Taylor, W. C. Aust. J. Chem. 1986, 39, 1629.

138. Carmely, S.; Cojocaru, M.; Loya, Y.; Kashman, Y. J. Org. Chem. 1988, 53, 4801.

139. Bobzin, S. C.; Faulkner, D. J. J. Nat. Prod. 1991, 54, 225.

140. Mayol, L.; Piccialli, V.; Sica, D. Gazz. Chim. Ital. 1988, 118, 559.

141. Rudi, A.; Kashman, Y. Tetrahedron 1990, 46, 4019.

142. Hirsch, S.; Kashman, Y. J. Nat. Prod. 1988, 51, 1243.

143. Gunasekera, S. P.; Schmitz, F. J. J. Org. Chem. 1991, 56, 1250.

144. Karuso, P.; Bergquist, P. R.; Cambie, R. C.; Buckleton, J. S.; Clark, G. R.; Rickard, C. E. F. Aust. J. Chem. 1986, 39, 1643.

145. Hambley, T. W.; Taylor, W. C.; Toth, S. Aust. J. Chem. 1997, 50, 903.

146. Hyosu, M.; Kimura, J. J. Nat. Prod. 2000, 63, 422.

147. Karuso, P.; Skelton, B. W.; Taylor, W. C.; White, A. H. Aust. J. Chem. 1984, $37,1081$.

148. Mayol, L.; Piccialli, V.; Sica, D. Tetrahedron Lett. 1985, 26, 1357.

149. Mayol, L.; Piccialli, V.; Sica, D. Tetrahedron Lett. 1985, 26, 1253.

150. Mayol, L.; Piccialli, V.; Sica, D. Tetrahedron 1986, 42, 5369.

151. Molinski, T. F.; Faulkner, D. J. J. Org. Chem. 1987, 52, 296.

152. Poiner, A.; Taylor, W. C. Aust. J. Chem. 1990, 43, 1713.

153. Goeller, F.; Heinemann, C.; Demuth, M. Synthesis 2001, 8, 1114.

154. Arno, M.; Gonzalez, M. A.; Marin, M. L.; Zaragoza, R. J. Tetrahedron lett. 2001, 42, 1669. 
155. Abad, A.; Agullo, C.; Arno, M.; Marin, M. L.; Zaragoza, R. J. J. Chem. Soc. Perkin 1 1996, 2193.

156. Betancur-Galvis, L.; Zuluaga, C.; Arno, M.; Gonzalez, M. A.; Zaragoza, R. J. J. Nat. Prod. 2002, 65, 189.

157. Zubia, E.; Gavagnin, M.; Scognamiglio, G.; Cimino, G. J. Nat. Prod. 1994, 57,725 .

158. Fenical, W. Diterpenoids. In Marine Natural Products: Chemical and Biological Perspectives; Scheuer, P. J. Ed.; Academic Press: New York, 1978; Vol. 2; pp. 392.

159. Buckleton, J. S.; Bergquist, P. R.; Cambie, R. C.; Clark, G. R.; Karuso, P.; Rickard, C. E. F. Acta Cryst. 1987, C43, 2430.

160. Silverstein, R. M.; Bassler, G. C.; Morrill, T. C. Spectrometric Identification of Organic Molecules, 5 ed.; John Wiley and Sons, Inc.: New York, 1991.

161. Bowden, B. F.; Coll, J. C.; Mitchell, S. J.; Skelton, B. W.; White, A. H. Aust. J. Chem. 1980, 33, 2737.

162. Cantillo-Ciau, Z.; Brito-Loeza, W.; Quijano, L. J. Nat. Prod. 2001, 64, 953.

163. Hadden, C. E.; Angwin, D. T. Magn. Reson. Chem. 2001, 39, 1.

164. Voelter, W.; Breitmaier, E. Carbon-13 NMR Spectroscopy, 3 ed.; VCH: New York, 1987.

165. Fokialakis, N.; Magiatis, P.; Terzis, A.; Tillequin, F.; Skaltsounis, A.-L. Tetrahedron Lett. 2001, 42, 5323.

166. Ortega, M. J.; Zubia, E.; Rodriguez, S.; Carballo, J. L.; Salva, J. Eur. J. Org. Chem. 2002, 3250 .

167. de Avellar, I. G. J.; Vierhapper, F. W. Tetrahedron 2000, 56, 9957.

168. Bobzin, S. C.; Faulkner, D. J. J. Org. Chem. 1991, 56, 4403.

169. Shimizu, Y.; Chou, H.-N.; Bando, H. J. Am. Chem. Soc. 1986, 108, 515.

170. Lin, Y.-Y.; Risk, M.; Ray, S. M.; van Engen, D.; Clardy, J.; Goik, J.; James, J. C.; Nakanishi, K. J. Am. Chem. Soc. 1981, 103, 6773.

171. Wichmann, C. F.; Boyer, G. L.; Divan, C. L.; Schantz, E. J.; Schnoes, H. K. Tetrahedron Lett. 1981, 22, 1941.

172. Schantz, E. J.; Ghazarossian, V. E.; Schnoes, H. K.; Strong, F. M.; Springer, J. P.; Pezzanite, J. O.; Clardy, J. J. Am. Chem. Soc. 1975, 97, 1238.

173. Bates, S. S.; Bird, C. J.; de Freitas, A. S. W.; Foxall, R.; Gilgan, M.; Hanic, L. A.; Johnson, G. R.; McCulloch, A. W.; Odense, P.; Pocklington, R.; Quilliam, 
M. A.; Sim, P. G.; Smith, J. C.; Subba Rao, D. V.; Todd, E. C. D.; Walter, J. A.; Wright, J. L. C. Can. J. Fish. Aquat. Sci. 1989, 46, 1203.

174. Subba Rao, D. V.; Quilliam, M. A.; Pocklington, R. Can. J. Fish. Aquat. Sci. 1988, 45, 2076.

175. Tachibana, K.; Scheuer, P. J.; Tsukitani, Y.; Kikuchi, H.; van Engen, D.; Clardy, J.; Gopichand, Y.; Schmitz, F. J. J. Am. Chem. Soc. 1981, 103, 2469.

176. Hu, T.; Curtis, J. M.; Walter, J. A.; Wright, J. L. C. J. Chem. Soc. Chem. Commun. 1995, 597.

177. Hu, T.; Curtis, J. M.; Walter, J. A.; Mclachlan, J. L.; Wright, J. L. C. Tetrahedron Lett. 1995, 36, 9273.

178. Arakawa, O.; Noguchi, T.; Shida, Y.; Onoue, Y. Toxicon 1994, 32, 175.

179. Koehn, F.; Hall, S.; Wichmann, C. F.; Schnoes, H. K.; Reichardt, P. B. Tetrahedron Lett. 1982, 23, 2247.

180. Onodera, H.; Satake, M.; Oshima, Y.; Yasumoto, T.; Carmichael, W. W. Natural Toxins 1997, 5, 146.

181. Shimizu, Y.; Hsu, C.-P.; Genenah, A. J. Am. Chem. Soc. 1981, 103, 605.

182. Zaman, L.; Arakawa, O.; Shimosu, A.; Shida, Y.; Onoue, Y. Toxicon 1998, 36,627 .

183. Wichmann, C. F.; Niemczura, W. P.; Schnoes, H. K.; Hall, S.; Reichardt, P. B.; Darling, S. D. J. Am. Chem. Soc. 1981, 103, 6977.

184. Laverty, R. Modes of Action of Shellfish Toxins. In Marine Toxins and New Zealand Shellfish; Royal Society of New Zealand: Wellington, 1993; pp. 31.

185. Murata, K.; Satake, M.; Naoki, H.; Kaspar, H. F.; Yasumoto, T. Tetrahedron 1998, 54, 735 .

186. Bates, M.; Baker, M.; Wilson, N.; Lane, L.; Handford, S. Epidemiologic Overview of the New Zealand Shellfish Toxicity Outbreak. In Marine Toxins and New Zealand Shellfish.; Royal Society of New Zealand.: Wellington, 1993; pp. 35.

187. Norte, M.; Gonzalez, R.; Fernandez, J. J.; Rico, M. Tetrahedron 1991, 47, 7437.

188. Takemoto, T.; Daigo, K. Chem. Pharm. Bull. 1958, 6, 578.

189. Quilliam, M. A.; Wright, J. L. C. Anal. Chem. 1989, 61, 1053A.

190. Halstead, B. W. Poisonous and Venomous Marine Animals of the World; United States Government Printing Office: Washington D.C., 1965; Vol. 2. 
191. Scheuer, P. J.; Takahashi, W.; Tsutsumi, J.; Yoshida, T. Science 1967, 155, 1267.

192. Murata, M.; Legrand, A.-M.; Ishibashi, Y.; Yasumoto, T. J. Am. Chem. Soc. $1989,111,8929$.

193. Murata, M.; Legrand, A.-M.; Scheuer, P. J.; Yasumoto, T. Tetrahedron Lett. $1992,33,525$.

194. Murata, M.; Naoki, H.; Iwashita, T.; Matsunaga, S.; Sasaki, M.; Yokoyama, A.; Yasumoto, T. J. Am. Chem. Soc. 1993, 115, 2060.

195. Murata, M.; Iwashita, T.; Yokoyama, A.; Sasaki, M.; Yasumoto, T. J. Am. Chem. Soc. 1992, 114, 6594.

196. Murata, M.; Yasumoto, T. Nat. Prod. Rep. 2000, 17, 293.

197. Murata, M.; Kumagi, M.; Lee, J.-S.; Yasumoto, T. Tetrahedron Lett. 1987, 28, 5869 .

198. Satake, M.; Terasawa, K.; Kadowaki, Y.; Yasumoto, T. Tetrahedron Lett. 1996, 37, 5955.

199. Takahashi, H.; Kusumi, T.; Kan, Y.; Satake, M.; Yasumoto, T. Tetrahedron Lett. 1996, 37, 7087.

200. Yasumoto, T.; Murata, M.; Oshima, Y.; Sano, M.; Matsumoto, G. K.; Clardy, J. Tetrahedron 1985, 41, 1019.

201. Personal Communication; Truman, P.; ESR; May; 2003.

202. Prasad, A. V.; Shimizu, Y.J. Am. Chem. Soc. 1989, 111, 6476.

203. Statistics New Zealand;

http://www.stats.govt.nz/domino/external/Web/nzstories.nsf/092edeb76ed5aa6 bcc256afe0081d84e/d00d6419b4c271dfcc256b1f0001aa49?OpenDocument; As at 18/09/02.

204. Chang, F. H.; Sharples, J.; Grieve, J. M. Harmful and Toxic Algal Blooms. 1996, 235.

205. Chang, F. H.; Mackenzie, L.; Till, D. G.; Hannah, D.; Rhodes, L. L. Harmful Marine Algal Blooms. 1995, 145.

206. Personal Communication; McFarlane, A.; New Zealand Fisheries Council; May; 1998.

207. Chang, F. H. Harmful Algae News 1999, 1.

208. Chang, F. H. NIWA Aquaculture Update 1998, 1.

209. Truman, P.; Lake, R. J. J. AOAC Int. 1996, 79, 1130. 
210. Truman, P.; Stirling, D. J.; Northcote, P. T.; Lake, R. J.; Seamer, C.; Hannah, D. J. J. AOAC Int. 2002, 85, 1057.

211. Personal Communication; Hay, B.; Aquabiotox; November; 2002.

212. Personal Communication; Truman, P.; ESR; July; 2002.

213. Igarashi, T.; Satake, M.; Yasumoto, T. J. Am. Chem. Soc. 1996, 118, 479.

214. Igarashi, T.; Satake, M.; Yasumoto, T. J. Am. Chem. Soc. 1999, 121, 8499.

215. Igarashi, T.; Oshima, Y.; Murata, M.; Yasumoto, T. Chemical Studies on Prymnesins Isolated from Prymnesium parvum. In Harmful Marine Algal Blooms; Lassus, P.; Arzul, G.; Erard, P.; Gentien, P.; Marcaillou, C. Eds.; Lavoisier: Paris, 1995; pp. 878.

216. Personal Communication; Stirling, D. J.; ESR; March; 2003.

217. Personal Communication; Zubkov, O.; VUW; March; 2003.

218. Seki, T.; Satake, M.; Mackenzie, L.; Kaspar, H. F.; Yasumoto, T. Tetrahedron Lett. 1995, 36, 7093.

219. Uemura, D.; Chou, T.; Haino, T.; Nagatsu, A.; Fukuzawa, S.; Zheng, S.-Z.; Chen, H.-S. J. Am. Chem. Soc. 1995, 117, 1155.

220. Torigoe, K.; Murata, M.; Yasumoto, T.; Iwashita, T. J. Am. Chem. Soc. 1988, 110,7876 .

221. Hu, T.; Curtis, J. M.; Oshima, Y.; Quilliam, M. A.; Walter, J. A.; WatsonWright, W. M.; Wright, J. L. C. J. Chem. Soc. Chem. Commun. 1995, 2159.

222. Morohashi, A.; Satake, M.; Murata, K.; Naoki, H.; Kaspar, H. F.; Yasumoto, T. Tetrahedron Lett. 1995, 36, 8995.

223. Sasaki, K.; Wright, J. L. C.; Yasumoto, T. J. Org. Chem. 1998, 63, 2475.

224. Macpherson, G. R.; Burton, I.; LeBlanc, P.; Walter, J. A.; Wright, J. L. C. J. Org. Chem. 2003, 68, 1659.

225. Dell'Aversano, C.; Walter, J. A.; Burton, I. W.; Stirling, D. J.; Fattorusso, E.; Quilliam, M. A. Isolation and structure elucidation of new and unusual saxitoxin analogues from mussels. In Harmful Algal Blooms; Intergovernmental Oceanographic Commission of UNESCO: Paris, 2003.

226. Kobayashi, J. i.; Yamaguchi, N.; Ishibashi, M. Tetrahedron Lett. 1994, 35, 7049 .

227. Kobayashi, J. i.; Kubota, T.; Takahashi, M.; Ishibashi, M.; Tsuda, M.; Naoki, H. J. Org. Chem. 1999, 64, 1478.

228. Ishibashi, M.; Takahashi, M.; Kobayashi, J. i. J. Org. Chem. 1995, 60, 6062. 
229. Kobayashi, J. i.; Yamaguchi, N.; Ishibashi, M. J. Org. Chem. 1994, 59, 4698.

230. Kobayashi, J. i.; Shigemori, H.; Ishibashi, M.; Yamasu, T.; Hirota, H.; Sasaki, T. J. Org. Chem. 1991, 56, 5221.

231. Kan, Y.; Uemura, D.; Hirata, Y.; Ishiguro, M.; Iwashita, T. Tetrahedron lett. 2001, 42, 3197.

232. Lewis, R. J.; Vernoux, J.-P.; Brereton, I. M. J. Am. Chem. Soc. 1998, 120, 5914.

233. Satake, M.; Fukui, M.; Legrand, A.-M.; Cruchet, P.; Yasumoto, T. Tetrahedron Lett. 1998, 39, 1197.

234. Satake, M.; Ofuji, K.; Naoki, H.; James, K. J.; Furey, A.; McMahon, T.; Silke, J.; Yasumoto, T. J. Am. Chem. Soc. 1998, 120, 9967.

235. Rudi, A.; Talpir, R.; Kashman, Y.; Benayahu, Y.; Schleyer, M. J. Nat. Prod. 1993, 56, 2178.

236. Satake, M.; Murata, M.; Yasumoto, T. Tetrahedron Lett. 1993, 34, 1975.

237. Personal Communication; Munday, R.; Ag Research; May; 2003.

238. Personal Communication; Beuzenberg, V.; Cawthron Institute; February; 2002.

239. Naoki, H.; Murata, M.; Yasumoto, T. Rapid Commun. Mass Spectrom. 1993, 7, 179 .

240. Matsumori, N.; Murata, M.; Tachibana, K. Tetrahedron 1995, 51, 12229.

241. Murata, M.; Matsuoka, S.; Matsumori, N.; Paul, G. K.; Tachibana, K. J. Am. Chem. Soc. 1999, 121, 870.

242. Matsumori, N.; Kaneno, D.; Murata, M.; Nakamura, H.; Tachibana, K. J. Org. Chem. 1999, 64, 866.

243. Riccio, R.; Bifulco, G.; Cimino, P.; Bassarello, C.; Gomez-Paloma, L. Pure Appl. Chem. 2003, 75, 295.

244. Moore, R. E.; Bartolini, G. J. Am. Chem. Soc. 1981, 103, 2491.

245. Moore, R. E.; Bartolini, G.; Barchi, J.; Bothner-By, A. A.; Dadok, J.; Ford, J. J. Am. Chem. Soc. 1982, 104, 3776.

246. Uemura, D.; Ueda, K.; Hirata, Y. Tetrahedron Lett. 1981, 22, 2781.

247. Gottlieb, H. E.; Kotlyar, V.; Nudelman, A. J. Org. Chem. 1997, 62, 7512. 